\title{
Les pensions dans les pays de l'OCDE 2009
}

PANORAMA DES SYSTÈMES DE RETRAITE 



\section{Les pensions \\ dans les pays de l'OCDE 2009}

PANORAMA DES SYSTÈMES DE RETRAITE 


\section{ORGANISATION DE COOPÉRATION ET DE DÉVELOPPEMENT ÉCONOMIQUES}

L'OCDE est un forum unique en son genre où les gouvernements de 30 démocraties œuvrent ensemble pour relever les défis économiques, sociaux et environnementaux que pose la mondialisation. L'OCDE est aussi à l'avant-garde des efforts entrepris pour comprendre les évolutions du monde actuel et les préoccupations qu'elles font naître. Elle aide les gouvernements à faire face à des situations nouvelles en examinant des thèmes tels que le gouvernement d'entreprise, l'économie de l'information et les défis posés par le vieillissement de la population. L'Organisation offre aux gouvernements un cadre leur permettant de comparer leurs expériences en matière de politiques, de chercher des réponses à des problèmes communs, d'identifier les bonnes pratiques et de travailler à la coordination des politiques nationales et internationales.

Les pays membres de l'OCDE sont : l'Allemagne, l'Australie, l'Autriche, la Belgique, le Canada, la Corée, le Danemark, l'Espagne, les États-Unis, la Finlande, la France, la Grèce, la Hongrie, l'Irlande, l'Islande, l'Italie, le Japon, le Luxembourg, le Mexique, la Norvège, la Nouvelle-Zélande, les Pays-Bas, la Pologne, le Portugal, la République slovaque, la République tchèque, le Royaume-Uni, la Suède, la Suisse et la Turquie. La Commission des Communautés européennes participe aux travaux de l'OCDE.

Les Éditions OCDE assurent une large diffusion aux travaux de l'Organisation. Ces derniers comprennent les résultats de l'activité de collecte de statistiques, les travaux de recherche menés sur des questions économiques, sociales et environnementales, ainsi que les conventions, les principes directeurs et les modèles développés par les pays membres.

Cet ouvrage est publié sous la responsabilité du Secrétaire général de l'OCDE. Les opinions et les interprétations exprimées ne reflètent pas nécessairement les vues de l'OCDE ou des gouvernements de ses pays membres.

Ce document a été produit avec le concours financier de l’Union européenne. Les opinions qui sont exprimées dans ce rapport ne peuvent en aucun cas être considérées comme reflétant la position officielle de l'Union européenne.

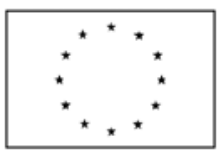

Publié en anglais sous le titre :

Pensions at a Glance 2009

RETIREMENT-INCOME SYSTEMS IN OECD COUNTRIES

Les corrigenda des publications de l'OCDE sont disponibles sur : www.oecd.org/editions/corrigenda.

(C) OCDE 2009

Vous êtes autorisés à copier, télécharger ou imprimer du contenu OECD pour votre utilisation personnelle. Vous pouvez inclure des extraits des publications, des bases de données et produits multimédia de l'OCDE dans vos documents, présentations, blogs, sites Internet et matériel d'enseignement, sous réserve de faire mention de la source OCDE et du copyright. Les demandes pour usage public ou commercial ou de traduction devront être adressées à rights@oecd.org. Les demandes d'autorisation de photocopier partie de ce contenu à des fins publiques ou commerciales peuvent être obtenue auprès du Copyright Clearance Center (CCC) info@copyright.com ou du Centre français d'exploitation du droit de copie (CFC) contact@cfcopies.com. 


\section{Avant-propos}

C ette troisième édition des Pensions dans les pays de l'OCDE : Panorama des politiques publiques propose toute une gamme d'indicateurs permettant de comparer les politiques des retraites menées par ces économies. Des analyses plus détaillées de nature à éclairer les débats sur la conception des systèmes de retraite et des réformes dans ce domaine sont présentées dans quatre chapitres thématiques.

La présente étude dresse un état des lieux à la fin mai 2009 étant donné que la situation économique et financière évolue constamment et que les systèmes de retraite sont fréquemment modifiés en conséquence.

Cette publication a été réalisée par une équipe dirigée par Edward Whitehouse, de la Division des politiques sociales de la Direction de l'emploi, du travail et des affaires sociales de l'OCDE. Anna Cristina D'Addio et Andrew Reilly ont été chargés de la gestion et de l'actualisation des modèles de retraite de l'OCDE, ainsi que de la rédaction de l'analyse des principaux indicateurs. Des responsables des différents pays ont apporté aux auteurs une aide active et précieuse pour la modélisation des informations relatives à leurs systèmes nationaux en matière de retraite et de fiscalité. Les résultats des modèles de retraite de l'OCDE ont été confirmés et validés par les autorités nationales.

Les chapitres spéciaux sur «Les systèmes de retraite pendant la crise économique et financière " et "Le déficit d'épargne-retraite et l'épargne-retraite facultative " ont été rédigés par Edward Whitehouse, avec le concours de collègues de la Direction de l'emploi, du travail et des affaires sociales et de Pablo Antolín, André Laboul, Robert Ley, Jean-Marc Salou, Fiona Stewart et Juan Yermo de la Direction des affaires financières et des entreprises de l'OCDE. Les délégués du Groupe de travail de l'OCDE sur la politique sociale ont utilement contribué à l'élaboration des précédentes versions de ces chapitres. Edward Whitehouse est l'auteur du chapitre consacré aux "Réformes récentes des retraites". Celui sur "Les revenus et la pauvreté des personnes âgées ", rédigé par Edward Whitehouse et Asghar Zaidi de la Division des politiques sociales de l'OCDE, s'appuie largement sur la base de données constituée pour la publication de 2008 de l'OCDE intitulée Croissance et inégalités.

Cette édition des Pensions dans les pays de l'OCDE a également bénéficié des suggestions et commentaires fournis par de nombreux collègues de l'OCDE, notamment Martine Durand, Michael Förster, John P. Martin, Mark Pearson et Monika Queisser.

Cette publication est le fruit d'un projet commun cofinancé par la Commission européenne et l'OCDE. Les modèles de retraite de l'OCDE, qui sous-tendent la majorité des indicateurs présentés, reposent sur l'infrastructure APEX (Analysis of Pension Entitlements across Countries), conçue par Axia Economics grâce à des financements de l'OCDE et de la Banque mondiale, notamment. 



\section{Table des matières}

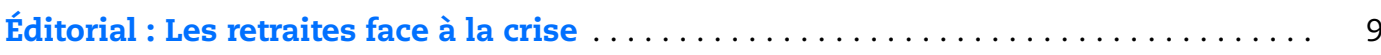

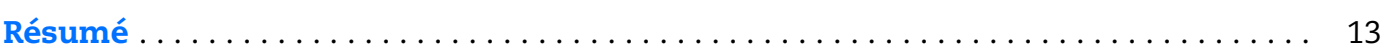

Le cadre conceptuel des Pensions dans les pays de l'OCDE . . . . . . . . . . 17

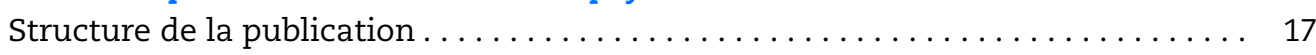

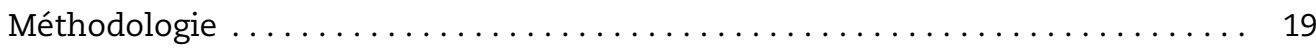

Tour d'horizon des régimes de retraite ...................... 21

Architecture des systèmes de retraite nationaux $\ldots \ldots \ldots \ldots \ldots \ldots \ldots \ldots .22$

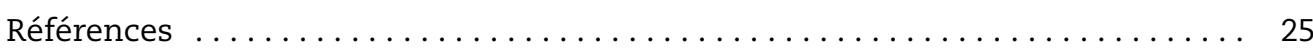

Partie I

Principaux enjeux des politiques

1. Les systèmes de retraite pendant la crise économique et financière . . . . . . . . 29

1. Quelles sont les catégories les plus durement touchées par la crise des retraites ? 29

2. Dans quels pays les retraites sont-elles le plus touchées ?........... 34

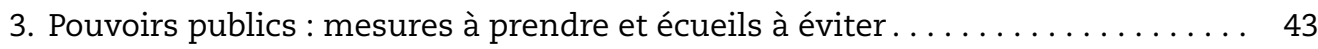

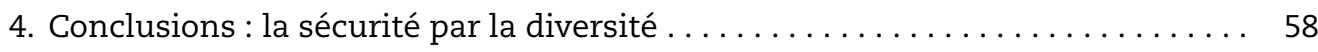

Notes ... . . . . . . . . . . . . . . . . . . . . . . . . . . . . . . . . . . . . 59

Références......................................... 61

2. Les revenus et la pauvreté des personnes âgées ..................... 65

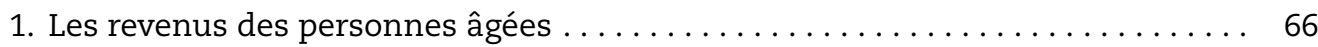

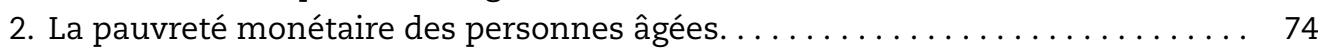

3. La fonction redistributive de l'État : impôts et prestations ............ 86

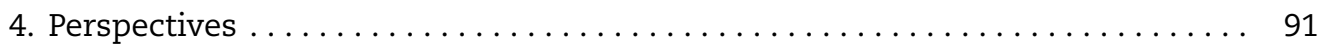

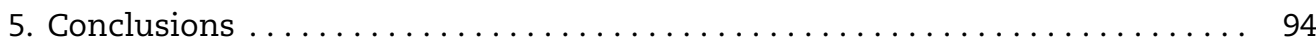

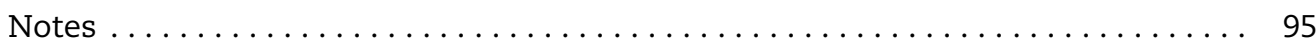

Références.......................................... 97

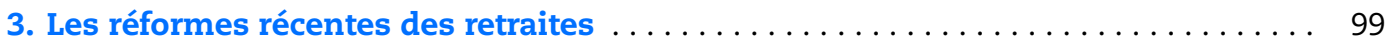

1. La couverture des systèmes de retraite $\ldots \ldots \ldots \ldots \ldots \ldots \ldots \ldots \ldots \ldots \ldots . \ldots \ldots$

2. L'adéquation des prestations de retraite $\ldots \ldots \ldots \ldots \ldots \ldots \ldots \ldots \ldots \ldots \ldots \ldots$

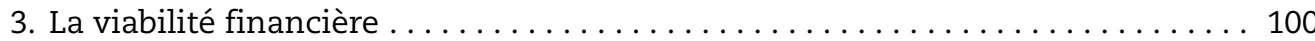

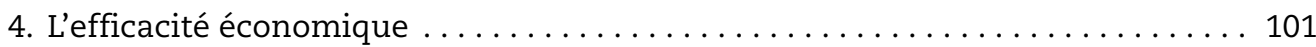

5. L'efficacité administrative . . . . . . . . . . . . . . . . . . . . . . . 102

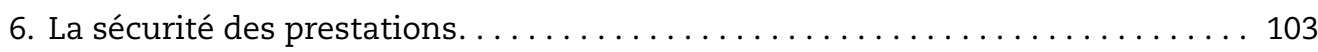

7. La réforme des retraites : processus et politique .................. 103

Notes . . . . . . . . . . . . . . . . . . . . . . . . . . . . . . . . . . . . . 112

Références.......................................... 112 
4. Le déficit d'épargne-retraite et l'épargne-retraite facultative $\ldots \ldots \ldots \ldots \ldots \ldots 113$

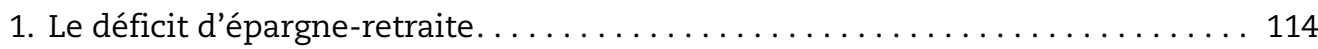

2. La couverture des régimes privés facultatifs en fonction de l'âge et du salaire 120

3. Les cotisations aux régimes de retraite privés . . . . . . . . . . . . . . . 122

4. Les mesures de nature à favoriser l'épargne-retraite privée. . . . . . . . . . 122

Notes . . . . . . . . . . . . . . . . . . . . . . . . . . . . . . . . . . . . . . . . . . . . 129

Références... . . . . . . . . . . . . . . . . . . . . . . . . . . . . . . . . . . . . . . 129

Partie II

Les indicateurs des politiques des retraites

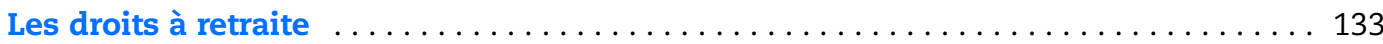

Taux de remplacement bruts . . . . . . . . . . . . . . . . . . . . . . 134

Taux de remplacement bruts : régimes publics et privés $\ldots \ldots \ldots \ldots \ldots \ldots \ldots$

Taux de remplacement nets. . . . . . . . . . . . . . . . . . . . . . . . . . . 138

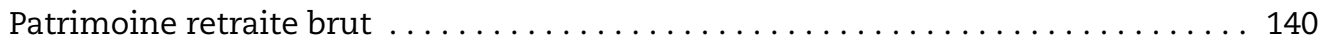

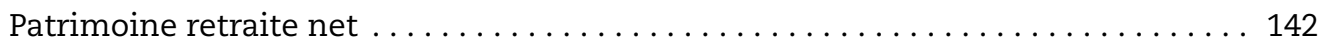

Progressivité de la formule de calcul des prestations de retraite . . . . . . . . . 144

Lien entre les pensions et les salaires. . . . . . . . . . . . . . . . . . . 146

Moyenne pondérée des pensions et des patrimoines retraite . . . . . . . . . . 148

Prestation globale de retraite . . . . . . . . . . . . . . . . . . . . . . . 150

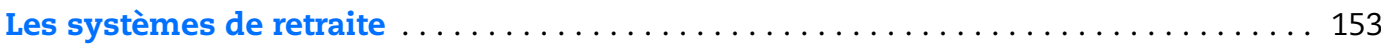

Cotisations ......................................... 154

Dépenses au titre des retraites. . . . . . . . . . . . . . . . . . . . . . . . 156

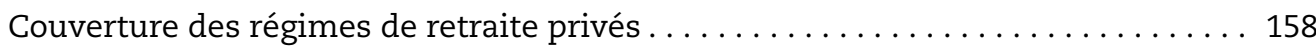

Actifs des fonds de pension et des fonds de réserve publics $\ldots \ldots \ldots \ldots \ldots \ldots .160$

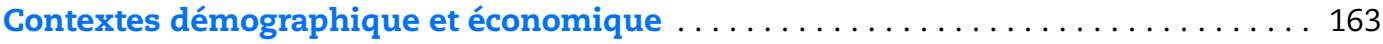

Espérance de vie........................................... 164

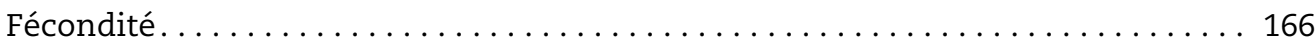

Taux de dépendance économique des personnes âgées . . . . . . . . . . . 168

Salaire moyen . . . . . . . . . . . . . . . . . . . . . . . . . . . 170

Partie III

Descriptifs par pays

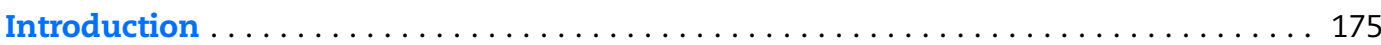

Principales caractéristiques des systèmes de retraite . . . . . . . . . . . . . . . 175

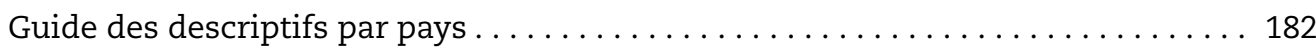

Notes . . . . . . . . . . . . . . . . . . . . . . . . . . . . . . . . . . . . . . . 183

Références........................................ 183

Allemagne. . . . . . . . . . . . . . . . . . . . . . . . . . . . . . . . . . . . . . . . 185

Australie . . . . . . . . . . . . . . . . . . . . . . . . . . . . . . . . . . . . 189

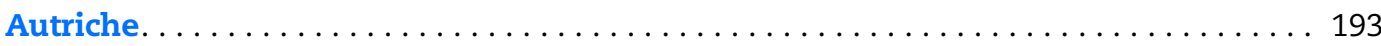




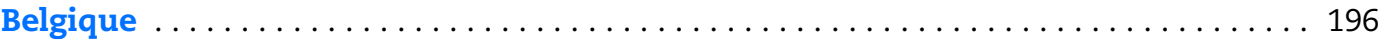

Canada . . . . . . . . . . . . . . . . . . . . . . . . . . . . . . . . 201

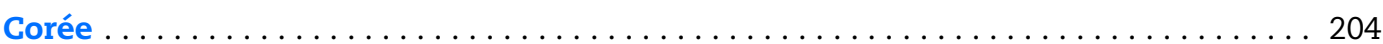

Danemark. . . . . . . . . . . . . . . . . . . . . . . . . . . . . . . . . 207

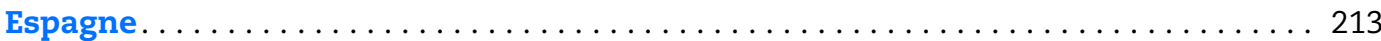

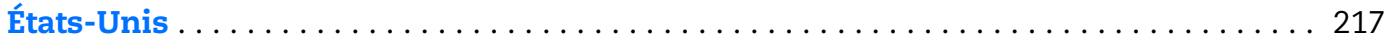

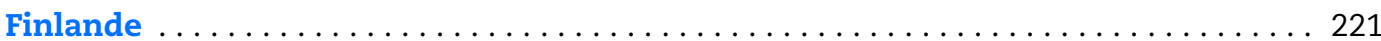

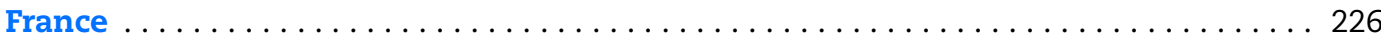

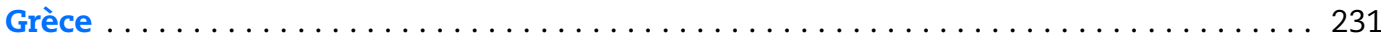

Hongrie . . . . . . . . . . . . . . . . . . . . . . . . . . . . 235

Irlande . . . . . . . . . . . . . . . . . . . . . . . . . . . . . . . . . . . . . 239

Islande. . . . . . . . . . . . . . . . . . . . . . . . . . . . . . . 242

Italie . . . . . . . . . . . . . . . . . . . . . . . . . . . . . . 246

Japon . . . . . . . . . . . . . . . . . . . . . . . . . . . . . . . . . . . . 250

Luxembourg . . . . . . . . . . . . . . . . . . . . . . . . . . . . . . . . . . . . . . . . . 254

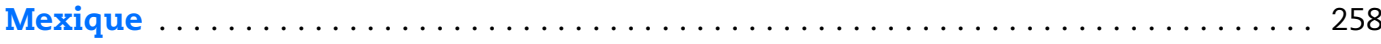

Norvège . . . . . . . . . . . . . . . . . . . . . . . . . . . . . 261

Nouvelle-Zélande . . . . . . . . . . . . . . . . . . . . . . . . . . . . . . . . . . . . . . 265

Pays-Bas ... . . . . . . . . . . . . . . . . . . . . . . . . . . . . . . . . . . 268

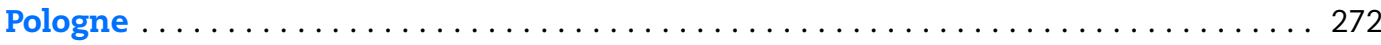

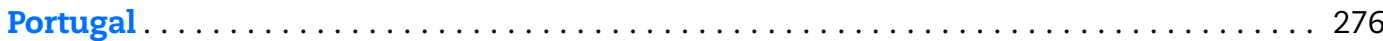

République slovaque $\ldots \ldots \ldots \ldots \ldots \ldots \ldots \ldots \ldots \ldots \ldots \ldots \ldots$

République tchèque . . . . . . . . . . . . . . . . . . . . . . . . . . . . . . 285

Royaume-Uni . . . . . . . . . . . . . . . . . . . . . . . . . . . . . . . . . 289

Suède. . . . . . . . . . . . . . . . . . . . . . . . . . . . . . . . . . . . . . 293

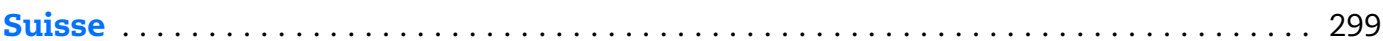

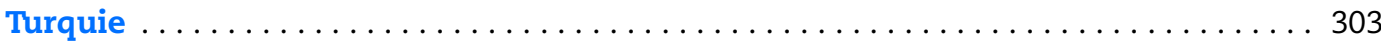

Ce livre contient des...

En bas à droite des tableaux ou graphiques de cet ouvrage, vous trouverez des StatLinks. Pour télécharger le fichier Excel ${ }^{\circledR}$ correspondant, il vous suffit de retranscrire dans votre

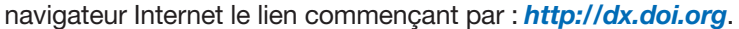

Si vous lisez la version PDF de l'ouvrage, et que votre ordinateur est connecté à Internet, il vous suffit de cliquer sur le lien.

Les StatLinks sont de plus en plus répandus dans les publications de l'OCDE. 



\section{Éditorial : Les retraites face à la crise}

$\mathrm{L}$ es chiffres publiés sont impressionnants. En raison de la crise financière, les fonds de pension ont vu la valeur de leurs investissements reculer de $23 \%$ en 2008 , soit de quelque 5400 milliards USD au total dans les pays de l'OCDE. Les marchés boursiers ont continué de chuter avant de se stabiliser à la mi-2009 pour remonter de 6.4\% le 21 mai 2009 par rapport au début de l'année. Les dernières prévisions de l'OCDE indiquent qu'en moyenne pour l'ensemble de la zone, la production pourrait se contracter de $4.3 \%$ en 2009 et que la reprise n'est pas attendue avant 2011. Le taux de chômage, qui avait atteint son point le plus bas en 2007 (5.6\%), pourrait atteindre $9.9 \%$ en 2010 dans la zone OCDE. Ainsi, ce qui était à l'origine une crise financière est désormais une crise économique et sociale.

Les régimes privés de retraite ont subi de plein fouet le repli du cours des actions et de l'immobilier. Les pays les plus touchés sont à l'évidence ceux où les pensions privées jouent un rôle important dans les systèmes de retraite, comme en Australie, aux États-Unis et aux Pays-Bas. Néanmoins, aucun pays, aucun système de retraite n'est à l'abri de la crise. Les régimes publics de retraite vont également être confrontés à des difficultés financières, dans la mesure où les recettes provenant des cotisations sociales s'amenuisent en raison de la montée du chômage et où les dépenses au titre de la redistribution augmentent pour compenser la baisse des pensions. De plus, là où ils existent, les fonds de réserve pour les retraites ont également subi des moins-values sur leurs placements. En outre, dans certains pays, comme on l'a vu tout récemment en Irlande et en Norvège, ces fonds sont mis à contribution pour recapitaliser les banques et financer des programmes de travaux publics dans le cadre des politiques mises en œuvre pour répondre à la crise.

Nombreuses sont les personnes qui ont perdu une part non négligeable de leur épargne-retraite investie dans des plans de retraite ou dans d'autres actifs financiers. La situation est particulièrement aiguë pour les salariés les plus âgés. Non seulement ils rencontrent des difficultés pour trouver un nouvel emploi en cas de licenciement, mais ils n'ont guère le temps de reconstituer leur épargne avant de devoir commencer à puiser dans leurs actifs pour financer leur retraite. Les revenus d'épargne, pensions privées comprises, représentent en moyenne un quart des revenus des retraités dans les pays de l'OCDE. Dans sept d'entre eux, ils atteignent plus de $40 \%$.

Les pertes subies vont-elles entraîner une augmentation de la pauvreté parmi les retraités? De nombreux pays de l'OCDE sont dotés de programmes sociaux faisant office de "stabilisateurs automatiques sociaux " qui atténuent l'impact sur le revenu global des retraités des moins-values enregistrées sur les placements financiers. Ainsi, des prestations soumises à conditions de ressources sont accordées aux personnes dont les retraites sont tombées en dessous d'un certain seuil. Dans certains pays, toutefois, les 
filets de protection sociale envers les personnes âgées risquent d'être insuffisants en cas d'effondrement des revenus de l'épargne privée. Dans certaines circonstances, il peut donc s'avérer nécessaire de renforcer temporairement les mesures de protection sociale afin de surmonter la crise actuelle.

Les pressions politiques à court terme exercées sur les pouvoirs publics pour qu'ils apportent une aide immédiate sont considérables et vont au-delà d'une simple prévention de la pauvreté des personnes âgées. Dans la situation actuelle, le danger est manifestement que les autorités soient tentées de faire basculer les chômeurs âgés vers les régimes d'assurance invalidité ou d'assurance maladie longue durée, ou de réintroduire les mesures de retraite anticipée. L'expérience montre, qu'une fois en place, il est très difficile de supprimer ces dispositifs qui pèsent particulièrement lourd sur les finances publiques. De telles mesures enverraient un signal erroné et iraient à l'encontre de l'objectif de relèvement de l'âge effectif de la retraite qui est nécessaire pour compenser les effets du vieillissement de la population. Jusqu'à présent, les pays ont toutefois résisté à de telles pressions.

La crise nous a confortés dans l'idée que la poursuite des réformes s'impose à la fois pour les régimes publics et privés de retraite. La priorité va notamment à un examen minutieux des systèmes publics de retraite afin de s'assurer qu'ils offrent une protection efficace contre la pauvreté, aujourd'hui comme demain. Dans certains pays, la crise a en effet révélé que les filets de protection sociale étaient très insuffisants et que le taux de pauvreté était élevé parmi les personnes âgées.

De nombreux pays ont introduit des mécanismes d'ajustement automatique des retraites liant les dépenses au titre des retraites à l'espérance de vie, à la hausse des salaires ou aux actifs des fonds de réserve. Ces mécanismes ont été conçus en des temps de croissance économique régulière. Ils se sont avérés pro-cycliques en période de récession. Leur application stricte contraindrait certains pays à réduire les pensions, y compris en valeur nominale dans certains cas. Les pouvoirs publics doivent donc envisager des ajustements aux règles d'application de ces mécanismes, leur suspension temporaire jusqu'au redémarrage de l'activité économique ou, de préférence, de les appliquer de façon sélective en en dispensant les groupes de retraités les plus vulnérables.

La confiance dans les régimes de pension privés a été très entamée par la crise. Dans plusieurs pays de l'OCDE, des voix s'élèvent de plus en plus en faveur de l'abandon des régimes mixtes de retraite ou pour un recours exclusif à un système public de retraite par répartition. En République slovaque, par exemple, les salariés affiliés aux nouveaux dispositifs de retraite à cotisations définies ont été autorisés à réintégrer le régime public. D'autres économies d'Europe orientale envisagent également de revenir sur les réformes engagées. Ces pays font fausse route. La crise économique et financière a détourné l'attention des questions liées au vieillissement démographique auxquelles sont confrontés les systèmes de retraite. Mais ces questions n'ont pas disparu pour autant et elles devront être résolues rapidement.

Cependant, pour empêcher tout retour en arrière, il faudra rétablir la confiance dans l'épargne-retraite privée. La crise a montré qu'il était impératif de modifier le fonctionnement des régimes privés, ce qui requiert une meilleure réglementation, une administration plus efficiente, une plus grande transparence sur les risques et avantages liés aux différentes options, ainsi qu'un basculement automatique vers des investissements moins risqués à mesure que l'on s'approche de l'âge de la retraite. Si les 
autorités échouent à démontrer de façon convaincante le bien fondé des systèmes de retraite diversifiés, associant des éléments publics et privés, par répartition et par capitalisation, individuels et collectifs, les efforts qu'elles auront déployés au cours des dernières années pour préserver la prospérité de sociétés vieillissantes risquent d'être réduits à néant.

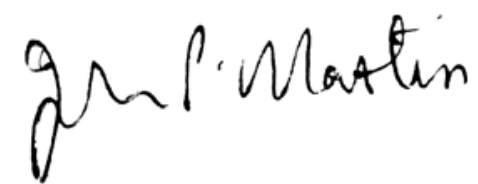

John P. Martin

Directeur,

Direction de l'OCDE sur l'emploi, le travail et les affaires sociales

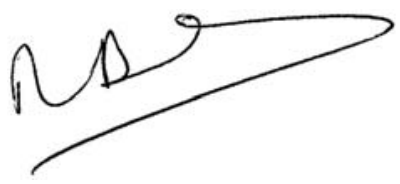

Martine Durand Directrice adjointe, Direction de l'OCDE sur l'emploi, le travail et les affaires sociales 



\section{Résumé}

$\mathrm{L}$

a crise financière et la profonde crise économique qui a suivi dominent l'actualité depuis plus d'un an. Le premier des chapitres de cette troisième édition de Pensions dans les pays de l'OCDE : Panorama des systèmes de retraite analyse les répercussions de la crise sur les systèmes de retraite. Dans les pays de l'OCDE, la crise financière a durement touché les fonds de pension dont les investissements se sont dépréciés de $23 \%$ en moyenne en 2008 , ce qui représente environ 5400 milliards USD. À l'échelon national, les retombées de la crise dépendent de l'importance des pensions privées dans l'ensemble des revenus des retraités. Leur part est particulièrement importante en Australie, au Danemark, aux ÉtatsUnis, aux Pays-Bas et au Royaume-Uni.

La crise économique et sociale se manifeste d'ores et déjà par un recul de la production, la montée du chômage et le ralentissement de la croissance (voire une baisse) des salaires. Cela signifie que les régimes de retraite publics vont également être mis à mal, en raison d'une diminution des recettes tirées des cotisations et de l'accentuation des pressions exercées sur les dépenses.

Ceux qui sont le plus affectés par la crise économique et financière sont les salariés les plus âgés, qui n'ont pas le temps de reconstituer leur épargne-retraite avant leur départ à la retraite et qui ont davantage de difficultés à trouver un nouvel emploi en cas de licenciement. Le chapitre spécial consacré aux retraites face à la crise contient une nouvelle évaluation chiffrée des effets du chômage de longue durée en fin de carrière sur les revenus perçus au titre de la retraite. Les salariés les plus jeunes sont affectés dans des proportions bien moindres, car en règle générale, ils épargnent moins à ce stade de leur vie et la durée attendue de leur vie active étant nettement plus longue, ils auront le temps de compenser la dépréciation éventuelle de leur épargne-retraite et de leurs droits à retraite. Les personnes qui ont déjà pris leur retraite et commencé à percevoir leur pension ont aussi tendance à être moins pénalisées.

S'appuyant sur les modèles de retraite de l'OCDE, ce chapitre montre de quelle manière l'impact négatif de la crise sur les revenus des retraités est atténué dans de nombreux pays par des filets de protection sociale et par le système fiscal. Ainsi, les prestations perçues par plus de $75 \%$ des personnes âgées en Australie et $65 \%$ environ au Danemark sont, pour une part au moins, soumises à conditions de ressources. Les droits à ce type de prestations augmentent à mesure que le rendement des pensions privées diminue, de sorte que les revenus des retraités à faible revenu et à revenu intermédiaire sont en grande partie protégés. En Australie, chaque dollar supplémentaire perçu dans le cadre d'un régime privé donne lieu à une réduction de 40 cents de la pension publique. Inversement, toute diminution des pensions privées de 1 dollar entraîne une revalorisation des prestations publiques à hauteur de 60 cents. Dans certains pays, toutefois, les filets de protection sociale des personnes âgées sont ou seront insuffisants 
lorsque l'épargne privée ne peut ou ne pourra pas venir compléter les faibles revenus perçus par les retraités.

Les mesures déjà prises par les pouvoirs publics pour atténuer les effets de la crise sont analysées et évaluées. Il apparaît dans ce chapitre que les programmes de relance économique introduits par de nombreux gouvernements ont eu deux retombées principales sur les systèmes de retraite: des transferts sociaux plus importants aux personnes âgées et l'utilisation des fonds de réserve publics pour les retraites pour atténuer les effets de la crise. On trouvera également dans ce chapitre une évaluation d'autres initiatives relevant de la politique des retraites, concernant le marché du travail, les filets de protection sociale, la réglementation des fonds de pension et le choix des supports d'investissement. En dépit de vives pressions politiques à court terme, les autorités doivent impérativement se garder de toute réaction opportuniste pouvant nuire à la stabilité à long terme et à la pérennité des régimes de retraite. Les difficultés à long terme dues à l'évolution démographique et au vieillissement de la population n'ont pas disparu et il faudra bien s'y atteler une fois la crise passée et la reprise amorcée.

Les revenus et la pauvreté des personnes âgées d'aujourd'hui sont étudiés dans le deuxième chapitre spécial de la Partie I. Au milieu des années 2000, le revenu net des plus de 65 ans représentait $82 \%$ de celui de la population totale, en moyenne dans les pays de l'OCDE (en tenant compte de la taille des ménages). Cependant, la situation varie considérablement d'un pays à l'autre. La pauvreté chez les personnes âgées est pratiquement inexistante dans certains pays, mais en Corée, par exemple, elles sont plus de $40 \%$ à percevoir des revenus inférieurs au seuil de pauvreté. Dans les pays de l'OCDE, le taux de pauvreté s'établit en moyenne à $13.2 \%$ pour les personnes âgées, contre $10.6 \%$ pour l'ensemble de la population. On s'intéresse également dans ce chapitre à l'évolution que devraient connaître, selon toute probabilité, les revenus et la pauvreté des personnes âgées sous l'effet de la réforme des retraites et des changements économiques et sociaux.

Les réformes récentes des retraites constituent le thème du troisième chapitre spécial de la Partie I. Ce chapitre, qui contient une version actualisée de l'analyse figurant dans la deuxième édition des Pensions dans les pays de l'OCDE montre que les pays ont continué de réformer leurs systèmes de retraite depuis 2004 ; en effet, les changements ont été rares voire inexistants dans cinq d'entre eux seulement. Ces réformes récentes sont regroupées en fonction des principaux objectifs poursuivis dans le cadre des systèmes de retraite: pourcentage de salariés couverts, adéquation des niveaux de pension, viabilité financière, efficience économique (minimisation des distorsions de l'offre de main-d'œuvre et des incitations à épargner), efficience administrative et sécurité des revenus des retraités face à différents risques et incertitudes.

L'évaluation des réformes montre que la période de 2004 à 2009 a été davantage marquée par une évolution que par une révolution. Aucune réforme systémique à grande échelle, comparable à celles des années 1995-2004, n’a été engagée. Dans certains pays comme les États-Unis, la Norvège, l'Autriche et l'Irlande, le processus de réforme est désormais au point mort. Dans d'autres, il connaît un ralentissement, voire un retour en arrière. Les modifications législatives du système de retraite en Italie, par exemple, ont été reportées. En République slovaque, les salariés affiliés aux nouveaux dispositifs de retraite à cotisations définies ont été autorisés à réintégrer le régime public et d'autres pays envisagent aussi de revenir sur les réformes engagées. La crise pourrait amener de 
nouveaux changements qui seraient incompatibles avec la stratégie à long terme nécessaire pour assurer la pérennité des retraites.

Le dernier chapitre spécial de la Partie I, qui présente également une actualisation et un enrichissement des travaux de la précédente édition des Pensions dans les pays de l'OCDE, s'intéresse à la couverture des pensions privées. Il s'intéresse principalement aux pays où les pensions publiques sont peu élevées, de sorte qu'il incombe davantage à chacun d'assurer sa propre retraite. Mais là encore, la crise financière soulève de réelles difficultés, notamment lorsqu'elle érode la confiance dans les pensions privées. Quoi qu'il en soit, en raison des contraintes budgétaires, les pensions privées doivent demeurer l'une des variables de l'équation des prestations de retraite. Les mesures visant à assurer que chacun se constitue une épargne-retraite, et notamment l'affiliation automatique et les incitations fiscales, sont évaluées dans ce chapitre.

Différents indicateurs relatifs aux retraites sont présentés dans la partie II de cette étude. Les neuf premiers concernent les droits individuels à retraite, calculés à l'aide des modèles de retraite de l'OCDE. La valeur des paramètres qui sous-tendent les estimations correspond à l'année 2006. Les calculs font apparaître les droits à retraite futurs des salariés qui sont entrés sur le marché du travail en 2006 et qui auront travaillé toute leur vie sans que les règles applicables aient été modifiées. Pour le salaire moyen, le taux de remplacement brut - prestations de retraite rapportées au salaire d'activité - s'établit en moyenne à 59 \% dans les 30 pays de l'OCDE. Néanmoins, de nombreux pays proposent aux personnes âgées des allègements au titre de l'impôt sur le revenu et la plupart des retraités sont exonérés des cotisations de sécurité sociale. Ainsi, pour le salaire moyen, le taux de remplacement net (après impôt et cotisations) est en moyenne de 70 \% environ. Les taux de remplacement sont présentés séparément pour les hommes et les femmes et pour différentes catégories de revenus. Un nouvel indicateur du taux de remplacement tenant compte des grands régimes de retraite privés facultatifs a été ajouté dans la présente édition.

Les autorités attachent de l'importance aux taux de remplacement, mais aussi à la " promesse globale » en matière de retraite, mesurée à l'aide des indicateurs du patrimoine retraite, qui représentent la valeur actualisée des prestations compte tenu de l'âge de la retraite, de l'espérance de vie et de l'indexation des pensions servies. En moyenne, au Luxembourg, les hommes ont un patrimoine retraite de quelque 825000 USD et les femmes d'environ 1 million USD. Le Luxembourg constitue sans doute un cas extrême. Le patrimoine retraite provenant des régimes obligatoires représente en moyenne 400000 USD pour les hommes et 475000 USD pour les femmes dans les pays de l'OCDE.

Un deuxième groupe de quatre indicateurs, également inédits dans Les Pensions dans les pays de l'OCDE, porte sur des composantes plus larges des systèmes de retraite. On trouvera dans cette partie des informations sur les cotisations et sur l'évolution passée des taux des cotisations retraite. En réalité, ces taux ont fait preuve d'une remarquable stabilité compte tenu des pressions démographiques pesant sur les systèmes de retraite et ils s'établissent en moyenne à $21 \%$ en 2007 , contre $20 \%$ en 1994 . Des pressions sont néanmoins manifestes lorsqu'on s'intéresse aux dépenses au titre des régimes publics qui ont progressé à un rythme supérieur de 17 \% à celui du revenu national entre 1990 et 2005, passant ainsi de $6.2 \%$ à $7.2 \%$ du produit intérieur brut. L'indicateur des dépenses au titre des retraites fournit également des renseignements sur les pensions privées obligatoires et sur les avantages en nature, comme les allocations et les aides au logement. Deux autres 
indicateurs relatifs aux systèmes de retraite portent sur les pensions privées, et contiennent des données sur la couverture des régimes privés facultatifs et la valeur des actifs des fonds de pension.

Le dernier groupe de quatre indicateurs concerne le contexte dans lequel fonctionnent les systèmes de retraite. Trois d'entre eux sont de nature démographique : espérance de vie, fertilité et taux de dépendance économique (nombre de retraités par personne d'âge actif). On y trouve également des données sur le salaire moyen, qui sous-tend bon nombre des autres indicateurs.

Enfin, les descriptifs pays de la Partie III fournissent les indicateurs essentiels des systèmes de retraite nationaux et présentent les paramètres et règles applicables de façon cohérente, ainsi que les principaux résultats obtenus pour les droits à retraite individuels : taux de remplacement et patrimoine retraite. On trouvera en début de la Partie III un tableau récapitulatif des règles et paramètres en vigueur dans les 30 pays de l'OCDE. 


\title{
Le cadre conceptuel des Pensions dans les pays de l'OCDE
}

\begin{abstract}
C
ette troisième édition des Pensions dans les pays de l'OCDE contient une nouvelle analyse des problématiques de la politique des retraites et constitue un précieux ouvrage de référence sur les systèmes des 30 pays de l'OCDE. Cette section présente la structure de la publication, qui se subdivise en trois grandes parties: les principaux enjeux, les indicateurs et les descriptifs pays, qui seront étudiés tour à tour. On trouvera ensuite les détails de la méthodologie utilisée pour calculer les droits à retraite individuels, ainsi qu'un aperçu des nombreux régimes de retraite, suivi d'une présentation succincte de l'architecture des systèmes nationaux.
\end{abstract}

\section{Structure de la publication}

Les quatre chapitres spéciaux de la partie I livrent un examen minutieux des grands enjeux de la politique des retraites. Le premier concerne les répercussions de la crise économique et financière sur les systèmes de retraite. Quels sont les pays et les individus les plus touchés? Quelles mesures les pouvoirs publics peuvent-ils prendre pour les aider et quels sont les écueils à éviter?

Le deuxième chapitre s'intéresse aux revenus et à la pauvreté des personnes âgées, en étudiant les tendances observées ces 20 dernières années. Dans de nombreux pays, la situation des retraités s'est améliorée par rapport à celle de la population dans son ensemble, mais il reste des poches de pauvreté chez les personnes âgées.

Le troisième chapitre spécial contient une analyse actualisée des réformes des retraites exposées dans l'édition 2007 des Pensions dans les pays de l'OCDE. En quoi les systèmes de retraite ont-ils changé entre 2004 et mai 2009 ?

Le dernier chapitre spécial traite de la couverture des régimes facultatifs privés, en élargissant le champ de l'étude réalisée dans l'édition 2007 des Pensions dans les pays de l'OCDE aux différences existant en fonction de l'âge et du salaire. L'analyse s'articule autour du concept de "déficit d'épargne-retraite ", c'est-à-dire la somme que différents individus devront épargner d'eux-mêmes pour atteindre un certain niveau de revenus une fois à la retraite. Ce chapitre évalue cinq types de mesures visant à développer ces dispositifs facultatifs privés, dont les incitations fiscales et l'affiliation automatique.

La partie II contient une version actualisée des grands indicateurs des systèmes de retraite élaborés pour la première et la deuxième éditions. La gamme des indicateurs retenus a également été élargie, et comprend une évaluation des actifs détenus par les fonds de pension privés et les fonds de réserve nationaux, ainsi que de la couverture des pensions privées. Les autres indicateurs inédits portent notamment sur les dépenses 
publiques au titre des retraites et sur le contexte et les perspectives démographiques relatives aux systèmes de retraite. Les informations nécessaires pour comparer les politiques menées dans le domaine des retraites sont présentées de façon claire et succincte.

Les neuf premiers indicateurs de la partie II concernent les droits à retraite individuels. L'approche générale adoptée est de nature "microéconomique ", en ce sens qu'elle s'intéresse aux prestations de retraite individuelles potentiellement servies dans le cadre des régimes des 30 pays membres de l'OCDE. Les trois premiers de ces indicateurs sont bien connus des analystes du secteur. Il s'agit des taux de remplacement, soit le rapport des prestations de retraite au salaire individuel. Le premier indicateur concerne le taux de remplacement brut - avant impôt - pour tous les mécanismes obligatoires, y compris les dispositifs privés obligatoires. Le deuxième fait apparaître le taux de remplacement brut en faisant ressortir les régimes publics et les mécanismes privés. Il comprend également des données sur les dispositifs privés facultatifs pour les pays où l'on dispose de données sur la conception "classique " de ce type de régimes. Le troisième indicateur du taux de remplacement est exprimé en termes nets, en prenant en considération les impôts et cotisations acquittés sur les salaires et les pensions perçus.

Les taux de remplacement sont suivis de deux indicateurs du "patrimoine retraite", également indiqués en termes bruts et nets. Le patrimoine retraite indique la valeur viagère du flux de prestations de retraite. C'est un indicateur des droits à retraite plus complet que le taux de remplacement, car il tient compte des différences existant entre les pays en matière d'âge de la retraite, d'indexation des pensions sur les salaires ou les prix et d'espérance de vie.

Les deux indicateurs suivants permettent d'évaluer les divers points d'équilibre trouvés par les pays de l'OCDE entre l'objectif consistant à assurer un revenu satisfaisant aux retraités et celui visant à remplacer une part donnée des revenus perçus avant le départ en retraite. Le premier représente la progressivité de la formule de calcul des prestations de retraite et le second le lien entre les pensions et les salaires.

Les deux derniers indicateurs des droits à retraite résument les conséquences du système de retraite pour les individus selon la place qu'ils occupent sur l'échelle de distribution des salaires, et présentent le niveau moyen des pensions, le patrimoine retraite et la contribution de chaque composante du régime de retraite aux prestations globales.

Si les neuf premiers indicateurs portaient essentiellement sur les droits à retraite individuels, les quatre suivants s'intéressent aux systèmes de retraite dans leur ensemble. Deux d'entre eux par exemple concernent le financement des retraites. Premièrement, à combien s'élèvent les taux de cotisation pour les retraites publiques et comment ont-ils évolué au fil du temps? Deuxièmement, quel est le montant des actifs des fonds de pension privés et des fonds de réserve nationaux?

Les dépenses au titre des retraites constituent aussi l'un de ces indicateurs, qui illustre la part du revenu national qui est consacrée au versement des pensions, ainsi que l'importance des retraites publiques dans le budget global de l'État. Cet indicateur rend également compte de la proportion du revenu national qui est versé par les régimes obligatoires privés et des dépenses correspondant aux avantages « en nature » accordés aux retraités.

Ce groupe d'indicateurs comprend également des données sur la couverture des pensions privées, qui concernent un plus grand nombre de pays de l'OCDE que l'analyse 
détaillée figurant dans le chapitre spécial sur « le déficit d'épargne-retraite et l'épargneretraite facultative » de la partie I.

Le dernier ensemble d'indicateurs illustre le contexte dans lequel s'inscrivent les politiques des retraites et celui dans lequel fonctionnent les systèmes de retraite. En particulier, trois d'entre eux ont trait aux contraintes démographiques auxquelles doivent faire face les autorités dans ce domaine. La baisse de la fertilité et l'allongement de l'espérance de vie sont à l'origine du vieillissement de la population, calculé à l'aide du ratio de dépendance économique, soit le nombre de personnes ayant atteint l'âge de la retraite par rapport au nombre de personnes d'âge actif.

Enfin, la partie III des Pensions dans les pays de l'OCDE contient des descriptifs pays. Elle s'ouvre sur une analyse comparative des paramètres et des règles appliqués par les régimes de retraite des 30 pays membres de l'OCDE, et introduit les concepts techniques utilisés dans les descriptifs pays qui suivent. Il s'agit de l'âge et d'autres conditions d'ouverture des droits, des règles de calcul des droits à retraite et du régime appliqué aux retraites anticipées et différées. Cette troisième édition des Pensions dans les pays de l'OCDE comporte aussi pour la première fois une présentation détaillée et complète du régime appliqué aux personnes ayant subi des interruptions de carrière dues au chômage ou à l'éducation des enfants. On trouvera également dans les descriptifs pays les principaux indicateurs de la partie II présentés dans des graphiques et des tableaux standardisés.

\section{Méthodologie}

\section{Droits futurs en fonction des paramètres et des règles d'aujourd'hui}

Les droits à retraite mentionnés sont ceux qui sont actuellement prévus par la loi dans les pays de l'OCDE. Les changements de réglementation qui ont déjà été votés, mais qui sont mis en application progressivement, sont supposés être intégralement introduits d'emblée. Les réformes adoptées après 2006 sont prises en compte lorsqu'on dispose d'informations suffisantes.

Les valeurs de tous les paramètres des systèmes de retraite reflètent la situation de l'année 2006. Les calculs correspondent aux droits d'un salarié qui adhère au système aujourd'hui et prend sa retraite à l'issue d'une carrière complète. Les résultats sont donnés pour une personne célibataire.

\section{Durée de la carrière}

On considère dans la présente publication qu'une carrière complète est celle d'un salarié qui entre sur le marché du travail à l'âge de 20 ans et travaille jusqu'à l'âge normal d'ouverture des droits à prestations qui, bien sûr, varie d'un pays à l'autre. Autrement dit, la longueur de la carrière varie avec l'âge légal de la retraite : 40 ans pour la retraite à l'âge de 60 ans, 45 ans pour la retraite à 65 ans, etc. (une analyse de sensibilité figurait également dans l'édition 2007 des Pensions dans les pays de l'OCDE pour les salariés qui sont entrés sur le marché du travail à 25 ans et non à 20 et dont la carrière a donc duré 5 ans de moins).

\section{Couverture}

Les résultats des modèles de retraite présentés ici incluent tous les régimes obligatoires des salariés du secteur privé, qu'ils soient publics (c'est-à-dire impliquant des versements de l'État ou d'organismes de sécurité sociale, selon la définition du Système de comptabilité nationale) ou privés. Pour chaque pays, on modélise le principal régime 
national applicable aux salariés du secteur privé. Les régimes applicables aux fonctionnaires, aux salariés du secteur public et à des catégories professionnelles particulières sont exclus.

Sont inclus également les systèmes à couverture quasi universelle, à condition qu'ils couvrent au moins $85 \%$ des salariés. Dans la présente publication, ces dispositifs sont qualifiés de " quasi obligatoires ». Ils sont particulièrement importants au Danemark, aux Pays-Bas et en Suède.

Dans un nombre croissant de pays de l'OCDE, les régimes de retraite professionnels ou individuels à participation facultative assurent une large couverture : ils jouent fréquemment un rôle important dans les revenus perçus par les retraités. Pour ces pays, on trouvera un deuxième ensemble de résultats pour les taux bruts de remplacement qui font ressortir les droits à retraite liés aux dispositifs facultatifs classiques.

Les prestations sous conditions de ressources auxquelles les retraités peuvent avoir droit sont aussi modélisées. Elles peuvent être soumises à des conditions qui prennent en compte aussi bien le patrimoine que les revenus, les seuls revenus, ou exclusivement les revenus perçus au titre de la retraite. Les calculs reposent sur l'hypothèse que tous les retraités qui y ont droit perçoivent ces prestations. Lorsque les conditions de ressources englobent également le patrimoine, le critère de revenus est considéré comme contraignant. On suppose que la totalité du revenu durant la retraite provient du régime de retraite obligatoire (auquel s'ajoutent les régimes de retraite facultatifs dans les pays pour lesquels ils sont modélisés).

Les droits à retraite sont comparés pour des salariés percevant différents niveaux de rémunération, soit entre 0.5 fois et deux fois la moyenne nationale. Cette fourchette permet d'examiner les prestations de retraite futures pour les salariés plus pauvres et celles de salariés plus aisés.

\section{Variables économiques}

Les comparaisons reposent sur un seul jeu d'hypothèses économiques pour les 30 pays. Dans la pratique, le niveau des pensions est influencé par la croissance économique, la hausse des salaires et l'inflation, qui varient d'un pays à l'autre, mais le fait d'avoir une seule série d'hypothèses garantit que les résultats modélisés obtenus pour différents régimes de retraite nationaux reflètent les différences de systèmes et de politiques des retraites.

Les hypothèses de base sont les suivantes :

- taux de croissance réel des salaires : 2 \% par an (soit une hausse nominale des salaires de $4.55 \%$ compte tenu de la hausse des prix retenue);

- salaire individuel : supposé croître comme la moyenne nationale (l'individu est supposé rester au même point sur l'échelle de distribution des revenus, et gagner le même pourcentage du salaire moyen durant toute sa vie professionnelle);

- hausse des prix : $2.5 \%$ par an ;

- taux de rendement réel compte tenu des frais administratifs prélevés sur les régimes de retraite par capitalisation à cotisations définies : $3.5 \%$ par an ;

- taux d'actualisation (pour les calculs actuariels) : $2 \%$ par an (pour plus de détails sur l'importance du taux d'actualisation dans l'analyse des retraites, voir Queisser et Whitehouse, 2006); 
- taux de mortalité : projections par pays de la base de données démographiques Nations Unies/Banque mondiale pour l'année 2040 ;

- distribution des salaires : les indicateurs composites utilisent la moyenne OCDE de la distribution des salaires (basée sur 18 pays) et les données spécifiques par pays lorsqu'elles sont disponibles.

Toute modification de ces hypothèses de base aura évidemment une incidence sur les droits à retraite en découlant. L'impact des variations de la hausse des salaires à l'échelle nationale, et pour une hausse du salaire individuel plus rapide ou plus lente que la moyenne, a été exposé dans la première édition des Pensions dans les pays de l'OCDE (OCDE, 2005), tandis que les effets des différents taux de rendement ont fait l'objet d'une simulation dans la deuxième édition des Pensions dans les pays de l'OCDE (OCDE, 2007a). On trouvera une nouvelle analyse, plus détaillée, des retombées de l'incertitude liée aux rendements des investissements sur les revenus des retraités dans Whitehouse et al. (2009).

Pour le versement des prestations de retraite, les calculs reposent sur l'hypothèse suivante : durant la retraite, les prestations au titre des régimes à cotisations définies sont perçues sous la forme d'une rente viagère indexée sur les prix à un taux actuariellement juste, calculé à partir des tables de mortalité. De même, le taux de rente théorique des régimes de comptes notionnels - ou virtuels - est (la plupart du temps) calculé à partir des tables de mortalité en utilisant les règles d'indexation et les hypothèses d'actualisation employées par chaque pays.

\section{Impôt et cotisations de sécurité sociale}

Les informations relatives à l'impôt sur le revenu des personnes physiques (IRPP) et aux cotisations de sécurité sociale acquittés par les retraités, qui ont été utilisées pour calculer les droits à retraite, sont disponibles à l'adresse www.oecd.org/els/social/ prestationsetsalaires.

La modélisation suppose que la fiscalité et les cotisations de sécurité sociale demeurent inchangées à l'avenir. Ce qui signifie implicitement que les paramètres de "valeur", tels que les abattements fiscaux ou les plafonds de cotisation, sont ajustés chaque année en fonction du salaire moyen, tandis que les paramètres de « taux ", tels que le barème de l'IRPP et les taux de cotisation de sécurité sociale ne subissent aucune variation. Les dispositions générales et le régime fiscal applicable aux salariés pour 2006 figurent dans la version 2007 de la publication de l'OCDE intitulée Les impôts sur les salaires. Les conventions utilisées dans cette étude, par exemple les versements à considérer comme des impôts, sont reprises ici.

\section{Tour d'horizon des régimes de retraite}

Les régimes procurant des revenus aux retraités des pays de l'OCDE sont variés et englobent souvent des programmes différents. Il est donc difficile de classer les systèmes de retraite et les différents mécanismes assurant des revenus aux retraités. La taxinomie utilisée ici est basée sur des travaux antérieurs (OCDE, 2004, 2005a et 2007) et s'appuie sur le rôle et l'objectif de chaque composante du système de retraite. On en trouvera une illustration au graphique 0.1 . 


\section{Graphique 0.1. Les différents types de régimes assurant des revenus aux retraités}

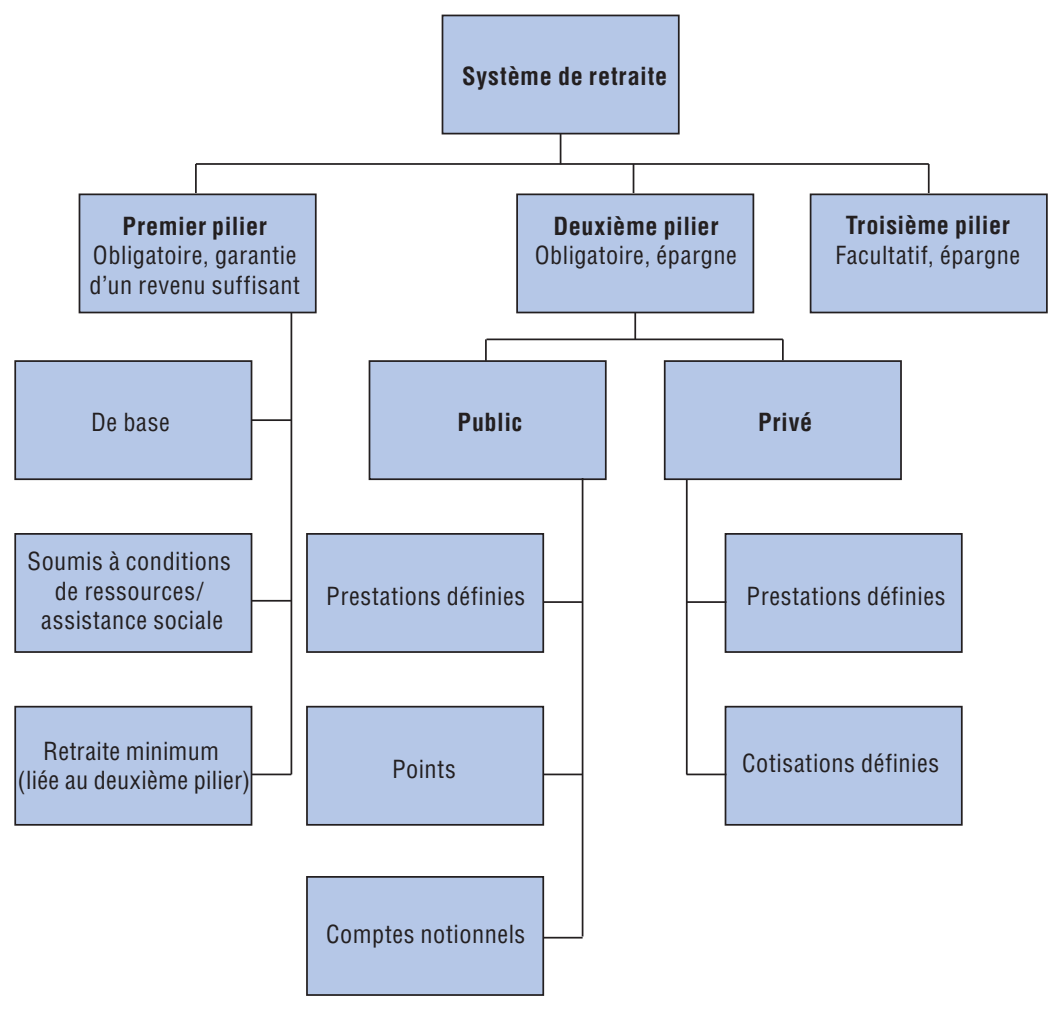

Le cadre comporte deux éléments - ou piliers - obligatoires : une composante redistributive et une composante "épargne ". Les composantes redistributives des systèmes de retraite sont conçues de manière à garantir aux retraités un niveau de vie minimum en termes absolus. Les composantes « épargne » doivent permettre aux retraités de conserver un niveau de vie en rapport avec celui qu'ils avaient lorsqu'ils travaillaient. Les composantes facultatives, qu'elles soient individuelles ou fournies par l'employeur, constituent un troisième volet. À l'intérieur de ces groupes, les régimes font l'objet d'une classification complémentaire en fonction du prestataire (régime public ou privé) et de la manière dont les prestations sont déterminées (régime à prestations définies ou à cotisations définies, par exemple).

\section{Architecture des systèmes de retraite nationaux}

Le tableau 0.1 fait apparaître la structure des régimes assurant des revenus aux retraités, pour les deux piliers obligatoires et les différents types de dispositifs. 


\section{Tableau 0.1. La structure des régimes assurant des revenus aux retraités} dans les pays de l'OCDE

\begin{tabular}{|c|c|c|c|c|c|}
\hline & \multicolumn{3}{|c|}{ Premier pilier } & \multicolumn{2}{|c|}{ Deuxième pilier } \\
\hline & \multicolumn{3}{|c|}{ Couverture universelle, redistributive } & \multicolumn{2}{|c|}{ Obligatoire, épargne } \\
\hline & \multicolumn{3}{|c|}{ Public } & Public & Privé \\
\hline & $\begin{array}{c}\text { Sous conditions } \\
\text { de ressources }\end{array}$ & De base & Minimum & \multicolumn{2}{|c|}{ Type } \\
\hline Allemagne & & & & Points & \\
\hline Australie & $\checkmark$ & & & & $C D$ \\
\hline Autriche & & & & PD & \\
\hline Belgique & $\checkmark$ & & $\checkmark$ & PD & \\
\hline Canada & $\checkmark$ & $\checkmark$ & & PD & \\
\hline Corée & & $\checkmark$ & & PD & \\
\hline Danemark & $\checkmark$ & $\checkmark$ & & & $C D$ \\
\hline Espagne & & & $\checkmark$ & PD & \\
\hline États-Unis & & & & PD & \\
\hline Finlande & & & $\checkmark$ & PD & \\
\hline France & & & $\checkmark$ & PD + points & \\
\hline Grèce & & & $\checkmark$ & PD & \\
\hline Hongrie & & & & PD & $C D$ \\
\hline Irlande & & $\checkmark$ & & & \\
\hline Islande & $\checkmark$ & $\checkmark$ & & & PD \\
\hline Italie & & & & NCD & \\
\hline Japon & & $\checkmark$ & & PD & \\
\hline Luxembourg & & $\checkmark$ & $\checkmark$ & PD & \\
\hline Mexique & & $\checkmark$ & $\checkmark$ & & $C D$ \\
\hline Norvège & & $\checkmark$ & $\checkmark$ & Points & $\mathrm{CD}$ \\
\hline Nouvelle-Zélande & & $\checkmark$ & & & \\
\hline Pays-Bas & & $\checkmark$ & & & PD \\
\hline Pologne & & & $\checkmark$ & NCD & $C D$ \\
\hline Portugal & & & $\checkmark$ & PD & \\
\hline Suède & & & $\checkmark$ & NCD & $\mathrm{CD}$ \\
\hline Suisse & & & $\checkmark$ & PD & PD \\
\hline Turquie & & & $\checkmark$ & PD & \\
\hline République slovaque & & & $\checkmark$ & Points & $C D$ \\
\hline République tchèque & & $\checkmark$ & $\checkmark$ & PD & \\
\hline Royaume-Uni & $\checkmark$ & $\checkmark$ & $\checkmark$ & PD & \\
\hline
\end{tabular}

$\mathrm{CD}=$ cotisations définies $; \mathrm{NCD}=$ comptes notionnels $; \mathrm{PD}=$ prestations définies.

Source : Descriptifs pays figurant en partie III de la présente publication.

Tous les pays de l'OCDE ont mis en place des programmes destinés à lutter contre la pauvreté des personnes âgées, qualifiés ici de "régimes redistributifs du premier pilier ». Tous ces dispositifs relèvent du secteur public et entrent dans trois grandes catégories.

- Premièrement, les régimes soumis à conditions de ressources, versent une prestation plus élevée aux retraités les plus pauvres et des prestations réduites aux plus aisés. Dans ces dispositifs, le montant des prestations dépend soit des revenus provenant d'autres sources, soit des revenus et du patrimoine. Tous les pays sont dotés de ce type de filets de protection sociale à caractère général, mais dans certains cas, ils couvrent uniquement un nombre restreint de personnes âgées ayant subi de nombreuses interruptions de carrière. Dans le tableau, plutôt que de faire figurer l'ensemble des pays de l'OCDE dans cette rubrique, seuls six pays sont indiqués dans cette colonne. Dans ces cas, seuls les salariés dont la carrière est complète et qui perçoivent de faibles 
revenus ( $50 \%$ de la moyenne) ont droit aux prestations soumises à conditions de ressources.

- Deuxièmement, dans les régimes de base, la prestation est soit forfaitaire (c'est-à-dire que c'est le même montant qui est versé à tous les retraités), soit fixée en fonction du nombre d'années d'activité et non des rémunérations passées. Les revenus supplémentaires perçus durant la retraite ne modifient pas non plus le montant de la pension de base. Treize pays ont mis en place un régime de base ou d'autres dispositions ayant un effet analogue.

- Troisièmement, les retraites minimum, qui présentent de nombreuses caractéristiques communes avec les régimes soumis à conditions de ressources, existent dans 16 pays. Dans ces dispositifs, la valeur des droits est déterminée en tenant compte des seuls revenus des retraités. Toutefois, à l'inverse des régimes soumis à conditions de ressources, ils ne prennent pas en considération les revenus tirés de l'épargne ou du patrimoine en dehors de la pension de retraite. Les " crédits minimums " prévus par les régimes liés à la rémunération, tels ceux de la Belgique et du Royaume-Uni, ont un effet similaire : les prestations versées aux salariés dont la rémunération est très basse sont calculées comme s'ils avaient gagné davantage.

Les programmes du "deuxième pilier " jouent un rôle d'épargne, en ce sens qu'ils visent à assurer aux retraités un revenu suffisant par rapport à celui qu'ils percevaient lorsqu'ils étaient en activité, et pas seulement un niveau de vie fixé en termes absolus audessus du seuil de pauvreté. Comme ceux du premier pilier, les régimes pris en compte ici sont obligatoires, qu'ils soient publics ou privés. Parmi les 30 pays de l'OCDE, seules l'Irlande et la Nouvelle-Zélande sont dépourvues de tout mécanisme obligatoire relevant du deuxième pilier.

- Les régimes à prestations définies (PD) sont publics dans 17 pays de l'OCDE. Les dispositifs privés (professionnels) sont obligatoires ou quasi obligatoires dans trois pays membres (l'Islande, les Pays-Bas et la Suisse). Dans les régimes publics, les revenus des retraités dépendent du nombre d'années où ils ont cotisé durant leur vie active, ainsi que de leur salaire. Aux Pays-Bas, le régime est explicitement à prestations définies. En Islande et en Suisse, les pouvoirs publics fixent le taux de cotisation, un rendement minimum et le taux de rente auquel les droits accumulés sont convertis en pension, l'ensemble de ces dispositions définissant les prestations de retraite servies.

- Les régimes à points existent dans quatre pays de l'OCDE: il s'agit des dispositifs professionnels français (qui sont gérés par le secteur public) et des régimes publics allemand, norvégien et slovaque. Les salariés acquièrent des points de retraite sur la base de leur rémunération individuelle pour chaque année de cotisations. Lors du départ en retraite, la somme des points de retraite est multipliée par la valeur du point et convertie en versement régulier d'une pension.

- Les régimes à cotisations définies (CD) sont obligatoires dans huit pays de l'OCDE (l'Australie, le Danemark, la Hongrie, le Mexique, la Norvège, la Pologne, la République slovaque et la Suède). Dans ces régimes, les cotisations sont versées sur un compte individuel. Les cotisations et les rendements des investissements accumulés sont habituellement convertis en flux de revenus versés sous forme de pension durant la retraite. Ces régimes sont gérés par le secteur privé, même si leur organisation varie considérablement d'un pays à l'autre. Ainsi, en Australie, les employeurs doivent couvrir leurs salariés, alors qu'en Hongrie, au Mexique et en Pologne, les salariés choisissent un 
prestataire sans intervention de l'employeur. En Suède, les salariés ne versent qu'une faible cotisation sur des comptes individuels obligatoires et on a prévu un dispositif complémentaire à cotisations définies pour la plupart des salariés dans le cadre des régimes professionnels quasi-obligatoires.

- Des systèmes de comptes notionnels ont été mis en place dans trois pays de l'OCDE (dans le cadre des régimes publics d'Italie, de Pologne et de Suède). On enregistre les cotisations de chaque salarié sur un compte individuel et on applique un taux de rendement à tous les comptes. Ces comptes sont « notionnels »- ou virtuels - en ce sens qu'aussi bien les cotisations que les intérêts versés n'existent que dans les livres de l'institution qui les gère. Lors du départ en retraite, le capital notionnel accumulé sur chaque compte est converti en flux de pension au moyen d'une formule basée sur l'espérance de vie. Ce dispositif étant conçu pour reproduire les régimes à cotisations définies, ils sont souvent qualifiés de régimes notionnels à cotisations définies (NCD).

\section{Références}

OCDE (2004), Les pensions privées - Classification et glossaire de l'OCDE, Éditions de l'OCDE, Paris.

OCDE (2005), Les pensions dans les pays de l'OCDE - Panorama des politiques publiques, Éditions de l'OCDE, Paris.

OCDE (2007a), Les pensions dans les pays de l'OCDE - Panorama des politiques publiques, Éditions de l'OCDE, Paris.

OCDE (2007b), Les impôts sur les salaires 2005/2006, Éditions de l'OCDE, Paris.

Queisser, M. et E.R. Whitehouse (2006), " Neutral or Fair? Actuarial Concepts and Pension-System Design ", Documents de travail de l'OCDE sur les affaires sociales, l'emploi et les migrations, $n^{\circ} 40$, Éditions de l'OCDE, Paris.

Whitehouse, E.R., A.C. D'Addio et A.P. Reilly (2009), "Investment Risk and Pensions: Impact on Individual Retirement Incomes and Government Budgets ", Documents de travail de l'OCDE sur les affaires sociales, l'emploi et les migrations, $n^{\circ} 87$, Éditions de l'OCDE, Paris. 



\section{PARTIE I}

\section{Principaux enjeux des politiques}

Cette partie de la publication s'intéresse de près aux questions auxquelles sont confrontés aujourd'hui les responsables de la politique des retraites. Elle s'articule autour de quatre chapitres.

Le premier est consacré aux répercussions de la crise économique et financière en cours sur les systèmes de retraite. Quels sont les pays et les individus les plus touchés? Quelles mesures les pouvoirs publics peuvent-ils prendre pour les aider et quels sont les écueils à éviter? Ce chapitre présente des données relatives aux performances des investissements des régimes de retraite privés.

Le deuxième chapitre traite des revenus et de la pauvreté des personnes âgées, en analysant les tendances observées ces 20 dernières années. Dans de nombreux pays, la situation des retraités s'est améliorée par rapport à la population dans son ensemble, mais il reste des poches de pauureté chez les personnes âgées. Ce chapitre va également plus loin dans l'analyse, en mettant en évidence les conséquences que pourrait avoir l'évolution des économies, des sociétés et des systèmes de retraite pour les revenus et la pauvreté des salariés d'aujourd'hui lorsqu'ils auront atteint l'âge de la retraite.

Le troisième chapitre présente les modifications des systèmes de retraite annoncées entre 2004 et la fin du mois de mai 2009. La quasi-totalité des pays de l'OCDE ont réformé leurs régimes de retraite ces dernières années, en s'attelant aux problèmes de l'adéquation des prestations, de la pérennité des systèmes et de l'efficacité des régimes assurant des revenus aux retraités.

Le quatrième et dernier chapitre spécial rend compte de la couverture des régimes facultatifs de pensions privées, en étudiant les différences existant en fonction de l'âge et du salaire. La crise financière en cours a porté un coup sévère à l'épargneretraite privée, mais les pensions privées resteront l'une des variables de l'équation des prestations de retraite. On évaluera dans ce chapitre cinq types de mesures. 



\section{Les systèmes de retraite pendant la crise économique et financière}

L a crise financière évolue rapidement vers une crise économique et sociale. La plupart des pays de l'OCDE sont déjà entrés en récession et d'autres vont suivre. Le taux de chômage s'inscrit en hausse dans le monde entier, alors que le salaire moyen amorce un repli. La crise financière a de profondes répercussions sur les revenus des retraités, qui sont touchés de plusieurs façons.

Les fonds de pension ont été durement frappés : en 2008, ils ont vu la valeur de leurs investissements reculer au total de $23 \%$, soit de quelque 5400 milliards USD. Cela signifie que de nombreuses personnes ont perdu une part importante de leur épargne-retraite, investie dans des plans de retraite ou d'autres actifs. Certains risquent également d'être affectés à double titre, la perte de leur emploi venant s'ajouter à celle d'une proportion substantielle de leur épargne. Ce problème est particulièrement sérieux pour les salariés les plus âgés qui rencontrent davantage de difficultés sur le marché du travail en cas de licenciement et n'ont guère le loisir d'attendre une appréciation de leur épargne-retraite.

Les systèmes publics de retraite ne s'en ressentent pas moins. La montée du chômage et la baisse des salaires vont peser sur les recettes que les régimes par répartition tirent des cotisations et, partant, sur leur aptitude à verser des prestations de retraite. Certains fonds de réserve publics pour les retraites ont aussi subi des plus-values considérables sur leurs placements. Par conséquent, la crise économique et financière met en relief et exacerbe tout à la fois les problèmes structurels à plus long terme auxquels sont confrontés les systèmes de retraite de nombreux pays en raison du vieillissement de la population.

La section 1 de ce chapitre s'intéresse aux catégories dont les revenus ont été ou vont être affectés par la crise économique et financière. La section 2 analyse plus en détail les systèmes de retraite qui ont été le plus mis à mal par la crise. Elle étudie dans quelle mesure l'ampleur des répercussions dépend de la conception nationale des systèmes de retraite (régimes publics et privés). Elle examine aussi les conséquences de la composition du portefeuille des fonds de pension. La section 3 passe en revue les mesures pouvant être adoptées par les pouvoirs publics afin d'atténuer les effets de la crise actuelle et de renforcer la résistance des systèmes de retraite aux crises futures. La section 4 formule une conclusion.

\section{Quelles sont les catégories les plus durement touchées par la crise des retraites?}

Pour évaluer les retombées sociales de la crise dans le domaine des retraites, il ne faut pas s'en tenir aux seuls fonds de pension. Les pertes moyennes sur les investissements 
masquent une grande diversité des répercussions observées sur le niveau de vie des salariés et des retraités. Ce chapitre porte donc essentiellement sur les individus et sur la vulnérabilité des personnes âgées, et non sur les organismes de retraite, comme les fonds de pension. Il analyse l'impact de la crise pour différentes catégories de salariés et de retraités, en fonction d'un certain nombre de critères, comme l'âge de la personne et le type de régime auquel elle est affiliée.

\section{Âge}

Le facteur le plus important est l'âge de l'individu. Dans le tableau 1.1, la population est répartie en trois catégories de personnes : i) celles qui ont déjà pris leur retraite ; ii) celles qui sont sur le point de le faire ; et iii) les salariés plus jeunes et appartenant aux tranches d'âge de forte activité qui sont encore loin de la retraite. Le tableau 1.1 montre dans quelle mesure les individus de chaque groupe d'âge souffrent de la crise, des plus affectés aux moins touchés.

\section{Tableau 1.1. Ampleur des répercussions sur les revenus perçus au titre de la retraite en fonction de l'âge et du régime}

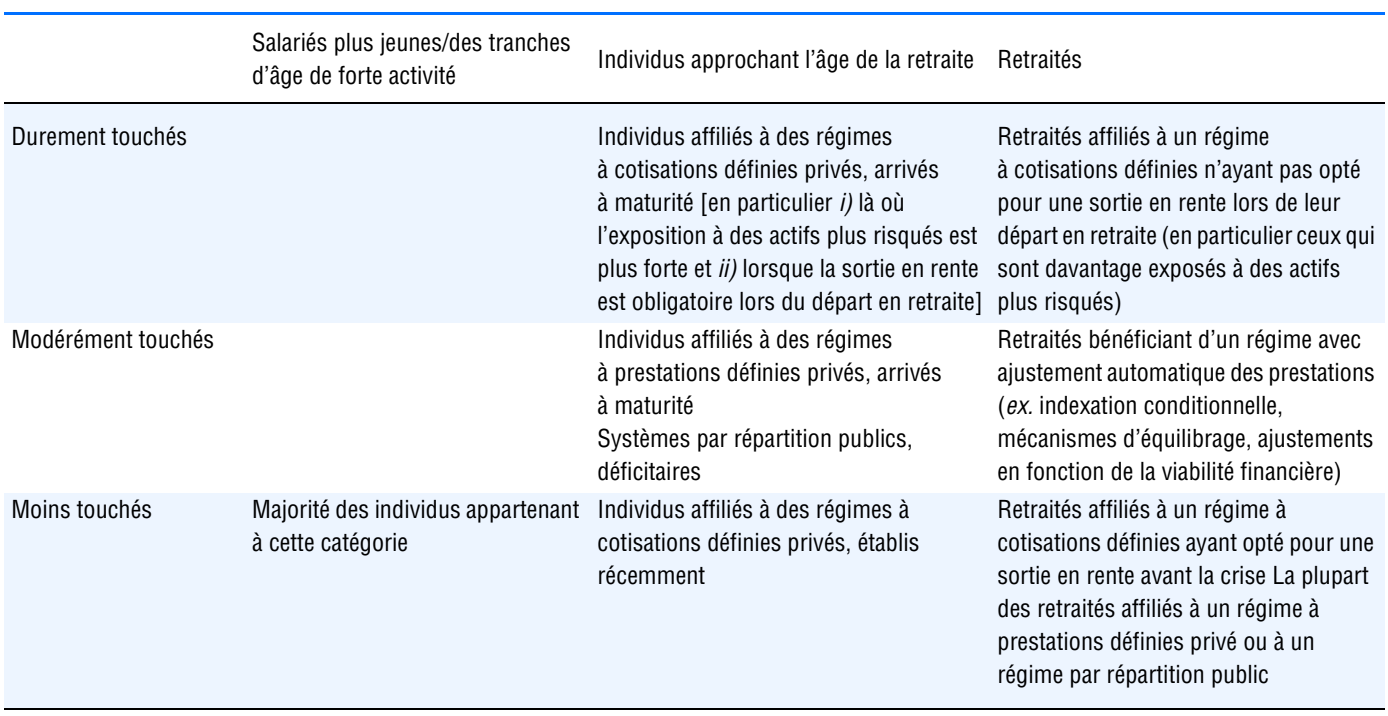

En toute logique, la crise économique et financière devrait avoir une incidence moindre sur les salariés plus jeunes et appartenant aux tranches d'âge de forte activité. Les plus jeunes auront en effet davantage de temps pour reconstituer leur capital-retraite une fois la reprise amorcée, même si à l'évidence les pertes subies seront d'autant plus lourdes que la récession sera longue.

Le solde de leur compte de retraite privé est généralement peu élevé et les moinsvalues exprimées en valeur absolue sont donc elles aussi peu importantes par rapport à celles enregistrées par d'autres catégories. Le graphique 1.1 fait apparaître une évolution du solde des comptes en 2008 pour le principal régime privé à cotisations définies aux États-Unis : les plans 401(k), qui tirent leur nom de la clause du code fiscal s'y rapportant. L'évolution du solde est présentée séparément en fonction de l'âge et de la durée de cotisation au dispositif. Pour les individus de 25-34 ans affiliés depuis au moins cinq ans, les cotisations supplémentaires versées durant l'année l'emportent sur les moins-values observées sur les placements, le solde progressant de près de $5 \%$. 


\section{Graphique 1.1. Évolution du solde des plans 401(k) aux États-Unis en 2008}

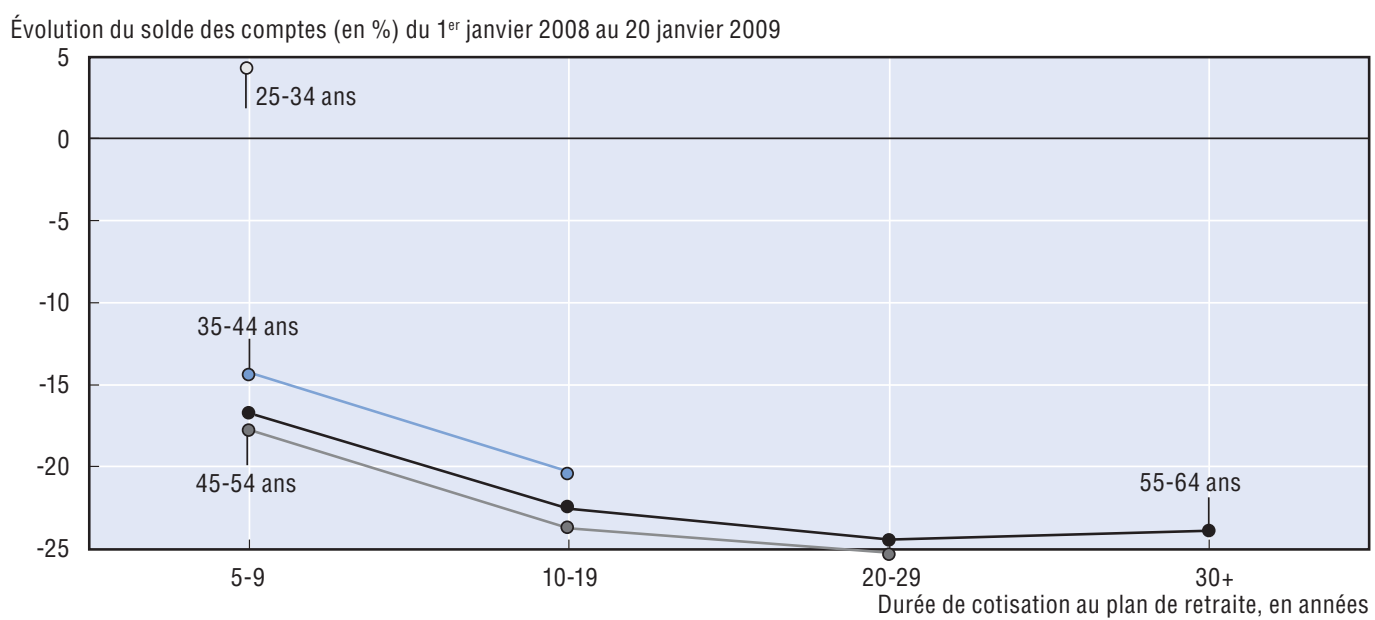

Note : Les données se rapportent à des comptes dont le solde est calculé au 31 décembre 2008, pour les 21 millions de participants à des plans $401(\mathrm{k})$ recensés dans la base de données de l'Employee Benefit Research Institute (EBRI) et de l'Investment Company Institute (ICI).

Source : VanDerhei, J. (2009), "The Impact of the Recent Financial Crisis on 401(k) Account Balances ", Issue Brief, $\mathrm{n}^{\circ}$ 326, Employee Benefit Research Institute, Washington, DC.

StatLink त्नाज्ञा $h t t p: / / d x . d o i . o r g / 10.1787 / 635204112635$

Dans le tableau 1.1, la catégorie la plus sévèrement touchée est généralement celle constituée des personnes qui sont sur le point de prendre leur retraite. À l'inverse des salariés plus jeunes, ces personnes n'ont pas le loisir d'attendre que les marchés se rétablissent. Cela signifie que les moins-values récemment enregistrées par les fonds de pension privés, les fonds de réserve publics pour les retraites et autres dispositifs d'épargne pourraient bien ne pas être récupérées. Même le fait de reporter leur départ à la retraite au-delà de la date prévue ne pourrait leur permettre de compenser qu'une partie des pertes subies. Comme le montre le graphique 1.1, le solde des comptes des pensions privées aux États-Unis a davantage diminué pour la tranche des 45-54 ans, les moinsvalues s'échelonnant de quelque $18 \%$, pour ceux qui cotisent depuis peu, à $25 \%$ pour ceux qui sont couverts depuis plus longtemps. Pour les différentes durées de cotisation, la dépréciation des actifs du compte est légèrement inférieure pour les 55-64 ans.

Les retraités actuels se situent à l'extrémité de la courbe des âges du tableau 1.1. La gravité des retombées de la crise sur cette catégorie dépend de la composition des revenus perçus. Les retraites publiques sont usuellement définies par un ensemble de règles et le pouvoir d'achat des retraités est protégé par un dispositif d'indexation automatique. Dans plusieurs pays, néanmoins, la crise aura des répercussions sur les retraites publiques, les mécanismes d'ajustement automatique pouvant entraîner une minoration des prestations de retraite (voir ci-dessous). Les prestations servies au titre des pensions privées sont elles aussi protégées de manière générale, les régimes de retraite professionnels et les organismes qui assurent le versement d'une rente détenant des actifs pour garantir ces prestations. C'est à d'autres intervenants qu'incombe la lourde tâche de combler les déficits : employeurs, prestataires de services financiers, programmes de garantie de l'État et cotisants. Cela étant, tous les dispositifs d'épargne-retraite facultatifs ou actifs immobiliers dans lesquels les retraités actuels espéraient pouvoir puiser durant leur retraite sont incontestablement frappés par la crise. Pour une partie des retraités d'aujourd'hui, les moins-values enregistrées sur ces actifs sont importantes et les taux 
d'intérêt sont au plus bas, ce qui pourrait se traduire par une nette détérioration de leur niveau de vie.

\section{Type de régimes de retraite}

Le deuxième grand facteur déterminant l'impact de la crise sur les retraites est le type de dispositifs composant le système de retraite national et l'épargne-retraite individuelle.

Dans les régimes à cotisations définies, chacun constitue une épargne pour sa retraite sur un compte individuel et la valeur des prestations de retraite est déterminée par les performances des investissements. En fonction de l'exposition à des actifs plus risqués, les personnes qui s'approchent de l'âge de la retraite peuvent être contraintes d'entériner les moins-values récentes et ne pas avoir le temps d'attendre que les marchés se redressent avant de devoir percevoir leurs revenus au titre de la retraite. Le solde des comptes des salariés plus jeunes est moins élevé, de sorte que les moins-values seront aussi relativement limitées (compte tenu de l'épargne-retraite qu'ils vont constituer toute leur vie durant). De surcroît, ces moins-values seront effacées par la reprise et eu égard à la faiblesse actuelle du prix des actifs, leurs cotisations actuelles devraient bénéficier à l'avenir de rendements appréciables. Pour les retraités qui étaient affiliés à un dispositif à cotisations définies, l'incidence de la crise dépend de ce qu'ils ont fait du capital accumulé sur leur compte lors de leur départ à la retraite. Bon nombre d'entre eux sont protégés car ils ont utilisé leurs avoirs pour acheter une rente avant la crise, entérinant ainsi les plusvalues antérieures et bénéficiant d'une prestation de retraite à vie. D'autres, cependant, n'ont pas acheté de rentes ou en ont différé l'acquisition et, en fonction de la composition de leur portefeuille, peuvent avoir subi des pertes importantes.

Les régimes privés à prestations définies ont également vu décliner la valeur de leurs actifs. Ces dispositifs sont la principale source de revenu pour les retraités en Islande, aux Pays-Bas et en Suisse. Pour les salariés approchant l'âge de la retraite, ils constituent aussi une source non négligeable de revenus au titre de la retraite dans d'autres pays comme le Canada, les États-Unis, l'Irlande, le Royaume-Uni et la Suède. Toutefois, il est nettement plus probable que dans ce deuxième groupe de pays les salariés plus jeunes soient affiliés à des dispositifs à cotisations définies (voir l'encadré 1.1).

Dans les régimes à prestations définies, les pensions sont, en théorie, « définies " par un ensemble de règles et devraient être versées quelles que soient les performances du fonds. Néanmoins, compte tenu de la baisse du prix des actifs, de nombreux régimes sont aujourd'hui déficitaires : leurs engagements envers les retraités actuels et à venir sont supérieurs à la valeur des actifs du fonds (auxquels s'ajoutent les "actifs " que représentent les cotisations futures des employeurs et des adhérents). Les promoteurs de dispositifs à prestations définies peuvent être en mesure de combler le déficit à l'aide de cotisations patronales supplémentaires tout en attendant une appréciation des actifs. Quoi qu'il en soit, comme on le verra plus en détail ci-dessous, le redressement devra venir pour une part d'une réduction des engagements du plan et d'un raffermissement du prix des actifs. Pour dire les choses clairement, il s'agit de diminuer les prestations versées aux retraités de demain, voire d'aujourd'hui.

Dans la majorité des pays, le volet financier de la crise n'a pas eu de répercussions directes sur les retraites publiques. Premièrement, seuls huit pays de l'OCDE disposent d'un fonds public pour les retraites dont les réserves s'élevaient à plus de $5 \%$ du revenu national en 2007 (voir OCDE, 2009a, chapitre 3, et l'indicateur des «Actifs des fonds de pension 


\section{Encadré 1.1. Le passage de régimes de retraite professionnels à prestations définies aux régimes professionnels à cotisations définies}

Le passage des prestations définies aux cotisations définies s'est amorcé en premier lieu aux États-Unis. En 1980, 32 \% des membres adhérents actifs d'un régime de retraite professionnel étaient couverts par un plan à cotisations définies. Cette proportion a doublé en 15 ans pour atteindre $64 \%$ en 1995, puis $71 \%$ en 2003 (Department of Labor, diverses années).

Au Canada, Statistique Canada fait état d'un recul de la couverture des retraites professionnelles depuis le début des années 80 . En effet, de nombreux employeurs proposent désormais des dispositifs individuels de retraite collectifs (connus sous le nom de Régimes enregistrés d'épargne-retraite ou REER) en lieu et place des dispositifs professionnels classiques. De plus, parmi ceux qui conservent des plans professionnels, les régimes à cotisations définies couvraient $24 \%$ des adhérents en 2003 , contre $14 \%$ dix ans plus tôt (Morisette et Johnson, 2003).

La proportion de salariés affiliés à un régime privé à prestations définies au Royaume-Uni a pratiquement diminué de moitié, passant de $23 \%$ à $12 \%$ de la population active totale entre 1988-89 et 2002-03. En 2003, $42 \%$ environ des adhérents cotisaient à des régimes fermés aux membres adhérents actifs. Selon une étude récente de la National Association of Pension Funds, $25 \%$ des grands régimes envisageraient de ne plus couvrir les adhérents existants et nouveaux.

En Irlande, la proportion des adhérents aux régimes professionnels privés à cotisations définies est passée de moins de 40 \% en 1999 à 50 \% en 2005 (Pensions Board, diverses années).

Enfin, la Suède a fait basculer le plus vaste régime professionnel (couvrant les employés et les cadres du secteur privé) vers un dispositif à cotisations définies en 2006. Le régime de retraite des ouvriers avait connu le même changement précédemment.

privés et des fonds de réserve publics » dans la présente publication. Deuxièmement, le fonds constitué aux États-Unis est intégralement investi en obligations d'État, lesquelles représentent plus de $80 \%$ du portefeuille du fonds coréen et plus de $60 \%$ de celui du Japon. À l'opposé, la part des obligations d'État s'établit à 35-40\% en Norvège et en Suède, et à moins de $20 \%$ en Nouvelle-Zélande et en Irlande. Le fonds de réserve irlandais pour les retraites a été encore plus touché par la crise, les autorités ayant proposé de s'en servir pour recapitaliser les banques en difficulté. Le gouvernement entend prélever 4 milliards EUR sur le fonds, auxquels s'ajoutent 3 milliards qui devront être versés en 2009 et 2010. Au total, cela correspond à plus de $40 \%$ des actifs du fonds au 31 décembre 2008. En échange, le fonds de réserve sera crédité des intérêts des actions préférentielles émises par les banques en faveur de l'État.

Cela étant, la crise économique, qui a débuté par les turbulences observées sur les marchés financiers, va peser de façon significative sur les systèmes de retraite publics. Les pays de l'OCDE entrent en phase de récession et le chômage a commencé de s'aggraver dans la plupart des pays membres. Selon les dernières prévisions de l'Organisation publiées le 31 mars 2009 pour l'ensemble de ses membres, le PIB devrait s'inscrire en retrait de 4.3 \% en 2009 et rester stable en 2010. Dans les pays de l'OCDE, le chômage a atteint un point bas en 2007, en s'établissant à $5.6 \%$ de la population active, pour atteindre $6.0 \%$ en 2008. Les prévisions font état de nouvelles hausses sensibles, les chiffres devant ainsi atteindre $8.4 \%$ en 2009 et $9.9 \%$ en 2010 . 
Le ralentissement de la croissance va également se répercuter sur les salaires. Les recettes tirées des impôts et des cotisations dont dépendent les systèmes de retraite publics vont s'en trouver réduites. Ce phénomène pourrait également avoir un impact sur les prestations de retraite puisque le nombre de salariés partant en retraite anticipée sera plus élevé compte tenu des difficultés rencontrées sur le marché du travail. Les dépenses consacrées au chômage et aux pensions d'invalidité pourraient également s'alourdir. Les budgets publics seront comprimés par un raffermissement de la demande de dépenses et un tassement de l'offre de recettes. Plusieurs pays ont réagi à ces pressions en diminuant les prestations de retraite (voir le chapitre spécial sur les « Réformes récentes des retraites").

\section{Dans quels pays les retraites sont-elles le plus touchées?}

Les pays de l'OCDE ont panaché de façons très diverses les dispositifs de retraite publics et privés, par capitalisation et par répartition, collectifs et individuels. L'impact de la crise économique et financière sur les systèmes de retraite dépend en grande partie de la formule choisie par le pays. Plus les composantes par capitalisation sont importantes, plus les retraites seront affectées. Et plus les dispositifs individuels sont développés, en particulier les régimes à cotisations définies, plus les retraités souffriront de la crise.

\section{Les pensions privées et la prestation globale de retraite}

Les pensions privées constituent une source importante et croissante de revenus pour les retraités, une évolution qui s'explique dans une large mesure par les réformes des retraites introduites depuis 20 ans $^{1}$. Le graphique 1.2 illustre le rôle joué par les pensions privées dans la prestation globale de retraite dans un certain nombre de pays. À l'instar des indicateurs de droits à retraite de la Partie II de la présente publication, les chiffres présentés ici sont prospectifs, faisant apparaître la structure des pensions de retraite que percevront les salariés qui entrent aujourd'hui sur le marché du travail. Dans certains pays, la situation des nouveaux entrant sur le marché du travail sera très différente de celle qui se présentera ces prochaines années, le basculement vers les régimes de retraite privés obligatoires étant relativement récent (voir l'encadré 1.2). On trouvera dans le graphique le pourcentage du revenu total des retraités qui provient de sources privées pour les personnes couvertes par des pensions privées: la composante du secteur public correspond tout simplement à la différence entre ce pourcentage et $100 \%$ (sont prises en compte les prestations soumises à conditions de ressources, les retraites de base et minimum, ainsi que les dispositifs publics liés à la rémunération). Les droits à retraite sont calculés pour les salariés qui gagnent entre $50 \%$ et $200 \%$ de la moyenne nationale, puis d'une moyenne pondérée fondée sur la distribution des revenus ${ }^{2}$.

Les calculs concernent huit pays dotés de régimes à cotisations définies obligatoires et trois pays disposant de régimes à prestations définies privés obligatoires (ou quasi obligatoires) : l'Islande, les Pays-Bas et la Suisse. Sont également inclus neuf pays où les régimes privés facultatifs couvrent un grand nombre de personnes (voir l'indicateur sur la " couverture des pensions privées " en partie II de cette publication) et pour lesquels on dispose de données sur les cotisations moyennes versées à ces régimes (voir les " descriptifs pays » en partie III). Il s'agit de l'Allemagne, de la Belgique, du Canada, des États-Unis de l'Irlande, de la Norvège, de la Nouvelle-Zélande, de la République tchèque et du Royaume-Uni ${ }^{3}$.

Dans la plupart des 11 pays de l'OCDE qui n'apparaissent pas dans le graphique 1.2, les retraites privées occupent une place mineure : dans huit d'entre eux, les actifs détenus par 


\section{Graphique 1.2. Le rôle des pensions privées dans la prestation globale de retraite} par type de régimes

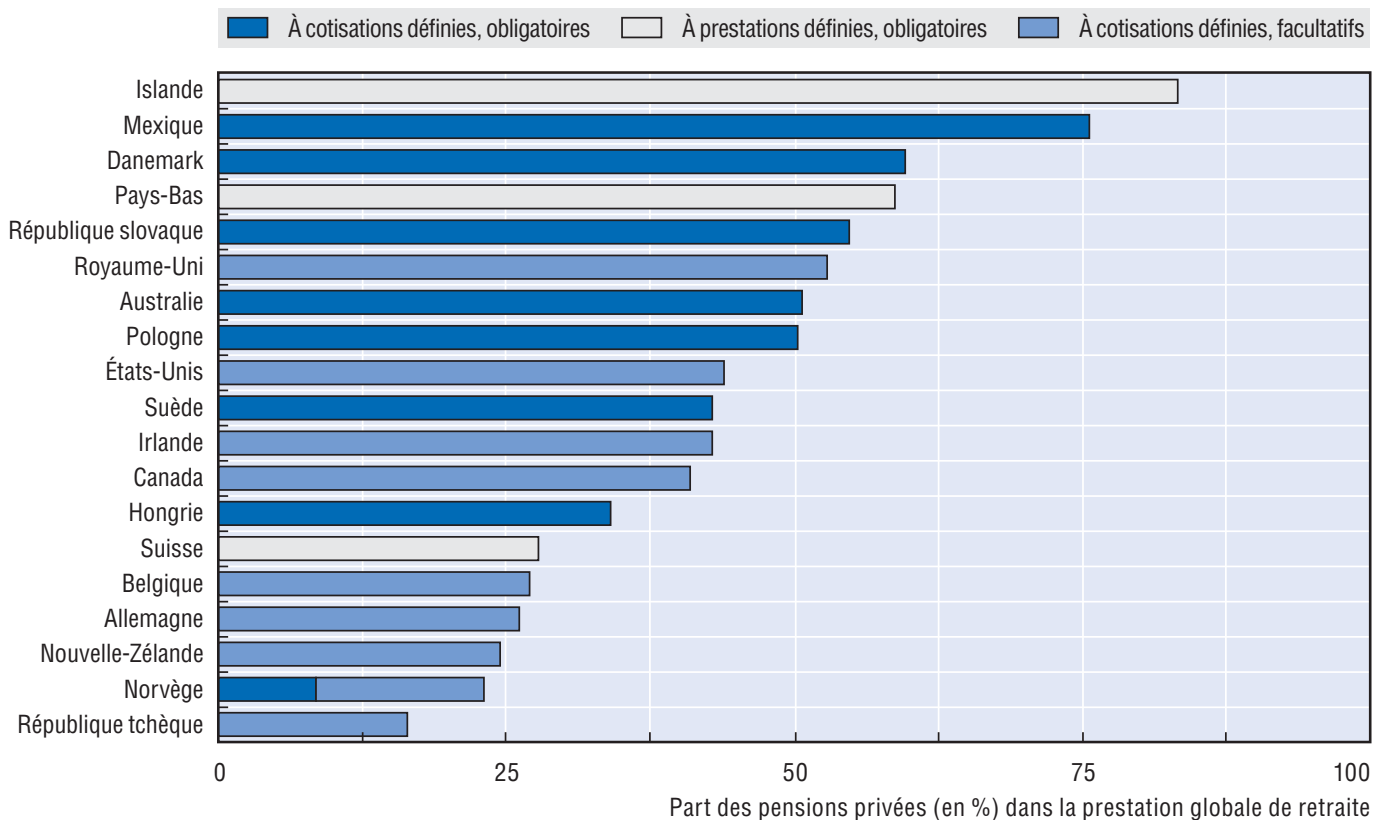

Note: Les régimes de retraite professionnels à prestations définies sont obligatoires en Islande et en Suisse. Ils sont " quasi obligatoires " aux Pays-Bas : grâce aux conventions collectives, la couverture est quasi universelle. Les régimes à cotisations définies au Danemark sont en partie obligatoires et en partie facultatifs. Les régimes privés facultatifs existant en Allemagne, au Canada, aux États-Unis, en Irlande et au Royaume-Uni sont à la fois à prestations définies et à cotisations définies. En l'occurrence, les résultats donnés concernent les dispositifs à cotisations définies, car il est bien plus probable que les nouveaux entrants sur le marché du travail seront couverts par ces régimes (voir l'encadré 1.1).

Source : Modèles de retraite de l'OCDE; voir aussi Whitehouse, E.R. et al. (2009), "Investment Risk and Pensions: Impact on Individual Retirement Incomes and Government Budgets ", Document de travail de l'OCDE sur les affaires sociales, l'emploi et les migrations, n 87, Éditions de l'OCDE, Paris, pour une description détaillée.

StatLink न्नारा http://dx.doi.org/10.1787/635210127154

ces régimes représentent moins de $5 \%$ du revenu national ${ }^{4}$. Dans tous les pays, cependant, les salariés se constituent d'eux-mêmes une épargne-retraite en recourant à des produits qui ne sont pas des retraites « classiques ». Dans certains, comme la France, l'assurance vie est utilisée de longue date comme une formule d'épargne à long terme et les actifs détenus par les compagnies d'assurance ont souffert de la crise. Dans de nombreux pays, les particuliers ont investi des sommes considérables dans l'immobilier, espérant financer leur retraite en emménageant dans un logement principal plus petit ou en vivant de la location d'autres biens. Malheureusement, là où ces pratiques étaient les plus répandues - Australie, Espagne, États-Unis, Irlande et Royaume-Uni - la bulle immobilière a éclaté.

Dans les 19 pays de l'OCDE répertoriés dans le graphique 1.2, les actifs des régimes privés représentaient avant la crise plus de $50 \%$ du revenu national global. La part des pensions privées dans la prestation globale de retraite pour les 19 pays retenus se situe juste en dessous de $50 \%$. C'est en Islande et au Mexique qu'elle est la plus importante, car la plupart des revenus futurs des retraités devraient provenir des régimes obligatoires à prestations définies et à cotisations définies, respectivement ${ }^{5}$. Le complément sera issu des prestations soumises à conditions de ressources et des retraites minimum versées par l'État aux salariés à faible revenu. Les pensions privées joueront également un rôle prééminent dans six autres pays : l'Australie, le Danemark, l'Islande, les Pays-Bas, la 


\section{Encadré 1.2. Basculement des régimes publics vers des dispositifs mixtes public-privé à cotisations définies}

Le Royaume-Uni a incité les adhérents au régime de retraite public lié à la rémunération à basculer vers les pensions privées à compter de 1988, une mesure qui a rencontré un succès bien plus vif que prévu, notamment en raison de la "vente abusive " des plans d'épargne-retraite individuels.

La Hongrie, le Mexique, la Pologne et la Suède ont pris la même initiative en 1997-99 et la République slovaque en 2005. Dans la plupart des cas (à l'exception du Mexique et de la Suède), de nombreux salariés pouvaient choisir leur régime de retraite. Ainsi, en Pologne, tous les salariés de moins de 30 ans et les futurs entrants sur le marché du travail ont dû basculer vers le nouveau régime mixte (voir Mattil et Whitehouse, 2009). Les salariés âgés de 30 à 50 ans avaient le choix : $90 \%$ environ des jeunes trentenaires ont choisi de basculer vers le nouveau régime, contre quelque $10 \%$ seulement des personnes proches de la cinquantaine. $\mathrm{Au}$ Mexique, les personnes déjà affiliées au régime public lors de la réforme ont reçu la garantie que leurs prestations ne seraient pas minorées par rapport à l'ancien système.

Il en résulte que sur les cinq à dix prochaines années au moins, seul un faible nombre de salariés affiliés à un régime à cotisations définies vont prendre leur retraite en Hongrie, en Pologne et en République slovaque. De surcroît, les salariés, dans tous les cas, seront loin d'avoir cotisé à ce régime pendant toute leur carrière, de sorte que le capital qu'ils auront constitué ne sera pas très important et que la plupart de leurs droits à retraite proviendront des régimes publics.

République slovaque et le Royaume-Uni. La quasi-totalité d'entre eux versent des prestations de retraite publiques largement ciblées sur les bas revenus. Il est intéressant de constater qu'aux États-Unis, le régime public - dit de Sécurité sociale - joue un rôle relativement plus important que dans ces autres pays.

À l'autre extrémité, le taux de cotisation type est généralement faible, soit de $2 \%$ à $4 \%$ environ en Allemagne, en Belgique, en Nouvelle-Zélande et en République tchèque. En Norvège, le taux de cotisation obligatoire s'élève à $2 \%$; même si les taux de cotisation obligatoires sont plus élevés, ils ne s'appliquent d'ordinaire qu'à une fraction de la rémunération. Pour la Suisse, il convient de noter que les calculs portent sur la part obligatoire des pensions privées. La plupart des employeurs proposent des prestations supérieures au minimum légal ${ }^{6}$.

Les mesures qui s'imposent pour remédier aux effets des turbulences financières sur les fonds de pension dépendent incontestablement du rôle que jouent ces dispositifs dans la prestation globale de retraite. D'une manière générale, plus les pays se situent dans la partie haute du graphique 1.2, plus les pressions politiques engageant les autorités à agir seront fortes. L'intensité de ces pressions dépendra néanmoins d'autres facteurs. Le Mexique, par exemple, qui se situe tout en haut de l'échelle, a observé des moins-values relativement faibles en 2008 , les portefeuilles ayant été investis de façon prudente. L'Irlande, en revanche - qui se situe au milieu du graphique - a subi les pertes les plus conséquentes.

\section{Types de régimes de retraite privés}

Comme on l'a vu plus haut, la crise financière produit sur les revenus des retraités des répercussions directes dans le cas des régimes à cotisations définies, mais indirectes dans 
celui des dispositifs à prestations définies. Le graphique 1.2 montre que huit pays de l'OCDE sont dotés de régimes obligatoires ou facultatifs à cotisations définies, alors que les régimes privés existants sont dans leur quasi-totalité à prestations définies dans trois pays seulement. Les pensions privées sont facultatives dans six pays. Dans ce cas, les prestations définies sont associées aux cotisations définies. Ce dosage a évolué au fil du temps, comme souligné à l'encadré 1.1. Les régimes privés à cotisations définies jouant un rôle croissant, les revenus des retraités sont plus directement liés aux performances des investissements : le risque d'investissement est ainsi reporté sur les individus à la retraite.

\section{Maturité des différents régimes de retraite}

Le graphique 1.2 repose sur une analyse prospective des droits à retraite des personnes entrées sur le marché du travail en 2006. Toutefois, on observe souvent un net décalage dans le temps entre les modifications apportées aux systèmes de retraite et leur incidence sur les revenus des retraités.

Deux évolutions fondamentales expliquent que la situation des salariés plus jeunes sera profondément différente de celles des plus âgés. Premièrement, le basculement d'un régime de retraite essentiellement public vers un dispositif mixte public-privé à cotisations définies ne remonte pas à plus de dix ans, en règle générale, de sorte que les jeunes retraités ne seront guère voire nullement couverts par un mécanisme à cotisations définies (voir l'encadré 1.2).

Deuxièmement, le processus du passage d'un régime privé à prestations définies à un dispositif privé à cotisations définies est loin d'être arrivé à maturité. Au Royaume-Uni, par exemple, la couverture des régimes professionnels à prestations définies s'est surtout contractée depuis le début des années 90. La majorité des dispositifs ont été simplement fermés aux nouveaux adhérents, si bien que la plupart des retraités affiliés à un régime professionnel prenant leur retraite dans les dix ans à venir, globalement, bénéficieront encore pour l'essentiel d'un mécanisme à prestations définies. Le phénomène a été plus précoce et plus rapide aux États-Unis, et légèrement plus tardif et plus lent au Canada et en Irlande (voir l'encadré 1.1).

\section{Performances récentes des investissements des fonds de pension}

Globalement, en 2008, les marchés boursiers internationaux (d'après l'indice MSCI) ont perdu pratiquement la moitié de leur valeur et ont connu une volatilité beaucoup plus marquée que par le passé. À l'inverse, l'indice mondial des obligations d'État (Citigroup) a progressé de quelque $7 \%$. Les marchés immobiliers ont fléchi dans de nombreuses économies de l'OCDE, parfois dans des proportions spectaculaires. Ces actifs, auxquels s'ajoutent les obligations de société et les dépôts, représentent la quasi-totalité des placements des fonds de pension. Cependant, les portefeuilles des fonds de pension varient fortement d'un pays à l'autre et les performances qu'ils ont enregistrées l'an dernier sont donc aussi très diverses.

Le graphique 1.3 présente les rendements des investissements des fonds de pension en termes réels (corrigés de l'inflation) pour l'année 2008. Les données concernent 23 pays de l'OCDE dans lesquels les régimes privés occupent une place importante dans l'économie (leurs actifs constituant $4 \%$ au moins du revenu national à la fin 2007, soit avant que la crise ne s'approfondisse). Le rendement réel moyen pondéré - soit -23\% témoigne du poids des États-Unis. La moyenne non pondérée (chacun des 23 pays ayant la même importance) ressort à $-17 \%$. 


\section{Graphique 1.3. Rendements réels des investissements des fonds de pension, 2008}

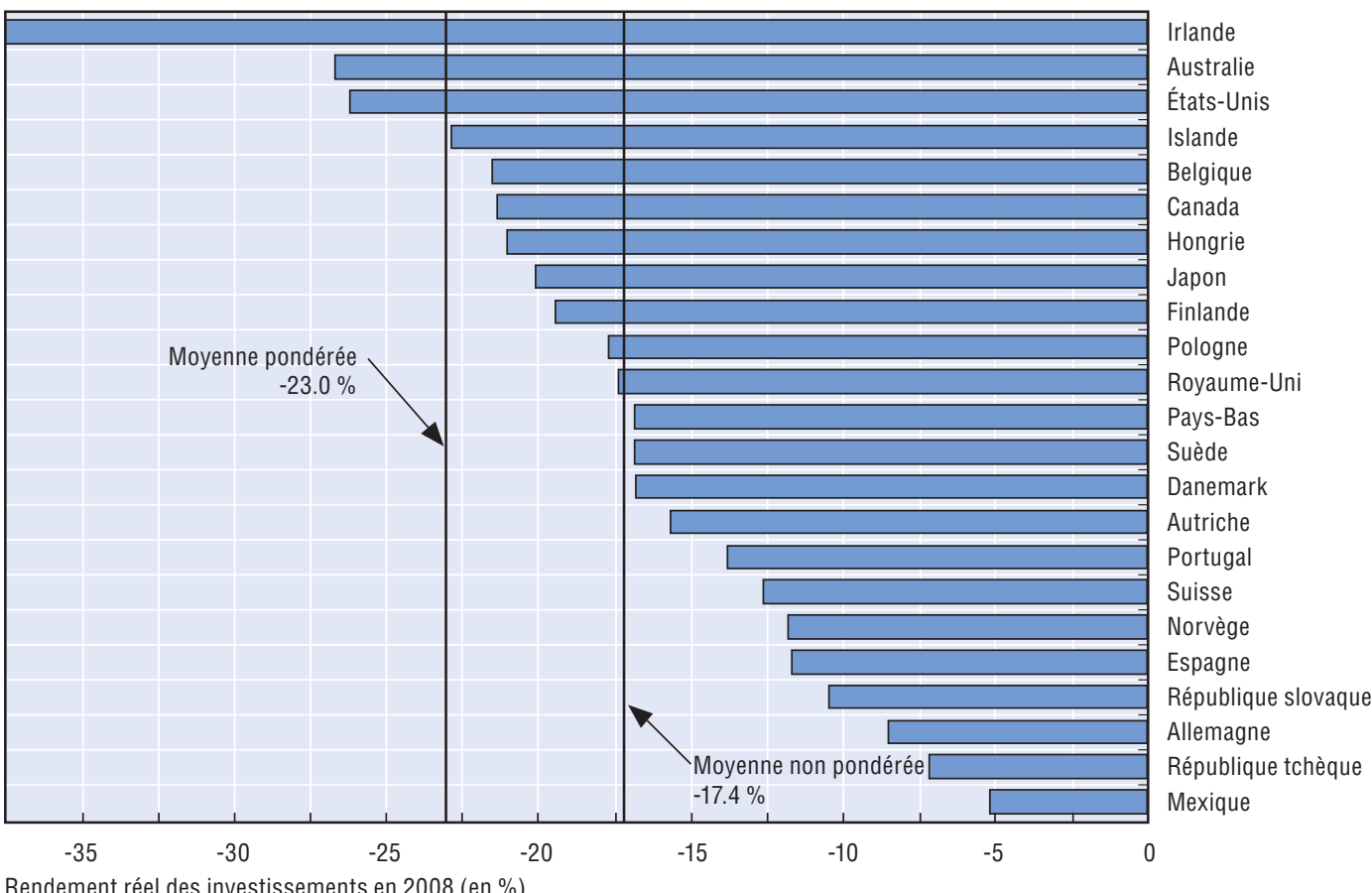

Note: Les rendements sont indiqués uniquement pour les pays où les actifs détenus par les fonds de pension représentaient plus de $4 \%$ du produit intérieur brut (PIB) en 2007. Les données proviennent de sources officielles pour l'Autriche, la Belgique, l'Espagne, la Finlande, la Hongrie, l'Irlande, le Mexique, la Norvège, la Pologne, la République slovaque et la Suisse. Lorsque les informations sur les performances effectives des fonds de pension faisaient défaut, on a estimé les rendements des investissements à l'aide des données sur l'allocation des actifs des fonds de pension et les rendements des différentes catégories d'actifs. Voir OCDE (2009a), Perspectives de l'OCDE sur les pensions privées 2008, note 1 à la fin du dossier spécial.

Belgique : Les données couvrent la période allant de janvier à fin septembre 2008. Finlande : les données concernent les régimes professionnels obligatoires du secteur public. Suède : les chiffres se rapportent aux régimes professionnels. Hongrie et République slovaque : pensions privées obligatoires uniquement.

Source: Statistiques de l'OCDE sur les pensions.

StatLink ants http://dx.doi.org/10.1787/635276166554

Parmi les pays ayant connu les plus fortes baisses, les États-Unis, qui représentent environ la moitié de l'ensemble des actifs détenus par les dispositifs de retraite privés dans les pays de l'OCDE, se classent en troisième position, avec un repli de $26 \%$ environ. Seule l'Irlande (près de $-38 \%$ ) et l'Australie (-27\%) affichent de moins bonnes performances en 2008. Dans cinq autres pays - la Belgique, le Canada, la Hongrie, l'Islande et le Japon l'investissement réel a fléchi de plus de $20 \%$.

À l'autre extrémité de l'échelle, les rendements s'inscrivent en retrait de quelque $10 \%$ en Allemagne, en Espagne, en Norvège, en République slovaque et en Suisse, et le recul observé est encore moins marqué en République tchèque et au Mexique.

Les différences entre les rendements des investissements constatées dans les pays considérés tiennent pour l'essentiel à la composition du portefeuille des fonds. Là où la réduction est moins importante, les fonds de pension sont en règle générale principalement investis en obligations, et en particulier en obligations d'État. Dans les pays qui enregistrent une baisse plus prononcée des fonds de pension, l'exposition aux actions est plus marquée. On en trouve une illustration au graphique 1.4, qui fait apparaître le rendement des investissements figurant au graphique 1.3 en fonction de 
la fraction du portefeuille investie en actions. Celle-ci est calculée pour 2007 afin de donner une vue d'ensemble de la situation avant l'éclatement de la crise financière : la part des actions dans le portefeuille des fonds aura fatalement fléchi dans le sillage des marchés boursiers.

On note incontestablement une très forte corrélation. L'Irlande affiche à la fois la plus forte baisse et la plus grande proportion d'actifs en portefeuille investis en actions, soit les deux tiers. La part des actions et le recul des rendements sont aussi relativement prononcés en Australie et aux États-Unis. Le Canada, le Japon et le Royaume-Uni, où la fraction du portefeuille constituée d'actions est importante et atteint au moins $50 \%$ des actifs, s'en sortent mieux.

Dans la plupart des pays les moins touchés par la baisse des rendements en 2008, les fonds détenaient des portefeuilles obligataires : la part des actions s'échelonnait de $6 \%$ à 12 \% seulement en Allemagne, au Mexique, en République slovaque et en République tchèque, par exemple. Néanmoins, il faut garder à l'esprit qu'à long terme, les actions ont offert des rendements supérieurs (quoique plus risqués), point qui sera traité plus en détail ci-dessous.

\section{Graphique 1.4. Rendements réels des investissements des fonds de pension en 2008 et exposition aux actions en 2007}

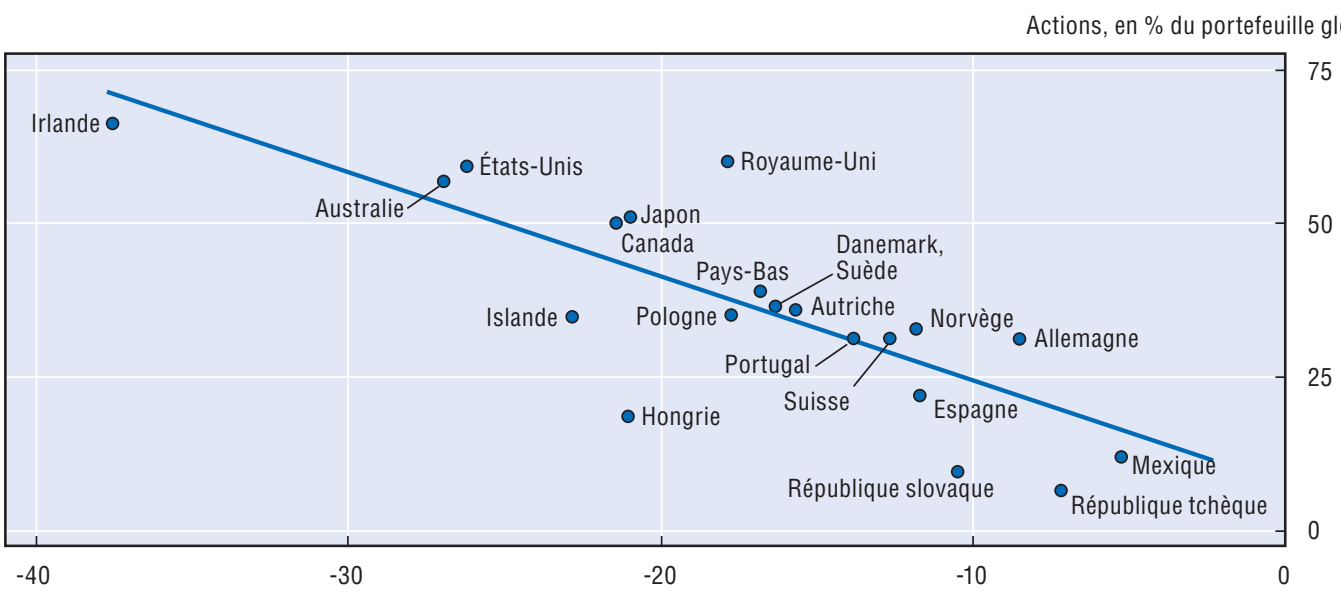

Rendement réel des investissements en 2008 (en \%)

Note: Lorsque les fonds de pension investissent dans des fonds communs de placement (FCP), on utilise la ventilation des actifs des FCP tirée de la base de données d'Institutional Investors pour répartir ces investissements entre les différentes catégories d'actifs. Voir également les notes relatives au graphique 1.3.

Source: La part des actions dans le portefeuille des fonds est tirée d'OCDE (2009a), Perspectives de l'OCDE sur les pensions privées 2008, graphique 2.12 et tableau 2.8; Australian Prudential Regulatory Authority (2007), Insight: Celebrating 10 Years of Superannuation Data Collection; International Financial Services London (2009), Pension Markets 2009 ; The Pensions Regulator (2008), The Purple Book: DB Pensions Universe Risk Profile.

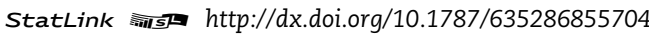

Ces moins-values ont une incidence directe sur les dispositifs de retraite individuels à cotisations définies; cela étant, elles ont aussi eu de profondes répercussions sur l'équilibre financier des régimes à prestations définies. De nombreux dispositifs privés à prestations définies sont déficitaires, c'est-à-dire que leurs engagements de retraite actuels et futurs sont supérieurs aux actifs en portefeuille. On utilise pour apprécier leur situation le "coefficient de capitalisation", soit les actifs du fonds ramenés aux engagements de retraite. 
- Belgique et Finlande : entre la fin 2007 et la fin 2008, le coefficient de capitalisation moyen est passé de $130 \%$ environ à $115 \%$.

- États-Unis : le coefficient de capitalisation moyen pour 100 dispositifs étendus s'établit à $78 \%$ en 2008, contre $109 \%$ précédemment, selon Watson Wyatt, un grand cabinet de conseil.

- Irlande : en décembre 2008 , le coefficient de capitalisation global atteignait $75 \%$, contre $120 \%$ un an auparavant.

- Pays-Bas : le coefficient de capitalisation pour les prestations de retraite nominales est passé de $144 \%$ au début 2008 à quelque $90 \%-95 \%$ au début 2009, d'après le ministère des Affaires sociales (les pensions servies, pas plus que les droits constitués par les salariés, ne sont corrigés de l'inflation : voir ci-dessous).

- Royaume-Uni : le niveau de financement a plongé, ressortant à $76 \%$ en février 2009 , contre $97 \%$ un an plus tôt et $118 \%$ au plus haut en juin 2007 (Pension Protection Fund, 2009).

- Suisse : le coefficient de capitalisation s'établissait en moyenne à $116 \%$ à la fin juin 2007 mais il était revenu à $102 \%$ à la fin 2008.

Les performances enregistrées sur les marchés financiers depuis le début de l'année 2009 n'ont pas apporté de réconfort aux adhérents des régimes de retraite privés ou aux gestionnaires des fonds de pension. La quasi-totalité des grands marchés d'actions ont poursuivi leur chute bien que l'indice mondial au 21 mai 2009 soit remonté de $6.4 \%$ par rapport au début de l'année. À l'inverse de 2008, les marchés obligataires se sont également infléchis, essentiellement par crainte des effets des plans de relance budgétaire sur la dette des États. La baisse au 21 mai était de $2.3 \%$.

\section{Stabilisateurs automatiques et filets de protection des personnes âgées}

L'impact global de la crise sur les revenus des salariés dépend des stabilisateurs économiques et des filets de protection destinés à lutter contre la pauvreté qui sont intégrés dans les systèmes de retraite nationaux. La plupart des pays disposent de mécanismes qui contribuent à empêcher les retraités de tomber dans la pauvreté, ce qui peut atténuer l'impact des pertes des investissements sur le revenu de certains retraités.

La plupart des programmes publics de retraite - les retraites de base et les dispositifs liés à la rémunération - prévoient des prestations identiques quelles que soient les répercussions sur les pensions privées. Néanmoins, de nombreux dispositifs soumis à conditions de ressources ont une incidence sur le montant des pensions privées, et inversement. En Australie et au Danemark, par exemple, la plupart des retraités actuels perçoivent des prestations sous conditions de ressources. Le montant de ces droits à prestation augmente lorsque les rendements des pensions privées diminuent, protégeant en grande partie les bas revenus et les revenus moyens. Par rapport à d'autres sources de revenu, le taux de dégressivité des prestations s'élève actuellement à $40 \%$ en Australie et à $30 \%$ au Danemark. En Australie, par exemple, chaque dollar supplémentaire perçu dans le cadre d'un régime privé donne lieu à une réduction de 40 cents de la pension publique. Inversement, toute diminution des pensions privées de 1 dollar entraîne une revalorisation des prestations publiques à hauteur de 60 cents. Les prestations perçues par plus de $75 \%$ des personnes âgées en Australie et $65 \%$ environ au Danemark sont, pour une part au moins, soumises à conditions de ressources. 
La proportion de personnes âgées bénéficiant de ce type de mécanisme est aussi relativement élevée au Canada, en Irlande et au Royaume-Uni (de $20 \%$ à $35 \%$ ). Les personnes à faible revenu voient ainsi leur retraite globale protégée grâce à des programmes de prestations sous conditions de ressources ${ }^{7}$. Dans tous ces cas de figure, les dispositifs de retraite publics font office de "stabilisateurs automatiques ", c'est-à-dire qu'une partie ou la majorité des retraités ne supportent pas dans leur intégralité les effets produits par la crise financière sur leurs revenus.

Cela étant, les régimes sous conditions de ressources ne tiennent pas tous compte des revenus tirés des régimes privés pour calculer les droits à prestation. En Suède, par exemple, le montant de la retraite garantie actuellement perçue par plus de la moitié des retraités dépend uniquement de celui des prestations versées par le régime public lié à la rémunération (fondé sur le principe des comptes notionnels). Les retraités suédois ne perçoivent donc aucune compensation pour les moins-values enregistrées sur leur épargne-retraite privée.

L'impôt sur le revenu des personnes physiques fait également office de stabilisateur automatique des revenus nets perçus par les retraités confrontés au risque d'investissement ${ }^{8}$. Dans la plupart des pays de l'OCDE, les pensions servies sont imposables. Les personnes dont le revenu s'inscrit dans la moyenne sont ainsi imposées à $30 \%$ environ sur leur retraite au Danemark et en Suède. En Allemagne, Belgique et Norvège, les prélèvements auxquels sont soumis ces retraités représentent $20 \%$ de leurs revenus et approximativement $15 \%$ en Hongrie et en Pologne. Si les rendements des investissements s'avèrent médiocres, les pouvoirs publics revoient à la baisse les impôts prélevés sur les retraites. Il en résulte que les revenus nets des retraités accusent un repli moins prononcé que la valeur des actifs des fonds de pension ${ }^{9}$. À l'inverse, les pensions ne sont pas imposables en République slovaque et compte tenu des crédits, des abattements et des allégements fiscaux spécifiques octroyés sur les revenus des retraités ou aux personnes âgées, seuls les retraités percevant des revenus très élevés de régimes facultatifs acquittent un impôt sur le revenu important en Australie, au Canada, aux États-Unis, en Irlande et au RoyaumeUni.

Si l'on tient compte de ces deux aspects - la fiscalité et les prestations soumises à conditions de ressources - c'est au Danemark que les stabilisateurs automatiques ont le plus d'incidence. L'effet correctif produit sur les revenus nets des retraités est aussi marqué en Belgique, en Pologne et en Suède, et sensible aux États-Unis et au RoyaumeUni.

\section{Ajustement automatique des prestations de retraite}

Trois pays de l'OCDE - l'Allemagne, le Canada et la Suède - ont mis en place des mécanismes d'ajustement automatique des prestations en vue d'assurer la solvabilité des régimes de retraite publics. On pourrait les qualifier de "déstabilisateurs automatiques ", car ils exercent un effet inverse à celui des stabilisateurs automatiques décrits précédemment. Ils protègent l'équilibre financier du régime de retraite, mais en contrepartie, les revenus des retraités varient. Dans chaque cas, l'ajustement s'effectue principalement par une indexation des pensions servies. Néanmoins, les prestations constituées par les salariés actuels peuvent aussi être affectées. Un mécanisme similaire a été introduit pour les dispositifs de retraite professionnels à prestations définies existant aux Pays-Bas (voir l'analyse à l'encadré 1.3). 


\section{Encadré 1.3. Ajustement automatique des prestations de retraite}

Au Canada, la viabilité financière du régime lié à la rémunération (dit "régime fondé sur le revenu ") est examinée tous les trois ans. Ce dispositif fonctionne partiellement par capitalisation : les provisions constituées ne sont pas destinées à couvrir la totalité des engagements, mais uniquement à lisser le taux de cotisation requis au fil du temps et, en particulier, à anticiper les effets du prochain départ en retraite de la vaste cohorte des enfants du baby boom. Lorsqu'on considère que le régime n'est pas viable, la loi impose un gel des retraites nominales et un relèvement du taux de cotisation (équivalent à la moitié de la hausse requise pour assurer sa solvabilité) sur trois ans, soit jusqu'à l'examen suivant. Les effets éventuels de la crise économique sur la solvabilité du dispositif doivent être supportés par les retraités et les cotisants d'aujourd'hui. Toutefois, les ministres des Finances des provinces sont habilités à prendre d'autres mesures pour garantir la solvabilité du régime ${ }^{1}$.

L'Allemagne a introduit en 2005 un «facteur de viabilité » dans son régime de retraite public - fondé sur des points de retraite. L'ampleur de l'ajustement de la valeur des points dépend du taux de dépendance économique, soit le rapport entre le nombre de bénéficiaires « normalisés » et le nombre de cotisants. Le taux de dépendance économique est "équivalent ", c'est-à-dire qu'il tient compte du fait que les hauts revenus cotisent davantage au régime que les bas revenus ${ }^{2}$. L'ajustement a une incidence sur l'évolution de la valeur du point de retraite. Ainsi, les pensions servies ne sont pas intégralement indexées sur la croissance des salaires, même si une clause de sauvegarde exclut toute réduction des prestations nominales. De plus, l'ajustement touche l'ensemble des salariés et retraités actuels, car aujourd'hui comme demain, les droits constitués seront également minorés ou majorés en proportion. Dans le cadre de la présente publication, l'indexation comme la revalorisation s'en ressentent (voir la définition et l'examen des systèmes nationaux dans la première section de la partie III). Compte tenu de perspectives économiques encourageantes au moment où la décision a été prise, le gouvernement a promis une hausse de la valeur du point de retraite de 0.6 à 0.7 points de pourcentage par rapport à ce que prévoyait la réglementation en 2008 et 2009. Du fait des conséquences budgétaires et financières de la crise (et du cycle électoral), il lui sera peut-être impossible de faire preuve d'une telle générosité.

En Suède, le "mécanisme d'équilibrage " du régime de comptes notionnels a également des conséquences pour les retraités actuels et futurs (à travers l'indexation et la revalorisation). Les pensions mises en paiement sont indexées sur la croissance des salaires minorée de la " norme de croissance " fixée à $1.6 \%$. Un taux d'intérêt notionnel correspondant à la croissance du salaire moyen est appliqué aux comptes notionnels des salariés actuels. La solvabilité du régime est évaluée en comparant ses actifs et ses engagements. La valeur des actifs correspond à celle du fonds de régulation, qui représente pour l'heure environ quatre fois les dépenses annuelles ${ }^{3}$, à laquelle s'ajoute la valeur actualisée du flux des cotisations (voir Settergren, 2001). La valeur des engagements est égale à la valeur actualisée du flux des retraites dues aux retraités et aux salariés actuels. Si la valeur des actifs est inférieure à celle des engagements, l'indexation des pensions servies et le taux d'intérêt notionnel sont tous deux réduits jusqu'à ce que la solvabilité du régime soit retrouvée. Le rapport entre ces deux indicateurs a atteint un point bas en 2004, soit 1.0014, et est resté légèrement supérieur à 1 jusqu'en 2007. En 2008, toutefois, il est revenu à 0.9672 . Conformément aux règles en vigueur, il convient de minorer les prestations de retraite des salariés d'aujourd'hui et de demain afin de rétablir l'équilibre du régime.

Aux Pays-Bas, les régimes de retraite professionnels peuvent également ajuster l'indexation et la revalorisation pour contribuer au rétablissement de leur solvabilité. La quasi-totalité des dispositifs professionnels demeurent à prestations définies (contrairement à d'autres pays, comme les États-Unis et le Royaume-Uni, ainsi qu'on l'a vu ci-dessus). Depuis quelques années, la plupart des régimes ne calculent plus le montant des pensions en fonction du dernier salaire, mais d'après les gains moyens perçus sur l'ensemble de la carrière, de sorte que la revalorisation a une large incidence sur les droits des individus. Un taux identique est généralement appliqué pour ajuster les droits à retraite constitués par les salariés et 


\section{Encadré 1.3. Ajustement automatique des prestations de retraite (suite)}

les pensions servies. Les régimes sont désormais tenus de faire état de leur objectif pour ce taux et la plupart des dispositifs ont opté pour une corrélation avec le salaire, à l'échelle du secteur ou au niveau national ${ }^{4}$. La réglementation protège sensiblement les prestations nominales des salariés et des retraités. Toutefois, la règle voulant que les provisions couvrent l'indexation et la revalorisation est moins stricte et nombre de régimes importants ont annoncé cette année des majorations inférieures à celles qui sont prévues par la politique qu'ils affichent en la matière ${ }^{5}$. Selon les estimations du Bureau central du Plan, la perte subie alors par les salariés d'une cinquantaine d'année (en raison des suspensions de l'indexation et des hausses de cotisations) s'établira aux alentours de $10 \%$ des prestations de retraite, et en deçà pour les salariés plus jeunes. Néanmoins, les autorités de tutelle ont alloué aux fonds de pension un délai supplémentaire pour assurer leur solvabilité et de ce fait, les suspensions de l'indexation pourraient être reportées (voir l'analyse sur " La réglementation des régimes à prestations définies » dans la section 3 ci-dessous).

1. Le Bureau du surintendant des institutions financières Canada (2007) et Brown (2008) proposent une analyse complète.

2. Pour une description détaillée, voir Börsch-Supan et Wilke (2006).

3. OCDE (2008), «Le point sur le marché des pensions ", Lettre d'information $n^{\circ} 5$ de la Division des affaires financières, décembre, graphique 11.

4. Voir Bikker et Vlaar (2006).

5. Cette réduction vient s'ajouter à l'indexation qui, sur la période 2003-06, s'est inscrite en retrait de $3 \%$ par rapport à la croissance des salaires et de $2 \%$ par rapport à l'inflation, dans un contexte de redressement des fonds de pension à la suite de l'effondrement des marchés boursiers intervenu en 2000-02 (Banque centrale néerlandaise, 2007).

Ces ajustements automatiques pourraient - à moins d'être supprimés - se traduire par une réduction des prestations réelles perçues par les retraités d'aujourd'hui. Ce phénomène s'explique à la fois par les répercussions de la crise financière sur l'investissement et par celles de la crise économique sur les salaires et l'emploi. Une dévalorisation des prestations de retraite pourrait jouer contre les plans de relance économique visant à soutenir la consommation en période de récession (voir ci-dessous). L'Allemagne a d'ores et déjà suspendu ces ajustements à titre temporaire et d'autres pays pourraient souhaiter lui emboîter le pas. Cela serait néanmoins beaucoup plus difficile aux Pays-Bas, car ces ajustements concernent des régimes professionnels par capitalisation à prestations définies (voir la discussion plus loin sur les questions de réglementation).

Il est cependant injuste de montrer du doigt ces quatre pays, car d'autres systèmes de retraite sont confrontés aux mêmes contraintes budgétaires et financières. Ces derniers sont simplement dépourvus de mécanismes automatiques permettant d'ajuster les droits à retraite en des temps aussi difficiles.

\section{Pouvoirs publics : mesures à prendre et écueils à éviter}

Depuis l'éclatement de la crise économique et financière, tous les pays de l'OCDE ont réagi à la montée du chômage et à l'aggravation de la détresse sociale en adoptant un certain nombre de mesures destinées à aider les personnes et les établissements en difficulté. Dans cette section, on examinera les différents domaines d'intervention et on formulera des recommandations concernant les mesures à court terme de nature à stabiliser les revenus des retraités et les systèmes de retraite sans perdre de vue les changements structurels nécessaires à plus long terme. La pression démographique et le vieillissement de la population n'ont nullement perdu leur caractère urgent et alarmant, même si la crise immédiate est aujourd'hui au centre de toutes les discussions. 


\section{Mesures concernant le marché du travail}

On note d'ores et déjà un essoufflement des marchés du travail et le chômage devrait remonter sensiblement à mesure que la crise économique se poursuit. Selon les dernières prévisions de l'OCDE publiées le 31 mars 2009 pour l'ensemble des pays membres, le chômage va probablement revenir de $6.0 \%$ de la population active en 2008 à $8.4 \%$ en 2009 et $9.9 \%$ en 2010 .

L'expérience des récessions précédentes laisse à penser que le fléchissement des marchés du travail touche plus durement les individus situés aux deux extrémités de la pyramide des âges. Les salariés les plus jeunes ont des difficultés à trouver un emploi à l'issue de leurs études. Les plus jeunes et les plus âgés sont souvent les premiers à perdre leur place en période de réductions d'effectifs et ils sont particulièrement exposés au chômage de longue durée. En ce qui concerne les revenus perçus une fois à la retraite, toutefois, les salariés les plus jeunes ont devant eux une longue carrière, ce qui leur permettra de compléter une retraite insuffisante. Ce n'est toutefois pas le cas pour les plus âgés. On étudiera dans cette section les effets potentiels des périodes de chômage sur les revenus qu'ils percevront durant leur retraite.

Les pouvoirs publics ont souvent assoupli les règles ou la gestion des retraites anticipées ou des pensions d'invalidité lors des précédentes phases de récession. Ils poursuivaient ainsi deux objectifs : premièrement, protéger les revenus des salariés les plus âgés qui perdent leur emploi et ne sont pas en mesure d'en trouver un autre; et deuxièmement, limiter l'augmentation du nombre de chômeurs inscrits. Si louables que soient les objectifs à court terme, les effets produits à long terme sur le marché du travail sont défavorables. Ainsi, de telles mesures ont été largement adoptées durant la récession du début des années 80 . Il en est résulté que les taux d'emploi sont restés faibles bien audelà de la reprise économique, essentiellement parce qu'il s'est avéré extrêmement difficile de revenir sur ces mesures ${ }^{10}$. Jusqu'à présent, les gouvernements n'ont cependant pas voulu adopter de telles politiques.

Dans les pays dotés de systèmes de retraite à cotisations définies étendus et ayant atteint une certaine maturité - les États-Unis en étant le principal exemple - les salariés pourraient souhaiter travailler plus longtemps pour reconstituer une partie de leur épargne-retraite. Une telle décision serait bénéfique sur trois plans : premièrement, en ajoutant des cotisations supplémentaires ; deuxièmement, en diminuant le nombre d'années de retraite devant être financées par le régime concerné ; et troisièmement, en laissant, peut-être, le temps nécessaire à un renchérissement des actifs. Un tel optimisme pourrait néanmoins être excessif. Les salariés plus âgés pourraient avoir de grandes difficultés à travailler plus longtemps car ils risquent de perdre leur emploi et, à mesure que le chômage s'aggrave, d'être dans l'incapacité d'en trouver un autre. De plus, 2009 a été à ce jour marquée par de nouvelles dépréciations des actifs et l'embellie pourrait bien se concrétiser trop tard pour changer la donne.

\section{Filets de protection des personnes âgées}

La crise économique et financière met en lumière et exacerbe la question des filets de protection assurant des prestations de retraite aux salariés à faible revenu et ayant subi des interruptions de carrière. Le graphique 1.5 fait apparaître les taux nets de remplacement pour les salariés ayant effectué une carrière complète dont le salaire est inférieur de moitié à la moyenne nationale. Le taux net de remplacement est le droit individuel à pension, 


\section{Graphique 1.5. Filets de protection des personnes âgées : taux nets de remplacement des salariés ayant effectué une carrière complète percevant $\mathbf{5 0} \%$ du salaire moyen}

Pourcentage des gains nets individuels

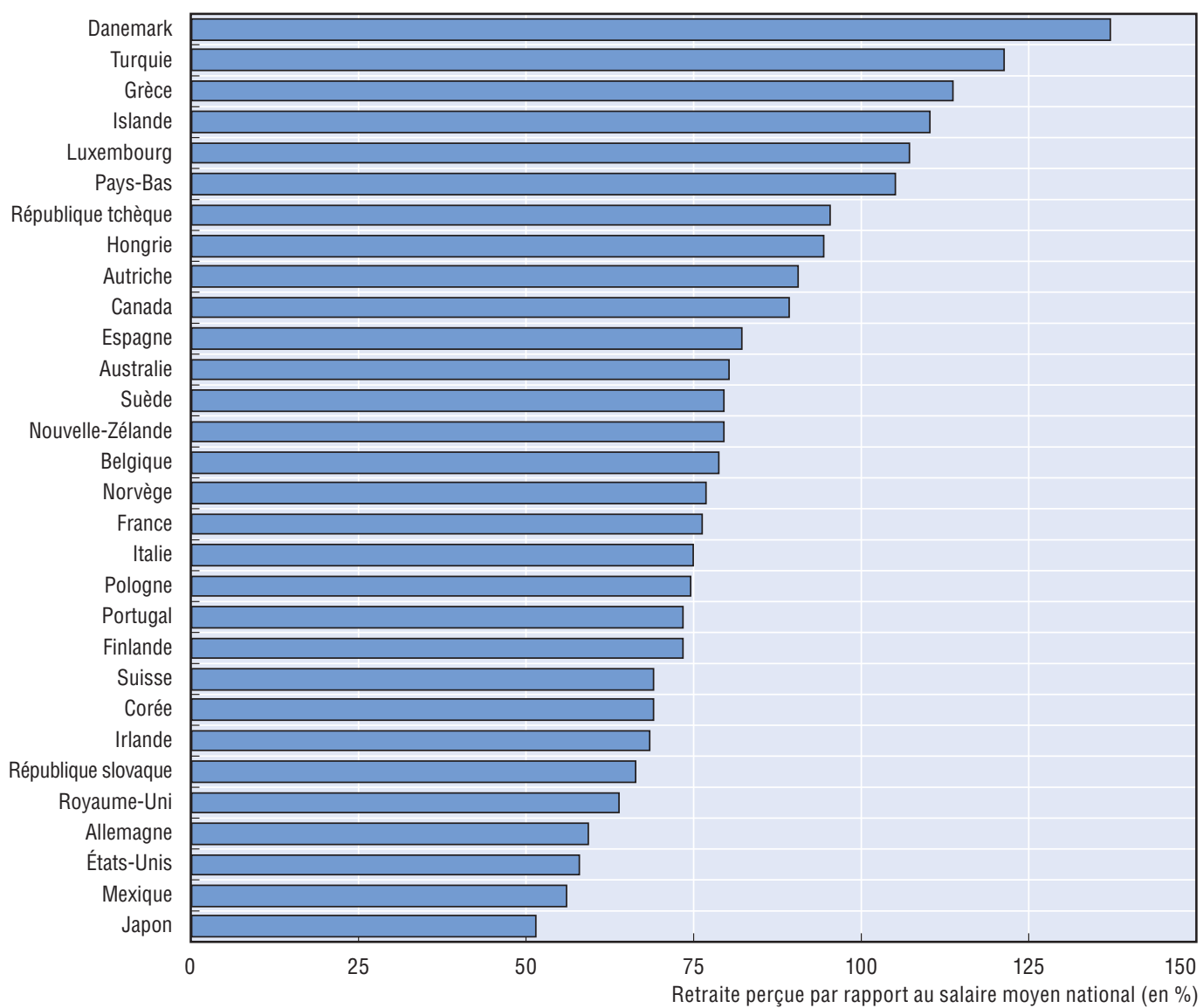

Source: Modèles de retraite de l'OCDE.

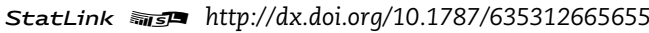

moins les impôts et cotisations, divisé par les gains individuels, là encore, nets : voir la discussion dans l'indicateur de la partie II).

L'Espagne a le même taux net de remplacement pour les travailleurs au bas de l'échelle salariale que la moyenne de l'OCDE, soit $82 \%$. Dans six pays, le taux de remplacement net pour ces salariés dépasse $100 \%$, ce qui signifie que le revenu net est plus élevé pendant la retraite que durant la période d'emploi. Cependant, les taux nets de remplacement ne dépassent pas $60 \%$ en Allemagne, au Japon, au Mexique et aux Etats-Unis. Quand on garde à l'esprit que ce calcul concerne les travailleurs à bas salaires, les revenus de remplacement ne représentent que la moitié du salaire moyen national : dans ces pays, les filets de protection pour les personnes âgées sont relativement faibles.

Si l'on tient compte d'une période de chômage de longue durée en fin de carrière ou d'une retraite anticipée, les revenus au titre de la retraite peuvent être encore plus bas. Sur des marchés du travail affaiblis, de nombreux travailleurs âgés pourraient être forcées de prendre une retraite anticipée ou subir des périodes de chômage de longue durée. Le chapitre spécial sur les "Réformes récentes des régimes de retraite " montre que 
l'Australie, la Belgique, la Corée, l'Espagne, la Finlande, la France, et le Royaume-Uni ont récemment décidé d'améliorer les filets de protection pour les personnes âgées (qui ne sont pas pris en compte dans ces calculs). D'autres pays, où les filets de protection sont faibles, devraient également prendre des mesures.

\section{Les retraites : une question à envisager à long terme}

Il ne fait aucun doute que 2008 aura été une mauvaise année pour les fonds de pension - voir le graphique 1.3 - et que 2009 n'aura, jusqu'à présent, été guère encourageante. Nombreux sont ceux qui ont à juste titre perdu confiance dans les pensions privées et certains responsables ont commencé à s'interroger sur le bien fondé du rôle croissant joué ces 20 dernières années par les régimes privés dans la majorité des pays de l'OCDE. Sans remettre en cause les difficultés réelles rencontrées par certaines personnes - en particulier celles qui s'approchent de l'âge de la retraite - il ne faut pas oublier que la question des retraites doit être envisagée sur le long terme.

Analysant 25 années de données sur les rendements des investissements dans les économies $\mathrm{du} G 7^{11}$ et en Suède, une simulation sur un horizon de 45 ans d'épargne-retraite d'une carrière à taux-plein montre un taux de rendement annuel réel de $5.5 \%$ pour les obligations et de $9.0 \%$ pour les actions. Le tableau 1.2 présente les résultats de la simulation pour un portefeuille " équilibré ", c'est-à-dire investi à parité en actions et en obligations. On suppose que ce portefeuille n'a pas évolué au fil de la carrière (on trouvera à l'encadré 1.4 les résultats obtenus pour différents portefeuilles). Les résultats tiennent compte des différences entre les rendements des marchés et ceux enregistrés par les particuliers sur leur épargne-retraite, en raison des frais administratifs, du coût de conversion du capital accumulé en annuités, etc. ${ }^{12}$. En conséquence, le rendement moyen (médian) calculé à l'aide de cette simulation s'établit à $5.0 \%$, contre $7.3 \%$ en moyenne sur les 25 dernières années.

\section{Encadré 1.4. Performances à long terme de différents types d'actifs}

Les données des graphiques 1.3 et 1.4 montraient que dans les pays où les retraites sont investies de façon prudente - en obligations d'État, par exemple - les pertes enregistrées en 2008 ont été nettement moins importantes que dans ceux où les actions occupent une plus grande place dans les portefeuilles des retraités.

L'analyse qui suit est davantage destinée à apprécier les effets des performances des investissements sur la durée de vie des cotisants qu'à se concentrer sur les résultats obtenus sur une année donnée. Cette simulation concerne des investissements réalisés sur 45 ans, sur la base de quelque 25 années de données correspondant à huit pays, soit jusqu'en 2006*. Notons que les résultats présentés dans le texte varient car les rendements utilisés tiennent compte des frais administratifs, etc. Les principaux résultats sont exposés au graphique 1.6. Celui-ci présente la fourchette des rendements simulés sur l'horizon à long terme de l'épargne-retraite en se concentrant sur les deux actifs fondamentaux des portefeuilles des fonds de pension : les actions et les obligations d'État. Aux extrémités gauche et droite de l'échelle horizontale, les rendements des portefeuilles composés exclusivement d'actions et d'obligations sont indiqués. Entre les deux, on trouve trois portefeuilles - qualifiés ici de "prudent ", d'“ équilibré » et de "dynamique »-qui associent ces deux types d'actifs dans des proportions diverses. 


\section{Encadré 1.4. Performances à long terme de différents types d'actifs (suite)}

La ligne blanche figurant au graphique 1.6 correspond au rendement réel médian : dans la moitié des cas, les rendements se situent au-delà et dans l'autre moitié, en deçà de cette ligne. Il s'établit à $7.3 \%$ pour un portefeuille équilibré, investi de façon égale en actions et en obligations d'État. Il est plus élevé - $8.9 \%$ - pour les actions et moins élevé - $5.2 \%$-pour les obligations. Les zones grisées du graphique correspondent à la probabilité d'occurrence de différents résultats, en fonction de l'expérience passée. Pour un portefeuille équilibré, les rendements réels ne devraient pas dépasser $5.5 \%$ par an dans $10 \%$ des cas. De même, ils devraient, selon les prévisions, excéder $9.0 \%$ par an dans $10 \%$ des cas également. Les zones grisées vont s'élargissant à mesure que s'accroît la part des actions dans le portefeuille, signe que les rendements sont soumis à une volatilité plus forte sur les marchés d'actions que sur ceux des obligations d'État.

\section{Graphique 1.6. Investissements : distribution des rendements moyens annuels simulés}

Taux de rendement réel annuel simulé (en \%)

Déciles de distribution

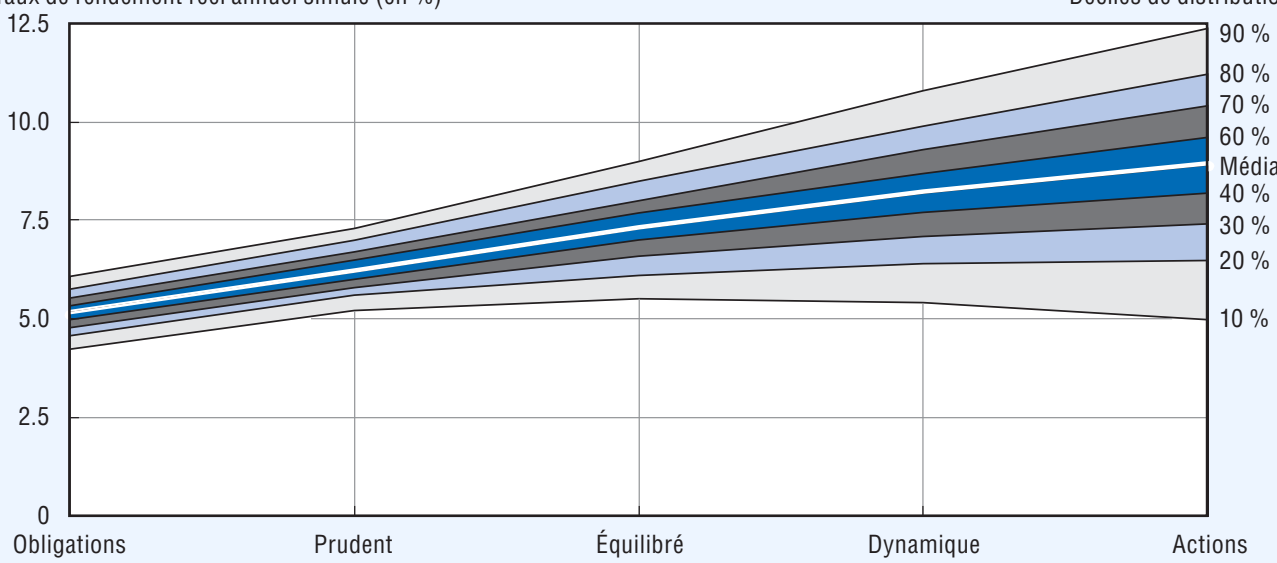

Note: Simulation effectuée à partir d'une série chronologique de données sur les rendements observés dans huit pays de l'OCDE, à savoir les économies du G7 plus la Suède. On suppose que le portefeuille dit prudent est investi à $25 \%$ en actions, le portefeuille équilibré à $50 \%$ et le portefeuille dynamique à $75 \%$. On présume que le solde est investi en obligations d'État à échéances diverses.

Source : D'Addio, A.C. et al. (2009), "Investment Risk and Pensions: Measuring Uncertainty in Returns ", Documents de travail de l'OCDE sur les affaires sociales, l'emploi et les migrations, $n^{\circ} 70$, sur la base de données de Thomson Financial Datastream.

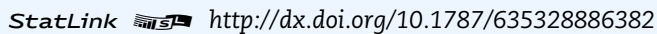

* Cette modélisation implique des calculs économétriques complexes fondés sur des séries chronologiques. Premièrement, on estime un modèle GARCH (Generalised Autoregressive Conditional Heteroskedasticity) à partir de la série chronologique des rendements mensuels des actions et des obligations (en tenant compte de l'évolution de la valeur des actifs et des sommes perçues au titre des dividendes et des intérêts). Deuxièmement, on applique aux résultats une méthode de simulation historique filtrée (Filtered Historical Simulation) pour obtenir les prévisions sur la gamme des résultats futurs. Les calculs présentés ici reposent sur 10000 simulations. Voir D'Addio, A.C. et al. (2009) pour plus de détails.

L'analyse porte également sur l'échelle des risques encourus et sur l'incertitude entourant les rendements des investissements. Ainsi, dans les $10 \%$ des cas les plus défavorables, les rendements devraient atteindre $3.2 \%$ par an à peine, voire moins. Dans les $10 \%$ des cas les plus propices, les rendements annuels s'élèvent au moins à $6.7 \%$ (première ligne du tableau 1.2). 


\section{Tableau 1.2. Distribution des rendements des investissements et des taux de remplacement futurs simulés}

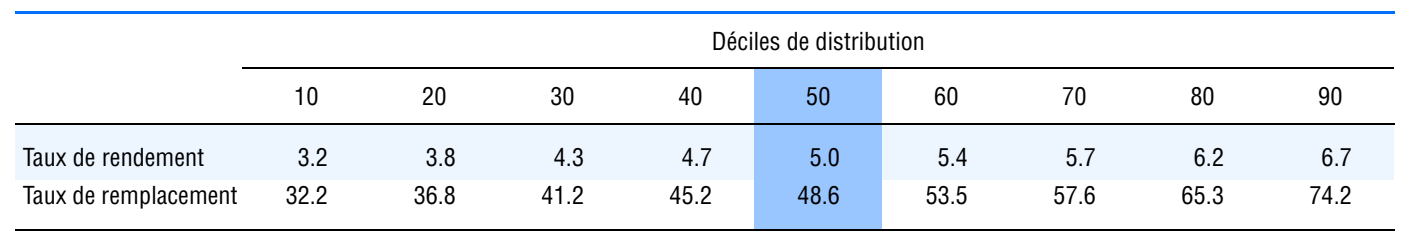

Note: Sur la base des taux de mortalité unisexe de la moyenne des pays de l'OCDE prévue pour 2040. La cotisation est présumée représenter $10 \%$ du salaire sur 45 ans.

Source : D'Addio, A.C. et al. (2009), « Investment Risk and Pensions: Measuring Uncertainty in Returns », Document de travail de l'OCDE sur les affaires sociales, l'emploi et les migrations, $n^{\circ} 70$, sur la base de données de Thomson Financial Datastream

Il faut cependant faire une mise en garde: les simulations reposent sur quelques 25 années de données, mais les séries analysées ne vont pas au-delà de la fin 2006. Plus récemment, on a observé à la fois des rendements sensiblement négatifs sur les marchés d'actions et une volatilité nettement supérieure. Cela étant, durant l'effondrement des marchés d'actions en 1987, pris en compte dans les données, les cours se sont repliés dans les mêmes proportions qu'en 2008. De plus, l'éclatement de la bulle technologique, qui a entraîné un net fléchissement des marchés boursiers en 2000-02, s'inscrit également dans la période considérée.

La fourchette des rendements des investissements à long terme ne semble pas particulièrement étendue. Néanmoins, le fait de calculer ces rendements sur l'horizon à long terme de l'épargne-retraite produit des effets très nets, ainsi qu'on le constate à la seconde ligne du tableau, qui présente le taux de remplacement pour ces différents niveaux de rendements. Le modèle repose sur des cotisations versées chaque année durant une carrière complète à hauteur de $10 \%$ du salaire individuel. L'épargne-retraite accumulée est convertie en flux de pension sur la base des taux de mortalité moyens de l'OCDE prévus pour 2040. Le résultat obtenu est présenté sous forme de taux de remplacement, soit le ratio des pensions de retraite sur le salaire perçu durant la vie active.

Le taux de remplacement correspondant au taux de rendement moyen (médian) pour cette cotisation de $10 \%$ du salaire s'établit à $49 \%$. Cela signifie que dans la moitié des cas, le taux de remplacement serait supérieur et, dans l'autre moitié, inférieur. Néanmoins, dans les $10 \%$ des cas les plus défavorables, le taux de remplacement n'excède pas $32 \%$. Dans les $10 \%$ les plus propices, la pension servie représente $74 \%$ du salaire. Cette fourchette de 32-74 \% est extrêmement étendue. Elle va d'une retraite « confortable » à une pension « à la limite du seuil de pauvreté ».

\section{Le choix des supports d'investissement}

Dans la majorité des régimes de retraite à cotisations définies, les adhérents peuvent choisir la composition de leur portefeuille. En Australie, par exemple, quelque $85 \%$ des habitants adhèrent à un dispositif proposant différents supports d'investissement, soit en règle générale cinq fonds différents. Néanmoins, les particuliers sont désormais libres de sélectionner leur prestataire de retraite : le nombre des produits proposés par les fonds communs d'épargne-retraite s'établit en moyenne à 58. On constate également un élargissement du choix offert par les régimes à cotisations définies les plus courants aux États-Unis. À la fin des années 70, 16 \% seulement proposaient différents supports, mais en 1994, ce chiffre avait déjà atteint $94 \%$. Plus de la moitié des dispositifs proposent 
désormais cinq types de supports ou davantage. Au Mexique et en République slovaque, chaque prestataire est tenu de présenter un échantillon de fonds assortis de différentes caractéristiques risque-rendement. En raison de la crise, la Pologne a annoncé récemment son intention d'introduire un éventail similaire de supports d'investissement.

Pour les régimes à prestations et à cotisations définies, la réglementation des investissements réalisés par les fonds de pension a été assouplie au cours des dix dernières années. Ainsi, seuls 12 pays de l'OCDE continuent d'imposer des limites quantitatives sur les investissements en actions. Néanmoins, elles sont relativement élevées et fixées en moyenne à $52 \%$ du portefeuille ${ }^{13}$. Les gestionnaires de fonds de pension peuvent de ce fait diversifier leurs portefeuilles.

Les choix d'investissement opérés par les particuliers auront des implications importantes sur les effets produits par la crise sur leur retraite. Le graphique 1.4 s'intéressait à cette question d'un point de vue global, en comparant les performances d'ensemble des fonds de pension à la proportion des actifs investis au total dans les actions. L'analyse qui suit concerne les choix d'investissement individuels et leur incidence sur l'action des pouvoirs publics.

Le graphique 1.7 montre qu'aux États-Unis, les particuliers ont tendance à délaisser les actions au profit de placements moins risqués à mesure qu'ils s'approchent de l'âge de la retraite. Ainsi, $55 \%$ environ des $36-45$ ans investissent à plus de $70 \%$ dans des actions, et ce chiffre revient à $43 \%$ pour les 56-65 ans. La part des actions dans le portefeuille de ces salariés plus âgés, proches de la retraite, semble très élevée : plus d'un sur cinq détient plus de $90 \%$ de son plan 401(k) sous forme d'actions. Il est naturellement impossible d'évaluer le caractère risqué des décisions financières des particuliers dans leur ensemble : ils peuvent, par exemple, détenir des dépôts et des obligations moins risqués hors de leur plan 401(k). Mais cette indication est préoccupante, car ces salariés proches de l'âge de la retraite auront subi une

\section{Graphique 1.7. Part des plans 401(k) investie en actions aux États-Unis par âge}

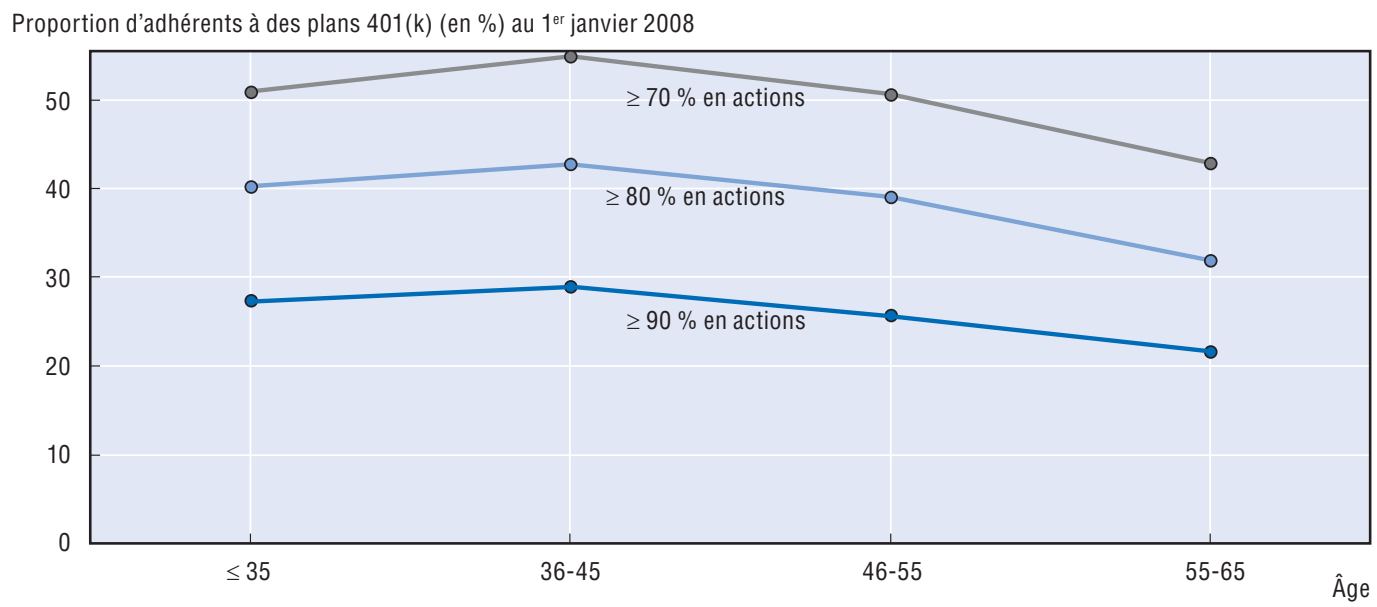

Note : Données concernant les 21 millions de participants à des plans 401(k) recensés dans la base de données de l'Employee Benefit Research Institute (EBRI) et de l'Investment Company Institute (ICI). La part totale investie en actions correspond à la somme des fonds en actions, des actions de chaque employeur détenues en portefeuille et de la fraction correspondante des supports d'investissement mixtes (fonds équilibrés et à horizon).

Source : VanDerhei, J. (2009), "The Impact of the Recent Financial Crisis on 401(k) Account Balances ", Issue Brief $\mathrm{n}^{\circ}$ 326, Employee Benefit Research Institute, Washington, DC. 
forte érosion de leur épargne-retraite par rapport à la minorité qui avait investi la plus grande partie de son portefeuille dans des actifs présentant un moindre risque.

Quelle est l'incidence de ce type de comportements sur l'action des pouvoirs publics ? Faut-il limiter les options proposées aux particuliers pour éviter que leur épargne-retraite soit réduite à néant ? Ou doit-on considérer qu'il appartient à chacun de prendre une telle décision et un tel risque?

Les salariés doivent en toute logique canaliser leur épargne-retraite vers des investissements moins risqués à mesure qu'ils s'approchent de la retraite (et donc de l'instant où ils devront puiser dans cette épargne). Les publications financières illustrent parfaitement ce point ${ }^{14}$.

Par conséquent, les pouvoirs publics devraient à tout le moins encourager les particuliers à adopter une telle stratégie, fréquemment qualifiée d'investissement fondé sur le cycle de vie, qui peut réduire le risque d'investissement encouru sur l'ensemble de la vie active sans sacrifier les rendements financiers offerts par un portefeuille plus diversifié en début de carrière. De fait, il serait logique que ce transfert soit automatique et corresponde à l'option par défaut. Adopter la stratégie fondée sur le cycle de vie par défaut revient à mettre les investissements sur "pilote automatique " et s'avère particulièrement utile pour les personnes qui ne souhaitent pas gérer leur portefeuille de façon active (soit la majorité, selon toute probabilité). Une telle mesure peut être prise tout en préservant la liberté de choisir entre différents portefeuilles assortis de différentes caractéristiques risque-rendement (pour la minorité qui souhaite prendre ses propres décisions quant aux supports d'investissement).

\section{Le choix des prestations}

Comme on l'a vu, les détenteurs de contrats de rente viagère ont été moins touchés par la crise, leur revenu au titre de la retraite étant déjà assuré et garanti à coup sûr jusqu'à la fin de leur vie. Les adhérents aux régimes à cotisations définies sont souvent tenus d'opter pour une sortie en rente au moment du départ en retraite. Une forte exposition du fonds aux actions juste avant la retraite risque d'entraîner un abaissement sensible de leur niveau de vie après une crise financière. Dans ces circonstances, les restrictions sur les choix d'investissement - les limitant aux options présentant un moindre risque - peuvent s'avérer judicieuses pour les personnes qui s'approchent de l'âge de la retraite.

Les autorités doivent aussi se demander s'il y a lieu d'imposer aux particuliers de convertir en rente le capital constitué dans le cadre d'un régime à cotisations définies à un instant ou à un âge donné. Le risque encouru est d'entériner des valeurs très faibles pour les actifs, et donc une diminution des prestations perçues durant toute la retraite. Certains pays autorisent d'ores et déjà les "retraits échelonnés ", une fraction déterminée des avoirs pouvant ainsi être retirée chaque année. D'autres pourraient aussi adopter cette approche plus souple. L'Irlande, par exemple, autorisera les retraités à retirer une somme forfaitaire de leurs comptes retraite et ils auront une période de deux ans pour transformer le solde de leurs comptes en annuités.

Les retraits échelonnés ne vont toutefois pas sans risque : s'agissant des personnes qui s'approchent de l'âge de la retraite, certaines restrictions sur les choix d'investissement s'imposent après le départ en retraite. De plus, les règles déterminant les sommes pouvant être retirées chaque année doivent être fixées avec soin pour éviter que les personnes se retrouvent dans la détresse à un âge très avancé. Les pouvoirs publics doivent également envisager d'associer retraits échelonnés et rente différée, une option qui serait choisie lors 
du départ à la retraite, et qui serait versée uniquement lorsque l'intéressé aurait atteint un âge avancé (entre 75 et 85 ans, par exemple).

À court terme, les autorités pourraient donc souhaiter assouplir l'obligation de conversion en rente en attendant un redressement du prix des actifs. À long terme, toutefois, cette formule doit être privilégiée pour protéger les individus contre un certain nombre d'incertitudes : risque d'investissement, risque de longévité (épargne-retraite insuffisante) et, pour les rentes indexées, risque d'inflation.

\section{Le choix du régime de retraite}

Compte tenu des turbulences qui ont frappé les marchés financiers et des pertes subies par les régimes de retraite privés, des pressions s'exercent d'ores et déjà pour que l'on autorise les cotisants à revenir dans le régime public ${ }^{15}$. C'est notamment le cas en République slovaque. Un délai de six mois, soit de janvier à juin 2008, a été laissé aux particuliers pour réintégrer, à leur choix, le régime public. Ce délai a été étendu par la suite, même si durant le premier semestre 2008, 6 \% seulement des adhérents aux dispositifs privés étaient revenus sur leur décision.

La lenteur de ce démarrage pourrait s'expliquer par un phénomène d'inertie, mais aussi indiquer que les particuliers apprécient la diversification des prestations de retraite apportée par les pensions privées. Quelque $60 \%$ des salariés slovaques ont opté pour le régime à cotisations définies lors de la réforme. Or, le risque de tarissement du flux de nouveaux cotisants pèse davantage sur le développement à long terme du système de retraite slovaque. Les nouveaux entrants sur le marché du travail ne sont plus tenus d'adhérer aux fonds privés. Ici, l'inertie pourrait produire l'effet inverse, en diminuant le nombre de personnes qui choisissent les pensions privées. Étant donné que, selon la réglementation en vigueur, la décision est irréversible, les répercussions sur le montant des retraites des nouveaux entrants sur le marché du travail se feront sentir à long terme.

Il a été longuement question d'autoriser les particuliers à réintégrer le régime public dans d'autres pays ayant mis en place des régimes privés obligatoires, et tout particulièrement en Europe centrale et orientale (l'Argentine est allée encore plus loin en nationalisant ses fonds de pension : voir l'encadré 1.5)

\section{Encadré 1.5. Nationalisation des pensions privées : le cas de l'Argentine}

Le gouvernement argentin a nationalisé ses fonds de pension obligatoires, valorisés à 30 milliards USD (23 milliards EUR), en octobre 2008. Il a présenté cette prise de contrôle au public comme un moyen de protéger les cotisants contre une gestion prétendument défectueuse durant la crise financière mondiale. Il est toutefois clair qu'il était notamment motivé par la volonté de remplir les coffres de l'État à l'heure où une part importante de la dette publique allait arriver à échéance.

L'impact économique de cette mesure n'est pas aussi net qu'on pourrait le croire à première vue, premièrement parce que l'Argentine gère un vaste régime de retraite public, à l'inverse d'autres pays d'Amérique latine comme le Chili et le Mexique (voir Whitehouse, 2007), et deuxièmement parce que beaucoup de ces actifs des fonds de pension étaient déjà investis dans des titres de dette publique non négociables.

Quoi qu'il en soit, ce revirement porte incontestablement atteinte à la stabilité et à la viabilité du régime de retraite. 
De telles mesures nuisent à la stabilité du système de retraite. Premièrement, elles ne font que différer les modifications nécessaires de la ligne d'action à tenir. Même si dans la morosité ambiante, une telle proposition peut sembler relever d'un optimisme excessif, si les rendements des investissements s'avéraient dans quelques années supérieurs et non inférieurs de 25 points à 0 , les particuliers pourraient souhaiter revenir au régime privé avec effet rétroactif.

Deuxièmement, le fait de permettre aux cotisants de racheter leurs droits à retraite au titre du régime public à l'aide des actifs de leur régime privé qui se sont dépréciés stimulera à court terme les finances publiques du fait du transfert des fonds. De nombreux gouvernements à court de liquidités accueilleront favorablement ces gains à court terme, mais à moyen et long terme, le coût induit pour les dépenses au titre des retraites publiques sera supérieur à ces gains à court terme.

Le motif de ces changements a d'abord été la crise fiscale qui a résulté de la crise économique et financière. Il n'est pas surprenant que les gouvernements veuillent combler les déficits par les revenus des cotisations qui vont aux retraites privées. Une façon plus rationnelle d'atteindre cet objectif est de réduire provisoirement les cotisations destinées aux retraites privées. Bien qu'aucun pays de l'OCDE n'ait adopté cette stratégie, elle va par exemple être mise en place en Lettonie et en Lituanie.

\section{Un choix éclairé}

La crise financière a également remis au centre du débat les questions de l'information des investisseurs et de l'éducation financière. Rares sont les personnes à être bien informées sur les retraites, et à disposer notamment de données générales sur la structure du système de retraite et d'informations précises sur leurs propres droits à retraite. Cela vaut pour tous les types de systèmes de retraite.

La responsabilité de la planification des revenus qu'ils percevront à la retraite incombe davantage aux particuliers dans les régimes privés que dans les dispositifs publics. Ils peuvent ainsi être tenus de sélectionner un prestataire de retraite parmi plusieurs concurrents ou un portefeuille d'investissements parmi d'autres. Et dans de nombreuses réformes des retraites, ils ont pu choisir d'adhérer au dispositif à cotisations définies ou de rester affiliés à un régime public lié à la rémunération (voir l'encadré 1.2 ci-dessus).

La mise en place d'une communication efficace de la part des prestataires et de programmes d'éducation financière tournés vers le plus grand nombre peut aider les personnes concernées à faire un choix éclairé quant à leur régime de retraite. Ce point a été mis en évidence par la crise actuelle, le risque étant que les intéressés prennent des décisions en fonction des perspectives à court terme produisant des effets négatifs à long terme. La crise a incontestablement érodé la confiance du public dans les pensions privées, entraînant un risque que les salariés soient plus réticents que jamais à constituer une épargne-retraite.

\section{Garanties publiques}

Les gouvernements doivent-ils sauver les comptes de retraite individuels comme ils l'ont fait pour les banques ? Ainsi qu'on l'a observé précédemment, ils soutiennent déjà les régimes professionnels à prestations définies dans de nombreux pays. L'analyse qui suit porte essentiellement sur les dispositifs à cotisations définies (le seul cas de sauvetage observé dans la pratique - celui d'Israël - est étudié à l'encadré 1.6). 


\section{Encadré 1.6. Anatomie d'un plan de sauvetage des retraites : le cas d'Israël}

Le gouvernement israélien va indemniser les salariés proches de l'âge de la retraite qui ont perdu de l'argent dans le cadre d'un régime de retraite professionnel facultatif (appartenant à trois types différents, à savoir les fonds de prévoyance, les régimes d'assurance des cadres dirigeants et les fonds de pension budgétés). Les salariés doivent être âgés de 57 ans au moins pour pouvoir prétendre à cette indemnisation, et avoir investi dans des fonds non garantis. La garantie s'appliquera au capital constitué à hauteur de 350000 USD et couvrira les pertes subies à concurrence de la moitié de ce montant.

Selon les prévisions du gouvernement, quelque 200000 personnes pourraient en bénéficier à terme (soit environ $15 \%$ des plus de 55 ans). Le coût total est estimé aux alentours de 37 milliards USD sur 13 ans, et devrait représenter approximativement $23 \%$ du produit intérieur brut (PIB) annuel.

Néanmoins, l'indemnisation se limite aux pertes subies depuis novembre 2008, de sorte qu'il s'agit davantage d'une garantie contre les pertes à venir que d'un renflouement des fonds de pension visant à compenser les effets de la crise financière à ce jour.

En Israël, les retraites professionnelles sont particulièrement développées ; il s'agit principalement de régimes à cotisations définies, un seul fonds couvre l'ensemble des salariés et les supports d'investissement sont imposés. Les actifs des fonds de pension étaient valorisés à $33.6 \%$ du PIB en 2007 (OCDE, 2009a, graphique 2.25).

Les arguments plaidant en faveur d'une intervention de l'État reposent principalement sur la conception du système des retraites. Ils sont moins probants dans les pays qui disposent d'un vaste régime public. Lorsque les adhérents peuvent choisir leurs supports d'investissement - en particulier quand les investissements sont reportés par défaut sur des actifs moins risqués à mesure qu'ils s'approchent de l'âge de la retraite il est là aussi plus difficile de justifier une telle intervention.

À l'opposé, les gouvernements peuvent être soumis à une obligation morale, sinon légale, d'intervenir lorsque les régimes à cotisations définies sont obligatoires et non facultatifs et que la conversion en rente lors du départ à la retraite est obligatoire.

Un plan de sauvetage direct, visant à renflouer les comptes de retraite des salariés, pourrait s'avérer extrêmement onéreux. De plus, ce coût devrait être supporté alors que les finances publiques sont mises à rude épreuve par la récession et les plans de relance économique. Selon les prévisions de l'OCDE, le besoin de financement net des pays membres, en moyenne, reviendra d'un point bas de $1.3 \%$ du produit intérieur brut (PIB) en 2006 à $7.2 \%$ en 2009 et $8.7 \%$ en 2010. Ces prévisions, publiées le 31 mars 2009, montrent déjà que l'état des finances publiques est parmi les plus catastrophiques enregistrés en temps de paix. En outre, à court terme du moins, les fonds mobilisés pour renflouer les comptes de retraite seraient utilisés à des fins d'épargne et ne viendraient donc guère à l'appui de la demande intérieure durant la phase de récession.

Inversement, soutenir l'épargne-retraite de ceux qui sont le plus touchés par la crise par le biais du système de retraite public présenterait l'avantage de répartir le coût de cette mesure dans le temps. Les sommes débloquées seraient dépensées tout au long de la retraite des personnes concernées et non en une seule fois aujourd'hui ou lors de leur départ en retraite. On gagnerait également en efficacité et en flexibilité, puisque l'aide pourrait être ciblée sur les retraités à faible revenu, par exemple. 
Tout sauvetage direct des fonds de pension présente également un risque d'aléa moral, car en comptant qu'une telle mesure sera prise au prochain incident, chacun sera incité à prendre davantage de risques une fois que la crise en cours sera passée.

Il serait particulièrement judicieux de renflouer les personnes qui sont proches de l'âge de la retraite. Toutefois, cela soulève des difficultés politiques considérables. En limitant ce plan aux salariés qui sont à quelques années de l'âge normal de la retraite, ceux qui sont légèrement en deçà de l'âge décisif se sentiraient lésés. De même, les retraités ayant converti leur capital en rente tout récemment, entérinant ainsi les moins-values observées sur les marchés financiers, reprocheraient aux autorités d'indemniser les personnes de leur âge dont l'argent est toujours investi sur ces marchés.

Pour toutes ces raisons, il convient d'éviter toute mesure ad hoc visant à garantir les rendements des investissements ou à indemniser les personnes ayant subi des moinsvalues. Par souci d'équité et compte tenu des obstacles budgétaires au financement direct des fonds de pension, les gouvernements doivent s'appuyer sur leurs régimes publics pour que les rendements négatifs enregistrés par les fonds de pension sur un an ne soient pas synonymes de pauvreté pour toute une génération de retraités. Indemniser les personnes concernées en leur versant des prestations de retraite publiques permet d'étaler le coût tout au long de la retraite des intéressés, d'apaiser les tensions politiques et de tempérer les problèmes d'aléa moral.

\section{Les systèmes de retraite, moteur de la reprise économique et financière}

Les systèmes de retraite pourraient contribuer au redressement de l'activité. Premièrement, les retraites publiques pourraient bénéficier de mesures budgétaires visant à stimuler l'économie. Deuxièmement, de nombreux pays disposent d'avoirs conséquents dans des dispositifs de retraite privés et des fonds de réserve publics. Troisièmement, les fonds de pension continuent de jouer un rôle important sur les marchés financiers en tant qu'investisseurs à long terme.

Les retraites publiques font partie intégrante des plans de relance économique annoncés dans certains pays. Au Royaume-Uni, par exemple, les retraités ont d'ores et déjà perçu au moins 110 GBP supplémentaires et l'indexation de la retraite de base et du crédit de retraite, ciblés sur les personnes âgées à faible revenu, sera plus généreuse, atteignant 300 millions GBP par an sur la période $2009-12^{16}$. En Australie, le plan de relance prévoit également des versements supplémentaires au profit des retraités. À titre exceptionnel, les retraités célibataires ont perçu 1400 AUD et les couples 2100 AUD en décembre 2008. Les États-Unis verseront 250 USD à tous les bénéficiaires de retraites publiques en mai 2009 pour un coût total de 13 milliards USD. La Grèce a également accordé une somme exceptionnelle aux faibles revenus, retraités inclus, comprise entre 100 et 200 EUR. La Belgique, l'Espagne et la France ont toutes trois annoncé des revalorisations supplémentaires des retraites minimum. En Espagne, par exemple, la hausse a atteint $6.4 \%$.

Dans tous ces cas de figure, ce coup de pouce supplémentaire sera particulièrement utile aux retraités à faible revenu, ce qui devrait contribuer à réduire la pauvreté des personnes âgées. Ainsi, en Australie, le taux de pauvreté des personnes âgées est plus de deux fois supérieur à celui de la population totale et les personnes âgées risquent aussi de s'appauvrir en Grèce (voir le chapitre spécial sur « Les revenus et la pauvreté des personnes âgées "). D'autres pays affichant un taux de pauvreté élevé parmi les personnes âgées pourraient également envisager d'effectuer des versements supplémentaires à court terme 
en leur faveur dans le cadre de plans de relance économique. Des mesures moins orthodoxes visant à assurer que ces plans soutiennent la demande intérieure sont analysées à l'encadré 1.7 .

Le deuxième rôle joué par les retraites en tant que moteur de la reprise économique serait de permettre aux autorités d'utiliser les avoirs des fonds de pension et de réserve pour soutenir la demande. Dans l'idéal, bien sûr, ces avoirs seraient affectés à une utilisation précise et préservés dans le seul souci de procurer des revenus aux retraités. Mais la situation actuelle est loin d'être idéale.

L'Australie autorise les particuliers à disposer de leur épargne-retraite privée pour éviter une saisie de leur bien immobilier en cas d'arriéré de remboursement de leur prêt. L'accès aux comptes de retraite est contrôlé afin de s'assurer que toutes les autres solutions permettant de remédier aux arriérés ont été épuisées. On peut difficilement faire valoir que les particuliers doivent utiliser leur épargne-retraite dans un but exclusif, au risque de perdre leur logement.

\section{Encadré 1.7. Relance économique, demande intérieure et consommation}

Le risque lié aux plans de relance budgétaire et monétaire est que les particuliers utilisent les sommes allouées pour équilibrer leurs comptes, atténuant ainsi les effets attendus sur la consommation et partant, sur la demande intérieure. Par le passé, le Japon a fait l'expérience des "bons d'achat", distribués afin de soutenir la consommation durant une phase de récession. Les bons remis aux ménages à faible revenu constitueront une part non négligeable du plan de relance de la Corée. Le taux de pauvreté des personnes âgées s'y établissant à $45 \%$ - contre moins de $15 \%$ pour la population totale - les retraités en seront probablement les principaux bénéficiaires (voir le chapitre spécial sur « Les revenus et la pauvreté des personnes âgées » dans la présente publication). L'Italie a émis 0.5 million de " cartes sociales » en faveur des personnes âgées et des familles avec enfants à faible revenu. Cette carte permet d'acquérir des biens et services de base à hauteur de 40 EUR par mois.

Le concept de ces programmes de bons, qui sont destinés à assurer que l'argent de la relance est dépensé et non épargné, s'inscrit dans une longue tradition. Pendant la Grande dépression des années 30, il avait été proposé, pour accroître la consommation, de verser chaque mois une somme forfaitaire aux retraités de 60 ans et plus. Cette somme aurait été versée sous forme de bons assortis d'un code couleurs garantissant qu'ils étaient arrivés à échéance et qu'ils seraient dépensés avant le 5 du mois suivant. Le plan Townsend, du nom de son fondateur, un médecin californien, a rapidement pris de l'ampleur après qu'il eut adressé un long courrier à un journal local. Un mouvement s'est bientôt développé à l'échelle nationale, sous le slogan allitératif " peace and prosperity thru pensions " (la paix et la prospérité par les pensions de retraite). L'introduction de la Sécurité sociale (retraites publiques) par l'administration Roosevelt, en 1935, est pour une bonne part à mettre au crédit de cette campagne (Amenta, 2006).

Le plan Townsend n'a pas été adopté pour deux grandes raisons. Premièrement, le montant de la pension forfaitaire était très élevé. Deuxièmement, on a estimé que le financement, qui devait être assuré par une taxe nationale sur les ventes, favorisait les grandes entreprises intégrées verticalement. Quoi qu'il en soit, c'est un exemple des moyens qui permettent de s'assurer que la relance budgétaire accroît la demande intérieure, ce qui revête une importance cruciale pour la majorité des économies asiatiques, au sein et hors de l'OCDE. 
Le Danemark autorisera l'accès anticipé au capital accumulé dans le plan "spécial retraite ". Le montant est relativement faible - 14600 DKK soit 2600 USD - car le taux de cotisation est de $1 \%$ des gains et que les cotisations ont été suspendues depuis 2004. Le gouvernement espère qu'environ un quart des personnes retireront leur capital.

L'Islande va autoriser les particuliers à puiser dans l'épargne-retraite qu'ils ont constituée dans le cadre de régimes professionnels, sans toucher à celle qui est nécessaire pour financer le taux de remplacement obligatoire. Elle va aussi leur donner la possibilité d'utiliser les avoirs constitués grâce aux cotisations facultatives pour atténuer leurs difficultés financières ${ }^{17}$. Selon les estimations du ministère des Finances (2009), quelque 75 milliards ISK pourront être mis à profit, soit plus de $5 \%$ du produit intérieur brut (PIB). En Islande, le taux de remplacement du régime privé obligatoire est nettement supérieur à la moyenne des pays de l'OCDE, si bien que le fait d'autoriser les intéressés à disposer de cette épargne-retraite supplémentaire n'est en rien préjudiciable à l'adéquation des revenus perçus au titre de la retraite.

Aux États-Unis, 90 \% environ des adhérents aux plans 401(k) sont autorisés à souscrire un emprunt sur leur compte. En 2007, seuls $18 \%$ des personnes habilitées avaient contracté un prêt dont le montant s'élevait en moyenne à $12 \%$ seulement du solde du compte (VanDerhei et al., 2008). Ces deux chiffres sont demeurés relativement constants dans le temps, malgré les fluctuations cycliques de l'économie. Une analyse détaillée donne à penser qu'il est fait un usage responsable de cette ligne de crédit : les emprunts souscrits sur les comptes d'épargne-retraite sont peu importants et remboursés (voir Kusko et al., 1998 ; et General Accounting Office, 1997). Aux termes de la loi, les remboursements sont soumis à intérêt au taux du marché, faute de quoi le prêt est considéré comme un retrait anticipé et soumis à des pénalités fiscales. Les retraits anticipés sont autorisés, sans pénalité fiscale, dans des situations particulièrement délicates qui sont rigoureusement définies. Cependant, les « fuites » de l'épargne-retraite déjà affectée se produisent pour l'essentiel lorsque les intéressés changent d'employeur. Les deux-tiers environ des personnes concernées ne transfèrent par les avoirs de leur plan 401(k) vers un autre régime de retraite, en dépit des pénalités fiscales, même s'il s'agit habituellement de montants peu importants (les deux tiers des avoirs sont transférés).

La Norvège, qui dispose du deuxième fonds souverain au monde, va puiser dans ces réserves pour financer un plan de relance budgétaire représentant au total $2.3 \%$ du PIB. Ce fonds, qui porte le nom de "Fonds de pension d'État - Mondial " peut aussi servir à renflouer les banques. Le plan norvégien de recapitalisation des banques a coûté à ce jour $13.8 \%$ du PIB, ce qui le place au deuxième rang mondial en proportion du revenu national. L'Irlande, qui va également utiliser son fonds de réserve pour les retraites afin de recapitaliser les établissements bancaires (voir ci-dessus), a dépensé à ce jour pratiquement autant que la moyenne des pays du G20, soit $5.3 \%$ du $\mathrm{PIB}^{18}$.

Une fois encore, dans l'idéal, ce fonds servirait exclusivement à couvrir le coût futur du vieillissement au titre des retraites et des dépenses de santé. Dans la pratique, la recapitalisation des banques va prélever un lourd tribut sur les finances publiques. Sur le plan économique, il est difficile de faire la différence entre les gouvernements qui puisent dans ces fonds et ceux qui émettent des obligations pour faire face à ces coûts, car la situation financière globale du secteur public demeure inchangée. Le principal danger est que cela crée un précédent et que ces fonds soient continuellement mis à contribution chaque fois que les gouvernements se retrouvent à court de liquidités, laissant ainsi les 
coffres vides au moment où les pressions financières dues au vieillissement de la population se feront plus vives.

Troisièmement, les fonds de pension pourraient contribuer à la stabilisation des marchés financiers. Les régimes de retraite privés ont généralement des besoins de liquidités très restreints (versement des prestations et couverture des frais administratifs) par rapport à la totalité de leurs actifs sous gestion et à d'autres investisseurs institutionnels. Les fonds de pension s'inscrivent également sur des horizons d'investissement lointains. La principale exception à la règle des faibles besoins de liquidités et de l'horizon lointain vient des régimes à prestations définies qui sont fermés aux nouveaux membres. Ceux-ci prélèvent fréquemment une part de leur stock d'actifs pour servir les prestations de retraite. Ils occupent une place importante dans des pays comme les États-Unis et le Royaume-Uni où le passage aux régimes à cotisations définies a été le plus rapide (voir l'analyse à l'encadré 1.1 ci-dessus).

En règle générale, les fonds de pension n'ont donc pas besoin de céder des actifs aux prix bas actuellement en vigueur pour honorer leurs engagements, car ils bénéficient d'un flux continu de cotisations et de produits de placements. Tout dépend néanmoins de la réglementation applicable aux fonds de pension et en particulier aux dispositifs à prestations définies (ce point sera examiné dans la section suivante). L'évolution des normes comptables et réglementaires, notamment celles qui contraignent les fonds de pension et les établissements promoteurs à tenir compte des accès de faiblesse du prix des actifs, pourrait limiter le rôle joué par les fonds de pension en vue de tempérer les turbulences financières.

\section{La réglementation des régimes à prestations définies}

Les pouvoirs publics imposent usuellement des règles de financement ou de solvabilité aux régimes professionnels à prestations définies. Ces règles, dont l'application est habituellement contrôlée par des organismes de tutelle indépendants, doivent servir à s'assurer que les actifs actuellement détenus par le fonds de pension suffiront à répondre aux engagements futurs, principalement sous la forme des pensions versées aux retraités d'aujourd'hui et de demain. Les règles de financement ont été durcies ces dernières années dans plusieurs pays. De fait, certains employeurs promoteurs versent encore des cotisations supplémentaires afin de compenser les carences dues au repli des marchés financiers en 2000-02.

Les autorités ont couramment fait face à la crise actuelle en prolongeant les « périodes de redressement » durant lesquelles les régimes à prestations définies doivent rétablir leur solvabilité. Cela paraît logique durant une phase de récession, où la rentabilité des entreprises diminue et où l'accès au crédit est sensiblement restreint. La trésorerie des sociétés est déjà soumise à rude épreuve, si bien que contraindre les employeurs à relever les cotisations à des régimes de retraite sous-capitalisés ne ferait qu'envenimer la situation. Une telle mesure pourrait même être préjudiciable à la solvabilité des établissements promoteurs, ce qui à l'évidence n'est pas dans l'intérêt des bénéficiaires ${ }^{19}$.

Les autorités de tutelle des Pays-Bas ont porté les périodes de redressement de trois à cinq ans, même si elles s'en sont tenues cette année à la date butoir du $1^{\mathrm{er}}$ avril pour les grandes lignes des plans de redressement des régimes. Les propositions récentes visant à relever l'âge de la retraite sont notamment destinées à limiter les ajustements nécessaires aux taux de cotisation et à l'indexation des retraites servies et des prestations de retraite 
constituées par les salariés (voir aussi l'encadré 1.3). En Irlande, les autorités de tutelle ont adopté un éventail de mesures destinées à aider les régimes professionnels insolvables: i) délai supplémentaire accordé pour remplir les propositions de redressement; ii) allongement des délais pour les plans de redressement, soit dix ans ou plus en cas de besoin ; iii) prise en compte des garanties facultatives de l'employeur. Le gouvernement a également récemment annoncé des plans de protection des travailleurs avec des droits à pension augmentés quand les plans professionnels sont abandonnés du fait de l'insolvabilité des employeurs. Aux États-Unis, le Pension Protection Act (Loi sur la protection des retraites) prévoyait un assouplissement temporaire des obligations de capitalisation pour les régimes mis en place par les employeurs. Au Canada, les autorités envisagent de porter de cinq à dix ans la période de recapitalisation requise pour assurer la solvabilité des dispositifs. En Norvège, la mise en œuvre d'une obligation de constituer des provisions supplémentaires du fait de l'allongement de l'espérance de vie a été reportée de trois à cinq ans. Enfin, par souci d'éviter les ventes forcées d'actions à un moment peu propice, la Finlande va également suspendre certaines obligations de solvabilité jusqu'à la fin 2010.

Certains pays s'interrogent également sur les récentes modifications des normes de valorisation des actifs des fonds de pension, notamment l'introduction des méthodes de la "juste valeur » et de la "valorisation au prix du marché » fondées, entre autres, sur des taux d'actualisation qui tiennent compte de l'échéance aussi bien des engagements de retraite que du niveau actuel des taux d'intérêt sur le marché. Au Danemark, par exemple, les fonds de pension vont être autorisés à calculer leur solvabilité sur la base d'un retour à des conditions "normales ». Une mesure analogue a été adoptée en Finlande et est à l'étude dans d'autres pays. Néanmoins, les autorités de tutelle des Pays-Bas ont résisté jusqu'à présent aux pressions exercées par les professionnels du secteur des retraites visant à modifier le taux d'actualisation des engagements de retraite futurs.

Cela étant, il importe que de telles mesures - en particulier l'allongement des périodes de redressement - soit nettement délimitées dans le temps et ne viennent pas affaiblir de façon permanente les règles de financement. Elles auraient pour effet ultime de réduire la protection des revenus perçus par les salariés une fois à la retraite. La présence de fonds de garantie des retraites ${ }^{20}$ pèse également sur les finances publiques.

\section{Conclusions : la sécurité par la diversitéé}

Dans presque tous les pays de l'OCDE, l'heure est à l'effondrement du prix des actifs, à la contraction de l'économie et à la montée du chômage. Les pressions politiques exercées à court terme sur les pouvoirs publics pour qu'ils apportent une réponse à ces questions sont considérables. Les autorités doivent néanmoins se garder de toute réaction hâtive pouvant nuire à la stabilité à long terme et à la pérennité des régimes de retraite. Il est aussi essentiel de ne pas oublier que les difficultés à long terme des systèmes de retraite résultant de l'évolution démographique et du vieillissement de la population n'ont pas disparu. Les pressions à court terme n'ont fait qu'envenimer ces problèmes à long terme.

En raison de la crise financière, le risque d'investissement est au centre des préoccupations du public et des autorités. Toutefois, il faut garder à l'esprit qu'un large éventail de risques et d'incertitudes pèsent sur les retraites, car il s'agit de contrats à long terme. La situation peut sensiblement évoluer durant les 40 années ou plus qui séparent l'entrée sur le marché du travail, et donc l'entrée dans le dispositif de retraite, du départ à la retraite. 
Les régimes publics, par exemple, s'accompagnent du risque de voir les autorités (ou plutôt les électeurs) changer d'avis sur ce qui constitue un revenu raisonnable au titre de la retraite et verser des prestations de retraite plus faibles que prévu. Les contribuables seront à la fois moins nombreux et moins disposés à mettre la main à la poche si la crise financière se transforme en crise économique profonde et prolongée. Les problèmes observés dans l'économie réelle se répercuteront également sur les revenus perçus au titre de la retraite en raison de l'aggravation du chômage et de la baisse des salaires.

Les difficultés rencontrées par les pensions privées découlant de la crise financière ne sont pas une raison suffisante pour remplacer les dispositifs privés par des régimes publics. De nombreux pays sont déjà dans une situation budgétaire inconfortable, qui devrait se dégrader plus encore au fil du ralentissement de l'activité. Compte tenu des coûts induits par le vieillissement de la population sur la santé, et sur les systèmes de retraite, une telle ligne de conduite remettrait en cause la viabilité des finances publiques à moyen et long termes.

La meilleure méthode en matière de retraites est de panacher les sources de revenu (publiques et privées), ainsi que les deux principaux modes de financement (par répartition et par capitalisation). Recourir uniquement ou largement à une seule source face à différents types de risque peut s'avérer imprudent.

L'OCDE préconise depuis longtemps une diversification des régimes de retraite, faisant valoir que "la diversité présente bien des avantages " (OCDE, 1998). L'étude intitulée Préserver la prospérité dans une société vieillissante indiquait également que "chacun des éléments du système présente ses forces et ses faiblesses, et un système souple associant ces divers éléments permet non seulement de diversifier les risques, mais aussi de mieux équilibrer la charge entre les générations ${ }^{22}$.

Des incertitudes économiques, démographiques, financières et sociales pèsent sur les systèmes de retraite et les individus. Il ne fait aucun doute que la meilleure méthode pour un individu - et, par extension, pour un gouvernement désireux d'agir au mieux de l'intérêt de ses administrés - est là encore de panacher les sources de revenu au titre de la retraite. La diversité en la matière est le meilleur moyen pour offrir une sécurité aux personnes âgées. La crise actuelle n'a fait que souligner la pertinence de ce message.

\section{Notes}

1. On trouvera dans OCDE (2007a), Martin et Whitehouse (2008), ainsi que dans Queisser et al. (2007) une analyse détaillée de ces réformes.

2. Voir l'analyse des indicateurs de la « moyenne pondérée des pensions et des patrimoines retraite » et de la "structure de la prestation globale de retraite » en partie II de la présente étude.

3. Il convient de noter que les taux de cotisation varient sensiblement entre les individus. Les plus jeunes et les personnes qui ont de faibles revenus, par exemple, ont tendance à moins cotiser en moyenne.

4. Pour les trois autres pays qui ne sont pas pris en compte dans le graphique 1.2 - à savoir le Japon (les actifs des pensions privées représentent $20.0 \%$ du PIB), le Portugal (13.7 \%) et l'Espagne (7.5 \%) - on ne dispose d'aucune information sur les règles types régissant les régimes. Quoi qu'il en soit, les actifs de ces pensions privées sont moins importants que dans la majorité des pays apparaissant dans le graphique 1.2. Voir l'indicateur des " actifs des fonds de pension privés et les fonds de réserve publics » figurant dans la partie II de cette publication.

5. Néanmoins, les salariés qui étaient déjà sur le marché du travail à l'époque de la réforme au Mexique continueront de percevoir la plus grande partie de leur retraite dans le cadre du régime 
public : voir le chapitre spécial sur « Le déficit d'épargne-retraite et l'épargne-retraite facultative » dans ce volume.

6. L'OCDE ne dispose malheureusement pas, sur les règles types, des données nécessaires pour modéliser ces droits.

7. Voir encadré 2.1 plus bas et Pearson et Whitehouse (2009) sur les retraités couverts par des régimes soumis à conditions de ressources.

8. Voir Keenay et Whitehouse (2003a et b) pour une analyse du rôle du système fiscal dans l'aide aux personnes âgées.

9. On trouvera des données détaillées dans Whitehouse et al. (2009), tableau 4. Ce document étudie également l'impact de la fiscalité sur les revenus nets des retraités pour différents rendements des investissements.

10. OCDE $(1996,2008)$ et Ebbinghaus (2006) contiennent une analyse détaillée. Voir OCDE (2009b) pour un tour d'horizon complet des répercussions de la crise sur les marchés du travail et les politiques sociales.

11. Allemagne, Canada, États-Unis, France, Italie, Japon et Royaume-Uni.

12. Voir l'analyse figurant dans D'Addio et al. (2009), sections 6 à 9.

13. Les restrictions les plus strictes sur les investissements en actions sont imposées en Corée et au Mexique (30\%), ainsi qu'en Allemagne et en Norvège (35\%) ; voir OCDE (2009a), graphique 2.18. Dans certains pays, les gestionnaires doivent proposer tout un éventail de fonds offrant différentes caractéristiques risque-rendement. À l'échelle internationale, la moyenne est calculée à l'aide du plafond défini pour les actions dans les fonds centraux ou équilibrés.

14. En termes clairs, les salariés plus jeunes détiennent habituellement peu d'actifs en dehors de leur capital humain (c'est-à-dire leur rémunération future). Ils ont tout intérêt à investir dans des actifs faiblement corrélés au salaire qu'ils devront percevoir. Pour les salariés plus âgés, c'est l'inverse. À mesure qu'ils approchent de la retraite, leur capital humain diminue, mais en règle générale, ils auront accumulé des actifs financiers dans le cadre d'un régime de retraite privé ou constitué un patrimoine sous la forme d'un flux de droits futurs à une retraite publique. Voir notamment Jagannathan et Kocherlakota (1996), ainsi que Samuelson (1998a et b).

15. Lorsque les pays basculent une partie de leurs prestations de retraite de régimes publics par répartition vers des pensions privées, plusieurs questions se posent. Il convient tout particulièrement de se demander dans quelle mesure les salariés actuels et futurs doivent être autorisés, encouragés ou obligés à basculer vers les dispositifs privés à cotisations définies : voir l'analyse à l'encadré 1.2 ci-dessus.

16. Premièrement, une somme de 60 GBP a été versée en janvier 2009, ce qui revenait à avancer l'indexation du mois d'avril au début de l'année. Deuxièmement, la retraite de base est désormais revalorisée à hauteur de la croissance de l'indice des prix de détail, ou de $2.5 \%$ si ce taux est supérieur. L'indice des prix de détail fait état d'une déflation en décembre 2008 et janvier 2009, et il devrait rester négatif durant la majeure partie de l'année. Cela signifie que la retraite de base va augmenter en termes réels. Troisièmement, l'aide à la cuve versée aux retraités a été majorée de $25 \%$ pour atteindre 250 GBP (les plus de 80 ans bénéficiant d'un coup de pouce supplémentaire).

17. Les particuliers sont autorisés à verser des cotisations sur un compte individuel facultatif à concurrence de $4 \%$ de leur salaire. D'une manière générale, l'employeur abonde ensuite à concurrence de $2 \%$ du salaire.

18. Selon le Fonds monétaire international (2009), toutefois, le gouvernement norvégien devrait recouvrer $98 \%$ de cet investissement, contre $52 \%$ seulement pour l'Irlande.

19. Il est préoccupant de constater qu'aux Pays-Bas, les entreprises les plus exposées (les sociétés de petite taille, fortement endettées) détiennent aussi des investissements plus risqués dans le cadre des dispositifs à prestations définies dont elles assurent la promotion (c'est-à-dire une proportion plus élevée d'actions dans les portefeuilles). Voir Davis et al. (2007).

20. Il s'agit notamment du Pension Protection Fund au Royaume-Uni et de la Pension Benefit Guaranty Corporation aux États-Unis.

21. "La sécurité par la diversité » - le slogan du processus de réforme des retraites mené en Pologne du milieu à la fin des années 90 - reste d'actualité. Voir Chlon et al. (1999).

22. Cette conclusion est largement étayée par les publications financières. Ainsi, Merton (1983) explique en se fondant sur la théorie du portefeuille pourquoi la diversification entre les régimes 
par répartition et par capitalisation est optimale. Ce modèle a été développé plus avant dans Bodie et al. (1992) et élargi au risque d'inflation dans Heeringa (2008).

\section{Références}

Amenta, E. (2006), When Movements Matter: The Townsend Plan and the Rise of Social Security, Princeton University Press.

Australian Prudential Regulatory Authority - Autorité australienne de contrôle prudentiel (2007), Insight: Celebrating 10 Years of Superannuation Data Collection, Sydney.

Bikker, J.A. et P.J.G. Vlaar (2006), " Conditional Indexation in Defined Benefit Pension Plans », Document de travail $\mathrm{n}^{\circ} 86$, De Nederlandsche Bank, Amsterdam.

Bodie, Z., R.C. Merton et W.F. Samuelson (1992), «Labor Supply Flexibility and Portfolio Choice in a Lifecycle Model ", Journal of Economic Dynamics and Control, vol. 16, n³, pp. 427-449.

Börsch-Supan, A. et C.B. Wilke (2006), «The German Public Pension System: How It Will Become an NDC System Look-Alike ", in R. Holzmann et E. Palmer (dir. pub.), Pension Reform: Issues and Prospects for Non-Financial Defined Contribution (NDC) Schemes, Banque mondiale, Washington, DC.

Brown, R.L. (2008), " Reforms to Canadian Social Security, 1996-7 », in S.J. Kay, et T. Sinha (dir. pub.), Lessons from Pension Reform in the Americas, Oxford University Press, Oxford.

Bureau du surintendant des institutions financières Canada (2007), "Capitalisation optimale du Régime de pensions du Canada ", Étude actuarielle $n^{\circ} 6$, Ottawa.

Chlon, A., M. Góra et M. Rutkowski (1999), "Shaping Pension Reform in Poland: Security through Diversity ", Pension Reform Primer series, Social Protection Discussion Paper n 9923, Banque mondiale, Washington, DC.

D’Addio, A.C., J. Seisdedos et E.R. Whitehouse (2009), "Investment Risk and Pensions: Measuring Uncertainty in Returns ", Documents de travail sur les affaires sociales, l'emploi et les migrations, $\mathrm{n}^{\circ} 70$, Éditions de l'OCDE, Paris.

Davis, E.P., S. Grob et L de Haan (2007), « Pension Fund Finance and Sponsoring Companies: Empirical Evidence on Theoretical Hypotheses ", Document de travail n 158 , De Nederlandsche Bank, Amsterdam

De Nederlandsche Bank - Banque centrale néerlandaise (2007), "Pension Contributions Stabilised, Indexation of Pensions Resumed », Statistical Bulletin, pp. 19-22, mars.

Department of Labor, Employee Benefits Security Administration - Ministère du Travail, Direction de la sécurité des prestations versées aux salariés (diverses années), Private Pension Plan Bulletin: Abstract of Form 5500 Annual Reports, Washington, DC.

Department of Work and Pensions - Ministère du Travail et des Retraites (2006), Second Tier Pension Provision $1978 / 79$ to $2003 / 04$, Londres.

Ebbinghaus, B. (2006), Reforming Early Retirement in Europe, Japan and the USA, Oxford University Press, Oxford.

Fonds monétaire international (2009), « The State of Public Finances: Outlook and Medium-Term Policies after the 2008 Crisis ", Département des finances publiques, Washington, DC.

General Accounting Office - Cour des comptes, États-Unis (1997), « 401(k) Pension Plans: Loan Provisions Enhance Participation but May Affect Income Security for Some », GAO/HEHS-98-5, Washington, DC.

Heeringa, W. (2008), « Optimal Lifecycle Investment with Pay-As-You-Go Pension Schemes: A Portfolio Approach ", Document de travail n 168, De Nederlandsche Bank, Amsterdam.

Holzmann, R. et R.P. Hinz (2005), Old-Age Income Support in the 21st Century: An International Perspective on Pension Systems and Reform, Banque mondiale, Washington, DC.

Hoskins, D., M.A. Pearson et E.R. Whitehouse (2009), " Social Pensions in High-Income Countries ", in R. Holzmann et N. Takayama (dir. pub.), Closing the Coverage Gap: The Role of Social Pensions, Banque mondiale, Washington, DC, à paraître.

International Financial Services London (2009), Pension Markets 2009, Londres.

Jagannathan, R. et Kocherlakota, N. (1996), «Why Should Older People Invest Less in Stocks than Younger People? ", Federal Reserve Bank of Minneapolis Quarterly Review, vol. 20, n³, pp. 11-23. 
Keenay, G. et E.R. Whitehouse (2003a), « Financial Resources and Retirement in Nine OECD Countries: the Role of the Tax System ", Documents de travail sur les affaires sociales, l'emploi et les migrations, $n^{\circ} 8$, Éditions de l'OCDE, Paris.

Keenay, G. et E.R. Whitehouse (2003b), "The Role of the Personal Tax System in Old-age Support: A Survey of 15 Countries », Fiscal Studies, vol. 24, n 1, pp. 1-21.

Kusko, A., J.M. Poterba et D. Wilcox (1998), « Employee Decisions with Respect to 401(k) Plans », in O.S. Mitchell et S. Schieber (dir. pub.), Living with Defined Contribution Pensions: Remaking Responsibility for Retirement, pp. 98-112, University of Pennsylvania Press, Philadelphie.

Martin, J.P. et E.R. Whitehouse (2008), « Reforming Retirement-Income Systems: Lessons from the Recent Experiences of OECD Countries ", Documents de travail sur les affaires sociales, l'emploi et les migrations, $n^{\circ} 66$, Éditions de l'OCDE, Paris.

Mattil, B. et E.R. Whitehouse (2009), « Rebalancing Retirement-income Systems: The Role of Individual Choice under Mixed Public/Private Pension Provision ", Documents de travail sur les affaires sociales, l'emploi et les migrations, Éditions de l'OCDE, à paraître.

Merton, R.C. (1983), " On the Role of Social Security as a Means for Efficient Risk Sharing in an Economy Where Human Capital Is Not Tradable ", in Z. Bodie et J.B. Shoven (dir. pub.), Financial Aspects of the United States Pension System, University of Chicago Press pour le National Bureau of Economic Research, pp. 325-358.

Ministry of Finance - Ministère des Finances, Islande (2009), "Weekly Web Release, 5 February », Reykjavik.

Morissette, R. et A. Johnson (2003), "Les bons emplois disparaissent-ils au Canada ? ", Document de recherche $n^{\circ} 239$, Direction des études analytiques, Statistique Canada, Ottawa.

OCDE (1996), La stratégie de l’OCDE pour l'emploi - Accélérer la mise en œuvre, Éditions de l'OCDE, Paris.

OCDE (1998), Préserver la prospérité dans une société vieillissante, Éditions de l'OCDE, Paris.

OCDE (2001), Vieillissement et revenus - Les ressources des retraités dans 9 pays de l'OCDE, Éditions de l'OCDE, Paris.

OCDE (2007a), Les pensions dans les pays de l'OCDE - Panorama des politiques publiques, Éditions de l'OCDE, Paris.

OCDE (2007b), Les impôts sur les salaires, Éditions de l'OCDE, Paris.

OCDE (2008), Perspectives de l'emploi de l'OCDE, Éditions de l'OCDE, Paris.

OCDE (2009a), Perspectives de l'OCDE sur les pensions privées 2008, Éditions de l'OCDE, Paris.

OCDE (2009b), « La crise de l'emploi : quelles sont les conséquences pour la politique de l'emploi et la politique sociale? ", chapitre 1, Perspectives de l'emploi de l'OCDE, Éditions de l'OCDE, Paris, à paraître.

Pensions Board - Office des pensions, Irlande (diverses années), Annual Report, Dublin.

Pension Protection Fund - Fonds de protection des pensions, Royaume-Uni (2009), « PPF 7800 Index March 2009 ", Croydon, Surrey.

Purcell, P. (2008), «Individual Retirement Accounts and 401(k) Plans: Early Withdrawals and Required Distributions », Rapport n RL31770, Congressional Research Service, Washington, DC.

Queisser, M., P. Whiteford et E.R. Whitehouse (2007), "The Public-Private Pension Mix in OECD Countries ", Industrial Relations Journal, vol. 38, $n^{\circ}$ 6, pp. 542-568.

Samuelson, P.A. (1989a), «The Judgement of Economic Science on Rational Portfolio Management: Indexing, Timing and Long-Horizon effects ", Journal of Portfolio Management, automne, pp. 4-12.

Samuelson, P.A. (1989b), "A Case at Last for Age-Phased Reduction in Equity ", Proceedings of the National Academy of Sciences, vol. 86, pp. 9048-9051.

Settergren, O. (2001), "The Automatic Balance Mechanism of the Swedish Pension System: A NonTechnical Introduction », Wirtschaftspolitische Blätter, n 4, pp. 339-349.

The Pensions Regulator - Autorité de tutelle des retraites, Royaume-Uni (2008), The Purple Book: DB Pensions Universe Risk Profile, Londres.

VanDerhei, J. (2009), "The Impact of the Recent Financial Crisis on 401(k) Account Balances ", Issue Brief n³26, Employee Benefit Research Institute, Washington, DC. 
VanDerhei, J., S. Holden, L. Alonso et C. Copeland (2008), « 401(k) Plan Asset Allocation, Account Balances, and Loan Activity in 2007 ", Issue Brief n³24, Employee Benefits Research Institute, Washington, DC.

Whitehouse, E.R. (2007), Pensions Panorama: Retirement-Income Systems in 53 Countries, Banque mondiale, Washington, DC.

Whitehouse, E.R., A.C. D’Addio et A.P. Reilly (2009), "Investment Risk and Pensions: Impact on Individual Retirement Incomes and Government Budgets ", Documents de travail sur les affaires sociales, Éditions de l'OCDE, Paris. 



\section{Les revenus et la pauvreté des personnes âgées}

\section{L'}

analyse présentée dans Les pensions dans les pays de l'OCDE est essentiellement prospective, en ce sens qu'elle évalue les droits à retraite futurs des salariés actuels. Ce chapitre spécial ${ }^{1}$ complète cette analyse en examinant les revenus des retraités actuels. Il s'interroge sur la situation de la génération actuelle des personnes âgées par rapport à la population actuelle d'âge actif.

Les politiques des retraites tentent de concilier deux objectifs. Le premier est d'assurer aux retraités un revenu décent qui les mette à l'abri de la pauvreté. Cet aspect est particulièrement significatif pour les personnes dont les revenus ont toujours été faibles, qui ont connu de longues périodes de travail temporaire ou à temps partiel ou dont la carrière a été interrompue par des périodes de chômage ou parce qu'elles ont élevé leurs enfants. Ces personnes n'auront probablement pas accumulé beaucoup de droits à prestations au titre des régimes publics, des régimes assis sur les salaires ou des régimes privés.

Le deuxième objectif est de faire en sorte que les revenus des retraités leur permettent de conserver un niveau de vie comparable à celui qu'ils avaient durant leur vie active. À titre d'exemple, le Canada, l'Irlande, la Nouvelle-Zélande et le Royaume-Uni donnent tous la priorité à la garantie d'un revenu suffisant. Par contraste, la Hongrie, l'Italie, la Pologne et la République slovaque sont des pays qui associent étroitement les prestations de retraite au niveau du salaire pendant la vie active ${ }^{2}$.

La section 1 de ce chapitre examine les revenus des personnes âgées (de 65 ans et plus) et les compare à ceux de la population. Ces mesures des revenus sont exhaustives en ce sens qu'elles comprennent toutes les sources de revenus en numéraire et toutes les catégories de personnes. Pour les personnes âgées, par exemple, elles incluent les prestations de retraites publiques et privées ainsi que les prestations soumises à conditions de ressources, comme dans le modèle de calcul des prestations individuelles utilisé dans l'analyse des droits à pension présentée dans les indicateurs de la section " Droits à retraite " en partie II du présent rapport. Néanmoins, elles tiennent également compte du rendement de produits d'épargne autres que l'épargne-retraite, ainsi que des gains ou revenus générés par l'emploi indépendant, qui jouent un rôle important pour subvenir aux besoins des "jeunes " retraités (de 66 à 75 ans) dans de nombreux pays de l'OCDE. Les calculs des revenus de la population intègrent toutes les personnes en âge de travailler, y compris celles qui n'exercent pas d'emploi.

La section 1 présente les revenus globaux des personnes âgées et illustre leurs variations en fonction de l'âge et du type de ménage (célibataires et couples, par exemple). 
Elle présente également des données sur les sources de revenus perçus par les personnes âgées et leurs différences d'un pays à l'autre. Enfin, elle compare la situation des personnes âgées au milieu des années 2000 et au milieu des années 80 afin de dégager des tendances dans l'évolution des revenus des retraités.

La section 2 s'intéresse aux retraités à faibles revenus et présente des données relatives à la pauvreté des personnes âgées dans les pays de l'OCDE. Elle compare également la prévalence de la pauvreté entre hommes et femmes, différents groupes d'âge, différents types de ménages et au cours du temps. La section 3 analyse le rôle redistributif de l'État: l'effet des prestations publiques et des impôts sur le revenu disponible. La section 4, de nature prospective, s'interroge sur l'évolution possible des revenus et de la pauvreté des personnes âgées sous l'effet des transformations socio-économiques et des réformes des retraites. Enfin, la section 5 formule une conclusion et examine les répercussions des principaux constats de ce chapitre sur l'élaboration des politiques.

\section{Les revenus des personnes âgées}

Les revenus sont mesurés à partir des données tirées des enquêtes nationales auprès des ménages ${ }^{3}$, effectuées au milieu des années 2000, et sont calculés comme suit:

- Les revenus incluent les salaires, les revenus des professions indépendantes, les revenus du capital (dividendes, intérêts, etc.), le revenu locatif et les transferts publics (comme les prestations de retraite, allocations familiales, prestations de chômage, de logement et d'invalidité).

- L'indicateur utilisé est le revenu « disponible ». Il s'agit du revenu net d'impôts sur le revenu des personnes physiques et des cotisations de sécurité sociale.

- Les revenus sont agrégés au niveau du ménage. En effet, on suppose que les membres du même ménage partagent leurs ressources - le logement en est l'exemple le plus évident - et il est souvent difficile d'attribuer tel ou tel type de revenu (comme le revenu de l'épargne) à des individus en particulier. Cette approche suppose implicitement que le revenu est partagé équitablement entre les membres du ménage.

- Le revenu total du ménage est ensuite divisé entre les individus qui le composent. Cette division s'opère sur une base " équivalente ", ce qui signifie que les revenus du ménage sont ajustés pour tenir compte des différences dans la taille des ménages. Selon le vieil adage, " on peut vivre à deux aussi chichement que tout seul ». Ce n'est pas entièrement vrai, mais les ménages de plus d'une personne réalisent sans nul doute des "économies d'échelle ". L'ajustement effectué par l'OCDE estime que deux personnes peuvent vivre avec aussi peu de moyens que 1.4 personne vivant seule.

Au milieu des années 2000, les revenus des personnes âgées de 65 ans et plus représentaient en moyenne $82.4 \%$ du revenu moyen de la population ${ }^{4}$. Il existe de fortes variations entre les pays de l'OCDE. Le revenu moyen de l'ensemble des personnes âgées de plus de 65 ans, représenté par la courbe noire dans le graphique 2.1, est le plus élevé au Mexique et en Autriche, où il équivaut à environ $97 \%$ du revenu moyen. Ce pourcentage tranche avec les $66 \%$ de l'Irlande. L'Australie, la Corée, le Danemark, la Finlande, la Nouvelle-Zélande et le Royaume-Uni sont d'autres pays où le revenu des personnes âgées est relativement faible - des deux tiers aux trois quarts de la moyenne nationale. À l'autre extrême, en France, au Luxembourg et en Pologne, les revenus des personnes âgées avoisinent $95 \%$ du revenu équivalent disponible national moyen. Les États-Unis, la 
Hongrie, l'Italie, la Suède et la Suisse se situent aux alentours de la moyenne de l'OCDE de $82.4 \%$.

Il existe une relation entre les revenus des personnes âgées et les dépenses publiques consacrées aux prestations de vieillesse, surtout lorsqu'on tient également compte des différences tenant à la situation démographique des pays ${ }^{5}$. Une hausse de $10 \%$ des dépenses publiques liées aux retraites est associée à une augmentation de 1.5 point de pourcentage du revenu relatif des personnes âgées. La conception spécifique des systèmes de retraite joue aussi un rôle. Par exemple, l'Australie, l'Irlande, la Nouvelle-Zélande et le Royaume-Uni ont tous en commun des revenus des personnes âgées relativement faibles et un régime obligatoire d'assurance vieillesse qui vise à garantir un revenu suffisant plutôt qu'à compenser l'écart par rapport à l'ancien revenu d'activité. Mais il en va de même pour le Canada et les États-Unis, où les revenus des personnes âgées sont supérieurs à la moyenne de la population. En Autriche, en France, au Luxembourg et en Pologne, du moins pour la génération actuelle de retraités, les régimes de retraite publics liés à la rémunération sont très développés et les revenus relatifs des personnes âgées comptent parmi les plus élevés. Néanmoins, l'Espagne et la Finlande garantissent des droits à retraite comparables, mais les revenus relatifs des personnes âgées y sont inférieurs à la moyenne de l'OCDE. En Corée, le faible niveau des revenus des personnes âgées s'explique principalement par le fait que le régime public de retraite n'a été introduit qu'en 1988, de sorte que les retraités actuels n'ont guère accumulé de droits.

Quelles sont les répercussions de ces résultats (basés sur les revenus disponibles équivalents) lorsqu'on compare le niveau de vie à la retraite et au cours de la vie active ${ }^{6}$ ? Il convient de prendre en considération d'autres facteurs pour interpréter ces statistiques. Premièrement, les retraités n'ont pas à supporter les coûts supplémentaires d'une vie active : transports quotidiens, habillement, repas, etc. ${ }^{7}$. Deuxièmement, les coûts de logement des retraités peuvent être très différents de ceux de la population active. Dans les pays de l'OCDE, beaucoup de personnes ayant atteint l'âge de la retraite, voire la plupart, sont propriétaires de leur logement. À la différence des personnes d'âge actif, les propriétaires retraités ont en général remboursé leur prêt immobilier ${ }^{8}$. Troisièmement, les personnes âgées possèdent en général plus d'actifs financiers que celles d'âge actif. Bien que les revenus des dividendes et intérêts sur ces actifs soient intégrés dans la mesure du revenu disponible, ce n'est pas le cas pour la valeur de ces actifs. De nombreuses personnes puisent dans ces actifs pour compléter leur consommation une fois à la retraite ${ }^{9}$. Enfin, la mesure du revenu monétaire exclut les transferts en nature sous forme de biens et services publics, comme les soins de santé, les services sociaux et de logement, qui peuvent être très importants pour les personnes âgées ${ }^{10}$. Malheureusement, tous ces effets sur le bienêtre économique relatif des personnes âgées sont difficiles à mesurer. Il convient néanmoins d'en tenir compte lorsqu'on interprète la statistique selon laquelle les revenus des personnes âgées représentent en moyenne 82.4 \% des revenus de la population dans les pays de l'OCDE.

\section{Les personnes les plus âgées ont-elles les revenus les plus bas?}

Les histogrammes dans le graphique 2.1 illustrent les résultats pour deux groupes différents de personnes âgées : celles âgées de 66 à 75 ans et celles de plus de 75 ans. En moyenne, dans l'OCDE, la première tranche (la plus jeune) perçoit des revenus qui représentent environ $86 \%$ de la moyenne de la population, tandis que pour la deuxième tranche (âgée de 75 ans et plus), ce pourcentage n'est que de $78 \%$. Néanmoins, il existe de 


\section{Graphique 2.1. Revenus relatifs des personnes âgées}

Revenu disponible équivalent des ménages, milieu des années 2000

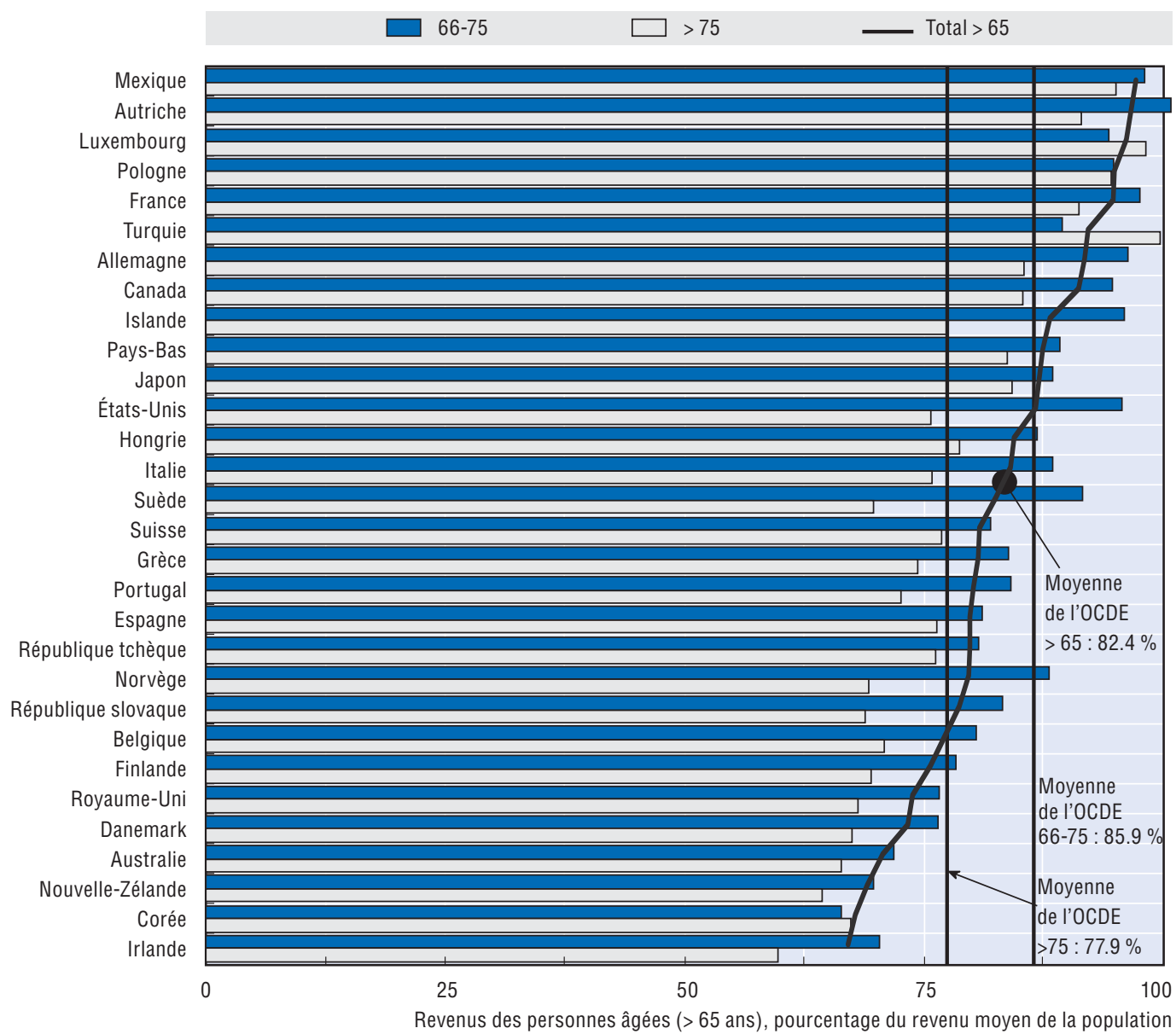

Note: Les pays sont classés en fonction du revenu relatif de toutes les personnes âgées de 65 ans et plus.

Source: Base de données de l'OCDE sur la distribution des revenus ; voir OCDE (2008), Croissance et inégalités, graphique 2.4.

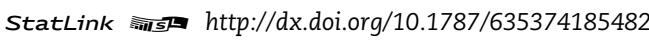

fortes variations d'un pays à l'autre. Les écarts de revenus en fonction de l'âge sont les plus marqués aux États-Unis, en Irlande, Islande, Norvège, République slovaque et Suède. Les retraités les plus âgés ont des revenus supérieurs aux retraités les plus jeunes en Corée, au Luxembourg et en Turquie, et des revenus légèrement inférieurs au Mexique et en Pologne.

De nombreuses raisons expliquent pourquoi les revenus relatifs des retraités les plus âgés sont inférieurs à ceux des retraités plus jeunes. Premièrement, il existe un effet de cohorte ou de génération. La quasi-totalité des pays de l'OCDE disposent, sous une forme ou une autre, d'un régime de retraite lié à la rémunération. Lorsque les personnes de plus de 75 ans sont parties à la retraite, leurs salaires étaient en moyenne $10 \%$ à $25 \%$ inférieurs, en termes réels, aux revenus moyens enregistrés lorsque les personnes appartenant à la tranche d'âge 66-75 ans ont pris leur retraite. Cet écart se retrouve dans les prestations de retraite des régimes assis sur les salaires. Dans les pays où le salaire réel a progressé très rapidement ces dernières années, comme en Espagne et en Irlande, les différentiels de revenus relatifs selon l'âge seront plus marqués. C'est l'une des principales raisons qui expliquent les écarts importants de revenus entre les deux tranches d'âge en Irlande et en République slovaque, par exemple. 
Deuxièmement, un effet strictement démographique se produit du fait de l'indexation des prestations de retraite servies afin de tenir compte des variations des coûts et des niveaux de vie (l'effet de cohorte tient au fait que les retraites des nouveaux retraités augmentent parallèlement à la croissance démographique ; l'effet démographique se produit en raison de l'évolution de la valeur relative de la pension après le départ en retraite). Les politiques et pratiques d'indexation ont évolué au cours du temps (voir cidessous). Toutefois, la plupart des pays de l'OCDE indexent désormais les prestations de retraite servies en fonction des prix, afin de préserver le pouvoir d'achat offert par ces prestations. Là encore, les pays qui ont vu leur salaire réel croître rapidement - comme l'Espagne, l'Irlande et la République slovaque - enregistrent aussi une forte baisse des revenus relatifs des retraités avec l'âge ${ }^{11}$. En revanche, le Luxembourg indexe les prestations de retraite sur les gains moyens. C'est une des raisons pour lesquelles les revenus des retraités les plus âgés sont supérieurs à ceux de 66 à 75 ans.

Troisièmement, il existe un effet de composition. Pour des raisons évidentes, la catégorie des plus âgés se compose principalement de personnes ayant une espérance de vie supérieure à la moyenne. Par exemple, les femmes ont une espérance de vie supérieure de 5.7 ans à celle des hommes ${ }^{12}$. C'est pourquoi elles sont prédominantes parmi les personnes âgées, et représentent $53 \%$ des 66 à 75 ans et $60 \%$ des plus de 75 ans en moyenne dans la zone OCDE. Elles perçoivent généralement des prestations de retraite inférieures à celles des hommes, en raison de salaires plus bas, d'un nombre d'heures travaillées plus faible et d'interruptions plus longues dans leur carrière. Beaucoup seront veuves, et leur situation dépendra du versement de pensions de réversion. Les différences de revenus les plus marquées selon l'âge (États-Unis, Islande, Norvège et Suède) sont probablement imputables aux règles relatives aux pensions de réversion (voir ci-dessous).

Un autre effet de composition provient du fait que les personnes plus riches ont tendance à vivre plus longtemps que celles plus pauvres ${ }^{13}$. Dans les pays de l'OCDE à haut revenu, cet effet est assez limité et les différences socio-économiques dans les caractéristiques de mortalité des retraités sont beaucoup moins marquées que parmi les personnes d'âge actif. Néanmoins, le fait que les hommes pauvres ont tendance à mourir plus tôt que la plupart des femmes et des hommes les plus riches signifie qu'il y a beaucoup de veuves âgées qui ont été mariées à des hommes percevant une retraite réduite et qui, par conséquent, reçoivent une pension de réversion d'un faible montant. L'incidence des différentiels de mortalité d'origine socio-économique est plus forte dans les pays de l'OCDE à plus bas revenu. En Turquie, par exemple, les personnes titulaires de bas revenus pendant leur vie active n'étaient souvent pas couvertes par le régime de retraite. Étant donné que les personnes plus riches vivent plus longtemps, plus de personnes âgées de plus de 75 ans ont des droits à retraite, ce qui explique le fait qu'elles perçoivent des revenus beaucoup plus élevés que la catégorie des 66-75 ans. Des effets analogues se manifestent en Corée et au Mexique.

\section{Quelles sont les différences de revenus entre personnes seules et couples?}

D'après l'examen des revenus par âge, les différences s'expliquent en grande partie par le fait que les femmes, et notamment les veuves, prédominent parmi les personnes âgées. Le graphique 2.2 compare les revenus des ménages dont le chef de famille est un adulte célibataire de plus de 65 ans à ceux des ménages, dont le chef de famille a plus de 65 ans, comptant deux adultes ou plus. Rappelons que ces chiffres de revenus des ménages sont déjà exprimés par unité de consommation afin de tenir compte des écarts de niveau de vie des 
Graphique 2.2. Revenus relatifs des personnes âgées célibataires

Pourcentage des revenus de ménages ayant atteint l'âge de la retraite comptant deux adultes ou plus, milieu des années 2000

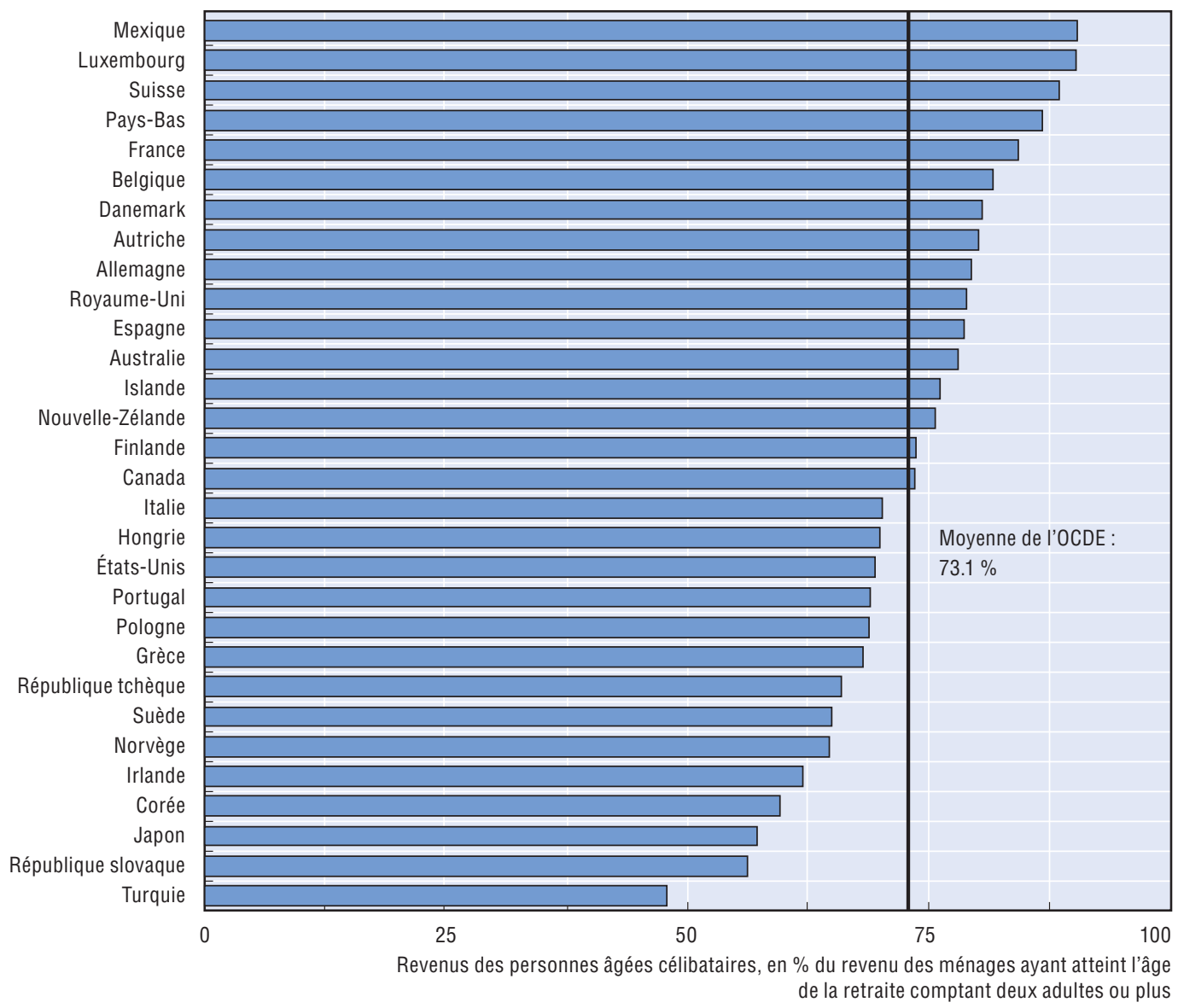

Source: Base de données de l'OCDE sur la distribution des revenus ; voir OCDE (2008), Croissance et inégalités, graphique 2.4. StatLink त्नाज्ञा $h t t p: / / d x . d o i . o r g / 10.1787 / 635414738676$

ménages de tailles différentes. Par exemple, un retraité célibataire ayant un revenu d'environ $70 \%$ de celui d'un couple est supposé, sur la base de la mesure de l'OCDE, bénéficier du même niveau de bien-être économique mesuré par le revenu équivalent. Dans cet exemple, la personne seule se situerait par conséquent à $100 \%$ sur l'échelle du revenu équivalent relatif.

La situation des revenus des retraités célibataires est excellente par rapport à celle des revenus des couples au Luxembourg, au Mexique, aux Pays-Bas et en Suisse. Cela s'explique par un ensemble de prestations de réversion relativement généreuses, d'autres dispositifs de protection des conjoints qui ne travaillent pas et de pratiques d'indexation (voir ci-dessus). Par contraste, les retraités célibataires sont défavorisés dans les pays d'Europe de l'Est, en Corée, en Irlande, au Japon et dans deux pays nordiques - Norvège et Suède - pour les raisons mentionnées plus haut.

\section{Quelles sont les sources de revenus des personnes âgées?}

Les transferts publics - sous la forme de prestations de retraite liées à la rémunération, de prestations sous conditions de ressources, etc. - représentent en moyenne $60 \%$ des revenus des personnes âgées dans les 27 pays de l'OCDE figurant dans le graphique 2.3. Les 


\section{Graphique 2.3. Sources de revenu des personnes âgées}

Pourcentage du revenu disponible des ménages, milieu des années 2000

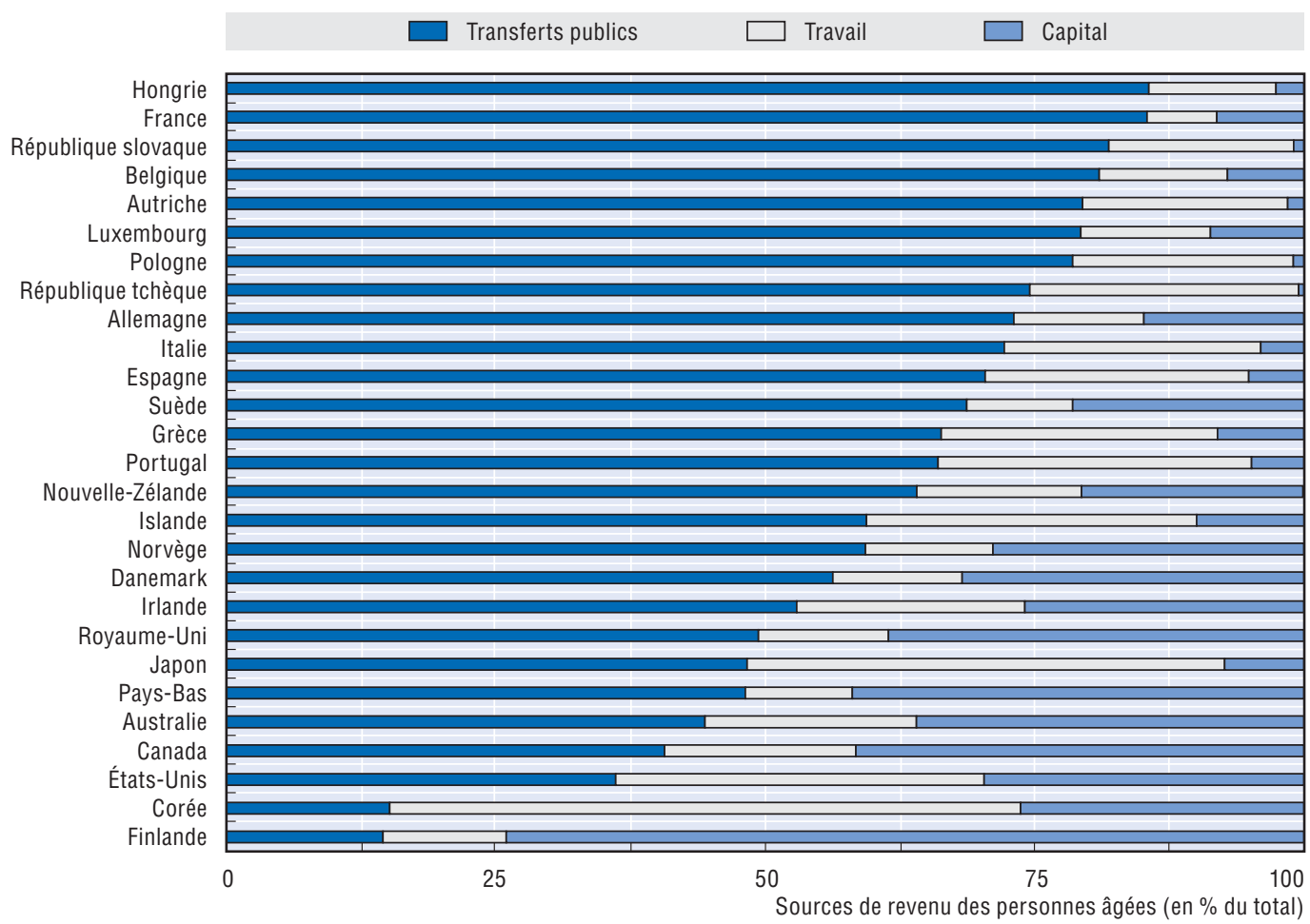

Note : Le revenu du travail inclut les revenus de l'emploi salarié et non salarié. Le revenu du capital inclut les pensions privées ainsi que le rendement de produits d'épargne autres que l'épargne-retraite.

Source: Base de données de l'OCDE sur la distribution des revenus.

StatLink न्ता $h$ ttp://dx.doi.org/10.1787/635426478286

personnes âgées de plus de 65 ans sont les plus tributaires de l'État pour leurs revenus en France et en Hongrie : 85 \% de leurs revenus proviennent des transferts publics. Les trois quarts ou plus des revenus des retraités sont assurés par l'État en Autriche, en Belgique, au Luxembourg, en Pologne et dans les Républiques tchèque et slovaque.

À l'autre extrême, les transferts publics couvrent à peine 15 \% des revenus moyens des retraités en Finlande. Néanmoins, cette situation s'explique par le fait que les régimes professionnels obligatoires sont assimilés à des revenus du capital, tandis que les comptes nationaux et Les pensions dans les pays de l'OCDE considèrent que ces régimes font partie du secteur public. La part des revenus des retraités générée par les transferts publics est également très faible en Corée : en effet, le régime public de retraite date seulement de 1988. Les transferts publics assurent moins de la moitié du revenu des retraités en Australie, au Canada, aux États-Unis, au Japon, aux Pays-Bas, au Royaume-Uni et en Suisse $\mathrm{e}^{14}$.

Dans les pays d'Asie de l'Est membres de l'OCDE, l'emploi - salarié et non salarié couvre une très large part du revenu des personnes âgées de plus de 65 ans : $44 \%$ au Japon et 59 \% en Corée. Le revenu du travail représente également un quart ou plus des revenus des retraités dans six autres pays de l'OCDE : Espagne, États-Unis, Grèce, Islande, Portugal et République tchèque. Dans certains de ces pays, cette situation traduit probablement le fait que de nombreuses personnes n'ont pas accumulé le nombre d'annuités requis dans le régime public de retraite et continuent donc de travailler pour compenser le manque à 
gagner. Aux États-Unis et en Islande, l'âge normal de la retraite est postérieur à 65 ans. En revanche, le revenu du travail (salarié et non salarié) représente moins de $10 \%$ des revenus des personnes âgées en France, aux Pays-Bas et en Suède.

Le revenu du capital - principalement sous la forme de plans de retraite privés constitue la principale source de revenu des personnes âgées en Australie, au Canada, au Danemark, aux États-Unis, aux Pays-Bas et au Royaume-Uni (la Finlande est un cas spécial pour les raisons exposées ci-dessus). Dans ces pays, le revenu du capital représente $30 \%$ ou plus du revenu des personnes âgées.

Il ne faut pas oublier que le graphique 2.3 présente des valeurs moyennes pour les personnes âgées considérées globalement. Toutefois, la composition des revenus varie énormément en fonction du statut socio-économique : les personnes âgées les plus pauvres tirent la quasi-totalité de leurs revenus des transferts publics, alors que les pensions privées et d'autres composantes du revenu du capital jouent un rôle plus important uniquement pour les retraités les plus riches ${ }^{15}$. Compte tenu de la progression de ces sources de revenus dans le total des gains perçus par les retraités, cette évolution pourrait accentuer les inégalités de revenus des personnes âgées (la section 3 ci-dessous analyse en détail la fonction redistributive des impôts et des prestations publiques dans différents pays).

\section{Comment évoluent les revenus relatifs des personnes âgées?}

Dans neuf pays de l'OCDE, les revenus des personnes âgées ont augmenté par rapport à ceux de la population totale entre le milieu des années 80 et le milieu des années 2000. C'est ce qu'illustre le graphique 2.4, qui compare les revenus relatifs des personnes âgées au milieu des années 2000 (axe horizontal) à leur situation au milieu des années 80 (axe vertical). Les résultats sont présentés pour les 20 pays de l'OCDE pour lesquels on dispose de données portant sur les deux intervalles de temps considérés. Dans les pays qui se situent à droite de la diagonale, les revenus des personnes âgées ont augmenté plus rapidement que ceux de la population totale. Dans ceux situés à gauche, les retraités n'ont pas pris toute leur part aux gains de prospérité. Là encore, les résultats sont divisés en deux groupes d'âge.

Les plus fortes progressions des revenus relatifs pour les personnes âgées de 66 à 75 ans sont enregistrées en Allemagne, Autriche et Norvège : 11 points de pourcentage environ. On observe également des gains significatifs - de 6 à 9 points - au Danemark, en Italie, au Luxembourg et au Royaume-Uni. Le revenu relatif des 66-75 ans n'a pas suivi la croissance des revenus moyens dans 11 pays, bien que les baisses soient relativement limitées en Finlande, Grèce et Irlande. Les dégradations les plus marquées entre le milieu des années 80 et le milieu des années 2000 concernent le Mexique et la Turquie, mais ces deux pays partaient de très haut. En Nouvelle-Zélande, le revenu des retraités âgés de 66 à 75 ans représentait $84 \%$ du revenu de la population au milieu des années 80 , contre seulement $73 \%$ au milieu des années 2000 , ce qui place ce pays à l'avant-dernier rang des pays de l'OCDE (graphique 2.1).

Concernant le groupe des retraités âgés de plus de 75 ans, leurs revenus ont également progressé dans neuf pays de l'OCDE par rapport à l'évolution pour l'ensemble de la population. Les retraités concernés en Allemagne, Autriche et Norvège ont vu leur revenu moyen sensiblement augmenter. Les gains au Canada, au Danemark, au Royaume-Uni et en Suède sont presque aussi élevés. Contrairement à la baisse du revenu relatif des 


\section{Graphique 2.4. Évolution des revenus du milieu des années 80 au milieu des années 2000}

Pourcentage des revenus de la population
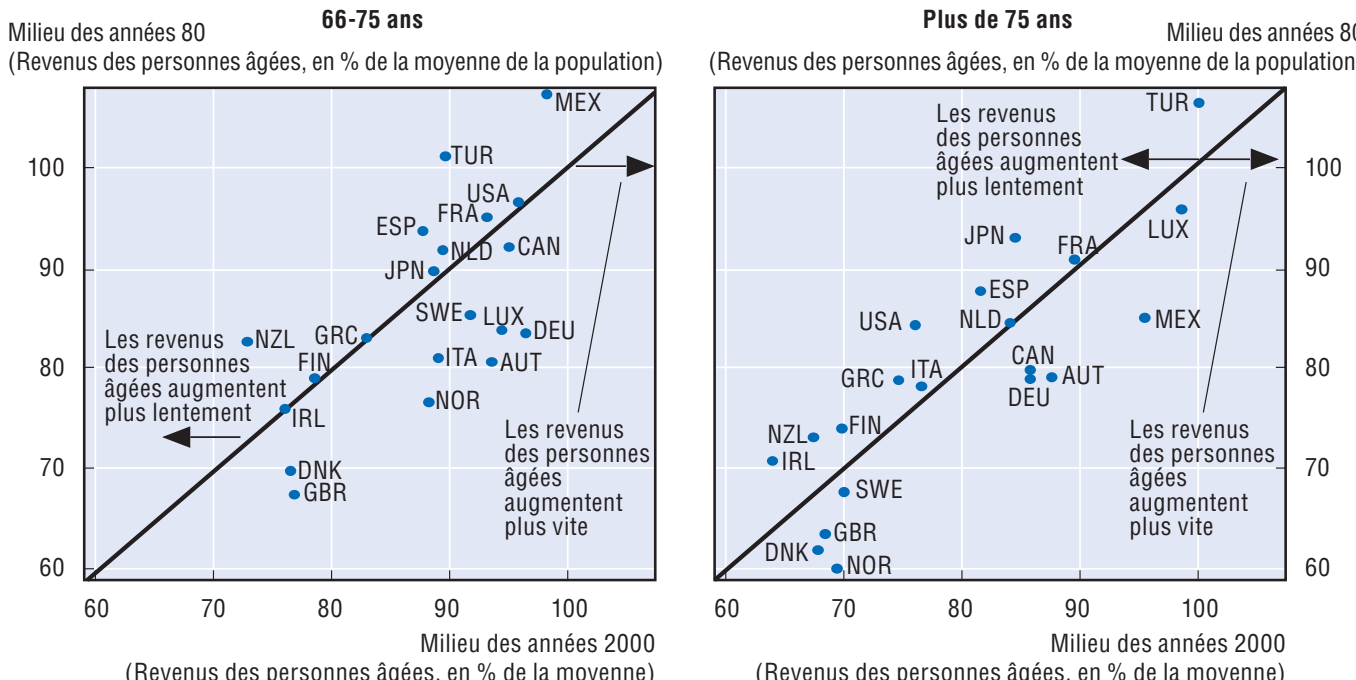

Note: Les données pour le milieu des années 2000 se situent autour de 2000 pour l'Autriche, l'Espagne et l'Italie. En Turquie, les revenus réels ont baissé de $15 \%$ pour l'ensemble de la population, de $20 \%$ pour les personnes de plus de 75 ans et de $25 \%$ pour celles âgées de 66 à 75 ans. Au Japon, les revenus réels ont globalement progressé, mais ont légèrement diminué pour les plus de 75 ans.

Source: Base de données de l'OCDE sur la distribution des revenus ; voir OCDE (2008), Croissance et inégalités, graphique 2.6. StatLink त्नाIs http://dx.doi.org/10.1787/635435501523

personnes de 66-75 ans, les revenus des retraités de plus de 75 ans ont augmenté plus rapidement au Mexique. Les revenus des retraités de plus de 75 ans en Espagne, aux ÉtatsUnis, en Irlande, au Japon, en Nouvelle-Zélande et en Turquie n'ont pas suivi, loin s'en faut, les gains pour l'ensemble de la population.

Ces évolutions des revenus des personnes âgées s'expliquent en grande partie par la maturation des systèmes de retraite. La cohorte des retraités des années 80 est entrée sur le marché du travail dans les années 20 et 30, lorsque les systèmes de retraite étaient beaucoup moins développés. La plupart des régimes publics de retraite tels que ceux que nous connaissons aujourd'hui datent de l'après-guerre : ils ont vu le jour à la fin des années 40 en Australie, en France, en Irlande, au Royaume-Uni et en Suisse, par exemple. D'autres sont plus récents : l'Autriche, la Finlande, la Grèce, l'Italie, le Japon et les Pays-Bas ont créé de nouveaux systèmes de retraite dans les années 50 , tandis que la Belgique, le Canada et la Suède l'ont fait dans les années 60. Par conséquent, les personnes âgées au milieu des années 80 avaient souvent accompli au mieux la moitié de leur carrière en bénéficiant de ces régimes. Les régimes liés à la rémunération, sous leur forme actuelle, ont été établis dans les années 70 au Mexique et au Royaume-Uni. Il est remarquable que la plupart des pays qui ont été les derniers à se doter de régimes de retraite modernes ont vu le revenu relatif des personnes âgées s'accroître fortement (graphique 2.4).

La période nécessaire à la mise en place de régimes publics de retraite se retrouve dans l'évolution des pensions privées dans les pays où ces régimes sont aujourd'hui largement développés. En Australie, au Canada, aux États-Unis, en Irlande et au Royaume-Uni, les régimes professionnels facultatifs ont vu le nombre de leurs adhérents croître rapidement dans les années 50 et 60. Les régimes de retraite privés sont devenus obligatoires en 1992 en Australie et en 1982 en Suisse. 
L'ajustement des pensions servies a entraîné une révision des politiques et des pratiques au fil du temps. De nombreux pays de l'OCDE ont connu une période de fortes augmentations réelles des pensions servies qui a pris fin récemment. En Allemagne et en France, par exemple, les pensions réelles ont plus que doublé entre 1960 et 1978. Dans d'autres pays, la période de forte hausse des prestations réelles s'est terminée plus tard: au début et au milieu des années 80 au Canada, aux États-Unis, en Italie et en NouvelleZélande, et au milieu des années 90 au Japon (Whitehouse, 2009). Les politiques d'indexation se répercutent davantage sur les retraités plus âgés. Parmi ces pays, l'incidence du changement de politique d'indexation sur les revenus des retraités est la plus manifeste aux États-Unis, au Japon et en Nouvelle-Zélande, mais est moins perceptible en France et en Italie. Au Canada et en Allemagne, cet effet a été compensé par d'autres facteurs économiques et changements dans les systèmes de retraite.

\section{La pauvreté monétaire des personnes âgées}

La question de la définition de la "pauvreté » a alimenté un vaste débat qui ne sera probablement jamais tranché. Pour pouvoir procéder à des comparaisons internationales, l'OCDE considère la pauvreté comme un concept relatif (et non absolu). Il est relatif dans deux sens du terme. Premièrement, la pauvreté est mesurée par rapport à un indicateur reposant sur le revenu médian des ménages. Deuxièmement, les seuils de pauvreté sont spécifiques à chaque pays, de sorte que la pauvreté est mesurée par rapport à des normes de niveau de vie en vigueur à un moment donné dans un pays donné. Cela signifie qu'une personne classée comme pauvre dans un pays prospère de l'OCDE aura un revenu supérieur à bien des personnes non considérées comme pauvres dans d'autres pays globalement moins prospères. L'approche générale qui consiste à mesurer la pauvreté relativement à une fraction du revenu médian, adoptée par l'OCDE pour ses études portant sur plusieurs pays, est largement utilisée ailleurs : par exemple, pour les taux de risque de pauvreté calculés dans le cadre du système de bilans sociaux de l'Union européenne.

Dans l'étude Croissance et inégalités (OCDE, 2008), les analyses reposent pour l'essentiel sur un seuil de pauvreté correspondant à $50 \%$ du revenu disponible équivalent médian des ménages. Les titulaires de revenus inférieurs à ce seuil sont considérés comme étant en situation de pauvreté monétaire ${ }^{16}$.

Au milieu des années 2000, 13.3 \% des personnes âgées (de plus de 65 ans) étaient en situation de pauvreté monétaire en moyenne dans les pays de l'OCDE. Le taux de pauvreté des personnes âgées était de loin le plus élevé en Corée, à $45 \%$ (voir le graphique 2.5). L'Australie (27\%), les États-Unis (24\%), la Grèce (23\%), l'Irlande (31 \%), le Japon (22\%) et le Mexique (28\%) connaissent eux aussi un taux de pauvreté des personnes âgées élevé (plus de $20 \%$ ). Un groupe de pays se situe juste un peu au-dessus de la moyenne de l'OCDE : Espagne, Portugal, Suisse et Turquie, avec un taux de personnes âgées vivant en situation de pauvreté compris entre $15 \%$ et $18 \%$. La Belgique, la Finlande et l'Italie se situent juste en dessous de la moyenne de l'OCDE. Les huit pays où le taux de pauvreté monétaire des personnes âgées est le plus bas - inférieur à $5 \%$ sont le Canada, la Hongrie, l'Islande, le Luxembourg, les Pays-Bas, la Nouvelle-Zélande, la Pologne et la République tchèque.

L'un des principaux déterminants des différences de taux de pauvreté des personnes âgées est le niveau auquel les prestations prévues par les filets de protection sont fixées. 
Graphique 2.5. Taux de pauvreté des personnes âgées, milieu des années $\mathbf{2 0 0 0}$

Pourcentage de personnes âgées de plus de 65 ans dont les revenus sont inférieurs à la moitié du revenu équivalent médian

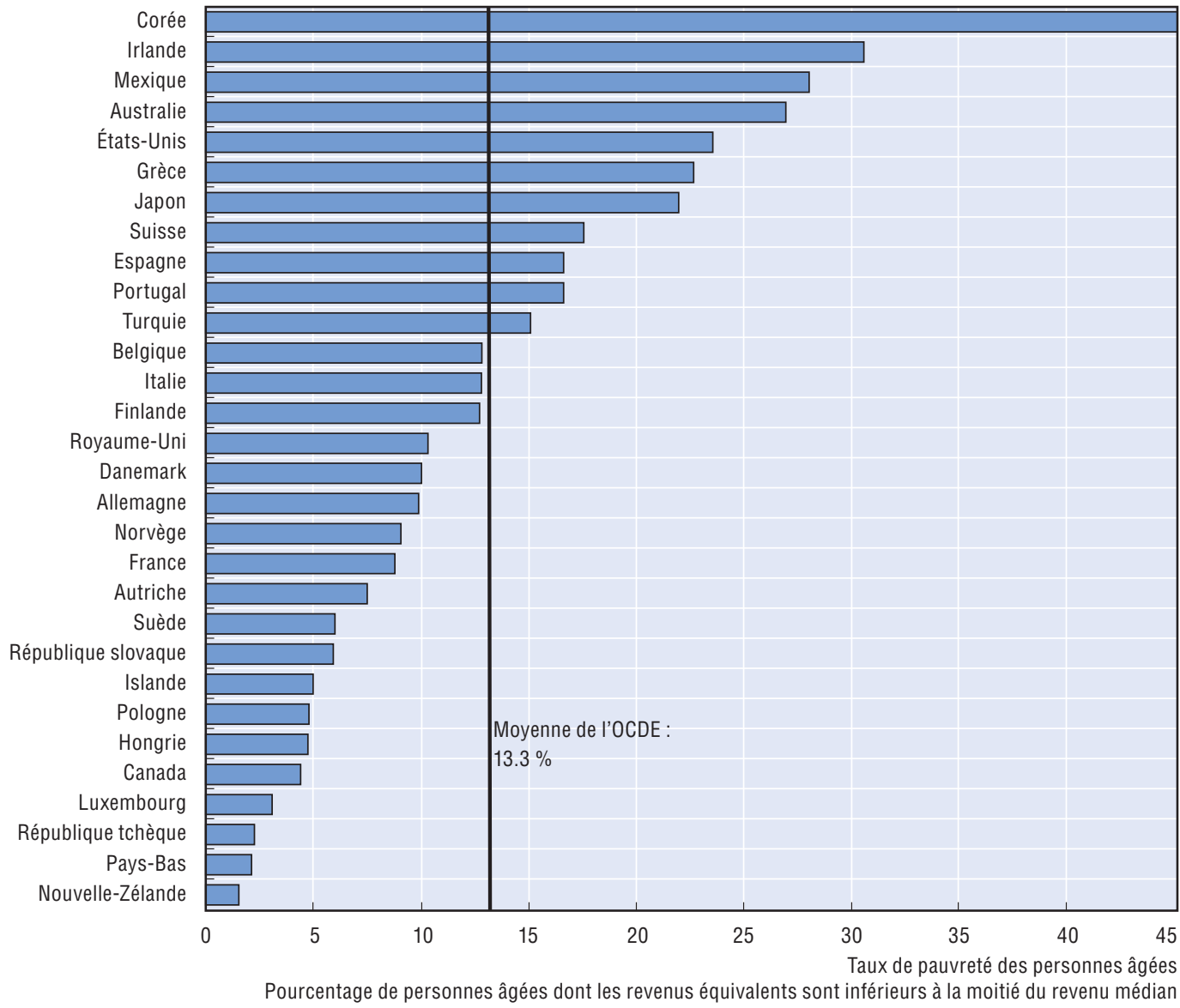

Source: Base de données de l'OCDE sur la distribution des revenus, voir OCDE (2008), Croissance et inégalités, tableau 5.3 .

En Australie, par exemple, le montant d'une retraite de base à taux plein en 2005 était de 12700 AUD par an, soit moins que le seuil de pauvreté fixé à 14770 AUD pour une personne seule ${ }^{17}$. L'écart entre ces deux valeurs est encore plus prononcé en Irlande : 8870 EUR pour la retraite de base contre 10775 EUR pour le seuil de pauvreté. Dans ces deux pays, de nombreuses personnes perçoivent un revenu qui se situe aux alentours du niveau de ces programmes, qui représentent $86 \%$ du seuil de pauvreté en Australie et $82 \%$ en Irlande. Cela explique pourquoi ces pays comptent parmi les quatre qui enregistrent les taux de pauvreté des personnes âgées les plus élevés.

En revanche, la retraite de base en Nouvelle-Zélande, d'un montant de 16100 NZD en 2005, était beaucoup plus élevée que le seuil de pauvreté de 13040 NZD pour une personne seule. Aux Pays-Bas, la retraite de base se situait un peu en dessous du seuil de pauvreté de 11500 EUR en 2005. Sachant que la couverture de ces régimes est pratiquement universelle (parce qu'ils sont conditionnés au lieu de résidence), il n'est pas surprenant que les taux de pauvreté des personnes âgées soient les plus bas dans ces deux pays (l'encadré 2.1 fournit plus de détails sur le niveau et la couverture des prestations de retraite de base, sous conditions de ressources et minimum). 


\section{Encadré 2.1. Filets de protection des personnes âgées*}

Les filets de protection visant les personnes âgées désignent les prestations versées par les « régimes de premier pilier, redistributifs " décrits dans le «Cadre conceptuel des Pensions dans les pays de l'OCDE ». Ils englobent donc toutes les retraites de base, sous conditions de ressources et minimum que les personnes âgées à faible revenu peuvent percevoir. Dans certains pays, les programmes ou niveaux de prestations varient en fonction de la durée de cotisation. Dans ce cas, les calculs indiquent le niveau de prestation le plus élevé qui suppose en général une certaine période de cotisation.

$\mathrm{Au}$ bas de l'échelle, l'assistance sociale, les retraites de base et minimum assurent un revenu minimum inférieur à $20 \%$ du salaire moyen en Allemagne, aux États-Unis, en Finlande, en Hongrie et au Japon.

\section{Graphique 2.6. Valeur des prestations de retraite de base, sous conditions de ressources et minimum}

Pourcentage du salaire moyen

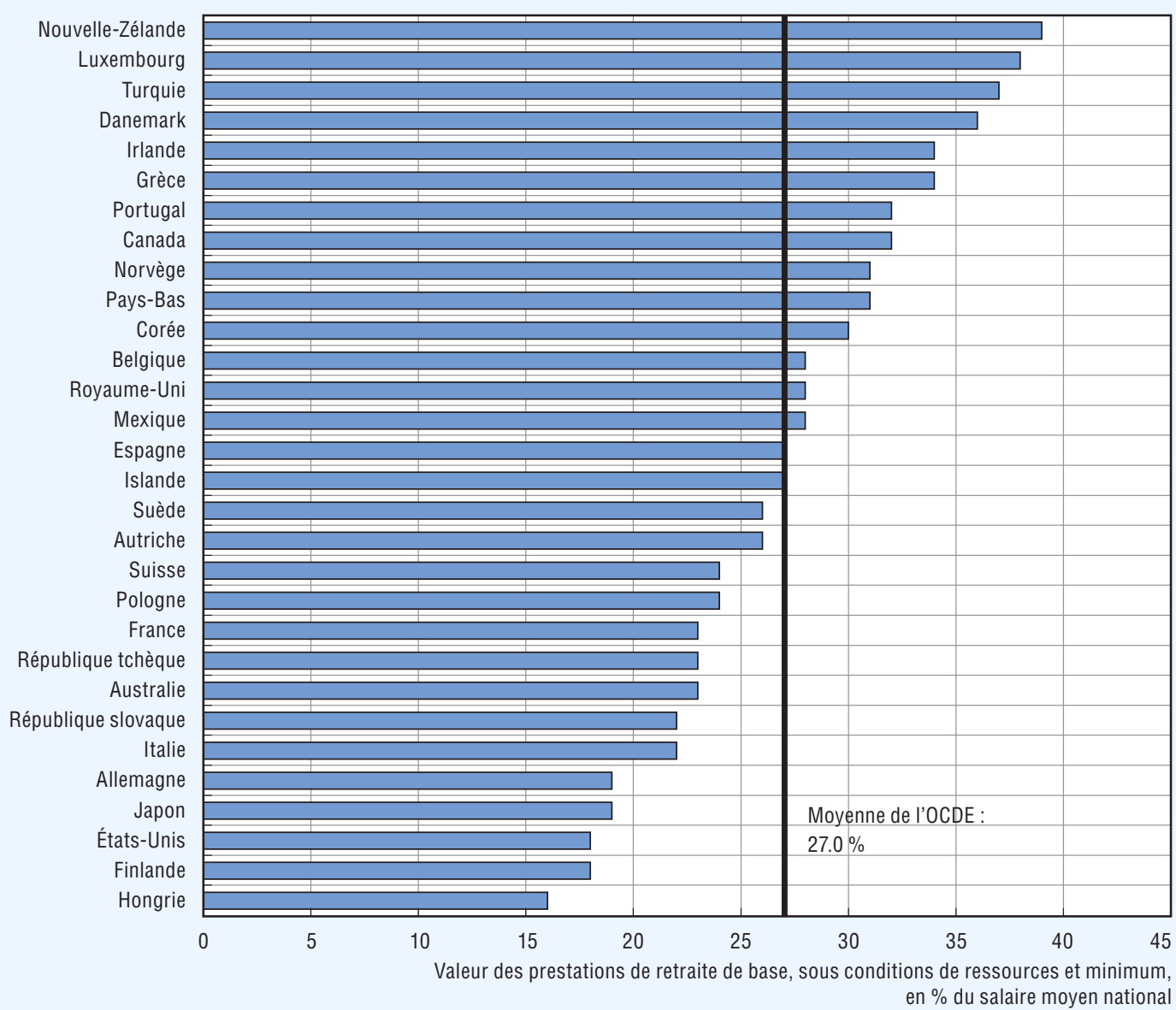

Source : Descriptifs pays en partie III de cette étude.

StatLink त्नाजा http://dx.doi.org/10.1787/635445585875

Le graphique 2.7 illustre le pourcentage de personnes âgées de plus de 65 ans qui perçoivent des prestations sous conditions de ressources, divisées, le cas échéant, entre différents régimes. Au Portugal, par exemple, près de $60 \%$ des retraités dépendent du régime de pension contributif minimum, et $17 \%$ perçoivent l'aide sociale ou les prestations de solidarité sociale. La couverture de ces deux régimes est 


\section{Encadré 2.1. Filets de protection des personnes âgées* (suite)}

même plus élevée en Grèce. En Finlande et en Suède, plus de la moitié des personnes âgées perçoivent aussi une retraite minimum. Toutefois, la couverture du filet de protection (assurée par l'assistance sociale) est très limitée dans ces deux pays par rapport à la Grèce et au Portugal. Cela reflète probablement le fait que les pays d'Europe du Sud comptent un secteur informel étendu qui est tributaire du revenu garanti par le filet de protection. En revanche, dans les pays nordiques, le secteur informel est très peu développé et les personnes ayant exercé un emploi rémunéré bénéficient d'une protection globale par le biais de crédits de retraite.

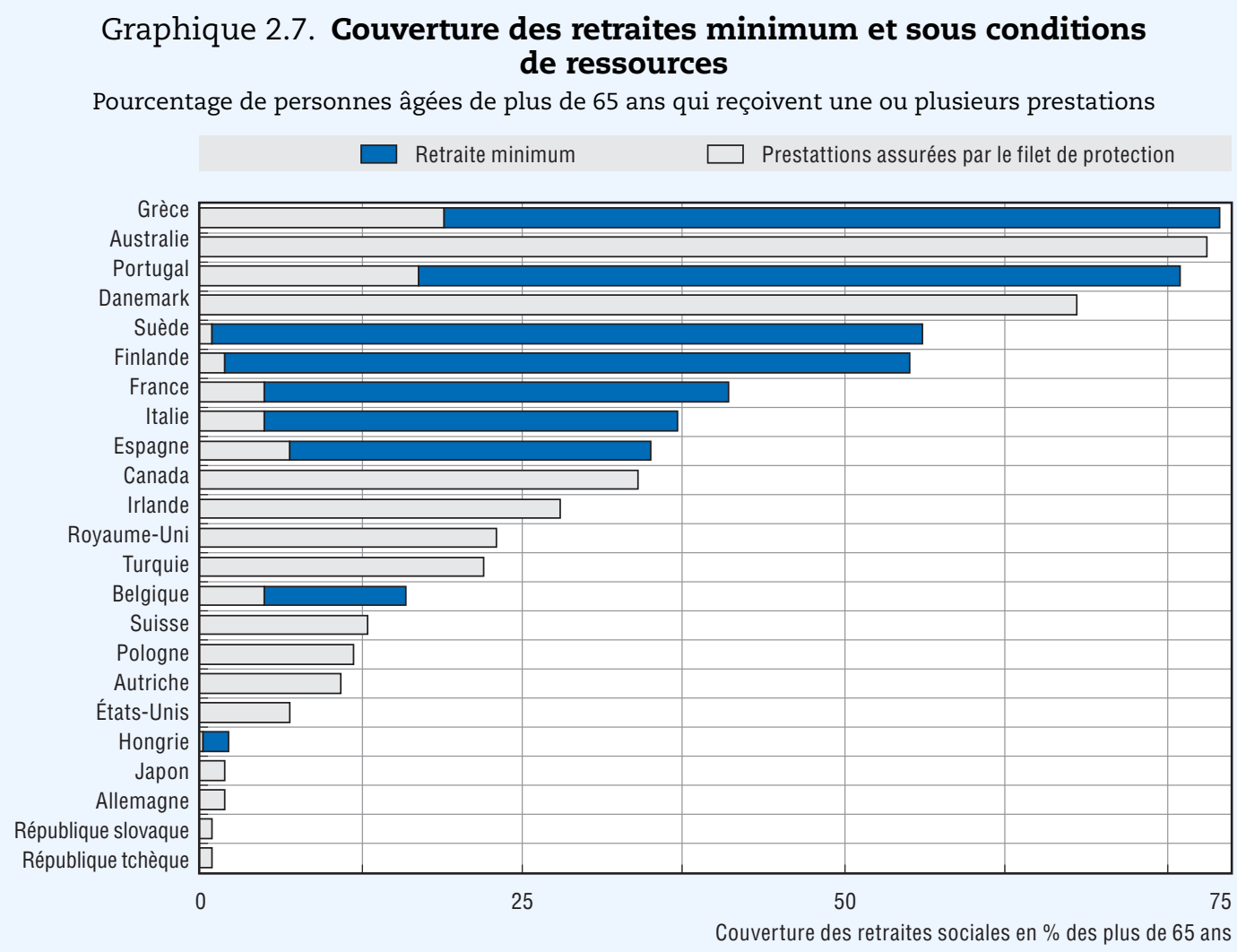

Source: Union européenne, Comité de politique sociale (2006); Pearson et Whitehouse (2009), "Social Pensions in High-Income Countries ", dans R. Holzmann et N. Takayama (dir. pub.), Closing the Coverage Gap: The Role of Social Pensions, Banque mondiale, Washington, DC, à paraître.

StatLink Anilst http://dx.doi.org/10.1787/635454065766

Dans la plupart des pays, les données du graphique 2.7 se rapportent à un dispositif de prestations unique. La fourchette des personnes couvertes par ces régimes est très large. En Australie, par exemple, le système public de retraite est conditionné aux ressources. Néanmoins, plus de $75 \%$ des personnes âgées peuvent y prétendre. Ce résultat tranche avec l'Allemagne et le Japon, où seulement $2 \%$ des personnes âgées perçoivent l'assistance sociale. Dans ces trois pays, les prestations sont versées sous conditions de ressources. Toutefois, leur fonctionnement dans la pratique est fondamentalement différent. Le régime australien a été qualifié de système " sous conditions de richesse " parce que les prestations sont versées à la majorité des personnes ayant l'âge de la retraite et refusées aux retraités les plus riches. En revanche, les régimes allemand et japonais sont dits "sous conditions de pauvreté ", parce que seules les personnes âgées les plus pauvres en bénéficient. 


\section{Encadré 2.1. Filets de protection des personnes âgées* (suite)}

Le graphique ne tient pas compte des bénéficiaires d'une retraite de base. En Nouvelle-Zélande et aux Pays-Bas, par exemple, la retraite de base est versée aux adultes résidant dans le pays, de sorte que la quasi-totalité des personnes âgées la perçoivent. Au Royaume-Uni, moins de 25 \% des personnes âgées bénéficient des programmes de prestations sous conditions de ressources - crédit de retraite et crédit d'épargne - mais 98 \% touchent une somme plus ou moins élevée au titre de la retraite de base. La situation est analogue au Canada, au Danemark, en Irlande, au Japon et en République tchèque, où la couverture de la retraite de base est pratiquement universelle.

* Cet encadré s'appuie sur l'analyse plus détaillée effectuée par Pearson et Whitehouse (2009).

\section{Les personnes âgées ont-elles plus de risques d'être pauvres?}

Une explication de la structure de la pauvreté, illustrée ci-dessus, réside dans le fait que dans certains pays, la répartition des revenus est plus inégale que dans d'autres, et ces inégalités persistent pendant la retraite. Le graphique 2.8 compare les taux de pauvreté monétaire des personnes âgées (axe vertical) avec ceux de l'ensemble de la population (axe horizontal). Dans les pays qui se situent au-dessus de la ligne, le taux de pauvreté des personnes âgées est plus élevé que pour l'ensemble de la population. Dans les pays situés en dessous de la ligne, le risque de pauvreté des personnes âgées est inférieur à celui de la population totale. Il existe certes une corrélation positive et forte entre la vieillesse et la pauvreté générale, mais la relation entre ces deux grandeurs reste très variable d'un pays à l'autre $^{18}$.

En moyenne, dans les pays de l'OCDE, le taux de pauvreté de la population - $10.6 \%$ est inférieur à celui des personnes âgées - $13.3 \%$. Dans 11 pays, le risque de pauvreté des personnes âgées est inférieur à celui de la population totale. L'écart est particulièrement important au Canada, en Nouvelle-Zélande et en Pologne, où le taux de pauvreté de la population oscille entre $10 \%$ et $15 \%$, alors que celui des personnes âgées de plus de 65 ans

\section{Graphique 2.8. Taux de pauvreté monétaire des personnes âgées et de la population, milieu des années 2000}

Pourcentage de personnes âgées dont les revenus sont inférieurs à la moitié du revenu équivalent médian

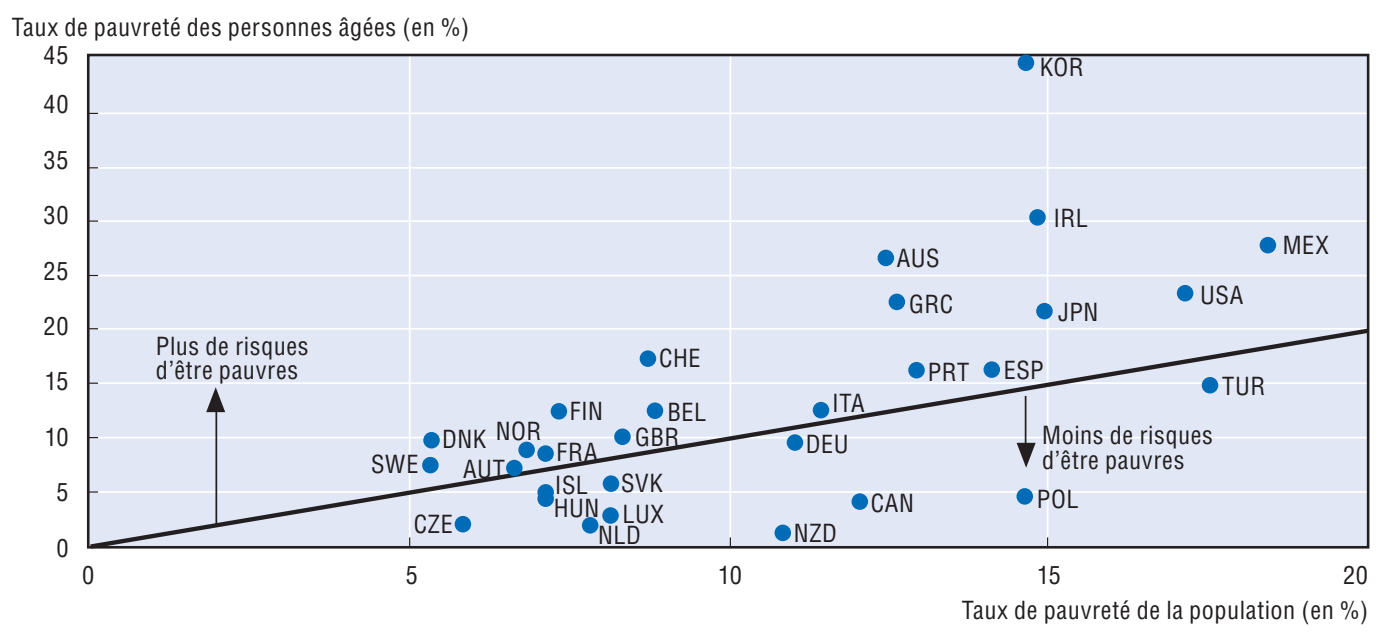

Source: Base de données de l'OCDE sur la distribution des revenus ; voir OCDE (2008), Croissance et inégalités, tableaux 5.1 et 5.3. 
est inférieur ou égal à $5 \%$. De même, le risque de pauvreté est beaucoup moins élevé pour les personnes âgées au Luxembourg, aux Pays-Bas et en République tchèque.

Parmi les 19 pays de l'OCDE dans lesquels la pauvreté des personnes âgées est relativement plus élevée, l'écart entre les taux de pauvreté des personnes de plus de 65 ans et de l'ensemble de la population est assez faible dans six d'entre eux : Espagne, France, Italie, Norvège, Royaume-Uni et Suède. Toutefois, la différence est significative dans huit pays. La Corée se distingue une fois encore, puisque trois fois plus de personnes âgées de plus de 65 ans vivent dans la pauvreté que la population totale. En Australie, Irlande et Suisse, les taux de pauvreté des personnes âgées sont deux fois plus élevés que ceux de la population ; l'écart est également marqué aux États-Unis, en Grèce, au Japon et au Mexique.

Comme on l'a vu plus haut, le niveau et le degré de couverture des prestations versées au titre du filet de protection sont l'une des principales causes de ces différences entre pays. Ces facteurs expliquent le risque de pauvreté relativement faible des personnes âgées au Canada, au Luxembourg, en Nouvelle-Zélande et aux Pays-Bas. De même, le risque de pauvreté des personnes âgées par rapport à la population est beaucoup plus élevé dans les pays où les filets de protection sont faibles. Outre l'Australie et l'Irlande (voir cidessus), les prestations versées au titre du filet de protection représentent seulement $34 \%$ environ du seuil de pauvreté en Grèce, 53 \% au Japon et aux États-Unis et 75 \% en Suisse.

Il convient de rappeler que ces chiffres sont basés sur des mesures de la pauvreté monétaire. L'encadré 2.2 montre que d'autres mesures de la pauvreté, qui tiennent compte de la satisfaction des besoins fondamentaux, dressent un tableau moins négatif des risques de pauvreté des personnes âgées.

\section{Encadré 2.2. Pauvreté monétaire, exclusion sociale et privation matérielle*}

De toute évidence, la pauvreté est un phénomène plus complexe que ne laisse entrevoir la seule pauvreté monétaire. Par exemple, les personnes ayant de faibles revenus peuvent bénéficier d'une aide en nature de la part de l'État ou de membres de leurs familles. Certaines personnes âgées sont « riches en patrimoine mais pauvres en revenu ", ce qui signifie qu'elles possèdent des actifs dans lesquels elles peuvent puiser pour subvenir à leurs besoins. Inversement, les revenus de certaines personnes peuvent être insuffisants pour leur permettre de satisfaire à leurs besoins en cas de maladie ou d'invalidité, par exemple. Les indicateurs de " privation matérielle » ou de « difficulté de vie » tentent de rendre compte de certains de ces effets.

La privation matérielle renvoie à l'incapacité de se procurer des biens ou des services considérés comme d'usage courant dans la société. Ces difficultés matérielles, qui peuvent être mesurées selon des modalités identiques dans plusieurs pays, sont par exemple :

- chauffage insuffisant ;

- limitation du choix de produits alimentaires ;

- manque d'espace ;

- nuisances environnementales ;

- retards de paiement de factures courantes ;

- retards de paiement de loyer ou de crédit immobilier ;

- incapacité de boucler le budget du ménage. 


\section{Encadré 2.2. Pauvreté monétaire, exclusion sociale et privation matérielle* (suite)}

Dans les 21 pays pour lesquels des données sont disponibles, $10.6 \%$ des personnes vivent dans des ménages qui souffrent de privations matérielles relevant d'au moins trois des catégories susmentionnées. Dans l'ensemble, la privation matérielle est la plus sévère en Europe de l'Est : 40 \% en Pologne, environ 25 \% en Hongrie et en République slovaque et $16 \%$ en République tchèque. Sa prévalence est également élevée en Grèce, en Italie et au Portugal.

\section{Graphique 2.9. Privation matérielle selon l'âge}

Pourcentage de personnes vivant dans des ménages qui se privent de trois biens matériels ou plus

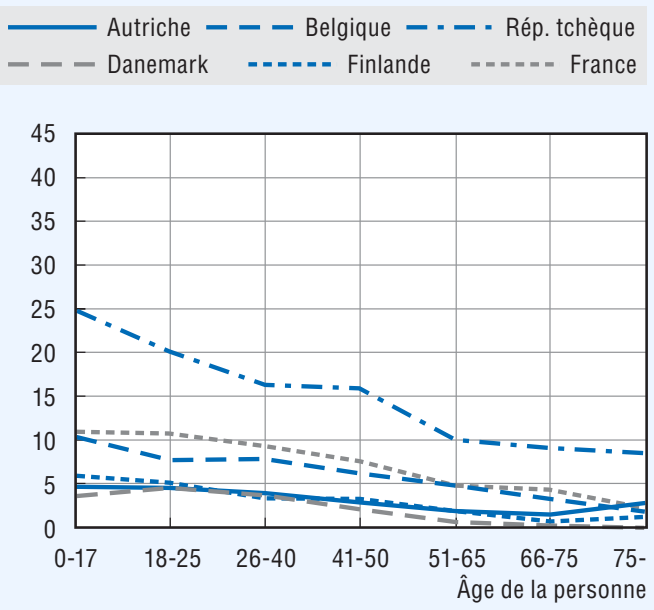

$$
\begin{array}{lll}
\hline & \text { Allemagne } \quad--- \text { Grèce } \quad-.- \text { Hongrie } \\
--- \text { Islande } & -. .-. \text { Irlande }
\end{array}
$$
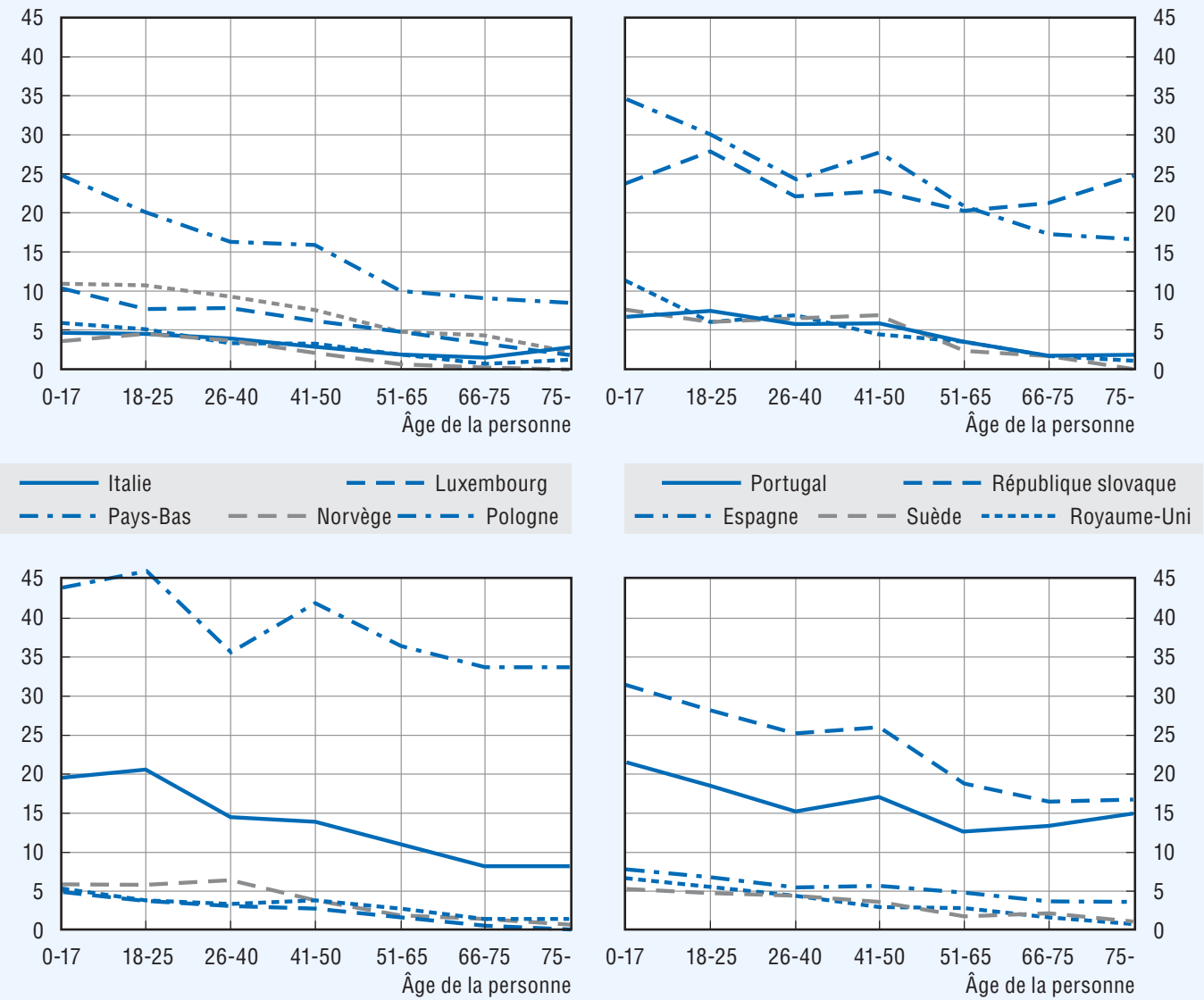

Source: Base de données de l’OCDE sur la distribution des revenus. Voir OCDE (2008), Croissance et inégalités, graphique 7.4.

StatLink न्ताs http://dx.doi.org/10.1787/635481167282

L'analyse des résultats par âge révèle que, dans les pays de l'OCDE, la privation matérielle touche surtout les enfants (de moins de 17 ans) et les jeunes adultes (de 18 à 25 ans), à un taux respectif de $14.1 \%$ et $13.1 \%$. Elle est beaucoup moins fréquente chez les personnes âgées (de plus de 65 ans), avec environ 6.8 \%. Dans la plupart des pays, la tendance générale est un recul de la privation matérielle avec l'âge (graphique 2.9), à l'exception notable de la Grèce, de la Pologne et du Portugal, où les différences entre groupes d'âge sont minimes. De même, la privation matérielle est assez élevée chez les personnes âgées par rapport à la population dans son ensemble en Espagne, Hongrie et République slovaque. Elle est pratiquement inexistante chez les personnes âgées au Danemark, en Islande, au Luxembourg, en Norvège, aux Pays-Bas et au Royaume-Uni.

* Voir OCDE (2008), Croissance et inégalités, chapitre III pour une analyse détaillée. 


\section{Les femmes âgées ont-elles plus de risques d'être pauvres que les hommes âgés ?}

En général, dans les pays de l'OCDE, le taux de pauvreté des femmes âgées est beaucoup plus élevé que celui des hommes âgés. En moyenne, il avoisine $15 \%$, contre $10 \%$ environ pour les hommes. Les seules exceptions concernent trois pays où le taux de pauvreté des personnes âgées est faible : l'Islande, le Luxembourg et la Nouvelle-Zélande (voir le panneau de gauche du graphique 2.10). Au Luxembourg et en Nouvelle-Zélande, cela tient probablement au fait que les retraites du système d'assistance sociale (respectivement minimum et de base) figurent parmi les quatre plus élevées comparativement au salaire moyen du pays. Toutefois, dans cinq autres pays, le taux de pauvreté des femmes âgées n'est que légèrement supérieur (d'un point de pourcentage ou moins) à celui des hommes: Belgique, Mexique, Pays-Bas, Portugal et Turquie. Les écarts les plus prononcés entre le taux de pauvreté des hommes et des femmes âgés sont observés en Finlande, en Irlande et en Norvège, où le taux pour les femmes est 10 points supérieur à celui des hommes. Les différences sont également significatives en Autriche, aux États-Unis, en Italie, au Japon et en République slovaque.

\section{Graphique 2.10. Taux de pauvreté monétaire selon l'âge et le sexe, milieu des années 2000}

Pourcentage de personnes dont les revenus sont inférieurs à la moitié du revenu équivalent médian de la population
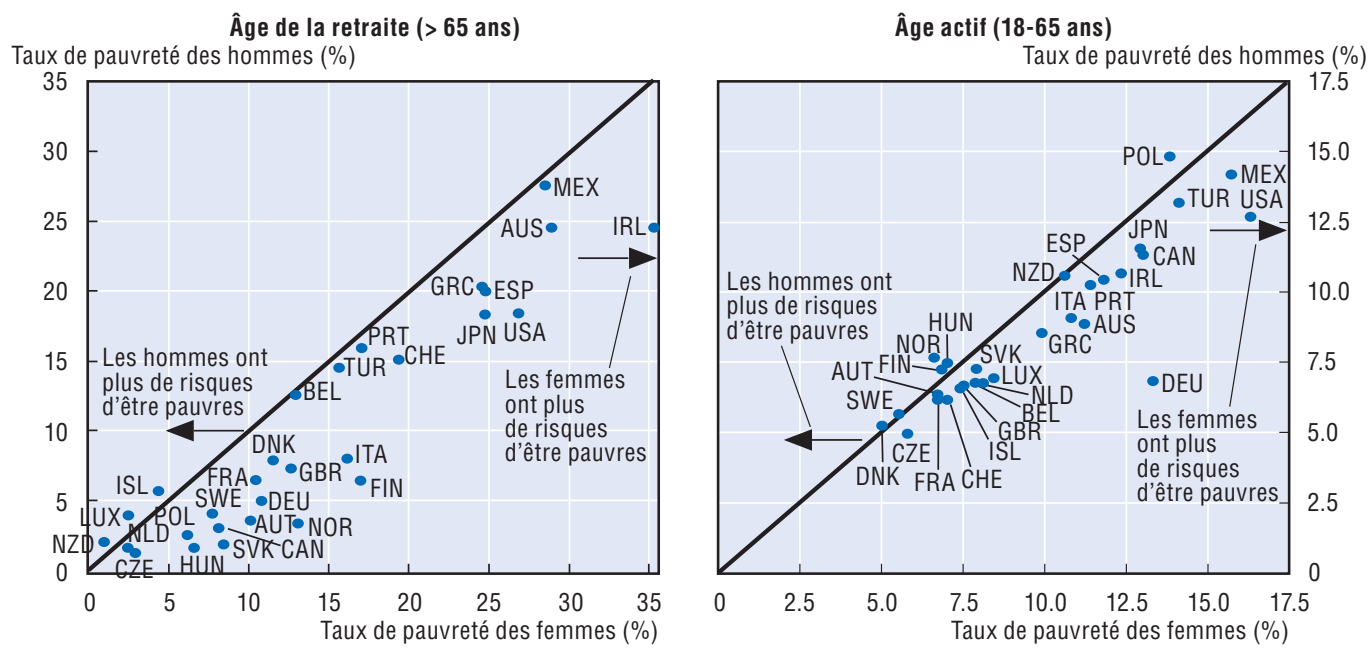

Note : Par souci de clarté, la Corée, qui constitue un cas atypique, est exclue des diagrammes. Le taux de pauvreté des hommes ayant l'âge de la retraite est de $41.8 \%$ contre $47.2 \%$ pour les femmes. Pour la population d'âge actif, ce taux est de $11.0 \%$ pour les hommes et de $12.4 \%$ pour les femmes.

Source : Base de données de l'OCDE sur la distribution des revenus ; voir OCDE (2008), Croissance et inégalités, graphique 5.6. StatLink न्तारा http://dx.doi.org/10.1787/635483036023

Le panneau de droite du graphique 2.10 procède à une comparaison identique des taux de pauvreté selon le sexe parmi les personnes d'âge actif (18-65 ans). Par souci de clarté, l'échelle utilisée pour les personnes d'âge actif correspond exactement à la moitié de celle employée pour les personnes ayant l'âge de la retraite. Comme le révèle la comparaison des taux de pauvreté des personnes âgées et de l'ensemble de la population, les personnes d'âge actif ont généralement moins de risques d'être pauvres. Les taux de pauvreté sont respectivement de $9.8 \%$ et de $8.8 \%$ pour les femmes et les hommes d'âge actif, contre $15.2 \%$ et 10.7 \% pour celles et ceux ayant l'âge de la retraite. La différence la plus marquée entre les sexes est observée en Allemagne, où le taux de pauvreté des femmes est de $13 \%$ et celui des hommes de $7 \%$. L'écart de pauvreté entre les sexes est 
relativement important en Australie et aux États-Unis. Néanmoins, le graphique 2.10 montre clairement que dans la plupart des pays, le taux de pauvreté des femmes d'âge actif n'est que légèrement supérieur à celui des hommes.

Le graphique 2.11, basé sur l'analyse des taux de pauvreté des personnes âgées de 66 à 75 ans et de plus de 75 ans, analyse plus en détail les différences entre hommes et femmes en termes de risques de pauvreté. Il illustre l'écart en points de pourcentage, entre les taux de pauvreté des hommes et des femmes âgés. Un chiffre positif indique que les femmes ont plus de risques d'être pauvres que les hommes. À titre de référence, le diagramme présente également l'écart de pauvreté pour l'ensemble des hommes et femmes de plus de 65 ans.

Quels sont les facteurs qui expliquent la féminisation croissante de la pauvreté avec l'âge ? Il est utile de reprendre la grille d'analyse fondée sur une division des effets démographiques, de cohorte et de génération employée pour décrire les caractéristiques du

\section{Graphique 2.11. Inégalités hommes-femmes en termes de pauvreté des personnes âgées : différentiel de pauvreté entre hommes et femmes selon l'âge, milieu des années $\mathbf{2 0 0 0}$}

Pourcentage de personnes dont les revenus sont inférieurs à la moitié du revenu équivalent médian de la population

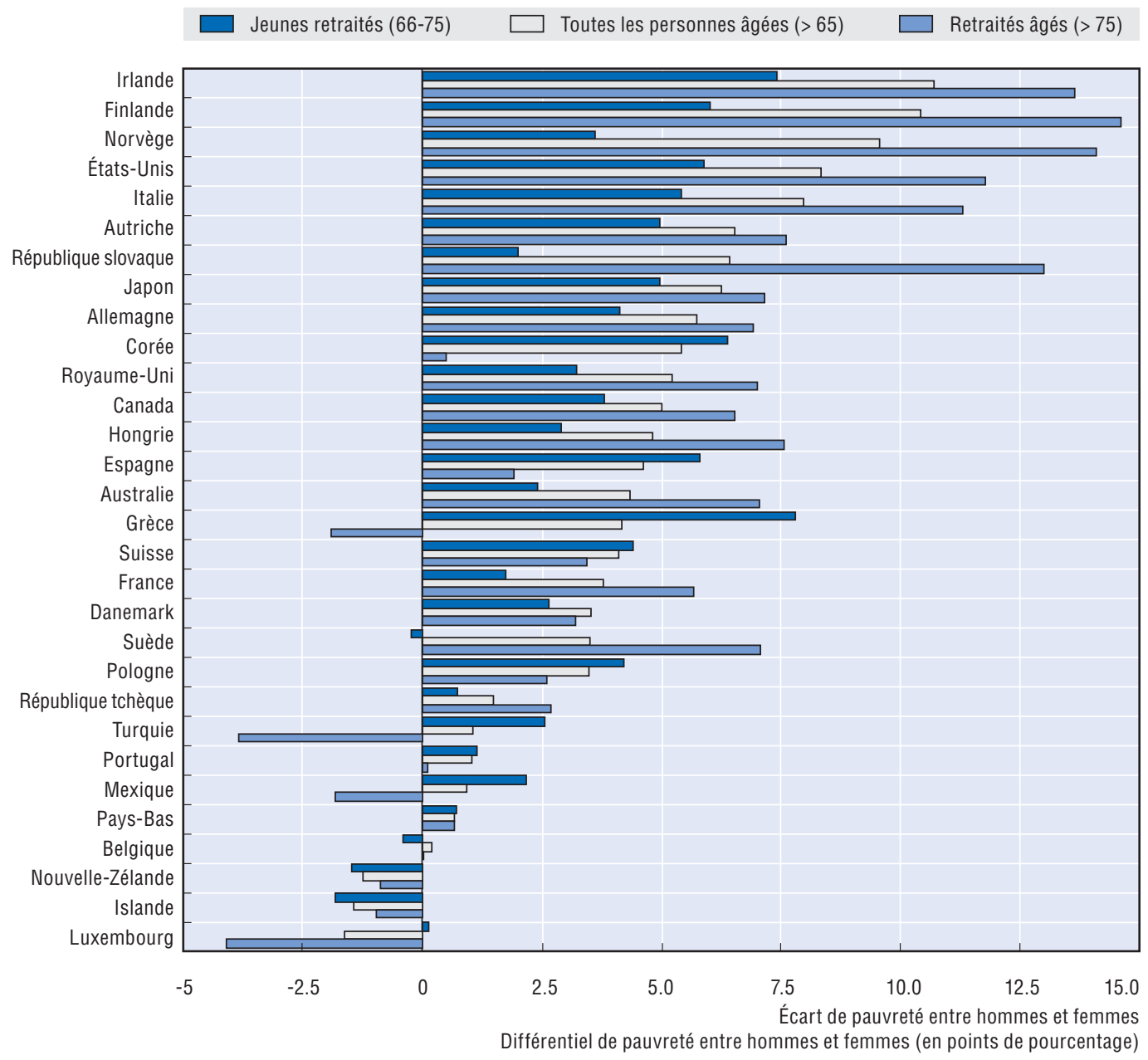

Source: Base de données de l'OCDE sur la distribution des revenus ; voir OCDE (2008), Croissance et inégalités, graphique 5.6. 
revenu relatif des personnes âgées dans la section 1 ci-dessus. Premièrement, l'effet démographique résulte des politiques d'indexation des pensions versées. La pauvreté étant considérée comme un concept relatif, l'indexation des prix peut faire en sorte que les retraités âgés passent sous le seuil de pauvreté à mesure qu'ils vieillissent. Ce phénomène touche davantage les femmes que les hommes parce qu'elles vivent plus longtemps en moyenne.

Toutefois, l'effet de cohorte ou effet générationnel est le plus significatif. Les jeunes retraités (66-75 ans) sont entrés sur le marché du travail dans les années 60, tandis que ceux plus âgés ont commencé à travailler dans les années 50. Les femmes de ces générations ont fondé une famille plus tôt qu'aujourd'hui : l'âge moyen auquel les femmes avaient leur premier enfant était de 24 ans en 1970, contre presque 28 ans en 2005. Ces femmes avaient aussi plus d'enfants que celles d'aujourd'hui : en moyenne, 2.7 enfants par femme en 1970 contre seulement 1.65 en 2005 (voir l'indicateur « Fertilité » dans la partie II de cette publication). Cette tendance étant pérenne, les personnes les plus âgées ont eu généralement plus d'enfants et ont fondé une famille plus tôt que celles plus jeunes. Cela signifierait que les jeunes retraitées ont interrompu moins fréquemment leur carrière pour s'occuper de leurs enfants.

De surcroît, la situation des femmes sur le marché du travail a radicalement changé au cours de cette période. En dépit des différences d'ampleur et de rythme d'un pays à l'autre, ce changement va partout dans le même sens : au cours de leur existence, les nouvelles générations de femmes consacrent plus de temps à l'exercice d'une activité rémunérée. De même, la législation antidiscrimination a permis un resserrement de l'écart salarial entre hommes et femmes (bien qu'il reste substantiel dans de nombreux pays). Par conséquent, les femmes sont de plus en plus nombreuses à parvenir à l'âge de la retraite en ayant plus de droits à pension, et ne sont plus tributaires des prestations versées au titre des cotisations acquittées par leurs conjoints. En outre, de nombreux pays ont pris des mesures visant à octroyer des crédits de retraite aux femmes qui quittent le marché du travail pour s'occuper de leurs enfants.

Par ailleurs, l'âge de départ à la retraite des femmes dans de nombreux pays de l'OCDE était généralement inférieur à celui des hommes, de sorte que les générations plus anciennes cotisaient moins longtemps au système de retraite et percevaient moins de prestations. En 1983, par exemple, l'âge de départ à la retraite des hommes dans la zone OCDE était en moyenne de 63.6 ans, soit 2.0 ans de plus que les femmes. Au cours de la dernière décennie, l'écart entre les deux s'est resserré à 1.5 an en 1993 : l'âge d'ouverture des droits a baissé pour les deux sexes, mais davantage pour les hommes. En 2002, une petite augmentation pour les hommes et un gain d'une année pour les femmes ont ramené l'écart de l'âge de départ à la retraite entre hommes et femmes à 0.7 an. En vertu de la législation actuelle, l'âge de la retraite sera relevé pour les deux sexes, et passera à terme à 65.3 ans pour les hommes et 64.8 ans pour les femmes en moyenne ${ }^{19}$.

Troisièmement, il existe un effet de composition. Les femmes vivent en moyenne plus longtemps que les hommes et sont souvent mariées à des hommes plus âgés qu'elles. Par conséquent, le groupe plus âgé se compose de nombreuses veuves, dont les revenus proviennent dans une large mesure des prestations de réversion. Les règles afférentes à ces pensions de réversion ont donc une influence considérable sur les taux de pauvreté des retraitées les plus âgées. En outre, la prévalence de femmes pauvres parmi les personnes les plus âgées reflète les différences de mortalité entre groupes socio-économiques : les femmes qui ont épousé des hommes plus pauvres ont plus de risques d'être veuves. 


\section{Le rôle des salaires}

La plupart des pays de l'OCDE majorent les droits à retraite pour les personnes qui reportent leur départ et qui continuent de travailler au-delà de l'âge normal de la retraite. Toutefois, les personnes âgées qui souhaitent travailler se heurtent souvent à la discrimination liée à l'âge et à d'autres obstacles, comme des barèmes de rémunération qui indexent le salaire sur l'ancienneté (recruter et faire travailler des personnes âgées revient donc plus cher à l'entreprise $)^{20}$.

Environ $27 \%$ des personnes de plus de 65 ans travaillent (ou font partie d'un ménage dont un membre travaille) en moyenne dans les pays de l'OCDE. Ce pourcentage est resté stable lors de la dernière décennie, lorsque l'âge effectif de départ à la retraite a cessé de baisser ${ }^{21}$.

Les ménages qui comptent au moins un membre ayant atteint l'âge de la retraite mais qui travaille enregistrent des taux de pauvreté beaucoup plus faibles $-7 \%$ en moyenne que ceux qui n'ont pas d'actifs en leur sein : 17 \% (tableau 2.1). Les écarts de pauvreté en fonction du statut professionnel du ou des membres du ménage sont surtout manifestes en Allemagne, Australie, France, Grèce, Irlande, Italie, Norvège, au Portugal et au RoyaumeUni. L'effet sur le taux de pauvreté est beaucoup plus faible en Autriche, en Finlande, en Nouvelle-Zélande, aux Pays-Bas et en Pologne. Toutefois, en Turquie, les ménages âgés qui ne travaillent pas enregistrent un taux de pauvreté inférieur à celui de ceux qui travaillent. Cela s'explique par la couverture relativement faible du système de retraite, dont bénéficient essentiellement les titulaires de salaires élevés.

\section{Le rôle des modes de vie}

Le tableau 2.1 évalue également la pauvreté des personnes âgées qui vivent dans différents types de ménages. Les deux premières colonnes indiquent par exemple le taux de pauvreté de l'ensemble des personnes âgées et des personnes qui vivent dans un ménage dont le chef a l'âge de la retraite (plus de 65 ans). La différence entre les deux est généralement faible, et n'est que d'un point de pourcentage en moyenne dans l'ensemble de l'OCDE. Ce n'est qu'en Espagne, au Portugal et en Turquie que le risque d'être pauvre est plus élevé dans les familles dont le chef est retraité. Cela reflète principalement le fait que certaines personnes âgées vivent dans des ménages où coexistent plusieurs générations, lorsqu'elles vivent avec leurs enfants, par exemple.

La différence la plus marquée en termes de risque de pauvreté se manifeste entre les personnes seules ayant l'âge de la retraite et les couples dans lesquels le chef de famille a atteint l'âge de la retraite, avec des taux de pauvreté respectivement de $25 \%$ et $9 \%$ en moyenne dans les pays de l'OCDE. Les personnes âgées vivant seules s'en sortent le moins bien en Corée et en Irlande, où plus de la moitié d'entre elles vivent dans la pauvreté. Les taux de pauvreté sont également élevés - 40 à $50 \%$ - pour les personnes âgées vivant seules en Australie, aux États-Unis, au Japon et au Mexique.

\section{Comment la pauvreté des personnes âgées a-t-elle évolué ?}

Les taux de pauvreté des personnes âgées ont baissé au cours des trois dernières décennies. Le graphique 2.12 illustre l'évolution des taux de pauvreté dans la durée pour une série de groupes d'âge par rapport au taux de pauvreté de l'ensemble de la population. Le panneau de gauche présente des données portant sur 23 pays de l'OCDE. Au milieu des années 80, les retraités les plus âgés (plus de 75 ans) avaient près de deux fois plus de risques d'être pauvres que la population. La pauvreté relative des personnes âgées a décliné pendant 
Tableau 2.1. Taux de pauvreté des personnes âgées (de plus de 65 ans) par type de ménage et statut d'activité

Pourcentage de personnes dont les revenus sont inférieurs à la moitié du revenu équivalent médian de la population

\begin{tabular}{|c|c|c|c|c|c|c|}
\hline & \multirow{2}{*}{ Total } & \multicolumn{5}{|c|}{ Le chef de famille a atteint l'âge de la retraite (plus de 65 ans) } \\
\hline & & Total & Occupé & Inoccupé & Célibataire & Couple \\
\hline Allemagne & 9 & 8 & 2 & 9 & 15 & 5 \\
\hline Australie & 27 & 27 & 4 & 32 & 50 & 18 \\
\hline Autriche & 7 & 8 & 7 & 9 & 16 & 4 \\
\hline Belgique & 13 & 12 & 4 & 13 & 17 & 10 \\
\hline Canada & 6 & 7 & 2 & 10 & 16 & 4 \\
\hline Corée & 45 & 49 & 35 & 69 & 77 & 41 \\
\hline Danemark & 10 & 10 & 2 & 12 & 17 & 4 \\
\hline Espagne & 23 & 27 & 12 & 32 & 39 & 24 \\
\hline États-Unis & 24 & 24 & 9 & 34 & 41 & 17 \\
\hline Finlande & 13 & 14 & 11 & 14 & 28 & 4 \\
\hline France & 9 & 9 & 1 & 9 & 16 & 4 \\
\hline Grèce & 23 & 21 & 7 & 31 & 34 & 18 \\
\hline Hongrie & 5 & 5 & {$[.]$.} & 5 & 11 & 1 \\
\hline Irlande & 31 & 25 & 5 & 36 & 65 & 9 \\
\hline Islande & 5 & 5 & 3 & 7 & 10 & 2 \\
\hline Italie & 13 & 13 & 3 & 17 & 25 & 9 \\
\hline Japon & 22 & 21 & 13 & 30 & 48 & 17 \\
\hline Luxembourg & 3 & 3 & [..] & 4 & 4 & 3 \\
\hline Mexique & 28 & 23 & 19 & 39 & 45 & 21 \\
\hline Norvège & 9 & 9 & 1 & 10 & 20 & 1 \\
\hline Nouvelle-Zélande & 2 & 4 & 1 & 2 & 3 & 1 \\
\hline Pays-Bas & 2 & 2 & 2 & 2 & 3 & 2 \\
\hline Pologne & 5 & 6 & 6 & 6 & 6 & 6 \\
\hline Portugal & 17 & 20 & 5 & 25 & 35 & 16 \\
\hline République slovaque & 6 & 4 & [...] & 7 & 10 & 3 \\
\hline République tchèque & 2 & 3 & {$[.]$.} & 3 & 6 & 2 \\
\hline Royaume-Uni & 10 & 10 & 1 & 12 & 17 & 7 \\
\hline Suède & 6 & 6 & 3 & 7 & 13 & 1 \\
\hline Suisse & 18 & 18 & [..] & {$[.]$.} & 24 & 15 \\
\hline Turquie & 15 & 18 & 20 & 16 & 38 & 17 \\
\hline OCDE & 13 & 14 & 7 & 17 & 25 & 9 \\
\hline
\end{tabular}

Note: La mention [. .] indique que la taille de l'échantillon est trop limitée.

Source: Base de données de l'OCDE sur la distribution des revenus ; voir OCDE (2008), Croissance et inégalités, tableau 5.3.

StatLink anish $h t t p: / / d x . d o i . o r g / 10.1787 / 651474216837$

les deux décennies suivantes, passant de $90 \%$ à moins de $45 \%$ de plus que la population totale. La situation des jeunes retraités (66 à 75 ans) a enregistré une amélioration comparable. Leur risque relatif de pauvreté était 33 \% supérieur à la moyenne au milieu des années 80 , mais était passé un peu en dessous de la moyenne au milieu des années 2000. Les taux de pauvreté des personnes en pleine période d'activité (26-50 ans) étaient assez stables. Ce sont donc les enfants et les jeunes adultes ( 25 ans ou moins) qui ont progressivement remplacé les personnes âgées en tant que groupe présentant un risque relativement élevé de pauvreté.

On dispose de séries de données plus longues - puisqu'elles remontent au milieu des années 70 - pour sept pays : Canada, États-Unis, Finlande, Grèce, Pays-Bas, Royaume-Uni et Suède. Les résultats pour ces sept pays, qui figurent dans le panneau de droite du graphique 2.12, montrent que la tendance à la baisse de la pauvreté des personnes âgées est durable. 


\section{Graphique 2.12. Évolution du risque relatif de pauvreté par groupe d'âge}

Risque de pauvreté de la population $=100$

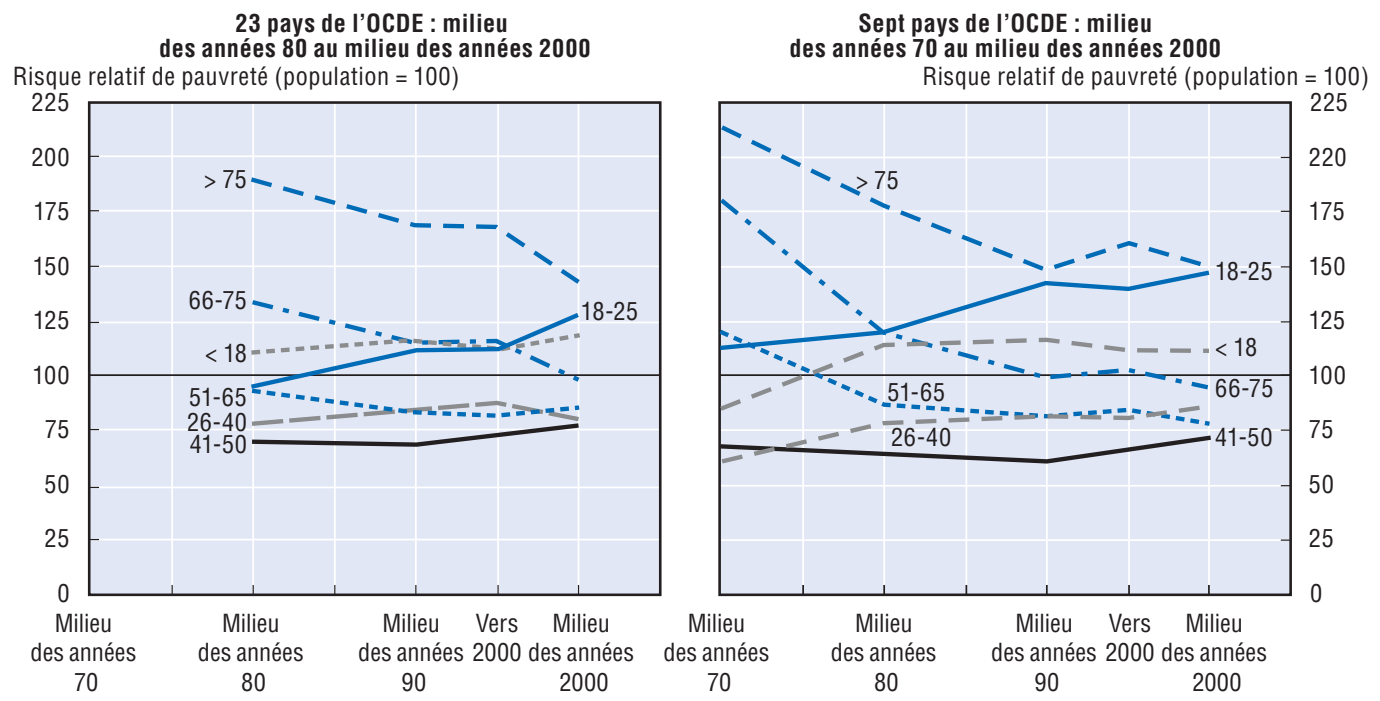

Note : Le panneau de droite contient des données pour le Canada, les États-Unis, la Finlande, la Grèce, les Pays-Bas, le Royaume-Uni et la Suède. Le panneau de gauche correspond aux 23 autres pays de l'OCDE.

Source: Base de données de l'OCDE sur la distribution des revenus ; voir OCDE (2008), Croissance et inégalités, graphique 5.5. StatLink ants $h t t p: / / d x . d o i . o r g / 10.1787 / 651227035011$

Ce déclin de la pauvreté monétaire des personnes âgées est commun à la plupart des pays de l'OCDE (même si aucun graphique n'en fait état dans la présente publication ; voir OCDE, 2008, tableau 5.3). Néanmoins, il existe des exceptions. L'Espagne et l'Irlande ont vu la part des personnes âgées pauvres augmenter fortement à la fin des années 80, dans les années 90 et au début des années 2000. Cette dégradation s'explique en partie par la croissance économique rapide et les gains de salaires réels qui ont en quelque sorte creusé l'écart avec la situation des personnes âgées. Au Mexique, l'augmentation du risque de pauvreté à l'âge de la retraite entre le milieu des années 80 et le milieu des années 90 a été compensée par une baisse entre cette dernière période et le milieu des années 2000. L'Australie, les États-Unis, la Finlande, la Suède et la Suisse ont tous enregistré une aggravation de la pauvreté des personnes âgées au fil du temps, avec la croissance économique du milieu des années 90 au milieu des années 2000.

Si l'on revient au rôle des modes de vie, au cours de la décennie allant du milieu des années 90 au milieu des années 2000, les taux de pauvreté des personnes âgées vivant seules ont diminué plus rapidement que les taux équivalents pour les couples âgés. Ce déclin est le plus important en République tchèque (19.1 points de pourcentage), suivie de la Norvège (13.8) et de l'Autriche (11.6). Toutefois, sept pays ont enregistré l'évolution inverse : notamment l'Espagne et la Finlande, où les taux de pauvreté des personnes âgées vivant seules ont augmenté respectivement de 32.7 et 12.5 points de pourcentage.

\section{La fonction redistributive de l'État : impôts et prestations}

L'analyse des revenus et de la pauvreté des personnes âgées, et leur comparaison avec la situation de la population dans son ensemble, sont fondées sur le revenu disponible. L'État influe considérablement sur ce revenu disponible. Il prélève l'argent des ménages sous la forme d'impôts sur le revenu des personnes physiques et de cotisations de sécurité 
sociale. Il restitue de l'argent sous la forme de prestations en espèces, y compris les pensions de retraite.

\section{Impôts et cotisations}

Les deux premières colonnes du tableau 2.2 illustrent la proportion du revenu des ménages payée sous forme d'impôts et de cotisations par des personnes ayant atteint l'âge de la retraite (plus de 65 ans) et en âge de travailler (18-65 ans). Dans les 24 pays pour lesquels on dispose de données, les personnes d'âge actif reversent en moyenne $31 \%$ de leurs revenus sous forme d'impôts directs ${ }^{22}$. Pour les personnes ayant l'âge de la retraite, la charge fiscale directe représente seulement $18 \%$ de leur revenu en moyenne dans la zone OCDE.

Tableau 2.2. Échelle et progressivité des impôts sur les ménages et des prestations en espèces Part des impôts sur les ménages et des transferts publics dans le revenu disponible et mesure de la progressivité des impôts et des transferts exprimées en pourcentages, milieu des années 2000

\begin{tabular}{|c|c|c|c|c|c|c|c|c|c|}
\hline & \multicolumn{2}{|c|}{$\begin{array}{l}\text { Impôts sur les ménages } \\
\text { (\% du revenu disponible) }\end{array}$} & \multicolumn{2}{|c|}{$\begin{array}{l}\text { Progressivité des impôts } \\
\text { sur les ménages }\end{array}$} & \multicolumn{2}{|c|}{$\begin{array}{c}\text { Transferts publics } \\
\text { (\% du revenu disponible) }\end{array}$} & \multicolumn{3}{|c|}{ Progressivité des transferts publics } \\
\hline & $\begin{array}{c}\text { Âge } \\
\text { de la retraite }\end{array}$ & Âge actif & $\begin{array}{c}\text { Âge } \\
\text { de la retraite }\end{array}$ & Âge actif & $\begin{array}{c}\text { Âge } \\
\text { de la retraite }\end{array}$ & Âge actif & $\begin{array}{c}\text { Âge } \\
\text { de la retraite }\end{array}$ & Âge actif & $\begin{array}{l}\text { Prestations } \\
\text { retraite }\end{array}$ \\
\hline Allemagne & 12.5 & 41.1 & 48.5 & 43.9 & 82.2 & 16.4 & 17.5 & -6.6 & 10.1 \\
\hline Australie & 9.7 & 24.8 & 81.6 & 49.2 & 48.7 & 10.1 & -8.0 & -43.1 & -47.0 \\
\hline Autriche & 27.5 & 35.0 & 46.4 & 36.5 & 101.3 & 27.4 & 25.6 & 13.0 & 24.9 \\
\hline Belgique & 19.6 & 42.1 & 42.0 & 36.3 & 96.9 & 22.3 & 16.9 & -14.1 & -8.9 \\
\hline Canada & 15.0 & 27.0 & 58.6 & 47.2 & 46.7 & 9.3 & -0.6 & -17.3 & -11.0 \\
\hline Corée & 5.0 & 8.1 & 46.2 & 36.3 & 15.7 & 3.0 & 28.2 & 4.0 & \\
\hline Danemark & 44.2 & 53.8 & 33.6 & 33.2 & 81.1 & 19.9 & -5.4 & -30.3 & -49.4 \\
\hline Espagne $^{1}$ & . & . & . & .. & 70.4 & 15.0 & 17.5 & 10.2 & 4.1 \\
\hline États-Unis & 16.4 & 27.7 & 65.8 & 54.9 & 42.1 & 5.6 & 10.5 & -11.5 & -3.8 \\
\hline Finlande & 24.8 & 31.0 & 44.4 & 41.9 & 18.1 & 12.4 & -13.8 & -25.8 & -44.1 \\
\hline France & 11.1 & 28.8 & 47.4 & 35.4 & 96.4 & 22.6 & 28.5 & 9.8 & 25.3 \\
\hline Grèce $^{1}$ & . & .. & .. & .. & 66.4 & 16.7 & 20.2 & 17.6 & 14.5 \\
\hline Hongrie $^{1}$ & .. & .. & .. & .. & 85.6 & 27.5 & 11.9 & -2.5 & 1.0 \\
\hline Irlande $^{1}$ & 5.4 & 20.7 & 78.2 & 53.1 & 55.8 & 13.3 & -0.1 & -20.5 & -32.0 \\
\hline Islande & 34.2 & 54.1 & 29.6 & 25.7 & 79.7 & 12.3 & 3.7 & 1.8 & \\
\hline Italie & 21.1 & 32.0 & 62.3 & 51.2 & 87.4 & 21.1 & 22.5 & 15.8 & 21.8 \\
\hline Japon & 15.4 & 21.0 & 42.9 & 35.6 & 55.8 & 11.0 & 12.1 & 2.0 & 2.3 \\
\hline Luxembourg & 14.8 & 26.3 & 43.0 & 40.4 & 91.0 & 22.4 & 14.5 & 7.5 & 17.3 \\
\hline Mexique $^{1}$ & . & . & .. & .. & 21.3 & 5.4 & 51.8 & 40.7 & \\
\hline Norvège & 22.7 & 35.0 & 43.3 & 35.5 & 72.7 & 15.4 & 7.4 & -17.7 & -26.7 \\
\hline Nouvelle-Zélande & 19.8 & 29.1 & 24.9 & 48.5 & 76.8 & 13.1 & -1.1 & -33.1 & -32.4 \\
\hline Pays-Bas & 10.0 & 26.9 & 70.5 & 43.6 & 53.0 & 12.7 & -1.4 & -22.3 & -16.0 \\
\hline Pologne ${ }^{1}$ & 17.9 & 28.8 & 32.5 & 38.2 & 92.6 & 30.4 & 19.8 & 17.3 & 26.0 \\
\hline Portugal $^{1}$ & . & . & .. & . & 74.2 & 20.3 & 29.5 & 31.5 & 33.2 \\
\hline République slovaque & 5.0 & 22.0 & 72.6 & 38.8 & 86.0 & 22.0 & 10.4 & -3.0 & -0.2 \\
\hline République tchèque & 6.1 & 23.9 & 78.9 & 42.4 & 79.1 & 17.0 & 3.7 & -15.1 & -10.8 \\
\hline Royaume-Uni & 10.0 & 26.2 & 61.4 & 48.6 & 54.3 & 8.7 & 3.5 & -34.7 & -20.6 \\
\hline Suède & 40.2 & 44.2 & 31.2 & 33.0 & 96.3 & 21.4 & 9.0 & -15.3 & -18.7 \\
\hline Suisse & 32.9 & 36.6 & 20.2 & 21.1 & 63.6 & 9.7 & 1.5 & -17.6 & -18.7 \\
\hline Turquie $^{1}$ & . & .. & . & .. & 46.0 & 18.6 & 28.8 & 32.0 & 37.3 \\
\hline OCDE 24 & 18.4 & 31.1 & 50.2 & 40.4 & 69.7 & 15.8 & 8.5 & -10.7 & -4.5 \\
\hline
\end{tabular}

1. Pour l'Espagne, la Grèce, la Hongrie, le Mexique, le Portugal et la Turquie, les données relatives aux transferts publics sont nettes d'impôts et les impôts sur les ménages ne peuvent pas être identifiés séparément. Par conséquent, la moyenne de l'OCDE exclut ces six pays. Pour ces pays ainsi que pour l'Irlande et la Pologne, la progressivité des transferts publics est calculée sur la base des valeurs nettes.

Source: Base de données de l'OCDE sur la distribution des revenus; voir OCDE (2008), Croissance et inégalités, tableaux 4.2-4.4 et l'analyse correspondante. 
Cette différence de charge fiscale pèse lourd lorsqu'on compare les revenus des personnes âgées avec ceux des personnes d'âge actif quelle que soit la période. Elle a également un effet notable sur le niveau de vie des personnes qui passent de la vie active à la retraite (voir l'indicateur relatif aux « taux de remplacement nets » dans la partie II de cette étude).

D'autres publications de l'OCDE examinent en détail les différentiels de charge fiscale entre pays pour les personnes d'âge actif ${ }^{23}$. La charge fiscale des personnes âgées est en général plus faible que celle supportée par les actifs. Il y a à cela trois principales raisons. Premièrement, les impôts sur le revenu dans les pays de l'OCDE sont progressifs : les personnes plus riches reversent une fraction plus élevée de leur revenu sous forme d'impôts. Les personnes âgées perçoivent en général des revenus inférieurs à celles d'âge actif. Deuxièmement, dans la plupart des pays de l'OCDE, les retraités ne paient pas de cotisations de sécurité sociale, ou bénéficient d'un taux de cotisation réduit destiné à financer des prestations spécifiques, comme l'assurance maladie. Troisièmement, dans la plupart des pays de l'OCDE, le régime de l'impôt sur le revenu prévoit des allégements visant soit les retraités, soit le revenu perçu au titre de la retraite.

La progressivité des impôts et transferts est mesurée en comparant leur répartition avec celle du revenu disponible équivalent des ménages. Le graphique 2.13 illustre ce concept. L'axe horizontal indique le pourcentage cumulé de la population, classée par revenu disponible équivalent, la catégorie la plus pauvre se situant à gauche et celle la plus riche à droite. L'axe vertical indique le pourcentage cumulé du total des impôts ou transferts payés ou perçus. Si les impôts et transferts étaient équitablement répartis, le résultat serait une diagonale. Dans la pratique, ce n'est pas le cas.

Le diagramme ci-dessous illustre l'effet d'un système d'imposition progressive dans lequel la part du revenu payée sous forme d'impôts augmente en fonction du revenu. Il s'agit de la courbe située en dessous de la diagonale. Il illustre également l'effet d'un système redistributif de transferts, dans lequel la fraction des prestations totales perçues diminue à mesure que le revenu augmente. Il s'agit de la courbe située au-dessus de la diagonale.

Les zones grisées illustrent l'ampleur de la progressivité des impôts et transferts. On parle officiellement de "coefficients de concentration ${ }^{24}$. Plus la zone située entre la diagonale et la part des impôts et transferts est étendue, plus ces systèmes sont progressifs. Dans l'illustration du graphique 2.13, les transferts publics sont redistributifs et le résultat relatif à la concentration est donc négatif.

Le tableau 2.2 présente une mesure empirique de la progressivité des impôts et des cotisations de sécurité sociale (colonnes deux et quatre). Une valeur zéro indique que tous les ménages ont payé une fraction identique d'impôts et de cotisations (la courbe relative aux impôts correspondrait à la diagonale dans le graphique 2.13). Plus cette valeur est élevée, plus le système d'imposition est progressif.

Pour les personnes d'âge actif, les pays anglophones - Australie, Canada, États-Unis, Irlande, Nouvelle-Zélande et Royaume-Uni - ainsi que l'Allemagne, les Pays-Bas et la République tchèque ont les systèmes d'imposition les plus progressifs. Les impôts directs sont les moins progressifs en France, en Suisse et dans les pays nordiques.

Pour les personnes ayant atteint l'âge de la retraite, les impôts sont beaucoup plus progressifs que pour les personnes d'âge actif dans 16 des 24 pays pour lesquels des données sont disponibles, et ce pour trois principales raisons. Premièrement, les allégements fiscaux de base (déductions, crédits, etc.) sont souvent plus importants pour les retraités que pour les personnes d'âge actif. Par conséquent, de nombreux retraités ne 


\section{Graphique 2.13. Une illustration de la mesure de la progressivité des impôts et transferts}

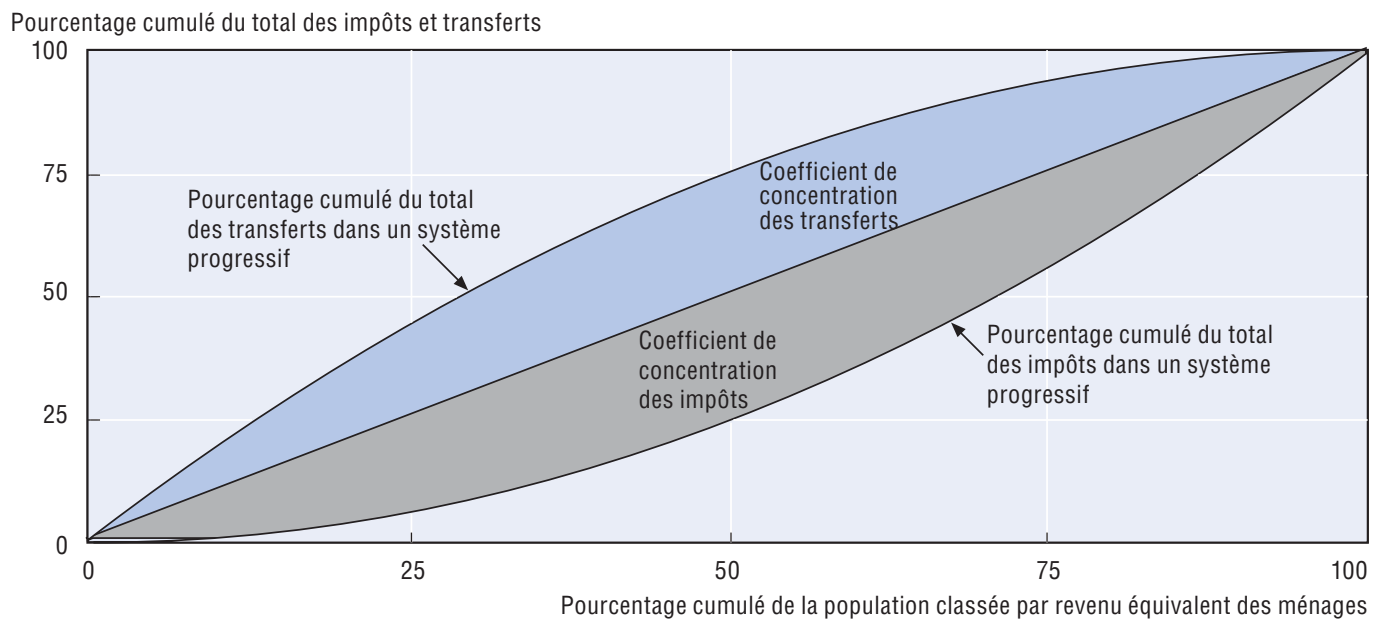

paient pas d'impôts. Deuxièmement, les retraités dans la plupart des pays de l'OCDE ne sont pas assujettis aux cotisations de sécurité sociale (ou le sont à taux très bas). Les cotisations de sécurité sociale sont généralement plus régressives que l'impôt sur les revenus des personnes physiques. Souvent, tous les salariés sont assujettis à un taux de cotisation unique. De plus, un plafonnement des cotisations limite les charges sociales supportées par les hauts salaires (voir le tableau III.1 en partie III).

Toutefois, les écarts de progressivité des impôts payés par les personnes d'âge actif et ayant atteint l'âge de la retraite sont faibles - l'imposition des salariés est plus progressive que celle des retraités - dans les pays nordiques, en Nouvelle-Zélande, en Pologne et en Suisse. Différentes raisons expliquent ce phénomène : une charge fiscale globale élevée dans les pays nordiques, l'absence de cotisations de sécurité sociale en Nouvelle-Zélande et les cotisations à la charge des retraités au titre de l'assurance maladie en Pologne.

\section{Transferts publics en espèces}

La partie droite du tableau 2.2 examine les prestations publiques en espèces. Elles englobent toute la gamme des prestations de retraite publiques : retraite de base, sous conditions de ressources et minimum, ainsi que régimes liés à la rémunération. Toutefois, elles incluent également d'autres prestations en espèces versées aux personnes âgées qui ne sont généralement pas traitées dans les autres parties de cette étude, comme les prestations familiales, les prestations de chômage, les allocations logement et les prestations d'invalidité ${ }^{25}$.

Les deux premières colonnes sur les cinq du tableau illustrent la part de ces prestations dans le revenu disponible. À la différence des données relatives aux sources de revenu du graphique 2.3 ci-dessus, il s'agit de chiffres bruts, avant déduction de l'impôt sur le revenu et des cotisations de sécurité sociale (ce qui explique pourquoi les transferts publics représentent plus de $100 \%$ du revenu disponible en Autriche).

Comme on pouvait s'y attendre, les transferts publics constituent une source de revenu plus importante pour les personnes ayant atteint l'âge de la retraite que pour celles d'âge actif dans tous les pays. En moyenne, les prestations publiques représentent $70 \%$ des revenus bruts des personnes âgées, contre seulement $16 \%$ pour celles d'âge actif. Les 
transferts publics visent surtout les personnes ayant l'âge de la retraite aux États-Unis, en Islande, en Nouvelle-Zélande, au Royaume-Uni et en Suisse. En effet, dans ces pays, les transferts publics aux personnes d'âge actif représentent une plus faible fraction du revenu disponible que dans la moyenne de l'OCDE.

Les dernières colonnes du tableau 2.2 illustrent la répartition des prestations publiques en espèces entre tranches de revenu, en faisant de nouveau la distinction entre personnes d'âge actif et personnes ayant atteint l'âge de la retraite. La mesure de la progressivité utilisée aurait pour valeur zéro si toutes les tranches de revenu recevaient la même fraction des prestations publiques. Toutefois, à la différence des impôts, les valeurs relatives aux prestations en espèces sont souvent négatives. Cela signifie que les personnes ayant des revenus plus faibles perçoivent une fraction proportionnellement plus élevée de l'ensemble des prestations (voir l'explication concernant le graphique 2.13 ci-dessus).

Dans tous les pays de l'OCDE à l'exception du Portugal et de la Turquie, les transferts en espèces payés aux personnes d'âge actif présentent une distribution plus progressive que les versements en faveur des personnes ayant l'âge de la retraite. En particulier, les prestations familiales et de chômage sont ciblées sur les catégories à plus faible revenu ${ }^{26}$. En revanche, 23 pays de l'OCDE sur 30 disposent d'un régime public de retraite lié à la rémunération (voir le tableau 0.1 dans la section intitulé "Le cadre des Pensions dans les pays de l'OCDE » ci-dessus). Par nature, ces régimes octroient des prestations plus généreuses aux personnes qui percevaient une rémunération plus élevée lorsqu'elles travaillaient. Cela explique en grande partie la différence de redistribution des prestations versées aux familles d'âge actif et aux retraités.

Pour examiner cet effet de plus près, la dernière colonne du tableau 2.2 distingue les prestations de retraite et fournit la mesure empirique de la progressivité (le coefficient de concentration) pour ces seules prestations. Dans six pays ${ }^{27}$ qui ne sont pas dotés de régimes publics de retraite liés à la rémunération - Australie, Danemark, Finlande ${ }^{28}$, Irlande, NouvelleZélande et Pays-Bas -, la mesure de la progressivité est fortement négative. En effet, les transferts publics sont soit des retraites de base, soit des prestations de retraite sous conditions de ressources. Il en résulte que la couverture de ces régimes est étendue, voire quasiment universelle dans certains cas (voir l'encadré 2.1 ci-dessus). Dans d'autres pays où les retraites minimum, de base et sous conditions de ressources, couvrent un nombre relativement élevé de personnes âgées, comme la Belgique, le Canada, le Royaume-Uni et la Suède, les transferts publics aux personnes âgées sont très progressifs. Aux États-Unis, en République tchèque et en Suisse, le système public de retraite est conçu de telle sorte que les titulaires de bas salaires perçoivent des retraites proportionnellement plus élevées par rapport à la rémunération, et la mesure de la progressivité au tableau 2.2 est là encore négative.

Par contraste, la mesure empirique de la progressivité des prestations de retraite est positive dans 12 pays. En effet, les prestations de retraite sont étroitement liées aux gains perçus pendant la période d'activité : c'est par exemple le cas en Autriche, en France et en Italie. Dans d'autres pays, cette situation s'explique par le fait que les travailleurs à bas salaires ont souvent accompli tout ou partie de leur carrière dans le secteur informel de l'économie. Cela explique les résultats pour la Grèce, le Portugal et la Turquie (ainsi que le résultat relatif à la progressivité de tous les transferts en faveur des personnes ayant atteint l'âge de la retraite au Mexique).

Il est également intéressant de comparer ces mesures empiriques de la répartition des prestations publiques de retraite payées aujourd'hui aux projections de répartition des 
retraites futures que toucheront les personnes qui entrent aujourd'hui sur le marché du travail et qui atteindront l'âge de la retraite dans 40 à 50 ans (indicateur de la " Progressivité de la formule de calcul des prestations de retraite » dans la partie II de cette publication). Les différences entre ces deux séries de résultats reflètent en partie les différences de types de prestations perçues : par exemple, les retraites publiques sont fortement redistributives en Finlande, aux Pays-Bas, en Suède et en Suisse. Toutefois, le système de revenus de retraite dans son ensemble l'est moins, en raison de l'exclusion des plans professionnels obligatoires ou quasi obligatoires de l'analyse qui sous-tend le tableau 2.2. Par ailleurs, de nombreux systèmes de retraite ont été réformés, de sorte que le niveau et la répartition des prestations seront très différents de ceux des retraités d'aujourd'hui.

\section{Perspectives}

Ce chapitre analyse la situation économique des personnes âgées aujourd'hui, en examinant le niveau de leurs revenus et leur pauvreté. Les revenus actuels des retraités dépendent des règles des systèmes de retraite en vigueur dans le passé, du parcours professionnel des retraités et de leur historique de gains. C'est pourquoi l'analyse des retraites est basée sur un horizon temporel lointain. La question se pose de savoir quelle sera la situation d'ici 20 à 40 ans, lorsque les salariés appartenant aux tranches d'âge de forte activité et ceux plus jeunes prendront leur retraite.

L'expérience des générations actuelles de salariés sera très différente de celle de leurs parents et grands-parents.

Premièrement, des transformations sociales de grande ampleur sont à l'œuvre : multiplication des divorces et des familles monoparentales, diminution du nombre d'enfants et déclin du modèle des ménages où l'homme est le seul apporteur de revenu à mesure que la participation des femmes au marché du travail augmente.

Deuxièmement, des mutations économiques profondes se sont produites et continuent de se manifester ; de nombreux pays ont connu des périodes de chômage persistent et de longue durée dans les années 90 . La situation du marché du travail s'est considérablement améliorée ces dernières années. Néanmoins, la crise économique actuelle devrait avoir de lourdes conséquences, du moins à court terme. De même, la crise financière aura des répercussions profondes pour de nombreux retraités au cours des cinq à dix prochaines années (voir le chapitre spécial « Les systèmes de retraite pendant la crise économique et financière " ci-dessus).

Troisièmement, les deux dernières décennies ont été caractérisées par une vague de réformes des retraites. Ces réformes auront des effets importants sur le niveau et les sources de revenu de retraite pour les salariés d'aujourd'hui, et donc sur l'évolution des revenus et de la pauvreté des personnes âgées.

\section{Changements socio-économiques}

L'évolution du rôle des femmes constitue le principal changement socio-économique. Les personnes âgées, dont cette étude analyse les revenus, sont entrées sur le marché du travail dans les années 40 ou avant. Le modèle du ménage avec l'homme comme seul apporteur de revenu était alors prédominant. Les femmes quittaient souvent le marché du travail lorsqu'elles se mariaient ou avaient des enfants (plus tôt qu'aujourd'hui) et passaient de longues périodes en dehors du marché du travail à élever leurs enfants. Certaines ne reprenaient jamais une activité rémunérée. 
L'écart entre les sexes en termes d'emploi a évolué, car les femmes ont moins d'enfants, les conçoivent plus tard et passent moins de temps en dehors du marché du travail à s'en occuper. L'écart de rémunération entre hommes et femmes s'est également réduit, grâce à l'adoption de lois contre la discrimination, à l'évolution des mentalités, au resserrement des différentiels entre hommes et femmes concernant l'éducation et les qualifications, et à l'allongement des heures de travail des femmes. L'effet de ce changement et que, génération après génération, les femmes seront de plus en plus nombreuses à bénéficier de retraites et le montant de ces retraites augmentera. La situation actuelle dans laquelle le revenu des retraitées est plus faible et le taux de pauvreté des femmes âgées est plus élevé devrait s'atténuer à l'avenir.

L'augmentation du nombre de divorces est une autre transformation sociale touchant les femmes. Quelques pays autorisent un partage du montant de la retraite entre les membres du couple en cas de divorce, mais ces règles sont relativement récentes. De nombreuses femmes qui atteindront l'âge de la retraite dans les années à venir ne pourront plus compter sur une pension de réversion, par exemple. La monoparentalité progresse à mesure que les divorces augmentent. Les parents isolés ont généralement des revenus plus faibles parce qu'ils doivent s'occuper de leurs enfants et les services de garde d'enfant sont trop onéreux.

Globalement, ces évolutions socio-économiques qui affectent la situation des femmes sur le marché du travail pourraient se traduire par une augmentation moyenne des revenus des femmes âgées. Toutefois, elles pourraient aussi aggraver leur pauvreté dans la mesure où les divorces et les familles monoparentales sont plus nombreux.

\section{Changements dans les systèmes de retraite}

Ces dernières années, les systèmes de retraite ont également connu des transformations touchant la plupart des pays de l'OCDE. Les pensions dans les pays de l'OCDE présentent les principaux éléments de ces réformes dans le chapitre spécial consacré aux "Réformes récentes des retraites " dans cette édition et l'avant-dernière (OCDE, 2007a, partie II.1). Cette section se concentre sur l'impact de ces réformes sur les droits à pension de personnes avec des revenus différents. Il s'agit en partie d'un exercice de style puisqu'il pose la question de savoir quels auraient été les droits à pension d'un salarié qui est entré sur le marché du travail en 2006 en l'absence de réforme. Il compare ensuite ces résultats avec ceux obtenus pour la même personne en présence des règles actuelles, en tenant compte des changements éventuels dont la mise en œuvre sera progressive. Si plusieurs réformes ont été menées au cours des 15 à 20 dernières années, l'analyse indique généralement l'effet cumulé de ces changements.

Le tableau 2.3 présente les résultats concernant les taux de remplacement : le rapport entre les prestations de retraite et le salaire pendant la vie active, à la fois en termes bruts et nets (déduction faite des impôts et cotisations) ${ }^{29}$. Les résultats sont présentés pour trois niveaux de revenu : $50 \%, 100 \%$ et $150 \%$ de la moyenne nationale. Les 20 pays du tableau peuvent être divisés en quatre groupes.

Dans un premier groupe, l'un des principaux objectifs des réformes était d'améliorer la viabilité financière à long terme des systèmes de retraite. Huit pays l'ont fait en procédant à des réductions globales des prestations qui s'appliquent de façon identique (ou quasiment identique) aux titulaires de revenus faibles, moyens et élevés. Ces pays sont l'Allemagne, l'Autriche, la Corée, la Finlande, l'Italie, le Japon, le Portugal et la Turquie. 
Tableau 2.3. Impact des réformes des retraites sur les droits individuels

Taux de remplacement bruts et nets avant et après les réformes, en pourcentage

\begin{tabular}{|c|c|c|c|c|c|c|c|c|c|c|c|c|}
\hline \multirow{3}{*}{$\begin{array}{l}\text { Rémunération } \\
\text { individuelle: }\end{array}$} & \multicolumn{6}{|c|}{ Valeur brute de remplacement } & \multicolumn{6}{|c|}{ Valeur nette de remplacement } \\
\hline & \multicolumn{3}{|c|}{ Avant la réforme } & \multicolumn{3}{|c|}{ Après la réforme } & \multicolumn{3}{|c|}{ Avant la réforme } & \multicolumn{3}{|c|}{ Après la réforme } \\
\hline & 0.5 & 1 & 1.5 & 0.5 & 1 & 1.5 & 0.5 & 1 & 1.5 & 0.5 & 1 & 1.5 \\
\hline Allemagne & 47.9 & 47.9 & 46.5 & 43.0 & 43.0 & 42.6 & 56.4 & 66.6 & 66.4 & 59.2 & 61.3 & 60.3 \\
\hline Australie & 46.2 & 23.1 & 15.4 & 67.0 & 41.6 & 33.1 & 55.3 & 30.4 & 21.8 & 80.2 & 53.1 & 41.8 \\
\hline Autriche & 90.0 & 90.0 & 85.9 & 80.1 & 80.1 & 76.4 & 98.4 & 99.2 & 95.1 & 90.5 & 90.3 & 86.3 \\
\hline Belgique & 54.8 & 40.4 & 31.4 & 58.1 & 42.0 & 32.5 & 74.2 & 62.1 & 50.6 & 78.7 & 63.7 & 51.7 \\
\hline Corée & 100.0 & 69.3 & 56.0 & 64.1 & 42.1 & 33.6 & 105.9 & 74.9 & 61.6 & 68.8 & 46.6 & 38.7 \\
\hline Finlande & 69.9 & 66.2 & 65.2 & 66.5 & 56.2 & 56.2 & 75.9 & 71.4 & 72.4 & 73.2 & 62.4 & 63.8 \\
\hline France & 64.7 & 64.7 & 58.4 & 61.7 & 53.3 & 48.5 & 79.7 & 78.2 & 70.8 & 76.2 & 65.7 & 60.2 \\
\hline Hongrie & 69.9 & 57.7 & 53.6 & 76.9 & 76.9 & 76.9 & 85.9 & 83.2 & 79.1 & 94.3 & 105.5 & 99.2 \\
\hline Italie & 90.0 & 90.0 & 90.0 & 67.9 & 67.9 & 67.9 & 99.1 & 99.1 & 99.2 & 74.8 & 74.8 & 77.1 \\
\hline Japon & 56.5 & 40.6 & 35.3 & 47.1 & 33.9 & 29.4 & 55.8 & 41.0 & 37.0 & 51.4 & 38.7 & 33.9 \\
\hline Mexique & 72.5 & 72.5 & 72.5 & 55.3 & 36.1 & 34.5 & 73.4 & 76.5 & 83.2 & 56.0 & 38.0 & 39.6 \\
\hline Norvège & 62.5 & 51.9 & 41.9 & 66.2 & 59.3 & 49.8 & 80.4 & 62.0 & 52.3 & 76.7 & 69.3 & 60.6 \\
\hline Nouvelle-Zélande & 77.5 & 38.7 & 25.8 & 79.3 & 41.1 & 29.0 & 77.5 & 38.7 & 25.8 & 79.3 & 41.1 & 29.0 \\
\hline Pologne & 81.2 & 62.9 & 56.8 & 61.2 & 61.2 & 61.2 & 97.1 & 76.9 & 69.7 & 74.4 & 74.9 & 75.0 \\
\hline Portugal & 91.3 & 89.9 & 88.5 & 63.0 & 53.9 & 53.1 & 106.1 & 112.0 & 110.8 & 73.2 & 69.6 & 72.0 \\
\hline République slovaque & 65.0 & 58.9 & 39.3 & 56.4 & 56.4 & 56.4 & 76.4 & 75.9 & 52.2 & 66.3 & 72.7 & 74.9 \\
\hline République tchèque & 72.1 & 45.0 & 32.9 & 79.2 & 49.7 & 36.4 & 86.7 & 58.1 & 44.6 & 95.3 & 64.1 & 49.4 \\
\hline Royaume-Uni & 41.1 & 29.7 & 20.6 & 51.0 & 30.8 & 21.3 & 51.9 & 39.8 & 28.3 & 63.8 & 40.9 & 29.2 \\
\hline Suède & 82.5 & 78.6 & 76.5 & 76.6 & 61.5 & 75.6 & 84.5 & 80.3 & 81.9 & 79.3 & 64.1 & 81.2 \\
\hline Turquie & 107.6 & 107.6 & 107.6 & 86.9 & 86.9 & 86.9 & 150.0 & 154.4 & 157.9 & 121.2 & 124.7 & 127.1 \\
\hline
\end{tabular}

Source : Modèles de retraite de l'OCDE ; voir également OCDE (2007), Les pensions dans les pays de l'OCDE, partie II.1.

Après la réforme, les droits bruts à retraite d'un salarié ayant effectué une carrière complète seront en moyenne $22 \%$ inférieurs à ce qu'ils étaient avant la réforme dans ces pays. Les plus fortes baisses, de l'ordre de $40 \%$, concerneront la Corée, tandis que d'autres pays de ce groupe connaîtront des diminutions plus modestes comprises entre 10 et $25 \%$.

Un deuxième groupe de pays a également revu les prestations à la baisse, mais les bas salaires échappent à tout ou partie de ces réductions. Ce groupe comprend la France, le Mexique et la Suède. Par exemple, la réforme menée au Mexique réduira de $50 \%$ les retraites des ménages à revenu moyen, contre moins de $25 \%$ des ménages à faible revenu (percevant la moitié du salaire moyen). Les baisses pour les ménages à revenu moyen en France et en Suède avoisinent $20 \%$, mais elles ne sont que de $5 \%$ pour les bas salaires.

Le troisième groupe de pays s'est employé à renforcer le lien entre retraite et rémunération, suivant ainsi la direction opposée au deuxième groupe. En Hongrie, en Pologne et en République slovaque, les caractéristiques redistributives du nouveau système de retraite sont beaucoup moins marquées que dans l'ancien système. Les retraites versées aux bas salaires seront considérablement réduites : $25 \%$ en Pologne et $13 \%$ en République slovaque. Les baisses pour les ménages à revenu moyen seront faibles : moins de $5 \%$. Les hauts salaires ( $150 \%$ du salaire moyen) toucheront des pensions plus élevées dans le nouveau système. En Hongrie, les taux de remplacement sont plus élevés après la réforme pour toutes les tranches de revenu, mais l'augmentation des retraites est plus importante pour les hauts salaires. 
Enfin, les pays qui versent des retraites majorées après la réforme constituent un quatrième groupe. En Australie et Norvège, les prestations de retraite privées sont désormais obligatoires. Auparavant, les pensions privées étaient très développées dans ces deux pays, mais grâce à cette mesure, les salariés ayant un revenu faible ou moyen sont désormais couverts par ces pensions. En Nouvelle-Zélande, l'âge de la retraite a été relevé, mais le niveau des prestations reste inchangé. En République tchèque et au Royaume-Uni, l'âge de la retraite sera également relevé, mais cela permettra d'accumuler des droits plus importants. En outre, au Royaume-Uni, le régime public de retraite lié à la rémunération passera progressivement à des prestations à taux uniforme. Les bas salaires verront leurs prestations augmenter fortement (près de $25 \%$ ), tandis que les gains pour les hauts et moyens salaires seront de seulement $4 \%$ environ. En Belgique, le taux de remplacement plus élevé suppose que les salariés partent à la retraite à l'âge normal (65 ans) du fait de la bonification de la retraite pour les personnes qui travaillent entre 62 et 65 ans.

\section{Conclusions}

On constate de grandes différences dans le bien-être économique des personnes âgées d'un pays de l'OCDE à l'autre. Dans certains de ces pays, les revenus des personnes âgées sont, en moyenne, pratiquement identiques à ceux de la population dans son ensemble une fois prises en compte les différences de taille des ménages. Dans d'autres, les personnes âgées doivent se contenter de revenus équivalents aux deux tiers seulement de la moyenne de la population. La proportion de retraités qui vivent dans une situation de pauvreté monétaire varie encore plus. Dans certains pays, la pauvreté des personnes âgées est pratiquement inexistante, tandis que dans d'autres elle dépasse $40 \%$. Même dans les pays où les retraités pauvres sont relativement peu nombreux, des poches de pauvreté subsistent, surtout parmi les plus âgés, les femmes et les personnes vivant seules.

Les résultats sont résumés dans le tableau 2.4 qui compare les taux de pauvreté et les revenus relatifs des personnes âgées. La section supérieure droite contient six pays où le taux de pauvreté des personnes âgées est faible et leurs revenus élevés. Pourtant, ces pays sont dotés de systèmes de retraite radicalement différents. À l'autre extrême se trouvent l'Australie, la Corée et l'Irlande, pays où les revenus des personnes âgées sont bas et leur taux de pauvreté élevé. L'Espagne, les États-Unis, la Grèce, le Japon, le Portugal et la Suisse enregistrent également des taux élevés de pauvreté des personnes âgées, mais leurs revenus se situent vers le milieu de l'échelle. En Belgique, au Danemark, en Finlande, en Norvège et au Royaume-Uni, les revenus relatifs des personnes âgés sont bas, tandis que leurs taux de pauvreté se situent vers le milieu de l'échelle. L'Allemagne, la France et la Turquie enregistrent des taux de pauvreté moyens, mais des revenus élevés.

Ces résultats constituent le point de départ d'un schéma d'action. Les pays confrontés à des revenus faibles et à une pauvreté élevée des personnes âgées pourraient envisager de redistribuer la richesse de la population d'âge actif vers les retraités. Étant donné que les données sur la répartition des revenus dans la zone OCDE datent du milieu des années 2000, l'Australie, la Corée et l'Irlande ont déjà pris des mesures dans cette direction. En revanche, moins d'arguments plaident en faveur d'une redistribution des salariés vers les retraités dans les pays où les retraités ont des revenus moyens à élevés. Néanmoins, lorsqu'à cette situation s'ajoutent des taux de pauvreté des personnes âgées moyens à élevés, il peut être justifié de mieux cibler les prestations sur les titulaires de petites retraites. Cela étant, il faut tenir compte de beaucoup d'autres facteurs pour concevoir la politique des retraites. 
Tableau 2.4. Résumé : taux de pauvreté et revenus relatifs des personnes âgées

\begin{tabular}{llll}
\hline \multirow{2}{*}{$\begin{array}{l}\text { Revenus relatifs } \\
\text { des personnes âgées }\end{array}$} & \multicolumn{2}{l}{ Taux de pauvreté des personnes âgées } \\
\cline { 2 - 4 } Élevés & Élevé & Moyen & Bas \\
Moyens & Mexique & Allemagne, France, Turquie & $\begin{array}{l}\text { Autriche, Canada, Islande, } \\
\text { Luxembourg, Pays-Bas, Pologne }\end{array}$ \\
Bas & $\begin{array}{l}\text { Espagne, États-Unis, Grèce, Japon, } \\
\text { Portugal, Suisse }\end{array}$ & Italie, Suède & Hongrie, République tchèque \\
& Australie, Corée, Irlande & $\begin{array}{l}\text { Belgique, Danemark, Finlande, } \\
\text { Norvège, Royaume-Uni }\end{array}$ & $\begin{array}{l}\text { Nouvelle-Zélande, République } \\
\text { slovaque }\end{array}$ \\
\hline
\end{tabular}

Source: Base de données de l’OCDE sur la distribution des revenus ; voir OCDE (2008), Croissance et inégalités.

À l'avenir, le nouveau rôle des femmes est la principale transformation socio-économique qui influencera les revenus et la pauvreté des personnes âgées : plus forte participation au marché du travail, rétrécissement de l'écart de rémunération avec les hommes et meilleure prise en charge des périodes de congé pour s'occuper des enfants (voir la section 4 cidessus). La réforme des retraites aura une incidence considérable sur l'évolution des revenus et de la pauvreté des personnes âgées ${ }^{30}$. Les pays qui ont procédé à des réductions globales des prestations devraient voir les revenus de leurs retraités baisser et leur pauvreté s'accroître à l'avenir, sauf si ces personnes compensent ces pertes en travaillant plus longtemps ou par une épargne-retraite facultative ${ }^{31}$. Les revenus moyens des personnes âgées pourraient bien décliner dans des pays qui n'appliquent pas ces réductions aux bas salaires, mais dans ce cas, la réforme n'aura pas d'effet sur le taux de pauvreté des retraités. Dans les pays qui ont décidé de renforcer le lien entre retraite et rémunération, le revenu moyen des personnes âgées pourrait croître, mais le manque de redistribution du nouveau système de retraite risque d'aggraver la pauvreté des retraités. Enfin, le groupe qui a renforcé les régimes retraite obligatoires devrait tout naturellement voir les revenus des personnes âgées s'améliorer. Dans tous ces cas, ces changements bénéficieront davantage aux bas salaires, et devraient donc avoir un effet plus prononcé sur la pauvreté des retraités.

\section{Notes}

1. Ce chapitre s'appuie sur la Base de données de l'OCDE sur la distribution des revenus qui sous-tend en grande partie les analyses effectuées dans la publication récente consacrée aux inégalités et à la pauvreté, Croissance et inégalités (OCDE, 2008).

2. Voir les indicateurs "Progressivité de la formule de calcul des prestations de retraite » et "Lien entre les retraites et les salaires » en partie II.

3. Voir OCDE (2008), tableau 1.A1.1, pour des informations sur les enquêtes utilisées et les ajustements des données effectués.

4. Les données concernent l'année 2004 pour la plupart des pays. Toutefois, elles se réfèrent à 2005 pour l'Allemagne, le Canada, la Corée, le Danemark, les États-Unis, la Hongrie, l'Irlande et le Royaume-Uni. L'enquête utilisée pour les Pays-Bas se rapporte à l'année 2003 et celle pour la Corée à l'année 2006 .

5. Une régression linéaire standard des revenus relatifs des personnes âgées génère les résultats suivants (erreurs types entre parenthèses) :

revenu $=2.128 \times$ dépenses publiques/PIB $-0.761 \times$ taux de dépendance économique

Les deux coefficients sont significatifs du point de vue statistique à un niveau de $5 \%$. Le coefficient $\mathrm{R}^{2}$ utilisé dans la régression est égal à 0.31. Les données sont tirées des indicateurs en partie II.

6. La plupart des personnes ayant atteint l'âge de la retraite n'ont plus d'enfants à charge, mais le coût des enfants est déjà pris en compte par l'application de l'échelle d'équivalence des revenus. 
7. Nous n'avons pas connaissance d'études ayant tenté de mesurer directement ces coûts. Toutefois, les ouvrages consacrés au comportement des consommateurs sur l'ensemble du cycle de vie étudient souvent ces aspects. Voir notamment Banks et al. (1995), et Browning et Crossley (2001).

8. L'analyse de l'endettement des ménages par âge du chef de famille pour cinq pays révèle un pic dans la fourchette d'âge comprise entre 35 et 44 ans. Les personnes de 65 ans et plus n'ont pratiquement aucune dette dans ces pays (voir OCDE, 2008, graphique 10.1).

9. Voir OCDE (2008), chapitre IV.10, pour des informations sur la distribution du patrimoine par tranche d'âge dans sept pays de l'OCDE. Disney et Whitehouse (2003) ainsi que Crystal et Shea (1990) examinent d'autres mesures qui tiennent compte de la valeur du patrimoine.

10. OCDE (2008), chapitre IV.9, analyse la distribution des services assurés par les pouvoirs publics. En particulier, voir le graphique 9.1 qui analyse la distribution des dépenses de santé en fonction de l'âge du bénéficiaire.

11. Voir Whitehouse (2009) pour un examen des politiques d'indexation des retraites et des aspects connexes.

12. Voir l'indicateur «Espérance de vie » en partie II de cette étude pour des données et une analyse portant sur les 30 pays de l'OCDE.

13. Voir Whitehouse et Zaidi (2008) pour un examen plus approfondi des travaux publiés sur ce sujet et des faits nouveaux relatifs aux différences socio-économiques en termes de mortalité des personnes âgées en Allemagne, aux États-Unis et au Royaume-Uni.

14. Le graphique 2.3 ne contient pas de données pour la Suisse. En effet, les revenus du capital (qui proviennent principalement des retraites privées) et du travail sont agrégés dans la base de données. Ensemble, ils représentent en moyenne 52 \% des revenus des personnes âgées, tandis que les $48 \%$ restants proviennent des transferts publics.

15. Voir les éléments correspondants dans Förster et Mira d'Ercole (2005) et Disney et Whitehouse (2001), chapitre 7, par exemple.

16. OCDE (2008), annexe 5.A1, indique les seuils de bas revenu pour différents types de familles, à la fois en monnaie nationale et en dollars des États-Unis (au taux de change à parité de pouvoir d'achat).

17. Les niveaux de prestations pour 2005 proviennent de la base de données qui sous-tend les calculs dans Whitehouse (2009). Les " descriptifs pays » en partie III indiquent les valeurs pour 2006. Le niveau des seuils de pauvreté pour 2005 provient de l'OCDE (2008), tableau 5.A1.1.

18. Le coefficient de corrélation est de 0.6425 , significatif au niveau de $0.01 \%$. Sur la base du coefficient $\mathrm{R}^{2}$ pour une régression simple, les niveaux généraux de pauvreté "expliquent » $41.3 \%$ des différences en termes de pauvreté des personnes âgées entre les pays.

19. Voir Whitehouse et al. (2009), Turner (2007) et la partie III de cette étude pour des informations sur les changements concernant l'âge normal de la retraite pour les hommes et les femmes.

20. OCDE (2006) procède à une analyse exhaustive de la situation des salariés âgés.

21. Voir OCDE (2006) et OCDE (2008), chapitre III.5.

22. Les calculs ne tiennent pas compte des cotisations salariales de sécurité sociale ou des impôts indirects comme les impôts sur la consommation (taxe sur la valeur ajoutée, taxe sur les biens et services et droits d'accise). Warren (2009) et OCDE (2008), chapitre V.11, étudient l'impact de la prise en compte des impôts sur la consommation sur l'analyse de la distribution des revenus.

23. OCDE (2007b et 2009), par exemple.

24. Néanmoins, les données relatives aux dépenses publiques consacrées à ces prestations sont indiquées pour dix pays où elles sont significatives selon l'indicateur "Dépenses au titre des retraites » en partie II.

25. Les détails techniques du calcul sont présentés dans OCDE (2008), chapitre II.4. La technique est comparable à celle employée pour calculer la distribution des prestations de retraite : voir l'indicateur « Progressivité de la formule de calcul des prestations de retraite » en partie II de cette étude et l'examen correspondant.

26. Voir OCDE (2008), tableau 2.4, pour des mesures de la progressivité divisées en huit catégories distinctes, comme les allocations familiales, prestations de chômage, de logement et d'invalidité.

27. L'Islande n'a pas non plus mis en place de régime public lié à la rémunération, mais on ne dispose pas de données sur la progressivité des prestations publiques de retraite. Au Mexique, le 
remplacement des régimes publics de retraite liés à la rémunération par des plans privés à cotisations définies a très peu d'impact sur les personnes déjà à la retraite.

28. Les plans professionnels en Finlande sont considérés comme « publics » dans les autres chapitres des Pensions dans les pays de l'OCDE et dans les comptes nationaux. Toutefois, ils sont considérés comme "privés " dans la base de données sur la distribution des revenus parce qu'il s'agit de régimes capitalisés à prestations définies.

29. Pour un examen plus poussé des taux de remplacement et une présentation des résultats après la réforme, voir les indicateurs " Taux bruts de remplacement » et " Taux nets de remplacement » en Partie II de cette étude).

30. Il faut noter que les mesures des revenus et de la pauvreté sont relatives : elles comparent les revenus des personnes âgées et ceux de la population dans son ensemble. Par conséquent, l'évolution des revenus des personnes d'âge actif - résultant des changements de taux d'emplois et de niveaux de salaires, par exemple - aura aussi un effet sur la situation des personnes âgées.

31. Voir le chapitre spécial intitulé « le déficit d'épargne-retraite et l'épargne-retraite facultative » ci-dessous.

\section{Références}

Banks, J., R. Blundell, R. et S. Tanner (1998), « Is There a Retirement Saving Puzzle? », American Economic Review, vol. 88, pp. 769-788.

Browning, M. et T.F. Crossley (2001), " The Life-Cycle Model of Consumption and Saving », Journal of Economic Perspectives, vol. 15, $\mathrm{n}^{\circ} 3$, pp. 3-22.

Crystal, S. et D. Shea (1990), «The Economic Well-Being of the Elderly », Review of Income and Wealth, vol. $36, n^{\circ} 3$, pp. $227-247$.

Disney, R.F. et E.R. Whitehouse (2001), Cross-Country Comparisons of Pensioners' Incomes, Research Report $\mathrm{n}^{\circ} 142$, Department of Social Security, Londres.

Disney, R.F. et E.R. Whitehouse (2003), « The Economic Well-Being of Older People in International Perspective: A Critical Review ", dans S. Crystal et D. Shea (dir. pub.), Economic Outcomes in Later Life, Annual Review of Gerontology and Geriatrics, vol. 22, Springer Publishing, New York.

Union européenne, Comité de politique sociale (2006), " Minimum Income Provision for Older People and their Contribution to Adequacy in Retirement ", étude spéciale sur les retraites, Bruxelles.

Förster, M. et M. Mira d'Ercole (2005), «Income Distribution and Poverty in OECD Countries in the Second Half of the 1990s ", Document de travail sur les affaires sociales, l'emploi et les migrations $\mathrm{n}^{\circ} 22$, Éditions de l'OCDE, Paris.

Martin, J.P. et E.R. Whitehouse (2008), « Reforming Retirement-Income Systems: Lessons from the Recent Experiences of OECD Countries ", Document de travail sur les affaires sociales, l'emploi et les migrations $n^{\circ} 66$, Éditions de l'OCDE, Paris.

OCDE (2006), Viure et travailler plus longtemps, Éditions de l'OCDE, Paris.

OCDE (2007a), Les pensions dans les pays de l'OCDE - Panorama des politiques publiques, Éditions de l'OCDE, Paris.

OCDE (2007b), Prestations et salaires, Éditions de l'OCDE, Paris.

OCDE (2008), Croissance et inégalités - Distribution des revenus et pauvreté dans les pays de l'OCDE, Éditions de l'OCDE, Paris.

OCDE (2009), Les impôts sur les salaires 2007/2008, Éditions de l'OCDE, Paris.

Pearson, M.A. et E.R. Whitehouse (2009), "Social Pensions in High-Income Countries ", dans R. Holzmann et N. Takayama (dir. pub.), Closing the Coverage Gap: The Role of Social Pensions, Banque mondiale, Washington, DC, à paraître.

Turner, J. (2007), « Social Security Pensionable Ages in OECD Countries: 1949-2035 », International Social Security Review, vol. 60, n 1, pp. 81-99.

Warren, N. (2008), « A Review of Studies on the Distributional Impact of Consumption Taxes in OECD Countries ", Document de travail sur les affaires sociales, l’emploi et les migrations $n^{\circ} 64$, Éditions de l'OCDE, Paris. 
Whitehouse, E.R. (2009), " Pensions, Purchasing-Power Risk, Inflation and Indexation », Document de travail sur les affaires sociales, l'emploi et les migrations $\mathrm{n}^{\circ} 77$, Éditions de l'OCDE, Paris.

Whitehouse, E.R. et A. Zaidi (2008), « Socio-Economic Differences in Mortality: Implications for Pension Policy », Document de travail sur les affaires sociales, l'emploi et les migrations n 71 , Éditions de l'OCDE, Paris.

Whitehouse, E.R., A.C. D'Addio, R. Chomik et A. Reilly (2009), « Two Decades of Pension Reform: What has been Achieved and what Remains to be Done ", Geneva Papers on Risk and Insurance, à paraître. 


\section{Les réformes récentes des retraites}

$\mathrm{T}$ ous les systèmes de retraite bien conçus ont en commun des objectifs et des principes clairement définis, qui ont été énoncés dans de nombreuses publications de l'OCDE ${ }^{1}$. L'analyse des réformes récentes des retraites présentée dans ce chapitre spécial s'articule autour de six objectifs principaux des régimes de retraite :

1. la couverture du système de retraite, par le biais de dispositifs obligatoires et facultatifs ;

2. l'adéquation des prestations de retraite ;

3. la viabilité financière et l'accessibilité économique des régimes de retraite pour les contribuables et les cotisants ;

4. l'efficacité économique: minimisation des distorsions produites sur le système de retraite par le comportement économique des individus, comme l'offre de main-d'œuvre et l'épargne constituée hors des dispositifs de retraite ;

5. l'efficacité administrative: maintien des coûts liés au recouvrement des cotisations, au versement des prestations et (si nécessaire) à la gestion des investissements, à un niveau aussi bas que possible ;

6. et la sécurité des prestations au regard de différents risques et incertitudes.

Ce cadre d'analyse illustre parfaitement les arbitrages inhérents à la conception des systèmes de retraite et aux réformes menées dans ce domaine. Ainsi, une revalorisation des pensions viendrait améliorer l'adéquation des prestations, mais aussi éroder la viabilité financière du système. On constate aussi parfois l'existence de synergies entre les différents objectifs. Inciter les salariés à partir plus tard à la retraite renforce à la fois l'efficience économique et la viabilité financière. De même, étendre la couverture des retraites devrait également améliorer l'adéquation des prestations qui seront servies demain aux salariés d'aujourd'hui.

Ces dernières années, les gouvernements des économies de l'OCDE ont continué de réformer activement leur système de retraite. L'édition 2007 des Pensions dans les pays de l'OCDE rendait compte des réformes adoptées dans ce domaine de 1990 à $2004^{2}$. Ce chapitre spécial présente une version actualisée de ces travaux. Les mesures prises par les pays de l'OCDE en vue d'atteindre les six objectifs à retenir sur la période 2004-09 sont exposées au tableau 3.1.

\section{La couverture des systèmes de retraite}

Les modifications apportées afin d'élargir la couverture des régimes de retraite ont principalement porté sur les dispositifs privés facultatifs. La France, la Hongrie, la Pologne et le Portugal ont introduit de nouveaux régimes privés, souvent assortis d'avantages fiscaux. L'Allemagne a prolongé les incitations fiscales qui auraient dû prendre fin en 2008. 
De 2004 à 2009, seule la Norvège a rejoint le groupe des pays de l'OCDE dotés de mécanismes privés obligatoires (il s'agit de l'Australie, de la Hongrie, de l'Islande, du Mexique, de la Pologne, de la République slovaque, de la Suède et de la Suisse).

La Corée et l'Italie ont poursuivi un processus de réforme engagé de longue date afin de transformer les dispositifs d'indemnités de fin de contrat actuellement gérés par les employeurs en plans de retraite professionnels. Dans ces nouveaux dispositifs, l'épargne constituée est exclusivement destinée à être utilisée après le départ à la retraite, et non retirée en cas de changement d'employeur ou de chômage. Cela étant, les plans professionnels n'ont rencontré le succès escompté par les pouvoirs publics dans aucun de ces deux pays, leur couverture restant relativement modérée.

La Nouvelle-Zélande a introduit son plan KiwiSaver, auquel les salariés sont automatiquement affiliés, mais avec possibilité d'en sortir. Le Royaume-Uni a légiféré afin de mettre en place un régime analogue, qui entrera en vigueur en $2012^{3}$.

Certains pays ont pris des dispositions pour étendre la couverture du système de retraite obligatoire. La Grèce, où cette couverture est relativement faible à l'heure actuelle, espère l'élargir au moyen de nouveaux mécanismes institutionnels. La Suisse a réduit le seuil de rémunération pour qu'un plus grand nombre de salariés à temps partiel faiblement rémunérés soient couverts. Le Royaume-Uni a assoupli les conditions d'ouverture des droits à la retraite de base à taux plein et renforcé les crédits accordés pour la garde d'une personne dépendante. L'un des résultats attendus de cette mesure est notamment que $90 \%$ des femmes aient droit à une retraite de base à taux plein en 2050, contre à peine $30 \%$ aujourd'hui.

\section{L’adéquation des prestations de retraite}

En règle générale, la revalorisation des prestations de retraite s'est essentiellement adressée aux personnes à faible revenu. Les modifications introduites comprennent un versement exceptionnel en Australie et en Grèce, ainsi qu'une hausse des retraites de base en Corée et au Royaume-Uni. L'Australie va également revaloriser les pensions de retraite en appliquant un taux de $10.8 \%$ pour les couples et un taux supérieur pour les célibataires. La Finlande va mettre en place une nouvelle retraite garantie à compter de 2011, d'un montant de 8200 EUR par an à prix courants. Les revenus perçus grâce à ce filet de protection seront ainsi supérieurs de $23 \%$ à la retraite nationale actuelle. La Belgique, l'Espagne et la France vont majorer les retraites minimum au-delà de ce que préconisent les règles d'indexation habituelles.

Du fait des revalorisations effectuées en 2008 et 2009 en Allemagne, toutes les pensions vont augmenter dans les mêmes proportions, contrairement aux autres pays où les hausses ont été ciblées.

Les autres changements visant à améliorer le niveau de vie des retraités ont été réalisés par le biais du système fiscal. L'Australie, la Finlande, ainsi que la Suède ont réduit les impôts auxquels est soumise cette catégorie de la population.

\section{La viabilité financière}

Les prévisions budgétaires pessimistes établies sur un horizon à long terme ont incité de nombreux pays à améliorer la viabilité financière à long terme des systèmes de retraite en révisant à la baisse les prestations futures. En France et au Mexique, les réformes ont essentiellement concerné les salariés du secteur public, dont le régime n'avait guère subi 
de changements lors des grandes modifications apportées dans les années 90 aux prestations perçues par les salariés du privé. L'Islande va réduire les retraites futures des hauts fonctionnaires, alors que l'Irlande va soumettre à cotisations les adhérents au régime de la fonction publique.

La Finlande et le Portugal ont pris des dispositions pour minorer les prestations futures en fonction de l'allongement de l'espérance de vie. Cela porte à 12 le nombre des pays de l'OCDE ayant adopté une telle mesure dans un ou plusieurs de leurs régimes de retraite obligatoires ${ }^{4}$.

La Corée, la Hongrie et la Suisse ont diminué plus directement les prestations de retraite. La Hongrie va supprimer le $13^{\mathrm{e}}$ mois perçu par les retraités les plus aisés, afin de pouvoir bénéficier d'un prêt substantiel du FMI. Le taux de remplacement visé par le régime public coréen va progressivement revenir de 60 \% à $40 \%$. En Suisse, le taux d'intérêt minimum légal applicable aux retraites privées obligatoires a été revu à la hausse et à la baisse durant la période considérée, mais il atteindra pratiquement $2 \%$ pour 2009. C'est le taux d'intérêt légal le plus bas depuis l'introduction du dispositif (il s'établissait alors à $4 \%$ ). Les nouvelles règles régissant la conversion en rente des avoirs détenus dans le cadre des régimes de retraite professionnels vont s'accompagner d'une baisse non négligeable des prestations à hauteur de $5.5 \%$. Parmi les autres mesures prises pour améliorer la viabilité financière figure une modification des procédures d'indexation des pensions servies. Afin d'atténuer ses difficultés budgétaires, la Hongrie va retarder d'un an la revalorisation de 2009. Les augmentations futures seront uniquement liées à l'inflation, et non plus à la hausse des salaires et à l'inflation. La France va adopter l'indexation sur les prix pour les régimes du secteur public.

\section{L'efficacité économique}

De nombreuses améliorations potentielles de la viabilité financière des systèmes de retraite tiennent à des mesures d'intensification des incitations relatives à la retraite. Ces modifications sont regroupées dans la présente publication sous la rubrique "efficacité économique ", car elles sont destinées à réduire les distorsions du marché du travail dues aux incitations à partir en retraite anticipée. L'âge effectif de départ à la retraite est actuellement inférieur à l'âge légal dans plus des deux tiers des pays de l'OCDE. En effet, il s'établit en moyenne à 60 ans pour les hommes dans huit pays membres, à savoir l'Autriche, la Belgique, la Finlande, la France, la Hongrie, l'Italie, le Luxembourg et la République slovaque ${ }^{5}$.

Sur la période 2004-09, l'Allemagne, le Danemark et le Royaume-Uni ont légiféré en vue de relever progressivement l'âge normal de la retraite à 67 ou 68 ans. La République tchèque va le fixer à 65 ans. Le gouvernement néerlandais a proposé de le porter de 65 à 67 ans (bien que ce sujet soit encore en cours d'examen avec les partenaires sociaux). Quoi qu'il en soit, il semble avoir l'intention d'agir plus vite que les autres pays, c'est-à-dire dans un délai de 24 mois, soit peut-être en 2011. La Hongrie va relever l'âge normal de la retraite en plusieurs étapes, en le portant de 62 à 65 ans à partir de 2012.

La Belgique, le Danemark, la Grèce, la Hongrie et l'Italie ont soit relevé l'âge de départ anticipé à la retraite, soit majoré le nombre d'années de cotisation requises pour avoir droit à une retraite anticipée, ou les deux. La France et l'Irlande ont appliqué de telles mesures dans les régimes de retraite du secteur public. Les Pays-Bas ont supprimé les incitations fiscales accordées dans le cadre des dispositifs professionnels privés de retraite anticipée. 
La Finlande, la France et le Royaume-Uni ont renforcé les mécanismes incitant les salariés à rester en activité au-delà de l'âge normal de la retraite. La Finlande vient également de proposer de relever l'âge de départ anticipé à la retraite.

La France et le Japon ont pris des mesures pour limiter les possibilités, pour les employeurs, de licencier les salariés qui souhaitent continuer de travailler après l'âge normal de la retraite. Cela étant, un salarié espagnol, qui faisait valoir que l'obligation de partir en retraite était contraire à la législation antidiscrimination, a été débouté par la Cour européenne de Justice (la plus haute cour de l'Union européenne).

L'Australie a introduit notamment une modification visant à améliorer les incitations à épargner (à l'inverse des autres changements qui concernaient des incitations à travailler). Le critère de patrimoine retenu pour les prestations publiques soumises à conditions de ressources sera moins strict, accroissant ainsi l'intérêt de l'épargne-retraite facultative.

\section{L'efficacité administrative}

$\mathrm{Au}$ Japon, une difficulté administrative non négligeable est apparue à l'été 2007. Jusqu'en 1997, lorsque les salariés japonais changeaient d'employeur, ils se voyaient fréquemment attribuer un nouveau numéro d'identification par la Social Insurance Agency (Agence de l'assurance sociale, ou SIA). Une nouvelle base de données de la SIA, introduite cette année-là, était destinée à regrouper les multiples comptes de retraite d'un salarié dans un compte unique assorti d'un numéro d'identification. Cependant, de nombreux enregistrements n'étaient pas liés à un autre, c'est-à-dire que quelque 50 millions de comptes n'étaient pas rattachés à un salarié ou à un retraité donné. Pour résoudre ce problème, le gouvernement a adopté plusieurs mesures, et notamment supprimé le délai de prescription applicable à la liquidation des droits pour les enregistrements recouplés. La SIA disparaîtra en 2010 et ses fonctions de tenue de registres et de recouvrement des cotisations seront assurées par une nouvelle société publique non gouvernementale, le ministère conservant la responsabilité financière et la gestion. Chaque adhérent a reçu ou va recevoir sous peu un relevé détaillé de ses droits à retraite.

La Grèce a rationalisé son système de retraite, en fusionnant 133 régimes en 13. La mise en place d'une base de données centralisée et l'attribution de numéros d'identification uniques devraient faire baisser les coûts administratifs, améliorer le respect des règles en vigueur et limiter les problèmes liés aux enregistrements non couplés qui ont suscité des difficultés au Japon. La Suède va fusionner les organes responsables des régimes obligatoires à cotisations définies (la Premium Pension Authority) et de la retraite publique (composée de comptes notionnels et d'une retraite minimum). Toutefois, chacun conservera la possibilité de choisir de quelle manière les retraites à cotisations définies seront investies.

D'autres mesures visent à réduire les coûts et les frais administratifs pour les régimes privés. Au Mexique, les salariés ont été encouragés à choisir des prestataires moins onéreux. La Pologne a annoncé récemment son intention d'avancer à l'année prochaine l'abaissement des plafonds des frais prélevés sur les pensions privées prévu en 2014. La République slovaque a proposé de diminuer les frais facturés et de lier davantage commissions et performances. Le Royaume-Uni a fixé un objectif à long terme pour les frais administratifs, soit $0.3 \%$ des actifs par an (et $0.5 \%$ lors de son introduction) pour le nouveau dispositif national d'épargne-retraite. En comparaison, les frais prélevés sur les "stakeholder pensions » (fonds d'épargne-retraite individuels) actuels sont plafonnés à $1 \%$. 
Pour contenir les coûts, le Royaume-Uni va mettre en place une central clearing house pour les cotisations, selon le principe de l'organe administratif suédois.

\section{La sécurité des prestations}

Les retraites présentent intrinsèquement un risque car il s'agit de contrats à long terme : en moyenne, il s'écoule pratiquement 60 ans entre le moment où les individus versent leur première cotisation et celui où ils perçoivent leur dernière prestation. La récente crise financière a souligné l'ampleur du risque d'investissement encouru à l'heure où un plus grand nombre de personnes sont couvertes par des régimes de retraite privés à cotisations définies dans bien des pays de l'OCDE ${ }^{6}$.

La diversification est l'arme idéale pour limiter ce risque d'investissement sans renoncer inutilement aux rendements offerts par les placements. En Belgique, au Canada et au Mexique, par exemple, les fonds de pension sont désormais libres d'investir dans un éventail d'actifs plus large qu'avant. Les Australiens disposent aujourd'hui d'un choix élargi quant au gestionnaire de leur plan de retraite et des supports d'investissement.

Il est également possible de réduire plus encore le risque d'investissement en encourageant les salariés à transférer leur retraite vers des actifs moins risqués à l'approche de l'âge de la retraite. La Hongrie a ainsi introduit un mécanisme de transfert automatique des investissements des salariés vers ce type de produits. Le Mexique a étoffé la gamme des supports d'investissement proposés aux adhérents aux régimes à prestations définies. La Pologne va mettre en place un système de trois fonds présentant différentes caractéristiques risque-rendement pour chaque prestataire. Seuls les salariés les plus jeunes pourront choisir le fonds le plus risqué. Le nouveau dispositif national d'épargne-retraite instauré au Royaume-Uni orientera par défaut les cotisants vers un produit "fondé sur le cycle de vie " qui diminuera automatiquement le risque de portefeuille à mesure que les salariés avanceront en âge. Aux États-Unis, grâce à une nouvelle réglementation, les employeurs auront plus de facilités à proposer ce type d'options d'investissement par défaut.

L'Islande va renforcer l'équilibre financier de ses régimes professionnels à prestations définies en relevant les cotisations patronales obligatoires ${ }^{7}$. Le Royaume-Uni a pris des dispositions pour protéger les régimes de retraite professionnels à prestations définies grâce à un Pension Protection Fund (analogue à la Pension Benefit Guaranty Corporation existant aux États-Unis) et à un dispositif d'assistance financière. Cela étant, la crise économique et financière en cours va mettre à plus rude épreuve encore les régimes de retraite professionnels de tous ces pays. Le chapitre spécial sur « les systèmes de retraite pendant la crise économique et financière " contient une analyse plus poussée des modifications dernièrement apportées de ce fait à la réglementation des pensions privées.

Les nouvelles procédures d'indexation mises en place au Portugal et en Turquie devraient protéger davantage le pouvoir d'achat des retraités ${ }^{8}$. Toutefois, la nouvelle politique adoptée en Turquie neutralise les effets d'autres réformes visant à améliorer la viabilité financière à long terme des régimes de retraite publics en réduisant les prestations et en relevant l'âge de la retraite.

\section{La réforme des retraites : processus et politique}

Sur le plan des retraites, les années 2004-09 ont été davantage marquées par une évolution que par une révolution. Ces toutes dernières années, la majorité des pays de 
l'OCDE ont pris de nouvelles dispositions pour que leur système de retraite puisse s'inscrire sur le long terme.

Quoi qu'il en soit, les mesures adoptées n'ont rien eu des vastes réformes systémiques adoptées du début des années 90 à 2004. Sur cette période, la Hongrie, le Mexique, la Pologne et la République slovaque ont remplacé tout ou partie des régimes de retraite publics par des dispositifs à cotisations définies. Il s'agit là d'une véritable rupture avec la situation antérieure, les retraites étant jusque là essentiellement assurées par des États en position de monopole. De plus, de profondes modifications ont été apportées aux systèmes de retraite entre le début des années 90 et 2004 et ont eu pour effet d'améliorer la viabilité financière à long terme des régimes publics. Dans les 16 pays de l'OCDE qui ont engagé des réformes de grande envergure à cette époque, les pensions viagères ont été minorées en moyenne de $22 \%$ pour les hommes et de $25 \%$ pour les femmes. L'Allemagne, la France, l'Italie et le Portugal, par exemple, ont sensiblement réduit les prestations qui seront versées aux futurs retraités.

Dans certains pays, le processus des réformes a marqué le pas. Aux États-Unis, la proposition formulée en 2001 par l'administration ${ }^{9}$ de substituer des dispositifs à cotisations définies à une partie du régime public lié à la rémunération n'a pas obtenu le soutien des Parlementaires. Les États-Unis sont en effet l'un des rares pays de l'OCDE où le système public n'a subi aucun changement marquant depuis les années 80 . En Norvège, également, les réformes plus profondes proposées par une commission indépendante ont été rejetées par le Parlement. Il en est ressorti un régime obligatoire à cotisations définies de modeste ampleur, qui étend la couverture à $40 \%$ des salariés qui n'étaient pas encore affiliés à un dispositif professionnel. En Autriche, le gouvernement de coalition s'est divisé sur la question de la réforme des retraites. En particulier, l'introduction d'un lien entre l'espérance de vie et les prestations de retraite a suscité des controverses. Enfin, les divers rapports établis par des groupes indépendants et les notes de réflexion gouvernementales n'ont pas encore permis de dégager un consensus sur l'orientation de la réforme des retraites en Irlande. D'aucuns sont favorables aux régimes obligatoires au détriment de la retraite de base existante, d'autres non. Parmi les partisans de la mise en place d'un deuxième régime de retraite obligatoire, certains privilégient un dispositif public lié à la rémunération, d'autres des régimes privés à cotisations définies.

Ailleurs, le processus des réformes a ralentit ou fait machine arrière. Les changements législatifs proposés en Italie afin de relever l'âge de la retraite et diminuer les prestations pour tenir compte de l'allongement de l'espérance de vie ont été différés. En République slovaque, les salariés couverts par les nouveaux plans à cotisations définies ont été autorisés à réintégrer le système public, mais rares sont ceux qui ont choisi cette option.

En conclusion, la crise économique et financière a focalisé plus que jamais l'attention des gouvernements sur le court terme. Cette situation porte en germe deux dangers. Le premier est que la planification stratégique à long terme - si décisive pour la politique des retraites - soit mise de côté. Le second est qu'un plus grand nombre de gouvernements puissent être tentés de faire preuve d'opportunisme à court terme en revenant sur les réformes antérieures, notamment en assouplissant les règles régissant le départ anticipé à la retraite à l'heure où les conditions se dégradent sur le marché du travail. Il reste nécessaire, en dépit de ces pressions, de prendre des mesures pour que les pouvoirs publics garantissent la mise en place d'un système de retraite à long terme qui soit sûr, adéquat, viable sur le plan financier et efficace d'un point de vue économique. 
Tableau 3.1. Les réformes des retraites : 2004-09

\begin{tabular}{|c|c|c|c|c|c|c|}
\hline Pays & Couverture & Adéquation & Viabilité financière & Efficacité économique & Efficacité administrative & Sécurité \\
\hline Allemagne & $\begin{array}{l}\text { Prolongation de l'exonération } \\
\text { des cotisations de sécurité sociale } \\
\text { (qui devrait être supprimée en } \\
\text { 2008) sur les versements effectués } \\
\text { au titre des régimes professionnels } \\
\text { à cotisations définies à } \\
\text { concurrence de } 4 \% \text { du salaire }\end{array}$ & $\begin{array}{l}\text { Revalorisation des retraites à hauteur } \\
\text { de } 1.1 \% \text { en } 2008 \text { (et non de } 0.46 \% \\
\text { en application des règles de } 2005 \text { ) } \\
\text { et de } 2.41 \% \text { en } 2009 \text { (au lieu de } \\
1.76 \% \text { ). Les retraites n'avaient pas été } \\
\text { revalorisées entre } 2003 \text { et } 2006\end{array}$ & & $\begin{array}{l}\text { Relèvement progressif de l'âge normal } \\
\text { de la retraite de } 65 \text { à } 67 \text { ans entre } 2012 \\
\text { et } 2029 \text { (toutefois, l'âge de la retraite } \\
\text { anticipée est maintenu à } 63 \text { ans, } \\
\text { moyennant une réduction } \\
\text { des prestations) }\end{array}$ & & $\begin{array}{l}\text { Assouplissement } \\
\text { des contraintes pesant } \\
\text { sur les investissements } \\
\text { réalisés à l'étranger } \\
\text { par les Pensionskassen }\end{array}$ \\
\hline Australie & & $\begin{array}{l}\text { Retraite ciblée portée de } 25 \% \text { à } 27.7 \% \\
\text { du salaire moyen } \\
\text { Revalorisation de la retraite } \\
\text { pour les célibataires, portée } \\
\text { aux deux-tiers de celle des couples } \\
\text { Versement exceptionnel } \\
\text { de } 1400 \text { AUD pour les retraités } \\
\text { célibataires et de } 2100 \text { AUD } \\
\text { pour les couples (déc. 2008) dans } \\
\text { le cadre du plan de relance économique }\end{array}$ & & $\begin{array}{l}\text { Âge de la retraite porté de } 65 \text { à } 67 \text { ans } \\
\text { en } 2017-23 \\
\text { La déduction pour actifs financiers } \\
\text { réalisée sur les droits à prestations } \\
\text { soumises à conditions de ressources } \\
\text { est ramenée de } 7.8 \% \text { de la valeur des } \\
\text { actifs à } 3.9 \% \text {, afin de promouvoir } \\
\text { l'épargne-retraite facultative }\end{array}$ & & $\begin{array}{l}\text { Projet de création d'un fonds } \\
\text { de précapitalisation } \\
\text { des prestations versées aux } \\
\text { salariés du secteur public. } \\
\text { Objectif : capitalisation } \\
\text { intégrale en } 2020 \\
\text { Possibilité de choisir } \\
\text { un prestataire pour } \\
\text { les régimes à cotisations } \\
\text { définies obligatoires }\end{array}$ \\
\hline \multicolumn{7}{|l|}{ Autriche } \\
\hline Belgique & & $\begin{array}{l}\text { Revalorisation des retraites minimum } \\
\text { supérieure à l'indexation standard }\end{array}$ & & $\begin{array}{l}\text { Relèvement de l'âge d'ouverture } \\
\text { des droits à une retraite anticipée } \\
\text { de } 58 \text { à } 60 \text { ans entre } 2008 \text { et } 2012 \\
\text { Suppression de l'exonération } \\
\text { des cotisations de sécurité sociale pour } \\
\text { les congés sabbatiques pris dans le } \\
\text { cadre du programme " crédit-temps " } \\
\text { Durcissement de l'obligation de } \\
\text { recherche d'emploi pour les chômeurs } \\
\text { plus âgés ayant droit aux prestations } \\
\text { de retraite anticipée }\end{array}$ & & $\begin{array}{l}\text { Adoption du principe } \\
\text { de prudence pour l'allocation } \\
\text { du portefeuille des régimes } \\
\text { privés }\end{array}$ \\
\hline Canada & & & & & & $\begin{array}{l}\text { Assouplissement } \\
\text { des contraintes relatives } \\
\text { aux investissements } \\
\text { à l'étranger }\end{array}$ \\
\hline
\end{tabular}


Tableau 3.1. Les réformes des retraites : 2004-09 (suite)

\begin{tabular}{|c|c|c|c|c|c|c|}
\hline Pays & Couverture & Adéquation & Viabilité financière & Efficacité économique & Efficacité administrative & Sécurité \\
\hline Corée & $\begin{array}{l}\text { Obligation pour les nouvelles } \\
\text { entreprises de mettre en place } \\
\text { des plans professionnels } \\
\text { à prestations ou à cotisations } \\
\text { définies au lieu } \\
\text { des régimes d'indemnités de fin de } \\
\text { contrat; les employeurs actuels } \\
\text { doivent consulter les salariés } \\
\text { sur le maintien du régime } \\
\text { d'indemnités ou le passage } \\
\text { aux plans professionnels }\end{array}$ & $\begin{array}{l}\text { Doublement en valeur de la retraite } \\
\text { de base, portée de } 5 \% \text { à } 10 \% \\
\text { du salaire moyen ; couverture portée } \\
\text { de } 60 \% \text { à } 70 \% \text { des personnes de plus } \\
\text { de } 65 \text { ans }\end{array}$ & $\begin{array}{l}\text { Objectif de taux } \\
\text { de remplacement } \\
\text { progressivement ramené } \\
\text { de } 60 \% \text { à } 40 \% \text { à compter } \\
\text { de } 2028\end{array}$ & $\begin{array}{l}\text { Système de plafonnement des salaires : } \\
\text { aides accordées par l'État aux plus } \\
\text { de } 53 \text { ans qui restent en activité tout } \\
\text { en acceptant une baisse de salaire. } \\
\text { Inciter les salariés à travailler plus } \\
\text { longtemps en avançant leur entrée } \\
\text { sur le marché du travail } \\
\text { (raccourcissement du service militaire, } \\
\text { de la formation) }\end{array}$ & & \\
\hline Danemark & & & & $\begin{array}{l}\text { Relèvement de l'âge de départ en } \\
\text { retraite anticipée de } 60 \text { à } 62 \text { ans entre } \\
2019 \text { et 2022, ainsi que de l'âge normal } \\
\text { de la retraite de } 65 \text { à } 67 \text { ans entre } 2024 \\
\text { et } 2027 \text {; ces deux âges seront ensuite } \\
\text { liés à l'espérance de vie }\end{array}$ & & \\
\hline Espagne & & $\begin{array}{l}\text { Revalorisation des retraites minimum } \\
\text { à hauteur de } 6.4 \%\end{array}$ & & & & \\
\hline États-Unis & $\begin{array}{l}\text { Autorisation pour les employeurs } \\
\text { d'affilier automatiquement } \\
\text { les salariés aux régimes de retraite }\end{array}$ & & & & & \\
\hline Finlande & & $\begin{array}{l}\text { Introduction d'une nouvelle retraite } \\
\text { garantie à partir de } 2011 \\
\text { Allégement des impôts prélevés } \\
\text { sur les retraites comprises entre } \\
15000 \text { EUR et } 30000 \text { EUR pour aligner } \\
\text { l'imposition des retraités } \\
\text { sur celle des salariés }\end{array}$ & $\begin{array}{l}\text { Salaire de référence : } \\
\text { dix dernières années } \\
\text { abandonnées au profit } \\
\text { d'une moyenne calculée } \\
\text { sur l'ensemble de la carrière } \\
\text { Introduction d'un lien entre } \\
\text { les prestations et l'espérance } \\
\text { de vie }\end{array}$ & $\begin{array}{l}\text { Modification des ajustements } \\
\text { applicables en cas de retraite anticipée } \\
\text { ou différée } \\
\text { Relèvement de l'âge de départ } \\
\text { en retraite anticipée de } 63 \text { à } 65 \text { ans } \\
\text { sur la période 2011-22 (proposition) }\end{array}$ & & \\
\hline
\end{tabular}


Tableau 3.1. Les réformes des retraites : 2004-09 (suite)

\begin{tabular}{|c|c|c|c|c|c|c|}
\hline Pays & Couverture & Adéquation & Viabilité financière & Efficacité économique & Efficacité administrative & Sécurité \\
\hline France & $\begin{array}{l}\text { Introduction d'un nouveau plan } \\
\text { d'épargne individuel pour la } \\
\text { retraite (PEIR) permettant de } \\
\text { déduire les cotisations du revenu } \\
\text { imposable dans la limite de } 10 \% \\
\text { des revenus, soit } 24000 \text { EUR par } \\
\text { an }\end{array}$ & $\begin{array}{l}\text { Revalorisation des retraites minimum } \\
\text { supérieure à l'indexation standard }\end{array}$ & $\begin{array}{l}\text { Indexation des retraites } \\
\text { du secteur public sur les prix } \\
\text { plutôt que sur les salaires }\end{array}$ & $\begin{array}{l}\text { Les employeurs peuvent imposer } \\
\text { le départ en retraite à } 70 \text { ans et non plus } \\
\text { à } 65 \text { ans } \\
\text { Durée de cotisation relevée de } 37.5 \\
\text { à } 40 \text { ans pour les salariés du secteur } \\
\text { public d'ici } 2012 \text {; réduction des } \\
\text { prestations pour les salariés du secteur } \\
\text { public en cas de retraite anticipée } \\
\text { Suppression progressive à horizon } \\
2010 \text { de la contribution « Delalande » } \\
\text { appliquée en cas de licenciement } \\
\text { d'un salarié de } 50 \text { ans ou plus } \\
\text { Surcote en cas de prolongation } \\
\text { d'activité portée de } 3 \% \text { à } 4 \% \text { de } 60 \text { à } \\
65 \text { ans et à } 5 \% \text { au-delà de } 65 \text { ans }\end{array}$ & & \\
\hline Grèce & $\begin{array}{l}\text { Introduction de nouvelles mesures } \\
\text { administratives (voir à droite) } \\
\text { visant à renforcer l'application } \\
\text { des règles régissant les régimes } \\
\text { publics ainsi que leur couverture }\end{array}$ & $\begin{array}{l}\text { 100-200 EUR versés aux retraités à titre } \\
\text { exceptionnel }\end{array}$ & & $\begin{array}{l}\text { Uniformisation de l'âge normal } \\
\text { de la retraite pour les hommes } \\
\text { et les femmes, soit } 65 \text { ans ; possibilité } \\
\text { de partir en retraite anticipée à partir } \\
\text { de } 55 \text { ans après } 15 \text { années de cotisation } \\
\text { au moins }\end{array}$ & $\begin{array}{l}\text { Fusion de } 133 \text { fonds } \\
\text { de pension en } 13 \text { dispositifs ; } \\
\text { base de données centralisée } \\
\text { des adhérents et des } \\
\text { employeurs : attribution } \\
\text { d'un numéro d'identification } \\
\text { unique à chaque individu }\end{array}$ & \\
\hline Hongrie & $\begin{array}{l}\text { Nouveaux comptes d'épargne- } \\
\text { retraite facultative avec } \\
\text { abondement de l'État à } \\
\text { concurrence de } 100000 \text { HUF } \\
\text { par an ; comptes également } \\
\text { exonérés de l'impôt sur les plus- } \\
\text { values (introduit en 1997) et } \\
\text { élargissement de la gamme } \\
\text { des supports d'investissements } \\
\text { proposés par rapport aux plans } \\
\text { actuels }\end{array}$ & & $\begin{array}{l}\text { Suppression du } 13^{\mathrm{e}} \text { mois } \\
\text { pour les retraites supérieures } \\
\text { à } 80000 \text { HUF par mois } \\
\text { Indexation des pensions } \\
\text { servies sur les prix et non } \\
\text { à la fois sur les salaires et les } \\
\text { prix } \\
\text { Indexation de } 2009 \text { reportée } \\
\text { à } 2010\end{array}$ & $\begin{array}{l}\text { Relèvement de l'âge de la retraite } \\
\text { de } 62 \text { à } 65 \text { ans à compter de } 2012 \\
\text { Durcissement des modalités de retraite } \\
\text { anticipée ramené de } 2013 \text { à } 2011\end{array}$ & & $\begin{array}{l}\text { Les fonds de pension doivent } \\
\text { proposer trois types de } \\
\text { fonds ; ajustement } \\
\text { automatique du portefeuille } \\
\text { en fonction du cycle de vie }\end{array}$ \\
\hline Irlande & & & $\begin{array}{l}\text { Adhérents au régime } \\
\text { de retraite de la fonction } \\
\text { publique soumis à cotisations } \\
\text { à hauteur de } 7.5 \% \text { en } \\
\text { moyenne }\end{array}$ & $\begin{array}{l}\text { Décote appliquée aux fonctionnaires } \\
\text { en cas de retraite anticipée }\end{array}$ & & $\begin{array}{l}\text { Les rentes au titre des } \\
\text { régimes professionnels qui } \\
\text { ont été liquidés seront } \\
\text { versées par le nouveau } \\
\text { Pensions Insolvency } \\
\text { Payment Scheme géré par } \\
\text { l'État }\end{array}$ \\
\hline
\end{tabular}


Tableau 3.1. Les réformes des retraites : 2004-09 (suite)

\begin{tabular}{|c|c|c|c|c|c|}
\hline Pays & Adéquation & Viabilité financière & Efficacité économique & Efficacité administrative & Sécurité \\
\hline Islande & & $\begin{array}{l}\text { Projet de réduire les pensions } \\
\text { des hauts fonctionnaires }\end{array}$ & & & $\begin{array}{l}\text { Relèvement des cotisations } \\
\text { patronales obligatoires } \\
\text { aux régimes professionnels } \\
\text { de } 6 \% \text { à } 8 \% \text {; obligation } \\
\text { de réduire les prestations } \\
\text { en cas de déficit actuariel } \\
\text { de } 10 \% \text { sur un an ou de } 5 \% \\
\text { par an sur } 5 \text { ans afin } \\
\text { de rétablir la solvabilité } \\
\text { des dispositifs professionnels }\end{array}$ \\
\hline Italie & $\begin{array}{l}\text { Les régimes d'indemnités de fin } \\
\text { de contrat des entreprises (de plus } \\
\text { de } 50 \text { salariés) doivent être } \\
\text { convertis en plans de retraite ; } \\
\text { possibilité de choisir parmi les } \\
\text { plans proposés par l'employeur, } \\
\text { un autre prestataire privé ou l'État } \\
\text { (cette dernière option est proposée } \\
\text { par défaut). Selon les prévisions } \\
\text { du gouvernement, environ un tiers } \\
\text { des cotisations seront versées sur } \\
\text { les nouveaux plans professionnels, } \\
\text { un tiers sur celui de l'État et un } \\
\text { tiers devrait continuer d'être versé } \\
\text { sur les dispositifs d'indemnités } \\
\text { de fin de contrat }\end{array}$ & $\begin{array}{l}\text { À compter de 2008, } \\
\text { minoration du coefficient } \\
\text { de conversion en pension du } \\
\text { solde des comptes notionnels } \\
\text { à cotisations définies afin } \\
\text { de tenir compte de l'évolution } \\
\text { de l'espérance de vie. } \\
\text { Les réductions des retraites } \\
\text { s'échelonnent de } 6.4 \% \text { pour } \\
\text { les nouveaux retraités âgés } \\
\text { de } 57 \text { ans à } 8.5 \% \text { pour les } \\
\text { retraités de } 65 \text { ans }\end{array}$ & $\begin{array}{l}\text { Relèvement de l'âge de la retraite à taux } \\
\text { plein de } 57 \text { à } 58 \text { ans en } 2008 \text { et à } 60 \text { ans } \\
\text { à partir de } 2011 \text {; la durée de cotisation } \\
\text { requise pour une retraite à taux plein est } \\
\text { portée de } 35 \text { à } 36 \text { ans (ce qui retarde } \\
\text { néanmoins l'application de lois } \\
\text { antérieures prévoyant de fixer l'âge } \\
\text { de la retraite à taux plein à } 60 \text { ans } \\
\text { à compter de 2008). } \\
\text { Relèvement échelonné de l'âge normal } \\
\text { de la retraite pour les femmes à } 65 \text { ans } \\
\text { (proposition) }\end{array}$ & & $\begin{array}{l}\text { Limitation des possibilités } \\
\text { offertes aux entreprises } \\
\text { de souscrire des prêts à court } \\
\text { ou moyen terme } \\
\text { sur les régimes d'indemnités } \\
\text { de fin de contrat }\end{array}$ \\
\hline Japon & & & $\begin{array}{l}\text { Relèvement de l'âge de départ } \\
\text { obligatoire à la retraite pouvant être } \\
\text { imposé par les employeurs aux salariés } \\
\text { du secteur privé de } 60 \text { à } 65 \text { ans sur la } \\
\text { période 2006-13 }\end{array}$ & $\begin{array}{l}\text { Problème soulevé par } \\
50 \text { millions d'enregistrement } \\
\text { non rattachés à des } \\
\text { individus : la Social Insurance } \\
\text { Agency devrait être } \\
\text { remplacée par une nouvelle } \\
\text { administration de tutelle à } \\
\text { partir de } 2010 ; \text { suppression } \\
\text { du délai de prescription } \\
\text { de cinq ans applicable } \\
\text { à la liquidation rétrospective } \\
\text { des droits pour les } \\
\text { enregistrements recouplés }\end{array}$ & \\
\hline
\end{tabular}

salariés) doivent être

possibilité de choisir parmi les

os par 'employeur,

prestataire privé ou l'État

r les dispositifs d'indemnités obligatoire à la retraite pouvant être

des droits pour les 
Tableau 3.1. Les réformes des retraites : 2004-09 (suite)

\begin{tabular}{|c|c|c|c|c|}
\hline Pays & Adéquation & Efficacité économique & Efficacité administrative & Sécurité \\
\hline Mexique & & $\begin{array}{l}\text { Introduction de régimes à } \\
\text { cotisations définies pour les } \\
\text { salariés du secteur public (à } \\
\text { l'image du dispositif du secteur } \\
\text { privé) ; affiliation obligatoire } \\
\text { pour les nouveaux venus ; les } \\
\text { salariés de moins de } 46 \text { ans } \\
\text { peuvent choisir ce type de } \\
\text { dispositif ou conserver leur } \\
\text { régime à prestations définies } \\
(7 \% \text { des salariés travaillent } \\
\text { dans le secteur public) }\end{array}$ & $\begin{array}{l}\text { Limitation des frais à ceux qui } \\
\text { sont prélevés sur le solde } \\
\text { des comptes ; } \\
\text { encouragement à opter pour } \\
\text { un prestataire à bas coût } \\
\text { (pour l'heure, les frais sont } \\
\text { deux fois plus élevés que la } \\
\text { moyenne des pays } \\
\text { d'Amérique latine). }\end{array}$ & $\begin{array}{l}\text { Élargissement de la gamme } \\
\text { des supports } \\
\text { d'investissement proposés } \\
\text { pour les régimes à cotisations } \\
\text { définies, soit } 5 \text { portefeuilles } \\
\text { par gestionnaire au lieu de } 2 \text {, } \\
\text { la part des actions ne pouvant } \\
\text { excéder } 30 \% \text {. }\end{array}$ \\
\hline Norvège & $\begin{array}{l}\text { Pour les régimes à cotisations } \\
\text { définies, cotisations patronales } \\
\text { minimum de } 2 \% \text { à partir de } 2006 \\
\text { sauf disposition plus avantageuse } \\
\text { déjà en vigueur; de ce fait, la } \\
\text { couverture est portée à } 25 \% \\
\text { de la population active }\end{array}$ & & & \\
\hline Nouvelle-Zélande & $\begin{array}{l}\text { KiwiSaver : plans à cotisations } \\
\text { définies avec affiliation obligatoire ; } \\
\text { contribution de l'État à concurrence } \\
\text { de } 1040 \text { NZD ; versement } \\
\text { exceptionnel de } 1000 \text { NZD à } \\
\text { l'ouverture du compte ; cotisations } \\
\text { salariales de } 4 \% \text { ou } 8 \% \text { (désormais } \\
\text { ramenées à } 2 \% \text { minimum); } \\
\text { cotisations patronales de } 1 \% \text {, } \\
\text { portées à } 2 \%\end{array}$ & & & \\
\hline
\end{tabular}


Tableau 3.1. Les réformes des retraites : 2004-09 (suite)

\begin{tabular}{|c|c|c|c|c|c|c|}
\hline Pays & Couverture & Adéquation & Viabilité financière & Efficacité économique & Efficacité administrative & Sécurité \\
\hline Pays-Bas & & & & $\begin{array}{l}\text { Suppression des avantages fiscaux } \\
\text { accordés pour les régimes } \\
\text { professionnels de retraite anticipée } \\
\text { Relèvement progressif de l'âge normal } \\
\text { de la retraite de } 65 \text { à } 67 \text { ans sur } 24 \text { mois } \\
\text { (proposition) }\end{array}$ & & $\begin{array}{l}\text { Gouvernance renforcée des } \\
\text { régimes professionnels : } \\
\text { énoncé explicite des politiques } \\
\text { d'indexation des prestations } \\
\text { des régimes professionnels; } \\
\text { système de régulation de la } \\
\text { solvabilité des dispositifs } \\
\text { professionnels en prévision } \\
\text { des engagements futurs; } \\
\text { valorisation des actifs des } \\
\text { régimes professionnels au prix } \\
\text { du marché }\end{array}$ \\
\hline Pologne & $\begin{array}{l}\text { Lancement d'un nouveau régime } \\
\text { à cotisations définies assorti } \\
\text { d'incitations fiscales }\end{array}$ & & & $\begin{array}{l}\text { Introduction de nouvelles règles } \\
\text { régissant les retraites anticipées, } \\
\text { ramenant le nombre d'ayants droit } \\
\text { de } 1.3 \text { million à } 0.25 \text { million de } \\
\text { personnes. Ces nouvelles règles sont } \\
\text { soumises à des délais }\end{array}$ & $\begin{array}{l}\text { Abaissement du plafond } \\
\text { pour les frais prélevés } \\
\text { dans le cadre des régimes } \\
\text { à cotisations définies }\end{array}$ & $\begin{array}{l}\text { Possibilité de choisir son } \\
\text { portefeuille entre trois } \\
\text { options } \\
\text { Exposé des règles de } \\
\text { versement des prestations au } \\
\text { titre des régimes à cotisations } \\
\text { définies : retraits échelonnés } \\
\text { et obligation de conversion en } \\
\text { rente à l'âge de } 65 \text { ans }\end{array}$ \\
\hline Portugal & $\begin{array}{l}\text { Introduction d'un nouveau régime } \\
\text { à cotisations définies facultatif } \\
\text { géré par un organisme central, les } \\
\text { cotisations s'élevant à } 2 \% \text { ou } 4 \% \\
\text { pour les moins de } 50 \text { ans et à } 6 \% \\
\text { pour les plus de } 50 \text { ans }\end{array}$ & & $\begin{array}{l}\text { Réduction des prestations de } \\
\text { retraite en fonction de } \\
\text { l'allongement de l'espérance } \\
\text { de vie à compter de } 2008 \text {; } \\
\text { accélération du basculement } \\
\text { au calcul fondé sur la } \\
\text { rémunération perçue sur } \\
\text { l'ensemble de la carrière }\end{array}$ & & & $\begin{array}{l}\text { Indexation des retraites } \\
\text { servies sur les prix } \\
\text { et la croissance du PIB et non } \\
\text { sur l'évolution du salaire } \\
\text { minimum }\end{array}$ \\
\hline $\begin{array}{l}\text { République } \\
\text { slovaque }\end{array}$ & & & & & $\begin{array}{l}\text { Abaissement du plafond pour } \\
\text { les frais prélevés dans le } \\
\text { cadre des régimes à } \\
\text { cotisations définies }\end{array}$ & \\
\hline République tchèque & & & & $\begin{array}{l}\text { Relèvement progressif de l'âge } \\
\text { de la retraite à } 65 \text { ans pour les hommes et } \\
\text { les femmes en } 2030 \text {; ainsi que de la durée } \\
\text { de cotisation requise de } 25 \text { à } 35 \text { ans }\end{array}$ & & \\
\hline
\end{tabular}


Tableau 3.1. Les réformes des retraites : 2004-09 (suite)

\begin{tabular}{|c|c|c|c|c|c|c|}
\hline Pays & Couverture & Adéquation & Viabilité financière & Efficacité économique & Efficacité administrative & Sécurité \\
\hline Royaume-Uni & $\begin{array}{l}\text { Mise en place d'un dispositif national } \\
\text { d'épargne-retraite à partir de 2012: } \\
\text { affiliation automatique des } \\
\text { personnes de } 22-65 \text { ans non } \\
\text { couvertes par un dispositif } \\
\text { professionnel ou individuel ; } \\
\text { cotisations salariales } \\
\text { de } 4 \% \text { et patronales de } 3 \% \text {, } \\
\text { introduction échelonnée d'une } \\
\text { contribution de l'État à hauteur de } \\
1 \% \\
\text { Durée de cotisation donnant droit à } \\
\text { une retraite à taux plein ramenée à } \\
30 \text { ans }\end{array}$ & $\begin{array}{l}\text { Projet d'indexation des retraites de base } \\
\text { sur le salaire moyen à partir de } 2012 \text {; } \\
\text { revalorisation de } 2004-08 \text { en fonction } \\
\text { des salaires } \\
\text { Accélération du basculement du State } \\
\text { Second Pension d'un régime lié à la } \\
\text { rémunération vers un régime à taux } \\
\text { uniforme, les prestations initiales étant } \\
\text { indexées sur le salaire moyen; } \\
\text { renforcement des crédits accordés pour } \\
\text { la garde d'une personne dépendante }\end{array}$ & & $\begin{array}{l}\text { Surcote pour retraite différée portée } \\
\text { de } 7.4 \% \text { à } 10.4 \% \text { par an ; désormais } \\
\text { disponible sous forme de prime } \\
\text { exceptionnelle }\end{array}$ & $\begin{array}{l}\text { Création d'une central } \\
\text { clearing house pour le } \\
\text { nouveau régime national } \\
\text { d'épargne-retraite ; objectifs } \\
\text { coûts équivalant à } 0.5 \% \\
\text { des avoirs, puis à } 0.3 \% \text {. } \\
\text { New Pensions Regulator } \\
\text { établi en } 2005 \text {, regroupant } \\
\text { les organismes existants }\end{array}$ & $\begin{array}{l}\text { Introduction en } 2004 \\
\text { du Pension Protection Fund, } \\
\text { afin de garantir les régimes } \\
\text { à prestations définies. } \\
\text { Les primes versées } \\
\text { par les régimes, en fonction } \\
\text { des risques, sont deux fois } \\
\text { plus élevées qu'initialement } \\
\text { prévu. Durcissement } \\
\text { des règles de redressement } \\
\text { pour les régimes déficitaires } \\
\text { Extension du Financial } \\
\text { Assistance Scheme aux } \\
\text { dispositifs professionnels } \\
\text { insolvables : } 140000 \text { salariés } \\
\text { supplémentaires couverts }\end{array}$ \\
\hline Suède & & $\begin{array}{l}\text { Réduction des impôts pour les } \\
\text { personnes de plus de } 65 \text { ans dont les } \\
\text { revenus ne dépassent pas } 363000 \\
\text { SEK à partir de 2009, soit } 90 \% \text { des } \\
\text { retraités }\end{array}$ & $\begin{array}{l}\text { Régime professionnel à } \\
\text { prestations définies des } \\
\text { cadres et employés du } \\
\text { secteur privé converti en } \\
\text { régime à cotisations définies }\end{array}$ & $\begin{array}{l}\text { Diminution de } 1 \% \text { des cotisations de } \\
\text { sécurité sociale des employeurs à } \\
\text { compter de } 2009\end{array}$ & $\begin{array}{l}\text { Fusion des organes gérant les } \\
\text { régimes à cotisations définies } \\
\text { public et privés }\end{array}$ & \\
\hline Suisse & $\begin{array}{l}\text { Abaissement du seuil de } \\
\text { rémunération pour couvrir un plus } \\
\text { grand nombre de salariés à temps } \\
\text { partiel faiblement rémunérés }\end{array}$ & & $\begin{array}{l}\text { S'agissant des régimes } \\
\text { professionnels obligatoires, } \\
\text { taux d'intérêt minimum } \\
\text { ramené de } 2.75 \% \text { à } 2 \% \text { pour } \\
2009 \text { (il avait toutefois été } \\
\text { porté de } 2.5 \% \text { en } 2007 \text { à } \\
2.75 \% \text { en } 2008 \text { ) } \\
\text { Pour les régimes } \\
\text { professionnels obligatoires, } \\
\text { taux de rente ramené de } \\
7.2 \% \text { à } 6.8-7.15 \% \text {, en } \\
\text { fonction de l'âge et du sexe }\end{array}$ & $\begin{array}{l}\text { Relèvement de l'âge normal de la } \\
\text { retraite pour les femmes de } 63 \text { à } 64 \text { ans } \\
\text { (pour les hommes, il reste inchangé à } \\
65 \text { ans) }\end{array}$ & & \\
\hline Turquie & & & & $\begin{array}{l}\text { Relèvement progressif de l'âge } \\
\text { de la retraite de } 58 \text { ans pour les femmes } \\
\text { et } 60 \text { ans pour les hommes à } 65 \text { ans } \\
\text { pour tous d'ici } 2048\end{array}$ & & $\begin{array}{l}\text { Ajustement des prestations } \\
\text { désormais effectué } \\
\text { en fonction de l'inflation } \\
\text { et de la croissance du PIB } \\
\text { et non de l'indice mensuel } \\
\text { des prix }\end{array}$ \\
\hline
\end{tabular}




\section{Notes}

1. OCDE (1998, 2001), par exemple. La Banque mondiale a également établi une liste analogue d'objectifs : voir Holzmann et Hinz (2005).

2. OCDE (2007), partie II.1. Voir aussi Martin et Whitehouse (2008), et Whiteford et Whitehouse (2006).

3. Voir le chapitre spécial sur "le déficit d'épargne-retraite et l'épargne-retraite facultative " dans la présente étude, Antolín et Whitehouse (2009) et Queisser et al. (2007) pour plus de détails sur cette mesure.

4. Voir Whitehouse (2007) pour de plus amples détails.

5. Voir OCDE (2006) pour une analyse détaillée de l'action publique.

6. Voir le chapitre spécial sur Les systèmes de retraite pendant la crise économique et financière dans la présente publication.

7. Cela étant, la crise économique et financière est particulièrement profonde en Islande, de sorte que l'on ignore encore comment les fonds de pension professionnels vont surmonter ces difficultés.

8. Voir Whitehouse (2009) pour une analyse détaillée du risque d'inflation et de l'indexation des retraites.

9. President's Commission to Strengthen Social Security [commission présidentielle chargée de renforcer la Sécurité sociale] (2001).

\section{Références}

Antolín, P. et E.R. Whitehouse (2009), « Filling the Pension Gap: Coverage and Value of Voluntary Retirement Savings ", Documents de travail sur les affaires sociales, l'emploi et les migrations, $\mathrm{n}^{\circ} 69$, Éditions de l'OCDE, Paris.

Holzmann, R. et R.P. Hinz (2005), Old-Age Income Support in the 21st Century: An International Perspective on Pension Systems and Reform, Banque mondiale, Washington, DC.

Martin, J.P. et E.R. Whitehouse (2008), « Reforming Retirement-Income Systems: Lessons from the Recent Experiences of OECD Countries », Documents de travail sur les affaires sociales, l'emploi et les migrations, $n^{\circ} 66$, Éditions de l'OCDE, Paris.

OCDE (1998), Préserver la prospérité dans une société vieillissante, Éditions de l'OCDE, Paris.

OCDE (2001), Vieillissement et revenus - Les ressources des retraités dans 9 pays de l'OCDE, Éditions de l'OCDE, Paris.

OCDE (2006), Viure et travailler plus longtemps, Éditions de l'OCDE, Paris.

OCDE (2007), Les pensions dans les pays de l'OCDE - Panorama des politiques publiques, Éditions de l'OCDE, Paris.

President's Commission to Strengthen Social Security [commission présidentielle chargée de renforcer la Sécurité sociale] (2001), Strengthening Social Security and Creating Personal Wealth for All Americans, Washington, DC.

Queisser, M., P. Whiteford et E.R. Whitehouse (2007), « The Public-Private Pension Mix in OECD Countries ", Industrial Relations Journal, vol. 38, $n^{\circ}$ 6, pp. 542-568.

Whiteford, P. et E.R. Whitehouse (2006), "Pension Challenges and Pension Reforms in OECD Countries ", Oxford Review of Economic Policy, vol. 22, n 1, pp. 78-94.

Whitehouse, E.R. (2007), « Life-Expectancy Risk and Pensions: Who Bears the Burden? », Documents de travail sur les affaires sociales, l'emploi et les migrations, $n^{\circ} 60$, Éditions de l'OCDE, Paris.

Whitehouse, E.R. (2009), " Pensions, Purchasing-Power Risk, Inflation and Indexation », Documents de travail sur les affaires sociales, l'emploi et les migrations, $n^{\circ} 77$, Éditions de l'OCDE, Paris. 


\section{Le déficit d'épargne-retraite et l'épargne-retraite facultative}

L

a plupart des pays de l'OCDE ont sensiblement réformé leur système de retraite au cours des 20 dernières années. Ces changements ont été principalement, mais pas uniquement, motivés par une volonté d'accroître la viabilité financière des régimes publics. Dans les 16 pays de l'Organisation ayant entrepris les réformes les plus vastes, les pensions viagères ont été diminuées en moyenne de $22 \%$ pour les hommes et de $25 \%$ pour les femmes ${ }^{1}$.

L'ampleur de ces réductions implique un profond rééquilibrage des responsabilités entre les secteurs public et privé au regard des prestations de retraite. Les salariés d'aujourd'hui vont devoir soit épargner davantage dans le cadre de dispositifs privés facultatifs, soit partir plus tard à la retraite, ou percevoir une fois à la retraite, un revenu inférieur à celui qui était prévu par les paramètres et les règles en vigueur avant la réforme, par rapport au revenu qu'ils touchaient lorsqu'ils étaient en activité. Cela correspond de fait à une " privatisation " implicite ou explicite d'une partie du système de retraite.

Ce chapitre spécial ${ }^{2}$ s'ouvre sur une analyse des différences de droits à la retraite au titre des régimes obligatoires existant entre les pays de l'OCDE. Il s'intéresse ensuite de plus près aux 16 pays dans lesquels les retraites obligatoires sont particulièrement peu élevées. Nombreux sont ceux qui, faute d'avoir constitué une épargne-retraite, vont y subir une chute brutale de leur niveau de vie lorsqu'ils quitteront la vie active pour faire valoir leurs droits à la retraite. Puis, compte tenu du fléchissement des taux de remplacement des régimes obligatoires observé dans ces pays par rapport à la moyenne des pays membres, on calcule la fraction des revenus qu'il conviendrait d'épargner pour combler ce déficit d'épargne-retraite à l'aide des modèles de retraite de l'OCDE. Les résultats de l'analyse sont donnés en premier lieu pour les autres moyens, puis pour les autres catégories de revenus considérées.

Après avoir déterminé les personnes qui doivent constituer une épargne-retraite et dans quelle proportion pour les différents pays, on s'intéresse aux données relatives aux comportements en la matière. Tout d'abord, on étudie l'évolution de la couverture des régimes privés facultatifs en fonction de l'âge et des revenus, puis on présente des données concernant les cotisations.

Ce chapitre s'achève sur un examen des différentes mesures introduites pour favoriser l'épargne-retraite privée. La plus évidente est de rendre l'adhésion obligatoire. Néanmoins, des approches plus souples peuvent être adoptées, par exemple en introduisant une obligation partielle (affiliation automatique avec possibilité de sortie, par exemple); en facilitant l'accès aux instruments d'épargne-retraite; en prenant des dispositions pour 
sensibiliser davantage le public aux questions financières; et en mettant en place un régime fiscal préférentiel pour l'épargne-retraite.

\section{Le déficit d'épargne-retraite}

Le taux de remplacement - rapport entre les revenus au titre de la retraite et le salaire perçu - est largement utilisé pour rendre compte des différences existant entre les systèmes de retraite des différents pays. Les données relatives aux 30 pays de l'OCDE, présentées en partie II de cette publication, montrent qu'en moyenne, le taux de remplacement brut s'établit à $59.0 \%$ pour les revenus moyens.

Le graphique 4.1 illustre le taux de remplacement brut attendu pour les revenus moyens dans les 16 pays où il se situe en deçà de la moyenne des pays de l'OCDE. Les calculs portent sur l'ensemble des programmes obligatoires assurant des revenus aux retraités, qui peuvent englober les régimes privés obligatoires et les dispositifs d'assistance sociale au sens large. Ce groupe de 16 pays comprend les 6 pays principalement anglophones de l'OCDE, soit l'Australie, le Canada, les États-Unis, l'Irlande, la Nouvelle-Zélande et le Royaume-Uni. Il compte aussi les deux économies membres situées en Asie de l'Est - la Corée et le Japon - et plusieurs pays d'Europe continentale, dont l'Allemagne et la Belgique.

\section{Graphique 4.1. Le déficit d'épargne-retraite}

Taux de remplacement brut, pour un salaire moyen, au titre des dispositifs de retraite obligatoires et différence par rapport au taux de remplacement moyen des pays de l'OCDE

Taux de remplacement : dispositifs obligatoires Taux de remplacement : déficit d'épargne-retraite

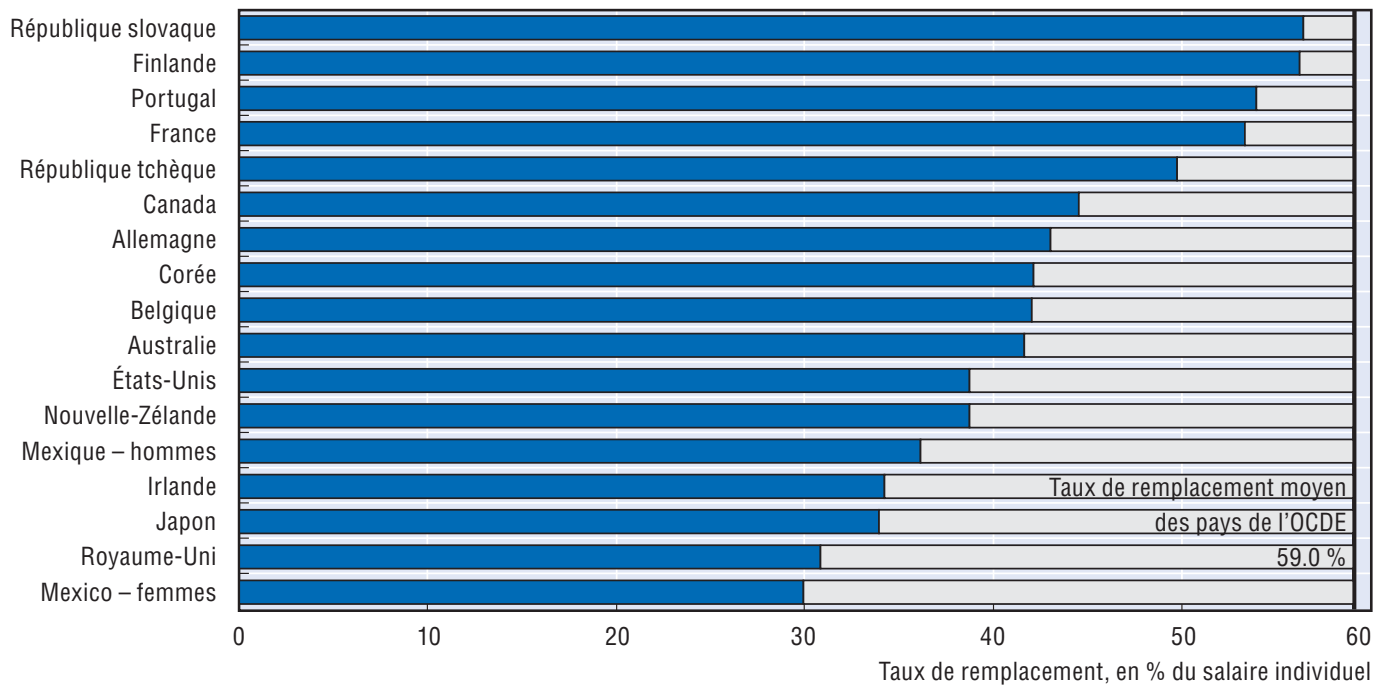

Source: Modèles de retraite de l'OCDE.

Dans nombre de ces pays, la couverture des dispositifs privés facultatifs est étendue parce que le régime obligatoire est relativement restreint. Au sein même de ces 16 pays, toutefois, la nécessité pour les retraités de percevoir des revenus supplémentaires dans le cadre d'un mécanisme d'épargne facultatif varie considérablement car les taux de remplacement au titre des régimes obligatoires diffèrent d'un pays à l'autre.

Pour évaluer l'épargne-retraite nécessaire dans les divers pays, il convient d'établir un objectif de taux de remplacement. Comme dans les précédentes analyses de l'OCDE, le 
taux de remplacement de référence utilisé est le taux de remplacement moyen des dispositifs obligatoires dans l'ensemble des pays de l'OCDE. La différence entre le taux de remplacement assuré par le système de retraite obligatoire et la moyenne des pays de l'OCDE est qualifiée de " déficit d'épargne-retraite ».

Au Royaume-Uni, il faudrait que le taux de remplacement des dispositifs de retraite privés ressorte à $28.2 \%$ pour que la pension globale d'une personne gagnant un salaire moyen atteigne la moyenne des pays de l'OCDE. La Finlande et la République slovaque sont les économies où le déficit d'épargne-retraite est le plus faible parmi les 16 pays étudiés, soit aux alentours de $2.5 \%$ du salaire. Pour les 16 pays considérés, le taux de remplacement des régimes obligatoires s'élève à $41.8 \%$ pour le salaire moyen, ce qui correspond à un déficit de $17.2 \%$ en moyenne. Pour le Mexique, les résultats diffèrent pour les hommes et pour les femmes, parce que le calcul des rentes varie selon le sexe et que de ce fait, une fois à la retraite, les femmes doivent étaler les sommes accumulées sur une période plus longue.

De plus, il est important de rappeler que comme dans le reste de l'étude, l'analyse repose sur les paramètres et règles du système de retraite en vigueur pour les personnes entrées sur le marché du travail en 2006. Cette hypothèse prend tout son sens lorsqu'on examine la situation au Mexique, car tous les salariés actuels ont eu l'assurance que leur retraite à cotisations définies serait complétée par le gouvernement pour atteindre le niveau assuré par le régime lié à la rémunération en place avant la réforme. Les modèles de retraite de l'OCDE donnent à penser que pour les personnes déjà présentes sur le marché du travail à l'époque de la réforme, le taux de remplacement sera pratiquement deux fois plus élevé que celui du régime à cotisations définies selon l'hypothèse standard de taux de rendement (voir OCDE, 2007, pp. 69-70).

Dans quelle proportion les intéressés doivent-ils cotiser à des dispositifs privés facultatifs pour porter dans leur ensemble les taux de remplacement assurés par les régimes obligatoires nationaux à la moyenne des pays de l'OCDE ? Pour faciliter les calculs et les comparaisons, on suppose que les personnes affiliées à un régime facultatif disposent d'un plan à cotisations définies, dans le cadre duquel les prestations dépendent des cotisations et du rendement des investissements ${ }^{3}$. La modélisation repose sur les mêmes hypothèses générales que les calculs figurant en partie II de la présente publication. On suppose en particulier que le rendement réel annuel s'élève à $3.5 \%$ de l'épargne constituée, nets de frais administratifs.

Le graphique 4.2 fait apparaître, pour chaque pays, le pourcentage de sa rémunération qu'une personne gagnant un salaire moyen devrait verser sur un plan de retraite privé pour combler le déficit d'épargne-retraite. Les pays sont classés dans le même ordre qu'au graphique 4.1, soit selon l'importance du déficit. Les barres plus sombres correspondent au taux de cotisation requis pour une carrière complète, ce qui correspond aux cotisations à acquitter chaque année de l'âge de 20 ans à l'âge normal de la retraite dans le pays concerné.

Le graphique montre l'impact de l'âge de la retraite dans chaque pays. Le taux de cotisation requis en Allemagne, aux États-Unis et au Royaume-Uni est plus bas, car l'âge de la retraite va y être porté de 67 à 68 ans. De même, lorsque l'âge de la retraite est inférieur à 65 ans - en France et en République slovaque - le nombre d'années de cotisation s'en trouve réduit et la durée de la retraite accrue. 


\section{Graphique 4.2. Comblement du déficit d'épargne-retraite}

Taux de cotisation requis pour un salaire moyen afin d'atteindre le taux de remplacement brut moyen des pays de l'OCDE

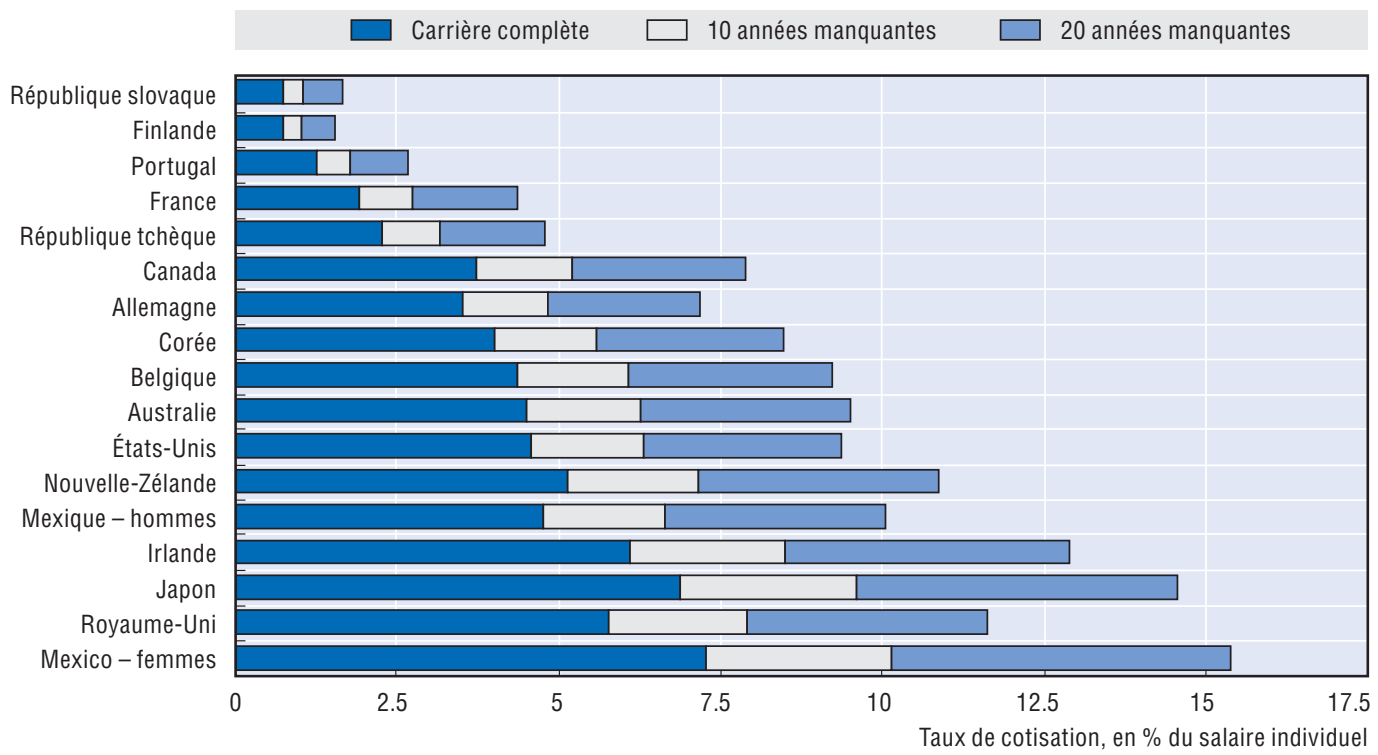

Source: Modèles de retraite de l'OCDE.

StatLink न्ना15t http://dx.doi.org/10.1787/651303243233

Les différences d'espérance de vie ont également des répercussions. Ainsi, au Mexique, les personnes âgées de 65 ans devraient vivre 17.6 années de plus, contre 22.2 années au Japon. Á l'évidence, l'allongement de l'espérance de vie majore le taux de cotisation requis, car les retraites doivent être versées plus longtemps.

\section{Les effets des carrières incomplètes}

Pour une carrière complète, la proportion du salaire qu'il conviendrait de verser sur un plan d'épargne-retraite afin de combler le déficit en la matière est généralement assez faible, soit approximativement $6 \%$ en Irlande et au Royaume-Uni, et $7 \%$ environ au Japon. Dans bien des cas - en Allemagne, en Australie, en Belgique, au Canada, en Corée et aux États-Unis -, le taux de cotisation requis s'établit à 3.5-4.5\%.

Cela étant, comme on le verra ci-après dans ce chapitre spécial, il est peu probable que les salariés effectuent une carrière complète. Les barres plus claires font apparaître deux scénarios : l'un tenant compte de 10 années de cotisation manquantes, l'autre de 20. On suppose que ces années manquantes se situent en début de carrière, c'est-à-dire que les personnes concernées ne souscrivent pas à un dispositif de retraite privé avant l'âge de 30 ou 40 ans. Pour les pays étudiés, le taux de cotisation requis en moyenne varie de $4.0 \%$ pour une carrière complète à $5.5 \%$ pour 10 années manquantes et $8.4 \%$ pour 20 années.

\section{Les effets du salaire individuel}

Jusqu'à présent, l'analyse a essentiellement porté sur la situation des revenus moyens. Pour les plus bas salaires, toutefois, les prestations assurées par le filet de sécurité jouent généralement un rôle plus important dans les revenus perçus au titre de la retraite. Pour cette catégorie de salariés, les taux de remplacement peuvent donc être supérieurs à ceux des salariés moyens. À l'autre extrémité, le plafonnement du salaire 
ouvrant droit à retraite peut entraîner une baisse des taux de remplacement pour les plus hauts salaires.

Le graphique 4.3 montre l'évolution, dans les 16 pays considérés, des taux de remplacement en fonction du salaire par rapport à la moyenne de la zone OCDE. Les salariés percevant $50 \%$ du salaire moyen bénéficient d'un taux de remplacement net moyen de $72 \%$ dans les 30 pays membres, contre $59 \%$ pour le salaire moyen. Pour ceux qui perçoivent le double du salaire moyen, le taux de remplacement atteint en moyenne $50 \%$. Comment les différents pays se situent-ils par rapport à ces taux de référence?

Graphique 4.3. Le déficit d'épargne-retraite et le salaire individuel

Écart entre le taux de remplacement du régime obligatoire national et le taux de remplacement moyen des pays de l'OCDE
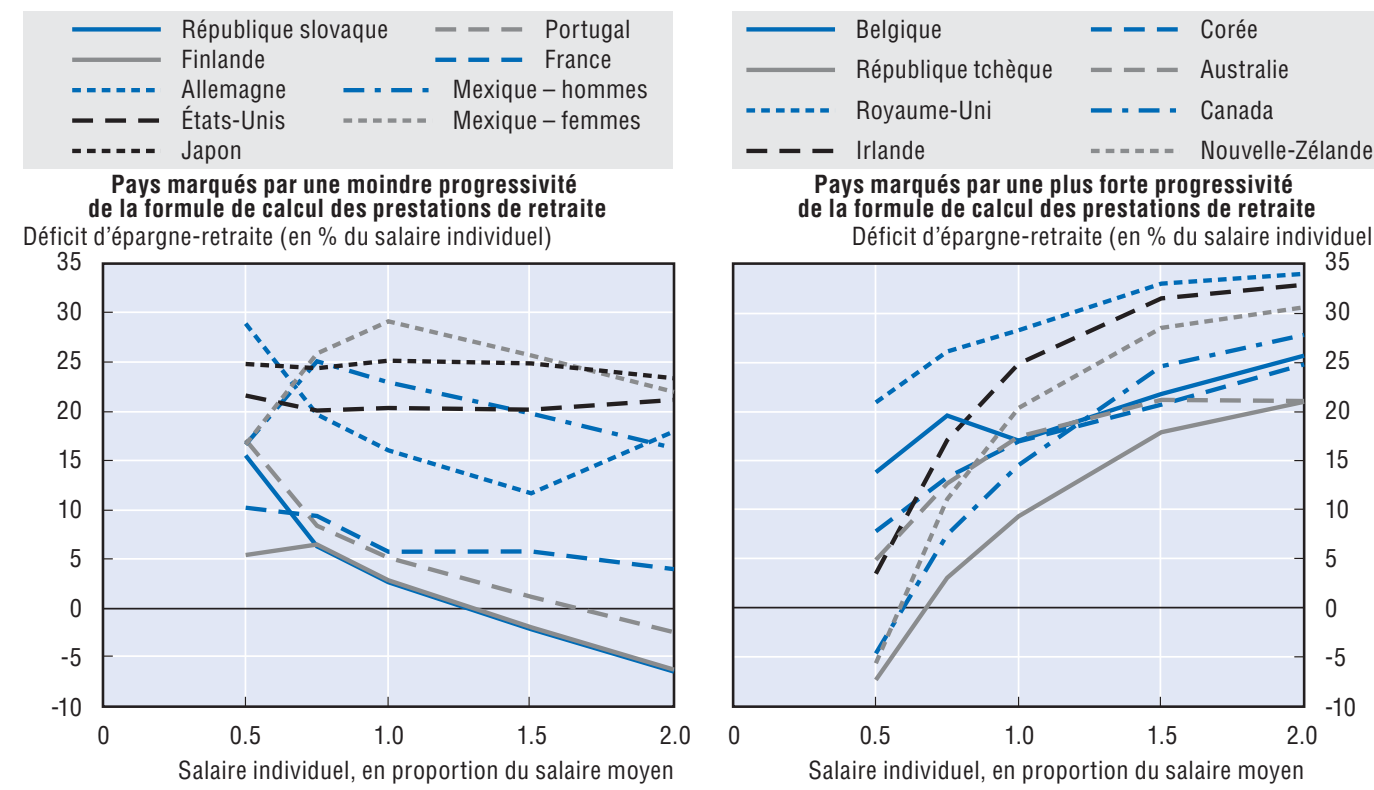

Note: Les pays ont été regroupés en fonction de l'indice de progressivité de la formule de calcul des prestations de retraite établi par l'OCDE.

Source: Modèles de retraite de l'OCDE.

StatLink त्नाजा http://dx.doi.org/10.1787/651315163361

Dans la partie gauche du graphique 4.3, l'évolution du déficit d'épargne-retraite est dans l'ensemble constante par rapport au salaire perçu aux États-Unis et au Japon : la progressivité des régimes obligatoires y est globalement conforme à l'évolution de la moyenne des pays de l'OCDE.

En Allemagne, le taux de remplacement du régime obligatoire suit une évolution constante à partir de $50 \%$ du salaire moyen au plafond fixé approximativement à quelque $150 \%$ du salaire moyen. Le déficit d'épargne-retraite est donc, par rapport au taux de remplacement moyen de l'OCDE en fonction du salaire, plus marqué pour les faibles revenus que pour les revenus moyens. En Finlande, au Portugal et en République slovaque, le taux de remplacement est pratiquement constant sur la gamme des salaires considérés, de sorte que les hauts revenus bénéficient d'un taux de remplacement supérieur à la moyenne de l'OCDE, tandis que les faibles revenus auraient intérêt à cotiser à des dispositifs de retraite facultatifs pour atteindre le taux de remplacement de l'OCDE à ce niveau de rémunération. 
Les pays figurant dans la partie droite suivent l'évolution inverse. Au Canada, en Nouvelle-Zélande et en République tchèque, en effet, le taux de remplacement du régime obligatoire pour les faibles revenus est supérieur à la moyenne de l'OCDE : le déficit d'épargne-retraite est donc nul pour ces salariés. Il est minime pour les faibles revenus en Australie et en Irlande, et il se creuse à mesure que le salaire augmente dans les huit pays figurant dans la partie droite du graphique 4.3. Pour les hauts salaires $(200 \%$ du salaire moyen), les taux de remplacement du régime obligatoire sont inférieurs à $25 \%$ en Belgique, au Canada, en Irlande, en Nouvelle-Zélande et au Royaume-Uni, contre $50 \%$ en moyenne dans les pays de l'OCDE. Le déficit d'épargne-retraite s'élève à $27 \%$ en moyenne pour les hauts revenus dans les huit pays du schéma de droite, contre pratiquement $4 \%$ pour les salariés percevant $50 \%$ du salaire moyen.

Le graphique 4.4 examine l'impact des différences de taux de remplacement en fonction du salaire sur la nécessité de constituer une épargne-retraite pour atteindre le taux de remplacement global de référence. Les pays sont regroupés selon le même principe que dans le graphique 4.3. On suppose ici qu'il manque 10 années de cotisation pour les régimes facultatifs (c'est-à-dire que les salariés cotisent à partir de 30 ans, puis chaque année jusqu'à l'âge normal de la retraite dans leur pays).

Dans la partie de droite, le taux de cotisation requis progresse en fonction du salaire. Ainsi, au Canada, en Nouvelle-Zélande et en République tchèque, les faibles revenus n'ont guère voire nullement besoin de pourvoir à leur retraite car les régimes obligatoires le font déjà. En Australie, en Corée et en Irlande, les taux de cotisation requis des personnes touchant $50 \%$ du salaire moyen représentent tout au plus $2.5 \%$ du salaire. Pour les huit

\section{Graphique 4.4. Comblement du déficit d'épargne-retraite pour différents niveaux de salaire}

Taux de cotisation requis pour 10 années manquantes afin d'atteindre le taux de remplacement brut moyen des pays de l'OCDE
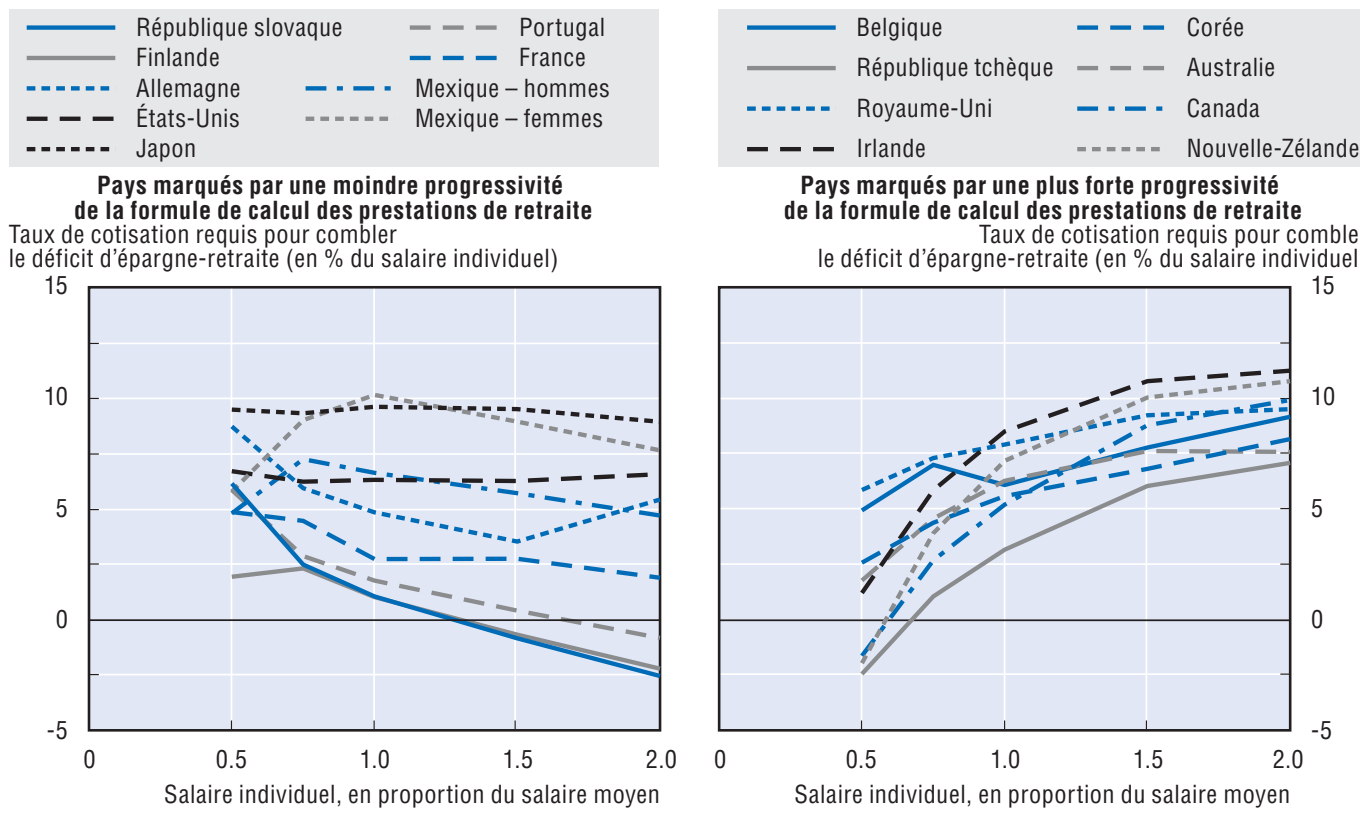

Note : Les pays ont été regroupés en fonction de l'indice de progressivité de la formule de calcul des prestations de retraite établi par l'OCDE.

Source: Modèles de retraite de l'OCDE. 
pays concernés, en moyenne, le taux de cotisation requis atteint moins de $1.5 \%$ pour les faibles revenus, $6 \%$ pour le salaire moyen et $9 \%$ pour les hauts revenus (pour ceux qui perçoivent le double du salaire moyen).

On trouvera dans la partie gauche du graphique 4.4 les pays affichant une progressivité du calcul des prestations de retraite inférieure à celle des pays figurant dans la partie droite. Les salariés américains et japonais doivent épargner une proportion analogue de leur salaire quel qu'il soit, à savoir $6.5 \%$ et $9.5 \%$ respectivement. La progressivité des systèmes de retraite obligatoires des États-Unis et du Japon est donc proche de la moyenne des pays de l'OCDE.

À l'inverse, les taux de remplacement suivent en Allemagne une évolution constante pour la plupart des salaires considérés. Pour atteindre un taux de remplacement plus élevé, les faibles revenus devraient épargner une proportion nettement plus importante que les salaires moyens, soit près de $9 \%$ contre $5 \%$ environ. La situation est analogue en Finlande et au Portugal.

\section{Effets de la fiscalité et de la mise sous conditions de ressources}

Jusqu'à présent, les calculs ont principalement porté sur les droits à retraite bruts alors que ce sont naturellement les revenus nets d'impôt et de cotisations qui déterminent le niveau de vie des intéressés durant leur vie active et leur retraite. Les répercussions de la fiscalité et des cotisations sur les résultats sont complexes. Lorsque les revenus au titre de la retraite sont soumis à des prélèvements relativement importants, les salariés sont davantage contraints de cotiser pour atteindre un niveau de vie donné. Inversement, le versement par les salariés d'impôts et de cotisations élevés accroît le taux de remplacement net, et la nécessité de cotiser est donc moindre par rapport à d'autres pays et à différentes catégories de salariés.

Les calculs ont également été fondés sur l'hypothèse selon laquelle l'épargne-retraite privée facultative a une influence directe sur les revenus au titre de la retraite, donc sans tenir compte du fait que les prestations sont soumises à conditions de ressources dans certains pays. Par conséquent, le fait de percevoir des sommes supplémentaires dans le cadre d'un dispositif de retraite privé entraîne une minoration des prestations publiques. La mise sous conditions de ressources contraint donc davantage les personnes concernées à épargner.

Le graphique 4.5 présente une comparaison des calculs bruts et nets du taux de cotisation requis afin d'atteindre le taux de remplacement (brut ou net) moyen des pays de l'OCDE pour différents revenus dans certains pays. Le cas de l'Australie illustre parfaitement l'impact d'une vaste mise sous conditions de ressources, car les revenus intermédiaires et les hauts revenus sont également touchés. Pour un salaire moyen, par exemple, il faudrait verser $13 \%$ de ce salaire sur un plan de retraite facultatif - en plus de la cotisation obligatoire de $9 \%$ - pour atteindre le taux de remplacement net moyen de l'OCDE. Percevoir des revenus au titre d'un dispositif de retraite privé donne en effet lieu à une diminution de la pension publique à hauteur de $40 \%$ de sa valeur à la marge.

En Allemagne, au contraire, le déficit net d'épargne-retraite est inférieur au déficit brut pour la quasi-totalité des salaires. Le taux de cotisation requis est particulièrement élevé pour les bas revenus en termes bruts et nets, le système de retraite allemand proposant aux salariés un taux de remplacement constant à concurrence du plafond, tandis que le taux de remplacement moyen de l'OCDE est de nature progressive, les faibles revenus 


\section{Graphique 4.5. Comblement du déficit d'épargne-retraite : impact de la fiscalité et de la mise sous conditions de ressources}

Taux de cotisation requis, pour une carrière complète, afin d'atteindre le taux de remplacement (brut et net) moyen des pays de l'OCDE en fonction du salaire en Allemagne, en Australie, en Belgique et aux États-Unis
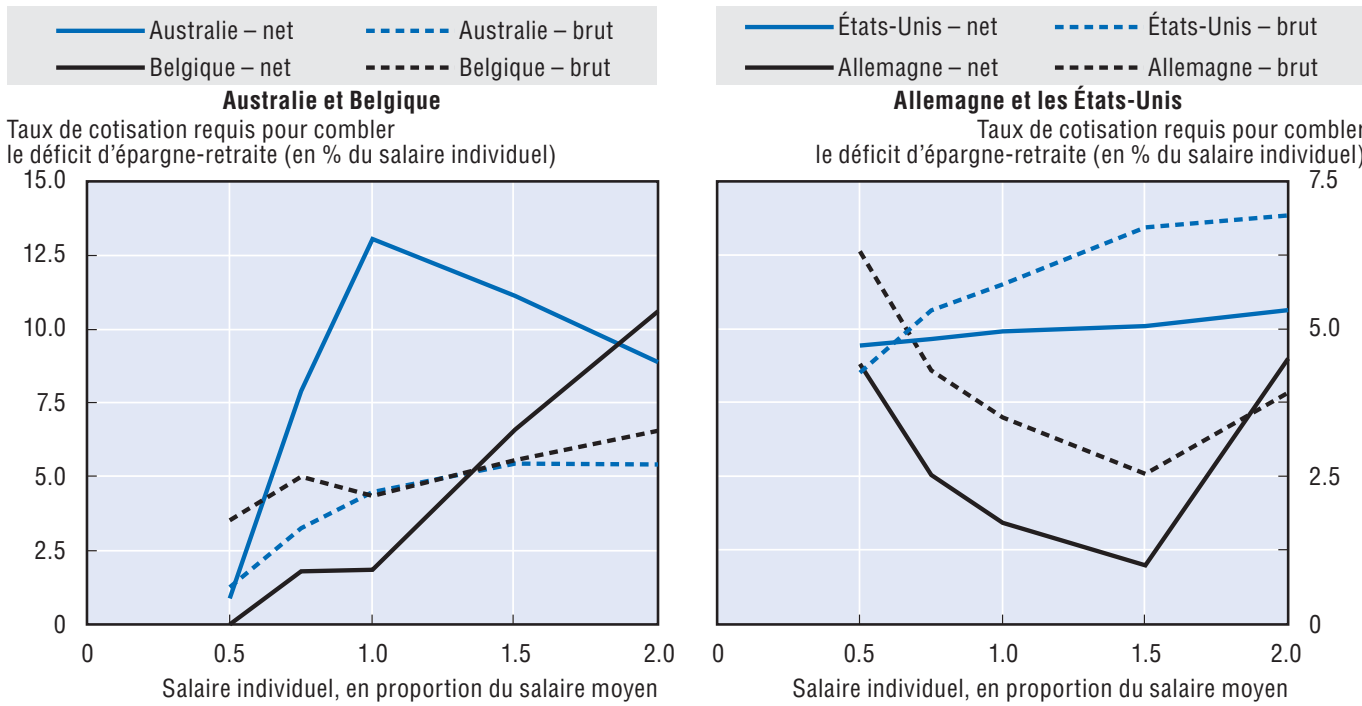

Note : Par souci de clarté, l'axe des ordonnées diffère entre les deux schémas.

Source: Modèles de retraite de l'OCDE.

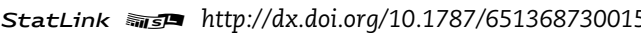

bénéficiant d'un taux plus élevé. La principale raison de cet écart sensible entre les chiffres bruts et nets du taux de cotisation nécessaire pour combler le déficit d'épargne-retraite est la pression fiscale relativement forte qui pèse sur les salariés et sur les retraités allemands.

L'impact de la fiscalité sur le calcul du déficit d'épargne-retraite est particulièrement marqué dans le cas de la Belgique. Pour les bas salaires et les revenus intermédiaires, les prélèvements et les cotisations sont relativement importantes, mais la fiscalité est nulle ou minime sur les pensions de retraite. Le taux de cotisation requis s'en trouve sensiblement réduit. Néanmoins, les hauts revenus consacreront également une part non négligeable de leurs revenus aux impôts durant leur retraite. D'où une hausse du taux de cotisation requis en termes nets par rapport aux calculs effectués en termes bruts.

Aux États-Unis, enfin, l'évolution des taux de cotisation requis est pratiquement identique en termes bruts et nets. C'est aussi le cas dans bon nombre des pays non représentés, comme le Canada, l'Irlande ou le Royaume-Uni.

\section{La couverture des régimes privés facultatifs en fonction de l'âge et du salaire 4}

Les données relatives à la couverture des régimes de retraite privés, tirées d'enquêtesménages, concernent huit pays de l'OCDE, dans la majorité desquels les régimes obligatoires offrent un taux de remplacement relativement bas. Les salariés doivent donc recourir aux dispositifs facultatifs pour compléter leurs revenus futurs au titre de la retraite.

La relation existant entre la couverture des dispositifs privés facultatifs et l'âge n'est pas linéaire ; elle atteint un pic pour les tranches d'âge de forte activité, à savoir 35-44 ans ou 45-54 ans selon le pays (partie gauche du graphique 4.6). En Allemagne, toutefois, les jeunes sont davantage susceptibles que les personnes âgées de souscrire à un dispositif 
privé, ce qui reflète très probablement l'introduction récente d'un plan d'épargne-retraite assorti d'avantages fiscaux. On observe parmi les salariés plus âgés une diminution des taux de couverture dans la plupart des pays (à l'exception notable du Canada et des ÉtatsUnis) ; ce phénomène pourrait être lié au départ en retraite anticipée des personnes affiliées à des régimes privés (leur patrimoine retraite étant plus élevé que celui des personnes dépourvues de plan d'épargne-retraite privé).

La couverture des régimes privés facultatifs tend à croître avec le salaire (partie droite du graphique 4.6). De manière générale, elle a néanmoins tendance à se stabiliser après les $7^{\mathrm{e}}$ et $8^{\mathrm{e}}$ déciles de la distribution. Parmi les plus démunis, la couverture est faible, soit de $10 \%$ à $20 \%$ dans l'ensemble des pays, à l'exception de l'Allemagne (40\%).

\section{Graphique 4.6. Couverture des régimes de retraite privés facultatifs}

En pourcentage de l'emploi total

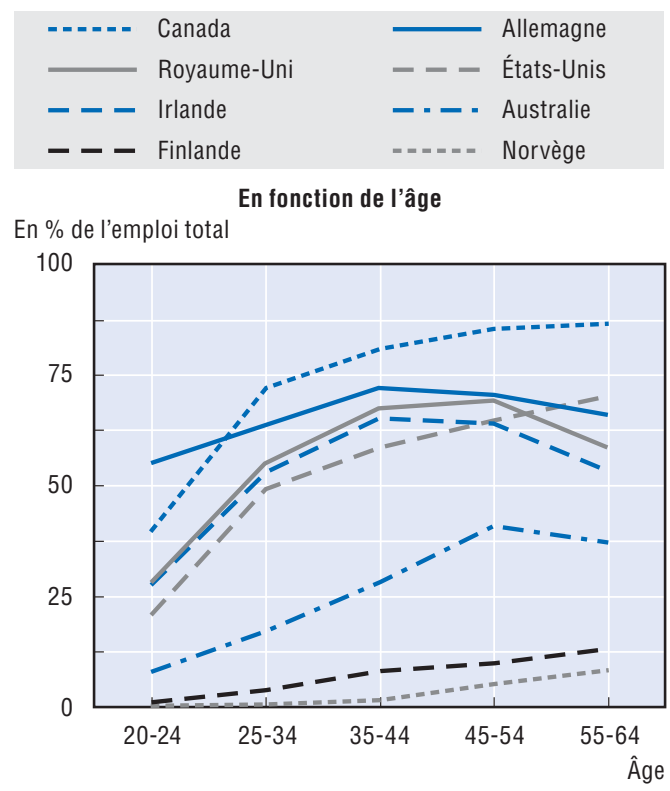

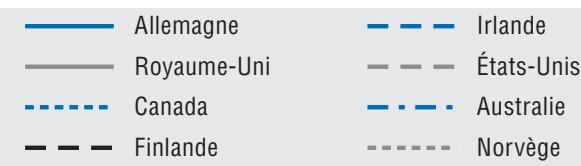

En fonction du salaire

En \% de l'emploi total

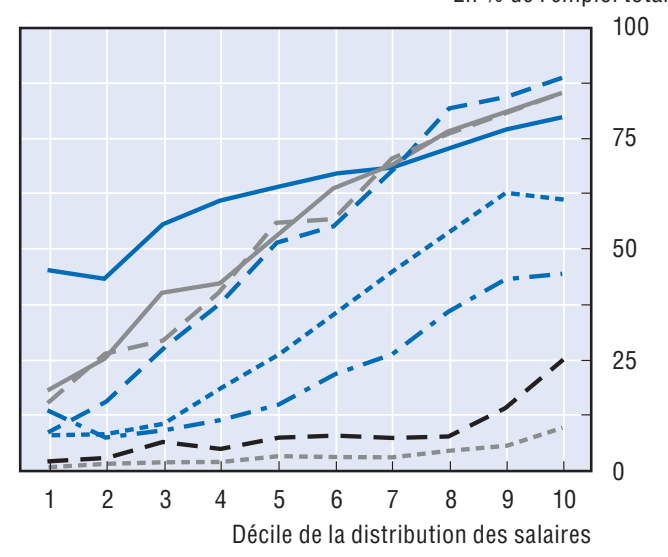

Source : Antolín, P. et E.R. Whitehouse (2009), « Filling the Pension Gap: Coverage and Value of Voluntary Retirement Savings ", Documents de travail sur les affaires sociales, l'emploi et les migrations, n 69, OCDE ; analyse des données nationales réalisée par l'OCDE (Finlande et Norvège).

Bien que les régimes privés facultatifs couvrent, dans l'ensemble, nettement plus de la moitié des personnes actives occupées dans bon nombre des pays étudiés, leur distribution est inégale. Il est beaucoup plus rare de voir les jeunes et les personnes à faible revenu adhérer à un régime facultatif. L'analyse des déficits d'épargne-retraite figurant dans la tableau $1 \mathrm{du}$ présent chapitre a montré qu'en Australie, au Canada et en Irlande, les bas revenus n'ont pas besoin de se constituer une épargne-retraite privée facultative pour atteindre le taux de remplacement moyen des pays de l'OCDE, ou alors uniquement dans des proportions minimes. Le faible taux de couverture des personnes à bas salaire y est donc moins préoccupant qu'aux États-Unis et au Royaume-Uni, où il leur faudrait épargner de $4 \%$ à $5 \%$ de leur salaire durant toute leur vie active pour atteindre le taux de remplacement de référence. Pour les faibles revenus, le déficit d'épargne-retraite est aussi relativement important en Allemagne, mais la couverture des régimes de retraite privés est également élevée dans cette catégorie de la population. Ces résultats donnent à penser 
qu'une partie, mais pas la totalité des pays de l'OCDE doivent s'attacher en priorité à étendre la couverture des faibles revenus.

L'évolution de la couverture en fonction de l'âge laisse supposer que la plupart de ceux qui finissent par souscrire à un régime privé ne commencent à cotiser qu'à l'âge de 30 ans, voire plus tard. Ces années manquantes accroissent sensiblement les efforts requis des intéressés, en termes d'épargne, durant les années où ils cotisent à un dispositif privé. Ainsi, le fait de souscrire à un tel régime à 30 ans au lieu de 20 entraîne une majoration du taux de cotisation requis de près de $50 \%$ (voir le graphique 4.2 ci-dessus). Il en ressort que les responsables de l'action publique doivent s'intéresser en tout premier lieu aux salariés les plus jeunes, en les incitant à commencer de cotiser à un régime privé plus précocement.

\section{Les cotisations aux régimes de retraite privés}

On dispose de données sur les cotisations facultatives aux régimes privés pour quelques-uns seulement des pays identifiés comme souffrant d'un déficit d'épargne-retraite dans la section 1 de ce chapitre spécial. Le tableau 4.1 montre que les taux de cotisation sont pratiquement identiques (Allemagne, Belgique) ou supérieurs au taux de cotisation requis pour combler le déficit d'épargne-retraite, sous réserve d'une carrière complète. Toutefois, si l'on tient compte de plusieurs années de cotisation manquantes, le nombre de pays où le taux de cotisation moyen est insuffisant (Allemagne, Belgique, République tchèque) est plus élevé. Au Royaume-Uni, le taux moyen est proche du taux de cotisation requis pour 10 années manquantes. Outre les salariés non couverts, toutefois, nombreux sont ceux qui cotisent moins que la moyenne. En Irlande, par exemple, le taux de cotisation moyen à des dispositifs à cotisations définies s'élève à $10 \%$, et se répartit à parts égales entre employeurs et salariés. Néanmoins, le taux de cotisation des salariés est inférieur à $5 \%$ dans $30 \%$ des dispositifs et c'est le cas du taux de cotisation des employeurs dans $18 \%$ d'entre eux.

\section{Tableau 4.1. Taux de cotisation total à des régimes de retraite à cotisations définies facultatifs}

En pourcentage du salaire

\begin{tabular}{lc}
\hline & Taux de cotisation moyen \\
\hline Allemagne & 4.0 \\
Belgique & 4.3 \\
États-Unis & 9.0 \\
Irlande & 10.0 \\
République tchèque & 2.5 \\
Royaume-Uni & 8.8 \\
\hline
\end{tabular}

Note : Pour le Royaume-Uni, les données se rapportent à des régimes professionnels à cotisations définies et ne comprennent pas les personnes possédant des plans de retraite individuels. Les chiffres ont été arrondis.

Source: Antolín, P. et E.R. Whitehouse (2009), « Filling the Pension Gap: Coverage and Value of Voluntary Retirement Savings ", Documents de travail sur les affaires sociales, l'emploi et les migrations, $n^{\circ} 69$, OCDE.

\section{Les mesures de nature à favoriser l'épargne-retraite privée}

Les salariés qui entrent sur le marché du travail aujourd'hui percevront une retraite publique nettement inférieure à celle de leurs parents et grands-parents. Autrement dit, ils doivent se prémunir en souscrivant à un régime privé facultatif pour conserver leur niveau de vie une fois à la retraite. En effet, un grand nombre des réformes des régimes publics ont été fondées sur l'hypothèse d'une progression de l'épargne-privée facultative. Dans certains 
pays comme le Canada, les États-Unis, le Japon et le Royaume-Uni, c'est le cas depuis longtemps. C'est toutefois un phénomène nouveau dans d'autres comme l'Allemagne et la France. De plus, la nécessité de constituer une épargne-retraite s'étend désormais à d'autres catégories de la population, comme les personnes à faible revenu qui n'ont guère pour habitude de prendre eux-mêmes des décisions dans ce domaine.

Certaines données laissent à penser que la couverture des régimes d'épargne-retraite est satisfaisante, de même que les cotisations qui y sont versées. D'autres indiquent que des déficits considérables pourraient exister à cet égard. Faute de preuves suffisantes, les responsables publics doivent donc éviter de relâcher leur vigilance. Heureusement, les gouvernements se sont activement employés à élaborer des mesures de nature à favoriser l'épargne-retraite privée.

\section{L'obligation d'affiliation}

Rendre obligatoire l'adhésion à un régime privé permet d'obtenir aisément un taux de couverture élevé et une distribution uniforme de la couverture en fonction de l'âge et du salaire. Dans des pays comme l'Australie, l'Islande, la Norvège et la Suisse, les dispositifs de retraite privés facultatifs bénéficient traditionnellement d'une vaste couverture (soit $50 \%$ des salariés au moins). Les gouvernements ont simplement imposé aux employeurs de mettre en place et de cotiser à des régimes privés pour le compte de leurs salariés. De manière générale, les prestations servies par les dispositifs obligatoires étaient toutefois inférieures à celles habituellement assurées par les régimes privés lorsqu'ils étaient encore facultatifs. Une deuxième mesure a consisté à rendre les cotisations privées obligatoires en remplacement de la retraite publique. La Hongrie, le Mexique, le Pologne, la République slovaque et la Suède ont tous emprunté cette voie.

D'autres pays tels le Danemark, la Suède et les Pays-Bas n'ont pas imposé directement l'affiliation à un régime privé, mais en vertu des contrats de travail, cette adhésion est de fait obligatoire (" quasi obligatoire ») et la couverture excède $85 \%$ des salariés. En Allemagne et en Belgique, la couverture des dispositifs facultatifs a également augmenté ces dernières années du fait de l'établissement de régimes de retraite sectoriels. Ce modèle est néanmoins difficilement exportable vers d'autres pays de l'OCDE, où la structure du marché du travail et les relations professionnelles se prêtent moins à une couverture quasi universelle des dispositifs de retraite privés.

Le principal argument en faveur du caractère obligatoire de ces régimes est qu'il empêche les intéressés de regretter de ne pas avoir suffisamment épargné pour leur retraite lorsqu'ils étaient plus jeunes. Il protège également la société en la préservant de devoir financer des prestations sociales au profit de ceux qui ont négligé de se prémunir. Mettre en œuvre cette approche paternaliste est simple : il suffit de choisir un objectif de taux de remplacement (variant ou non en fonction du salaire) puis de veiller à ce que les retraités atteignent cet objectif grâce aux prestations servies par le régime public ou à leur dispositif de retraite privé obligatoire.

La nécessité d'imposer ou non l'affiliation à un régime est une question importante, malheureusement non résolue. Les salariés font-ils preuve d'un manque de prévoyance? Laissés à eux-mêmes, manqueront-ils d'épargner suffisamment pour leur retraite? L'analyse des revenus des retraités présentée dans le précédent chapitre spécial sur "Les revenus et la pauvreté des personnes âgées » donne à penser qu'en tenant compte de la taille des ménages, ils représentent en règle générale de $75 \%$ à $85 \%$ du revenu moyen de 
la population dans son ensemble. Il n'existe cependant aucun lien entre les revenus relatifs et le type de système de retraite. Les régimes privés facultatifs jouent un rôle important au Canada et aux États-Unis, où le revenu des personnes âgées, en termes relatifs, est supérieur à la moyenne des pays de l'OCDE. C'est également le cas en Irlande, où le revenu est le plus bas, et au Royaume-Uni. L'OCDE (2001) a qualifié ce phénomène de " convergence de résultats, diversité des moyens ». Ces données infirment pour une part l'hypothèse d'un manque de prévoyance.

Plusieurs arguments plaident en défaveur de l'obligation d'affiliation à un régime privé.

- Premièrement, même si les individus ne sont pas suffisamment prévoyants, accentuer le caractère obligatoire des pensions n'est pas toujours indiqué. Si l'on rend l'épargneretraite obligatoire, il convient de fixer un objectif de taux de remplacement, exercice difficile qui ne donne pas droit à l'erreur. La perte de bien-être individuel enregistrée lorsqu'on contraint les salariés à épargner de façon excessive peut être équivalente à celle qui découle d'un manque de prévoyance ou d'une épargne insuffisante. Ainsi, compte tenu des ressources mobilisées par l'épargne-retraite, les sommes consacrées à l'éducation des enfants pourraient s'avérer trop maigres.

- Deuxièmement, les plans de retraite formels ne sont pas les seuls instruments dont les salariés disposent et se servent pour épargner en vue de leur retraite. Certains peuvent souhaiter investir dans la pierre ou dans leur propre entreprise. Or, ces comportements tout à fait rationnels peuvent être prohibés par l'obligation de constituer une épargneretraite conséquente dans le cadre de régimes de retraite formels.

- Troisièmement, les cotisations retraite à caractère obligatoire sont souvent perçues comme un impôt, ce qui risque de dissuader les actifs de travailler.

- Enfin, les prestataires de dispositifs de retraite facultatifs - en particulier les régimes professionnels - se sont souvent élevés contre cette obligation d'affiliation par crainte qu'elle ne vide de leurs adhérents les plans existants. Il y a également un risque que les prestations servies diminuent si l'objectif fixé par le gouvernement est inférieur aux normes en vigueur.

\section{L'obligation partielle d'affiliation}

L'obligation présente des inconvénients tandis que les systèmes purement facultatifs sont assortis d'un risque d'épargne insuffisante. En cas d'affiliation automatique à un dispositif privé, les personnes concernées doivent choisir de sortir du régime plutôt que d'y adhérer. L'objectif est d'accroître la participation tout en préservant le libre arbitre. De nombreuses personnes indiquent dans des enquêtes qu'il est important d'épargner en vue de leur retraite et avoir le sentiment qu'elles devraient la préparer (OCDE, 2005). Malheureusement, il est rare qu'elles passent des paroles aux actes en raison, naturellement, de la longueur et de la complexité manifestes du processus de souscription à un dispositif de retraite, et faute d'informations et de connaissances sur les différentes formules d'épargne-retraite. L'affiliation automatique sert à amener les temporisateurs à épargner pour leurs vieux jours.

Aux États-Unis et au Royaume-Uni, plusieurs employeurs proposant des régimes de retraite recourent de longue date à l'affiliation automatique pour accroître le nombre de salariés couverts. Aux États-Unis, on a observé récemment une progression rapide de l'affiliation automatique aux régimes professionnels à cotisations définies (connus sous le 
nom de plans 401(k) d'après la clause du Code fiscal s'y rapportant), qui a atteint $16.9 \%$ en 2005 contre $8.4 \%$ en 2003. Les dispositifs les plus étendus ont tout particulièrement contribué à ce phénomène. En 2002, ils étaient près de $17 \%$ à pratiquer l'affiliation automatique et pas moins de $41.3 \%$ en 2006. Cette progression devrait se poursuivre eu égard aux modifications législatives qui ont supprimé les obstacles à l'affiliation automatique.

Une analyse de la situation au Royaume-Uni fait apparaître quatre procédures d'affiliation. Quelque 44 \% des employeurs utilisent une procédure d'« adhésion simplifiée ", consistant tout simplement à signer un formulaire prérempli (McKay, 2006). Seuls $19 \%$ des salariés étaient couverts par un régime à affiliation intégralement automatique, c'est-à-dire nécessitant de choisir expressément de ne pas être affilié. Ainsi qu'aux États-Unis, ces deux procédures étaient plus répandues parmi les gros employeurs. L'adhésion traditionnelle concernait $19 \%$ des dispositifs, pondérés par le nombre d'adhérents.

L'affiliation automatique est aujourd'hui mise en place à l'échelle nationale. La Nouvelle-Zélande l'a déjà adoptée et le Royaume-Uni s'apprête le faire. L'introduction de tels programmes a déjà fait l'objet d'un débat animé en Allemagne, aux États-Unis et en Irlande.

En ce qui concerne l'affiliation automatique, la question cruciale est savoir si elle est efficace. En dépit d'un enthousiasme croissant, on ne dispose guère de données sur ses effets. L'un des ouvrages les plus largement cités - Madrian et Shea (2001) - analyse l'expérience d'un employeur américain. Avant l'affiliation automatique, seuls $57 \%$ des personnes travaillant dans cette entreprise depuis moins de trois ans avaient adhéré au régime professionnel, contre $80 \%$ au moins pour les salariés ayant au moins 10 ans d'ancienneté. Durant les 18 mois suivant l'introduction de l'affiliation automatique, le taux de couverture est grimpé à 86 \%. De même, Beshears et al. (2006) mettent en évidence une hausse de 35 points de la couverture des personnes employées depuis trois mois dans une autre entreprise, contre 25 points seulement pour celles qui ont deux années d'ancienneté. Ces résultats donnent à penser que l'affiliation automatique hâte la décision des intéressés d'adhérer à un régime de retraite d'entreprise, mais que la couverture des salariés de longue date ne s'accroît guère.

Horack et Wood (2005) ont étudié, au Royaume-Uni, 11 régimes de retraite d'entreprise ayant modifié leurs modalités d'adhésion. Deux sociétés ayant introduit l'affiliation automatique ont élargi leur couverture, la portant de $25 \%$ à $58 \%$ et de $45 \%$ à $62 \%$ respectivement. Les deux autres entreprises affichaient déjà des taux de couverture extrêmement élevés, soit $86 \%$ et $88 \%$, très probablement parce que les salariés n'étaient pas tenus de cotiser. L'affiliation automatique a permis de porter la couverture à $92 \%$ et $100 \%$ respectivement. Une autre enquête, réalisée pour le Department of Work and Pensions [ministère du Travail et des Retraites], fait état d'une couverture de $41 \%$ pour l'adhésion traditionnelle et de $60 \%$ pour l'affiliation automatique (McKay, 2006). Ces chiffres concernent des employeurs plus importants (plus de 20 salariés). Parmi les plus petites entreprises, la couverture était pratiquement la même dans les deux cas (67\%).

En Nouvelle-Zélande, le plan KiwiSaver, introduit en juillet 2007, marque la première mise en place de l'affiliation automatique à l'échelle nationale (voir Rashbrooke, 2009). Les employeurs doivent y affilier les nouveaux salariés et les particuliers ont deux mois pour en sortir. À ce jour, la proportion des salariés ayant choisi de ne pas y adhérer s'établissait 
en moyenne à un tiers. Il n'est pas surprenant de constater que les personnes optant pour la sortie du régime sont davantage les jeunes (37\% des 25-34 ans, par exemple) que les plus âgés ( $25 \%$ des 55 ans et plus). Cela étant, l'analyse de la mesure prise par la NouvelleZélande est rendue complexe par les incitations généreuses à adhérer au plan KiwiSaver Ainsi, $47 \%$ des adhérents sont passés par un prestataire de services financiers et $17 \%$ par leur employeur. On peut donc affirmer avec certitude que $36 \%$ seulement des détenteurs de plans KiwiSaver y ont adhéré du fait de l'affiliation automatique.

Ces études laissent à penser, dans l'ensemble, que l'affiliation automatique aurait un impact non négligeable sur la couverture des dispositifs de retraite privés. Néanmoins, il est toujours préférable de ne pas généraliser à partir d'un nombre restreint d'études de cas. Qui plus est, de nombreux éléments portent à croire que les expériences acquises en la matière par les régimes nationaux seront différentes de celles des dispositifs proposés par les employeurs. Il convient à l'évidence de réunir de nouvelles données avant de pouvoir affirmer que l'obligation partielle permet d'étendre ou non la couverture des régimes de retraite privés.

- Premièrement, si l'affiliation automatique ne fait qu'atténuer la procrastination, en précipitant la constitution d'une épargne-retraite, les répercussions sur les retraites futures seront limitées.

- Deuxièmement, l'évaluation de la pérennité de la couverture des retraites doit se fonder sur des données à plus long terme. Ainsi, les salariés peuvent, au fil du temps, vaincre leur inertie et faire le choix inverse en se rendant compte qu'en sortant du régime, ils peuvent accroître rapidement leur revenu.

- Troisièmement, il faut s'intéresser à la façon dont les individus financent les cotisations aux régimes d'épargne-retraite à affiliation automatique (voir l'analyse des incitations fiscales ci-dessous).

- Quatrièmement, enfin, les dispositifs à affiliation automatique étaient également assortis d'aides substantielles à l'épargne individuelle. L'abondement de l'État néozélandais aux comptes KiwiSaver en est le plus bel exemple. Aux États-Unis et au Royaume-Uni, toutefois, le montant des cotisations patronales aux régimes professionnels était extrêmement divers. Il faut donc isoler avec soin une retombée "parfaite " de l'affiliation automatique sur la couverture, indépendamment de l'influence des allégements fiscaux, des cotisations patronales et autres aides.

Les arguments jouant en défaveur de l'obligation partielle sont de deux ordres : ceux qui vont dans le sens d'une obligation « rigoureuse » et ceux qui sont à l'appui d'une approche purement facultative. La validité du premier type d'arguments repose sur l'incapacité de l'affiliation automatique d'étendre sensiblement la couverture. Les arguments en faveur d'une approche purement facultative rappellent ceux qui militent à l'encontre de l'obligation d'affiliation, à savoir que les régimes à affiliation automatique vont faire disparaître les dispositifs existants et entraîner un nivellement par le bas des revenus des retraités. Par exemple, les études de cas menées sur l'affiliation automatique aux États-Unis ont montré que le taux de cotisation par défaut constitue un bon indicateur des adhérents et que l'affiliation automatique fait baisser le taux de cotisation moyen (voir, par exemple, Beshears et al., 2006 ; et Madrian et Shea ; 2001). Les employeurs sont en effet nombreux à fixer délibérément les taux de cotisation par défaut à un faible niveau pour minimiser les risques de sortie. 
Quoi qu'il est soit, il est probable que l'on recourra davantage à l'affiliation automatique afin d'élargir la couverture des régimes de retraite privés. Les enquêtes menées laissent à penser qu'au Royaume-Uni, l'affiliation automatique recueille auprès des particuliers une adhésion plus large que l'obligation d'affiliation (Bunt et al., 2006 ; Hall et al., 2006). Et le point de vue des électeurs est partagé par de nombreux hommes politiques, qui craignent que les salariés ne considèrent les cotisations obligatoires aux régimes privés comme une ponction intempestive sur leur revenu.

\section{L'éducation financière}

L'éducation financière peut également permettre de sensibiliser les salariés à la nécessité de l'épargne-retraite et, il faut l'espérer, d'accroître la couverture des régimes par capitalisation facultatifs. Il est établi, par exemple, que les campagnes d'information menées par les employeurs ont entraîné une augmentation de la participation et des cotisations aux régimes de retraite privés (OCDE, 2005). Des études menées aux États-Unis ont ainsi montré que les salariés dotés d'une meilleure éducation financière sur les plans 401(k) semblent davantage devoir adhérer à ce plan (ou risquent moins de sortir d'un régime à affiliation automatique). Les relevés des droits à retraite individuels - qui viennent d'être introduits ou améliorés en Allemagne, en France, au Royaume-Uni et en Suède, notamment - peuvent aider les salariés à mieux planifier leur retraite et à faire des choix éclairés en matière d'épargne-retraite privée facultative.

\section{Un accès simplifié aux régimes de retraite privés}

Il est possible d'accroître la participation aux dispositifs de retraite privés en en facilitant l'accès. Les régimes de retraite professionnels sont essentiellement représentés parmi les salariés des grands groupes. Pratiquement $50 \%$ des employés d'entreprises de moins de 25 personnes ont accès à un régime de retraite professionnelle au Royaume-Uni, contre plus de $95 \%$ dans les sociétés de plus de 1000 salariés (Office of National Statistics [Office national de statistique], 2009, tableau 6.10). Aux États-Unis, deux tiers environ des entreprises de plus de 500 personnes proposent un régime de retraite, contre $28 \%$ dans les structures moins importantes comptant moins de 25 salariés. La situation est équivalente dans d'autres pays. Les personnes qui travaillent dans des sociétés de petite taille perçoivent un salaire relativement bas, de sorte que les personnes à faible revenu ont moins de chances de bénéficier d'un régime de retraite professionnel. Aux États-Unis, par exemple, 30 \% environ des salariés touchant moins de 20000 USD sont employés par une entreprise dotée d'un régime de retraite, contre près de $70 \%$ pour ceux dont le salaire dépasse 50000 USD (Copeland, 2007).

On évoque souvent, afin d'expliquer pourquoi les petites entreprises ont moins tendance à mettre en place des dispositifs de retraite professionnels, les coûts fixes de gestion d'un tel régime, attribuables en partie aux difficultés de se conformer à la réglementation. Les États-Unis ont fait face à ce problème en autorisant les petits employeurs à établir des plans de retraite soumis à des contraintes administratives moins strictes que ceux des grandes entreprises. Aux États-Unis, par exemple, les employeurs peuvent créer un régime simplifié d'épargne-retraite (Simplified Employee Pension ou SEP), qui regroupe de fait un ensemble de comptes de retraite individuels gérés par une institution financière agissant en qualité d'administrateur fiduciaire. 


\section{Les incitations fiscales}

Pour promouvoir l'épargne-retraite facultative privée, il est fréquent de voir les pouvoirs publics soumettre à un régime fiscal préférentiel les cotisations aux dispositifs de retraite et les rendements des investissements réalisés dans ce cadre. Ils estiment en effet qu'un taux de rendement net plus élevé encouragera les salariés à épargner davantage. En règle générale, ces incitations fiscales sont assorties de conditions, habituellement applicables pendant la durée de l'épargne, et de restrictions quant aux modalités de versement des prestations. Ce sont ces conditions qui permettent de parler d'« épargneretraite » (voir Engen et al., 1994 et 1996, par exemple).

La principale question qui se pose aux responsables est de savoir si ces incitations fiscales permettent d'accroître le volume de l'épargne spécifiquement constituée en vue de la retraite. L'OCDE a évalué les incitations à constituer une épargne dans le cadre d'un régime de retraite en comparant le taux effectif d'imposition sur les pensions à celui qui s'applique à l'« épargne de référence "; il s'agit le plus souvent d'un dépôt en banque (voir Yoo et de Serres, 2004). L'ampleur des incitations fiscales est calculée en pourcentage des cotisations, mais en tenant compte du manque à gagner fiscal dû aux cotisations déductibles et aux rendements d'investissement exonérés d'impôt, ainsi que des recettes collectées lors du versement des prestations. Il convient de noter que l'étude est fondée sur les règles et paramètres fiscaux de 2003 : dans bien des pays, le régime fiscal des retraites a sensiblement évolué depuis.

Les résultats donnent à penser qu'il existe de fait une incitation à épargner dans le cadre d'un dispositif de retraite (graphique 4.7). Cette incitation varie considérablement, allant de près de zéro au Mexique et en Nouvelle-Zélande à plus de $40 \%$ des cotisations en République tchèque. Hormis ces deux pays, les incitations représentent $10 \%$ au moins des cotisations dans la majorité des économies et la moyenne des pays de l'OCDE est supérieure à $20 \%$.

Selon certaines enquêtes, l'incitation fiscale à souscrire à un régime de retraite privé accroît le montant de l'épargne-retraite constituée (voir Engen et al., 1994 et 1996, par

\section{Graphique 4.7. Incitation fiscale en faveur des régimes de retraite privés, par rapport à l'épargne-retraite de référence \\ Paramètres et règles de 2003}

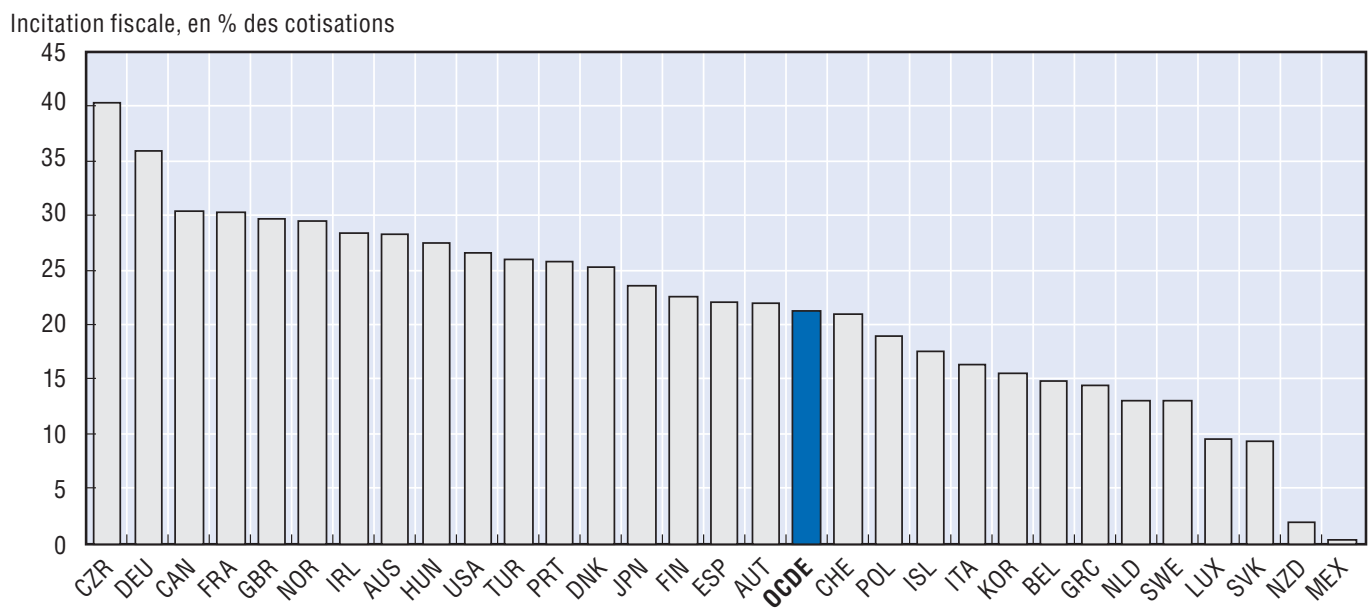

Source: Yoo, K.Y. et A. de Serres (2004), « Le régime fiscal de l'épargne-retraite privée dans les pays de l'OCDE », Revues économiques de l’OCDE, vol. 39, n², pp. 81-120. 
exemple). Cette progression pourrait néanmoins être le fait de personnes qui, en réalité, épargnent davantage de façon globale (épargne nouvelle), ou qui transfèrent l'épargne accumulée sur d'autres supports (réallocation) sans variation de leur épargne totale. Malheureusement, les données empiriques recueillies pour tenter de savoir si l'épargne constituée dans le cadre de régimes de retraite présentant des avantages fiscaux est nouvelle ou issue d'une réallocation ne sont pas concluantes et reposent en grande partie sur le cas des États-Unis.

Si l'épargne nouvellement constituée prédomine, l'épargne nationale (tenant compte de la réduction de l'épargne publique due à l'incitation fiscale) a de fortes chances d'augmenter. En revanche, si la réallocation est plus importante, l'épargne nationale devrait s'inscrire en repli. Cette substitution de l'épargne privée à l'épargne publique n'a guère de répercussions sur le plan macroéconomique. Elle peut néanmoins servir l'action publique en bloquant l'épargne individuelle dans des dispositifs à long terme exclusivement destinés à financer les retraites.

La conception des incitations fiscales a aussi son importance. En permettant simplement aux particuliers de déduire leurs cotisations aux régimes de retraite privés des sommes dues au titre de l'impôt sur le revenu, les autorités avantagent tout particulièrement les hauts revenus, qui sont soumis à des taux marginaux plus élevés. À l'inverse, les bas salaires, qui n'acquittent pas d'impôt sur le revenu ou sont soumis à un taux d'imposition minime, bénéficient d'une moindre incitation fiscale à épargner en vue de la retraite. De plus, leurs droits à retraite étant relativement faibles, leurs prestations de retraite peuvent être soumises à conditions de ressources. Cela représente de fait une "ponction " supplémentaire sur l'épargne-retraite, comme on l'a vu précédemment. Néanmoins, il est possible de concevoir des incitations fiscales bénéficiant également aux faibles revenus ou axées sur les bas salaires. Ainsi, les allégements fiscaux sur les cotisations pourraient se limiter au taux standard ou réduit de l'impôt sur le revenu. L'État peut aussi verser un abondement ou propo ser un crédit d'impôt aux personnes qui ne sont pas assujetties à l'impôt sur le revenu.

\section{Notes}

1. Voir OCDE (2007, section II.1), Martin et Whitehouse (2008), ainsi que le chapitre spécial sur « Les réformes récentes des retraites » de la présente publication.

2. Ce chapitre spécial est inspiré de l'analyse plus approfondie exposée dans Antolín et Whitehouse (2009). Comme dans le reste de la publication, les calculs figurant dans la section 1 du présent chapitre sont une mise à jour des modélisations des paramètres et des règles des systèmes de retraite de 2004 à 2006. Queisser et al. (2008) analysent également le rééquilibrage des retraites publiques et privées.

3. Une analyse des calculs détaillée, étape par étape, figure dans OCDE (2007), pp. 88-90.

4. La couverture d'ensemble des régimes de retraite privés est présentée dans la partie II de cette publication, qui est consacrée aux indicateurs.

\section{Références}

Antolin, P. et E.R. Whitehouse (2009), « Filling the Pension Gap: Coverage and Value of Voluntary Retirement Savings ", Documents de travail sur les affaires sociales, l'emploi et les migrations, $\mathrm{n}^{\circ} 69$, Éditions de l'OCDE, Paris. 
Beshears, J., J.J. Choi, D. Laibson et B.C. Madrian (2006), "The Importance of Default Options for Retirement Saving Outcomes: Evidence from the United States ", Document de travail n 12009 , National Bureau of Economic Research, Cambridge, Massachusetts.

Bunt, K., L. Adams, Z. Koroglu et E. O’Donnell (2006), Pensions and Pension Reform, Rapport $n^{\circ} 357$, Department for Work and Pensions, Londres.

Copeland, C. (2007), «Employment-Based Retirement Plan Participation: Geographic Differences and Trends », Issue Brief n³11, Employee Benefits Research Institute, Washington, DC.

Engen, E.M., W.G. Gale et J.K. Scholz (1994), « Do Saving Incentives Work? », Brookings Papers on Economic Activity, $\mathrm{n}^{\circ} 1$, pp. 85-151.

Engen, E.M., W.G. Gale et J.K. Scholz (1996), « The Illusory Effect of Saving Incentives on Saving », Journal of Economic Perspectives, vol. 10, n 4, pp. 113-138.

Hall, S., N. Pettigrew et P. Harvey (2006), « Public Attitudes to Personal Accounts: Report of a Qualitative Study », Rapport n³70, Department for Work and Pensions, Londres.

Horack, S. et A. Wood (2005), An Evaluation of Scheme Joining Techniques in Workplace Pension Schemes with an Employer Contribution, Rapport $n^{\circ}$ 292, Department for Work and Pensions, Londres.

Madrian, B.C. et D. Shea (2001), " The Power of Suggestion: Inertia in 401(k) Participation and Savings Behavior ", Quarterly Journal of Economics, vol. 116, n 4, pp. 1149-1525.

Martin, J.P. et E.R. Whitehouse (2008), « Reforming Retirement-Income Systems: Lessons from the Recent Experiences of OECD Countries ", Documents de travail sur les affaires sociales, l'emploi et les migrations, $n^{\circ} 66$, Éditions de l'OCDE, Paris.

McKay, S. (2006), Employers’ Pension Provision Survey 2005, Rapport n³29, Department for Work and Pensions, Londres.

OCDE (2001), Vieillissement et revenus - Les ressources des retraités dans 9 pays de l'OCDE, Éditions de l'OCDE, Paris.

OCDE (2005), Pour une meilleure éducation financière : enjeux et initiatives, Éditions de l'OCDE, Paris.

OCDE (2007), Les pensions dans les pays de l'OCDE - Panorama des politiques publiques, Éditions de l'OCDE, Paris.

Office of National Statistics (2009), General Household Survey 2007, Londres.

Queisser, M., P. Whiteford et E.R. Whitehouse (2007), « The Public-Private Pension Mix in OECD Countries ", Industrial Relations Journal, vol. 38, n 6, pp. 542-568.

Rashbrooke, G. (2009), "Automatic Enrolment: KiwiSaver in New Zealand », The Changing Pensions Landscape in Asia and the Pacific, OCDE, Paris, à paraître.

Yoo, K.Y. et A. de Serres (2004), "Le régime fiscal de l'épargne-retraite privée dans les pays de l'OCDE ", Reuue économique de l'OCDE, vol. 39, n², pp. 81-120. 


\section{PARTIE II}

\section{Les indicateurs des politiques des retraites}

La partie II contient une version actualisée des grands indicateurs des systèmes de retraite élaborés pour les première et deuxième éditions des Pensions dans les pays de l'OCDE. Elle propose aussi une gamme élargie d'indicateurs. Ces informations - présentées de façon claire et succincte - constituent un cadre de référence complet et cohérent permettant de comparer et d'évaluer les systèmes de retraite et les politiques adoptées dans ce domaine.

Les 17 indicateurs sont répartis en trois catégories. La première regroupe les indicateurs relatifs aux droits à retraite individuels octroyés par les régimes de retraite des 30 pays de l'OCDE. Elle comprend, outre les traditionnels taux de remplacement, des indicateurs du patrimoine retraite, de la progressivité des systèmes de retraite et du point d'équilibre trouvé entre les dispositifs publics et privés.

Le deuxième groupe porte sur les systèmes de retraite dans leur ensemble. Il contient des données sur les taux de cotisation aux régimes publics, les actifs des fonds de pension et des fonds de réserve nationaux, la couverture des régimes de retraite privés et les dépenses au titre des prestations de retraite.

La troisième et dernière catégorie d'indicateurs a trait au contexte dans lequel s'inscrivent nécessairement les systèmes de retraite. Elle regroupe des indicateurs démographiques essentiels - comme l'espérance de vie et la fécondité - et le salaire moyen. 



\section{Les droits à retraite}

Les calculs des droits à retraite, effectués à l'aide des modèles de retraite de l'OCDE, sont fondés sur les règles et les paramètres nationaux en vigueur en 2006. Ils se réfèrent à un salarié entré cette année-là sur le marché du travail.

Les trois premiers indicateurs concernent le traditionnel taux de remplacement, qui se définit comme le rapport des prestations de retraite au salaire individuel. Le premier de ces indicateurs porte sur le taux de remplacement brut (avant impôt) de tous les régimes obligatoires, y compris les dispositifs privés obligatoires. Le deuxième fait ressortir les régimes de retraite publics et les mécanismes privés, et comprend des données sur les dispositifs privés facultatifs pour les pays où leur couverture est étendue. Le troisième indicateur du taux de remplacement est exprimé en termes nets, en prenant en considération les impôts et cotisations acquittés sur les salaires et les pensions de retraite.

Viennent ensuite deux indicateurs $d u$ " patrimoine retraite ", qui désigne la valeur sur la durée de vie du flux de prestations de retraite. Ce sont des indicateurs plus complets que le taux de remplacement, car ils tiennent compte de l'âge de la retraite, de l'indexation des pensions sur les salaires ou les prix et de l'espérance de vie.

Les points d'équilibre trouvés entre deux objectifs - assurer un revenu satisfaisant aux retraités et remplacer une part donnée des revenus perçus avant le départ en retraite - sont évalués dans les deux indicateurs suivants, qui présentent de façon synthétique la progressivité de la formule de calcul des prestations de retraite et le lien existant entre les pensions et les salaires.

Les deux derniers indicateurs des droits à retraite résument les conséquences du système de retraite pour les individus en fonction de différents niveaux de salaire, en mettant en évidence le niveau moyen des pensions, le patrimoine retraite et la contribution de chaque composante du système de retraite. 


\section{Principaux résultats}

Le taux de remplacement brut correspond au montant de la pension perçue au titre de la retraite par rapport au salaire. Pour les personnes touchant un salaire moyen, le taux de remplacement brut s'établit en moyenne à 59 \% dans les 30 pays de l'OCDE. Néanmoins, il varie considérablement d'un pays à l'autre. Dans le bas du classement, l'Irlande, le Japon et le Royaume-Uni offrent aux nouveaux venus sur le marché du travail des taux de remplacement inférieurs à $35 \%$. L'Islande et la Grèce, situés dans le haut du classement, proposent des taux de remplacement supérieurs à $90 \%$. Les autres pays affichant un taux de remplacement attendu élevé (entre 70 \% et $90 \%$ ) sont l'Autriche, le Danemark, l'Espagne, la Hongrie, le Luxembourg et les Pays-Bas, alors que la Finlande, la Norvège et la Suisse assurent des taux de remplacement bruts proches de la moyenne des pays de l'OCDE.

La majorité des pays de l'OCDE protègent les personnes à faible revenu contre la pauvreté en leur proposant des taux de remplacement plus élevés que pour celles dont le salaire s'inscrit dans la moyenne. Le tableau montre ainsi que les personnes ne touchant que la moitié de la rémunération moyenne bénéficient d'un taux de remplacement de $72 \%$ en moyenne, contre $59 \%$ pour celles qui perçoivent le salaire moyen. Dans neuf pays, toutefois, le taux de remplacement est identique pour les revenus moyens et les rémunérations deux fois moins élevées que la moyenne; il s'agit de l'Allemagne, de l'Autriche, de l'Espagne, de la Grèce, de la Hongrie, de l'Italie, de la Pologne, de la République slovaque et de la Turquie. En haut du classement, trois pays assurent aux personnes à faible revenu une pension de retraite égale voire supérieure au salaire qu'elles percevaient lorsqu'elles étaient en activité : le Danemark (avec un taux de remplacement de $124.0 \%)$, l'Islande $(108.3 \%)$ et le Luxembourg (99.4\%). En bas du classement, l'Allemagne et le Japon proposent aux personnes à faible revenu des taux de remplacement de $43 \%$ et $47 \%$, respectivement. Certains pays, comme le Canada et la Nouvelle-Zélande, versent des prestations relativement peu élevées aux salaires moyens, mais s'inscrivent dans la moyenne pour les salariés à faible revenu.

Dans les 30 pays de l'OCDE, le taux de remplacement brut pour un revenu correspondant au salaire moyen multiplié par 1.5 (qualifié ici de « haut revenu ") ressort en moyenne à $54.3 \%$, soit légèrement en deçà des $59.0 \%$ proposés pour un salaire moyen. Pour les hauts revenus, on note une fois encore des variations considérables entre les pays. Le taux de remplacement est supérieur à $80 \%$ dans six pays : l'Espagne, la Grèce, l'Islande, le Luxembourg, les PaysBas et la Turquie. À l'autre extrémité, l'Irlande et la Nouvelle-Zélande (qui versent des retraites publiques forfaitaires), ainsi que le Royaume-Uni offrent des taux de remplacement inférieurs à $26 \%$.

Pour un salaire médian - niveau au-dessus duquel se situe la moitié des salariés et en deçà duquel se situe l'autre moitié - le taux de remplacement brut s'établit à $60.8 \%$ pour les pays de l'OCDE. En général, il ne diffère guère du taux de remplacement pour le salaire moyen (le salaire médian s'échelonne de $75 \%$ à $90 \%$ de la moyenne ; voir l'indicateur sur le "salaire moyen ").
Pour les femmes, les taux de remplacement bruts sont différents (l'âge d'ouverture des droits étant plus bas pour les femmes que pour les hommes) dans trois pays : l'Italie, la Pologne et la Suisse. On note de nettes différences entre les sexes en Italie et en Pologne, les taux de remplacement pour les femmes étant inférieurs d'un tiers environ à ceux des hommes. Au Mexique, les taux de remplacement offerts aux femmes sont également plus bas que pour les hommes, mais dans des proportions bien moindres qu'en Pologne et en Italie. En Suisse, enfin, les taux de remplacement sont légèrement supérieurs pour les femmes, car les régimes professionnels obligatoires leur confèrent des droits plus élevés à certains âges.

\section{Définition et calcul}

Le taux de remplacement indique dans quelle mesure un système de retraite assure un revenu aux retraités en remplacement de leur salaire, principale source de revenu avant leur départ en retraite. Le taux de remplacement est souvent exprimé sous la forme du ratio de la pension de retraite sur le salaire final (juste avant la retraite). Dans le cas présent, toutefois, les prestations de retraite sont exprimées en proportion du salaire individuel perçu en moyenne sur l'ensemble de la carrière (revalorisé en fonction de la hausse des salaires à l'échelle nationale). Selon les hypothèses de base, les salariés gagnent le même pourcentage du salaire moyen à l'échelle nationale durant toute leur vie active. En l'occurrence, le salaire revalorisé perçu en moyenne sur l'ensemble de la carrière et le salaire final individuel sont identiques. Si le salarié grimpe sur l'échelle de distribution des salaires avec l'âge, il percevra juste avant de prendre sa retraite un salaire supérieur à celui qu'il a touché en moyenne sur l'ensemble de sa carrière et le taux de remplacement calculé en fonction du salaire final individuel sera moins élevé.

Le taux de remplacement brut se définit comme les droits à retraite bruts divisés par le salaire brut avant le départ en retraite. Il est présenté ici pour le salaire médian et pour le salaire moyen multiplié par $0.5,0.75$, $1,1.5$ et 2, à l'aide du concept de "salarié moyen " nouvellement élaboré par l'OCDE (voir l'indicateur sur le « salaire moyen »). 


\section{Taux de remplacement bruts en fonction du salaire}

\begin{tabular}{|c|c|c|c|c|c|c|c|c|c|c|c|c|c|}
\hline & \multirow{2}{*}{$\begin{array}{l}\text { Salarié } \\
\text { à revenu } \\
\text { médian }\end{array}$} & \multicolumn{5}{|c|}{ Salaire individuel, en multiple de la moyenne } & & \multirow{2}{*}{$\begin{array}{c}\text { Salarié } \\
\text { à revenu } \\
\text { médian }\end{array}$} & \multicolumn{5}{|c|}{ Salaire individuel, en multiple de la moyenne } \\
\hline & & 0.5 & 0.75 & 1 & 1.5 & 2 & & & 0.5 & 0.75 & 1 & 1.5 & 2 \\
\hline Hommes & & & & & & & Hommes (suite) & & & & & & \\
\hline Australie & 45.7 & 67.0 & 50.0 & 41.6 & 33.1 & 28.9 & Nouvelle-Zélande & 45.6 & 77.5 & 51.6 & 38.7 & 25.8 & 19.4 \\
\hline Autriche & 80.1 & 80.1 & 80.1 & 80.1 & 76.4 & 57.3 & Norvège & 59.6 & 66.2 & 61.0 & 59.3 & 49.8 & 42.2 \\
\hline Belgique & 42.4 & 58.1 & 43.1 & 42.0 & 32.5 & 24.3 & Pologne & 61.2 & 61.2 & 61.2 & 61.2 & 61.2 & 61.2 \\
\hline Canada & 50.2 & 76.5 & 55.2 & 44.5 & 29.7 & 22.2 & Portugal & 54.1 & 63.0 & 54.3 & 53.9 & 53.1 & 52.4 \\
\hline Rép. tchèque & 54.9 & 79.2 & 59.6 & 49.7 & 36.4 & 29.0 & Rép. slovaque & 56.4 & 56.4 & 56.4 & 56.4 & 56.4 & 56.4 \\
\hline Danemark & 88.0 & 124.0 & 94.9 & 80.3 & 67.5 & 63.7 & Espagne & 81.2 & 81.2 & 81.2 & 81.2 & 81.2 & 66.7 \\
\hline Finlande & 56.2 & 66.5 & 56.2 & 56.2 & 56.2 & 56.2 & Suède & 61.5 & 76.6 & 64.6 & 61.5 & 75.6 & 81.3 \\
\hline France & 53.3 & 61.7 & 53.3 & 53.3 & 48.5 & 46.0 & Suisse & 62.0 & 62.5 & 62.1 & 58.3 & 40.5 & 30.4 \\
\hline Allemagne & 43.0 & 43.0 & 43.0 & 43.0 & 42.6 & 32.0 & Turquie & 86.9 & 86.9 & 86.9 & 86.9 & 86.9 & 86.9 \\
\hline Grèce & 95.7 & 95.7 & 95.7 & 95.7 & 95.7 & 95.7 & Royaume-Uni & 33.5 & 51.0 & 36.6 & 30.8 & 21.3 & 16.0 \\
\hline Hongrie & 76.9 & 76.9 & 76.9 & 76.9 & 76.9 & 76.9 & États-Unis & 40.8 & 50.3 & 42.6 & 38.7 & 34.1 & 28.8 \\
\hline Islande & 91.7 & 108.3 & 93.0 & 90.2 & 87.5 & 86.1 & & & & & & & \\
\hline Irlande & 39.8 & 68.4 & 45.6 & 34.2 & 22.8 & 17.1 & OCDE & 60.8 & 72.2 & 62.7 & 59.0 & 54.3 & 50.0 \\
\hline Italie & 67.9 & 67.9 & 67.9 & 67.9 & 67.9 & 67.9 & & & & & & & \\
\hline Japon & 35.7 & 47.1 & 38.3 & 33.9 & 29.4 & 26.6 & Femmes & & & & & & \\
\hline Corée & 45.1 & 64.1 & 49.4 & 42.1 & 33.6 & 25.2 & Italie & 52.8 & 52.8 & 52.8 & 52.8 & 52.8 & 52.8 \\
\hline Luxembourg & 90.1 & 99.4 & 91.9 & 88.1 & 84.3 & 82.5 & Mexique & 32.5 & 55.3 & 36.8 & 29.9 & 28.6 & 28.0 \\
\hline Mexique & 36.9 & 55.3 & 37.6 & 36.1 & 34.5 & 33.7 & Pologne & 44.5 & 49 & 44.5 & 44.5 & 44.5 & 44.5 \\
\hline Pays-Bas & 88.9 & 93.4 & 90.0 & 88.3 & 86.6 & 85.8 & Suisse & 62.6 & 62.8 & 62.6 & 59.0 & 41.0 & 30.7 \\
\hline
\end{tabular}

Note: Les chiffres sont mentionnés pour les femmes uniquement lorsqu'ils sont différents de ceux des hommes.

Source: Modèles de retraite de l'OCDE.

\section{Taux de remplacement bruts (TRB) en fonction du salaire}

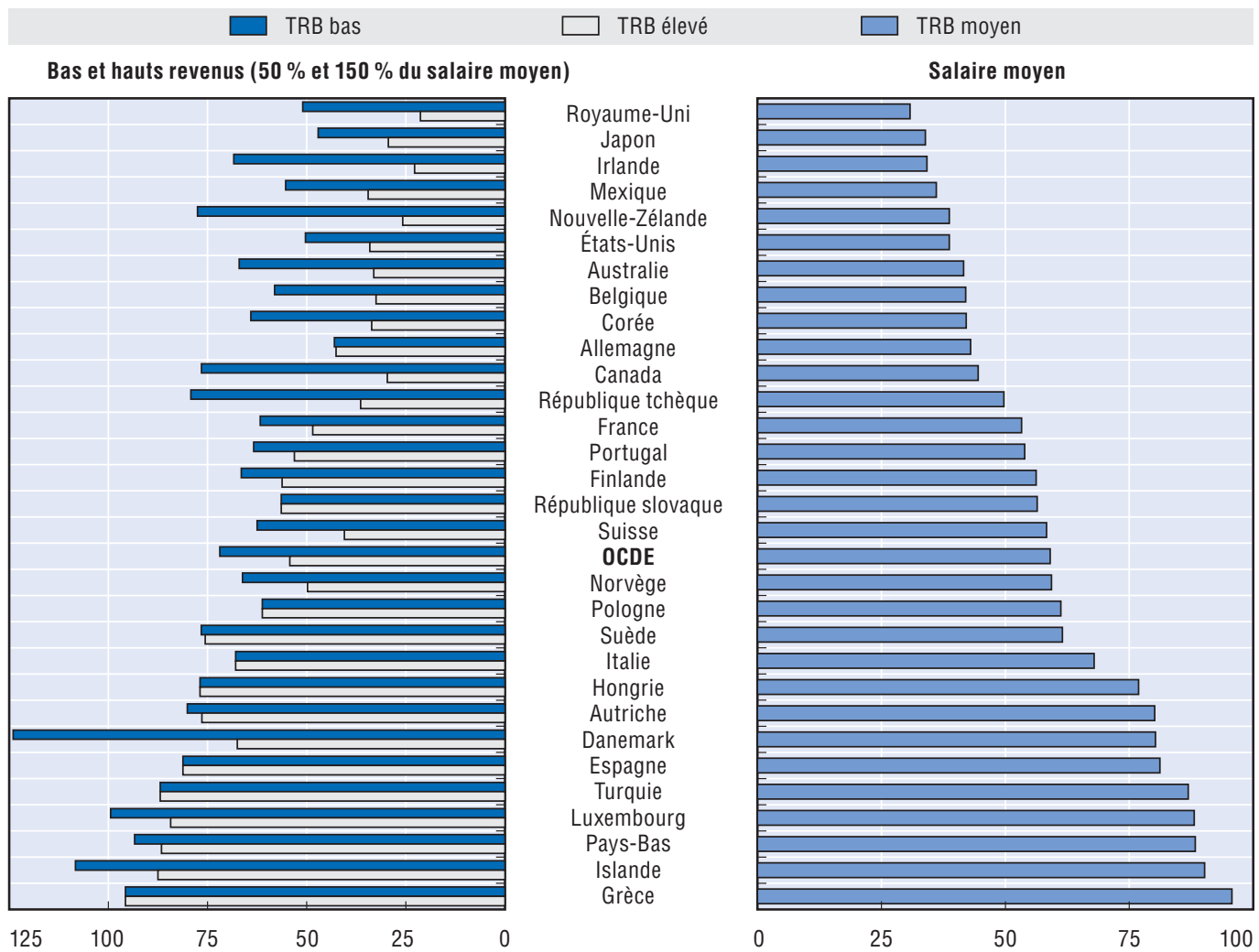

Note : Les pays sont classés d'après le taux de remplacement brut (TRB) pour le salaire moyen, soit le TRB moyen apparaissant dans le graphique.

Source: Modèles de retraite de l'OCDE. 


\section{Principaux résultats}

Les régimes privés assurent une part importante et croissante des revenus des personnes âgées, comme en témoignent les calculs des taux de remplacement bruts présentés séparément pour les dispositifs publics et les mécanismes privés. Dans les pays de l'OCDE, le taux de remplacement moyen assuré par les seuls régimes publics pour un salaire moyen s'établit à $46 \%$, contre $59 \%$ si l'ont tient également compte des dispositifs privés obligatoires. Lorsqu'on ajoute les mécanismes privés facultatifs, pour les règles types, le taux de remplacement moyen atteint $68 \%$ pour une personne touchant le salaire moyen.

Concernant les 11 pays pour lesquels les calculs portent uniquement sur les retraites publiques, le taux de remplacement ressort à $67 \%$ en moyenne pour le salaire moyen. S'agissant des 22 pays pour lesquels on dispose de données sur les régimes publics et les dispositifs privés obligatoires, le taux de remplacement moyen s'inscrit à $66 \%$. Pour les 30 pays de l'OCDE, en tenant compte des dispositifs publics, privés obligatoires et privés facultatifs, le taux de remplacement s'élève là encore en moyenne à $67 \%$.

Cela témoigne d'un phénomène de substitution entre différents types de régimes. L'Australie, le Danemark et l'Islande sont dotés de programmes publics extrêmement ciblés, de sorte que les taux de remplacement des régimes publics qui sont particulièrement bas pour les salariés à revenu moyen et élevé sont complétés à l'aide de dispositifs privés obligatoires. En Hongrie, au Mexique, en Pologne, en République slovaque et en Suède, on a assisté à une substitution pure et simple : au terme des réformes, les prestations des dispositifs privés obligatoires ont remplacé une partie des prestations publiques. Le Canada, les États-Unis, l'Irlande et le Royaume-Uni se sont longtemps caractérisés par des retraites publiques relativement peu élevées et une vaste couverture des dispositifs facultatifs.

\section{Les régimes privés obligatoires}

Le premier groupe de 11 pays est doté de régimes de retraite privés obligatoires, ou de régimes privés dont la couverture est quasi universelle et qualifiés par conséquent de « quasi obligatoires » (Danemark, Pays-Bas et Suède).

En Islande, aux Pays-Bas et en Suisse, les régimes privés sont à prestations définies, alors que dans les autres pays, ils sont à cotisations définies. Les taux de remplacement des mécanismes privés obligatoires s'échelonnent de $23 \%$ à $33 \%$ pour le salaire moyen dans sept des 11 pays. Ils s'inscrivent néanmoins bien au-delà au Danemark, en Islande et aux Pays-Bas, et nettement en deçà en Norvège.

Dans cinq pays, les taux de remplacement sont identiques pour les salariés percevant de $50 \%$ à $150 \%$ de la moyenne nationale. Dans certains pays, toutefois, des dispositifs privés couvrent les salariés au-delà du plafond du régime public. C'est la raison pour laquelle à tous les niveaux de rémunération, les taux de remplacement des régimes privés augmentent avec les salaires aux Pays-Bas et en Norvège. Cela explique aussi pourquoi les taux de remplacement des salariés touchant $150 \%$ du salaire moyen sont nettement plus élevés en Suède.
En Suisse, la situation est complexe. Une fois encore, les bas salaires bénéficient d'un taux de remplacement plus faible afin de tenir compte des prestations publiques. Mais le plafond imposé sur les salaires qui doivent être couverts par les régimes professionnels est relativement bas.

\section{Les régimes privés facultatifs}

Les taux de remplacement apparaissent pour les neuf pays où les régimes privés facultatifs sont développés, c'est-à-dire qu'ils couvrent entre $40 \%$ et $65 \%$ de la population active (voir l'indicateur sur la " couverture des régimes de retraite privés"). Le seul pays où la population active est couverte par des dispositifs privés facultatifs dans une proportion comparable est le Japon, mais on ne dispose d'aucune information sur les règles types. On suppose que les salariés affiliés à un mécanisme privé facultatif effectuent une carrière complète dans ce cadre (les éléments relatifs aux carrières incomplètes, ainsi que leurs répercussions sont analysés dans le chapitre spécial sur « Le déficit d'épargne-retraite et l'épargneretraite facultative ").

Les règles qui ont été modélisées sont présentées dans les "descriptifs pays " figurant en partie III. Dans cinq pays, la modélisation concerne un régime à cotisations définies. Dans quatre autres - le Canada, les États-Unis, l'Irlande et le Royaume-Uni-, on a calculé séparément le taux de remplacement des régimes à cotisations définies et celui des mécanismes à prestations définies. Les informations relatives à ce deuxième type de dispositifs sont principalement données à titre d'exemple : il est peu probable en effet qu'un salarié du secteur privé entré sur le marché du travail en 2006 se voie proposer un régime à prestations définies (voir l'encadré 1 du chapitre spécial sur « Les systèmes de retraite pendant la crise économique et financière $")$.

En général, les régimes à cotisations définies proposent un taux de remplacement constant en fonction du salaire (les données sur les taux de cotisation effectifs en fonction du salaire ne sont pas disponibles pour la majorité des pays et on pose donc un taux moyen ou standard sur toute l'échelle des salaires). L'Allemagne et la Belgique constituent des exceptions, en raison du plafonnement du salaire de référence donnant droit à des incitations fiscales. En Norvège, comme pour le régime obligatoire à cotisations définies, les taux de remplacement augmentent avec les salaires, car les régimes privés sont destinés à compenser en partie la redistribution effectuée sous forme de prestations de retraite publiques. 


\section{Taux de remplacement bruts des régimes de retraite publics, privés obligatoires et privés facultatifs}

En pourcentage du salaire individuel

\begin{tabular}{|c|c|c|c|c|c|c|c|c|c|c|c|c|c|c|c|c|c|c|}
\hline & \multicolumn{3}{|c|}{ Publics } & \multicolumn{3}{|c|}{ Privés obligatoires } & \multicolumn{3}{|c|}{ CD facultatifs } & \multicolumn{3}{|c|}{ PD facultatifs } & \multicolumn{3}{|c|}{ Total obligatoires } & \multicolumn{3}{|c|}{ Total facultatifs inclus } \\
\hline & 0.5 & 1 & 1.5 & 0.5 & 1 & 1.5 & 0.5 & 1 & 1.5 & 0.5 & 1 & 1.5 & 0.5 & 1 & 1.5 & 0.5 & 1 & 1.5 \\
\hline Allemagne & 43.0 & 43.0 & 42.6 & & & & 18.3 & 18.3 & 18.1 & & & & 43.0 & 43.0 & 42.6 & 61.3 & 61.3 & 60.8 \\
\hline Australie & 40.1 & 14.6 & 6.2 & 26.9 & 26.9 & 26.9 & & & & & & & 67.0 & 41.6 & 33.1 & & & \\
\hline Autriche & 80.1 & 80.1 & 76.4 & & & & & & & & & & 80.1 & 80.1 & 76.4 & & & \\
\hline Belgique & 58.1 & 42.0 & 32.5 & & & & 16.6 & 16.6 & 13.0 & & & & 58.1 & 42.0 & 32.5 & 74.7 & 58.7 & 45.4 \\
\hline Canada & 76.5 & 44.5 & 29.7 & & & & 33.2 & 33.2 & 33.2 & 26.4 & 26.4 & 30.8 & 76.5 & 44.5 & 29.7 & 93.2 & 72.6 & 59.4 \\
\hline Corée & 64.1 & 42.1 & 33.6 & & & & & & & & & & 64.1 & 42.1 & 33.6 & & & \\
\hline Danemark & 61.5 & 22.9 & 11.7 & 62.5 & 57.4 & 55.8 & & & & & & & 124.0 & 80.3 & 67.5 & & & \\
\hline Espagne & 81.2 & 81.2 & 81.2 & & & & & & & & & & 81.2 & 81.2 & 81.2 & & & \\
\hline États-Unis & 50.3 & 38.7 & 34.1 & & & & 40.1 & 40.1 & 40.1 & 30.6 & 30.6 & 30.6 & 50.3 & 38.7 & 34.1 & 90.4 & 78.8 & 74.2 \\
\hline Finlande & 66.5 & 56.2 & 56.2 & & & & & & & & & & 66.5 & 56.2 & 56.2 & & & \\
\hline France & 61.7 & 53.3 & 48.5 & & & & & & & & & & 61.7 & 53.3 & 48.5 & & & \\
\hline Grèce & 95.7 & 95.7 & 95.7 & & & & & & & & & & 95.7 & 95.7 & 95.7 & & & \\
\hline Hongrie & 50.7 & 50.7 & 50.7 & 26.2 & 26.2 & 26.2 & & & & & & & 76.9 & 76.9 & 76.9 & & & \\
\hline Irlande & 68.4 & 34.2 & 22.8 & & & & 40.8 & 40.8 & 40.8 & 0.0 & 15.7 & 27.1 & 68.4 & 34.2 & 22.8 & 109.2 & 75.0 & 63.6 \\
\hline Islande & 26.4 & 8.3 & 5.5 & 81.9 & 81.9 & 81.9 & & & & & & & 108.3 & 90.2 & 87.5 & & & \\
\hline Italie & 67.9 & 67.9 & 67.9 & & & & & & & & & & 67.9 & 67.9 & 67.9 & & & \\
\hline Japon & 47.1 & 33.9 & 29.4 & & & & & & & & & & 47.1 & 33.9 & 29.4 & & & \\
\hline Luxembourg & 99.4 & 88.1 & 84.3 & & & & & & & & & & 99.4 & 88.1 & 84.3 & & & \\
\hline Mexique & 23.8 & 4.6 & 3.1 & 31.4 & 31.4 & 31.4 & & & & & & & 55.3 & 36.1 & 34.5 & & & \\
\hline Norvège & 60.1 & 51.9 & 41.9 & 6.0 & 7.4 & 7.9 & 9.1 & 12.8 & 18.1 & & & & 66.2 & 59.3 & 49.8 & 75.2 & 72.1 & 67.9 \\
\hline Nouvelle-Zélande & 77.5 & 38.7 & 25.8 & & & & 15.9 & 15.9 & 15.9 & & & & 77.5 & 38.7 & 25.8 & 93.3 & 54.6 & 41.7 \\
\hline Pays-Bas & 60.5 & 30.2 & 20.2 & 32.9 & 58.1 & 66.5 & & & & & & & 93.4 & 88.3 & 86.6 & & & \\
\hline Pologne & 30.0 & 30.0 & 30.0 & 31.3 & 31.3 & 31.3 & & & & & & & 61.2 & 61.2 & 61.2 & & & \\
\hline Portugal & 63.0 & 53.9 & 53.1 & & & & & & & & & & 63.0 & 53.9 & 53.1 & & & \\
\hline Rép. slovaque & 24.0 & 24.0 & 24.0 & 32.4 & 32.4 & 32.4 & & & & & & & 56.4 & 56.4 & 56.4 & & & \\
\hline Rép. tchèque & 79.2 & 49.7 & 36.4 & & & & 11.6 & 11.6 & 11.6 & & & & 79.2 & 49.7 & 36.4 & 90.8 & 61.3 & 48.0 \\
\hline Royaume-Uni & 51.0 & 30.8 & 21.3 & & & & 39.2 & 39.2 & 39.2 & 38.4 & 38.4 & 38.4 & 51.0 & 30.8 & 21.3 & 89.3 & 70.0 & 60.6 \\
\hline Suède & 52.9 & 37.8 & 27.9 & 23.7 & 23.7 & 47.7 & & & & & & & 76.6 & 61.5 & 75.6 & & & \\
\hline Suisse & 52.4 & 35.6 & 23.8 & 10.1 & 22.7 & 16.7 & & & & & & & 62.5 & 58.3 & 40.5 & & & \\
\hline Turquie & 86.9 & 86.9 & 86.9 & & & & & & & & & & 86.9 & 86.9 & 86.9 & & & \\
\hline OCDE & 60.0 & 45.7 & 40.1 & & & & & & & & & & 72.2 & 59.0 & 54.3 & 81.1 & 68.4 & 63.6 \\
\hline
\end{tabular}

$\mathrm{CD}=$ à cotisations définies $; \mathrm{PD}=$ à prestations définies.

Source: Modèles de retraite de l'OCDE. 


\section{Principaux résultats}

Pour les salaires moyens, le taux de remplacement net s'établit aux alentours de $70 \%$ dans les pays de l'OCDE. Il est donc supérieur de 11 points au taux de remplacement brut, car les impôts et cotisations versés sur les salaires sont plus élevés que ceux qui sont prélevés sur les pensions de retraite. Les taux de remplacement nets varient aussi très sensiblement entre les différents pays, de moins de $40 \%$ au Mexique et au Japon à un chiffre largement supérieur à $100 \%$ en Grèce et en Turquie pour la rémunération moyenne.

Concernant les personnes à faible revenu (percevant la moitié du salaire moyen), le taux de remplacement net ressort à $82 \%$ dans la zone OCDE. Pour les hauts revenus ( $150 \%$ du salaire moyen), il s'inscrit en moyenne à $65 \%$, soit en deçà de celui dont bénéficient les bas salaires. Comme pour les taux de remplacement bruts, cette disparité en fonction de la rémunération témoigne du caractère progressif des systèmes de retraite, notamment à travers les prestations minimum et les plafonds.

Le régime de l'impôt sur le revenu des personnes physiques joue un rôle important dans les mesures en faveur des personnes âgées. Il est fréquent que les retraités ne versent pas de cotisations de sécurité sociale et, l'IRRP étant progressif et les droits à retraite souvent inférieurs au salaire perçu avant la retraite, le taux d'imposition moyen des revenus des retraités est généralement moins élevé que celui des salaires. De plus, la majorité des régimes d'imposition soumettent à un traitement préférentiel les revenus perçus au titre de la retraite ou les retraités eux-mêmes, en accordant des crédits ou des abattements supplémentaires aux personnes âgées. Les taux de remplacement nets sont donc habituellement plus élevés que les taux de remplacement bruts.

Pour le salaire moyen, les taux de remplacement diffèrent selon qu'ils sont exprimés en termes nets ou bruts. En Allemagne et en Belgique, les taux de remplacement nets des régimes de retraite sont sensiblement supérieurs aux taux bruts. Cette situation s'explique premièrement par le régime favorable dont bénéficient les revenus au titre de la retraite au regard des cotisations de sécurité sociale. Deuxièmement, puisque les taux de remplacement sont relativement bas par rapport aux pays de l'OCDE et que l'IRPP est extrêmement progressif dans ces pays, les retraités acquittent un impôt sur le revenu nettement moins élevé que celui qu'ils payaient lorsqu'ils étaient en activité, et ce en dépit du retrait progressif du régime fiscal particulièrement généreux appliqué en Allemagne aux revenus des retraités.

À l'inverse, l'écart entre les chiffres bruts et nets est moins important en Nouvelle-Zélande et en Suède. En effet, ces pays imposent les revenus des retraités et les salaires à des taux pratiquement identiques (bien que la Suède ait réintroduit des avantages fiscaux pour les retraités en 2009 : voir le chapitre spécial sur "les réformes récentes des retraites » en partie I).

Pour les bas revenus, les effets des impôts et des cotisations sur les taux de remplacement nets sont moins marqués que pour les salariés situés plus haut sur l'échelle des salaires, car de manière générale, ils paient moins d'impôts et de cotisations que les personnes dont la rémunération s'inscrit dans la moyenne. Dans bien des cas, leurs revenus au titre de la retraite sont inférieurs aux allégements d'impôt traditionnellement accordés au titre de l'impôt sur le revenu des personnes physiques (abattements, crédits, etc.). Ils ne peuvent donc pas profiter pleinement des avantages supplémentaires octroyés sur les retraites ou aux retraités dans le cadre de l'IRPP.

L'écart entre les taux de remplacement brut et net est en moyenne de 10 points pour les bas salaires. La Belgique et la République tchèque offrent aux salariés faiblement rémunérés des taux de remplacement nettement plus élevés en termes nets.

Le taux de remplacement net pour les personnes gagnant $150 \%$ du salaire moyen est plus élevé en Turquie, car les revenus des retraités ne sont pas imposables. Sans surprise, on observe les taux de remplacement les plus faibles dans les régimes de retraite forfaitaire de Nouvelle-Zélande et d'Irlande. Dans ces deux pays, les salariés touchant $150 \%$ de la rémunération moyenne percevront des retraites correspondant à moins d'un tiers de leur salaire net.

On note des disparités régionales pour ce qui est de l'écart entre les taux bruts et nets. Pour les salariés à salaire médian de l'UE15, le taux de remplacement net est en moyenne supérieur de 11 points à celui du taux brut. En Europe méridionale, l'écart est de 13 points, alors que dans les pays nordiques, il ressort à 7 points seulement. L'impôt sur le revenu joue en effet dans les pays nordiques un rôle plus important qu'ailleurs.

\section{Définition et calcul}

Le taux de remplacement net se définit comme les droits à retraite individuels nets divisés par le salaire net, compte tenu de l'IRPP et des cotisations de sécurité sociale versés par les salariés et les retraités. Pour le reste, la définition et le calcul du taux de remplacement net sont les mêmes que pour le taux de remplacement brut (voir indicateur précédent). Une fois encore, les résultats concernent les salariés ayant effectué une carrière complète percevant le salaire médian ou le salaire moyen multiplié par $0.5,0.75,1,1.5$ et 2 . 


\section{Taux de remplacement nets en fonction du salaire}

\begin{tabular}{|c|c|c|c|c|c|c|c|c|c|c|c|c|c|}
\hline & \multirow{2}{*}{$\begin{array}{c}\text { Salarié } \\
\text { à revenu } \\
\text { médian }\end{array}$} & \multicolumn{5}{|c|}{ Salaire individuel, en multiple de la moyenne } & & \multirow{2}{*}{$\begin{array}{c}\text { Salarié } \\
\text { à revenu } \\
\text { médian }\end{array}$} & \multicolumn{5}{|c|}{ Salaire individuel, en multiple de la moyenne } \\
\hline & & 0.5 & 0.75 & 1 & 1.5 & 2 & & & 0.5 & 0.75 & 1 & 1.5 & 2 \\
\hline Hommes & & & & & & & Hommes (suite) & & & & & & \\
\hline Australie & 59.2 & 80.2 & 63.7 & 53.1 & 41.8 & 36.8 & Nouvelle-Zélande & 47.3 & 79.3 & 53.5 & 41.1 & 29.0 & 22.8 \\
\hline Autriche & 90.3 & 90.5 & 90.3 & 90.3 & 86.3 & 64.8 & Norvège & 70.2 & 76.7 & 72.3 & 69.3 & 60.6 & 52.8 \\
\hline Belgique & 65.3 & 78.7 & 69.0 & 63.7 & 51.7 & 41.2 & Pologne & 74.8 & 74.4 & 74.7 & 74.9 & 75.0 & 77.0 \\
\hline Canada & 63.6 & 89.1 & 68.9 & 57.9 & 40.0 & 30.9 & Portugal & 68.0 & 73.2 & 66.7 & 69.6 & 72.0 & 72.6 \\
\hline Rép. tchèque & 69.8 & 95.3 & 74.7 & 64.1 & 49.4 & 41.1 & Rép. slovaque & 71.5 & 66.3 & 70.4 & 72.7 & 74.9 & 75.9 \\
\hline Danemark & 98.7 & 137.0 & 106.2 & 91.3 & 82.7 & 77.7 & Espagne & 84.2 & 82.1 & 84.1 & 84.7 & 85.3 & 72.2 \\
\hline Finlande & 62.0 & 73.2 & 62.7 & 62.4 & 63.8 & 64.5 & Suède & 64.1 & 79.3 & 67.4 & 64.1 & 81.2 & 85.9 \\
\hline France & 65.3 & 76.2 & 65.6 & 65.7 & 60.2 & 57.5 & Suisse & 69.5 & 68.8 & 79.4 & 64.5 & 44.3 & 33.4 \\
\hline Allemagne & 61.5 & 59.2 & 61.1 & 61.3 & 60.3 & 44.4 & Turquie & 124.0 & 121.2 & 123.4 & 124.7 & 127.1 & 130.4 \\
\hline Grèce & 110.4 & 113.6 & 110.1 & 110.8 & 106.7 & 104.2 & Royaume-Uni & 44.3 & 63.8 & 48.0 & 40.9 & 29.2 & 22.8 \\
\hline Hongrie & 95.4 & 94.3 & 95.4 & 105.5 & 99.2 & 99.2 & États-Unis & 47.1 & 57.9 & 49.2 & 44.8 & 39.5 & 33.3 \\
\hline Islande & 96.5 & 110.1 & 97.7 & 95.1 & 92.1 & 90.6 & & & & & & & \\
\hline Irlande & 45.6 & 68.4 & 50.1 & 40.1 & 30.3 & 24.4 & OCDE & 71.8 & 82.4 & 74.0 & 70.3 & 65.5 & 60.8 \\
\hline Italie & 74.8 & 74.8 & 74.8 & 74.8 & 77.1 & 78.7 & & & & & & & \\
\hline Japon & 40.3 & 51.4 & 42.8 & 38.7 & 33.9 & 30.8 & Femmes & & & & & & \\
\hline Corée & 49.2 & 68.8 & 53.5 & 46.6 & 38.7 & 29.6 & Italie & 58.1 & 76.6 & 58.1 & 58.1 & 59.9 & 63 \\
\hline Luxembourg & 98.1 & 107.1 & 99.7 & 96.5 & 93.5 & 91.8 & Mexique & 33.5 & 56 & 37.3 & 31.5 & 32.8 & 32.9 \\
\hline Mexique & 38.0 & 56.0 & 38.1 & 38.0 & 39.6 & 39.7 & Pologne & 55.2 & 60.6 & 55.3 & 55.2 & 55 & 56.4 \\
\hline Pays-Bas & 105.5 & 105.0 & 107.4 & 103.2 & 98.6 & 95.5 & Suisse & 70.2 & 69.1 & 67.3 & 65.3 & 44.9 & 33.8 \\
\hline
\end{tabular}

Source: Modèles de retraite de l'OCDE.

\section{Taux de remplacement nets (TRN) en fonction du salaire}

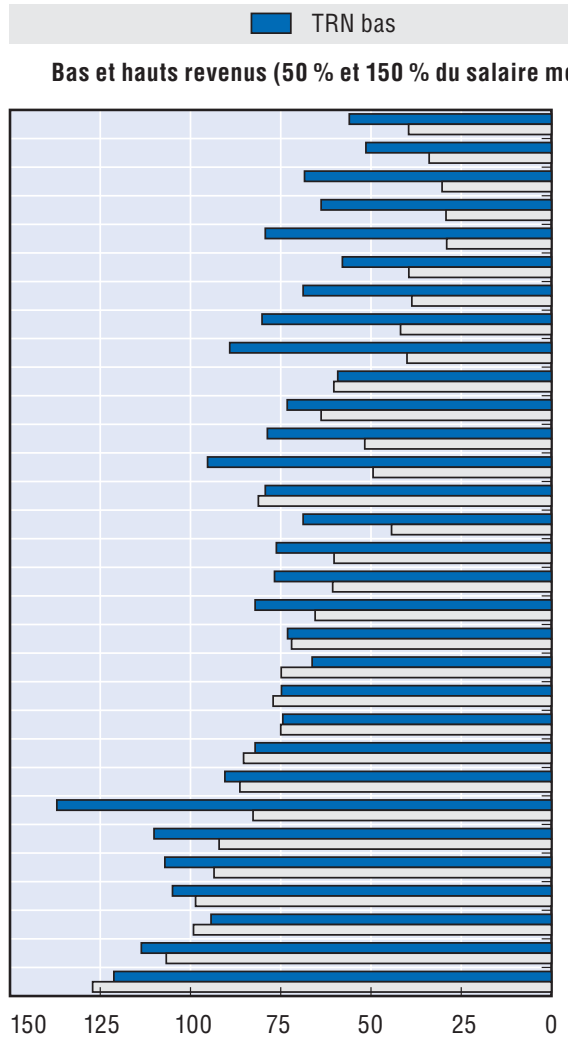

$\square$ TRN élevé

Mexique
Japon
Irlande
Royaume-Uni
Nouvelle-Zélande
États-Unis
Corée
Australie
Canada
Allemagne
Finlande
Belgique
République tchèque
Suède
Suisse
France
Norvège
OCDE
Portugal
République slovaque
Italie
Pologne
Espagne
Autriche
Danemark
Islande
Luxembourg
Pays-Bas
Hongrie
Grèce
Turquie

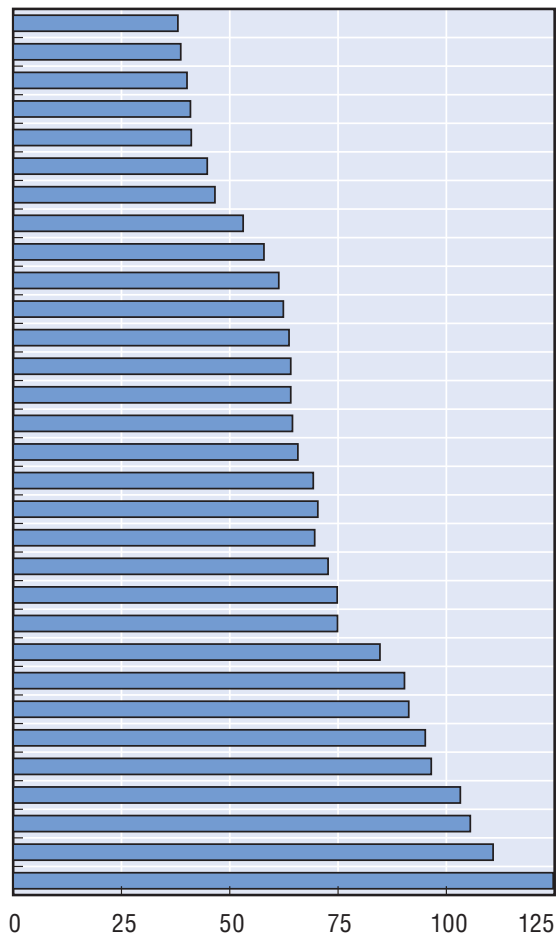

Note : Les pays sont classés d'après le taux de remplacement net (TRN) pour le salaire moyen, soit le TRN moyen apparaissant dans le graphique.

Source: Modèles de retraite de l'OCDE. 


\section{Principaux résultats}

Le patrimoine retraite correspond à la valeur viagère totale du flux de revenus perçus au titre de la retraite. Le patrimoine retraite des personnes touchant le salaire moyen correspond au salaire individuel annuel perçu en moyenne dans les pays de l'OCDE multiplié par 9.3. Pour les femmes, la moyenne est plus élevée - soit la rémunération moyenne multipliée par 10.9 - à l'image de leur espérance de vie.

Les taux de remplacement donnent une indication de l'ampleur des promesses de retraite, mais ces indicateurs sont incomplets, car ils rendent uniquement compte du montant des prestations au moment du départ en retraite. Pour avoir une vue d'ensemble, il faut également prendre en considération l'espérance de vie, l'âge de la retraite et l'indexation des pensions qui, à eux trois, déterminent la durée de versement des prestations et leur évolution dans le temps. Le patrimoine retraite - qui mesure le «stock » des flux de prestations de retraite à venir - tient compte de tous ces éléments. On peut donc considérer qu'il s'agit de la somme nécessaire pour acheter un contrat de rente donnant droit au même flux de prestations que celui que l'on peut attendre des régimes de retraite obligatoires.

En ce qui concerne les hommes percevant une rémunération moyenne, c'est au Luxembourg que le patrimoine retraite brut est le plus élevé, suivi des PaysBas, de la Grèce et de l'Islande. Dans ces pays, le patrimoine retraite correspond en moyenne au salaire individuel multiplié par 15.9 , soit $70 \%$ de plus que la moyenne des pays de l'OCDE qui s'établit à 9.3. Le patrimoine retraite pour les hommes qui gagnent le salaire moyen est le plus bas au Royaume-Uni, en raison d'un taux de remplacement relativement faible et du relèvement de l'âge de la retraite à 68 ans. Le RoyaumeUni est suivi de près par le Mexique ; dans ces deux pays, le patrimoine retraite représente moins de cinq fois le salaire individuel.

Le fait que le taux de remplacement soit plus élevé pour les bas salaires indique que le patrimoine retraite tend à être plus important pour cette catégorie que pour les revenus moyens. Pour les hommes percevant la moitié du salaire moyen, le patrimoine retraite représente 11.5 fois le salaire individuel en moyenne, contre 9.3 pour ceux dont la rémunération s'inscrit dans la moyenne. De même, le patrimoine retraite pour les femmes faiblement rémunérées correspond au salaire individuel multiplié par 13.4 , contre 10.9 pour celles qui touchent un salaire moyen. Pour les hommes, dans les quatre pays où le patrimoine retraite des faibles revenus est le plus élevé (le Danemark, l'Islande, le Luxembourg et les Pays-Bas), il est égal au salaire individuel multiplié par 17.0.

Dans les pays où l'espérance de vie est plus brève, comme la Hongrie, le Mexique, la Pologne, la République slovaque et la Turquie, les prestations sont versées moins longtemps, de sorte que toutes choses étant égales, les promesses sont plus accessibles d'un point de vue financier. On observe l'effet inverse en Suisse et dans les pays nordiques, où l'espérance de vie est élevée. À la différence des indicateurs des taux de remplacement, le patrimoine retraite rend compte du lien existant entre le coût et l'espérance de vie.

Pour cette même raison, l'espérance de vie des femmes étant plus longue que celle des hommes, le patrimoine retraite des femmes est relativement plus important dans l'ensemble des pays. En effet, on escompte tout simplement que les prestations de retraite seront versées sur une période plus longue. De plus, l'âge de la retraite demeure plus bas pour les femmes dans certain pays, ce qui prolonge encore la durée des versements.

L'âge de la retraite joue également sur le patrimoine retraite. L'Allemagne, le Danemark, les États-Unis, l'Islande, la Norvège et le Royaume-Uni, par exemple, ont tous relevé l'âge de la retraite au-delà de 65 ans ou en ont l'intention, ce qui réduit le patrimoine retraite.

Les règles d'indexation ont elles aussi une incidence sur le patrimoine retraite. Bien que la plupart des pays de l'OCDE indexent désormais les retraites mises en paiement sur les prix, on note des exceptions: le Luxembourg établit ainsi un lien entre les pensions de retraite et le salaire moyen, alors que cinq pays, dont la Finlande, la Hongrie, la République slovaque, la République tchèque et la Suisse, les indexent à la fois sur l'inflation et sur la hausse des salaires. En temps normal, du moins, les salaires progressent généralement plus vite que les prix, de sorte que le patrimoine retraite est plus élevé selon ces méthodes d'indexation plus généreuses qu'avec l'indexation des prix.

Diverses mesures d'indexation influent également sur le patrimoine retraite des femmes par rapport aux hommes. L'espérance de vie des femmes étant plus longue, elles bénéficient davantage de méthodes d'indexation plus généreuses (à un taux supérieur à l'inflation, par exemple).

\section{Définition et calcul}

Le patrimoine retraite est calculé à l'aide d'un taux d'actualisation uniforme de $2 \%$ et de tables de mortalité nationales. Les comparaisons ayant trait à des droits à retraite prospectifs, les calculs sont effectués sur la base de projections pour l'année 2040.

Le patrimoine retraite est calculé et exprimé sous forme de multiple du salaire individuel annuel brut. Il est donné ici pour les personnes touchant le salaire moyen multiplié $0.5,1$ et 1.5 , en établissant une distinction entre les femmes et les hommes. 


\section{Patrimoine retraite brut en fonction du salaire}

\begin{tabular}{|c|c|c|c|c|c|c|c|c|c|c|c|c|c|}
\hline & \multicolumn{6}{|c|}{ Salaire individuel, en multiple de la moyenne } & & \multicolumn{6}{|c|}{ Salaire individuel, en multiple de la moyenne } \\
\hline & 0.5 & 1.0 & 1.5 & 0.5 & 1.0 & 1.5 & & 0.5 & 1.0 & 1.5 & 0.5 & 1.0 & 1.5 \\
\hline & \multicolumn{3}{|c|}{ Hommes } & \multicolumn{3}{|c|}{ Femmes } & & \multicolumn{3}{|c|}{ Hommes } & \multicolumn{3}{|c|}{ Femmes } \\
\hline Allemagne & 7.2 & 7.2 & 7.1 & 8.5 & 8.5 & 8.4 & Japon & 7.8 & 5.6 & 4.9 & 8.8 & 6.3 & 5.5 \\
\hline Australie & 11.7 & 6.9 & 5.3 & 13.7 & 8.1 & 6.2 & Luxembourg & 21.7 & 19.2 & 18.4 & 26.5 & 23.5 & 22.5 \\
\hline Autriche & 12.2 & 11.6 & 10.5 & 14.2 & 13.5 & 12.1 & Mexique & 7.3 & 4.8 & 4.6 & 8.9 & 4.8 & 4.6 \\
\hline Belgique & 8.9 & 6.4 & 5.0 & 10.3 & 7.5 & 5.8 & Norvège & 11.4 & 10.2 & 8.5 & 13.4 & 11.9 & 9.9 \\
\hline Canada & 11.7 & 6.8 & 4.5 & 13.6 & 7.9 & 5.3 & Nouvelle-Zélande & 14.3 & 7.2 & 4.8 & 16.8 & 8.4 & 5.6 \\
\hline Corée & 8.9 & 5.9 & 4.7 & 10.7 & 7.0 & 5.6 & Pays-Bas & 17.2 & 16.3 & 16.0 & 20.1 & 19.1 & 18.7 \\
\hline Danemark & 18.5 & 11.6 & 9.6 & 21.3 & 13.3 & 11.0 & Pologne & 8.4 & 8.4 & 8.4 & 9.5 & 8.6 & 8.6 \\
\hline Espagne & 12.2 & 12.2 & 12.2 & 14.3 & 14.3 & 14.3 & Portugal & 9.2 & 8.1 & 8.0 & 10.7 & 9.5 & 9.3 \\
\hline États-Unis & 7.2 & 5.5 & 4.9 & 8.3 & 6.4 & 5.7 & Rép. slovaque & 8.8 & 8.8 & 8.8 & 10.6 & 10.6 & 10.6 \\
\hline Finlande & 10.4 & 8.8 & 8.8 & 12.3 & 10.5 & 10.5 & Rép. tchèque & 12.1 & 7.6 & 5.6 & 14.3 & 9.0 & 6.6 \\
\hline France & 10.8 & 9.3 & 8.5 & 12.5 & 10.8 & 9.8 & Royaume-Uni & 6.8 & 4.1 & 2.9 & 7.8 & 4.7 & 3.3 \\
\hline Grèce & 14.3 & 14.3 & 14.3 & 16.6 & 16.6 & 16.6 & Suède & 12.2 & 9.9 & 12.0 & 14.0 & 11.3 & 13.7 \\
\hline Hongrie & 12.4 & 12.4 & 12.4 & 15.4 & 15.4 & 15.4 & Suisse & 10.7 & 9.8 & 6.8 & 13.1 & 12.0 & 8.3 \\
\hline Irlande & 12.1 & 6.1 & 4.0 & 14.5 & 7.2 & 4.8 & Turquie & 11.0 & 11.0 & 11.0 & 12.9 & 12.9 & 12.9 \\
\hline Islande & 17.0 & 13.7 & 13.2 & 19.1 & 15.4 & 14.8 & & & & & & & \\
\hline Italie & 10.0 & 10.0 & 9.9 & 10.7 & 10.7 & 10.7 & OCDE & 11.5 & 9.3 & 8.5 & 13.4 & 10.9 & 9.9 \\
\hline
\end{tabular}

Source: Modèles de retraite de l'OCDE.

\section{Patrimoine retraite brut en fonction du salaire et du sexe}

Hommes bas revenus $\square$ Femmes bas revenus $\square$ Hommes salaire moyen $\square$ Femmes salaire moyen

Bas revenus (hommes et femmes)

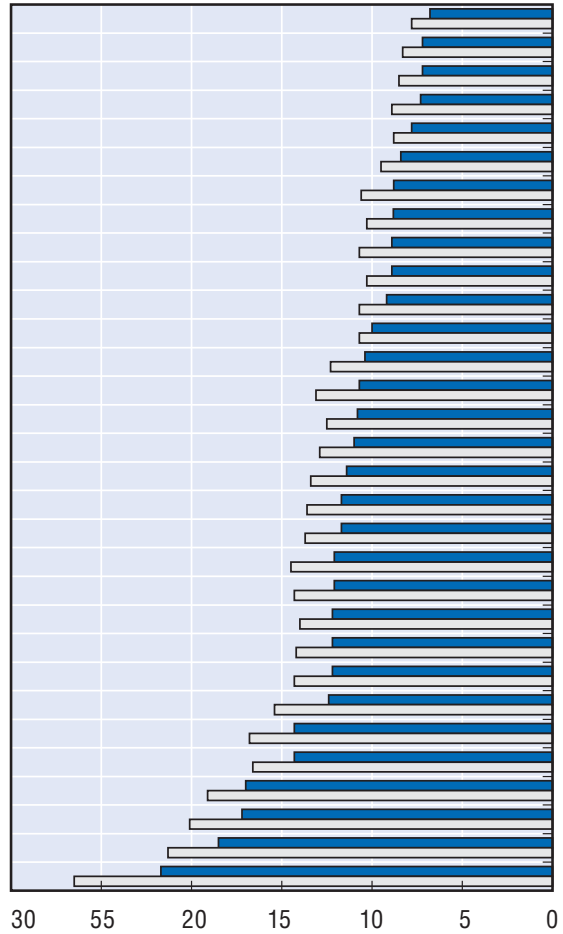

Salaire moyen (hommes et femmes)
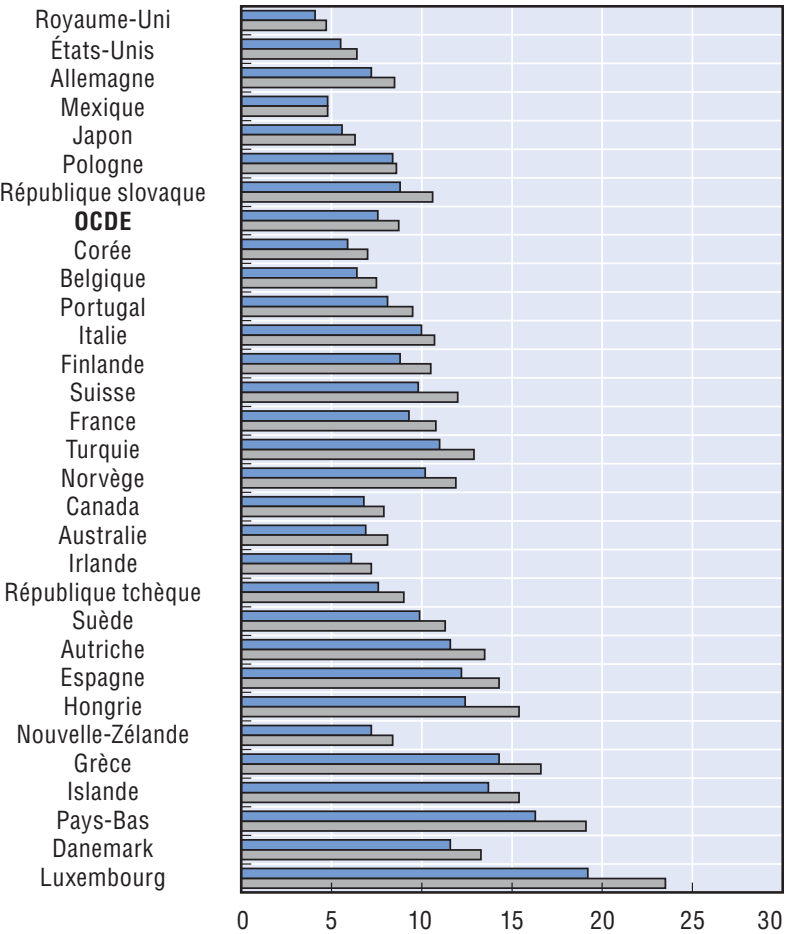

Note: Les pays sont classés d'après le taux de remplacement brut (TRB) des bas revenus (hommes).

Source: Modèles de retraite de l'OCDE. 


\section{Principaux résultats}

Le patrimoine retraite net, à l'instar de l'indicateur équivalent exprimé en termes bruts, correspond à la valeur actualisée du flux des prestations de retraite viagères, mais en tenant compte des impôts et cotisations acquittés sur les revenus perçus au titre de la retraite. Dans un cas comme dans l'autre, le patrimoine retraite est exprimé en multiple du salaire individuel brut.

Pour un salaire moyen, le patrimoine retraite net correspond en moyenne dans les pays de l'OCDE au salaire individuel brut multiplié par 7.9 pour les hommes et 9.2 pour les femmes. Les chiffres sont plus élevés pour les femmes que pour les hommes, en raison principalement des différences d'espérance de vie.

Le patrimoine retraite net, indiqué dans la partie gauche du tableau, est immanquablement inférieur au patrimoine retraite brut (lorsque les retraités sont assujettis à l'impôt) ou identique (lorsque les pensions de retraite ne pas soumises à l'impôt ou que les revenus au titre de la retraite s'inscrivent en deçà du seuil d'imposition). Ainsi, le patrimoine retraite net est égal au patrimoine retraite brut en République slovaque et en Turquie car les pensions ne sont pas imposables.

Dans le tableau, les colonnes de droite font apparaître la part des pensions qui est consacrée aux impôts et aux cotisations pour les retraités qui percevaient différents niveaux de rémunération lorsqu'ils étaient en activité. Les salariés à revenu moyen exclusivement affiliés à un régime obligatoire aux États-Unis, en Irlande, au Mexique, au Portugal et en République tchèque ne seront pas assujettis à l'impôt une fois à la retraite, car les taux de remplacement des dispositifs obligatoires sont faibles par rapport à d'autres pays de l'OCDE. Par conséquent, les personnes touchant une rémunération moyenne n'accumulent pas assez de droits pour être soumises à l'impôt pendant leur retraite, car elles bénéficient d'allégements d'impôt à la base et sont exonérées de cotisations de sécurité sociale. C'est également le cas des hauts revenus ( $150 \%$ du salaire moyen) dans tous ces pays à l'exception du Portugal, où leur pension de retraite est imposée à un taux de $2.2 \%$, de sorte que le patrimoine retraite net est légèrement en deçà du résultat brut.

Le classement des patrimoines retraite varie sensiblement lorsqu'on prend en compte les chiffres nets et non les valeurs brutes. Ainsi, pour un salaire moyen, la République slovaque se classe en $8^{\mathrm{e}}$ position d'après le patrimoine retraite net, mais au $15^{\mathrm{e}}$ rang en termes bruts. Le Danemark connaît la situation inverse, car c'est là que le taux d'imposition des retraites obligatoires est le plus élevé sur toute l'échelle des rémunérations. Il se classe $7^{\mathrm{e}}$ en termes bruts et $14^{\mathrm{e}}$ en termes nets.

Dans les cinq pays nordiques, ainsi qu'en Autriche, en Italie, au Luxembourg et aux Pays-Bas, les retraités subissent une forte pression fiscale. Cette situation est due pour une part au niveau élevé du taux de remplacement brut du système obligatoire, mais aussi à la forte ponction fiscale observée dans l'ensemble du pays.
Les personnes à faible revenu ne sont pas soumises à l'impôt ni au versement de cotisations dans dix pays, soit l'Australie, la Belgique et le Canada, ainsi que les sept pays où les retraités percevant un revenu moyen ne sont pas assujettis à l'impôt. Dans quatre autres pays - la Corée, la Grèce, la Hongrie et le Royaume-Uni -, le taux d'imposition pour les retraités à faible revenu est modique, soit moins de $1 \%$ des pensions.

Il convient de noter que ces calculs portent sur le volet « prestations » du système de retraite uniquement. Les répercussions des impôts et des cotisations versés par les personnes d'âge actif sur leur niveau de vie une fois à la retraite, comparé à celui qu'elles avaient lorsqu'elles étaient en activité, sont examinées ci-dessus dans l'indicateur " taux de remplacement nets ».

\section{Définition et calcul}

Le patrimoine retraite net correspond à la valeur actualisée du flux des prestations de retraite, compte tenu des impôts et cotisations de sécurité sociale dus par les retraités sur leur pension. Il est calculé et exprimé sous forme de multiple du salaire individuel annuel brut du pays concerné. On utilise le salaire brut comme élément de référence afin d'isoler les effets des impôts et des cotisations payés à la retraite de ceux qui ont été versés durant la vie active. Selon cette définition, le patrimoine retraite brut et le patrimoine retraite net sont identiques lorsqu'aucun impôt sur le revenu ni cotisations ne sont prélevés sur les pensions de retraite.

Les impôts et les cotisations versés par les retraités sont calculés en fonction des prestations de retraite auxquelles donnent droit les régimes obligatoires pour différents niveaux de rémunération. Les calculs tiennent compte de l'ensemble des abattements fiscaux et allégements d'impôts standard, ainsi que des avantages accordés sur les revenus au titre des retraites ou aux personnes ayant atteint l'âge de la retraite. On trouvera le détail des règles appliquées aux retraités par les systèmes fiscaux nationaux dans les descriptifs pays disponibles en ligne à l'adresse www.oecd.org/els/social/pensions/pag-fr.

Le patrimoine retraite net est donné ici pour les personnes touchant le salaire moyen multiplié $0.5,1$ et 1.5 . 
Patrimoine retraite net et impôts et cotisations acquittés par les retraités

\begin{tabular}{|c|c|c|c|c|c|c|c|c|c|}
\hline \multirow{3}{*}{ Salaire individuel } & \multicolumn{6}{|c|}{$\begin{array}{c}\text { Patrimoine retraite net } \\
\text { En multiple du salaire individuel brut annuel }\end{array}$} & \multirow{2}{*}{\multicolumn{3}{|c|}{$\begin{array}{l}\text { Impôts et cotisations acquittés } \\
\text { par les retraités (en \% de leur pension) }\end{array}$}} \\
\hline & \multicolumn{3}{|c|}{ Hommes } & \multicolumn{3}{|c|}{ Femmes } & & & \\
\hline & 0.5 & 1 & 1.5 & 0.5 & 1 & 1.5 & 0.5 & 1 & 1.5 \\
\hline Allemagne & 6.6 & 5.8 & 5.3 & 7.8 & 6.8 & 6.3 & 8.4 & 19.6 & 25.2 \\
\hline Australie & 11.7 & 6.7 & 4.8 & 13.7 & 7.8 & 5.5 & 0.0 & 2.8 & 10.7 \\
\hline Autriche & 10.9 & 8.8 & 7.4 & 12.6 & 10.1 & 8.5 & 11.1 & 24.7 & 29.7 \\
\hline Belgique & 8.9 & 5.7 & 4.1 & 10.3 & 6.6 & 4.8 & 0.0 & 12.0 & 16.5 \\
\hline Canada & 11.7 & 6.7 & 4.5 & 13.6 & 7.8 & 5.2 & 0.0 & 1.0 & 1.0 \\
\hline Corée & 8.9 & 5.8 & 4.6 & 10.6 & 6.9 & 5.5 & 0.8 & 1.6 & 2.2 \\
\hline Danemark & 12.7 & 7.8 & 6.1 & 14.6 & 8.9 & 7 & 31.5 & 33.2 & 36.3 \\
\hline Espagne & 10.9 & 10.1 & 9.7 & 12.8 & 11.8 & 11.3 & 10.1 & 17.1 & 20.6 \\
\hline États-Unis & 7.2 & 5.5 & 4.9 & 8.3 & 6.4 & 5.7 & 0.0 & 0.0 & 0.0 \\
\hline Finlande & 9 & 6.6 & 6.2 & 10.6 & 7.9 & 7.3 & 13.6 & 24.9 & 30.0 \\
\hline France & 10.2 & 8.2 & 7.1 & 11.7 & 9.4 & 8.2 & 5.9 & 12.6 & 15.7 \\
\hline Grèce & 14.3 & 12.3 & 11.1 & 16.5 & 14.3 & 12.9 & 0.3 & 13.9 & 22.3 \\
\hline Hongrie & 12.4 & 11 & 9.5 & 15.3 & 13.6 & 11.7 & 0.2 & 11.2 & 23.8 \\
\hline Irlande & 12.1 & 6.1 & 4 & 14.5 & 7.2 & 4.8 & 0.0 & 0.0 & 0.0 \\
\hline Islande & 13.9 & 10.2 & 9.3 & 15.6 & 11.4 & 10.5 & 18.2 & 25.6 & 29.1 \\
\hline Italie & 7.6 & 7.6 & 7.5 & 10.7 & 8.1 & 8.1 & 24.1 & 24.1 & 24.1 \\
\hline Japon & 7.1 & 5.2 & 4.4 & 7.9 & 5.8 & 4.9 & 9.7 & 8.0 & 10.7 \\
\hline Luxembourg & 19.2 & 15.2 & 13.3 & 23.5 & 18.5 & 16.3 & 11.3 & 21.1 & 27.5 \\
\hline Mexique & 7.3 & 4.8 & 4.6 & 8.9 & 4.8 & 4.6 & 0.0 & 0.0 & 0.0 \\
\hline Norvège & 10.3 & 8.4 & 6.8 & 12.1 & 9.9 & 7.9 & 9.8 & 17.3 & 20.1 \\
\hline Nouvelle-Zélande & 11.8 & 5.9 & 3.9 & 13.9 & 6.9 & 4.6 & 17.6 & 17.6 & 17.6 \\
\hline Pays-Bas & 14.2 & 12.1 & 11 & 16.6 & 14.2 & 12.8 & 17.4 & 25.6 & 31.4 \\
\hline Pologne & 7.2 & 7 & 6.9 & 8.3 & 7.2 & 7.1 & 14.1 & 17.0 & 18.0 \\
\hline Portugal & 9.2 & 8.1 & 7.8 & 10.7 & 9.5 & 9.1 & 0.0 & 0.0 & 2.2 \\
\hline Rép. slovaque & 8.8 & 8.8 & 8.8 & 10.6 & 10.6 & 10.6 & 0.0 & 0.0 & 0.0 \\
\hline Rép. tchèque & 12.1 & 7.6 & 5.6 & 14.3 & 9 & 6.6 & 0.0 & 0.0 & 0.0 \\
\hline Royaume-Uni & 6.8 & 4 & 2.8 & 7.8 & 4.6 & 3.2 & 0.9 & 2.8 & 3.6 \\
\hline Suède & 9.3 & 7.1 & 8 & 10.6 & 8.1 & 9.1 & 23.8 & 27.9 & 33.3 \\
\hline Suisse & 10.4 & 7.9 & 5.5 & 12.7 & 9.6 & 6.7 & 2.6 & 19.6 & 19.2 \\
\hline Turquie & 11 & 11 & 11 & 12.9 & 12.9 & 12.9 & 0.0 & 0.0 & 0.0 \\
\hline OCDE & 10.5 & 7.9 & 6.9 & 12.3 & 9.2 & 8.0 & 7.7 & 12.7 & 15.7 \\
\hline
\end{tabular}

Source: Modèles de retraite de l'OCDE.

\section{Patrimoine retraite net et brut par sexe, pour un salaire moyen}
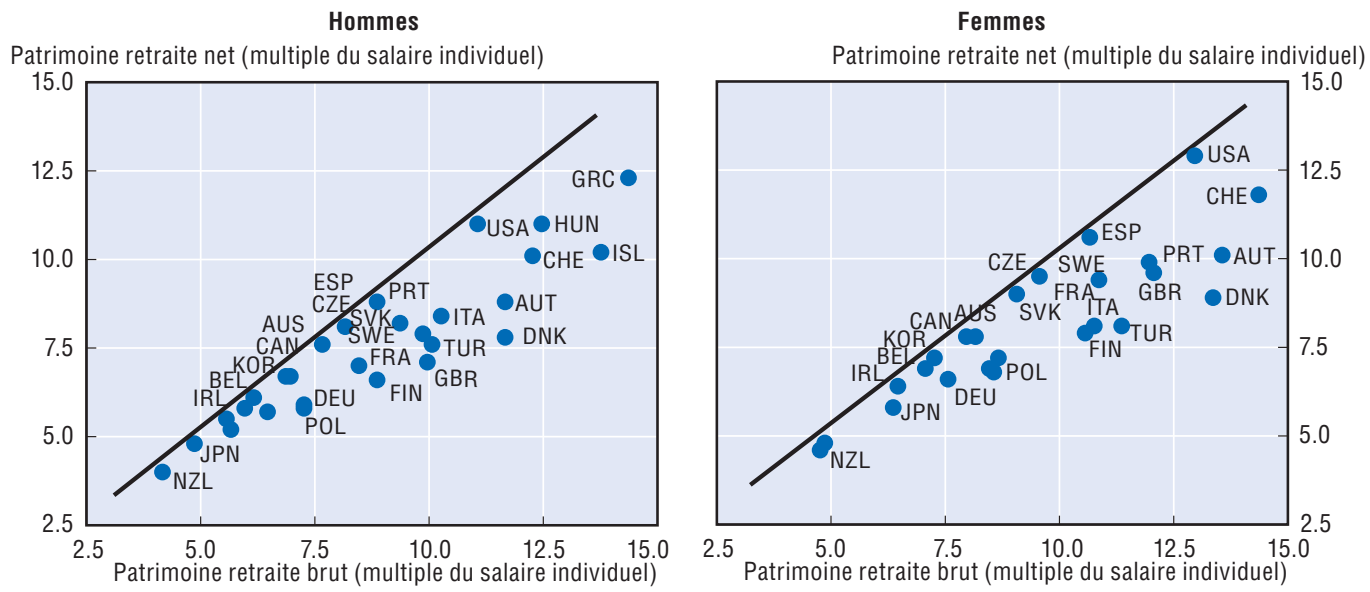

Note : Les échelles des deux graphiques ont été limitées à un patrimoine retraite brut égal à 15 fois le salaire individuel, ce qui exclut le Luxembourg et les Pays-Bas des deux graphiques, et la Grèce, la Hongrie et l'Islande du graphique concernant les femmes.

Source: Modèles de retraite de l'OCDE. 


\section{Principaux résultats}

L'indice de progressivité varie de 100 dans les régimes de base purs (Irlande et Nouvelle-Zélande) à un résultat négatif en Suède, ce qui indique que le système de retraite dans son ensemble est régressif. L'indice moyen des pays de l'OCDE s'établit à 34.8. Les différences existant entre les régions sont frappantes. L'indice ressort en moyenne à 80 dans les pays anglophones, signe que leurs systèmes sont fortement progressifs. Dans les pays d'Europe méridionale, toutefois, il atteint pratiquement 6 en moyenne, témoignant d'une corrélation très étroite entre le salaire et les prestations de retraite.

Les systèmes de retraite « de base purs " servent les mêmes prestations aux retraités quels qu'aient été leur salaire et autres sources de revenu. La valeur relative de la pension de retraite est indépendante de la rémunération et le taux de remplacement décroît avec le salaire. À l'inverse, les régimes "strictement assurantiels " ont pour objectif de servir le même taux de remplacement à tous les salariés lorsqu'ils prennent leur retraite. Les régimes à cotisations définies relèvent généralement du modèle strictement assurantiel, à l'instar des dispositifs liés à la rémunération qui proposent le même taux d'accumulation quels que soient le salaire, le nombre d'années de service ou l'âge.

Ces deux régimes de référence sous-tendent "l'indice de progressivité » utilisé pour comparer les formules de calcul des prestations de retraite des différents pays. L'indice est conçu de telle façon qu'un système de base pur prend la valeur 100 et un régime strictement assurantiel la valeur zéro. Le premier est celui qui présente la progressivité maximale et le second n'est nullement progressif, car le taux de remplacement est constant. Un score élevé n'est pas forcément " meilleur » qu'un score bas et réciproquement. Les pays ayant un score élevé ont tout simplement des objectifs différents de ceux des pays dont le score est bas. "

La première colonne du tableau fait apparaître le coefficient de Gini pour les prestations de retraite brutes. La seconde colonne présente l'indice de progressivité de la formule de calcul des prestations. Outre les deux pays affichant un indice de 100, l'Australie, le Canada, la République tchèque et le Royaume-Uni sont dotés de systèmes de retraite extrêmement progressifs avec un indice proche de 70 ou supérieur. Ces pays sont tous dotés d'importants régimes de retraite de base ou ciblés.

À l'autre extrémité, la Finlande, la Grèce, la Hongrie, l'Italie, les Pays-Bas, la Pologne, le Portugal, la République slovaque et la Turquie disposent de régimes presque strictement proportionnels et donc très peu progressifs. L'indice y est inférieur à 10. Ce groupe comprend deux pays à comptes nationaux, dans lesquels les cotisations sont, à dessein, étroitement liées aux prestations. D'autres pays se situent entre ces deux groupes. La Suède se caractérise par un indice de progressivité négatif. Cette régressivité ressort dans le graphique du taux de remplacement brut figurant dans le « descriptif pays » de la partie III qui montre que les hauts et les bas revenus bénéficient de taux de remplacement supérieurs à ceux des revenus moyens.

Les deux dernières colonnes permettent de savoir si les inégalités en matière de droits à retraite s'expliquent par une inégalité dans la distribution des salaires à l'échelle nationale ou par des différences de formule de calcul des prestations. Les graphiques font apparaître la distribution des revenus pour une sélection de pays. De fait, l'indice de progressivité s'établit en moyenne à 40 pour les deux indicateurs dans les 18 pays pour lesquels on dispose de données complètes.

Enfin, il convient de noter que l'indice de progressivité de la formule de calcul des prestations de retraite mesure uniquement les volets obligatoires des systèmes de retraite. Certains pays sont dotés de vastes dispositifs individuels et professionnels privés. Si on en tenait compte, la distribution des revenus des retraités serait plus ouverte.

\section{Définition et calcul}

Les systèmes de retraite des pays de l'OCDE accordent une importance diverse au rôle de l'assurance et de la redistribution. L'indice de progressivité est conçu de telle façon qu'un régime de base pur prend la valeur 100 et un régime strictement assurantiel la valeur zéro. Les calculs reposent sur le coefficient de Gini, un indicateur courant des inégalités. Formellement, l'indice de progressivité correspond à 100 minoré du ratio du coefficient de Gini des droits à retraite sur le coefficient de Gini des salaires, pondéré dans les deux cas par la distribution des revenus. Les calculs ont été réalisés sur la base des données nationales (lorsque ces chiffres étaient disponibles) et de la distribution moyenne des revenus dans les pays de l'OCDE.

Cet indicateur est fondé sur l'analyse de Musgrave et Thin (1948). Il a été adopté par d'autres chercheurs (voir Biggs et al., 2009).

\section{Références}

Biggs, A.G., M. Sarney et C.R. Tamborini (2009), «A Progressivity Index for Social Security ", Issue Paper $n^{\circ}$ 2009-01, United States Social Security Administration, Washington, DC.

Musgrave, R.A et T. Thin (1948), "Income Tax Progression 1924-48 ", Journal of Political Economy, vol. 56, pp. 498-514. 


\section{Coefficients de Gini des droits à retraite et des salaires}

Données sur la distribution de la moyenne de l'OECD et la distribution des salaires à l'échelle nationale

\begin{tabular}{|c|c|c|c|c|c|}
\hline & \multicolumn{2}{|c|}{ Distribution de la moyenne de l'OCDE } & \multicolumn{3}{|c|}{ Distribution des salaires à l'échelle nationale } \\
\hline & Retraite Gini & Indice de progressivité & Retraite Gini & Indice de progressivité & Salaire Gini \\
\hline Australie & 8.1 & 70.3 & 8.1 & 70.1 & 27.2 \\
\hline Autriche & 18.7 & 31.2 & & & \\
\hline Belgique & 11.8 & 56.7 & 10.2 & 52.6 & 21.6 \\
\hline Canada & 3.3 & 87.7 & & & \\
\hline Rép. tchèque & 8.5 & 69 & 8.8 & 65.5 & 25.5 \\
\hline Danemark & 12.8 & 53.1 & & & \\
\hline Finlande & 24.9 & 8.5 & 22.6 & 5.9 & 24 \\
\hline France & 21.9 & 19.5 & & & \\
\hline Allemagne & 20.6 & 24.2 & 19.8 & 24.7 & 26.3 \\
\hline Grèce & 26.1 & 4.3 & & & \\
\hline Hongrie & 27.2 & 0 & 33.6 & 0 & 33.6 \\
\hline Islande & 22.5 & 17.2 & & & \\
\hline Irlande & 0 & 100 & 0 & 100 & 29.6 \\
\hline Italie & 26.8 & 1.6 & 23.3 & 1.8 & 23.7 \\
\hline Japon & 14.6 & 46.5 & 14.3 & 46 & 26.4 \\
\hline Corée & 9.3 & 65.8 & 10.2 & 65.5 & 29.6 \\
\hline Luxembourg & 22.5 & 17.3 & & & \\
\hline Mexique & 18.5 & 31.9 & & & \\
\hline Pays-Bas & 25.7 & 5.8 & 24.3 & 5.7 & 25.7 \\
\hline Nouvelle-Zélande & 0 & 100 & 0 & 100 & 27.7 \\
\hline Norvège & 16.8 & 38.4 & 13.6 & 38.1 & 22 \\
\hline Pologne & 26.3 & 3.4 & 29.2 & 4.1 & 30.5 \\
\hline Portugal & 26.2 & 3.8 & & & \\
\hline Rép. slovaque & 27 & 0.9 & & & \\
\hline Espagne & 22.4 & 17.9 & 25.7 & 17.1 & 31.1 \\
\hline Suède & 29.6 & -8.8 & 26.4 & -14.4 & 23.1 \\
\hline Suisse & 12.7 & 53.4 & & & \\
\hline Turquie & 26.5 & 2.8 & & & \\
\hline Royaume-Uni & 5.1 & 81.3 & 5.1 & 82.4 & 28.9 \\
\hline États-Unis & 16.1 & 40.8 & 16.1 & 50.8 & 32.7 \\
\hline OCDE moyenne & 17.8 & 34.8 & & & \\
\hline OCDE 18 & 16.3 & 40.1 & 16.2 & 39.8 & 27.2 \\
\hline
\end{tabular}

Note : “ OCDE 18 » désigne les pays pour lesquels on dispose de données sur la distribution des salaires à l'échelle nationale. Source: Modèles de retraite de l'OCDE ; base de données de l'OCDE sur la distribution des revenus.

Distribution des salaires : moyenne de l'OCDE et sélection de pays
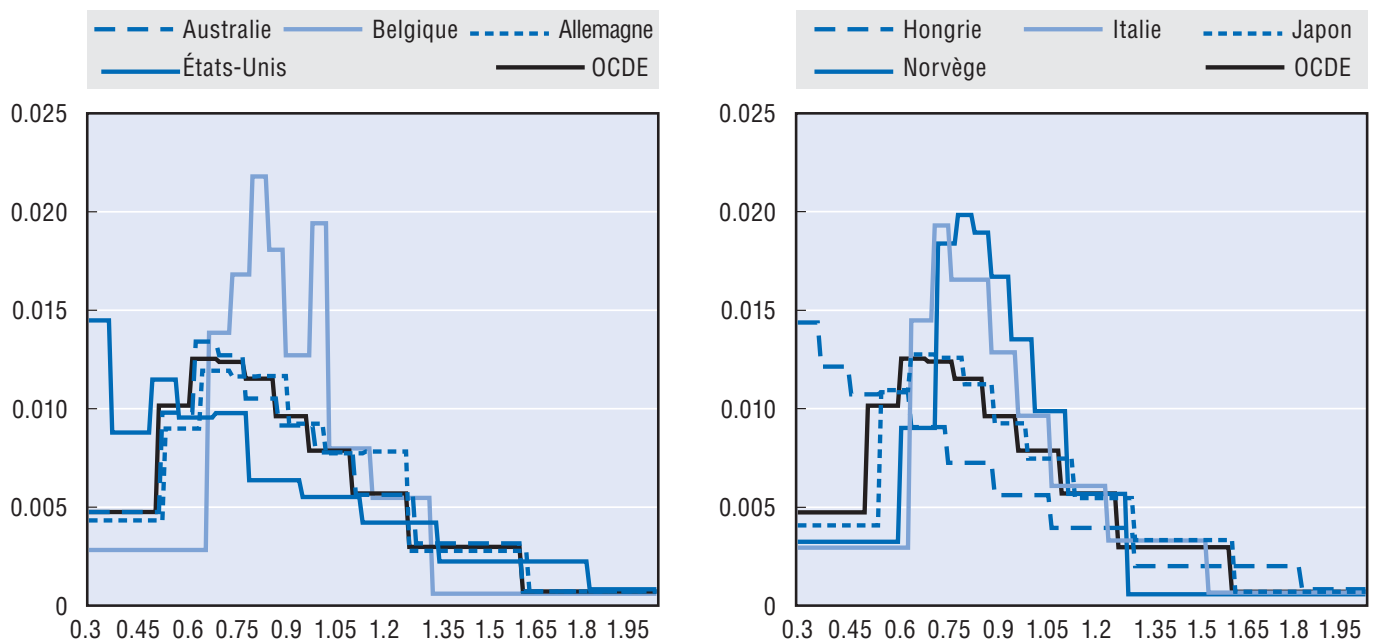

Source: Base de données de l'OCDE sur la distribution des revenus. 


\section{Principaux résultats}

Dans certains pays, comme la Hongrie, l'Italie ou la République slovaque, on observe une corrélation très étroite entre les droits à retraite et le salaire. Inversement, les prestations étant forfaitaires en Irlande et en Nouvelle-Zélande, les pensions de retraite ne sont pas liées à la rémunération.

Dans le graphique, le niveau relatif des pensions dans les pays de l'OCDE figure en ordonnée et le salaire individuel en abscisse. Les pays ont été regroupés en fonction de la corrélation (ou non) entre les prestations de retraite et le salaire individuel, sur la base de la distribution des prestations de retraite au regard de la distribution des salaires, exposée dans l'indicateur précédent : "progressivité de la formule de calcul des prestations de retraite ".

Dans le premier groupe de cinq pays (panel A), les prestations de retraite ne sont guère voire nullement liées au salaire. Outre les systèmes à taux uniforme en place en Irlande et en Nouvelle-Zélande, le niveau relatif des pensions varie peu au Canada, passant de $38 \%$ pour les faibles revenus à $44 \%$ pour le salaire moyen et au-delà. Bien que le Canada soit pourvu d'un régime de retraite lié à la rémunération (dit "régime fondé sur le revenu), son objectif de taux de remplacement est très faible, son plafond correspond au salaire moyen national et une prestation soumise à conditions de ressources peut être versée en fonction des revenus assurés par ce régime. Au Royaume-Uni, le dispositif lié à la rémunération est fondé sur une formule extrêmement progressive et un programme de retraite de base a également été mis en place. En Australie, le tracé relativement plat de la courbe s'explique principalement par le programme de retraite publique soumise à conditions de ressources. De plus, le salaire pour lequel les employeurs doivent cotiser dans le cadre du régime à cotisations définies est plafonné.

À l'autre extrémité se situent cinq pays où les droits à retraite sont très fortement corrélés au salaire (panel F). Aux Pays-Bas, le salaire ouvrant droit à retraite n'est pas plafonné dans les régimes professionnels quasi obligatoires. En République slovaque et en Italie, les limites imposées sur le salaire ouvrant droit à retraite correspondent au salaire moyen multiplié par trois ou plus. Dans ces pays, le niveau relatif de la pension augmente avec le salaire de façon linéaire sur la majeure partie de la fourchette indiquée.

La corrélation entre le salaire individuel et la pension est légèrement moins forte pour les cinq pays composant le panel E que pour ceux du panel $\mathrm{F}$, notamment parce que le Luxembourg et la Suède sont dotés de programmes à caractère redistributif visant un revenu minimum relativement élevé, soit $38 \%$ du salaire moyen.

L'autre moitié des pays de l'OCDE constituent des cas intermédiaires (entre ceux où il n'existe guère voire nullement de lien entre le salaire individuel et les pensions, et ceux où cette corrélation est forte ou très forte). Dans les dix pays des panels $B$ et $C$, le lien entre la pension de retraite et le salaire est plus étroit que dans le premier groupe de pays, mais le système de retraite repose sur une formule nettement plus progressive que celui des cinq pays du panel F. Aux États-Unis, en Norvège et en République tchèque, cette redistribution en faveur des faibles revenus tient essentiellement à une formule de calcul des prestations progressive, grâce à laquelle la fraction du salaire qui est remplacée est plus élevée pour les salariés plus démunis que pour ceux qui perçoivent un salaire moyen ou élevé. Pour ce faire, l'Islande a mis en place des programmes de retraite ciblés. Le Danemark est doté de vastes régimes ciblés et de base.

Le panel $\mathrm{D}$ contient cinq pays qui se situent dans la moyenne des économies de l'OCDE au regard de la corrélation entre les droits à retraite et le salaire. La France et le Portugal se caractérisent par des programmes de retraite redistributifs - minimum et ciblés - pour les bas revenus, et par une forte corrélation salaire-prestations pour les plus hauts revenus.

\section{Définition et calcul}

Le lien existant entre les droits à retraite et le salaire individuel est évalué à l'aide du niveau relatif des pensions, c'est-à-dire la retraite individuelle brute divisée par le salaire moyen brut à l'échelle nationale (et non le salaire individuel comme c'était le cas pour les taux de remplacement). Cet indicateur illustre davantage l'adéquation des pensions de retraite, car il fait apparaître le montant des prestations qui seront servies à un retraité par rapport au salaire moyen dans le pays concerné. Le niveau relatif des pensions rend compte de la corrélation existant entre le salaire individuel et les prestations de retraite, pour des personnes dont le salaire représente de 0.5 à 2 fois le salaire moyen. 


\section{Le lien entre le salaire et les droits à retraite}

Droits à retraite bruts en proportion du salaire moyen national

Panel A
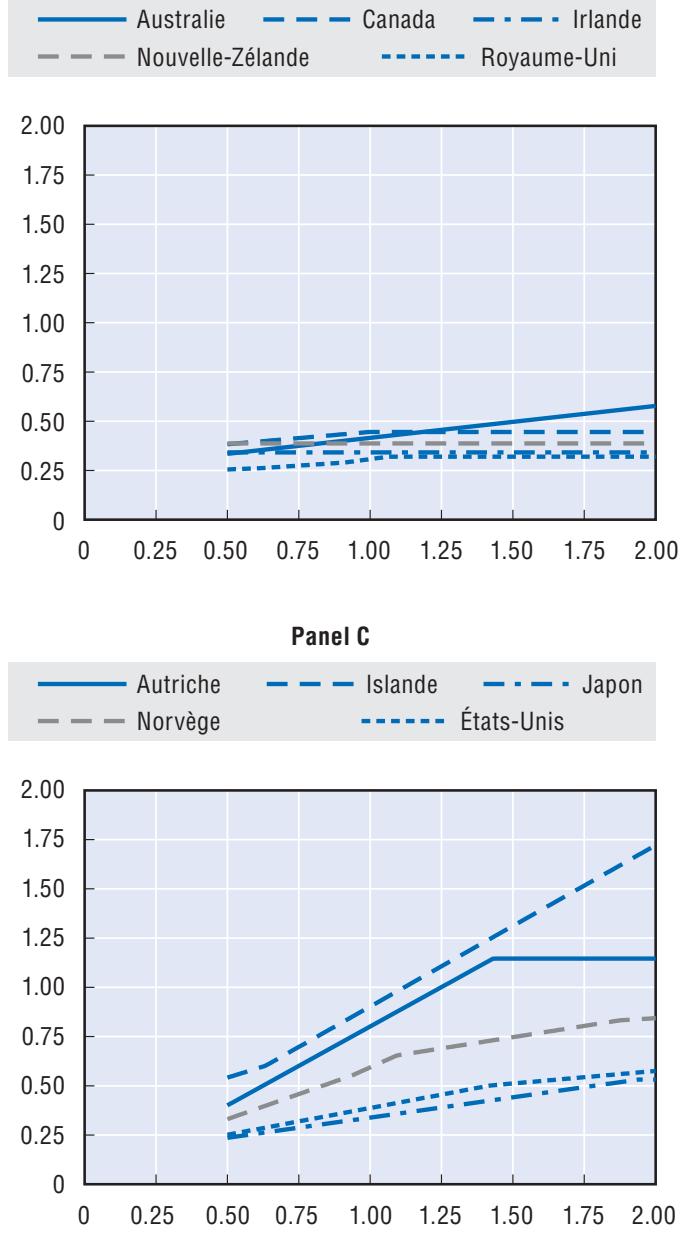

Panel E
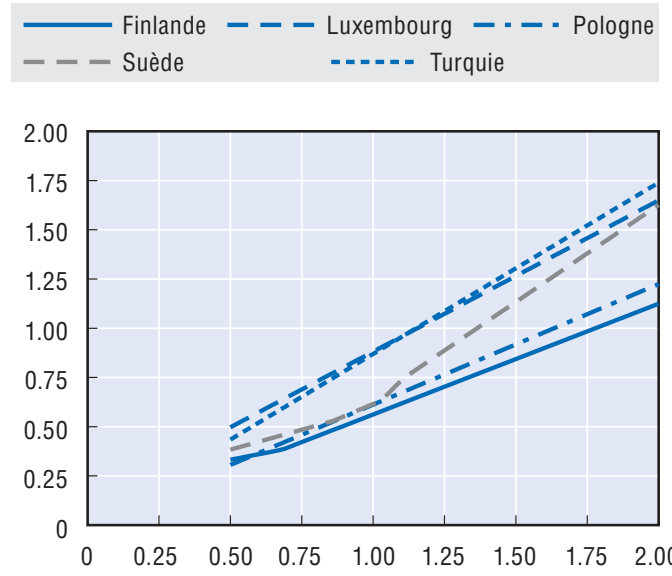

Panel B
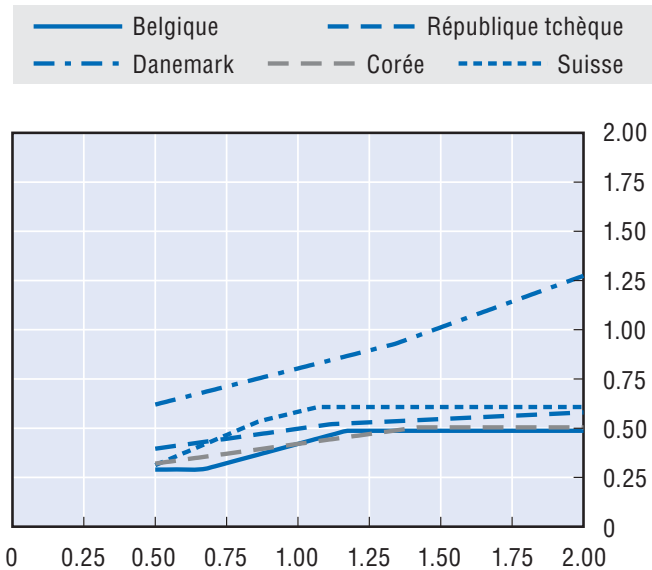

Panel D

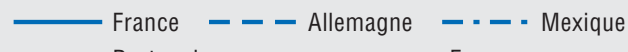

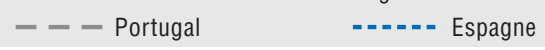

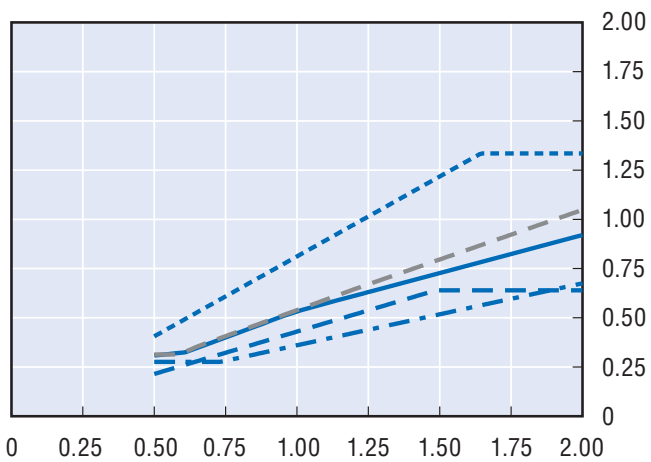

Panel F
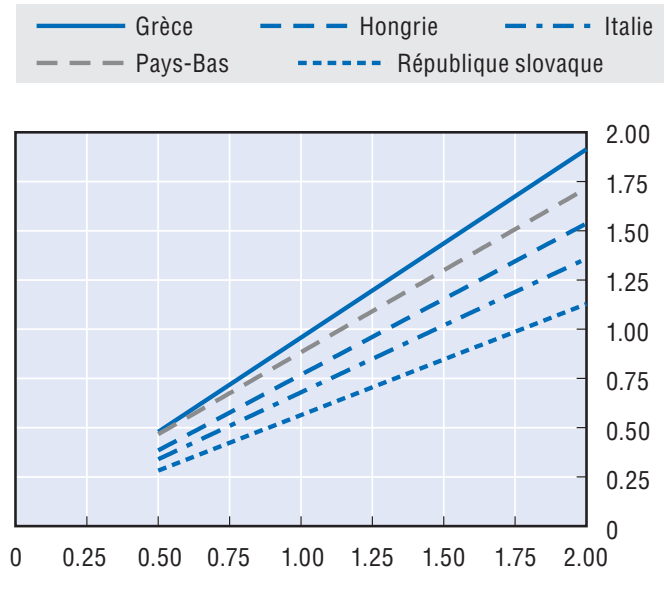

StatLink त्नाls http://dx.doi.org/10.1787/651411374141 


\section{Principaux résultats}

Les indicateurs présentés jusqu'à présent ont porté sur les taux de remplacement, le niveau relatif des pensions et le patrimoine retraite pour différents niveaux de rémunération. Fondés sur la moyenne pondérée de ces indicateurs sur toute la fourchette des revenus, les chiffres présentés ici correspondent à la pension moyenne au moment du départ en retraite et au patrimoine retraite moyen. Le premier indicateur rend compte du revenu moyen perçu au titre de la retraite, en prenant en considération les différences de régimes appliqués aux salariés percevant des revenus différents. Le second est destiné à évaluer de façon synthétique le coût total des prestations de retraite.

Dans les pays de l'OCDE, la pension moyenne pondérée représente $57.6 \%$ du salaire moyen national. Le patrimoine retraite moyen pondéré correspond au salaire moyen multiplié par 9.8 pour les hommes et 11.4 pour les femmes.

La mesure du niveau relatif moyen pondéré des pensions associe des données sur la distribution des salaires aux calculs des droits à retraite. Le niveau relatif des pensions est calculé en moyenne sur toute l'échelle de distribution des revenus et pondéré pour tenir compte du fait que les salariés sont plus nombreux à percevoir un salaire inférieur à la moyenne. La pension moyenne pondérée est exprimée en pourcentage du salaire moyen national. Les résultats apparaissent dans les première et deuxième colonnes du tableau pour les hommes et les femmes, respectivement.

En tête du classement, les pensions moyennes pondérées de la Grèce et de l'Islande, suivies de près par les Pays-Bas et le Luxembourg, dépassent $86 \%$ du salaire moyen. Dans cinq pays - le Danemark, l'Espagne, l'Autriche, la Hongrie et la Suède -, la pension moyenne pondérée représente plus de $70 \%$ du salaire moyen. En bas du classement, elle est inférieure à $40 \%$ dans sept pays de l'OCDE (la Nouvelle-Zélande, la Belgique, le Mexique, les États-Unis, l'Irlande, le Japon et le Royaume-Uni).

Cette méthode de pondération peut aussi s'appliquer au patrimoine retraite, qui est l'indicateur le plus complet de l'ampleur des promesses de retraite faites aux salariés d'aujourd'hui (troisième et quatrième colonnes du tableau). Les moyennes des pays de l'OCDE s'élèvent à 407000 USD pour les hommes et 476000 USD pour les femmes (cinquième et sixième colonnes du tableau).

Pour le patrimoine retraite moyen pondéré, on observe des valeurs nettement supérieures à la moyenne - soit le salaire moyen multiplié par 13.6 à 16.5 pour les hommes et 15.6 à 19.3 pour les femmes - au Danemark, en Grèce, en Islande et aux Pays-Bas. L'Autriche, l'Espagne, la Hongrie, l'Italie et la Turquie sont au coude à coude, avec des valeurs de quelque 10 à 12 fois le salaire moyen. Converties en USD, les promesses de retraite s'élèvent dans ces neuf pays à 565000 USD pour les hommes et à plus de 650000 USD pour les femmes. Il s'agit de la valeur actualisée des transferts que les sociétés promettent d'effectuer en moyenne en faveur des futurs retraités selon les règles actuelles du système de retraite.

À l'autre extrémité, le patrimoine retraite s'inscrit nettement en deçà de la moyenne de l'OCDE dans quatre pays (les États-Unis, le Japon, le Mexique et le Royaume-Uni), avec moins de 6 fois le salaire moyen pour les hommes et 7 fois pour les femmes.

Les promesses de retraite évaluées à l'aide du patrimoine retraite moyen pondéré sont également moindres dans les pays où l'espérance de vie est plus courte, comme en Pologne.

\section{Définition et calcul}

En s'appuyant sur les résultats obtenus pour les taux de remplacement et le niveau des pensions sur toute la gamme des rémunérations individuelles, il est possible d'élaborer des indicateurs composites des systèmes de retraite nationaux qui agrègent les résultats pour des salaires plus ou moins élevés. Ces indicateurs sont la pension moyenne pondérée et le patrimoine retraite moyen pondéré. Ils sont fondés sur les calculs des droits à retraite de personnes touchant entre 0.3 et 3 fois le salaire moyen national.

Chaque niveau de rémunération individuelle reçoit une pondération en fonction de son importance dans la distribution des salaires. Les calculs s'appuient sur la distribution moyenne des salaires fondée sur des données concernant 18 pays de l'OCDE. La distribution des revenus est asymétrique. Le mode (ou pic) de la distribution se situe aux alentours des deux tiers du salaire moyen. La médiane (soit le salaire de part et d'autre duquel les salariés se répartissent en nombre égal) représente généralement de $80 \%$ à $85 \%$ du salaire moyen. Les deux tiers des salariés gagnent moins que le salaire moyen. On compte donc un grand nombre de salariés faiblement rémunérés et une moins grande proportion de personnes à haut revenu, de sorte que les bas salaires se voient octroyer une pondération plus forte que les hauts revenus dans les calculs de l'indicateur. 


\section{Moyennes pondérées}

\begin{tabular}{|c|c|c|c|c|c|c|}
\hline & \multicolumn{2}{|c|}{ Pension moyenne pondérée } & \multicolumn{2}{|c|}{ Patrimoine retraite moyen pondéré } & \multicolumn{2}{|c|}{ Patrimoine retraite moyen (USD) } \\
\hline & Hommes & Femmes & Hommes & Femmes & Hommes & Femmes \\
\hline Allemagne & 40.5 & 40.5 & 7 & 8.3 & 291000 & 345000 \\
\hline Australie & 41.5 & 41.5 & 8.1 & 9.5 & 337000 & 395000 \\
\hline Autriche & 72.4 & 72.4 & 11.4 & 13.2 & 474000 & 549000 \\
\hline Belgique & 38.6 & 38.6 & 6.5 & 7.6 & 270000 & 316000 \\
\hline Canada & 42 & 42 & 7.9 & 9.2 & 328000 & 382000 \\
\hline Corée & 40.2 & 40.2 & 6.7 & 8 & 278000 & 333000 \\
\hline Danemark & 81.8 & 81.8 & 13.6 & 15.6 & 565000 & 648000 \\
\hline Espagne & 73 & 73 & 12 & 14 & 499000 & 582000 \\
\hline États-Unis & 37.1 & 37.1 & 5.9 & 6.8 & 245000 & 283000 \\
\hline Finlande & 57.6 & 57.6 & 9.1 & 10.8 & 378000 & 449000 \\
\hline France & 51.2 & 51.2 & 9.5 & 11 & 395000 & 457000 \\
\hline Grèce & 93.6 & 93.6 & 14.2 & 16.5 & 590000 & 686000 \\
\hline Hongrie & 72.3 & 72.3 & 12.4 & 15.4 & 515000 & 640000 \\
\hline Irlande & 34.2 & 34.2 & 8 & 9.5 & 333000 & 395000 \\
\hline Islande & 90.9 & 90.9 & 14.6 & 16.4 & 607000 & 682000 \\
\hline Italie & 69.3 & 53.9 & 10 & 10.8 & 416000 & 449000 \\
\hline Japon & 33.5 & 33.5 & 6 & 6.8 & 249000 & 283000 \\
\hline Luxembourg & 86.4 & 86.4 & 19.8 & 24.2 & 823000 & 1006000 \\
\hline Mexique & 37.8 & 33.5 & 5.5 & 6.1 & 229000 & 254000 \\
\hline Norvège & 54.2 & 54.2 & 10.2 & 12 & 424000 & 499000 \\
\hline Nouvelle-Zélande & 38.7 & 38.7 & 9.1 & 10.6 & 378000 & 441000 \\
\hline Pays-Bas & 89.4 & 89.4 & 16.5 & 19.3 & 686000 & 802000 \\
\hline Pologne & 59.3 & 44.2 & 8.5 & 9.2 & 353000 & 382000 \\
\hline Portugal & 53.6 & 53.6 & 8.1 & 9.5 & 337000 & 395000 \\
\hline Rép. tchèque & 47.2 & 47.2 & 8.5 & 10 & 353000 & 416000 \\
\hline Rép. slovaque & 56.1 & 56.1 & 8.8 & 10.7 & 366000 & 445000 \\
\hline Royaume-Uni & 28.9 & 28.9 & 4.7 & 5.4 & 195000 & 224000 \\
\hline Suède & 70.6 & 70.6 & 10.9 & 12.5 & 453000 & 520000 \\
\hline Suisse & 49.2 & 49.7 & 9.4 & 11.5 & 391000 & 478000 \\
\hline Turquie & 86.4 & 86.4 & 11.1 & 13 & 461000 & 540000 \\
\hline OCDE moyenne & 57.6 & 56.4 & 9.8 & 11.4 & 407000 & 476000 \\
\hline
\end{tabular}

\section{Comparaison des moyennes pondérées : pension et patrimoine retraite hommes-femmes}
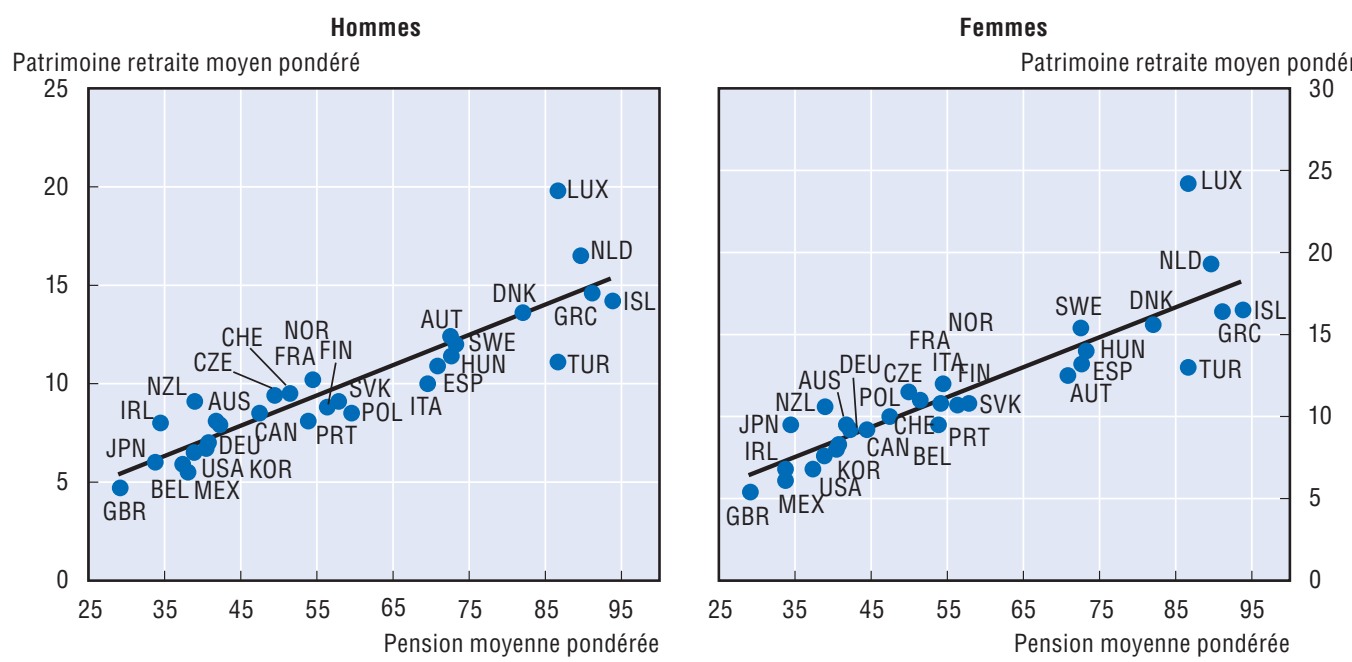

Source: Modèles des pensions de l'OCDE ; Base de données de l'OCDE sur la distribution des salaires. 


\section{Principaux résultats}

La prestation globale de retraite se subdivise en différentes composantes conformément à la taxinomie du "Cadre des Pensions dans les pays de l'OCDE " présenté ci-dessus. Les systèmes de retraite comportent ainsi deux piliers obligatoires : i) une composante redistributive, destinée à garantir aux retraités un niveau de vie minimum en termes absolus ; et ii) une composante assurantielle, afin qu'ils perçoivent un revenu cible comparable à celui qu'ils touchaient lorsqu'ils étaient en activité. Cet indicateur, qui montre l'articulation des systèmes de retraite nationaux en piliers et en différentes prestations au sein de ces piliers, fait une fois encore ressortir de fortes disparités entre les politiques menées par les pays.

Les prestations du premier pilier, à vocation redistributive, se subdivisent en trois.

Tout d'abord, les retraites minimums, importantes dans 13 pays, visent à empêcher les prestations de retraite (fréquemment servies par un seul dispositif) de tomber en deçà d'un certain seuil. En Belgique et au Royaume-Uni, les crédits minimums de retraite produisent un effet analogue : les prestations versées aux salariés faiblement rémunérés sont calculées en fonction d'un salaire plus élevé. Ces crédits constituent une très large part de l'ensemble des prestations servies au Royaume-Uni. Les retraites minimum occupent également une place importante en Belgique, en France, au Mexique et en Suède.

Les régimes de base, dans lesquels le montant des prestations ne dépend ni du salaire ni du niveau d'autres pensions, relèvent également du premier pilier. Ces régimes (ou des dispositifs ayant des effets similaires en Corée et au Mexique) existent dans 13 pays de l'OCDE. En Irlande et en Nouvelle-Zélande, les retraites obligatoires s'inscrivent exclusivement dans ce cadre. En Corée, au Japon, aux Pays-Bas et au Royaume-Uni, les retraites de base contribuent à la promesse totale de retraite à hauteur de $40 \%$ à $60 \%$. Elles jouent également un grand rôle au Canada, au Danemark et en Norvège, avec de $25 \%$ à $35 \%$ des ressources allouées aux retraités.

Tous les pays de l'OCDE ont mis en place un filet de protection des retraités à faible revenu. Dans la plupart d'entre eux, toutefois, les salariés ayant réalisé une carrière complète n'y ont pas droit, même si leur rémunération est basse. On note sept exceptions. L'Australie est en l'exemple le plus frappant, puisque les prestations au titre du premier pilier sont intégralement soumises à conditions de ressources et qu'elles constituent pratiquement la moitié de la prestation globale de retraite. Au Canada et au Danemark, elles ont également une fonction capitale, puisqu'elles représentent entre $14 \%$ et $21 \%$ de la promesse de retraite, respectivement.

L'importance relative des régimes du premier et du deuxième piliers dans la prestation globale de retraite apparaît dans le graphique de gauche. Le deuxième pilier représente $95 \%$ au moins dans plus de la moitié des pays de l'OCDE. Dans certains d'entre eux - l'Autriche, l'Espagne, l'Italie, la Pologne et la Turquie -, cela correspond à des objectifs de taux de remplacement ambitieux. Dans d'autres, comme les États-Unis ou la
Suisse, la formule de calcul des prestations du régime public revêt un caractère progressif : la redistribution assurée dans les autres pays par le biais du premier pilier est réalisée ici par les dispositifs du deuxième pilier. À l'autre extrémité, il n'existe aucun régime de retraite obligatoire au titre du deuxième pilier en Irlande et en Nouvelle-Zélande, et au Royaume-Uni, le régime lié à la rémunération sert principalement à verser des prestations calculées à partir des crédits minimums.

Le deuxième pilier, à caractère obligatoire, se répartit, comme on le voit dans le tableau, entre les prestations publiques et privées et, dans le deuxième cas, entre dispositifs à cotisations définies (CD) et à prestations définies (PD). Des régimes publics liés à la rémunération ont été mis en place dans 23 pays de l'OCDE. Ils servent la quasi-totalité des prestations dues aux salariés ayant réalisé une carrière complète dans neuf pays : l'Allemagne, l'Autriche, l'Espagne, les États-Unis, la Finlande, la Grèce, l'Italie, le Portugal et la Turquie.

Les régimes privés sont obligatoires ou quasi obligatoires dans 11 pays. Il s'agit de dispositifs à prestations définies en Islande, aux Pays-Bas et en Suisse, mais à cotisations définies dans la plupart des cas. Dans cinq pays - l'Australie, le Danemark, les Pays-Bas, la Pologne et la République slovaque - ils représentent de $50 \%$ à $60 \%$ de l'ensemble de la prestation de retraite obligatoire. Ils jouent un rôle bien plus substantiel en Islande et au Mexique. L'importance relative des prestations publiques et privées apparaît dans le graphique de droite.

\section{Définition et calcul}

La structure de la prestation globale de retraite est présentée à l'aide de l'indicateur du patrimoine retraite moyen pondéré expliqué précédemment, en fonction des différentes composantes du système de retraite. Les pondérations sont tirées de la distribution des revenus.

En règle générale, les cellules vides indiquent qu'un pays ne dispose pas de ce type de dispositif de retraite. Cela étant, il ne faut pas oublier que les calculs correspondent à des carrières complètes. L'ensemble des programmes du premier pilier sont bien plus décisifs pour les personnes dont la carrière est incomplète. Néanmoins, il n'est guère facile d'obtenir des informations sur la distribution des prestations perçues, et encore moins d'établir des prévisions en la matière. 


\section{Structure de la prestation globale de retraite}

Contribution (en \%) des composantes du système de retraite au patrimoine retraite moyen pondéré

\begin{tabular}{|c|c|c|c|c|c|c|c|c|c|c|c|c|c|c|c|}
\hline & \multicolumn{3}{|c|}{ Premier pilier } & \multicolumn{3}{|c|}{ Deuxième pilier } & \multirow[b]{2}{*}{ Total } & \multicolumn{4}{|c|}{ Premier pilier } & \multicolumn{3}{|c|}{ Deuxième pilier } & \multirow[b]{2}{*}{ Tota } \\
\hline & $\begin{array}{c}\text { Soumis à } \\
\text { conditions } \\
\text { de } \\
\text { ressources }\end{array}$ & De base & Minimum & Public & $\begin{array}{l}\text { PD } \\
\text { privé }\end{array}$ & $\begin{array}{c}\text { CD } \\
\text { privé }\end{array}$ & & & $\begin{array}{c}\text { Soumis à } \\
\text { conditions } \\
\text { de } \\
\text { ressources }\end{array}$ & De base & Minimum & Public & $\begin{array}{l}\text { PD } \\
\text { privé }\end{array}$ & $\begin{array}{l}\text { CD } \\
\text { privé }\end{array}$ & \\
\hline Allemagne & 1.5 & & & 98.5 & & & 100 & Italie & & & & 100.0 & & & 100 \\
\hline Australie & 49.2 & & & & & 50.8 & 100 & Japon & & 44.3 & & 55.7 & & & 100 \\
\hline Autriche & & & & 100.0 & & & 100 & Luxembourg & & $15.8^{6}$ & 0.2 & 84.1 & & & 100 \\
\hline Belgique & & & $4.4^{1}$ & 95.5 & & & 100 & Mexique & & 14.0 & $10.3^{7}$ & & & 75.7 & 100 \\
\hline Canada & 21.4 & 35.2 & & 43.4 & & & 100 & Norvège & & 32.4 & 1.2 & 56.5 & & 10.0 & 100 \\
\hline Corée & & $60.1^{5}$ & & 39.9 & & & 100 & Nouvelle-Zélande & & 100 & & & & & 100 \\
\hline Danemark & 13.8 & 26.3 & & & & $59.7^{2}$ & 100 & Pays-Bas & & 41.2 & & & 58.8 & & 100 \\
\hline Espagne & & & 1.2 & 98.8 & & & 100 & Pologne & & & 1.5 & 48.2 & & 50.3 & 100 \\
\hline États-Unis & & & & 100.0 & & & 100 & Portugal & & & 1.8 & 98.2 & & & 100 \\
\hline Finlande & & & 2.9 & 97.1 & & & 100 & Rép. slovaque & & & 0.3 & 44.9 & & 54.8 & 100 \\
\hline France & & & 4.7 & $95.3^{3}$ & & & 100 & Rép. tchèque & & 17.1 & & 82.9 & & & 100 \\
\hline Grèce & & & & $100^{4}$ & & & 100 & Royaume-Uni & 0.7 & 50 & $36.4^{9}$ & 12.9 & & & 100 \\
\hline Hongrie & & & & 65.9 & & 34.1 & 100 & Suède & & & 4.5 & 52.6 & & $42.9^{8}$ & 100 \\
\hline Irlande & & 100 & & & & & 100 & Suisse & 0.2 & & & 72.0 & 27.8 & & 100 \\
\hline Islande & 3.5 & 13.0 & & & 83.4 & & 100 & Turquie & & & 1.1 & 98.9 & & & 100 \\
\hline
\end{tabular}

$\mathrm{CD}=$ à cotisations définies $; \mathrm{PD}=$ à prestations définies.

1. Belgique : retraite minimum et crédits minimums inclus.

2. Danemark : les régimes privés à cotisations définies comprennent les dispositifs professionnels quasi obligatoires (51.0 \%) et le régime spécial $(5.0 \%)$.

3. France : les pensions publiques correspondent au régime général (64.2\%) et au mécanisme professionnel complémentaire (31.1 \%)

4. Grèce : les pensions publiques sont composées des composantes principale (73.0\%) et complémentaire (27\%).

5. Corée : la retraite de base représente la part de la retraite publique qui est fondée sur le salaire moyen et non sur la rémunération individuelle.

6. Luxembourg : la retraite de base inclut également l'allocation de fin d'année.

7. Mexique : la composante de base est calculée à partir de l'abondement forfaitaire de l'État aux comptes à cotisations définies qui s'élève à $5.5 \%$ du salaire minimum réel à partir de 1997.

8. Suède : les régimes privés à cotisations définies comprennent les deux dispositifs à cotisations définies (12 \% et $30.9 \%$ ).

9. Royaume-Uni : la retraite minimum correspond aux crédits minimums prévus par le régime public lié à la rémunération.

Source: Modèles de retraite de l'OCDE.

Importance relative des programmes redistributifs du premier pilier et des régimes d'assurance du deuxième pilier

En \% du patrimoine retraite moyen pondéré

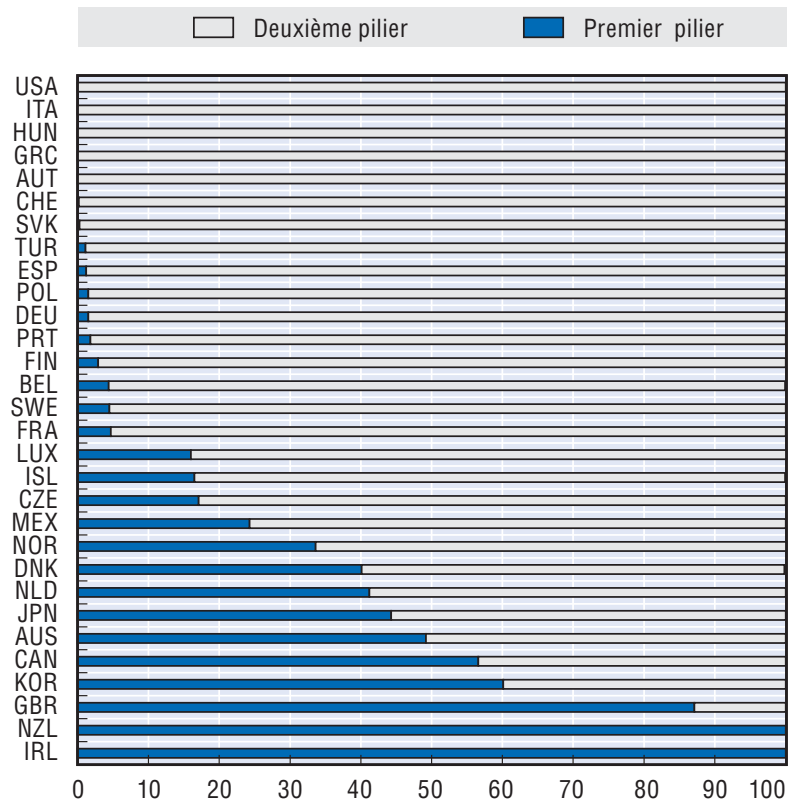

Source: Modèles de retraite de l'OCDE.

\section{Importance relative des prestations publiques et des prestations privées au titre des régimes obligatoires}

En \% du patrimoine retraite moyen pondéré

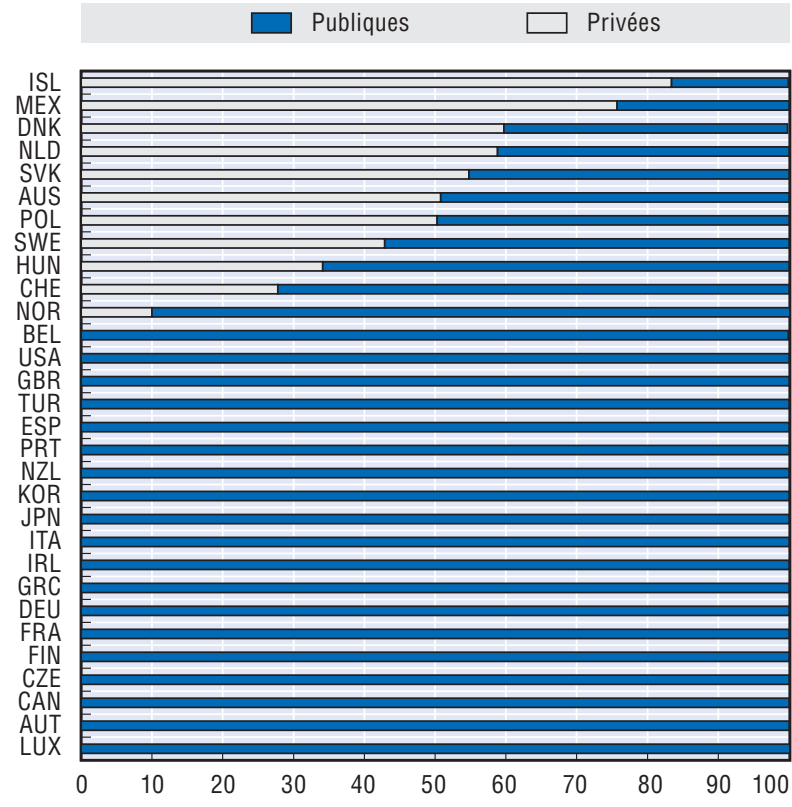

Source: Modèles de retraite de l'OCDE. 



\section{Les systèmes de retraite}

Le premier jeu d'indicateurs concernait essentiellement les droits à retraite individuels. Ce deuxième groupe, en revanche, porte sur les systèmes de retraite dans leur ensemble.

Les deux premiers indicateurs de cette section s'intéressent au financement des retraites, c'est-à-dire aux cotisations pour les régimes publics et aux dépenses consacrées au versement des prestations de retraite. Les taux de cotisation des régimes publics, lorsqu'il est possible de les identifier, sont présentés pour plusieurs années comprises entre 1994 et 2007. L'indicateur sur les dépenses correspond à la proportion du revenu national qui est affectée au paiement des retraites. Il témoigne également de la place qu'occupent les retraites publiques dans le budget global de l'État.

Le troisième indicateur a trait aux régimes de retraite privés, et met en évidence la proportion de la population active qui est couverte par les dispositifs obligatoires, quasi obligatoires et facultatifs. Dans de nombreux pays, les régimes de retraite privés obligatoires et les dispositifs publics de prestations " en nature " (pour le logement, par exemple) représentent d'importantes sources de revenu pour les personnes âgées. Lorsque celles-ci sont disponibles, les données relatives aux dépenses réalisées dans ces domaines sont également indiquées. L'évolution de tous ces indicateurs du coût des retraites est exposée de 1990 à 2006.

Le dernier indicateur porte sur les actifs des fonds de pension auxquels sont adossées les promesses de retraite publiques et privées. Il présente des chiffres pour l'année 2007. 


\section{Principaux résultats}

Les taux des cotisations de retraite sont restés globalement stables depuis le milieu des années 90 . Le taux moyen pour les 21 pays de l'OCDE qui prélèvent des cotisations distinctes au titre des régimes publics est revenu de $20.0 \%$ en 1994 à $21.0 \%$ en 2007, vraisemblablement parce que les pouvoirs publics s'inquiétaient des conséquences pour l'emploi d'une lourde fiscalité du travail. Ces préoccupations semblent en effet avoir pris le pas sur les pressions exercées sur l'équilibre financier des systèmes de retraite par le vieillissement de la population et la maturation des dispositifs.

Dans les 23 pays pour lesquels les données sont disponibles, les recettes issues de ces cotisations représentent en moyenne $5.0 \%$ du revenu national, soit $14.1 \%$ du total des recettes que tire l'État des prélèvements et des cotisations.

La majorité des indicateurs présentés dans Les pensions dans les pays de l'OCDE concernent l'aspect "prestations » des systèmes de retraite. Les présents indicateurs s'intéressent à l'aspect « cotisations ».

On trouvera dans la partie gauche du tableau l'évolution des taux de cotisation. Dans un tiers environ des pays qui prélèvent des cotisations de retraite distinctes, les taux sont demeurés inchangés entre 2004 et 2007 : l'Autriche, la Belgique, les États- Unis, la Grèce, le Luxembourg et la Turquie. De plus, ils n'ont que fort peu évolué en Allemagne et en Suisse. On relève des augmentations sensibles au Canada, en Corée et en République tchèque, même si dans les deux premiers cas, les taux étaient assez bas à l'origine. Parmi les variations les moins marquées, on note de légères hausses en Finlande, en France, en Italie et en Pologne, probablement sous l'effet du gonflement des dépenses publiques au titre des retraites. On observe en revanche une réduction des taux de cotisation en Espagne, au Japon et en République slovaque, souvent motivée par une volonté d'alléger la fiscalité du travail afin de développer l'emploi.

La partie droite du tableau concerne les fonds collectés grâce aux cotisations aux régimes de retraite publics. Les chiffres des recettes viennent en complément de ceux du taux de cotisation, car ils témoignent des répercussions d'autres paramètres du système de retraite. Ainsi, la plupart des pays de l'OCDE ont fixé un plafond pour les cotisations de retraite, qui s'échelonnent du salaire moyen, environ, au salaire moyen multiplié par 3.7 en Italie et 5.9 au Mexique. L'abaissement du plafond entraînera naturellement une contraction des recettes pour un taux de cotisation donné. Dans d'autres pays, les cotisations sont soumises à des seuils, de sorte que les cotisations des faibles revenus peuvent être faibles ou nulles. Enfin, les recettes de certains pays peuvent se ressentir de la taille du secteur informel ou d'un phénomène de sousdéclaration des revenus.

Les recettes publiques issues des cotisations de retraite sont les plus élevées en Italie, avec $9.4 \%$ du produit intérieur brut (PIB). Malgré un taux de cotisation pratiquement équivalent à la moyenne des pays de l'OCDE, la Turquie ne tire que $2.2 \%$ de son revenu national des cotisations, en raison de l'ampleur du secteur informel. Les recettes provenant des cotisations sont également peu importantes au Canada - $2.7 \%$ du PIB - le taux de cotisation étant assez bas (soit la moitié de la moyenne de l'OCDE), à l'instar du plafond (pratiquement égal au salaire moyen).

En moyenne, les cotisations salariales représentent au total $1.8 \%$ du PIB, contre $2.9 \%$ pour les cotisations patronales. Les salariés versent $36 \%$ des cotisations totales, en moyenne, contre $58 \%$ pour les employeurs (le solde provient essentiellement des travailleurs indépendants, bien qu'il inclue également les cotisations versées par d'autres catégories, comme les chômeurs). La majorité des cotisations sont acquittées par les employeurs en Espagne, en Finlande, en Hongrie, en Italie et en République tchèque. Il ne faut pas oublier toutefois que comme l'ont montré de nombreuses analyses économiques, les sommes prélevées auprès des employeurs sont répercutées, en totalité ou en partie, sur les salariés, par exemple sous la forme d'une diminution des salaires ou du nombre d'emplois. Les cotisations se répartissent équitablement entre employeurs et salariés dans bien des pays, dont l'Allemagne, l'Autriche, la Belgique, le Canada, les États-Unis, le Japon et la Suisse.

La dernière colonne du tableau présente les cotisations de retraite exprimées en pourcentage du total des recettes que tire l'État des prélèvements et des cotisations. L'Italie ne se classe pas en tête dans ce cas. En Espagne, en Grèce et en Pologne, les cotisations de retraite représentent de $23 \%$ à $24 \%$ des recettes totales, contre $22.4 \%$ en Italie. En Australie, au Danemark et en Nouvelle-Zélande, les retraites sont financées par les recettes générales. Pour les raisons exposées ci-dessus, les cotisations de retraite constituent une part relativement faible des recettes de l'État au Canada, en Corée et en Turquie. 


\section{Taux de cotisation aux régimes publics et recettes tirées des cotisations de retraite}

\begin{tabular}{|c|c|c|c|c|c|c|c|c|}
\hline & \multirow{2}{*}{\multicolumn{4}{|c|}{ Taux des cotisations de retraite (en \% du salaire brut) }} & \multicolumn{4}{|c|}{ Recettes tirées des cotisations de retraite, 2006} \\
\hline & & & & & \multicolumn{3}{|c|}{ (en \% du PIB) } & \multirow{2}{*}{$\begin{array}{c}\text { (en \% } \\
\text { du total des } \\
\text { prélèvements }\end{array}$} \\
\hline & 1994 & 1999 & 2004 & 2007 & Salariés & Employeurs & Total & \\
\hline Allemagne & 19.2 & 19.7 & 19.5 & 19.5 & 2.6 & 2.7 & 5.8 & 16.4 \\
\hline Australie & \multicolumn{4}{|c|}{ Régimes privés uniquement } & 0.0 & 0.0 & 0.0 & 0.0 \\
\hline Autriche & 22.8 & 22.8 & 22.8 & 22.8 & 3.5 & 3.7 & 7.9 & 18.9 \\
\hline Belgique & 16.4 & 16.4 & 16.4 & 16.4 & 2.2 & 2.0 & 4.6 & 10.4 \\
\hline Canada & 5.2 & 7.0 & 9.9 & 9.9 & 1.3 & 1.3 & 2.7 & 8.1 \\
\hline Corée & 6.0 & 9.0 & 9.0 & 9.0 & 1.6 & 1.0 & 2.6 & 9.8 \\
\hline Danemark & \multicolumn{4}{|c|}{ Régimes privés uniquement } & 0.0 & 0.0 & 0.0 & 0.0 \\
\hline Espagne & 29.3 & 28.3 & 28.3 & 28.3 & 1.3 & 6.6 & 8.5 & 23.3 \\
\hline États-Unis & 12.4 & 12.4 & 12.4 & 12.4 & 2.3 & 2.3 & 4.6 & 17.2 \\
\hline Finlande & 18.6 & 21.5 & 21.4 & 20.9 & 1.6 & 6.9 & 8.9 & 20.5 \\
\hline France & 21.5 & 24.0 & 24.0 & 24.0 & & & & \\
\hline Grèce & 20.0 & 20.0 & 20.0 & 20.0 & 2.9 & 3.5 & 7.5 & 23.9 \\
\hline Hongrie & 30.5 & 30.0 & 26.5 & 26.5 & 1.0 & 4.8 & 5.8 & 15.7 \\
\hline Irlande & \multicolumn{4}{|c|}{ Pas de cotisation de retraite distincte } & & & & \\
\hline Islande & \multicolumn{4}{|c|}{ Pas de cotisation de retraite distincte } & & & & \\
\hline Italie & 28.3 & 32.7 & 32.7 & 32.7 & 2.2 & 7.3 & 9.4 & 22.4 \\
\hline Japon & 16.5 & 17.4 & 13.9 & 14.6 & 2.9 & 2.9 & 5.9 & 21.0 \\
\hline Luxembourg & 16.0 & 16.0 & 16.0 & 16.0 & 2.5 & 2.2 & 4.8 & 13.3 \\
\hline Mexique & \multicolumn{4}{|c|}{ Régimes privés uniquement } & 0.0 & 0.0 & 0.0 & 0.0 \\
\hline Norvège & \multicolumn{4}{|c|}{ Pas de cotisation de retraite distincte } & & & & \\
\hline Nouvelle-Zélande & \multicolumn{4}{|c|}{ Pas de cotisations } & 0.0 & 0.0 & 0.0 & 0.0 \\
\hline Pays-Bas & 33.1 & 37.7 & 28.1 & 31.1 & & & & \\
\hline Pologne & & 32.5 & 32.5 & 35.0 & 4.3 & 3.7 & 8.1 & 24.0 \\
\hline Portugal & \multicolumn{4}{|c|}{ Pas de cotisation de retraite distincte } & & & & \\
\hline Rép. slovaque & 28.5 & 27.5 & 26.0 & 24.0 & 1.3 & 2.3 & 5.2 & 17.4 \\
\hline Rép. tchèque & 26.9 & 26 & 28.0 & 32.5 & 1.7 & 5.7 & 7.8 & 21.2 \\
\hline Royaume-Uni & \multicolumn{4}{|c|}{ Pas de cotisation de retraite distincte } & & & & \\
\hline Suède & 19.1 & 15.1 & 18.9 & 18.9 & 2.5 & 3.6 & 6.2 & 12.7 \\
\hline Suisse & 9.8 & 9.8 & 9.8 & 10.1 & 2.8 & 2.7 & 6.0 & 20.4 \\
\hline Turquie & 20.0 & 20.0 & 20.0 & 20.0 & 1.1 & 1.1 & 2.2 & 8.8 \\
\hline OCDE & 20.0 & 20.7 & 20.2 & 21.0 & 1.8 & 2.9 & 5.0 & 14.1 \\
\hline
\end{tabular}

Note: Tous les chiffres sont arrondis à une décimale. Concernant les taux de cotisation, la moyenne de l'OCDE ne tient pas compte des pays où les cotisations de retraite n'existent pas ou sont incluses dans les cotisations aux dispositifs de protection sociale au sens large. S'agissant des recettes tirées des cotisations, pour la moyenne de l'OCDE, la valeur retenue dans les calculs pour les pays ne prélevant pas de cotisations est zéro.

Dans certains cas, les recettes tirées des cotisations de retraite ont été calculées en partant de l'hypothèse que ces recettes sont réparties entre différents programmes de protection sociale dans les mêmes proportions que les taux de cotisation. Les cotisations totales englobent les sommes versées par des non-salariés (principalement les travailleurs indépendants).

Finlande : les taux de cotisation ont été relevés depuis pour les salariés de 53 ans et plus. Les employeurs sont soumis à un prélèvement supplémentaire, qui varie de $0.8 \%$ à $3.9 \%$ de la masse salariale en fonction de leur capital. France et Pays-Bas : pour les recettes tirées des cotisations, il est impossible d'établir une distinction entre celles qui sont affectées aux retraites et celles qui servent d'autres fins. Pologne : le taux des cotisations de retraite a été diminué de 3 points en juillet 2007 ; on a retenu ici le chiffre précédent, qui est plus élevé.

Source: OCDE (diverses années), Les impôts sur les salaires; OCDE (2008), Statistiques des recettes publiques ; Social Security Administration, États-Unis (diverses années), Social Security Programs throughout the World ; Modèles de retraite et modèles impôts-prestations de l'OCDE. 


\section{Principaux résultats}

Dans les pays de l'OCDE, les dépenses publiques au titre des pensions de retraite et prestations de réversion en espèces ont progressé entre 1990 et 2005 à un rythme supérieur de $16.7 \%$ à la hausse du revenu national, grimpant ainsi d'une moyenne de $6.2 \%$ du produit intérieur brut (PIB) à $7.2 \%$. Ce phénomène s'explique par le vieillissement de la population et par la maturation des systèmes de retraite.

L'Italie est le pays qui a le plus dépensé au titre des retraites publiques en 2005 , soit $14.0 \%$ du PIB. Les dépenses publiques au titre des prestations de retraite en espèces s'inscrivent également bien au-delà de $10 \%$ du PIB dans d'autres pays européens: l'Allemagne, l'Autriche, la France, la Grèce et la Pologne. À l'autre extrémité, la Corée et le Mexique consacrent quelque $1.5 \%$ seulement de leur PIB aux prestations de retraite et de réversion. En Corée, en effet, le régime de retraite public n'a été introduit qu'en 1988, mais les dépenses ont fortement progressé de 1990 à 2005 - et ont plus que doublé par rapport au revenu national - du fait de la maturation du régime et du rapide vieillissement de la population. Au Mexique, la faiblesse des dépenses témoigne d'une couverture relativement peu importante des dispositifs de retraite $35 \%$ environ des salariés seulement) et de la relative jeunesse de la population.

Les dépenses sont aussi relativement faibles dans d'autres pays bénéficiant d'un contexte démographique favorable, comme l'Australie, le Canada, les États-Unis, l'Irlande et la Nouvelle-Zélande. Ce n'est toutefois pas toujours le cas : bien qu'en deuxième position parmi les pays de l'OCDE les plus jeunes d'un point de vue démographique, la Turquie consacre $7.8 \%$ de son PIB aux retraites publiques - soit plus que la moyenne des pays de l'OCDE, qui s'élève à $7.2 \%$.

Dans certains pays de l'OCDE, les dépenses publiques au titre des retraites sont peu élevées du fait des prestations servies par les régimes privés obligatoires (premier poste pour mémoire du tableau). En témoigne tout particulièrement le cas de la Suisse, où les dépenses de ces régimes, soit $6.0 \%$ du PIB, ne sont guère éloignées des dépenses publiques, qui s'établissent à $6.8 \%$ du PIB. Si l'on additionne ces deux chiffres, la Suisse, avec des dépenses totales atteignant $12.8 \%$ du revenu national, n'est devancée que par l'Italie, et dépasse de peu l'Autriche et la France. En Australie, le régime obligatoire à cotisations définies a été introduit en 1992, si bien que les retraités d'aujourd'hui n'ont guère voire nullement accumulé de droits dans ce cadre. En 2005, l'enveloppe globale des dépenses représentait au total pratiquement $0.4 \%$ du PIB, mais elle va gonfler rapidement dans les années qui viennent. De même, les prestations servies par les dispositifs privés obligatoires en Pologne et en Hongrie (mis en place à la fin des années 90), ainsi qu'en République slovaque (en 2005) seront faibles ou nulles pendant au moins dix ans.

Les dépenses de retraite, en proportion du revenu national, ont été stables sur la période 1990-2005 dans cinq pays de l'OCDE, à savoir la Belgique, le Canada, l'Espagne, les États-Unis et la Suède. Dans six pays, les dépenses publiques au titre des retraites ont cru à un rythme moins soutenu que le revenu national. En Irlande, cette situation est due à la progression exceptionnelle du PIB sur cette période. En Nouvelle-Zélande, la diminution des dépenses de retraites au regard du revenu national, supérieure à $40 \%$, s'explique par deux mesures : le gel de la retraite de base de 1992 à 1994 et le relèvement de l'âge de la retraite de 60 à 65 ans. Les autres pays enregistrant un recul sensible des dépenses de retraite sont l'Islande, le Luxembourg, la Norvège et les Pays-Bas.

Dans cinq pays de l'OCDE, les dépenses publiques au titre des retraites ont été multipliées par plus de deux par rapport au revenu national. Dans le cas de la Corée et du Mexique (et, dans une moindre mesure, de la Turquie), cela est dû au fait que les dépenses étaient peu élevées en 1990. La Pologne et le Portugal, dont les dépenses s'inscrivaient à l'origine en deçà de la moyenne des pays de l'OCDE, l'ont cependant nettement dépassée aujourd'hui.

Les colonnes de droite du tableau présentent les sommes affectées aux prestations de retraite et de réversion en espèces au regard du total des dépenses publiques (et non du revenu national). Une fois encore, l'Italie arrive en tête, les retraites absorbant pratiquement $30 \%$ du budget. En Allemagne, en Autriche, en France, en Grèce et en Pologne, un quart environ des dépenses publiques vont aux retraites. Le risque, dans ces pays, est que les dépenses publiques au titre des retraites prennent le pas sur des dépenses souhaitables, en matière sociale (prestations en faveur des enfants et des parents) et dans d'autres domaines (comme l'éducation).

Les aides publiques à l'intention des personnes âgées ne se limitent pas aux prestations en espèces. Le deuxième poste pour mémoire fait apparaître les dépenses publiques totales en faveur des personnes âgées, prestations en nature comprises. Les plus importantes sont les allocations logement et les aides au logement. Elles sont qualifiées de " prestations en nature " car elles dépendent de dépenses spécifiques effectuées par les particuliers. Elles jouent un rôle particulièrement important dans les cinq pays nordiques, où elles s'élèvent en moyenne à $1.8 \%$ du PIB. Le logement constitue également un aspect non négligeable des mesures prises aux Pays-Bas et au Royaume-Uni en faveur des personnes âgées, les sommes dépensées par l'Australie concernant principalement la santé.

\section{Définition et calcul}

Les données et les définitions sont exposées plus en détail dans la Base de données sur les dépenses sociales disponible en ligne : www.oecd.org/els/social/depenses. 
Dépenses au titre des prestations de retraite et de réversion

\begin{tabular}{|c|c|c|c|c|c|c|c|}
\hline & \multicolumn{4}{|c|}{ En \% du PIB } & \multirow{2}{*}{$\begin{array}{c}\text { Variation } \\
1990-2005 \\
\%\end{array}$} & \multicolumn{2}{|c|}{ En \% des dépenses publiques } \\
\hline & 1990 & 1995 & 2000 & 2005 & & 1990 & 2005 \\
\hline \multicolumn{8}{|c|}{ Prestations publiques en espèces } \\
\hline Allemagne & 10.0 & 10.5 & 11.0 & 11.4 & +14.0 & & 24.3 \\
\hline Australie & 3.1 & 3.7 & 3.9 & 3.5 & +10.6 & 8.6 & 9.9 \\
\hline Autriche & 11.7 & 12.6 & 12.3 & 12.6 & +7.8 & 22.7 & 25.3 \\
\hline Belgique & 9.1 & 9.3 & 8.9 & 9.0 & -0.9 & 17.4 & 17.3 \\
\hline Canada & 4.2 & 4.7 & 4.3 & 4.1 & -2.9 & 8.7 & 10.6 \\
\hline Corée & 0.8 & 1.2 & 1.4 & 1.6 & +108.5 & 3.8 & 5.4 \\
\hline Danemark & 5.1 & 6.2 & 5.3 & 5.4 & +6.1 & 9.2 & 10.3 \\
\hline Espagne & 7.9 & 9.0 & 8.6 & 8.1 & +1.9 & & 21.0 \\
\hline États-Unis & 6.1 & 6.3 & 5.9 & 6.0 & -0.7 & 16.1 & 16.2 \\
\hline Finlande & 7.3 & 8.8 & 7.6 & 8.4 & +16.4 & 15.1 & 16.7 \\
\hline France & 10.6 & 12.0 & 11.8 & 12.4 & +16.3 & 21.5 & 23.0 \\
\hline Grèce & 9.9 & 9.6 & 10.7 & 11.5 & +16.6 & & 26.6 \\
\hline Hongrie & & & 7.3 & 8.5 & & & 17.1 \\
\hline Irlande & 3.9 & 3.5 & 3.1 & 3.4 & -12.1 & 9.0 & 10.0 \\
\hline Islande & 2.2 & 2.4 & 2.2 & 2.0 & -10.5 & & 4.7 \\
\hline Italie & 10.1 & 11.4 & 13.6 & 14.0 & +37.9 & 19.2 & 29.0 \\
\hline Japon & 4.9 & 6.2 & 7.4 & 8.7 & +75.5 & & 22.7 \\
\hline Luxembourg & 8.2 & 8.8 & 7.5 & 7.2 & -11.2 & 21.6 & 17.3 \\
\hline Mexique & 0.5 & 0.8 & 0.9 & 1.3 & +161.6 & & \\
\hline Norvège & 5.6 & 5.5 & 4.8 & 4.8 & -14.3 & & 11.5 \\
\hline Nouvelle-Zélande & 7.5 & 5.8 & 5.1 & 4.4 & -41.8 & 14.0 & 10.9 \\
\hline Pays-Bas & 6.7 & 5.8 & 5.0 & 5.0 & -26.3 & 12.2 & 11.0 \\
\hline Pologne & 5.1 & 9.4 & 10.5 & 11.4 & +121.6 & & 26.3 \\
\hline Portugal & 5.0 & 7.4 & 8.2 & 10.2 & +102.1 & & 22.0 \\
\hline Rép. slovaque & & 6.3 & 6.3 & 6.2 & & & 16.2 \\
\hline Rép. tchèque & 6.1 & 6.2 & 7.5 & 7.3 & +20.0 & & 16.3 \\
\hline Royaume-Uni & 4.9 & 5.4 & 5.4 & 5.7 & +15.4 & 11.9 & 12.8 \\
\hline Suède & 7.7 & 8.2 & 7.3 & 7.7 & -0.3 & & 13.9 \\
\hline Suisse & 5.6 & 6.6 & 6.6 & 6.8 & +21.6 & 18.3 & 19.1 \\
\hline Turquie & 3.2 & 3.7 & & 7.8 & +146.1 & & \\
\hline OCDE & 6.2 & 6.8 & 6.9 & 7.2 & +16.7 & & \\
\hline \multicolumn{8}{|c|}{ Pour mémoire : Dépenses totales, régimes privés obligatoires inclus } \\
\hline Australie & 3.1 & 4.4 & 4.8 & 3.9 & +23.5 & & \\
\hline Italie & 12.9 & 14.5 & 14.8 & 15.1 & +17.3 & & \\
\hline Japon & 5.1 & 6.4 & 7.9 & 9.0 & +76.6 & & \\
\hline Royaume-Uni & 5.0 & 5.6 & 5.9 & 6.2 & +23.4 & & \\
\hline Suisse & 8.7 & 11.3 & 12.4 & 12.8 & +47.2 & & \\
\hline \multicolumn{8}{|c|}{ Pour mémoire : Dépenses publiques totales, prestations en nature incluses } \\
\hline Australie & 3.7 & 4.2 & 5.1 & 4.7 & +25.1 & & \\
\hline Danemark & 7.4 & 8.4 & 7.1 & 7.3 & -1.5 & & \\
\hline Finlande & 8.0 & 9.7 & 8.4 & 9.4 & +17.8 & & \\
\hline Hongrie & & & 7.8 & 9.1 & & & \\
\hline Islande & 3.5 & 3.7 & 3.5 & 3.8 & +10.4 & & \\
\hline Japon & 5.1 & 6.4 & 8.1 & 9.9 & +94.4 & & \\
\hline Norvège & 7.5 & 7.5 & 6.8 & 6.6 & -12.4 & & \\
\hline Pays-Bas & 7.3 & 6.3 & 5.7 & 5.8 & -19.7 & & \\
\hline Royaume-Uni & 5.2 & 5.9 & 5.9 & 6.3 & +19.6 & & \\
\hline Suède & 9.2 & 10.5 & 9.8 & 10.2 & +11.0 & & \\
\hline
\end{tabular}

Note: Les pays figurent dans les postes pour mémoire uniquement lorsque la valeur correspondante - dépenses des régimes privés obligatoires ou dépenses publiques au titre des prestations en nature - est significative.

Source: Base de données de l'OCDE sur les dépenses sociales (SOCX), Base de données de l'OCDE sur les principaux indicateurs économiques. 


\section{Principaux résultats}

Puisque les pensions de retraite publiques que percevront demain les salariés d'aujourd'hui ont été réduites pour tenter de rétablir la viabilité financière des régimes publics, la charge des prestations en faveur des personnes âgées a été reportée sur les dispositifs privés. Dans 11 pays de l'OCDE, les régimes de retraite privés sont obligatoires ou quasi obligatoires (c'est-à-dire qu'ils couvrent pratiquement la totalité des salariés par le biais de conventions collectives). Dans huit autres, les dispositifs facultatifs couvrent une part importante de la population active, soit plus de $55 \%$.

En Islande, Norvège et Suisse, les régimes de retraite professionnels sont obligatoires: les employeurs doivent ainsi gérer un dispositif et les taux de cotisation sont définis par les pouvoirs publics. Aux Pays-Bas et en Suède, les mécanismes professionnels sont " quasi obligatoires " : aux termes des conventions collectives, les employeurs doivent mettre en place des dispositifs auxquels les salariés sont tenus d'adhérer. En conséquence, $90 \%$ au moins de la population active est couverte.

Six pays de l'OCDE - le Danemark, la Hongrie, le Mexique, la Pologne, la République slovaque et la Suède - sont pourvus de régimes individuels obligatoires. La couverture est quasi universelle au Danemark et en Suède. En Europe de l'Est, toutefois, ces dispositifs ont été introduits ces dix dernières années. D'une manière générale, les salariés âgés ne sont pas couverts par ces nouveaux mécanismes. Le taux de couverture, qui avoisine les $65-75 \%$, va donc progresser à mesure que les nouveaux venus sur le marché du travail adhéreront aux dispositifs individuels et que les salariés exclusivement couverts par le régime public feront valoir leurs droits à la retraite.

$\mathrm{Au}$ Mexique, le taux de couverture des régimes individuels obligatoires est peu élevé en raison de l'ampleur du secteur informel.

Le système australien associe dispositifs professionnels et individuels. À l'origine, les employeurs choisissaient un prestataire, spécialisé dans les mécanismes de retraite sectoriels ou les services financiers. Les salariés peuvent désormais sortir du régime choisi par leur employeur et en sélectionner un autre, ou se constituer d'eux-mêmes une épargneretraite. Il est difficile de subdiviser la couverture globale de $85 \%$ en dispositifs professionnels et individuels.

En ce qui concerne les régimes privés facultatifs, les pays où la couverture est la plus élevée - $55 \%$ au moins - sont l'Allemagne, la Belgique, le Canada, les États-Unis, l'Irlande, le Japon, la Norvège et le RoyaumeUni. Les dispositifs professionnels sont la seule ou la principale source de retraite privée. Ils sont "facultatifs " en ce que les employeurs sont libres de les mettre en place et que les salariés peuvent souvent choisir d'y adhérer ou non.

Lorsque l'OCDE dispose de données issues d'enquêtes-ménages, la couverture globale des régimes de retraite privés facultatifs se caractérise par un important phénomène de "double comptage » des personnes affiliées à la fois à un régime professionnel et à un dispositif individuel. C'est aux États-Unis que ce phénomène est le plus net : $46 \%$ des salariés y sont en effet affiliés à un régime professionnel et près de 35 \% à un mécanisme individuel, tandis que la couverture globale des régimes privés est légèrement inférieure à 58 \%. En d'autres termes, les deux tiers des salariés affiliés à un dispositif individuel sont également affiliés à un mécanisme professionnel.

La couverture des régimes de retraite individuels facultatifs est généralement peu étendue : l'Allemagne obtient le meilleur résultat (44\%), suivie des États-Unis (35\% environ). Bien souvent, cela s'explique probablement par le fait que la demande de pensions privées est principalement satisfaite par des régimes professionnels, et exclut ainsi toute demande de dispositifs individuels.

L'impact du nouveau régime "KiwiSaver » en Nouvelle-Zélande est patent (voir en Partie I les chapitres spéciaux sur « les réformes récentes des retraites » et « le déficit d'épargne-retraite et l'épargne-retraite facultative "). La couverture des régimes privés s'est fortement réduite après la diminution des incitations fiscales. En 2007, $13 \%$ des salariés étaient affiliés à un régime professionnel et $5.5 \%$ adhéraient à un dispositif individuel. Dorénavant, KiwiSaver couvre pratiquement un tiers des salariés à travers un régime professionnel et $10.7 \%$ de plus par le biais d'un dispositif individuel. Voilà les premiers éléments attestant de l'efficacité des mécanismes d'affiliation automatique à ce régime.

\section{Définition et calcul}

Il est extrêmement difficile de réunir des données précises et comparables sur les régimes de retraite privés en raison des profondes différences institutionnelles existant entre les pays au regard de la constitution de ces dispositifs. Le tableau suit, dans toute la mesure du possible, la catégorisation des différents types de dispositifs utilisée dans les autres sections des Pensions dans les pays de l'OCDE. La classification n'est donc pas tout à fait identique à celle des Perspectives de l'OCDE sur les pensions privées 2008 (2009). On cherche ici à exprimer la couverture des salariés en pourcentage de l'emploi total. Dans certains pays, toutefois, les personnes couvertes sont pour une part des travailleurs indépendants ou sans activité et, pour les pourcentages, elles sont donc prises en compte dans le numérateur, mais pas dans le dénominateur. 


\section{Couverture des régimes de retraite privés par type de dispositif}

En pourcentage

\begin{tabular}{|c|c|c|c|c|c|}
\hline & \multicolumn{2}{|c|}{ Obligatoire/quasi obligatoire } & \multicolumn{2}{|c|}{ Facultatif } & \multirow{2}{*}{ Total } \\
\hline & Professionnel & Individuel & Professionnel & Individuel & \\
\hline Allemagne & & & 64.0 & 44.0 & \\
\hline Australie & & & 18.8 & 9.7 & \\
\hline Autriche & & & 13.9 & & \\
\hline Belgique & & & 55.6 & & \\
\hline Canada & & & 39.4 & & 57.3 \\
\hline \multicolumn{6}{|l|}{ Corée } \\
\hline Danemark & $>90.0 / 76.1$ & 88.6 & & & \\
\hline Espagne & & & 8.7 & & \\
\hline États-Unis & & & 46.0 & 34.7 & 57.7 \\
\hline Finlande & & & 8.7 & 7.3 & \\
\hline France & & & 15.0 & & \\
\hline \multicolumn{6}{|l|}{ Grèce } \\
\hline Hongrie & & 64.5 & & & \\
\hline Irlande & & & 42.9 & 14.9 & 55.0 \\
\hline Islande & $>90.0$ & & & & \\
\hline Italie & & & 10.6 & 5.1 & \\
\hline Japon & & & 45.0 & & \\
\hline Luxembourg & & & 5.6 & & \\
\hline Mexique & & 34.5 & & & \\
\hline Norvège & $>90.0$ & & 60.0 & 3.0 & \\
\hline Nouvelle-Zélande & & & $13.0 / 32.6$ & $5.5 / 10.7$ & n.d. $/ 43.3$ \\
\hline Pays-Bas & $>90.0$ & & & & \\
\hline Pologne & & 71.7 & & & \\
\hline Portugal & & & 4.0 & & \\
\hline Rép. slovaque & & 65.8 & & & \\
\hline Rép. tchèque & & & & & \\
\hline Royaume-Uni & & & 47.1 & 18.9 & 59.1 \\
\hline Suède & $>90.0$ & $>90.0$ & & & \\
\hline Suisse & $>90.0$ & & & & \\
\hline Turquie & & & & & \\
\hline
\end{tabular}

Note: Les cellules vides indiquent que ce type de régime ne repose sur aucune base juridique dans le pays concerné ou que sa couverture est négligeable (inférieure à $1 \%$ ). L'entrée " > 90.0 » indique que la couverture est quasi universelle. La colonne correspondant à la couverture totale est remplie uniquement lorsqu'on dispose de données adéquates permettant de remédier au double comptage des salariés affiliés à un régime professionnel et à un dispositif individuel.

Note : Allemagne : la couverture des dispositifs professionnels est exprimée en pourcentage des salariés couverts par le régime public. Australie : le régime obligatoire de garantie de retraite (superannuation guarantee) permet aux salariés de choisir d'adhérer à un régime d'entreprise, un fonds sectoriel ou un prestataire de services financiers, ou bien d'investir eux-mêmes dans le fonds : ce dispositif est ainsi à la fois professionnel et individuel. Corée : le gouvernement a l'intention de transformer les dispositifs d'indemnités de fin de contrat en plans professionnels (voir le chapitre spécial sur "Les réformes récentes des retraites » dans la partie I; jusqu'à présent, toutefois, les conversions ont été limitées, même si on ne dispose pas encore des chiffres exacts. Danemark : pour les dispositifs professionnels obligatoires, le premier chiffre concerne l'ATP (retraite complémentaire du marché du travail) et le second les régimes professionnels quasi obligatoires à cotisations définies. Le chiffre indiqué dans la colonne "obligatoire, individuel "se rapporte au régime spécial (SP). Voir le descriptif pays relatif au Danemark pour plus de précisions. Hongrie, Pologne et République tchèque : les retraites privées facultatives sont assurées par les régimes professionnels et individuels : il est donc impossible de déterminer la couverture de chaque type de dispositif. Nouvelle-Zélande : dans chaque cellule, le second chiffre correspond aux salariés couverts par KiwiSaver (par l'intermédiaire de leur employeur - régime professionnel - ou d'un prestataire de services financiers - dispositif individuel). Le premier chiffre correspond à la couverture des dispositifs professionnels et individuels traditionnels (à l'exclusion des personnes de plus de 65 ans cotisant à des dispositifs individuels pour des raisons fiscales).

Source: OCDE (2009), Perspectives de l'OCDE sur les pensions privées 2008 ; OCDE (2007), Les pensions dans les pays de l'OCDE : panorama des politiques publiques; Union européenne, Comité de la protection sociale (2008), Privately Managed Funded Pension Provision and their Contribution to Adequate and Sustainable Pensions; Antolín, P. et E.R. Whitehouse (2009), "Filling the Pension Gap: Coverage and Value of Voluntary Retirement Savings ", Documents de travail sur les affaires sociales, l'emploi et les migrations, n 69, Éditions de l'OCDE, Paris ; base de données de la Banque mondiale sur les retraites; autorités nationales. 


\section{Principaux résultats}

Des actifs substantiels ont été accumulés dans la plupart des pays de l'OCDE afin de faire face aux engagements de retraite futurs. Au total, les actifs des régimes de retraite privés représentaient près de $75 \%$ du produit intérieur brut (PIB) en 2007. La moitié des pays de l'OCDE ont créé des fonds de réserve publics pour faciliter le versement des retraites. Dans ces pays, les réserves constituées atteignent pratiquement $15 \%$ du PIB.

Cela étant, il ne faut pas oublier que ces chiffres concernent l'année 2007 et ne tiennent donc pas compte de l'impact de la crise financière sur la valeur des actifs.

En 2007, la valeur des actifs des régimes de retraite privés était supérieure au revenu national annuel dans quatre pays de l'OCDE : l'Australie, l'Islande, les PaysBas et la Suisse. Les fonds de pension occupaient également une place non négligeable aux États-Unis et au Royaume-Uni, avec $75 \%$ au moins du PIB.

Compte tenu du poids des États-Unis dans l'ensemble des économies de l'OCDE, les actifs de retraite privés représentent au total plus de $75 \%$ du PIB total des pays de l'OCDE. Néanmoins, si l'on accorde la même pondération à chaque pays membre, les actifs de retraite privés représentent en moyenne $33 \%$ du PIB.

Là encore, il convient de souligner que ces chiffres sont antérieurs à la crise, car ils se rapportent essentiellement à l'année 2007. Les répercussions de la crise financière sur les investissements des fonds de pension, étudiées en Partie I dans le chapitre spécial sur "les systèmes de retraite pendant la crise économique et financière ", ont été considérables. Dans les pays de l'OCDE, les fonds de pension ont vu la valeur de leurs investissements reculer de $23 \%$ en 2008, l'Australie, les États-Unis et l'Islande enregistrant des moins-values particulièrement importantes. En 2009, les cours des actifs ont poursuivi leur repli.

Les pays où les fonds de pension occupent la plus grande place au sein de l'économie sont tous dotés de régimes de retraite privés arrivés à maturité, mis en place de longue date. Il s'agit, en plus des six pays mentionnés précédemment, du Canada, du Danemark et de l'Irlande.

Dans d'autres pays, les systèmes de retraite privés ont été élaborés bien plus récemment. La Hongrie, le Mexique, la Pologne et la République slovaque, par exemple, ont introduit des régimes privés obligatoires, destinés à remplacer en partie les retraites publiques, à la fin des années 90 et au début des années 2000. L'encours de ces régimes a connu une forte croissance depuis, atteignant quelque $11-12 \%$ du PIB en Hongrie, au Mexique et en Pologne. Il va progresser à un rythme soutenu durant les prochaines années et décennies, puisque le nombre des personnes affiliées au nouveau système de retraite va aller croissant et que les adhérents actuels vont continuer de cotiser.

La Nouvelle-Zélande pourrait également connaître une croissance rapide. Bien que des régimes professionnels privés soient en place depuis longtemps, la couverture a fortement diminué à partir du début des années 80 , pour revenir à $13 \%$ environ aujourd'hui. Au bout d'un an d'exploitation, le nouveau dispositif privé facultatif KiwiSaver couvrait plus de $40 \%$ des salariés, ce qui laisse à penser que les actifs de retraite privés vont enregistrer une hausse sensible dans les années qui viennent.

Quinze pays de l'OCDE sont dotés de fonds de réserve publics pour les retraites. Un grand nombre de ces fonds occupent une place relativement modeste : en 2007 , ils représentaient plus de $5 \%$ du revenu national dans huit pays seulement. Le fonds américain est intégralement investi en obligations d'État. D'aucuns ont fait valoir qu'il s'agit simplement d'un moyen détourné de financer les retraites selon le principe de la répartition, en vertu duquel les cotisations d'aujourd'hui financent les prestations d'aujourd'hui. En effet, les cotisations versées au fonds de réserve sont simplement prêtées au gouvernement pour financer les dépenses engagées dans le cadre d'autres programmes.

Les obligations d'État constituent également plus de $80 \%$ du portefeuille du fonds de réserve public coréen pour les retraites et plus de $60 \%$ du fonds japonais.

Cela étant, la part des obligations d'État atteint tout juste $35-40 \%$ en Norvège et en Suède et moins de $20 \%$ en Nouvelle-Zélande et en Irlande. Ces fonds sont aussi relativement importants.

Des arguments similaires à ceux de la maturité des régimes privés récemment instaurés peuvent être invoqués pour les fonds de réserve publics. En Australie, en Irlande et en Nouvelle-Zélande - trois pays de l'OCDE qui sont jeunes d'un point de vue démographique les fonds de réserve pour les retraites ont été créés dans un passé relativement récent. Les actifs devraient s'étoffer au cours des prochaines années, mais ils s'amenuiseront lorsque le vieillissement de la population s'accélèrera.

\section{Définition et calcul}

L'OCDE a établi un ensemble de principes de classification des pensions privées (voir OCDE, 2004). L'analyse présentée ici repose sur ce cadre. Pour plus de précisions, voir OCDE (2008 et 2009).

\section{Références}

OCDE (2005), Les Pensions privées : classification et glossaire de l'OCDE, OCDE, Paris.

OCDE (2008), " Le point sur les marchés des pensions ", Lettre d'information, $n^{\circ} 5$, OCDE, Paris, décembre.

OCDE (2009), Perspectives de l'OCDE sur les pensions privées 2008, OCDE, Paris. 
Les actifs des fonds de pension et des fonds de réserve publics

\begin{tabular}{|c|c|c|}
\hline & Valeur d & B), 2007 \\
\hline & Fonds de pension privés & Fonds de réserve publics \\
\hline Allemagne & 4.1 & \\
\hline Australie & 105.4 & 4.9 \\
\hline Autriche & 4.8 & \\
\hline Belgique & 4.0 & \\
\hline Canada & 55.3 & 7.9 \\
\hline Corée & 3.1 & 23.9 \\
\hline Danemark & 32.4 & 0.3 \\
\hline Espagne & 7.5 & 4.5 \\
\hline États-Unis & 76.7 & 16.6 \\
\hline Finlande & 71.0 & \\
\hline France & 1.1 & 1.9 \\
\hline Grèce & 0.0 & \\
\hline Hongrie & 10.9 & \\
\hline Irlande & 46.6 & 11.5 \\
\hline Islande & 134.0 & \\
\hline Italie & 3.3 & \\
\hline Japon & 20.0 & 26.2 \\
\hline Luxembourg & 1.0 & \\
\hline Mexique & 12.1 & 0.9 \\
\hline Norvège & 7.0 & 79.7 \\
\hline Nouvelle-Zélande & 11.1 & 7.8 \\
\hline Pays-Bas & 138.1 & \\
\hline Pologne & 12.2 & 0.3 \\
\hline Portugal & 13.7 & 4.3 \\
\hline Rép. tchèque & 4.7 & \\
\hline Rép. slovaque & 4.2 & \\
\hline Royaume-Uni & 78.9 & \\
\hline Suède & 8.7 & 31.7 \\
\hline Suisse & 119.2 & \\
\hline Turquie & 1.2 & \\
\hline Total OCDE & 74.5 & 14.5 \\
\hline Moyenne non pondérée & 33.1 & 14.8 \\
\hline
\end{tabular}

Note : Les données sur les fonds de réserve publics pour la Norvège, le Mexique et le Portugal concernent 2006. Pour la Norvège, le Government Pension Fund - Global, qui était auparavant un fonds d'État appelé Government Petroleum Fund, tire ses revenus du pétrole et a un mandat qui dépasse le financement des dépenses au titre des pensions; il n'est donc pas comptabilisé comme fond de réserve d'Etat pour les pensions. Les données de ce tableau ne portent donc que sur le Government Pension Fund - Norway, auparavant dénommé le National Insurance Scheme Fund (5.2\%). Par contraste, les actifs du Government Pension Fund - Global, se monteraient à $79.7 \%$ du PIB.

«Total OCDE » agrège l'ensemble des pays membres. Contrairement à la « moyenne non pondérée », le résultat rend donc compte des différences de montant du PIB entre les pays. S'agissant des fonds de réserve publics, les chiffres " total OCDE » et « moyenne non pondérée » couvrent uniquement les 15 pays pour lesquels les données sont indiquées.

Source : OCDE (2008), « Le point sur les marchés des pensions », Lettre d'information, $n^{\circ}$ 5, graphique 6, OCDE, Paris ; OCDE (2009), Perspectives de l'OCDE sur les pensions privées 2008, tableau 3.1, sources nationales. 



\section{Contexte démographique et économique}

Depuis deux décennies, le vieillissement de la population est l'un des principaux facteurs qui conditionnent les politiques des retraites et leurs réformes. Le vieillissement est la résultante de deux changements démographiques.

Le premier facteur à l'origine du vieillissement de la population est l'augmentation de l'espérance de vie. Un chapitre analyse l'évolution de l'espérance de vie - à la naissance et à l'âge de 65 ans - dans la durée. L'évolution future probable de l'espérance de vie est également esquissée. Le deuxième est une baisse du nombre de naissances. Un second chapitre examine les taux de fécondité et leur évolution au fil du temps, et analyse brièvement les raisons à l'origine de ces changements.

Le troisième indicateur concerne directement le vieillissement démographique. Le vieillissement est mesuré par le taux de dépendance économique: nombre de personnes ayant atteint l'âge de la retraite rapporté au nombre de personnes d'âge actif. Le taux de dépendance des personnes âgées est indiqué sur un siècle: les données historiques remontent à 1950 et des projections sont effectuées jusqu'en 2050.

Le dernier indicateur porte sur le contexte économique. Il fournit des données sur le salaire moyen, calculé en utilisant la mesure du "salarié moyen "établie par l'OCDE pour 2006. Ces données sont largement utilisées dans cette étude: de nombreux paramètres et résultats concernant les droits à la retraite sont exprimés en pourcentages des salaires moyens nationaux. 


\section{Principaux résultats}

L'allongement considérable de l'espérance de vie est l'un des accomplissements les plus remarquables du siècle dernier. On vit de plus en plus longtemps. Depuis 1960, l'espérance de vie des femmes a augmenté de près de 11 ans, atteignant 81.7 ans. Pour les hommes, l'augmentation est d'un peu plus de 10 ans, à 76.0 ans. En 2006 , l'espérance de vie à la naissance pour les femmes était la plus élevée au Japon (85.8 ans), suivi de la France, de l'Espagne, de la Suisse et de l'Italie. Pour les hommes, l'Islande arrivait en tête (79.4 ans), suivie de la Suisse, du Japon, de l'Australie et de la Suède. L'espérance de vie des personnes âgées - indicateur plus pertinent pour les systèmes de retraite - a elle aussi fortement progressé.

La hausse générale de l'espérance de vie dans les pays de l'OCDE s'est accompagnée d'une convergence entre les pays. En Corée et en Turquie, l'espérance de vie à la naissance des femmes et des hommes pris globalement a augmenté respectivement de 26.7 et 23.3 ans entre 1960 et 2006, tandis qu'au Mexique, le gain a dépassé 18 ans. Les rattrapages opérés par ces pays traduisent principalement une baisse de la mortalité infantile.

Il ne semble pas que cette évolution soit sur le point de cesser. Après la période de rattrapage, les gains d'espérance de vie des Japonaises ont certes été divisés par deux, mais se poursuivent depuis lors au taux d'environ $3 \%$ par an.

L'écart d'espérance de vie entre hommes et femmes s'est légèrement amplifié, passant de 5.0 ans en 1960 à 5.7 ans en 2006 en moyenne dans l'OCDE. Toutefois, les tendances n'étaient pas les mêmes d'une décennie à l'autre. Alors que l'écart d'espérance de vie entre hommes et femmes a considérablement augmenté pendant les années 60 et 70 (culminant à 6.7 ans en moyenne en 1980), il s'est résorbé au cours des 25 dernières années. Ce resserrement reflète en partie le rapprochement entre hommes et femmes en termes de prise de risques (tabagisme par exemple) et la diminution des décès dus aux maladies cardiovasculaires chez les hommes.

Les personnes âgées vivent plus longtemps. En 2006, en moyenne, les femmes âgées de 65 ans pouvaient espérer vivre encore 20.1 ans, soit 5.3 ans de plus qu'en 1960. Les hommes du même âge pouvaient espérer vivre encore 16.7 ans, soit un gain de 4.0 ans depuis 1960. Dans plusieurs pays de l'OCDE, les écarts de longévité entre hommes âgés et femmes âgées se sont réduits depuis le milieu des années 80 .

Dans l'ensemble, les gains de longévité s'expliquent par l'élévation du niveau de vie, mais aussi par un meilleur accès à des services de santé de qualité. Toutefois, les gains d'espérance de vie sont plus faibles pour les personnes appartenant aux catégories socioéconomiques les moins favorisées.

Les analyses divergent sur l'évolution probable de l'espérance de vie à l'avenir. Les optimistes insistent sur les progrès de la biotechnologie et d'autres disciplines de pointe. Les pessimistes soulignent les dangers d'une pandémie mondiale de grippe, l'augmentation de l'obésité et l'incapacité de guérir des affections chroniques liées à l'âge, comme la maladie d'Alzheimer. Le tableau ci-dessous présente des calculs de l'OCDE basés sur l'évolution des taux de mortalité depuis 1945. À partir de 2002, la prévision centrale mise sur un allongement de l'espérance de vie à 65 ans d'environ 3.5 ans au cours des 50 prochaines années. Toutes choses égales par ailleurs, les coûts des retraites s'en trouveraient majorés d'environ $20 \%$. Toutefois, dans le scénario le plus défavorable, l'augmentation serait de seulement deux ans environ, tandis qu'elle atteindrait cinq ans dans le scénario optimiste. Compte tenu de cette incertitude, la plupart des pays de l'OCDE ont intégré, dans leur régime de retraite, des mécanismes d'ajustement automatique des retraites à l'évolution de l'espérance de vie.

\section{Espérance de vie à 65 ans en 2002 : distribution des prévisions sur 50 ans}

\begin{tabular}{lrrrrrr}
\hline & Base & $5 \%$ & $25 \%$ & $50 \%$ & $75 \%$ & $95 \%$ \\
\hline $\begin{array}{l}\text { Espérance de vie (années) } \\
\text { Hommes }\end{array}$ & 15.1 & 20.1 & 19.1 & 18.5 & 18.0 & 17.1 \\
$\quad$ Femmes & 18.7 & 23.7 & 22.8 & 22.2 & 21.7 & 20.9 \\
Variation (années) & & & & & & \\
$\quad$ Hommes & 0.0 & +5.0 & +4.0 & +3.4 & +2.9 & +2.0 \\
$\quad$ Femmes & 0.0 & +5.0 & +4.1 & +3.5 & +3.0 & +2.2 \\
\hline
\end{tabular}

Source: Whitehouse, E.R. (2007), «Life-expectancy Risk and Pensions: Who Bears the Burden? ", Document de travail sur les affaires sociales, l'emploi et les migrations $n^{\circ} 60$, OCDE, Paris. Les calculs utilisent la base de données sur la mortalité humaine (Université de Californie, Berkeley et Max Planck Institute for Demographic Research). Les taux de mortalité de base pour 2002 proviennent de la base de données démographiques des Nations Unies/Banque mondiale.

\section{Définition et calcul}

Les indicateurs présentés dans cette section, à savoir l'espérance de vie à la naissance et à 65 ans, désignent le nombre moyen d'années qu'une personne peut espérer vivre compte tenu du taux de mortalité par groupe d'âges qui prévaut dans un pays donné une année donnée : en l'espèce, 1960 et 2006. Chaque pays calcule son espérance de vie en employant des méthodologies variables. Toutefois, l'impact de ces différences méthodologiques est relativement faible et ne modifie l'espérance de vie mesurée que d'une fraction d'une année. 


\section{Espérance de vie des hommes et des femmes à la naissance, en années, 1960 et 2006}

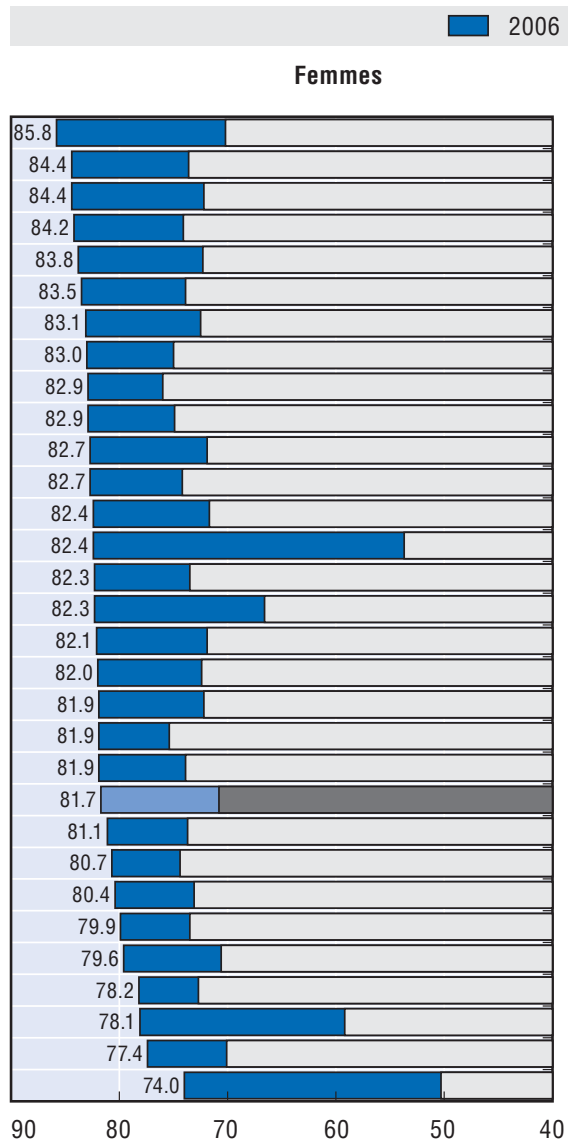

Japon
France
Espagne
Suisse
Italie
Australie
Finlande
Islande
Norvège
Suède
Autriche
Canada
Allemagne
Corée
Belgique
Portugal
Irlande
Grèce
Luxembourg
Pays-Bas
Nouvelle-Zélande
OCDE
Royaume-Uni
Danemark
États-Unis
République tchèque
Pologne
République slovaque
Mexique
Hongrie
Turquie

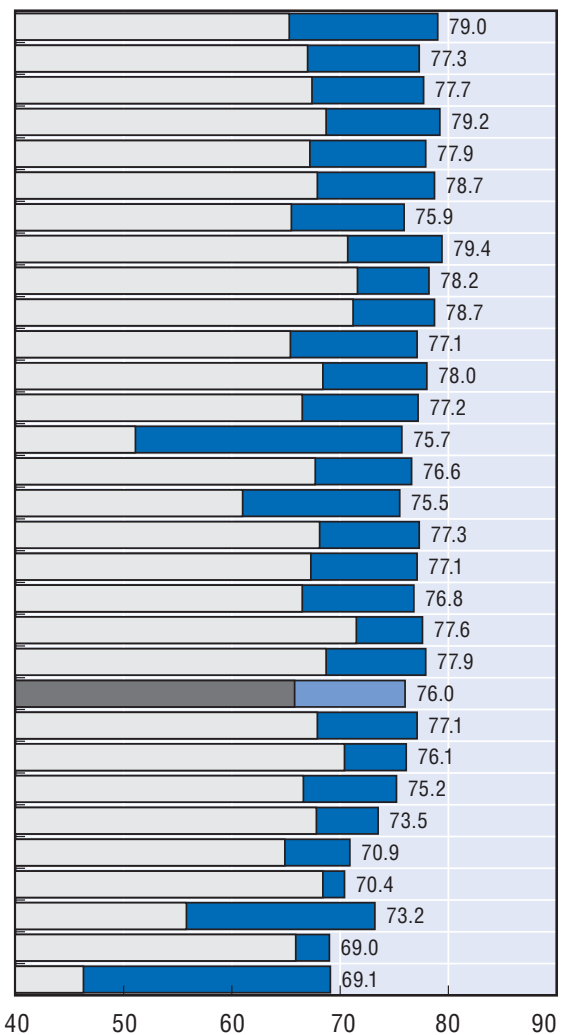

Source : OCDE (2008), Éco-Santé OCDE 2008, OCDE, Paris (www.oecd.org/sante/donnees) et OCDE (2009), Panorama de la société.

Espérance de vie à 65 ans, en années, pour les hommes et les femmes, en 2006

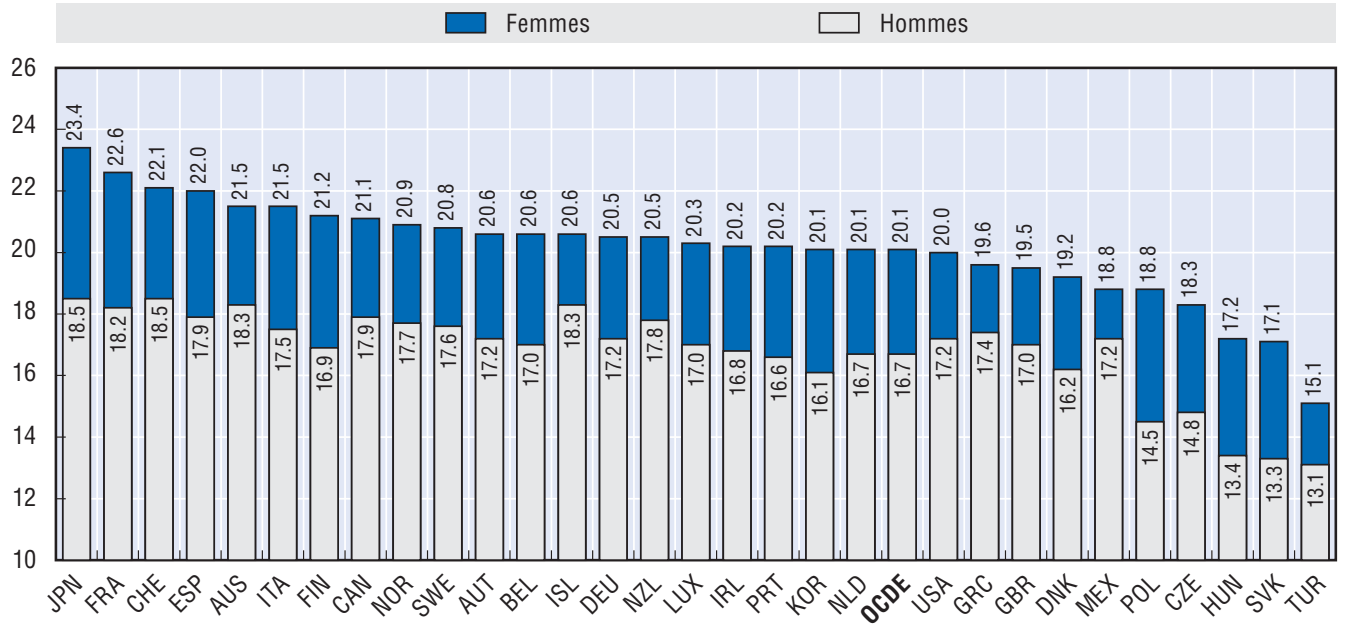

Note : Les données portent sur l'année 2005 pour le Canada, le Royaume-Uni et les États-Unis, et sur 2004 pour l'Italie.

Source : OCDE (2008), Éco-Santé OCDE 2008, OCDE, Paris (www.oecd.org/sante/donnees) et OCDE (2009), Panorama de la société. 


\section{Principaux résultats}

L'indicateur conjoncturel de fécondité est inférieur au seuil de renouvellement de la population - nombre d'enfants nécessaire pour que la population totale reste constante - dans 26 des 30 pays de l'OCDE. Les seules exceptions sont le Mexique et la Turquie (avec 2.2 enfants par femme) ainsi que l'Islande et les États-Unis (aux environs de 2.1). Toutefois, dans plus des deux tiers des pays de l'OCDE, les taux de fécondité enregistrent une augmentation modérée depuis 2002.

Les taux de fécondité ont des répercussions considérables sur les systèmes de retraite parce qu'ils comptent parmi les principaux déterminants du vieillissement démographique, aux côtés de l'espérance de vie.

En 2006, les taux de fécondité s'établissaient en moyenne à 1.65 dans les pays de l'OCDE, soit bien audessous du niveau nécessaire au renouvellement de la population. La tendance à la baisse du nombre d'enfants se poursuit depuis les années 70 . La chute des taux de fécondité est le reflet des transformations dans les modes de vie des individus et des contraintes de la vie quotidienne, comme la précarité de l'emploi, la difficulté de trouver un logement convenable et le coût prohibitif des services de garde d'enfant.

L'écart positif (et croissant) entre le nombre d'enfants que les femmes déclarent vouloir et celui qu'elles ont effectivement montre l'influence de ces contraintes (D'Addio et Mira d'Ercole, 2005).

L'évolution du statut marital des femmes a également des conséquences. L'augmentation du nombre de femmes non mariées a peut-être pesé sur les taux de fécondité, notamment dans les pays où mariage et maternité sont étroitement liés. Le lien le plus fort se manifeste au Japon et en Corée, bien qu'il soit aussi significatif dans plusieurs pays européens, comme la Grèce, l'Italie, la Pologne et la Suisse. Toutefois, les caractéristiques de procréation des femmes non mariées ont elles aussi changé. Par exemple, au moins la moitié des naissances ont aujourd'hui lieu hors mariage en France, Islande, Norvège et Suède (selon Panorama de la société). Dans la zone OCDE, la part moyenne des naissances hors mariage est désormais de une sur trois.

Ces dernières années, la tendance à la baisse du nombre d'enfants s'est inversée dans certains pays de l'OCDE. Le Royaume-Uni, la France, la Suède, l'Espagne et la République tchèque sont les pays où le redressement est le plus marqué. Plusieurs raisons expliquent ce retournement: il est possible que les politiques publiques, notamment l'augmentation des aides aux familles et aux femmes qui travaillent, aient joué un rôle. Ce rebond pourrait aussi s'expliquer par l'augmentation du nombre de naissances chez les femmes ayant retardé l'âge de leur première maternité jusqu'à la trentaine ou au-delà.

La faiblesse des taux de fécondité a plusieurs conséquences socio-économiques de grande ampleur. Premièrement, le déclin démographique peut s'autoalimenter, car le nombre de femmes en âge de procréer diminue. Deuxièmement, il y a moins de soignants familiaux susceptibles de prendre soin des personnes âgées. Troisièmement, les personnes d'âge actif supportent une charge fiscale plus lourde au titre du financement des retraites et des soins de santé. Quatrièmement, la main-d'œuvre vieillira elle aussi et risque de s'adapter plus difficilement aux changements technologiques, ce qui freinera la productivité et la croissance économique. Enfin, le vieillissement pourrait éroder l'épargne servant à financer l'investissement dans l'économie, car les personnes âgées puisent dans leur épargne pour consommer.

Le report de l'âge de la maternité accompagne et explique en partie la tendance à la baisse des taux de fécondité. En moyenne, L'âge de la mère à la naissance de son premier enfant est passé de 24 ans en 1970 à près de 28 ans en 2005. Ce report de l'âge de procréation à des conséquences durables. Premièrement, il augmente la probabilité que les femmes restent sans enfant ou aient moins d'enfants que souhaité. Deuxièmement, il accroît les risques de santé pour les mères comme pour les enfants.

\section{Définition et calcul}

L'indicateur conjoncturel de fécondité est le nombre d'enfants que chaque femme pourrait mettre au monde jusqu'à la fin de sa période de fécondité si, à tout âge, sa probabilité de mettre au monde des enfants correspondait au niveau de fécondité de l'année considérée pour cet âge. On le calcule en faisant la somme des taux de fécondité à chaque âge sur une période de cinq ans. Un indicateur conjoncturel de fécondité de 2.1 enfants par femme (seuil de renouvellement) assure une stabilité générale de la population, en supposant qu'il n'y ait pas de flux migratoires ni de variation de la mortalité.

\section{Références}

D'Addio, A.C. et M. Mira d'Ercole (2005), « Trends and Determinants of Fertility Rates in OECD Countries: The Role of Policies ", Document de travail de l'OCDE sur les affaires sociales, l'emploi et les migrations, $n^{\circ} 27, \mathrm{OCDE}_{\text {, Paris. }}$

OCDE (2009), Panorama de la société, OCDE, Paris. 


\section{Évolution des taux de fécondité de 1970 à 2006}
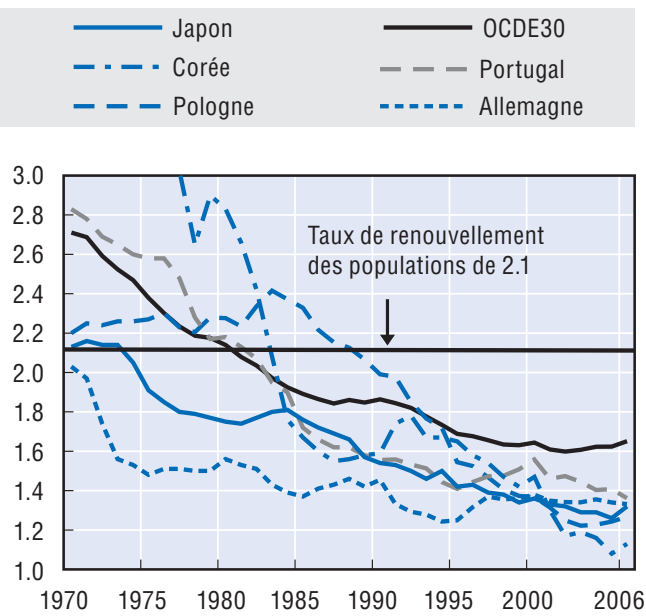

- Royaume-Uni _ - - - République tchèque

- - - France

-- Suède

OCDE30

-.--... Espagne

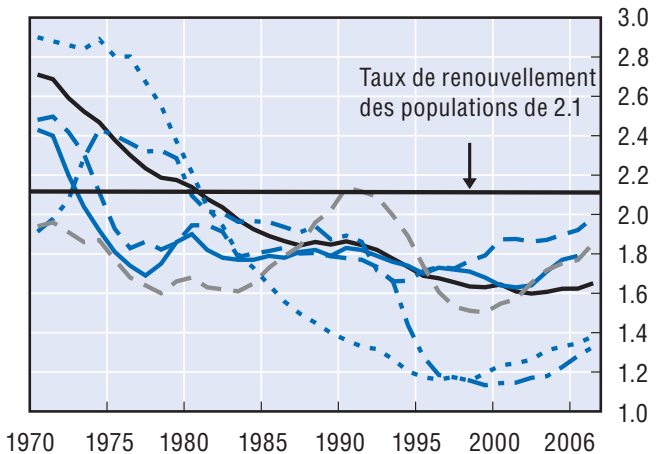

L'âge des mères à la naissance de leur premier enfant augmente

Âge moyen des femmes à la naissance du premier enfant

\begin{tabular}{|c|c|c|c|c|}
\hline & 1970 & $1995^{1}$ & $2000^{2}$ & $2005^{3}$ \\
\hline Allemagne & 24.0 & 27.5 & 28.2 & 28.1 \\
\hline Australie & 23.2 & 26.8 & .. & 28.0 \\
\hline Autriche & .. & 25.6 & 26.4 & 27.2 \\
\hline Belgique & 24.3 & 27.3 & .. & 27.4 \\
\hline Corée & .. & .. &.. & 29.1 \\
\hline Danemark & 23.8 & 27.4 & 27.7 & 28.4 \\
\hline Espagne & .. & 28.4 & 29.1 & 29.3 \\
\hline États-Unis & 24.1 & 24.5 & 24.9 & 25.1 \\
\hline Finlande & 24.4 & 27.2 & 27.4 & 27.9 \\
\hline France & 24.4 & 28.1 & 27.9 & 28.5 \\
\hline Grèce & 25.0 & 26.6 & 27.5 & 28.5 \\
\hline Hongrie & 22.8 & 23.8 & 25.1 & 26.7 \\
\hline Irlande & .. & 27.3 & 27.6 & 28.5 \\
\hline Islande & 21.3 & 25.0 & 25.5 & 26.3 \\
\hline Italie & 25.0 & 28.0 & .. & 28.7 \\
\hline Japon & 25.6 & 27.5 & 28.0 & 29.1 \\
\hline Luxembourg & 24.7 & 27.4 & 28.4 & 29.0 \\
\hline Mexique & .. & 20.9 & 21.0 & 21.3 \\
\hline Norvège & .. & 26.4 & 26.9 & 27.7 \\
\hline Nouvelle-Zélande & .. & .. & 28.0 & 28.0 \\
\hline Pays-Bas & 24.8 & 28.4 & 28.6 & 28.9 \\
\hline Pologne & 22.8 & 23.8 & 24.5 & 25.8 \\
\hline Portugal & .. & 25.8 & 26.5 & 27.4 \\
\hline République slovaque & 22.6 & 23.0 & 24.2 & 25.7 \\
\hline République tchèque & 22.5 & 23.3 & 25.0 & 26.6 \\
\hline Royaume-Uni & .. & 28.3 & 29.1 & 29.8 \\
\hline Suède & 25.9 & 27.2 & 27.9 & 28.7 \\
\hline Suisse & 25.3 & 28.1 & 28.7 & 29.5 \\
\hline OCDE16 & 24.0 & 26.2 & 26.8 & 27.7 \\
\hline
\end{tabular}

1. 1992 pour le Mexique.

2. 2001 pour la Nouvelle-Zélande ; 2003 pour le Mexique.

3. 2003 pour la Finlande, la Grèce, l'Espagne et le Royaume-Uni ; 2002 pour les États-Unis ; 2004 pour la Nouvelle-Zélande ; et 2006 pour le Mexique.

Source : OCDE (2009), Panorama de la société. 


\section{Principaux résultats}

Le vieillissement de la population est l'un des principaux facteurs à l'origine de la vague de réformes des systèmes de retraite menées ces dernières années. Le taux de dépendance économique des personnes âgées est un indicateur important des pressions que l'évolution démographique fait peser sur les systèmes de retraite. Il mesure le nombre de personnes ayant atteint l'âge de la retraite (65 ans et plus) rapporté au nombre de personnes d'âge actif. En moyenne, dans les pays de l'OCDE, ce rapport est de $24 \%$. Autrement dit, il y a 4.2 personnes d'âge actif pour une personne retraitée.

Depuis un certain temps, les pays de l'OCDE vieillissent : entre 1950 et 1980, le taux de dépendance est passé de $14 \%$ à $21 \%$. Toutefois, l'accélération dans le passé récent et durant la période actuelle est plutôt modérée. En 2010, par exemple, ce taux devrait être de $25 \%$, soit une progression beaucoup plus lente qu'entre 1950 et 1980. À partir de 2010, le vieillissement devrait s'accélérer, et le taux de dépendance économique doublera, atteignant 50 \%, voire plus, dès 2047. Il y a aura alors à peine deux personnes d'âge actif pour une personne retraitée.

En 2007, le Japon était le pays de l'OCDE comptant le plus de personnes âgées, avec un taux de dépendance de $36 \%$. L'Allemagne, la Grèce et l'Italie enregistraient des taux de dépendance supérieurs à $30 \%$. Les pays les plus jeunes étaient le Mexique et la Turquie, avec des taux de dépendance de seulement $10 \%$, suivis de la Corée $(15 \%)$. Quatre des cinq membres de l'OCDE principalement anglophones - Australie, Canada, ÉtatsUnis et Irlande - connaissent tous une situation démographique relativement favorable. Les taux de dépendance y sont compris entre $17 \%$ et $22 \%$. Cette situation résulte probablement de l'immigration de travailleurs. De nombreux autres pays dont la population est actuellement jeune se trouvent en Europe de l'Est : en République slovaque, en République tchèque et en Pologne, les taux de dépendance économique oscillent entre $18 \%$ et $22 \%$.

L'évolution des taux de dépendance dépend de la mortalité, des taux de fécondité et des soldes migratoires. Comme le montrent les deux indicateurs précédents, les pays de l'OCDE ont enregistré une hausse continue de l'espérance de vie qui devrait se poursuivre à l'avenir. Ce phénomène accroît le nombre de personnes âgées et donc de retraités. Parallèlement, les taux de fécondité ont fortement chuté, ce qui réduira bien évidemment le nombre d'entrants sur le marché du travail. Étant donné que les bébés sont déjà nés, nous connaissons l'ampleur des variations du nombre de personnes d'âge actif sur les deux prochaines décennies. Par exemple, en moyenne, les taux de fécondité sont devenus inférieurs au seuil de renouvellement vers 1980 dans les pays de l'OCDE, ce qui signifie que chaque nouvelle génération sera moins nombreuse que la précédente. En 2000, par exemple, compte tenu du nombre de naissances, la taille de la cohorte des «bébés du millénaire " sera $20 \%$ à $25 \%$ inférieure à celle de leurs parents. Toutefois, de grandes incertitudes pèsent sur l'évolution future des taux de fécondité.
Pour l'OCDE dans son ensemble, le vieillissement de la population s'accélérera, passant du point le plus bas en 2006 à un pic en 2013. Le taux de dépendance atteindra $30 \%$ en 2018 , contre $24 \%$ actuellement. À partir de 2030, le processus de vieillissement démographique ralentira. Néanmoins, les taux de dépendance continueront de croître, atteignant 52 \% en moyenne en 2050. À cette date, il y aura un peu moins de deux personnes d'âge actif pour une personne ayant l'âge de la retraite, contre plus de quatre aujourd'hui.

La Corée sera de loin le pays de l'OCDE qui connaîtra le vieillissement le plus rapide. Le taux de dépendance devrait augmenter de $15 \%$ en 2007 à $77 \%$ en 2050. La Corée, qui était alors le troisième pays le plus jeune de l'OCDE, deviendra le deuxième plus âgé, derrière le Japon. Les autres pays de l'OCDE dont la population est actuellement jeune - le Mexique et la Turquie - vieilliront plus rapidement car leur évolution démographique converge avec celle des autres pays. Toutefois, à la différence de la Corée, ils continueront de faire partie des plus jeunes en 2050, avec des taux de dépendance qui se situeront respectivement à $38 \%$ et $30 \%$.

Selon les prévisions, certains pays de l'OCDE dont la population est actuellement âgée - Belgique France, Norvège, Pays-Bas, Royaume-Uni et Suède, par exemple - devraient voir leurs taux de dépendance faiblement augmenter au cours des 40 prochaines années environ. Là encore, cette évolution traduit une convergence dans les caractéristiques de vieillissement de la population des pays de l'OCDE.

\section{Définition et calcul}

Les projections des taux de dépendance économique des personnes âgées utilisées dans cette section sont basées sur les dernières projections de la "variante moyenne " de la population de chaque pays de l'OCDE, telles qu'elles figurent dans la Base de données de l'OCDE sur la démographie et la population active. 


\section{Taux de dépendance économique des personnes âgées - valeurs historiques et projections, $1950-2050$}
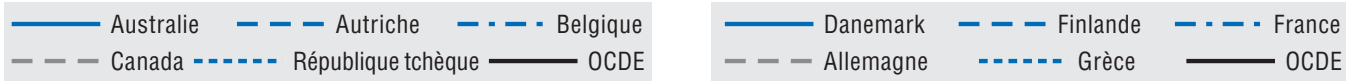

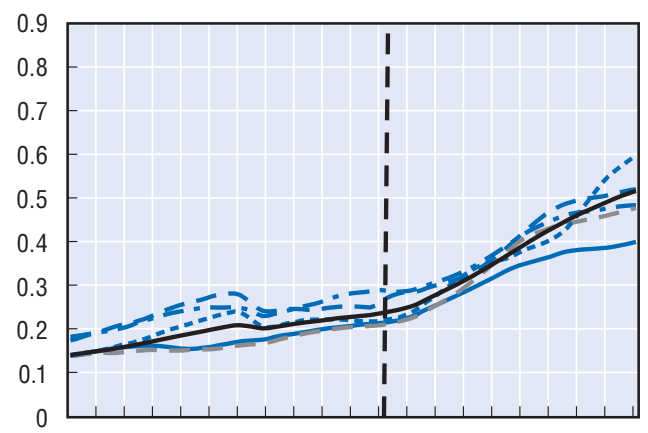

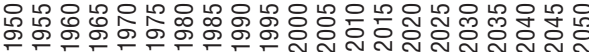

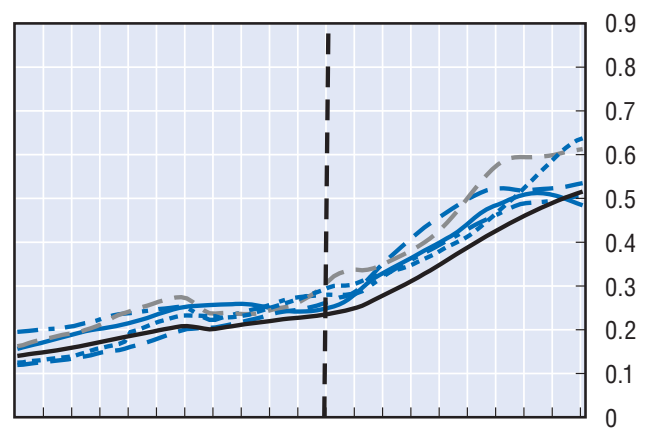

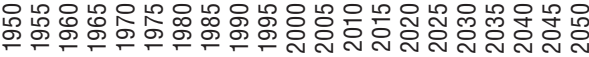
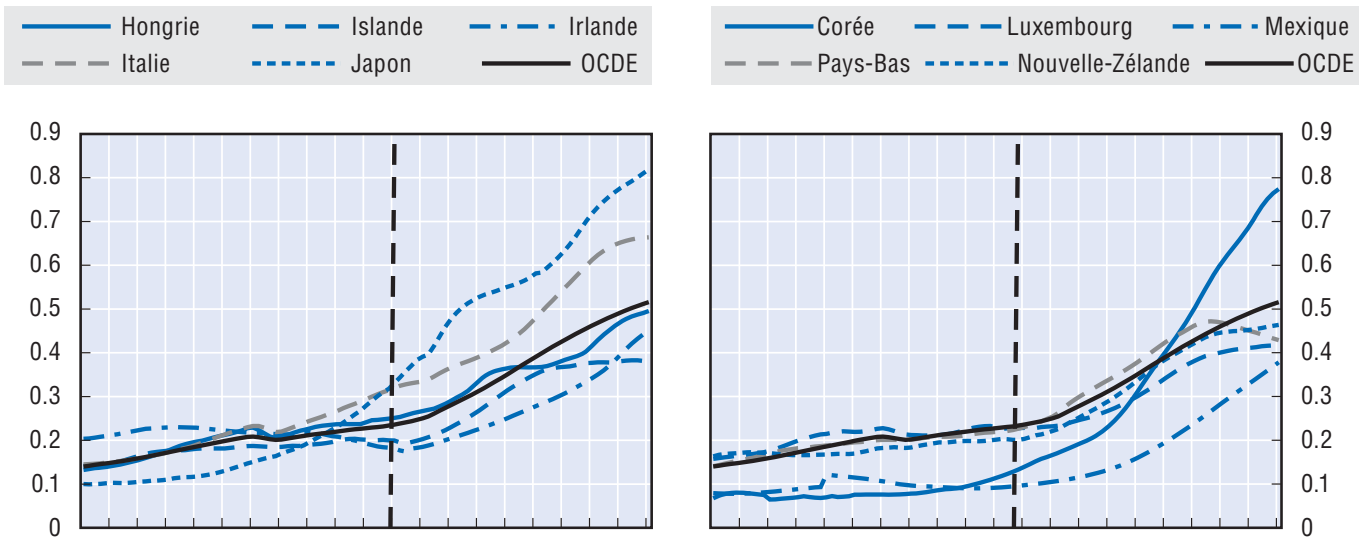

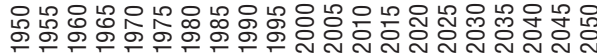

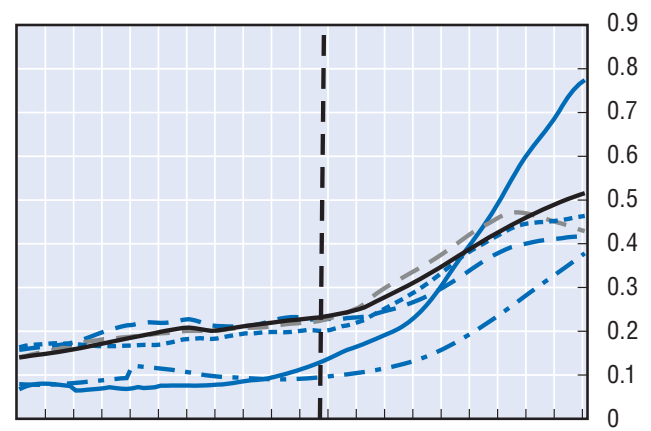

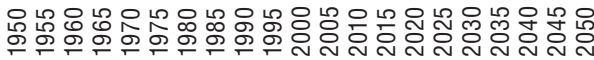
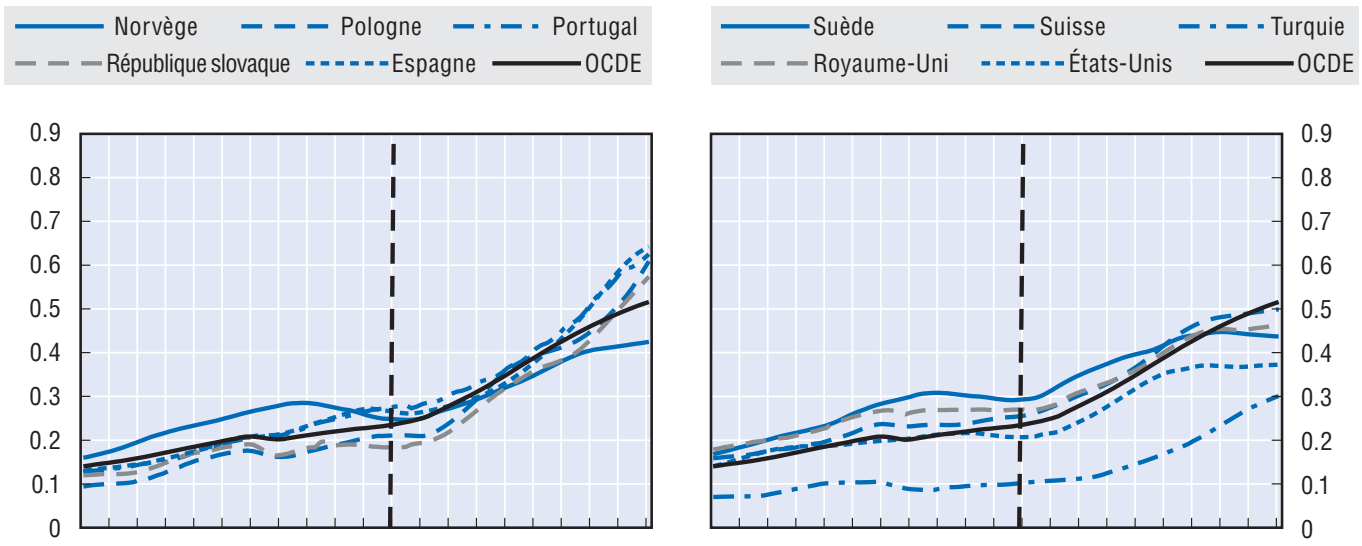

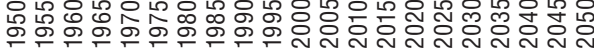

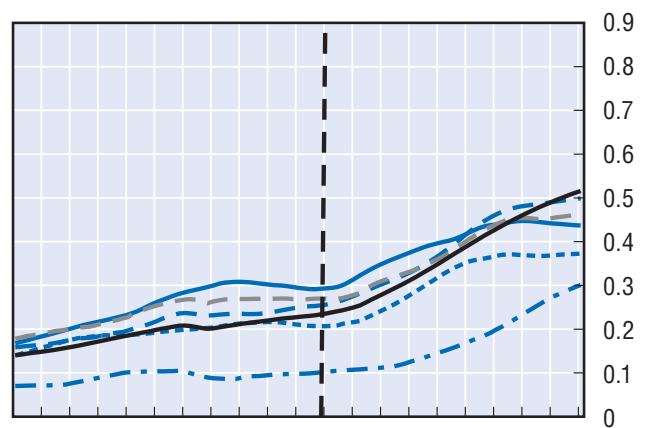

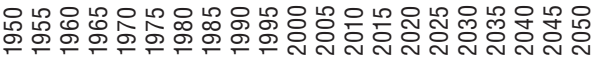

Source: Base de données de l’OCDE sur la démographie et la population active. 


\section{Principaux résultats}

Le salaire moyen est un indicateur important qui sous-tend la présentation des paramètres du système et les résultats de la modélisation des retraites. Néanmoins, il est très difficile de se procurer des données fiables et comparables pour différents pays.

Dans les années 70, l'OCDE a élaboré une méthode de calcul du salaire moyen en mesure de générer des résultats comparables pour les pays membres. Toutefois, cette comparabilité s'est faite au prix de résultats non représentatifs de l'ensemble des salariés et qui, au fil du temps, sont devenus de moins en moins représentatifs.

Un nouvel indicateur, adopté en 2004, calcule le salaire moyen en s'appuyant sur un éventail plus large de salariés, tout en conservant la comparabilité de l'ancienne mesure.

À partir de la deuxième édition des Pensions dans les pays de l'OCDE, le système de modélisation de l'OCDE adopte un indicateur nouveau et plus complet du salaire moyen, celui du "salarié moyen ", notion plus large que l'" ouvrier moyen ». Ce nouveau concept, qui a été introduit dans Les impôts sur les salaires, a également été repris dans la publication Prestations et salaires.

L'idée est que, compte tenu du déclin régulier de l'emploi manuel dans l'industrie manufacturière observé dans la plupart des pays de l'OCDE, un ouvrier moyen n'est plus représentatif du " contribuable type ». La nouvelle assiette de calcul du salaire moyen englobe davantage de secteurs économiques et se réfère à la fois aux travailleurs manuels et non manuels. Le concept et la définition du salaire, cependant, restent les mêmes : salaire brut du "salarié moyen", mesuré avant toute déduction, mais en incluant les heures supplémentaires et autres suppléments monétaires versés aux salariés.

Le tableau donne les niveaux du salaire moyen selon la nouvelle définition du salaire moyen pour l'année 2006. Seuls deux pays (Irlande et Turquie) ne sont pas encore en mesure de fournir des données selon la nouvelle définition, de sorte que la modélisation est basée sur l'ancienne mesure du salaire de l'ouvrier moyen. Les salaires moyens sont exprimés en monnaie nationale et en dollar américain (aux taux de change du marché et en parités de pouvoir d'achat, PPA). Le taux de change à PPA tient compte du fait que le pouvoir d'achat d'un dollar varie d'un pays à l'autre : il prend en compte des différences de prix d'un panier de biens et services entre pays. Le magazine The Economist publie régulièrement une version populaire et facile à comprendre de la PPA, l'indice "Big-Mac", qui illustre le niveau de surévaluation ou de sous-évaluation des monnaies par rapport au niveau auquel le coût d'un hamburger serait identique partout dans le monde.

Aux taux de change du marché, les salaires dans les pays de l'OCDE s'établissaient en moyenne à 35800 USD en 2006 dans les pays de l'OCDE. Exprimés en PPA, le salaire moyen était de 32800 USD. Ce chiffre inférieur laisse penser que le taux de change de monnaies de nombreux pays de l'OCDE par rapport au dollar américain était supérieur au taux qui permettrait d'égaliser le coût d'un panier standard de biens et services.

\section{Salaire moyen et médian}

La plupart des résultats présentés dans cette publication sont basés sur le salaire moyen. Toutefois, de nombreux indicateurs clés s'appuient aussi sur des estimations du salaire " médian ", c'est-à-dire le salaire tel que la moitié des salariés de la population considérée gagne moins et l'autre moitié gagne plus. Le tableau qui se trouve au bas de la page suivante, extrait de la base de données de l'OCDE sur la distribution des revenus, exprime le salaire médian en pourcentage du salaire moyen. On constate de grandes variations d'un pays à l'autre. Aux États-Unis et en Hongrie, la fourchette très large de distribution des salaires signifie que la médiane représente seulement les trois quarts du salaire moyen. Par contraste, la médiane atteint près de $90 \%$ de la moyenne en Allemagne, en Belgique, aux Pays-Bas et en Suède. Ce tableau fait également apparaître le décile des salaires le plus bas : $10 \%$ des salariés gagnent moins que ce montant. Pour les pays étudiés, ce niveau se situe à environ $50 \%$ du salaire moyen, niveau qui désigne un «bas salaire ». Le décile supérieur $-10 \%$ des salariés gagnent plus - représente en moyenne $160 \%$ du salaire moyen. Un « haut salaire " est supposé être une personne qui perçoit $150 \%$ du salaire moyen.

\section{Révisions des données relatives aux salaires depuis 2004}

Depuis la deuxième édition des Pensions dans les pays de l'OCDE, les estimations du "salarié moyen " ont été révisées. Les résultats de huit pays seulement s'en trouvent modifiés et, hormis le Royaume-Uni et la Turquie, l'effet est relativement faible. Au Royaume-Uni, par exemple, le salaire moyen a été révisé à hausse, passant de 27150 GBP (49 747 USD) à 29312 GBP (53 708 USD). La retraite de base représentant un volet important de la prestation obligatoire de revenus des personnes âgées, cette hausse réduit considérablement le taux de remplacement pour le titulaire du salaire moyen.

\section{Références}

OCDE (2007), Prestations et salaires, OCDE, Paris.

OCDE (2007), Les pensions dans les pays de l'OCDE, OCDE, Paris.

OCDE (2008), Les impôts sur les salaires 2006-2007, OCDE, Paris. 
Mesures du salaire moyen dans l'OCDE, 2006

En monnaie nationale et en USD au taux de change du marché et à parité de pouvoir d'achat

\begin{tabular}{|c|c|c|c|c|c|c|c|c|c|c|c|}
\hline & \multicolumn{3}{|c|}{$\begin{array}{c}\text { Mesures OCDE } \\
\text { du salaire moyen }\end{array}$} & \multicolumn{2}{|c|}{$\begin{array}{l}\text { Taux de change avec } \\
\text { le dollar américain (USD) }\end{array}$} & & \multicolumn{3}{|c|}{$\begin{array}{l}\text { Mesures OCDE } \\
\text { du salaire moyen }\end{array}$} & \multicolumn{2}{|c|}{$\begin{array}{l}\text { Taux de change avec } \\
\text { le dollar américain (USD) }\end{array}$} \\
\hline & $\begin{array}{c}\text { Monnaie } \\
\text { nationale } \\
\text { (salaire } \\
\text { moyen) }\end{array}$ & $\begin{array}{l}\text { USD, taux } \\
\text { du marché }\end{array}$ & $\begin{array}{l}\text { USD, } \\
\text { PPA }\end{array}$ & $\begin{array}{l}\text { Taux du } \\
\text { marché }\end{array}$ & PPA & & $\begin{array}{c}\text { Monnaie } \\
\text { nationale } \\
\text { (salaire } \\
\text { moyen) }\end{array}$ & $\begin{array}{l}\text { USD, taux } \\
\text { du marché }\end{array}$ & $\begin{array}{l}\text { USD, } \\
\text { PPA }\end{array}$ & $\begin{array}{l}\text { Taux du } \\
\text { marché }\end{array}$ & PPA \\
\hline Allemagne & 42400 & 53200 & 48700 & 0.8 & 0.87 & Japon & 4988900 & 42900 & 40100 & 116.35 & 124.46 \\
\hline Australie & 55200 & 41600 & 39100 & 1.33 & 1.41 & Luxembourg & 43600 & 54800 & 45900 & 0.8 & 0.95 \\
\hline Autriche & 36700 & 46100 & 42600 & 0.8 & 0.86 & Mexique & 73200 & 6700 & 10600 & 10.9 & 7.22 \\
\hline Belgique & 37700 & 47300 & 42400 & 0.8 & 0.89 & Norvège & 397800 & 62000 & 43200 & 6.41 & 9.21 \\
\hline Canada & 40600 & 35800 & 33700 & 1.13 & 1.2 & Nouvelle-Zélande & 43000 & 27500 & 28200 & 1.54 & 1.52 \\
\hline Corée & 30440200 & 32000 & 40000 & 951.82 & 762 & Pays-Bas & 39700 & 49900 & 44800 & 0.8 & 0.888 \\
\hline Danemark & 330900 & 55700 & 39200 & 5.94 & 8.44 & Pologne & 29300 & 9400 & 15700 & 3.1 & 1.87 \\
\hline Espagne & 21200 & 26500 & 27300 & 0.8 & 0.774 & Portugal & 15300 & 19300 & 21700 & 0.8 & 0.706 \\
\hline États-Unis & 39400 & 39400 & 39400 & 1 & 1 & République slovaque & 231200 & 7800 & 13500 & 29.65 & 17.13 \\
\hline Finlande & 33500 & 42100 & 34800 & 0.8 & 0.97 & République tchèque & 234800 & 10400 & 16500 & 22.59 & 14.19 \\
\hline France & 31000 & 38900 & 34200 & 0.8 & 0.91 & Royaume-Uni & 31500 & 58000 & 49200 & 0.54 & 0.645 \\
\hline Grèce & 23000 & 28900 & 32800 & 0.8 & 0.7 & Suède & 324600 & 44000 & 35600 & 7.37 & 9.12 \\
\hline Hongrie & 1988700 & 9500 & 15400 & 210.4 & 129.19 & Suisse & 72400 & 57800 & 42200 & 1.25 & 1.71 \\
\hline Irlande & 30000 & 37600 & 29500 & 0.8 & 1.01 & Turquie & 15600 & 10900 & 16700 & 1.43 & 0.939 \\
\hline Islande & 3480000 & 49800 & 34000 & 69.9 & 102.49 & & & & & & \\
\hline Italie & 24600 & 30900 & 27100 & 0.8 & 0.86 & OCDE & & 35800 & 32800 & & \\
\hline
\end{tabular}

Note : PPA = parité de pouvoir d'achat. Le salaire moyen selon le nouveau concept du salarié moyen n'est pas disponible pour l'Irlande et la Turquie, pour qui on utilise la définition de l'ouvrier moyen. Les valeurs monétaires pour la Turquie sont divisées par un million. Le salaire moyen est arrondi au centième le plus proche et les taux de change sont arrondis à une décimale.

Source : OCDE (2008), Les impôts sur les salaires 2006-2007 et Principaux indicateurs économiques de l'OCDE.

\section{Échelle de distribution des salaires (\% du salaire moyen)}

\begin{tabular}{lccc}
\hline & Décile inférieur & Médiane & Décile supérieur \\
\hline Allemagne & 53.0 & 89.3 & 161.1 \\
Australie & 51.1 & 86.1 & 159.3 \\
Belgique & 65.5 & 88.7 & 132.6 \\
Corée & 44.0 & 88.0 & 170.0 \\
Espagne & 39.9 & 80.3 & 168.5 \\
États-Unis & 36.9 & 77.4 & 179.1 \\
Finlande & 61.6 & 87.9 & 148.7 \\
Hongrie & 36.9 & 74.4 & 180.4 \\
Irlande & 43.9 & 85.6 & 172.7 \\
Italie & 63.6 & 87.7 & 152.2 \\
Japon & 54.4 & 88.0 & 160.2 \\
Norvège & 60.7 & 87.4 & 128.3 \\
Nouvelle-Zélande & 48.5 & 86.4 & 165.2 \\
Pays-Bas & 53.6 & 89.1 & 156.4 \\
Pologne & 41.2 & 81.7 & 168.4 \\
République tchèque & 52.5 & 87.1 & 153.9 \\
Royaume-Uni & 46.5 & 82.9 & 162.5 \\
Suède & 63.7 & 88.1 & 147.3 \\
OCDE18 & $\mathbf{5 1 . 0}$ & $\mathbf{8 5 . 3}$ & $\mathbf{1 5 9 . 3}$ \\
\hline
\end{tabular}

Source: Base de données de l'OCDE sur la distribution des revenus.
Effet sur les taux de remplacement bruts pour les titulaires du salaire moyen des révisions des données relatives aux salaires, 2004

\begin{tabular}{lcc|c}
\hline & \multicolumn{2}{c|}{ Taux de remplacement (\%) } & \multirow{2}{*}{ Différence } \\
\cline { 2 - 3 } & Avant & Après & \\
\hline États-Unis & 41.2 & 38.7 & -2.5 \\
Irlande & 32.5 & 31.9 & -0.6 \\
Islande & 77.5 & 77.1 & -0.4 \\
Luxembourg & 88.3 & 87.9 & -0.4 \\
Mexique & 35.8 & 36.6 & +0.8 \\
Portugal & 54.1 & 54.0 & -0.1 \\
Royaume-Uni & 30.8 & 29.9 & +0.9 \\
Turquie & 72.5 & 80.9 & +8.4 \\
\hline
\end{tabular}

Source: Modèles de retraite de l'OCDE. 



\section{PARTIE III}

\section{Descriptifs par pays}

Cette partie des Pensions dans les pays de l'OCDE décrit les paramètres et les règles qui régissent les systèmes de retraite nationaux. Toutefois, avant de présenter les descriptifs par pays, l'introduction procède à une comparaison internationale des caractéristiques essentielles et fournit un guide sur le contenu de ces descriptifs. En fin d'introduction, on trouvera un tableau récapitulatif des paramètres et des règles qui régissent les systèmes de retraite. 



\section{Introduction}

\section{Principales caractéristiques des systèmes de retraite}

Le «Cadre conceptuel des Pensions dans les pays de l'OCDE » exposé un peu plus haut classe les principales composantes des systèmes nationaux de retraite en piliers (voir graphique 0.1). Les deux premiers piliers sont obligatoires. Le premier relève du secteur public et se compose de régimes visant à garantir un revenu de retraite suffisant. Le deuxième relève du secteur public ou du secteur privé et se compose de régimes dont le principal objectif est d'assurer des revenus de retraite offrant un certain taux de remplacement des revenus perçus pendant la vie active. Le tableau III.1 résume les principales caractéristiques de ces deux piliers, c'est-à-dire les paramètres essentiels et les règles qui déterminent le montant de revenus de retraite que les personnes percevront.

\section{Premier pilier, régimes redistributifs}

Le niveau des prestations dans les régimes redistributifs du premier pilier est exprimé en pourcentage du salaire moyen dans chaque pays (voir l'indicateur du «Salaire moyen » à la partie II). Du fait que certains pays associent différents programmes, le niveau des prestations est présenté séparément pour chacun. Les régimes de base versent une prestation forfaitaire soumise à une condition de résidence (aux Pays-Bas et en Nouvelle-Zélande, par exemple) ou à un certain nombre d'années de cotisation (Irlande et Royaume-Uni). Les régimes soumis à conditions de ressources sont ciblés sur un niveau de revenu et réduisent les prestations au prorata de toutes les autres sources de revenus. Au contraire, la pension minimum ne prend en compte que la valeur du revenu de retraite pour le calcul des droits à prestations.

Dans le cas du régime de base et de la pension minimum, les droits à prestations sont présentés pour un travailleur qui entre sur le marché du travail à 20 ans et travaille sans interruption jusqu'à l'âge normal ouvrant droit à la retraite. Dans la plupart des pays de l'OCDE, cet âge est de 65 ans, comme l'indiquent les dernières lignes du tableau III.1. Seuls les travailleurs à très faible revenu ayant effectué une carrière complète seront admissibles aux programmes soumis à conditions de ressources ; les bénéficiaires seront en majorité des travailleurs ayant eu un parcours professionnel bref et ponctué d'interruptions ${ }^{1}$. La dernière ligne indique la prestation totale de premier pilier pour un travailleur ayant effectué une carrière complète. Dans certains cas, les travailleurs peuvent percevoir plusieurs types différents de prestations de premier pilier tandis que dans d'autres ils n'ont droit qu'à un seul programme.

Les prestations de premier pilier représentent en moyenne, dans les pays de l'OCDE, 27 \% du salaire moyen national. Les prestations sont particulièrement élevées par rapport au salaire moyen en Belgique, au Luxembourg et en Nouvelle-Zélande. C'est en Finlande, en Allemagne, en Hongrie, au Japon et aux États-Unis qu'elles sont les plus faibles (inférieures à $20 \%$ du salaire moyen national). 
Tableau III.1. Résumé des paramètres et des règles des régimes de retraite

\begin{tabular}{|c|c|c|c|c|c|c|c|c|c|c|}
\hline & Australie & Autriche & Belgique & Canada & $\begin{array}{l}\text { République } \\
\text { tchèque }\end{array}$ & Danemark & Finlande & France & Allemagne & Grèce \\
\hline \multicolumn{11}{|l|}{ Premier pilier (\% salaire moyen) } \\
\hline Soumis à conditions de ressources & 23 & 26 & 22 & 18 & 23 & 18 & 18 & 22 & 19 & 11 \\
\hline De base & - & - & - & 14 & 8 & 18 & - & - & - & - \\
\hline Minimum & - & - & 28 & - & 11 & - & - & 23 & - & 34 \\
\hline Droit total (carrière complète) & 23 & 26 & 28 & 32 & 23 & 36 & 18 & 23 & 19 & 34 \\
\hline \multicolumn{11}{|l|}{ Deuxième pilier } \\
\hline \multicolumn{11}{|l|}{ Lié à la rémunération } \\
\hline Type & Néant & PD & PD & PD & PD & Néant & PD & PD/points & points & $\mathrm{PD}$ \\
\hline Taux d'acquisition (\% salaire individuel) & - & 1.78 & 1.33 & 0.63 & $0.45[w]^{1}$ & - & $1.5[a]^{2}$ & $1.75[w]^{3}, 4$ & 1.00 & $2.57^{3}$ \\
\hline Salaire de référence & - & 40 & $\mathrm{~L}$ & b34 & $\mathrm{f} 30$ & - & $\mathrm{L}$ & $\mathrm{b} 25 / \mathrm{L}$ & $\mathrm{L}$ & f5 \\
\hline Revalorisation & - & $w^{5}$ & $\mathrm{p}$ & w & w & - & $80 w / 20 p$ & $\mathrm{p} / \mathrm{p}$ & $w^{6}$ & 7 \\
\hline Indexation & - & $d$ & $\mathrm{p}$ & $p(c)$ & $33 w / 67 p$ & - & $20 w / 80 p$ & $\mathrm{p} / \mathrm{p}$ & $w^{6}$ & $d$ \\
\hline \multicolumn{11}{|l|}{ Cotisations définies } \\
\hline Taux de cotisation & 9 & - & - & - & - & $1+10.8^{8}$ & - & - & - & \\
\hline \multicolumn{11}{|l|}{ (\% salaire individuel) } \\
\hline \multicolumn{11}{|l|}{ Plafonds (\% salaire moyen) } \\
\hline Public & - & 146 & 118 & 104 & Néant & - & - & 99 & 149 & $325^{9}$ \\
\hline Privé/professionnel & 244 & - & - & - & - & - & Néant & 298 & - & - \\
\hline \multicolumn{11}{|l|}{ Âge de départ en retraite } \\
\hline Normal & 65 & 65 & 65 & 65 & 65 & 65 & 65 & 61 & 67 & 65 \\
\hline (femmes) & & 65 & & & $62-65^{10}$ & & & & & \\
\hline Anticipé & 55 & & 60 & 60 & 60 & & 62 & & 63 & 55 \\
\hline (femmes) & & & & & $59-60^{10}$ & & & & & \\
\hline
\end{tabular}

Les paramètres concernent l'année 2006 mais tiennent compte de tous les changements de législation qui prendront effet dans le futur. Par exemple, certains pays relèvent l'âge de départ à la retraite et élargissent le salaire de référence pour le calcul des prestations ; pour les femmes, l'âge de la retraite n'est indiqué que s'il est différent de celui des hommes. L'âge de départ anticipé n'est indiqué que lorsqu'une retraite anticipée est possible.

- = ne s'applique pas $;[a]$ = varie avec l'âge $; b=$ nombre de meilleures années ; $(c)=$ indexation dépendant des régimes de financement ; $\mathrm{d}=$ indexation discrétionnaire $; \mathrm{f}=$ nombre des dernières années $; \mathrm{fr}=$ revalorisation à un taux fixé par voie règlementaire $; \mathrm{L}=$ moyenne sur l'ensemble de la carrière ; $\mathrm{p}=$ revalorisation/indexation sur les prix ; $\mathrm{w}=$ revalorisation/indexation sur le salaire moyen ; [w] = varie avec le salaire; $[y]$ = varie avec le nombre d'années d'activité.

$\mathrm{CD}=$ cotisations définies $; \mathrm{NCD}=$ comptes notionnels $; \mathrm{PIB}=$ lié au produit intérieur brut $; \mathrm{PD}=$ prestations définies.

1. République tchèque : taux d'acquisition plus élevés pour les faibles revenus et moins élevés pour les hauts revenus.

2. Finlande : les taux d'acquisition augmentent avec l'âge.

3. France et Grèce : les chiffres indiqués associent deux programmes différents.

4. France : taux d'acquisition plus élevé pour les hauts revenus dans les régimes professionnels.

5. Autriche : la revalorisation est supposée se faire désormais davantage en fonction de l'évolution des salaires du fait de l'allongement de la période sur laquelle est établie la moyenne pour le salaire de référence.

6. Allemagne : l'indexation et la revalorisation sont toutes deux sujettes à un ajustement de durabilité.

7. Grèce : revalorisation alignée sur les augmentations des pensions pour les salariés du secteur public.

8. Danemark : $1 \%$ est le taux de la retraite spéciale obligatoire, $10.8 \%$ est le taux de cotisation type pour les régimes professionnels quasi obligatoires.

9. Grèce : plafond effectif calculé à partir de la pension maximum.

10. République tchèque : pour les femmes l'âge de départ à la retraite varie avec le nombre d'enfants.

\section{Deuxième pilier, régimes d'assurance}

Les informations sur le deuxième pilier (régime d'assurance) des systèmes de retraite sont présentées séparément pour les régimes liés à la rémunération et pour les plans à cotisations définies $(C D)$.

Les informations sur les régimes liés à la rémunération commencent par le type de régime: prestations définies (PD), points ou comptes notionnels (NCD). Le principal paramètre auquel sont imputables les différences de valeur de ces régimes est le taux 
Tableau III.1. Résumé des paramètres et des règles des régimes de retraite (suite)

\begin{tabular}{|c|c|c|c|c|c|c|c|c|c|c|}
\hline & Hongrie & Islande & Irlande & Italie & Japon & Corée & Luxembourg & Mexique & Pays-Bas & $\begin{array}{l}\text { Nouvelle- } \\
\text { Zélande }\end{array}$ \\
\hline \multicolumn{11}{|l|}{ Premier pilier (\% salaire moyen) } \\
\hline Soumis à conditions de ressources & - & $18^{11}$ & 32 & - & 19 & - & - & - & - & - \\
\hline De base & - & 9 & 34 & - & 16 & 22 & 10 & 4.6 & 31 & 39 \\
\hline Minimum & 16 & - & - & - & - & - & 38 & 28 & - & - \\
\hline Droit total & 16 & 27 & 34 & 22 & 19 & 22 & 38 & 28 & 31 & 39 \\
\hline \multicolumn{11}{|l|}{ Deuxième pilier } \\
\hline \multicolumn{11}{|l|}{ Lié à la rémunération } \\
\hline Type & PD & PD & Néant & n.acs & PD & PD & PD & Néant & PD & Néant \\
\hline Taux d'acquisition (\% salaire individuel) & 1.22 & 1.40 & - & 1.75 & 0.55 & 1 & $1.85[y]^{12}$ & - & $1.75^{13}$ & - \\
\hline Salaire de référence & L & L & - & L & L & $\mathrm{L}$ & L & - & $L^{14}$ & - \\
\hline Revalorisation & w & (fr) & - & PIB & w & w & w & - & w (c) & - \\
\hline Indexation & $50 w / 50 p$ & $p$ & - & $p^{15}$ & $\mathrm{p}$ & $\mathrm{p}$ & w & - & w (c) & - \\
\hline \multicolumn{11}{|l|}{ Cotisations définies } \\
\hline Taux de cotisation (\% salaire individuel) & 8 & - & - & - & - & - & - & $6.5^{16}$ & - & - \\
\hline \multicolumn{11}{|l|}{ Plafonds (\% salaire moyen) } \\
\hline Public & 220 & - & - & 367 & 149 & 142 & 231 & - & - & - \\
\hline Privé/professionnel & 220 & Néant & - & - & - & - & - & 607 & Néant & - \\
\hline \multicolumn{11}{|l|}{ Âge de départ en retraite } \\
\hline Normal & 62 & 67 & 66 & 65 & 65 & 65 & 65 & 65 & 65 & 65 \\
\hline (femmes) & & & & 60 & & & & & & \\
\hline Anticipé & & & 65 & 60 & 60 & 60 & 57 & 60 & 60 & \\
\hline
\end{tabular}

11. Islande : englobe deux programmes différents.

12. Luxembourg : taux d'acquisition plus élevé pour des durées de cotisation plus longues.

13. Pays-Bas : le taux d'acquisition varie d'un régime professionnel à l'autre.

14. Pays-Bas : les gains sont mesurés par le salaire moyen pour environ les deux tiers des régimes professionnels et par le dernier salaire pour le tiers restant.

15. Italie : l'indexation se fait intégralement sur les prix pour les pensions modestes et à $90 \%$ ou à $75 \%$ sur les prix pour les pensions plus élevées.

16. Mexique : la cotisation supplémentaire de $5.5 \%$ du salaire minimum est présentée comme une pension de base. La valeur plus faible de la rente calculée concerne les femmes.

d'acquisition des droits par année de cotisation, c'est-à-dire le rythme auquel un travailleur acquiert des droits à prestations pour chaque année de couverture. Le taux d'acquisition est exprimé en pourcentage de la rémunération « couverte " par le régime de retraite. La plupart des régimes de retraite ne prennent en compte qu'une partie de la rémunération pour le calcul des prestations de retraite.

Pour les systèmes à points, le taux effectif d'acquisition des droits qui apparaît dans le tableau III.1 est le ratio entre le coût et la valeur du point de retraite, exprimé en pourcentage du salaire individuel. Comme le taux d'acquisition dans les régimes à prestations définies, ce ratio indique la prestation acquise chaque année en proportion des gains de l'année en question. Dans les régimes de comptes notionnels, le taux effectif d'acquisition des droits est calculé à peu près de la même façon pour obtenir le droit annuel à prestations de retraite en proportion des gains d'une année donnée. Les calculs, qui dépendent du taux de cotisation, du taux d'intérêt théorique et des facteurs de rente, sont décrits en détail dans Queisser et Whitehouse (2006).

Dans un peu moins de la moitié des pays ayant des régimes liés à la rémunération (des trois types), les taux d'acquisition sont linéaires. Dans les autres, la prestation de retraite acquise pour chaque année de couverture varie avec la rémunération, le nombre d'années de cotisation ou l'âge de l'individu. 
Tableau III.1. Résumé des paramètres et des règles des régimes de retraite (suite)

\begin{tabular}{|c|c|c|c|c|c|c|c|c|c|c|}
\hline & Norvège & Pologne & Portugal & $\begin{array}{c}\text { Rép. } \\
\text { slovaque }\end{array}$ & Espagne & Suède & Suisse & Turquie & $\begin{array}{l}\text { Royaume- } \\
\text { Uni }\end{array}$ & États-Unis \\
\hline \multicolumn{11}{|l|}{ Premier pilier (\% salaire moyen) } \\
\hline Soumis à conditions de ressources & 31 & - & 16 & - & - & 26 & 24 & 5 & 19 & 18 \\
\hline De base & 16 & - & - & - & - & - & - & - & 14 & - \\
\hline Minimum & - & 24 & 32 & $22^{17}$ & 27 & - & 18 & 37 & $14^{17}$ & - \\
\hline Droit total (carrière complète) & 31 & 24 & 32 & 22 & 27 & 26 & 24 & 37 & 28 & 18 \\
\hline \multicolumn{11}{|l|}{ Deuxième pilier } \\
\hline \multicolumn{11}{|l|}{ Lié à la rémunération } \\
\hline Type & Points & n.d. & PD & Points & PD & n.acs & PD & PD & PD & PD \\
\hline Taux d'acquisition (\% salaire indivuel) & $1.05[w]^{18}$ & 0.67 & $2.25[w]^{19}$ & 1.19 & $3.0[y]^{20}$ & $1.21[w]$ & {$[\mathrm{w} / \mathrm{a}]$} & 2.0 & $0.89[w]^{21}$ & $0.91[w]^{19}$ \\
\hline Salaire de référence & b20 & $\mathrm{L}$ & L & L & $\mathrm{f} 15$ & $\mathrm{~L}$ & L & $\mathrm{L}$ & L & b35 \\
\hline Revalorisation & w & $w^{22}$ & $25 w / 75 p$ & w & $\mathrm{p}$ & w & $\operatorname{Fr}(2.0 \%)$ & $\mathrm{PIB}$ & w & $w^{24}$ \\
\hline Indexation & $\mathrm{p}$ & $p^{22}$ & $\mathrm{p} / \mathrm{PIB}^{23}$ & $50 w / 50 p$ & $\mathrm{p}$ & $w-1.6(c)$ & $50 w / 50 p$ & $\mathrm{p}$ & $\mathrm{p}$ & $\mathrm{p}$ \\
\hline \multicolumn{11}{|l|}{ Cotisations définies } \\
\hline Taux de cotisation (\% salaire individuel) & 2.0 & 7.3 & - & 9 & - & $2.5+4.5^{25}$ & - & - & - & - \\
\hline \multicolumn{11}{|l|}{ Plafonds (\% salaire moyen) } \\
\hline Public & 188 & 250 & Néant & 300 & 164 & 111 & 106 & 265 & 105 & 240 \\
\hline Privé/professionnel & - & - & - & - & - & - & 106 & - & - & - \\
\hline \multicolumn{11}{|l|}{ Âge de départ en retraite } \\
\hline Normal & 67 & 65 & 65 & 62 & 65 & 65 & 65 & 65 & 68 & 67 \\
\hline (femmes) & & 60 & & & & & 64 & & & \\
\hline Anticipé & & & 55 & & 60 & 61 & 63 & & & 62 \\
\hline (femmes) & & & & & & & 62 & & & \\
\hline
\end{tabular}

17. République slovaque et Royaume-Uni : prestation minimum calculée à partir du crédit minimum.

18. Norvège : taux d'acquisition plus faible pour les hauts revenus.

19. Portugal et États-Unis : taux d'acquisition plus élevés pour les faibles revenus et moins élevés pour les hauts revenus.

20. Espagne : taux d'acquisition plus élevé pour les premières années d'activité, plus faible ensuite.

21. Royaume-Uni : c'est pour les bas revenus que le taux d'acquisition est le plus élevé ; il baisse ensuite pour monter à nouveau.

22. Pologne : revalorisation sur la croissance réelle de la masse salariale et à tout le moins sur la hausse des prix. L'indexation se fait à $80 \%$ sur les prix et à $20 \%$ sur les salaires mais depuis 2005 elle se fait davantage sur la base des prix.

23. Portugal : l'indexation sera plus forte que la hausse des prix pour les pensions modestes et vice versa. Plus la croissance du PIB sera forte, plus l'indexation sera généreuse.

24. États-Unis : revalorisation sur les salaires jusqu'à 60 ans ; aucun ajustement entre 60 et 62 ans; revalorisation sur les prix entre 62 et 67 ans.

25. Suède : le taux de cotisation est de $2.5 \%$ pour les plans individuels. Pour les plans professionnels quasi obligatoires, le taux de cotisation est de $4.5 \%$ sur la tranche inférieure des gains et de $30 \%$ sur la tranche supérieure (dans le régime général des salariés du secteur privé).

Source: Informations fournies par les autorités nationales et calculs de l'OCDE. Voir les descriptifs par pays ci-après.

Dans huit cas, le taux d'acquisition varie avec la rémunération (indiqué au tableau III.1 par [w]). Dans les régimes publics de la République tchèque, du Portugal, de la Suisse et des Etats-Unis, le schéma est progressif, les taux de remplacement étant plus élevés pour les travailleurs à faible revenu. Au Royaume-Uni, la courbe des taux d'acquisition a une forme en $\mathrm{U}$ : très élevée pour les travailleurs à faible revenu, elle baisse ensuite pour remonter à nouveau. En Norvège, les taux d'acquisition sont moindres pour les revenus élevés. En France et en Suède, les régimes professionnels sont conçus pour compenser la redistribution instaurée par le régime public ; le taux de remplacement est plus élevé pour les hauts revenus sur la partie de leur rémunération se situant au-dessus du plafond du régime public.

Dans les régimes professionnels de Finlande et de Suisse, le taux d'acquisition des droits à pension augmente avec l'âge (indiqué par [a]).

Deux pays ont des taux d'acquisition qui varient avec la durée d'activité ([y]). Au Luxembourg, le taux d'acquisition augmente avec le nombre d'années de cotisation. En 
Espagne, il existe trois taux d'acquisition. Le schéma est inverse de celui du Luxembourg en ce sens que le taux d'acquisition est au plus haut pour les premières années de couverture et au plus bas pour les dernières dans le cas de personnes ayant cotisé un grand nombre d'années.

\section{Mesure de la rémunération pour le calcul des prestations}

Dans les régimes à prestations définies, les droits à pension dépendent de la rémunération passée de l'individu mais la manière dont celle-ci est évaluée diffère. Le tableau III.1 indique si l'on utilise les gains moyens perçus sur l'ensemble de la carrière, les salaires d'un nombre limité de meilleures années ou les salaires des dernières années. Il est important de se souvenir que les informations présentées ici concernent les règles à long terme du système.

Pour calculer les prestations, dix-sept pays de l'OCDE utilisent la moyenne des gains perçus sur l'ensemble de la carrière. Le Canada, la République tchèque et les États-Unis utilisent la moyenne des gains perçus sur la grande majorité de la carrière (30 à 35 ans). En Autriche, la durée prise en compte pour le calcul des prestations est portée progressivement à 40 ans. Il existe toutefois quelques exceptions. Les derniers salaires seront utilisés pour le calcul des prestations en Grèce et en Espagne (salaires des cinq et 15 dernières années respectivement). En France et en Norvège, les prestations des régimes publics de retraite seront établies respectivement sur la base des 20 et 25 meilleures années.

\section{Revalorisation}

La politique de revalorisation ou de réévaluation est étroitement liée au salaire de référence. Elle consiste à ajuster les gains passés pour prendre en compte l'évolution du niveau de vie entre le moment où les droits à pension sont acquis et celui où ils sont liquidés. (Elle est parfois appelée indexation de préretraite.) Si les prestations sont établies sur la base des salaires de la dernière année, la revalorisation est inutile. En revanche, elle est nécessaire pour préserver la valeur des droits à pension lorsque les prestations sont basées sur les gains d'une période plus longue. Le relèvement du point de retraite et le taux d'intérêt théorique dans les régimes à points et les régimes de comptes notionnels sont respectivement les exacts corollaires de la revalorisation dans les régimes à prestations définies (voir encadré 4 dans Queisser et Whitehouse, 2006 pour une explication détaillée).

L'incidence de la politique de revalorisation sur les droits à pension est importante en raison de l'effet "intérêt composé ". Pour les hypothèses économiques de base retenues dans ce rapport, à savoir taux réel de progression des salaires de $2 \%$ et hausse des prix de $2.5 \%$, la revalorisation sur la base des prix pour une carrière complète (entre 20 et 65 ans) conduit à une pension qui serait inférieure de $40 \%$ à celle que donnerait une politique d'ajustement total de la rémunération des années antérieures en fonction du salaire moyen à l'échelle nationale.

La pratique la plus courante, adoptée par quinze pays de l'OCDE, consiste à réévaluer la rémunération des années antérieures en fonction de la progression du salaire moyen à l'échelle nationale. Toutefois, la Belgique, la France, l'Islande et l'Espagne ne réévaluent les gains qu'en fonction de la hausse des prix, bien qu'en Espagne l'incidence soit relativement faible car la formule de calcul des prestations ne porte que sur le salaire des 15 dernières années contre 25 dans le régime public français et la moyenne des gains perçus sur l'ensemble de la carrière en Belgique et dans les régimes professionnels 
français. La Finlande, le Portugal et la Turquie réévaluent les gains des années antérieures selon une formule associant la hausse des prix et celle des salaires.

\section{Régimes à cotisations définies}

Le principal paramètre des régimes à cotisations définies (CD) est la proportion $d u$ salaire qui doit être versée sur les comptes individuels par les salariés, les employeurs ou l'État. Les taux de cotisation à ces régimes varient sensiblement d'un pays à l'autre. Dans les pays nordiques, les taux de cotisation aux régimes $C D$ obligatoires sont relativement bas : $1 \%$ au Danemark, $2 \%$ en Norvège et $2.5 \%$ en Suède. Toutefois, au Danemark et en Suède les régimes professionnels sont classés en "quasi obligatoires " car ils couvrent la quasi-totalité de la population active. Les taux de cotisation à ces régimes sont habituellement de $10.8 \%$ du salaire au Danemark; en Suède, ils sont de $4.5 \%$ sur la tranche de gains inférieure et de $30 \%$ sur la tranche supérieure (dans le régime général des salariés du secteur privé). Le taux de cotisation moyen pour les huit pays présentés est de $8.3 \%$.

\section{Plafonnement de la rémunération ouvrant droit à pension}

La plupart des pays n'exigent pas que les salariés à haut revenu contribuent au régime de retraite sur l'intégralité de leur rémunération. Habituellement, une limite est fixée à la rémunération utilisée à la fois pour le calcul des cotisations et des prestations de retraite. Ce plafonnement des salaires couverts par le régime de retraite a un impact important sur la structure, la taille et le coût des régimes du deuxième pilier. Le plafond moyen imposé aux retraites publiques de 20 pays est de $190 \%$ du salaire moyen à l'échelle nationale. (Cette moyenne exclut les huit pays dans lesquels il n'existe pas de régime public de retraite, tels que programmes de base ou ciblés, pour lequel un tel plafond serait pertinent et les deux pays dans lesquels la rémunération ouvrant droit à une pension publique n'est pas plafonnée.)

Le tableau III.1 montre également (de façon séparée) les plafonds des régimes de retraite privés obligatoires et des régimes professionnels publics en France et en Finlande. Sur les neuf pays s'étant doté de ce type de programme, trois (Finlande, Islande et Pays-Bas) n'ont pas de système de plafonnement. En France et en Suède, les plafonds des régimes professionnels sont égaux respectivement à trois et 2.8 fois le plafond de la rémunération ouvrant droit à pension dans le programme public. Le plafond global de la rémunération ouvrant droit à pension dans les 22 pays pour lesquels cet élément est pertinent et où il existe un système de plafonnement est en moyenne de $230 \%$, soit nettement supérieur au plafond des seuls régimes publics.

\section{Âge d'ouverture des droits à pension}

Dans la majorité des pays membres de l'OCDE, l'âge normal de départ à la retraite est de 65 ans pour les hommes comme pour les femmes. L'âge ouvrant droit à la retraite demeure plus bas pour les femmes dans plusieurs pays mais, dans la plupart d'entre eux (Belgique, Hongrie et Royaume-Uni, par exemple), il sera progressivement relevé et aligné sur celui des hommes. Seuls l'Italie, la Suisse et la Pologne envisagent le maintien à long terme d'un âge de retraite inférieur pour les femmes.

En Islande, en Norvège et aux États-Unis, l'âge normal de la retraite est de 67 ans et d'autres pays, comme le Danemark, l'Allemagne et le Royaume-Uni, vont relever l'âge de la retraite au-delà de 65 ans. 
Toutefois, plus de la moitié des pays de l'OCDE autorisent le départ en retraite avant l'âge normal mais généralement assorti d'une réduction des prestations ou décote.

\section{Indexation des pensions mises en paiement}

L'indexation fait référence à la revalorisation des paiements à compter de la liquidation des droits à pension (voir Whitehouse, 2009). Les prestations de retraite sont généralement ajustées sur la base de l'indice des prix à la consommation. Cinq pays ont un système mixte de revalorisation des prestations qui associe la hausse des prix et celle des salaires : il s'agit de la République tchèque, la Finlande, la Hongrie, la République slovaque et la Suisse.

L'Autriche, la Grèce, l'Italie et le Portugal ont adopté des mécanismes progressifs d'indexation qui augmentent davantage les pensions modestes que les prestations élevées. L'Italie ajuste entièrement les petites pensions sur la hausse des prix tandis que pour les pensions élevées le pourcentage d'ajustement varie. Au Portugal, les pensions sont ajustées selon une formule combinant la hausse des prix et la croissance du PIB; les augmentations exactes dépendent à la fois du niveau des pensions et des taux de croissance du PIB.

\section{Indexation des paramètres des systèmes de retraite}

L'indexation affecte non seulement les pensions mises en paiement mais également les paramètres des systèmes de retraite. Dans les régimes de base et dans les régimes soumis à conditions de ressources, l'ajustement des prestations lors de la liquidation des droits est plus important que celui des prestations mises en paiement.

Prenons l'exemple du régime de base du Royaume-Uni. La pension de base est liée à l'évolution des prix depuis 1981 ; à cette date, elle correspondait à $24 \%$ du salaire moyen. Aujourd'hui, elle correspond à peine à $14 \%$ du salaire moyen. La modification de la procédure d'indexation a réduit non seulement les droits à prestations des retraités mais aussi les prestations de tous les futurs travailleurs. Si la procédure est maintenue, la pension de base des nouveaux entrants ne représentera plus qu'un très faible pourcentage du salaire moyen lorsqu'ils partiront à la retraite dans 45 ans.

Le Canada et la Suède également indexent les prestations de leurs régimes soumis à conditions de ressources sur les prix (tandis que le Royaume-Uni les indexe désormais sur le salaire moyen). La conséquence c'est que, sur les longues périodes qu'impliquent les politiques de retraite, ces programmes vont à pratiquement disparaître. Pour les nouveaux entrants, le revenu minimum de retraite dans 45 ans sera très faible. Il est difficile de croire qu'il sera politiquement possible de verser des revenus aussi faibles à des personnes âgées pauvres. En conséquence, ces politiques ont peu de chances d'être tenables ou d'être maintenues.

Un argument analogue s'applique aux autres paramètres du régime de retraite, par exemple au plafond du régime lié à la rémunération qui, dans certains pays (le Canada, par exemple) est indexé sur les prix.

En conséquence, la modélisation utilisée dans ce rapport suppose explicitement que ces prestations et paramètres sont liés au salaire moyen et non pas indexés sur les prix même si c'est ce que spécifie la législation. Manifestement, cette hypothèse a une forte incidence sur les résultats lorsqu'on calcule la valeur de la promesse de retraite. 


\section{Guide des descriptifs par pays}

Les descriptifs par pays utilisent un cadre commun. Ils commencent par un bref résumé du système national de retraite et un tableau des indicateurs essentiels. Ce tableau indique le salaire moyen, les dépenses publiques au titre des retraites, l'espérance de vie et le taux de dépendance économique (nombre de retraités pour 100 actifs). Des données sont présentées pour le pays en question et pour la moyenne de l'ensemble des pays de l'OCDE.

Ils décrivent ensuite dans le détail les règles et les paramètres des régimes de retraite qui composent le système de revenus des retraites de chaque pays. Ils sont structurés comme suit.

- Conditions d'ouverture des droits : âge d'ouverture des droits à la retraite (ou « de départ à la retraite ") et nombre d'années de cotisation requises pour percevoir une pension.

- Calcul des prestations : règles pour chacun des régimes composant le système de retraite tels que régime de base, régime soumis à conditions de ressources et pension minimum mais aussi régime public, régime lié à la rémunération et régimes privés obligatoires.

- Pensions privées facultatives : les paramètres des plans facultatifs types sont indiqués pour les pays pour lesquels les taux de remplacement dans ces régimes ont été modélisés dans l'indicateur des " Taux bruts de remplacement des pensions publiques et privées " à la partie II.

- Variante de carrière 1 : règles et conditions dans lesquelles les travailleurs peuvent partir à la retraite anticipée ou continuer à travailler au-delà de l'âge normal de la retraite et impact sur les droits à pension.

- Variante de carrière 2 : règles visant à protéger les retraites des personnes en situation de non emploi rémunéré pour cause de chômage ou d'interruption d'activité liée aux enfants.

Par manque de place, la présente édition ne décrit pas le traitement des retraités au regard de l'impôt sur le revenu des personnes physiques et des cotisations de sécurité sociale ${ }^{2}$. Cependant, la version en ligne des descriptifs par pays, disponible à l'adresse www.oecd.org/els/social/pensions/pag-fr, contient ces informations. Pour plus de détails sur l'impôt et les cotisations de sécurité sociale payés par les travailleurs, voir OCDE (2007).

Les valeurs de tous les paramètres des retraites et autres chiffres pertinents comme le salaire minimum sont indiqués dans la monnaie nationale et en proportion du salaire moyen. (Voir à la partie II l'indicateur du « Salaire moyen ».)

Dans chaque descriptif pays, un tableau donne les valeurs relatives attendues des pensions, les taux de remplacement et le patrimoine retraite à différents niveaux de salaire individuel pour les régimes de retraite obligatoires. (Voir la partie I de ce rapport pour les définitions et les mesures des différents indicateurs). Ces données sont indiquées en valeur brute et en valeur nette (cette dernière tenant compte des impôts et cotisations payés pendant l'activité et lors de la liquidation de la retraite).

Des graphiques résumés montrent la ventilation de la valeur brute relative des pensions entre les différentes composantes du système de retraite (première ligne des graphiques). Dans la mesure du possible, la même terminologie est utilisée pour décrire ces régimes. On trouvera le système national particulier décrit dans le corps du texte. Les légendes des graphiques utilisent quelques abréviations types :

- AS : aide sociale ; 
- Ciblé : régime distinct soumis à conditions de ressources pour les personnes âgées ;

- Minimum : pension minimum dans un régime lié à la rémunération ;

- De base: pension basée uniquement sur un nombre d'années de couverture ou de résidence ;

- Lié à la rémunération: désigne tous les programmes publics liés à la rémunération, englobant les régimes de comptes notionnels et les régimes à points mais aussi les régimes traditionnels à prestations définies ;

- $C D$ : régimes privés obligatoires à cotisations définies ;

- Professionnel : retraites obligatoires ou quasi obligatoires qui peuvent être proposées par l'employeur, par des régimes au niveau du secteur d'activité (Pays-Bas), par des régimes professionnels (Suède) ou par le secteur public (Finlande, France).

La deuxième ligne des graphiques pays, qui indique les valeurs brutes et nettes, montre l'incidence de l'impôt sur le revenu et des cotisations de sécurité sociale sur la valeur relative des pensions et sur les taux de remplacement.

Les graphiques utilisent une échelle type afin de faciliter les comparaisons entre pays : pour les taux de remplacement l'échelle va jusqu'à $125 \%$ tandis que pour les valeurs relatives des pensions elle va jusqu'à 2.5 fois le salaire moyen. Les graphiques indiquent également les droits à pension pour les personnes gagnant entre $50 \%$ et $200 \%$ du salaire moyen à l'échelle nationale.

\section{Notes}

1. Pour plus d'informations sur les prestations destinées à ces travailleurs, voir la discussion sur les filets de sécurité pour les personnes âgées dans l'encadré 2.1 du chapitre spécial sur " Les revenus et la pauvreté des personnes âgées » et les descriptifs par pays qui suivent dans la partie III.

2. Pour tous les pays de l'OCDE, les impôts et cotisations de sécurité sociale acquittés par les travailleurs sont ceux en vigueur en 2006.

\section{Références}

OCDE (2007), Les impôts sur les salaires, OCDE, Paris.

Queisser, M. et E.R. Whitehouse (2006), " Neutral or Fair? Actuarial Concepts and Pension-system Design ", Document de travail de l'OCDE sur les affaires sociales, l'emploi et les migrations $\mathrm{n}^{\circ} 40$, OCDE, Paris.

Whitehouse, E.R. (2009), "Pensions, Purchasing-power Risk, Inflation and Indexation », Document de travail de l'OCDE sur les affaires sociales, l'emploi et les migrations $n^{\circ} 77, \mathrm{OCDE}$, Paris. 



\section{Allemagne}

\section{Allemagne : système de retraite en 2006}

Le système public légal de retraite comporte un seul pilier; c'est un régime par répartition lié à la rémunération. Les pensions sont calculées selon un système de points. Pour les retraités à faible revenu, il existe toutefois un dispositif d'assistance sociale qui constitue un filet de sécurité.

\section{Indicateurs essentiels}

\begin{tabular}{llrr}
\hline & & Allemagne & OCDE \\
\hline Salaire moyen & EUR & 42400 & 28600 \\
& USD & 53200 & 35800 \\
$\begin{array}{l}\text { Dépenses publiques } \\
\text { au titre des retraites }\end{array}$ & $\%$ du PIB & 11.4 & 7.2 \\
Espérance de vie & à la naissance & 79.8 & 78.9 \\
& à 65 ans & 83.9 & 83.4 \\
Population de plus de 65 ans & \% de la population & 32.2 & 23.8 \\
& d'âge actif & & \\
\hline
\end{tabular}

\section{Conditions d'ouverture des droits}

La pension est payable à partir de 67 ans aux personnes justifiant de cinq années de cotisation et, pour celles qui sont nées en 1964 et au-delà, à partir de 63 ans avec 35 années de cotisation. Les personnes ayant cotisé moins de cinq ans n'ont droit à aucune prestation.

\section{Calcul des prestations}

\section{Régime lié à la rémunération}

Une année de cotisation au salaire moyen vaut un point de retraite. Le salaire moyen considéré est à peu près identique au salaire moyen selon la comptabilité nationale. Les cotisations basées sur un revenu inférieur ou supérieur donnent droit à un nombre proportionnellement inférieur ou supérieur de points. En 2006, les cotisations sont prélevées sur le salaire annuel jusqu'à un plafond de 63000 EUR équivalant à $214 \%$ du salaire moyen. Le salaire moyen pris en compte en 2006 était de 29494 EUR, soit l'équivalent de $70 \%$ du salaire moyen selon l'OCDE.

Lors du départ à la retraite, on additionne les points de retraite de chaque année et on multiplie cette somme par la valeur du point, qui était pour l'année civile 2006 de 313.56 EUR. La valeur du point est applicable aux nouveaux retraités comme aux anciens. Elle est réévaluée chaque année en fonction de l'évolution des salaires bruts mais aussi de deux facteurs supplémentaires. Le premier facteur prend en compte l'évolution des taux de cotisation au régime légal de retraite et aux régimes professionnels et individuels facultatifs subventionnés. Une augmentation des taux de cotisation réduira l'ajustement de la valeur du point. Le deuxième, appelé " facteur de viabilité ", relie la revalorisation du point de retraite au taux de dépendance économique du système, c'est-à-dire au ratio retraités/cotisants.

Ces facteurs ont été intégrés aux règles d'indexation afin de plafonner à $22 \%$ l'augmentation du taux de cotisation qui est actuellement de $19.9 \%$. L'augmentation du ratio retraités/cotisants se traduira par une indexation sur les salaires inférieurs à la moyenne. À long terme, on s'attend à ce que l'ajustement de la valeur du point soit inférieur de $18 \%$ à l'augmentation du salaire moyen. 
Dans les nouveaux Länder, les salaires moyens pris en compte pour calculer les points de retraite et la valeur du point sont légèrement différents. On suppose que sur le long terme cette différence s'estompera avec l'alignement des salaires.

\section{Aide sociale}

Pour les personnes à faible revenu, il existe un système d'aide sociale qui s'applique également aux retraités. En 2006, le montant de l'aide sociale dans les Länder de l'Ouest était de 8172 EUR par an ; ce montant, qui inclut les allocations moyennes de logement et de chauffage, est équivalent à $19.3 \%$ du salaire moyen.

\section{Pensions privées facultatives}

Une pension privée facultative complémentaire peut être souscrite auprès d'une banque, d'une compagnie d'assurances ou d'un fonds d'investissement. Le taux de cotisation à cette pension (Riester) est relevé progressivement pour atteindre $4 \%$ en 2008. Les plans professionnels couvrent $64 \%$ des salariés, les plans individuels $44 \%$. Il existe en Allemagne de nombreux types de plans individuels et professionnels facultatifs et les doubles comptages d'individus entre ces deux catégories sont nombreux. Désormais, l'essentiel de l'offre se fait sur une base de cotisations définies et on suppose un taux de cotisation de $4 \%$ : c'est le taux le plus courant car il s'agit du taux maximum qui déclenche une aide publique importante.

\section{Variantes de carrière}

\section{Retraite anticipée}

Pour les personnes nées à partir de 1964, un départ anticipé à la retraite est possible à partir de 63 ans avec 35 années de cotisation dont huit immédiatement avant le départ à la retraite avec décote. En cas de départ avant 67 ans, les prestations sont réduites de $3.6 \%$ par année d'anticipation. En outre, par rapport à quelqu'un partant à 65 ans, les droits à pension sont sensiblement inférieurs du fait qu'il manque deux années de travail et les points de retraite correspondants. Un départ en retraite anticipée sans décote à 65 ans est possible avec 45 années de cotisation.

\section{Retraite différée}

Un départ à la retraite après 67 ans génère une augmentation des droits à pension de $6 \%$ par année de travail supplémentaire.

\section{Enfants}

L'État prend en charge trois années de cotisation de retraite par enfant né en 1992 et au-delà. Le paiement peut être effectué par parent, en situation d'emploi ou de non emploi, ou partagé entre eux. Ces années sont créditées d'un point de pension (égal aux cotisations calculées sur la base du salaire moyen) par enfant. Les périodes consacrées à l'éducation d'enfants de moins de 10 ans sont également validées. Ces années sont prises en compte dans le nombre d'années requis pour avoir droit à une retraite (Berücksichtigungszeit) ; elles affectent également les droits à pension. Si des parents d'enfants de moins de 10 ans travaillent et cotisent ou si des parents élèvent au minimum deux enfants de moins de 10 ans, ils perçoivent une bonification allant jusqu'à 0.33 point de retraite par an sous réserve que l'on n'aboutisse pas à un total excédant un point de retraite par an. 


\section{Chômage}

L'assurance chômage cotise au régime de retraite pour le compte des chômeurs. Pendant la première période d'indemnisation du chômage (Arbeitslosengeld I), les cotisations sont payées sur la base de $80 \%$ du salaire brut antérieur. Cette première période dure entre 6 et 24 mois selon l'âge et le nombre d'années de cotisation. Le chômeur entre ensuite dans une deuxième période d'indemnisation (Arbeitslosengeld II) à un taux moindre, soumise à conditions de ressources. Pendant cette période, l'assurance chômage règle les cotisations sur une base de 205 EUR par mois, de sorte que 0.0834 point de pension est acquis pour chaque année de la deuxième période de chômage. 


\section{Résultats de la modélisation des retraites : Allemagne}

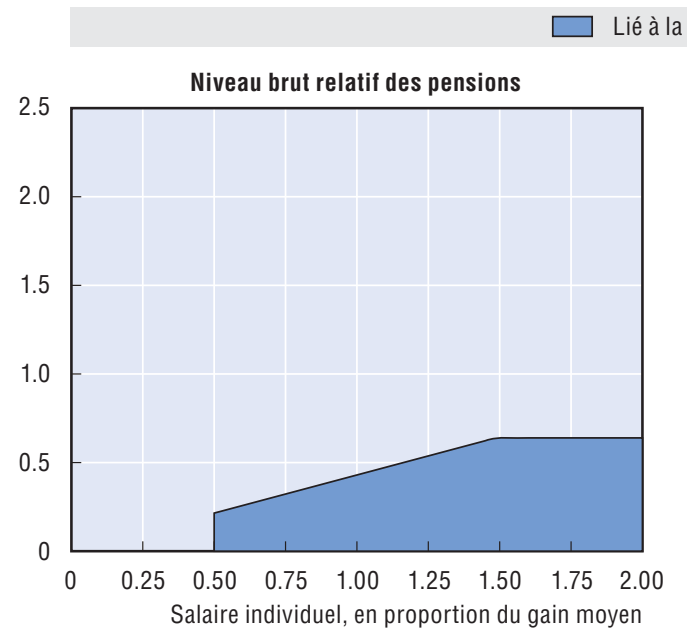

ié à la rémunération
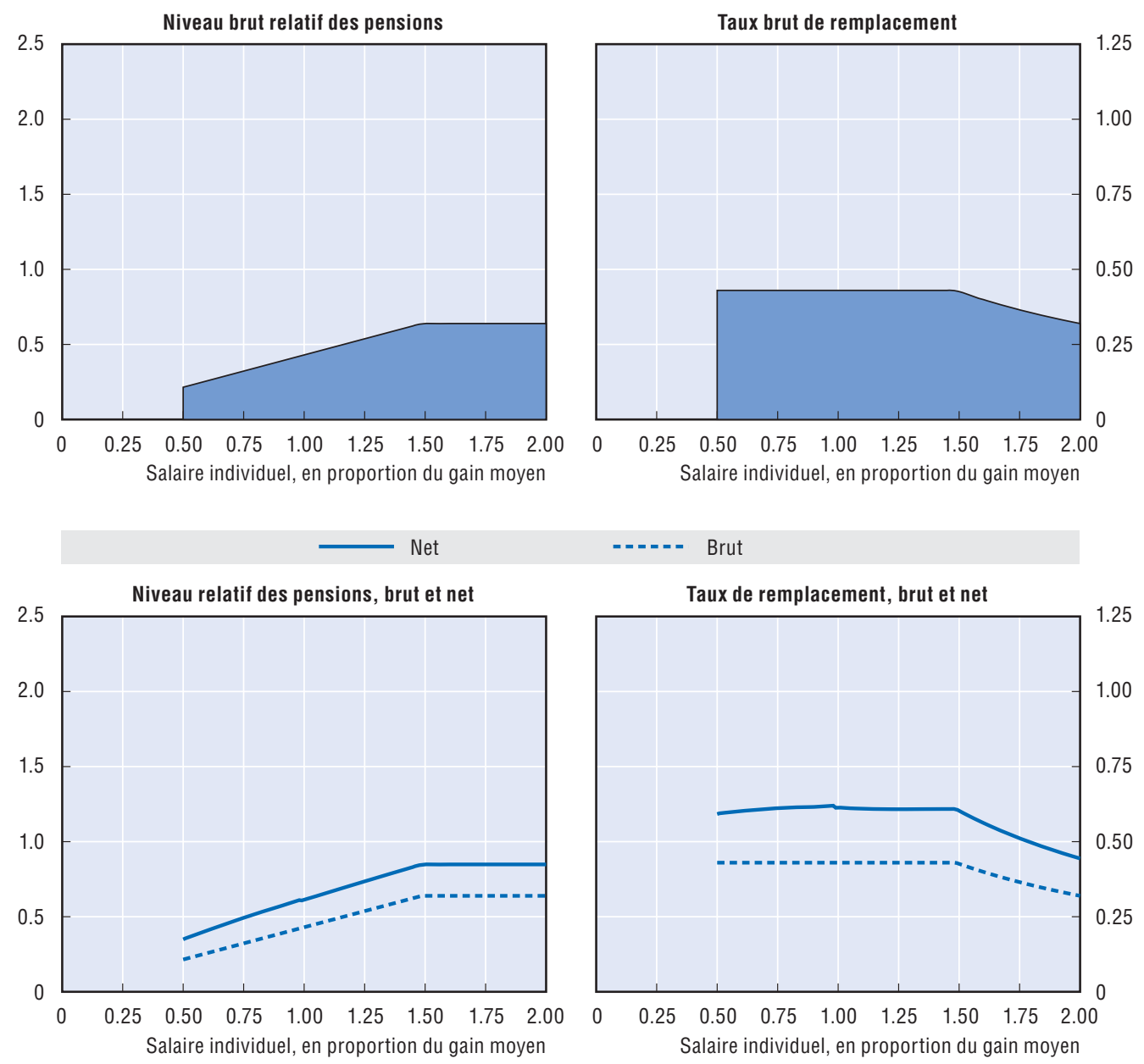

\begin{tabular}{|c|c|c|c|c|c|c|}
\hline \multirow{2}{*}{$\begin{array}{l}\text { Hommes } \\
\text { Femmes (si différent) }\end{array}$} & \multirow{2}{*}{$\begin{array}{l}\text { Salarié à revenu } \\
\text { médian }\end{array}$} & \multicolumn{5}{|c|}{ Salaire individuel, en multiple du salaire moyen } \\
\hline & & 0.5 & 0.75 & 1 & 1.5 & 2 \\
\hline Niveau brut relatif des pensions & 38.3 & 21.5 & 32.3 & 43.0 & 63.9 & 63.9 \\
\hline \multicolumn{7}{|l|}{ (\% salaire moyen brut) } \\
\hline Niveau net relatif des pensions & 56.5 & 34.9 & 49.3 & 61.3 & 84.8 & 84.8 \\
\hline \multicolumn{7}{|l|}{ (\% salaire moyen net) } \\
\hline Taux brut de remplacement & 43.0 & 43.0 & 43.0 & 43.0 & 42.6 & 32.0 \\
\hline \multicolumn{7}{|l|}{ (\% salaire individuel brut) } \\
\hline Taux net de remplacement & 61.5 & 59.2 & 61.1 & 61.3 & 60.3 & 44.4 \\
\hline \multicolumn{7}{|l|}{ (\% salaire individuel net) } \\
\hline Patrimoine retraite brut & 7.2 & 7.2 & 7.2 & 7.2 & 7.1 & 5.3 \\
\hline (multiple du salaire individuel brut) & 8.5 & 8.5 & 8.5 & 8.5 & 8.4 & 6.3 \\
\hline Patrimoine retraite net & 6.0 & 6.6 & 6.2 & 5.8 & 5.3 & 4.0 \\
\hline (multiple du salaire individuel brut) & 7.1 & 7.8 & 7.3 & 6.8 & 6.3 & 4.7 \\
\hline
\end{tabular}




\section{Australie}

\section{Australie : système de retraite en 2006}

Le système de retraite australien repose sur trois composantes : une pension de vieillesse soumise à condition de ressources et financée par l'impôt; la garantie de retraite (superannuation guarantee), qui est une cotisation obligatoire de l'employeur à une épargneretraite privée (principalement à travers des plans à cotisations définies); enfin, des cotisations facultatives et autres formes d'épargne privée, qui sont encouragées pour soutenir l'autonomie à la retraite.

\section{Indicateurs essentiels}

\begin{tabular}{llrr}
\hline & & Australie & OCDE \\
\hline Salaire moyen & AUD & 55200 & 47600 \\
& USD & 41600 & 35800 \\
$\begin{array}{l}\text { Dépenses publiques } \\
\text { au titre des retraites }\end{array}$ & \% du PIB & 3.5 & 7.2 \\
$\begin{array}{l}\text { Espérance de vie } \\
\text { Population de plus de } 65 \text { ans }\end{array}$ & $\begin{array}{l}\text { \%̀ la naissance } \\
\text { d'âge actif }\end{array}$ & 81.1 & 78.9 \\
& & 21.5 & 23.8 \\
\hline
\end{tabular}

\section{Conditions d'ouverture des droits}

La pension de vieillesse est versée à partir de 65 ans pour les hommes. Pour les femmes, l'âge d'ouverture des droits, qui est actuellement de 63 ans, va être relevé progressivement pour atteindre 65 ans en 2014. L'âge minimum pour percevoir les prestations de la garantie de retraite est actuellement de 55 ans ; d'ici à 2025, il sera porté progressivement à 60 ans.

\section{Calcul des prestations}

\section{Régime à cotisations définies}

Mise en place en 1992, la garantie de retraite consiste en une cotisation obligatoire de l'employeur à un régime de retraite privé. Ce régime peut être géré par l'employeur, des associations de branche, des sociétés de services financiers ou encore par les individus eux-mêmes. Depuis l'exercice 2002/03, le taux de cotisation obligatoire est de $9 \%$ de la rémunération des salariés.

Les employeurs ne sont pas tenus de cotiser pour les salariés qui gagnent moins de 450 AUD par mois (soit 5400 AUD par an) mais ils peuvent choisir de le faire (on notera que ce minimum n'a jamais été revalorisé). Il existe aussi une limite à la rémunération couverte par la garantie de retraite et les employeurs ne sont pas tenus de cotiser pour la fraction de la rémunération dépassant cette limite. Pour l'exercice 2004/05, ce plafond trimestriel était de 32180 AUD et pour l'exercice 2005/06, il est de 33720 AUD. Ce plafond, évalué à environ $2 \frac{1}{2}$ fois le salaire moyen, est indexé sur une mesure des salaires moyens.

Le stade de liquidation de la garantie de retraite complique les calculs. Bien qu'il existe quelques plans professionnels à prestations définies, la plupart des salariés relèvent de régimes à cotisations définies. Les adhérents peuvent sortir le capital accumulé sous la forme d'une somme globale ou de flux de revenus. Actuellement, la plupart des prestations 
sont prises sous la forme d'une somme globale. Pour les besoins de la comparaison avec les autres pays (dans lesquels prédominent les plans à prestations définies), le capital provenant de la garantie de retraite est supposé converti en rente indexée sur les prix. Le calcul de la rente est effectué sur la base des statistiques australiennes de la mortalité.

\section{Régime ciblé}

La pension de vieillesse est destinée à offrir un filet de sécurité aux personnes dans l'incapacité d'épargner tout au long de leur vie professionnelle et un complément à l'épargne-retraite des autres. Les cconditions de revenu et de patrimoine (condition de ressources) sont utilisées pour cibler les paiements sur ceux qui en ont besoin.

La valeur de la pension de vieillesse est ajustée semestriellement. Elle est versée par quinzaine ; en septembre 2005, le taux maximum unique était de 489 AUD par quinzaine ; il a été porté à 500 AUD en mars 2006 et à 512 AUD en septembre 2006. (Toutes les valeurs ont été arrondies au dollar le plus proche). Cela donne une moyenne de prestation annuelle pour l'exercice de 12737 AUD, soit 23\% du salaire moyen.

La valeur de la pension de vieillesse suit l'évolution des prix mesurée par l'indice des prix à la consommation (IPC). Lorsque nécessaire, il s'y ajoute une augmentation supplémentaire afin qu'elle ne tombe pas en dessous de $25 \%$ du salaire hebdomadaire moyen total avant impôts d'un travailleur de sexe masculin selon la définition nationale (qui est légèrement différent du salaire de référence utilisé dans les analyses de l'OCDE).

La pension de vieillesse est réduite dès lors que le revenu annuel provenant d'autres sources excède un seuil dit de "franchise ". Ce seuil est ajusté tous les ans en juillet. Pour 2006, ce seuil (calculé là encore par quinzaine) s'est établi à 124 AUD pour le premier semestre et à 128 AUD pour le second. Pour l'ensemble de l'exercice 2006, il a donc été de 3224 AUD, soit 5.8\% du salaire moyen. Le taux de dégressivité est de $40 \%$ (personnes seules et couples confondus). Il existe également une condition de patrimoine. Toutefois, plus de $90 \%$ des retraités concernés voient leurs prestations réduites par la condition de revenu plutôt que par la condition de patrimoine (on a donc retenu dans la modélisation l'hypothèse que la condition de revenu est contraignante). Près de $40 \%$ des retraités voient leurs prestations réduites par la condition de ressources et perçoivent donc une retraite à taux partiel. Les un peu plus de $60 \%$ restants perçoivent une retraite à taux maximum.

\section{Variantes de carrières}

\section{Retraite anticipée}

L'accès aux prestations de retraite (y compris la garantie de retraite) est actuellement possible à partir de 55 ans (seuil qui sera porté progressivement à 60 ans). Les personnes qui travaillent encore peuvent également recevoir leurs prestations à compter de 55 ans mais uniquement sous la forme d'un flux de revenus non convertibles. La pension de vieillesse ne peut être perçue avant l'âge ouvrant droit aux prestations, à savoir 65 ans pour les hommes et 63 ans pour les femmes (sera porté à 65 ans d'ici à 2014).

\section{Retraite différée}

Il est possible de différer la liquidation de la garantie de retraite après 65 ans. Les employeurs sont tenus de verser les cotisations à la garantie de retraite pour leurs salariés éligibles à cette prestation jusqu'à l'âge de 70 ans. 
Il est également possible de différer la liquidation des droits à la pension de vieillesse après 65 ans. Le système de bonification verse un montant forfaitaire unique et non imposable aux adhérents éligibles qui diffèrent la liquidation de leurs droits et continuent à travailler. Cette bonification est versée au moment où l'adhérent admissible liquide ses droits et perçoit sa pension de vieillesse. L'intéressé doit s'inscrire et travailler au minimum 12 mois à compter de la date d'inscription et faire au minimum 960 heures de travail rémunéré par an. La bonification peut s'accumuler jusqu'à cinq ans. Son montant dépend du taux de la pension de vieillesse à laquelle l'intéressé aura droit lorsqu'il liquidera et touchera sa retraite. Il est de $9.4 \%$ des droits à la retraite de base pour la première année de report. Il est de quatre fois ce montant pour deux ans, neuf fois pour trois ans, 16 fois pour quatre ans et 25 fois pour cinq ans. La bonification maximum sur cinq ans équivaut à 2.35 fois les droits à pension maximums d'une année.

\section{Enfants}

La garantie de retraite ne prévoit pas de protection spécifique pour les périodes de non-emploi. Des cotisations facultatives sont possibles pour les périodes de non-emploi.

La structure de la pension de vieillesse, qui est soumise à conditions de ressources, offre une certaine protection aux personnes ayant des périodes de non-emploi, en ce sens qu'elle fournit un filet de sécurité et un complément de retraite pour ceux qui n'ont pas été en mesure d'épargner suffisamment au cours de leur vie professionnelle.

\section{Chômage}

La garantie de retraite ne comporte aucun dispositif spécifique de protection pour les périodes de non emploi. Des cotisations facultatives sont possibles pour les périodes de non-emploi.

Dans le régime australien de retraite, les périodes de chômage ne sont pas validées. 
Résultats de la modélisation des retraites : Australie
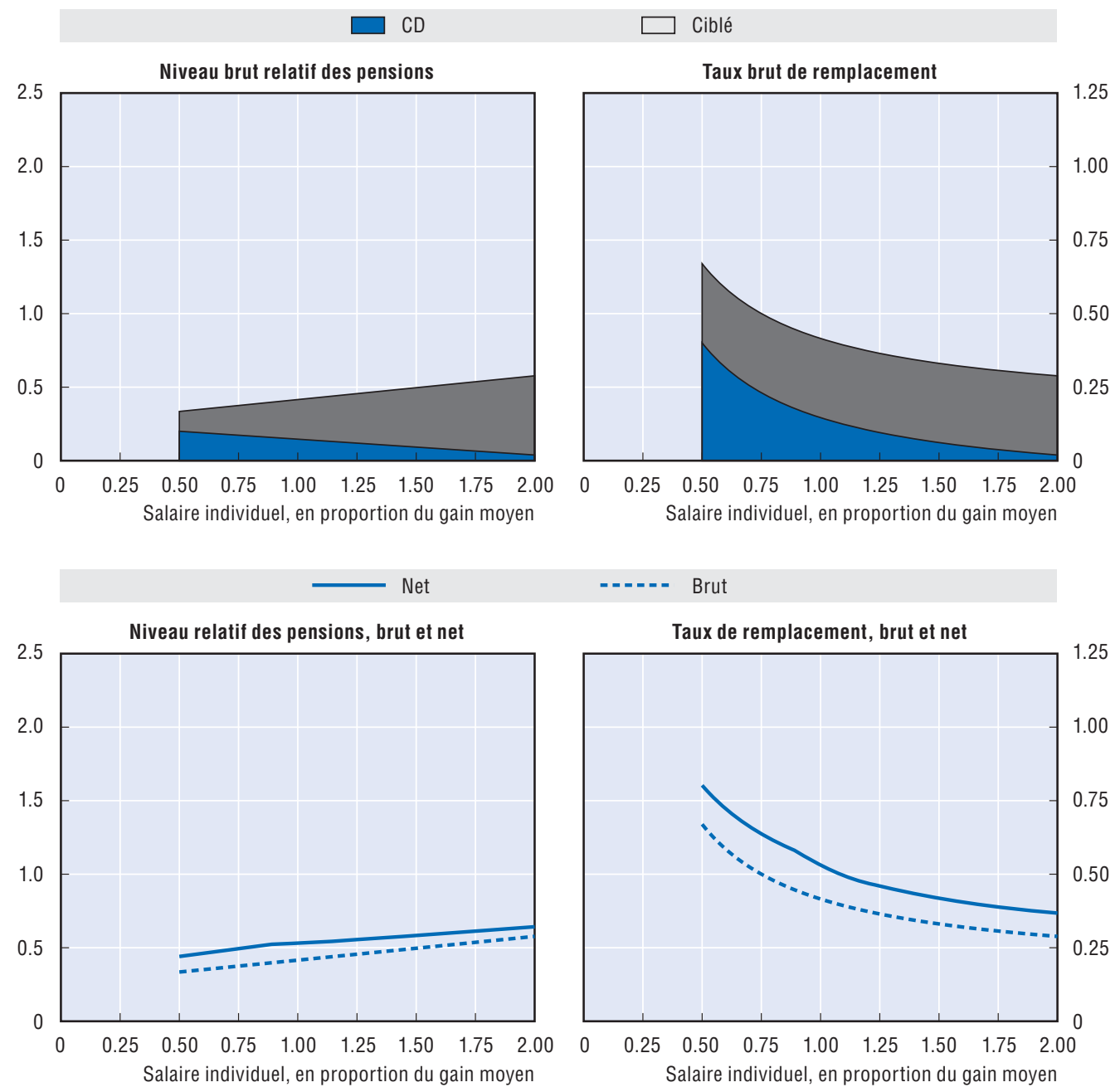

\begin{tabular}{|c|c|c|c|c|c|c|}
\hline \multirow{2}{*}{$\begin{array}{l}\text { Hommes } \\
\text { Femmes (si différent) }\end{array}$} & \multirow{2}{*}{$\begin{array}{l}\text { Salarié à revenu } \\
\text { médian }\end{array}$} & \multicolumn{5}{|c|}{ Salaire individuel, en multiple du salaire moyen } \\
\hline & & 0.5 & 0.75 & 1 & 1.5 & 2 \\
\hline Niveau brut relatif des pensions & 39.3 & 33.5 & 37.5 & 41.6 & 49.7 & 57.7 \\
\hline \multicolumn{7}{|l|}{ (\% du salaire moyen brut) } \\
\hline Niveau net relatif des pensions & 51.7 & 44.1 & 49.4 & 53.1 & 58.3 & 64.3 \\
\hline \multicolumn{7}{|l|}{ (\% du salaire moyen net) } \\
\hline Taux brut de remplacement & 45.7 & 67.0 & 50.0 & 41.6 & 33.1 & 28.9 \\
\hline \multicolumn{7}{|l|}{ (\% du salaire individuel brut) } \\
\hline Taux net de remplacement & 59.2 & 80.2 & 63.7 & 53.1 & 41.8 & 36.8 \\
\hline \multicolumn{7}{|l|}{ (\% du salaire individuel net) } \\
\hline Patrimoine retraite brut & 7.7 & 11.7 & 8.5 & 6.9 & 5.3 & 4.5 \\
\hline (multiple du salaire individuel brut) & 9.0 & 13.7 & 9.9 & 8.1 & 6.2 & 5.2 \\
\hline Patrimoine retraite net & 7.7 & 11.7 & 8.5 & 6.7 & 4.8 & 3.8 \\
\hline (multiple du salaire individuel brut) & 9.0 & 13.7 & 9.9 & 7.8 & 5.5 & 4.4 \\
\hline
\end{tabular}




\section{Autriche}

\section{Autriche : système de retraite en 2006}

Le système autrichien de retraite est un régime public à prestations définies avec un complément soumis à conditions de ressources pour les retraités à faible revenu.

\section{Conditions d'ouverture des droits}

Indicateurs essentiels

\begin{tabular}{llrr}
\hline & & Autriche & OCDE \\
\hline Salaire & EUR & 36700 & 28600 \\
moyen & USD & 46100 & 35800 \\
Dépenses publiques & \% du PIB & 12.6 & 7.2 \\
au titre des retraites & & 79.9 & 78.9 \\
Espérance de vie & à la naissance & 83.9 & 83.4 \\
& à 65 ans & 27.1 & 23.8 \\
Population de plus de 65 ans & \% de la population & & \\
& d'âge actif & & \\
\hline
\end{tabular}

L'âge normal de départ à la retraite est de 65 ans pour les hommes. Pour les femmes, il est actuellement de 60 ans mais sera porté à 65 ans entre 2024 et 2033 . La couverture requise est de 180 mois ( 15 ans) sur les 30 dernières années ou de 300 mois ( 25 ans) sur l'ensemble de la vie active. Cent quatre-vingt mois de cotisations effectivement versées (par opposition à la couverture seule) peuvent également suffire. Les mois assurés sont soit les mois de cotisations (du fait de l'emploi ou de cotisations facultatives) soit les mois supplémentaires (c'est-à-dire les mois assimilés ou Ersatzzeiten) pour lesquels ne sont acquittés que des cotisations limitées. Dans le cadre de la réforme de 2005, le nombre d'années de cotisation (dans le cadre d'un emploi lucratif) exigé pour percevoir la pension de vieillesse a été ramené de 15 à sept ans. La période d'assurance minimum résiduelle de huit ans peut être atteinte, par exemple avec des périodes validées au titre de l'éducation des enfants.

\section{Calcul des prestations}

\section{Régime lié à la rémunération}

Le taux d'acquisition des droits à la retraite est actuellement de $1.88 \%$ du salaire pour chaque année de cotisation mais il va être progressivement ramené à 1.78 \% d'ici à 2009 .

Le salaire de référence est actuellement le salaire des 18 meilleures années. La procédure de revalorisation est complexe mais, dans la pratique, les ajustements ont été jusqu'ici plus alignés sur la hausse des prix que sur celle des salaires. La période de référence pour le calcul des droits est actuellement rallongée ; à partir de 2008, elle sera de 40 ans. Les modalités de revalorisation dans le cadre de cette nouvelle procédure font encore débat. La modélisation retient comme période de référence l'ensemble de la carrière et suppose que les gains des années antérieures sont réévalués en fonction de l'évolution des salaires.

Les cotisations sont payables jusqu'à un plafond de 52500 EUR par an, correspondant à $143 \%$ du salaire moyen.

En 2005, les pensions mises en paiement ont été ajustées en fonction de l'évolution des prix à hauteur de la pension médiane ; au-delà de ce seuil, elles ont été augmentées d'un montant uniforme égal à l'augmentation absolue de la retraite médiane. De 2006 à 
2009, on envisage d'indexer totalement les pensions sur les prix à hauteur de 15 fois le plafond de cotisation journalière, ce qui pour 2006 aurait donné 125 EUR x $15=1875$ EUR. La modélisation suppose la poursuite de cette pratique.

\section{Régime ciblé}

Un complément soumis à conditions de ressources (Ausgleichszulage) assure un revenu de retraite minimum de 690 EUR par mois pour une personne seule et de 1056 EUR pour un couple, ceci sur 14 mois. Là encore, l'ajustement est discrétionnaire ; la modélisation suppose implicitement qu'il suivra l'évolution du salaire moyen.

\section{Variantes de carrière}

\section{Retraite anticipée}

Le départ en retraite anticipée est actuellement possible à partir de 62 ans pour les hommes et de 57 ans pour les femmes sous réserve de justifier de 37.5 années de cotisation ou années validées. À partir de 2017, l'âge de départ en retraite anticipée pour les femmes sera porté à 60 ans. Les retraites prises avant 65 ans subissent une décote de $4.2 \%$ par année d'anticipation.

\section{Retraite différée}

Pour un départ à la retraite entre 65 et 68 ans, la pension est majorée de $4.2 \%$ par an ; au-delà de 68 ans, il n'y a plus de surcote. Les travailleurs qui diffèrent leur départ à la retraite continuent à cotiser et donc à acquérir des droits à pension.

Le cumul retraite-travail est possible mais dans une certaine limite de gains. Si des retraités de moins de 65 ans gagnent plus de 333.16 EUR (en 2006), leur pension est intégralement suspendue. Après 65 ans, le cumul de la retraite et d'un travail dont les revenus ne sont plus soumis à aucune limite est autorisé.

\section{Enfants}

Les périodes de non-emploi liées aux enfants sont prises en compte de deux façons différentes. Des périodes d'au maximum quatre ans par enfant sont validées sur la base d'un salaire fictif ouvrant droit à pension de 1350 EUR par mois. Mais seules deux années par enfant sont considérées comme couvertes et prises en compte dans l'établissement de la période ouvrant droit à pension.

\section{Chômage}

Les périodes de chômage indemnisé au titre de l'assurance chômage et de l'assistance chômage à 70 \% de la base d'évaluation comptent en années de cotisation. 


\section{Résultats de la modélisation des retraites : Autriche}

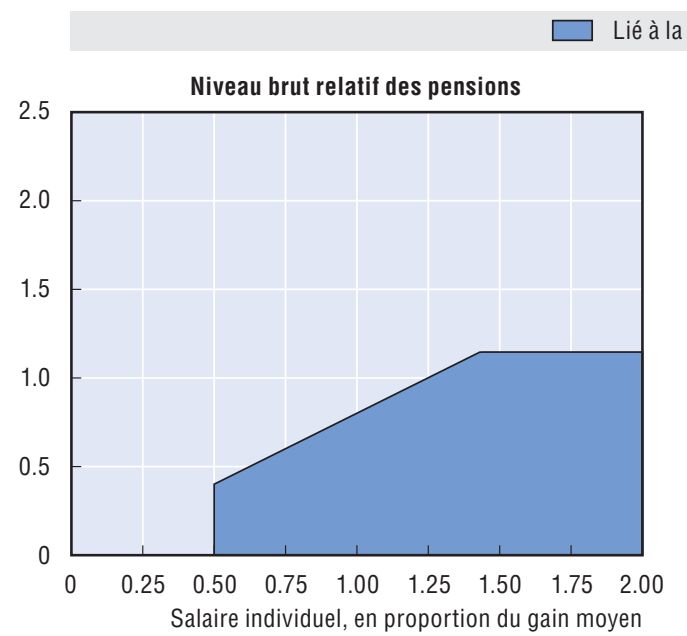

à la rémunération
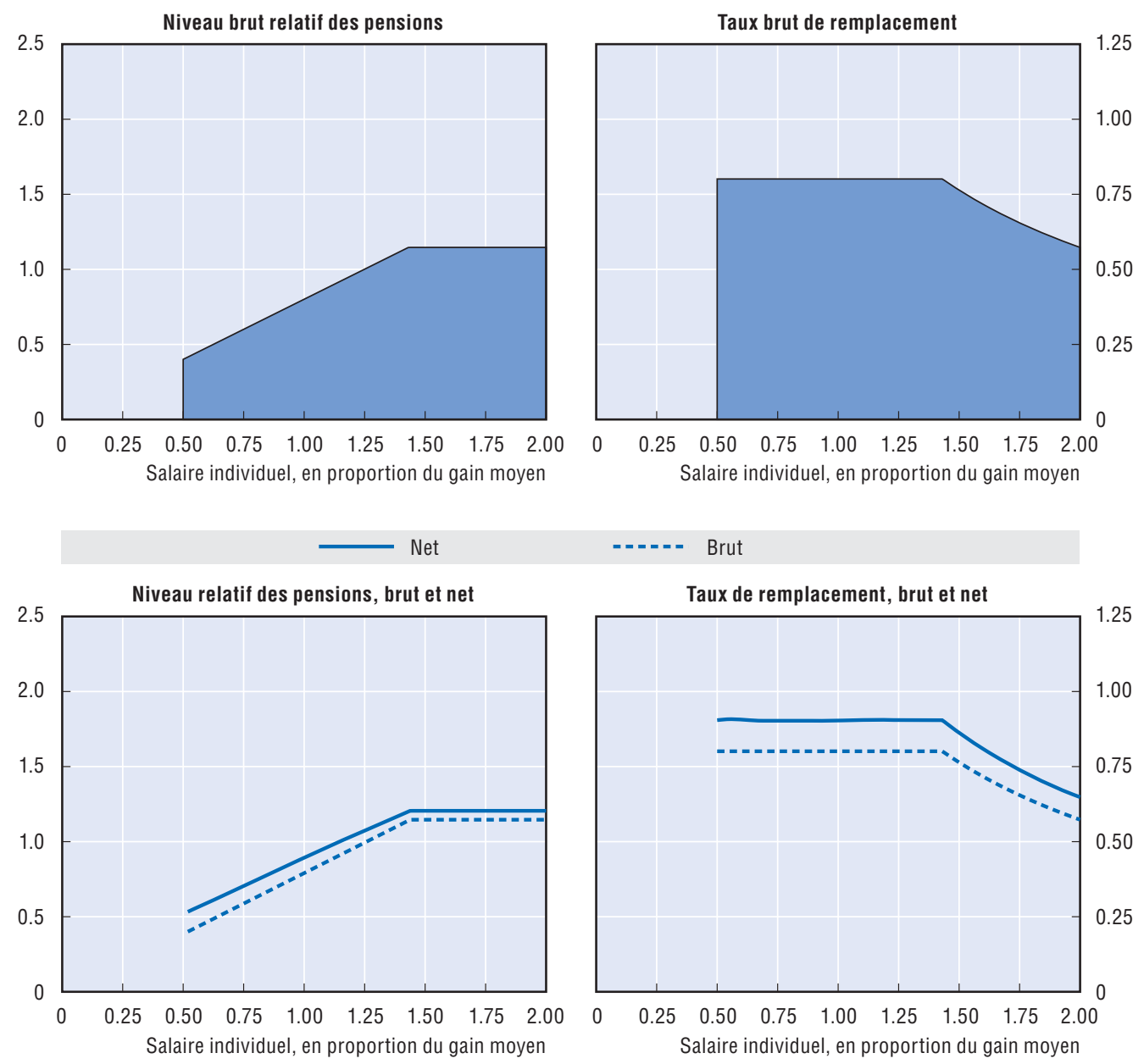

\begin{tabular}{|c|c|c|c|c|c|c|}
\hline \multirow{2}{*}{$\begin{array}{l}\text { Hommes } \\
\text { Femmes (si différent) }\end{array}$} & \multirow{2}{*}{$\begin{array}{l}\text { Salarié à revenu } \\
\text { médian }\end{array}$} & \multicolumn{5}{|c|}{ Salaire individuel, en multiple du salaire moyen } \\
\hline & & 0.5 & 0.75 & 1 & 1.5 & 2 \\
\hline Niveau brut relatif des pensions & 68.1 & 40.0 & 60.1 & 80.1 & 114.6 & 114.6 \\
\hline \multicolumn{7}{|l|}{ (\% du salaire moyen brut) } \\
\hline Niveau net relatif des pensions & 79.1 & 53.3 & 71.7 & 90.3 & 120.6 & 120.6 \\
\hline \multicolumn{7}{|l|}{ (\% du salaire moyen net) } \\
\hline Taux brut de remplacement & 80.1 & 80.1 & 80.1 & 80.1 & 76.4 & 57.3 \\
\hline \multicolumn{7}{|l|}{ (\% du salaire individuel brut) } \\
\hline Taux net de remplacement & 90.3 & 90.5 & 90.3 & 90.3 & 86.3 & 64.8 \\
\hline \multicolumn{7}{|l|}{ (\% du salaire individuel net) } \\
\hline Patrimoine retraite brut & 12.0 & 12.2 & 12.2 & 11.6 & 10.5 & 7.9 \\
\hline (multiple du salaire individuel brut) & 13.9 & 14.2 & 14.2 & 13.5 & 12.1 & 9.1 \\
\hline Patrimoine retraite net & 9.3 & 10.9 & 9.7 & 8.8 & 7.4 & 5.6 \\
\hline (multiple du salaire individuel brut) & 10.8 & 12.6 & 11.3 & 10.1 & 8.5 & 6.4 \\
\hline
\end{tabular}




\section{Belgique}

\section{Belgique : système de retraite en 2006}

Le système belge de retraite repose sur deux éléments : un régime public lié à la rémunération avec une retraite minimum et un filet de sécurité soumis à conditions de ressources.

\section{Conditions d'ouverture des droits}

Indicateurs essentiels

\begin{tabular}{llrr}
\hline & & Belgique & OCDE \\
\hline Salaire moyen & EUR & 37700 & 28600 \\
& USD & 47300 & 35800 \\
Dépenses publiques & \% du PIB & 9.0 & 7.2 \\
au titre des retraites & & & \\
Espérance de vie & à la naissance & 79.5 & 78.9 \\
& à 65 ans & 83.8 & 83.4 \\
Population de plus de 65 ans & \% de la population & 28.7 & 23.8 \\
& d'âge actif & & \\
\hline
\end{tabular}

L'âge normal ouvrant droit à la retraite est de 65 ans pour les hommes. Pour les femmes, il est de 64 ans depuis le 1.1.2006 et sera porté à 65 ans à compter du 1.12.2009. Selon les règles en vigueur en Belgique, une carrière complète suppose 45 années de cotisation pour un homme et 44 pour une femme (depuis le 1.1.2006). À compter du 1.1.2009, elle supposera également 45 années de cotisation pour les femmes.

Les hommes et les femmes peuvent partir en retraite anticipée à partir de 60 ans sous réserve de justifier du nombre requis d'années de cotisation. Depuis le 1.1.2005, la retraite peut être liquidée à 60 ans avec 35 années de cotisation.

\section{Calcul des prestations}

\section{Régime lié à la rémunération}

Pour le calcul de la pension, le taux plein est de $60 \%$ pour une personne seule; pour un retraité ayant un conjoint à charge il est de $75 \%$. Le taux annuel d'acquisition des droits est donc de $60 \% / 45=1.33 \%$ pour les hommes (et pour les femmes à compter de 2009). Le salaire de référence est la moyenne des gains perçus sur l'ensemble de la carrière. Les salaires des années antérieures sont revalorisés en fonction de l'évolution des prix et parallèlement les salaires des personnes âgées sont revalorisés en fonction de la hausse du coût de la vie à l'aide d'un coefficient qui est différent pour chaque année. L'application de ce coefficient de revalorisation n'est pas modélisée.

La pension à taux plein est versée si les conditions d'ouverture des droits énoncées cidessus sont remplies. Pour des durées de cotisations plus courtes, la pension sera servie mais elle sera calculée sur le nombre (moindre) d'années d'activité.

Le salaire ouvrant droit à pension est plafonné à 44081.27 EUR pour 2006 (soit environ $117 \%$ du salaire moyen).

Les pensions mises en paiement sont revalorisées en fonction d'un indice des prix à la consommation (d'où certains biens sont exclus). Des augmentations réelles discrétionnaires (appelées " adaptations à l'évolution du bien-être général ") ont été également opérées mais, dans la période récente, elles ont été davantage ciblées sur les pensions les plus basses ou les plus anciennes. À compter de 2008, l’État est tenu par la 
législation de prendre des décisions sur la revalorisation de toutes les pensions tous les deux ans en concertation avec les partenaires sociaux.

Des prestations complémentaires (" pécule de vacances " et " allocations supplémentaires ") sont payables une fois par an. Elles sont égales à un mois de pension jusqu'à un plafond de 525.50 EUR pour une personne seule et de 656.88 EUR pour les retraités ayant un conjoint à charge (montants payables en mai 2006).

\section{Crédit annuel minimum}

Un système de crédit annuel minimum a été mis en place afin d'accroître les droits à pension des travailleurs à bas salaire ou ayant travaillé à temps partiel au cours de leur carrière. Les rémunérations annuelles inférieures à 14810.70 EUR (au 1.1.2006, soit $39 \%$ du salaire moyen) sont portées à ce niveau. À compter du 1.10.2006, ce crédit annuel minimum a été porté à 17674.73 EUR suite aux mesures dites du " pacte de solidarité entre les générations ». Pour être admis à bénéficier du crédit minimum, 15 ans au moins de cotisation sont nécessaires pour l'équivalent d'au minimum un tiers de plein-temps. (Cela donne une pension effective minimum pour une carrière complète et pour une personne seule justifiant de 45 années de cotisation, portée à ce niveau pour chaque année de la carrière.) L'application de ce crédit annuel minimum ne peut conduire à l'attribution d'une pension supérieure à 15553.48 EUR pour une pension au taux « famille " ou de 12442.78 EUR pour une pension au taux "personne isolée ". Si le calcul de la pension aboutit à un montant de cet ordre, le "crédit annuel minimum " ne sera pas appliqué à toutes les années de carrière éligibles de manière à ramener le montant de la pension sous ce plafond.

\section{Pension minimum liée à la rémunération}

Il existe également une pension minimum liée à la rémunération dont le montant était de 10 232.50 EUR au 1.1.2006 (10 603.65 EUR à compter du 1.10.2006) pour une personne seule ou de 12990.85 EUR pour un retraité ayant un conjoint à charge (13 250.39 EUR à compter du 1.10.2006) et remplissant la condition requise de 45 années de cotisation. Pour une personne seule, cela correspond à environ $28 \%$ du salaire moyen. Pour les personnes n'ayant pas une carrière complète, le montant de la prestation sera réduit au prorata sous réserve qu'elles justifient au minimum des deux tiers du nombre complet d'années. Dans le cas contraire, le montant de la prestation sera simplement obtenu en appliquant la formule de calcul des prestations (sans «plafonnement » de la prestation aligné sur la pension minimum).

La pension minimum est indexée sur les prix, certains biens étant exclus de l'indice. Les prestations sont augmentées de $2 \%$ à chaque fois que le taux d'inflation cumulé depuis le dernier ajustement dépasse un certain seuil (2\%).

Les retraités percevront soit la pension minimum décrite ici soit la retraite calculée selon le système du crédit annuel minimum, si elle est supérieure.

\section{Régime ciblé : revenu du filet de sécurité}

Dans le cas de personnes âgées n'ayant pas de droits à la retraite fondés sur une activité professionnelle ou dont les droits sont très faibles, un revenu soumis à conditions de ressources peut être attribué. Ce filet de sécurité appelé Garantie de revenu aux personnes âgées (GRAPA) s'inscrit dans un ensemble de mesures d'aide sociale 
complémentaires de l'offre de sécurité sociale (par exemple retraite légale pour les travailleurs du secteur privé modélisés).

Le revenu soumis à conditions de ressources pour les personnes âgées est de 8234.87 EUR (8 399.39 EUR à compter du 1.10.2006) pour un retraité vivant seul (22\% du salaire moyen) et de 5489.91 EUR (5 599.59 EUR à compter du 1.10.2006) pour une personne âgée vivant avec d'autres. Là encore, il est indexé sur les prix, certains biens étant exclus de l'indice. Pour la condition de ressources, le revenu " normal » de retraite est pris en compte pour $90 \%$ seulement de son montant réel.

Les limites d'âge correspondent à l'âge légal de 65 ans. Pendant une période transitoire (entre le 1.1.2006 et le 31.12.2008 pour la GRAPA attribuée), il est possible de faire une demande de GRAPA à 64 ans (pour les femmes). À partir du 1.1.2009, l'âge limite sera de 65 ans dans tous les cas.

\section{Pensions privées facultatives}

Un nouveau régime de " retraites complémentaires sectorielles " a été mis en place en 2003 pour élargir le deuxième pilier du système de retraite. Les taux de cotisation sont fixés dans le cadre de conventions collectives (sectorielles) et peuvent varier d'un secteur à l'autre. Ces plans professionnels sont à cotisations définies et environ $56 \%$ des salariés y adhèrent. La modélisation suppose un taux de cotisation de $4.25 \%$, ce qui correspond approximativement à la moyenne nationale.

\section{Variantes de carrière}

\section{Retraite anticipée}

Depuis 2005, un départ en retraite anticipée est possible à partir de 60 ans, sous réserve d'avoir à son actif 35 années de cotisation. Il n'y a pas de réduction actuarielle dans le calcul des retraites mais la pension peut être incomplète du fait d'une carrière incomplète (moins de 45 ans). La possibilité de cumuler une pension de retraite anticipée avec un travail est soumise à un plafond de salaire plus strict que celui appliqué après l'âge normal de la retraite (voir ci-dessous).

Pour les pensions démarrant entre le 1.1.2007 et 2013, le travail au-delà de 62 ans ou de 44 années de cotisation sera crédité d'une bonification de deux euros pour chaque journée travaillée, dans la limite de 624 EUR (non indexés) pour chaque année complète de travail, suite au « pacte de solidarité entre les générations ».

\section{Retraite différée}

Il est possible de différer le départ à la retraite au-delà de l'âge normal. En continuant à travailler après l'âge normal de la retraite, certaines personnes ayant eu des interruptions de carrière peuvent les combler de façon à s'assurer une pension (plus) complète ou à améliorer son montant, dans la mesure où seules les 45 dernières années (44 pour les femmes) sont prises en compte dans le calcul.

Il est également possible, dans certaines limites, de cumuler une pension de retraite et un salaire (après l'âge normal de la retraite). Pour un salaire annuel inférieur à 15 590.18 EUR (pour une personne seule) ou 19300.98 EUR (avec un enfant à charge), la pension ne subira pas de décote. Au-delà de ce plafond, elle sera réduite du montant du dépassement. Si le salaire réel dépasse de $15 \%$ les limites mentionnées ci-dessus, la pension sera totalement suspendue. 
Avant l'âge légal (normal) de la retraite, les limites fixées pour le cumul emploi-retraite sont respectivement de 7421.57 EUR ou de 11 132.37 EUR avec la même restriction de 15 \%.

\section{Enfants}

$\mathrm{Au}$ maximum trois années entièrement consacrées à élever des enfants peuvent être validées en emploi lucratif si l'intéressé bénéficie du tijdskrediet, c'est-à-dire du droit pour tous les salariés du secteur privé de suspendre totalement leur activité professionnelle ou de ne travailler qu'à mi-temps sous réserve d'avoir travaillé à plus de trois-quarts de temps pendant un minimum de 12 mois avant le début du tijdskrediet. Pour en bénéficier, il faut également avoir travaillé plus d'un an pour le même employeur dans les 15 mois qui ont précédé la demande de tijdskrediet. Lorsqu'une personne sort totalement du marché du travail, elle ne reçoit aucune compensation. Ces années sont prises en compte au numérateur de la formule de calcul des prestations. Le salaire retenu dans la formule est le dernier salaire avant la sortie du marché du travail.

\section{Chômage}

Les périodes de chômage indemnisé par l'assurance chômage sont créditées/validées dans le régime belge de retraite. Les années de chômage sont prises en compte au numérateur de la formule de calcul des prestations et c'est le salaire perçu avant l'épisode de chômage qui est pris dans la base de calcul pour l'ensemble de la période de chômage. Le nombre d'années validées n'est soumis à aucune limite. L'application de ce système conduira toutefois à une prestation de retraite légèrement inférieure à celle obtenue dans le cas d'une carrière totalement active car ce montant de crédits ne suit pas nécessairement et intégralement la progression réelle des salaires sur la période validée. Les épisodes de chômage après 62 ans ou après 42 années de carrière ne permettront pas l'application de la « bonification de retraite » au titre de ces années. 
Résultats de la modélisation des retraites : Belgique
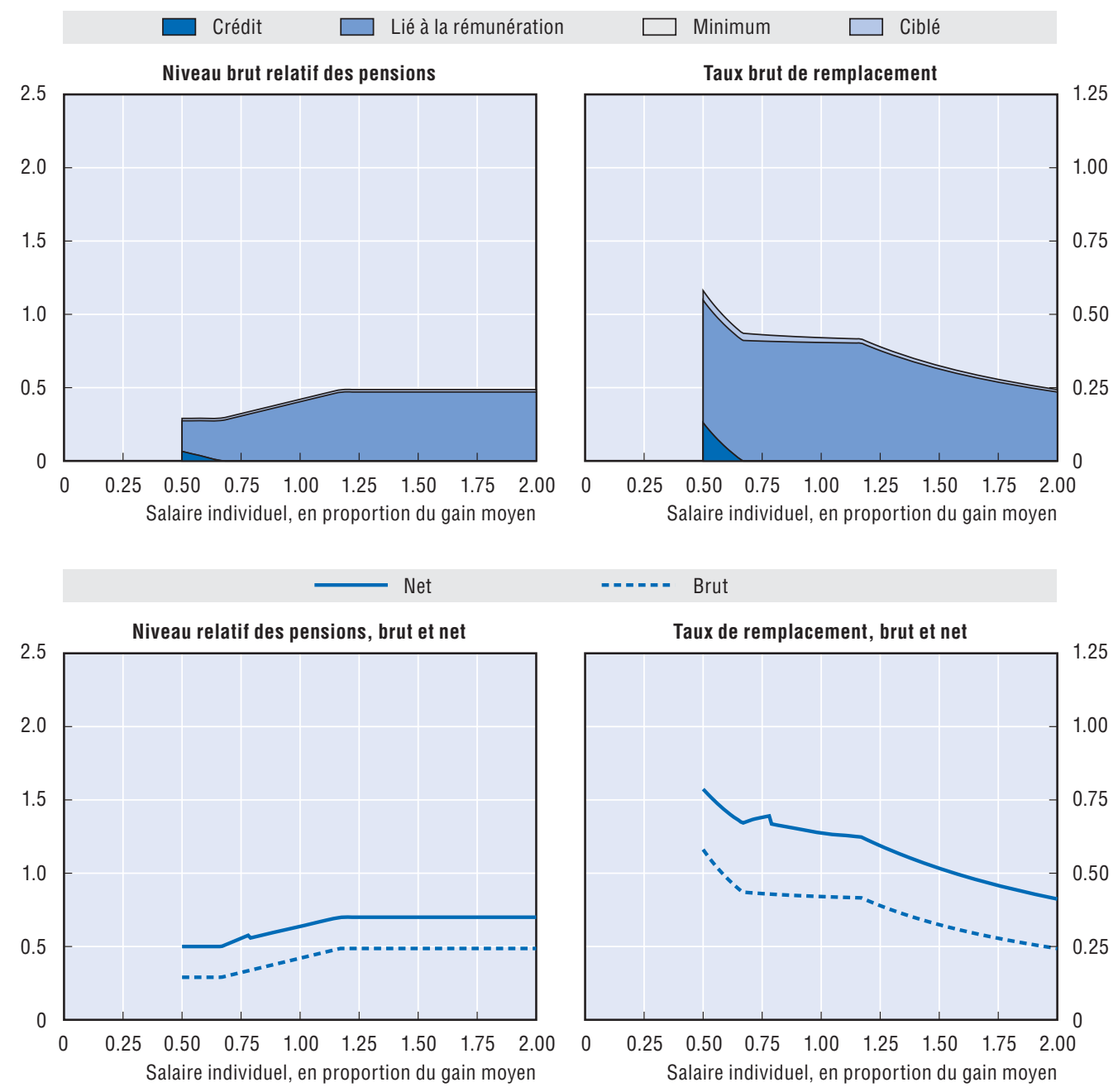

\begin{tabular}{|c|c|c|c|c|c|c|}
\hline \multirow{2}{*}{$\begin{array}{l}\text { Hommes } \\
\text { Femmes (si différent) }\end{array}$} & \multirow{2}{*}{$\begin{array}{l}\text { Salarié à revenu } \\
\text { médian }\end{array}$} & \multicolumn{5}{|c|}{ Salaire individuel, en multiple du salaire moyen } \\
\hline & & 0.5 & 0.75 & 1 & 1.5 & 2 \\
\hline Niveau brut relatif des pensions & 37.8 & 29.0 & 32.3 & 42.0 & 48.7 & 48.7 \\
\hline \multicolumn{7}{|l|}{ (\% du salaire moyen brut) } \\
\hline Niveau net relatif des pensions & 59.7 & 50.0 & 55.6 & 63.7 & 70.0 & 70.0 \\
\hline \multicolumn{7}{|l|}{ (\% du salaire moyen net) } \\
\hline Taux brut de remplacement & 42.4 & 58.1 & 43.1 & 42.0 & 32.5 & 24.3 \\
\hline \multicolumn{7}{|l|}{ (\% du salaire individuel brut) } \\
\hline Taux net de remplacement & 65.3 & 78.7 & 69.0 & 63.7 & 51.7 & 41.2 \\
\hline \multicolumn{7}{|l|}{ (\% du salaire individuel net) } \\
\hline Patrimoine retraite brut & 6.5 & 8.9 & 6.6 & 6.4 & 5.0 & 3.7 \\
\hline (multiple du salaire individuel brut) & 7.5 & 10.3 & 7.6 & 7.5 & 5.8 & 4.3 \\
\hline Patrimoine retraite net & 6.0 & 8.9 & 6.6 & 5.7 & 4.1 & 3.1 \\
\hline (multiple du salaire individuel brut) & 6.9 & 10.3 & 7.6 & 6.6 & 4.8 & 3.6 \\
\hline
\end{tabular}




\section{Canada}

\section{Canada : système de retraite en 2006}

Le système canadien de retraite comporte un régime universel forfaitaire, qui peut être complété par une prestation soumise à une condition de revenu, et des régimes publics liés à la rémunération.
Indicateurs essentiels

\begin{tabular}{llrr}
\hline & & Canada & OCDE \\
\hline Salaire moyen & CAD & 40600 & 40500 \\
& USD & 35800 & 35800 \\
Dépenses publiques & \% du PIB & 4.1 & 7.2 \\
au titre des retraites & à la naissance & 80.4 & 78.9 \\
Espérance de vie & à 65 ans & 84.5 & 83.4 \\
& \% de la population & 21.1 & 23.8 \\
Population de plus de 65 ans & d'âge actif & & \\
& & & \\
\hline
\end{tabular}

\section{Conditions d'ouverture des droits}

La pension de base de la SV (Sécurité de la vieillesse) est soumise à une condition de résidence : $1 / 40^{\mathrm{e}}$ de la pension maximum est acquis pour chaque année de résidence audelà de l'âge de 18 ans jusqu'à un maximum de 40 ans. Un minimum de dix ans de résidence est exigé pour percevoir une quelconque prestation. Celle-ci est payable à partir de 65 ans.

Pour le régime lié à la rémunération, environ 40 années de cotisation sont exigées pour percevoir une retraite à taux plein mais une seule année de cotisation validée suffit à générer un droit à prestations. L'âge normal de la retraite est de 65 ans mais un départ en retraite anticipée est possible à partir de 60 ans.

\section{Calcul des prestations}

\section{Régime de base}

En 2006, le montant de la retraite à taux plein pour la SV était de 5846.19 CAD. Le montant de la retraite de base est indexé sur les prix.

Cette retraite est soumise à une condition de revenu qui est mise en oeuvre par le biais de la fiscalité (récupération fiscale). En 2006, pour un revenu supérieur à 62144 CAD par an, la retraite de base était dégressive au taux de $15 \%$. Elle est également indexée sur les prix.

\section{Régime ciblé}

Le supplément de revenu garanti (SRG) vient s'ajouter à la pension SV de base. Le cumul des deux donnait en 2006 une prestation maximum de 13011.33 CAD.

Le SRG se voit appliquer une décote au taux de $50 \%$ au titre des revenus autres que la retraite de base. Le niveau objectif de la prestation est indexé sur les prix.

\section{Régime lié à la rémunération}

Les pensions et prestations liées à la rémunération relèvent du Régime de Pensions du Canada (RPC) ou du Régime de Rentes du Québec (RRQ). Le RPC et le RRQ offrent des prestations assez analogues. Ils visent un taux de remplacement de $25 \%$ du salaire moyen calculé sur toute la vie active (à l'exclusion des $15 \%$ d'années de rémunérations les plus faibles). Les rémunérations des années antérieures sont réévaluées en fonction de 
l'évolution des salaires à l'échelle nationale. Comme indiqué précédemment, environ 40 années de cotisation sont nécessaires pour percevoir des prestations à taux plein, avec des réductions au prorata pour des durées d'activité plus courtes. Pour 2006, la pension maximum de retraite liée à la rémunération a été de 844.58 CAD par mois.

Les personnes qui gagnent moins de $3500 \mathrm{CAD}$ par an ne sont pas tenues de cotiser. En 2006, le plafond de cotisation était de 42100 CAD. Ce plafond est indexé sur le salaire moyen tandis que le plancher de cotisation est gelé en valeur nominale.

Après le départ à la retraite, le montant de la pension liée à la rémunération est revalorisé chaque année en fonction de la hausse des prix.

\section{Pensions privées facultatives}

Environ $40 \%$ des salariés sont couverts par des plans de retraite professionnels dont environ 80 \% étaient, en 2003, des plans à prestations définies. Le plan à prestations définies modélisé, établi sur la base des résultats d'une enquête nationale sur les régimes de retraite, a un taux d'acquisition des droits de $1.3 \%$ du salaire jusqu'au plafond du régime public et de $2 \%$ au-delà. Les pensions sont calculées sur la base des derniers salaires.

La couverture globale des pensions privées facultatives (qui regroupent à la fois les plans individuels et les plans professionnels) est d'environ $57 \%$. Pour calculer les montants de pension des régimes à cotisations définies, on suppose un taux de cotisation de $8.5 \% \mathrm{du}$ salaire.

\section{Variantes de carrière}

\section{Retraite anticipée}

Dans le régime public lié à la rémunération, il est possible de partir en retraite anticipée à partir de 60 ans, moyennant une réduction de la prestation de $6 \%$ par an. Dans les deux autres régimes publics (de base et sous conditions de ressources), le départ en retraite anticipée n'est pas possible.

\section{Retraite différée}

La pension liée à la rémunération peut être différée jusqu'à cinq ans avec une surcote de $6 \%$ par an après 65 ans. La prestation de base et la prestation soumise à conditions de resources ne peuvent être différées. Pour la seconde, la condition de revenu inclut le salaire ; pour la première, un dispositif de récupération sur les gros revenus englobe, là encore, le salaire.

\section{Enfants}

Les années consacrées à l'éducation d'enfants de moins de 7 ans sont exclues de la période de référence dans le calcul des pensions; elles sont également exclues de la période de cotisation dans le régime lié à la rémunération.

\section{Chômage}

Dans le calcul du salaire moyen on peut exclure jusqu'à $15 \%$ de la durée de cotisation. Cet abattement est destiné à compenser les périodes de chômage, de maladie, de scolarité, etc. Les périodes de chômage ne sont pas validées. 
Résultats de la modélisation des retraites : Canada
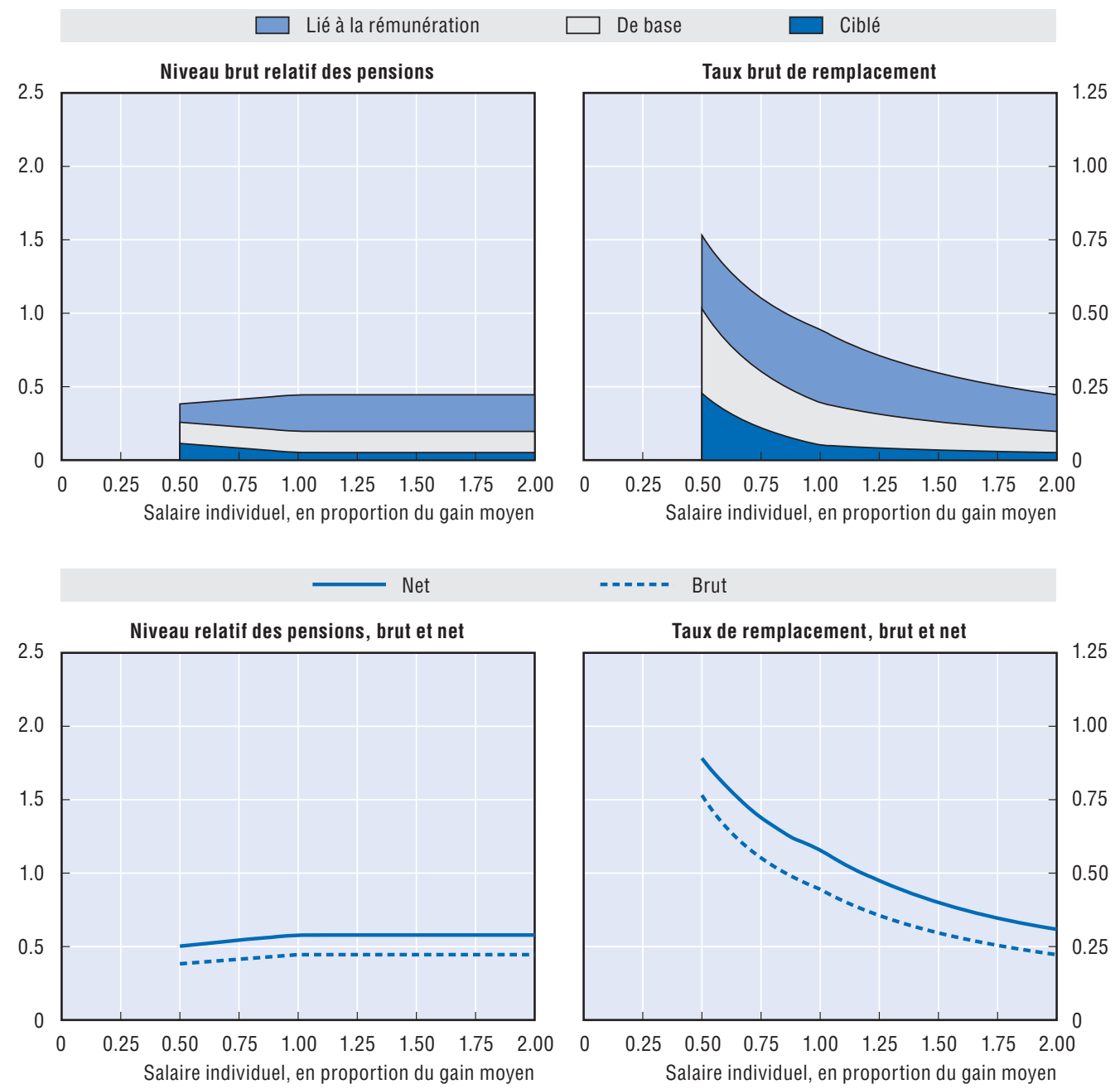

\begin{tabular}{|c|c|c|c|c|c|c|}
\hline \multirow{2}{*}{$\begin{array}{l}\text { Hommes } \\
\text { Femmes (si différent) }\end{array}$} & \multirow{2}{*}{$\begin{array}{l}\text { Salarié à revenu } \\
\text { médian }\end{array}$} & \multicolumn{5}{|c|}{ Salaire individuel, en multiple du salaire moyen } \\
\hline & & 0.5 & 0.75 & 1 & 1.5 & 2 \\
\hline Niveau brut relatif des pensions & 42.6 & 38.3 & 41.4 & 44.5 & 44.5 & 44.5 \\
\hline \multicolumn{7}{|l|}{ (\% salaire moyen brut) } \\
\hline Niveau net relatif des pensions & 55.9 & 50.3 & 54.4 & 57.9 & 57.9 & 57.9 \\
\hline \multicolumn{7}{|l|}{ (\% salaire moyen net) } \\
\hline Taux brut de remplacement & 50.2 & 76.5 & 55.2 & 44.5 & 29.7 & 22.2 \\
\hline \multicolumn{7}{|l|}{ (\% salaire individuel brut) } \\
\hline Taux net de remplacement & 63.6 & 89.1 & 68.9 & 57.9 & 40.0 & 30.9 \\
\hline \multicolumn{7}{|l|}{ (\% salaire individuel net) } \\
\hline Patrimoine retraite brut & 7.7 & 11.7 & 8.4 & 6.8 & 4.5 & 3.4 \\
\hline (multiple du salaire individuel brut) & 8.9 & 13.6 & 9.8 & 7.9 & 5.3 & 4.0 \\
\hline Patrimoine retraite net & 7.6 & 11.7 & 8.4 & 6.7 & 4.5 & 3.4 \\
\hline (multiple du salaire individuel brut) & 8.9 & 13.6 & 9.8 & 7.8 & 5.2 & 3.9 \\
\hline
\end{tabular}




\section{Corée}

\section{Corée : système de retraite en 2006}

En Corée, le régime public de retraite n'a été mis en place que relativement récemment. Il s'agit d'un régime lié à la rémunération qui applique une formule progressive dans la mesure où les prestations sont calculées sur la base à la fois du salaire individuel et du salaire moyen à l'échelle nationale.
Indicateurs essentiels

\begin{tabular}{llrr}
\hline & & Corée & OCDE \\
\hline Salaire & KRW (millions) & 30.44 & 34.08 \\
moyen & USD & 32000 & 35800 \\
Dépenses publiques & $\%$ du PIB & 1.6 & 7.2 \\
au titre des retraites & & & \\
Espérance de vie & à la naissance & 79.1 & 78.9 \\
& à 65 ans & 83.1 & 83.4 \\
Population de plus de 65 ans & \% de la population & 14.5 & 23.8 \\
& d'âge actif & &
\end{tabular}

\section{Conditions d'ouverture des droits}

Actuellement, la retraite peut être prise à partir de 60 ans sous réserve que l'intéressé ait cotisé pendant au minimum dix ans. À partir de 55 ans, il est possible de faire valoir ses droits à une retraite anticipée avec décote.

L'âge normal de la retraite est progressivement relevé ; il atteindra 65 ans en 2033. La modélisation suppose pour le long terme un âge de la retraite de 65 ans ; l'âge de départ anticipé à la retraite sera également relevé (de 55 à 60 ans).

\section{Calcul des prestations}

\section{Régime lié à la rémunération}

Pour 40 années de cotisation, le taux de remplacement était de $60 \%$ du salaire en 2007 mais il sera ramené à $50 \%$ en 2008 puis abaissé de 0.5 point de pourcentage par an à compter de 2009 pour tomber à $40 \%$ en 2028. Le modèle suppose que ce taux de $40 \%$ est calculé sur une période de 45 ans. Le salaire de référence est la moyenne des gains moyens perçus sur l'ensemble de la carrière, revalorisés en fonction de la hausse des salaires, et du salaire moyen des assurés du régime national de retraite, mesuré sur les trois années précédentes et revalorisé en fonction de la hausse des prix. Le salaire ouvrant droit à pension est plafonné à 3.6 millions KRW par mois, soit $142 \%$ du salaire moyen des assurés en 2006.

Le niveau maximum de la prestation est de $100 \%$ du salaire individuel. Après le départ à la retraite, la prestation est indexée sur les prix. Les personnes de 60 ans et plus ne cotisent plus et n'acquièrent plus de droits supplémentaires à prestations.

\section{Pension de vieillesse de base}

À partir de 2008, environ $60 \%$ des personnes de 65 ans et plus peuvent percevoir la "pension de vieillesse de base " soumise à conditions de ressources. Il était prévu de porter à $70 \%$ en 2009 le nombre des futurs bénéficiaires. Il s'agit d'une prestation à taux uniforme de $5 \%$ par an du salaire moyen sur trois ans des affiliés au régime national de retraite. La prestation est réduite progressivement en fonction du revenu et du patrimoine des personnes âgées. Pour les couples, le taux de pension est fixé pour chaque membre du couple à $80 \%$ du taux appliqué aux personnes seules. 


\section{Variantes de carrière}

\section{Retraite anticipée}

Lorsqu'en 2013, l'âge normal de la retraite passera de 60 à 65 ans, on suppose que l'âge de départ anticipé à la retraite passera de 55 à 60 ans. À 60 ans, la pension de retraite anticipée sera alors de $70 \%$ de la pension de vieillesse normale. La prestation augmente de $6 \%$ par an, de sorte qu'une personne prenant sa retraite à 64 ans aura droit à $94 \%$ de la pension de vieillesse à taux plein.

\section{Retraite différée}

Les personnes peuvent acquérir des droits à pension supplémentaires en différant leur départ à la retraite. La prestation est majorée de $6 \%$ par an et le report maximum est de cinq ans jusqu'à 70 ans.

Si des retraités entre 65 et 69 ans ont des revenus supérieurs au salaire moyen de l'ensemble des assurés, la pension qu'ils percevront à 65 ans sera égale à $50 \%$ de la retraite à taux plein et la prestation augmentera de $10 \%$ par an; ce dispositif est connu sous le nom de " pension de vieillesse active ". Par conséquent, si des retraités ayant entre 65 et 69 ans travaillent, ils peuvent choisir entre la " pension différée " et la " pension de vieillesse active ».

\section{Enfants}

Une personne qui ne travaille pas pour élever ses enfants peut être exemptée de cotisations durant la période pour laquelle elle demande cette exemption. La période assurée peut être augmentée en réglant, après la reprise du travail, le montant total (y compris la part patronale) des cotisations ayant fait l'objet d'exemption.

Excepté pour le premier enfant, une assurée ayant un enfant après janvier 2008 peut bénéficier de crédits de retraite. Les crédits attribués sont de 12 mois avec un maximum de 50 mois selon le nombre d'enfants nés après cette date.

\section{Chômage}

Un chômeur peut être exempté du paiement de ses cotisations durant la période pour laquelle il demande cette exemption. La période assurée peut être augmentée en réglant, après la reprise du travail, le montant total (y compris la part patronale) des cotisations ayant fait l'objet d'exemption. 


\section{Résultats de la modélisation des retraites : Corée}
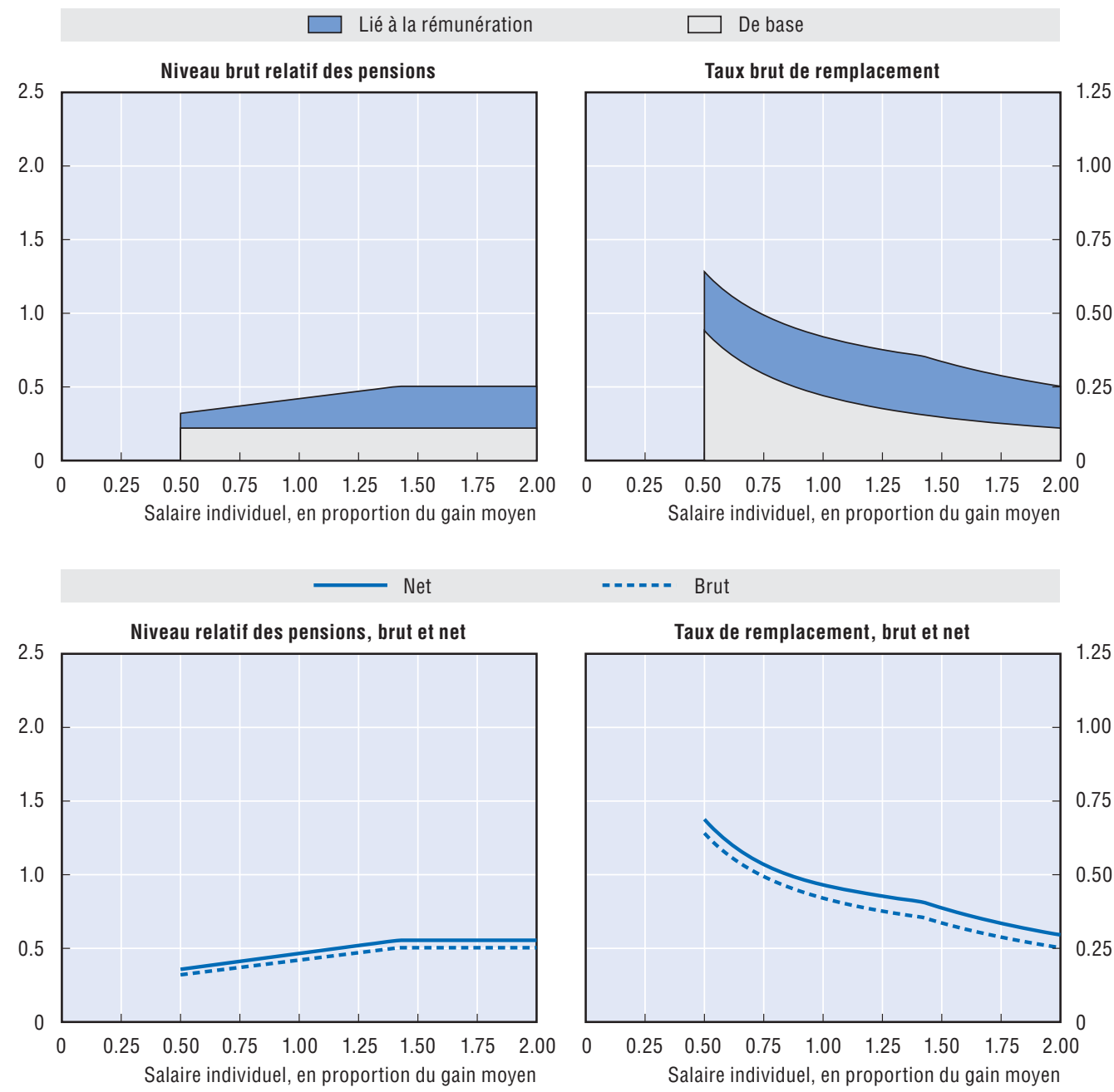

\begin{tabular}{|c|c|c|c|c|c|c|}
\hline \multirow{2}{*}{$\begin{array}{l}\text { Hommes } \\
\text { Femmes (si différent) }\end{array}$} & \multirow{2}{*}{$\begin{array}{l}\text { Salarié à revenu } \\
\text { médian }\end{array}$} & \multicolumn{5}{|c|}{ Salaire individuel, en multiple du salaire moyen } \\
\hline & & 0.5 & 0.75 & 1 & 1.5 & 2 \\
\hline Niveau brut relatif des pensions & 39.7 & 32.1 & 37.1 & 42.1 & 50.4 & 50.4 \\
\hline \multicolumn{7}{|l|}{ (\% salaire moyen brut) } \\
\hline Niveau net relatif des pensions & 44.0 & 35.8 & 41.2 & 46.6 & 55.5 & 55.5 \\
\hline \multicolumn{7}{|l|}{ (\% salaire moyen net) } \\
\hline Taux brut de remplacement & 45.1 & 64.1 & 49.4 & 42.1 & 33.6 & 25.2 \\
\hline \multicolumn{7}{|l|}{ (\% salaire individuel brut) } \\
\hline Taux net de remplacement & 49.2 & 68.8 & 53.5 & 46.6 & 38.7 & 29.6 \\
\hline \multicolumn{7}{|l|}{ (\% salaire individuel net) } \\
\hline Patrimoine retraite brut & 6.3 & 8.9 & 6.9 & 5.9 & 4.7 & 3.5 \\
\hline (multiple du salaire individuel brut) & 7.5 & 10.7 & 8.2 & 7.0 & 5.6 & 4.2 \\
\hline Patrimoine retraite net & 6.2 & 8.9 & 6.8 & 5.8 & 4.6 & 3.4 \\
\hline (multiple du salaire individuel brut) & 7.4 & 10.6 & 8.1 & 6.9 & 5.5 & 4.1 \\
\hline
\end{tabular}




\section{Danemark}

\section{Danemark : système de retraite en 2006}

Le régime public de base (Folkepension) est constitué d'une prestation de base et d'un complément de retraite soumis à condition de revenu. Une pension complémentaire soumise à conditions de ressources est versée aux retraités les plus défavorisés. À cela s'ajoutent deux régimes reposant sur des cotisations individuelles : l'ATP (retraite complémentaire du marché du travail) et le SP (régime spécial d'épargne retraite). En outre, environ $90 \%$ des salariés à pleintemps sont couverts par des plans professionnels obligatoires négociés dans le cadre de conventions collectives.

\section{Indicateurs essentiels}

\begin{tabular}{llrr}
\hline & & Danemark & OCDE \\
\hline Salaire moyen & DKK & 330900 & 212700 \\
& USD & 55700 & 35800 \\
Dépenses publiques & \% du PIB & 5.4 & 7.2 \\
au titre des retraites & & 78.4 & 78.9 \\
Espérance de vie & à la naissance & 82.7 & 83.4 \\
& à 65 ans & 25.3 & 23.8 \\
Population de plus de 65 ans & \% de la population & & \\
& d'âge actif & & \\
\hline
\end{tabular}

\section{Conditions d'ouverture des droits}

L'âge normal de la retraite est actuellement de 65 ans ; il sera porté à 67 ans sur la période 2024-27. Quarante années de résidence sont exigées pour percevoir une pension publique de vieillesse à taux plein. Pour des durées de résidence plus courtes, les prestations sont calculées au prorata.

Une durée complète de cotisations (carrière complète) est exigée pour avoir pleinement droit à la retraite complémentaire du marché du travail (ATP) et au régime spécial d'épargne retraite (SP). Le régime ATP a été mis en place en 1964. Il s'agit d'un régime à cotisations définies fondé sur une assurance collective. Le régime SP a été mis en place en 1999. Il s'agit d'un régime obligatoire purement fondé sur l'épargne dans lequel les cotisations sont versées sur des comptes individuels.

\section{Calcul des prestations}

\section{Régime de base}

Le montant de la retraite de base à taux plein est de 4836 DKK par mois ou 58032 DKK par an, équivalant à $17.5 \%$ du salaire moyen. L'existence d'une condition de revenu individuel signifie que le montant de la retraite de base sera réduit si le revenu du travail excède 246500 DKK (approximativement les trois-quarts du salaire général annuel d'un ouvrier moyen). Au-delà de ce montant, la prestation se voit appliquer un taux de dégressivité de $30 \%$.

\section{Régime ciblé}

Le complément de retraite à taux plein est de 4868 DKK par mois ou 58416 DKK par an pour une personne seule et de 27276 DKK pour un couple. Les montants réels sont soumis à 
conditions de ressources, toutes sources confondues (y compris l'ATP, le SP et les pensions professionnelles) autres que la retraite publique. Au-delà d'un revenu de 54400 DKK pour une personne seule, le complément de retraite ciblé est dégressif au taux de $30 \%$ de l'excédent. Pour un couple, le montant est de 109200 DKK et le taux de dégressivité est de $15 \%$.

Une nouvelle prestation complémentaire de 6300 DKK (2006) liée à la pension de vieillesse publique a été mise en place en 2004. Ce complément imposable et versé une fois par an, est soumis à conditions de ressources et ciblée sur les retraités les plus modestes ne disposant pas d'une épargne importante (au maximum 56800 DKK).

La pension de vieillesse publique (régimes de base et ciblé plus complément de retraite) et la prestation complémentaire sont ajustées chaque année en fonction du salaire moyen. L'ajustement est effectué sur la base d'un indice des hausses de salaires des deux années précédentes. Si la hausse des salaires nominaux est supérieure à $2 \%$, un maximum de 0.3 point de l'excédent est alloué à une réserve pour dépenses sociales. Ainsi, l'indexation des retraites et autres prestations sociales est basée sur les hausses de salaires nettes d'éventuelles affectations à la réserve.

\section{Régimes professionnels}

Il s'agit de régimes par capitalisation intégrale et à cotisations définies convenus entre les partenaires sociaux La couverture de ces régimes est quasi universelle. Les taux de cotisation se situent habituellement entre $9 \%$ et $17 \%$ de la rémunération. En 2006, le pourcentage applicable à la majorité des travailleurs danois a été porté à $10.8 \%$; c'est ce taux de cotisation qui a été retenu pour la modélisation. Les prestations sont habituellement versées sous forme de rente. Le taux d'intérêt supposé est de $1.5 \%$ pour les cotisations récentes ou les nouveaux régimes. Toutefois, les régimes fonctionnant sur un modèle " à but lucratif ", l'augmentation des retraites dépend du rendement des actifs et des statistiques du fonds en matière de mortalité. De nombreux régimes autorisent également les retraits de montants forfaitaires. Depuis 2000, le calcul du montant des rentes doit se faire sur la base de tables de mortalité unisexe.

\section{Régime à cotisations définies}

L'ATP est un régime obligatoire, par capitalisation intégrale et à cotisations définies reposant sur une assurance collective. Il assure une pension à vie à compter de 65 ans et une pension de réversion d'un montant forfaitaire aux personnes à charge en cas de décès de l'adhérent. L'ATP couvre tous les salariés et la quasi totalité des bénéficiaires des allocations de sécurité sociale. L'adhésion à l'ATP est facultative pour les travailleurs indépendants. L'ATP couvre pratiquement la totalité de la population et s'approche de l'universalité absolue.

Techniquement, la pension de vieillesse de l'ATP repose sur une rente différée garantie. La cotisation est un montant fixe, pas un pourcentage du revenu; elle varie en fonction du nombre d'heures travaillées. En 2006, elle était de 2924 DKK pour un salarié à plein-temps. Les cotisations sont payées par l'employeur pour les deux tiers et par le salarié pour le tiers restant. Le tableau ci-dessous indique la somme des cotisations patronales et salariales payées par rapport au nombre d'heures travaillées (pour les travailleurs mensualisés) :

\begin{tabular}{lrrrr}
\hline Heures travaillées par mois & $<39$ & $39-77$ & $78-116$ & $>116$ \\
\hline Cotisation, DKK/mois jusqu'en 2009 & 0 & 81.3 & 162.6 & 243.9 \\
Cotisation, DKK/mois après 2009 & 0 & 90 & 180 & 270 \\
\hline
\end{tabular}


La cotisation est ajustée sur décision des partenaires sociaux dans le cadre de conventions collectives. Sur les vingt dernières années, elle a été relevée par étapes plus ou moins sur la base du salaire moyen. La modélisation prend pour hypothèse que le montant de la cotisation augmentera sur la base du salaire moyen. Une augmentation de l'ordre de $10 \%$ a été convenue pour 2009.

Jusqu'en 2002, 396 DKK de cotisations donnaient droit à 100 DKK de prestations à partir de 65 ans quel que soit l'âge auquel étaient versées ces cotisations, ce qui impliquait un taux d'intérêt moyen (pour toutes les cohortes) d'environ $4.5 \%$. Depuis 2002, on a supposé un taux d'intérêt nominal de $1.5 \%$. Dans le modèle, on suppose que l'ATP rapporte le même taux d'intérêt que celui retenu pour les régimes par capitalisation à cotisations définies dans d'autres pays de l'OCDE.

Si sa situation financière le permet, le régime ATP augmente de la même façon les pensions mises en paiement et les droits à pension. Il le fait sous la forme d'allocations de bonus. Les augmentations sont garanties au même titre que les droits acquis.

La modélisation suppose une indexation totale sur les prix.

Un système ATP entièrement nouveau de rentes constituées a été mis en place en 2008. Le modèle repose sur des swaps de taux d'intérêt et non pas sur un taux d'intérêt nominal fixe de $1.5 \%$, par exemple.

\section{Régime à cotisations définies (régime spécial d'épargne retraite, SP)}

Les salariés, les travailleurs indépendants et les bénéficiaires d'indemnités de chômage et de maladie cotisent à ce régime obligatoire individuel d'épargne retraite à hauteur de $1 \%$ de leur rémunération. La gestion des placements est actuellement centralisée. Depuis 2005, les adhérents sont en mesure de choisir leur gestionnaire et leur portefeuille. Les rémunérations couvertes par ce régime ne sont pas plafonnées. Les prestations sont versées à l'âge normal de la retraite. Si le solde du compte est inférieur à 15000 DKK, il est versé en une seule fois et imposé au taux de $40 \%$. S'il se situe entre 15000 et $120000 \mathrm{DKK}$, son paiement est étalé sur une période de dix ans et s'il est supérieur à 120000 DKK à l'âge normal de la retraite, il est versé sous la forme de paiements mensuels étalés sur dix ans. Ces paiements périodiques sont imposés au taux de l'impôt sur le revenu des personnes physiques.

Dans le cadre d'accords, les cotisations au régime SP ont été suspendues depuis 2004. La modélisation, qui adopte une perspective de long terme, suppose une reprise de ces cotisations.

\section{Variantes de carrière}

\section{Retraite anticipée}

Il existe une pension de retraite anticipée partielle pour les travailleurs de 60 à 65 ans qui continuent à travailler entre 12 et 30 heures par semaine. Le régime est progressivement supprimé. Désormais, il ne s'applique plus qu'aux travailleurs nés avant le $1^{\text {er }}$ janvier 1959. Le bénéficiaire doit réduire son nombre d'heures de travail hebdomadaire d'au moins sept heures ou d'au moins un quart du nombre total d'heures travaillées dans une semaine moyenne. La pension partielle est calculée sur la base d'un montant fixe pour chaque heure de travail en moins. Ce montant est d'environ 76 DKK par heure pour 2007. Depuis 1999, les bénéficiaires se voient appliquer une dégressivité. 
Par ailleurs, un programme facultatif de retraite anticipée lié à l'assurance chômage verse des prestations entre l'âge de 60 ans (qui passera progressivement à 62 ans sur la période 2019-22) et l'âge normal de la retraite. Pour être admissibles à ces prestations, les personnes doivent avoir été membres de la caisse d'assurance chômage pendant au moins 25 ans au cours des 30 dernières années et avoir cotisé au programme facultatif de retraite anticipée durant cette période. Elles doivent également remplir les conditions ouvrant droit aux prestations de chômage si elles étaient au chômage au moment de leur entrée dans le régime facultatif de retraite anticipée. Le montant des prestations correspond au taux d'indemnisation du chômage dans la limite de 91 \% du taux maximum, soit 3110 DKK par semaine pour des travailleurs à plein-temps et 2075 DKK pour des travailleurs à temps partiel (chiffres de 2006). Il n'est pas possible de cumuler des prestations de retraite anticipée facultative avec la pension sociale.

Les personnes qui diffèrent la liquidation de leurs prestations de retraite anticipée facultative d'au minimum deux ans après l'âge normal de la retraite et qui continuent à travailler perçoivent une prestation plus élevée dont le taux est équivalent au taux maximum de l'indemnité de chômage (3 415 DKK par semaine en 2006). Une personne éligible à un programme facultatif de retraite anticipée ou équivalent, reçoit un montant forfaitaire payé en une fois d'un montant maximum de 124860 DKK en 2006.

Les personnes couvertes par l'un des deux programmes de retraite anticipée retrouvent la pension de vieillesse standard lorsqu'elles arrivent à l'âge normal de la retraite, à savoir 65 ans (du fait de leur âge, elles ne seront pas concernées par le relèvement de l'âge de la retraite prévu par la législation sur la période 2024-27).

\section{Retraite différée}

Il est possible de différer jusqu'à dix ans la pension de vieillesse publique. La surcote pour une année est égale au ratio entre la durée du report et l'espérance de vie moyenne au moment de la liquidation des droits. Par exemple, selon les projections démographiques Banque mondiale/Nations Unies, l'espérance de vie pour une personne de 68 ans est de 17.1 ans. La surcote par année de report au-delà de 67 ans serait donc de 1/17.1 = 5.8\%.

\section{Enfants}

Pour les périodes de congé maternité/paternité/parental, le montant des cotisations à l'ATP est multiplié par deux. La cotisation est payée par le bénéficiaire à hauteur d'un tiers et par les pouvoirs publics/la municipalité à hauteur des deux tiers. Les indemnités de maternité/paternité/parentales peuvent être versées pendant une durée totale pouvant atteindre 52 semaines. Les quatre semaines qui précèdent la naissance et les 14 semaines qui suivent sont réservées à la mère. Le père a droit à deux semaines de congé durant les 14 semaines suivant immédiatement la naissance (congé de paternité). Les trois dernières semaines peuvent être divisées ou partagées entre le père et la mère (congé parental). Habituellement, les personnes qui restent en dehors du marché du travail au-delà du congé de maternité pour s'occuper de leurs enfants passent dans un autre système qui suppose également une cotisation à l'ATP. Les jeunes parents reprennent habituellement le travail dès la fin de leur congé sauf si l'enfant est malade ou handicapé, auquel cas ils auront normalement la possibilité de bénéficier d'une sorte de prestation publique avec une cotisation à l'ATP. Les parents percevant des indemnités de maternité/paternité/ parentales ont cotisé à la SP et cotiseront à nouveau lorsque ces cotisations. Pour les plans 
professionnels de retraite, les périodes de non-emploi liées aux enfants ne donnent lieu ni à crédits ni à cotisations.

\section{Chômage}

Pendant les périodes de chômage, l'assurance chômage (ou à défaut la municipalité) prend à sa charge l'obligation de paiement de l'employeur et le taux de cotisation à l'ATP est doublé lorsque le chômeur est indemnisé par l'assurance chômage (taux normal si prestation d'aide sociale). Les pouvoirs publics prennent en charge les deux tiers du paiement lorsque l'assurance chômage est épuisée et que le chômeur bascule sur le chômage/l'aide sociale. Pour les regimes professionnels de retraite, les périodes de chômage ne donnent lieu ni à crédits ni à cotisations. 
Résultats de la modélisation des retraites : Danemark
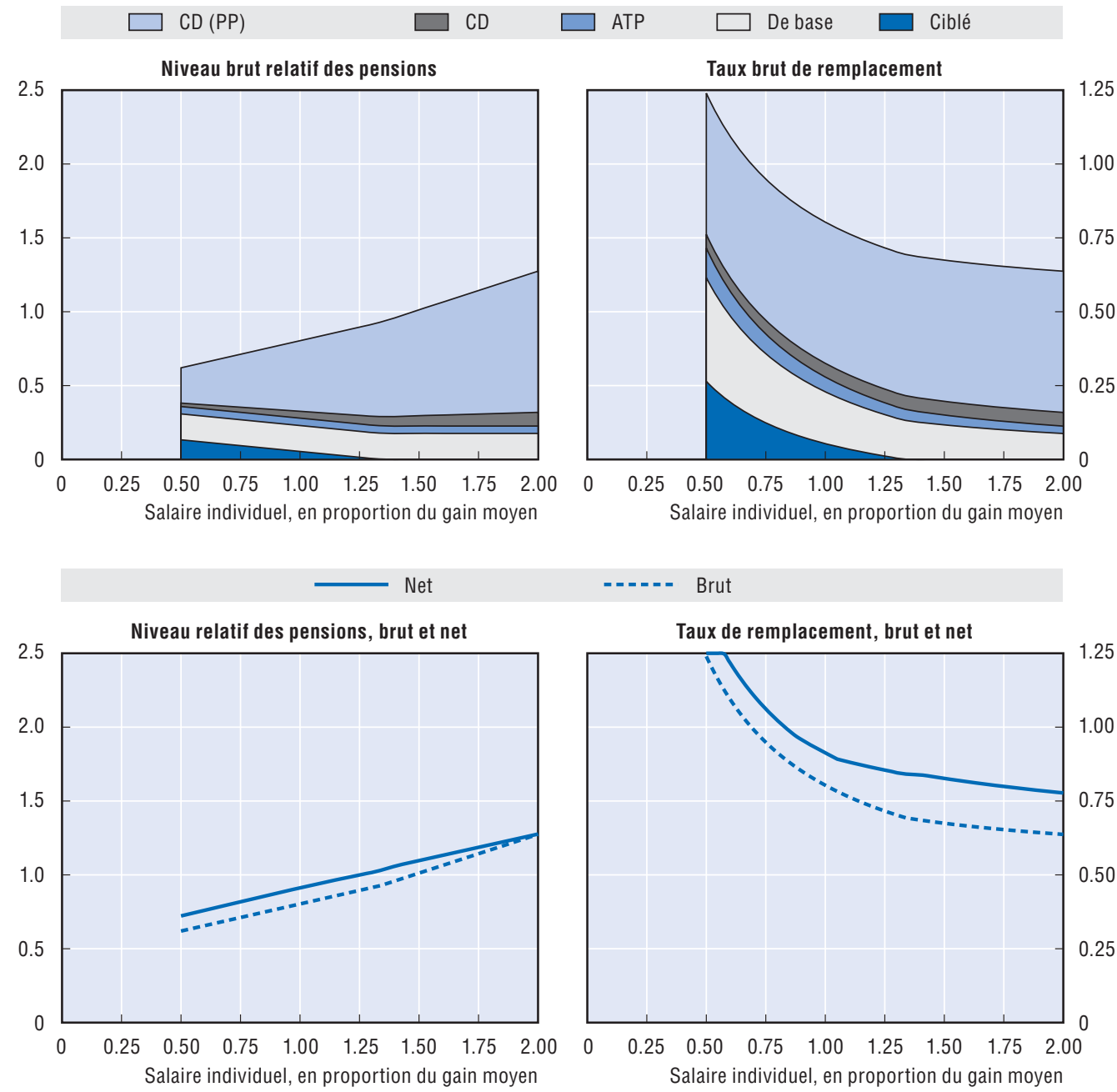

\begin{tabular}{|c|c|c|c|c|c|c|}
\hline \multirow{2}{*}{$\begin{array}{l}\text { Hommes } \\
\text { Femmes (si différent) }\end{array}$} & \multirow{2}{*}{$\begin{array}{l}\text { Salarié à revenu } \\
\text { médian }\end{array}$} & \multicolumn{5}{|c|}{ Salaire individuel, en multiple du salaire moyen } \\
\hline & & 0.5 & 0.75 & 1 & 1.5 & 2 \\
\hline Niveau brut relatif des pensions & 74.8 & 62.0 & 71.2 & 80.3 & 101.2 & 127.4 \\
\hline \multicolumn{7}{|l|}{ (\% salaire moyen brut) } \\
\hline Niveau net relatif des pensions & 85.6 & 72.2 & 81.8 & 91.3 & 109.7 & 127.6 \\
\hline \multicolumn{7}{|l|}{ (\% salaire moyen net) } \\
\hline Taux brut de remplacement & 88.0 & 124.0 & 94.9 & 80.3 & 67.5 & 63.7 \\
\hline \multicolumn{7}{|l|}{ (\% salaire individuel brut) } \\
\hline Taux net de remplacement & 98.7 & 137.0 & 106.2 & 91.3 & 82.7 & 77.7 \\
\hline \multicolumn{7}{|l|}{ (\% salaire individuel net) } \\
\hline Patrimoine retraite brut & 12.8 & 18.5 & 13.9 & 11.6 & 9.6 & 9.0 \\
\hline (multiple du salaire individuel brut) & 14.7 & 21.3 & 16.0 & 13.3 & 11.0 & 10.3 \\
\hline Patrimoine retraite net & 8.6 & 12.7 & 9.4 & 7.8 & 6.1 & 5.3 \\
\hline (multiple du salaire individuel brut) & 9.9 & 14.6 & 10.8 & 8.9 & 7.0 & 6.1 \\
\hline
\end{tabular}




\section{Espagne}

\section{Espagne : système de retraite en 2006}

Le régime public de retraite espagnol comporte une prestation unique liée à la rémunération (niveau contributif) avec une retraite minimum soumise à conditions de ressources. Il comporte également un niveau non contributif soumis à conditions de ressources qui remplace le régime d'aide sociale antérieur.
Indicateurs essentiels

\begin{tabular}{llrr}
\hline & & Espagne & OCDE \\
\hline Salaire moyen & EUR & 21200 & 28600 \\
& USD & 26500 & 35800 \\
$\begin{array}{l}\text { Dépenses publiques au titre des } \\
\text { retraites }\end{array}$ & $\%$ du PIB & 8.1 & 7.2 \\
Espérance de vie & à la naissance & 81.1 & 78.9 \\
& à 65 ans & 85.0 & 83.4 \\
Population de plus de 65 ans & \% de la population & 26.2 & 23.8 \\
& d'âge actif & &
\end{tabular}

\section{Conditions d'ouverture des droits}

Pour une pension à taux plein, l'âge de départ à la retraite est de 65 ans pour les hommes comme pour les femmes. Quinze années de cotisations sont nécessaires pour être éligible à une pension.

\section{Calcul des prestations}

\section{Régime lié à la rémunération}

L'acquisition des droits à prestations se fait selon un barême. Après 15 années de cotisation, le taux est de $50 \%$ du salaire de référence. Il est majoré de $3 \%$ par an pour les dix années suivantes puis de $2 \%$ par la suite. Le taux d'acquisition maximum, de $100 \%$, est atteint après 35 années de cotisation.

Le salaire de référence retenu est le salaire des 15 dernières années, revalorisé en fonction de l'évolution des prix, sauf pour les deux dernières années. Cela signifie que le taux de remplacement par rapport au dernier salaire est inférieur à $100 \%$. Sur la base des hypothèses types de hausse des salaires et des prix, il est de $88 \%$.

La rémunération retenue pour le calcul des cotisations et des prestations est plafonnée à 34772.4 EUR, ce qui correspond à $164 \%$ du salaire moyen.

Les prestations sont indexées sur les prix.

\section{Pension minimum et pension maximum}

Une pension minimum de 469.73 EUR par mois, soit $31.1 \%$ du salaire moyen, est payable à partir de 65 ans aux retraités n'ayant pas de conjoint à charge ; pour les retraités ayant un conjoint à charge son montant est de 569.07 EUR par mois, soit $37.7 \%$ du salaire moyen. Les paiements sont effectués sur 14 mois.

Suite à la politique mise en place en 2004 , la pension minimum a augmenté plus vite que l'indice des prix au cours des dernières années.

La pension maximum, qui était de 2245.67 EUR par mois en 2006, est versée sur 14 mois. 


\section{Variantes de carrière}

\section{Retraite anticipée}

Un départ en retraite anticipée est possible à partir de 61 ans pour les personnes entrées dans le régime à partir de 1967 qui perdent leur emploi, à condition qu'elles aient cotisé au moins 30 ans. La réduction actuarielle dépend du nombre d'années de cotisation : $8 \%$ pour 30 ans, $7.5 \%$ pour $31-34$ ans, $7 \%$ pour $35-37$ ans, $6.5 \%$ pour $38-39$ ans et $6 \%$ pour plus de 40 ans.

Pour les personnes entrées dans le régime avant 1967, une retraite anticipée est possible à partir de 60 ans. Si le départ est volontaire, la réduction est de $8 \%$ par an. Sinon, elle est la même que pour les personnes de 61 ans et plus entrées dans le régime à partir de 1967.

La pension minimum de retraite anticipée est de 437.68 EUR, soit $29 \%$ du salaire moyen pour les retraités n'ayant pas de conjoint à charge et de 531.84 EUR par mois, soit $35 \%$ du salaire moyen pour les retraités ayant un conjoint à charge ; après 65 ans, elle est plus élevée.

Entre 60 et 64 ans, il est possible de cumuler une retraite partielle et un emploi à temps partiel si le nombre d'heures de travail est réduit d'un pourcentage se situant entre 25 et $85 \%$. Un autre salarié doit effectuer les heures de travail restantes. Quinze années de cotisation sont exigées.

Avec la nouvelle loi 40/2007, les règles relatives à la retraite partielle ont changé à partir du $1^{\mathrm{er}}$ janvier 2008:

1. Le nombre d'heures de travail doit être réduit de 25 à $75 \%$.

2. Les candidats à une retraite partielle doivent avoir passé au moins six ans chez leur dernier employeur et justifier d'un total de 30 années ou plus de cotisation.

3. Pour les personnes entrées dans le régime après 1967, la retraite partielle peut être prise à partir de 61 ans.

\section{Retraite différée}

Il est possible de différer la pension au-delà de l'âge normal de la retraite. Pour les personnes de 65 ans ayant cotisé pendant 35 années, le montant de la pension peut excéder $100 \%$ de la base de calcul. La prestation augmente de $2 \%$ par année de report.

À partir de 65 ans, il est également possible de cumuler une retraite partielle et un emploi à temps partiel. Dans ce cas, il n'y a pas d'obligation de remplacer les heures de travail restantes.

Avec la nouvelle loi 40/2007, les travailleurs ayant cotisé 15 années et plus qui continuent de travailler après 65 ans voient leurs prestations augmenter de $2 \%$ de la base de calcul par année supplémentaire. Avec 40 années de cotisation, l'augmentation est de $3 \%$. Les retraités ayant droit à une pension maximum qui partent à la retraite à 66 ans et plus recevront une somme forfaitaire annuelle $(2 \%$ de la pension maximum par année supplémentaire au-delà de 65 ans et $3 \%$ avec 40 années de cotisation).

\section{Enfants}

Une couverture est prévue pendant la maternité. Deux années d'interruption de carrière pour élever des enfants sont validées dans le calcul des prestations de retraite. 


\section{Chômage}

Durant les périodes de chômage indemnisé, l'État prend à sa charge l'intégralité de la part patronale et $35 \%$ de la part salariale des cotisations au régime de l'assurance retraite. Les $65 \%$ restants sont à la charge du travailleur. Le salaire de base pour le calcul des cotisations est le salaire moyen des six mois précédant l'épisode de chômage. La durée dépend du nombre de jours de cotisation dans les six mois précédant l'épisode de chômage : elle varie entre quatre mois et deux ans. L'assistance chômage qui est versée ensuite ne donne lieu à aucun crédit de retraite, excepté pour les personnes de 52 ans et plus. Pour ces personnes, les cotisations à la de la pension de vieillesse sont prises en charge par l'État jusqu'à l'âge de la retraite. Ces cotisations sont calculées sur la base minimum de 631.20 EUR par mois. 


\section{Résultats de la modélisation des retraites : Espagne}

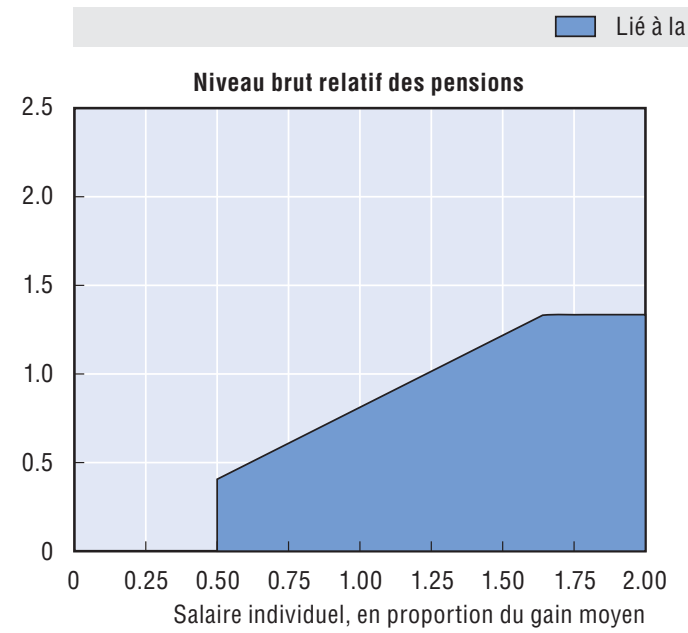

ié à la rémunération
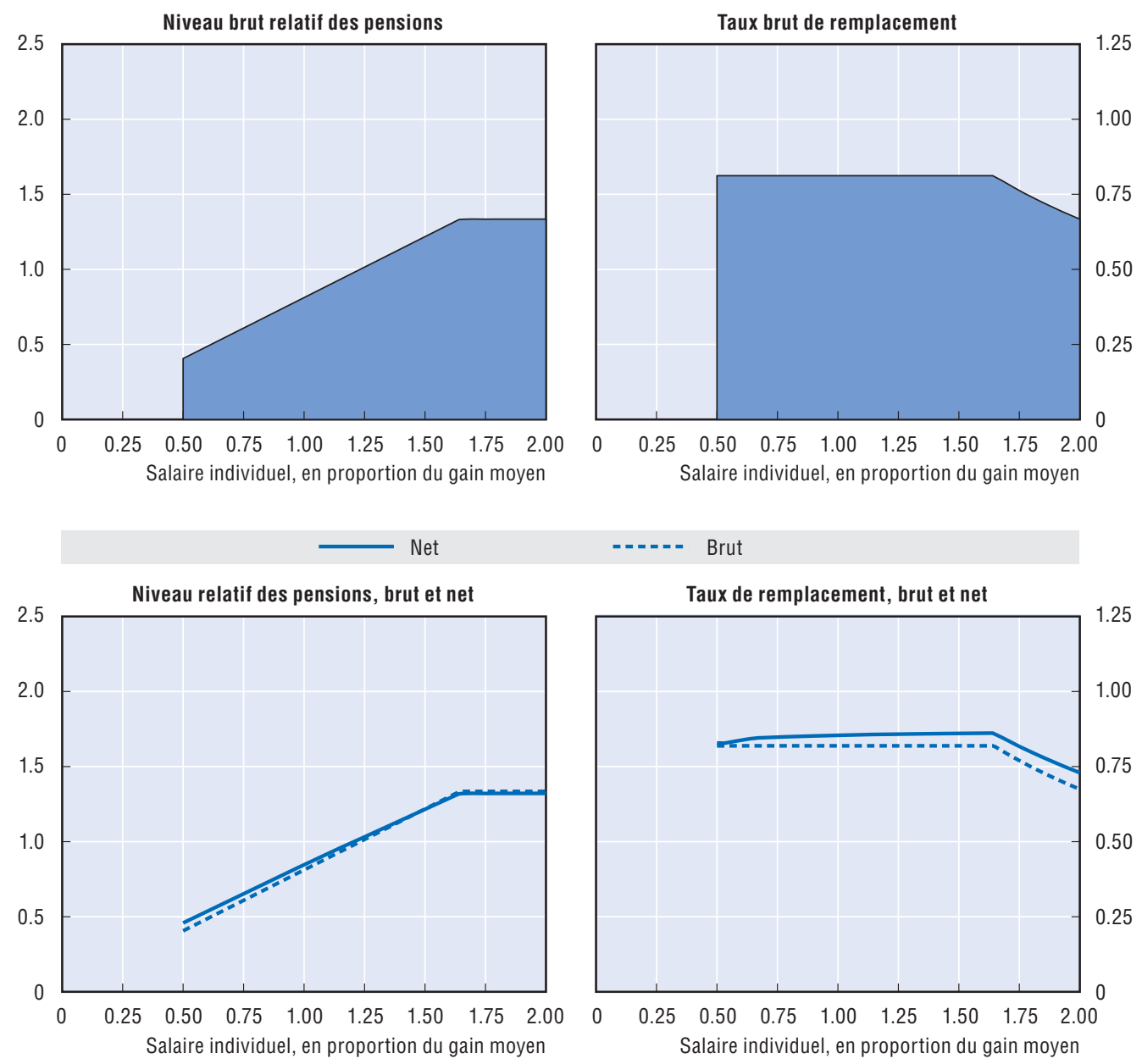

\begin{tabular}{|c|c|c|c|c|c|c|}
\hline \multirow{2}{*}{$\begin{array}{l}\text { Hommes } \\
\text { Femmes (si différent) }\end{array}$} & \multirow{2}{*}{$\begin{array}{l}\text { Salarié à revenu } \\
\text { médian }\end{array}$} & \multicolumn{5}{|c|}{ Salaire individuel, en multiple du salaire moyen } \\
\hline & & 0.5 & 0.75 & 1 & 1.5 & 2 \\
\hline Niveau brut relatif des pensions & 64.9 & 40.6 & 60.9 & 81.2 & 121.8 & 133.5 \\
\hline \multicolumn{7}{|l|}{ (\% salaire moyen brut) } \\
\hline Niveau net relatif des pensions & 69.1 & 45.9 & 65.3 & 84.7 & 121.6 & 132.1 \\
\hline \multicolumn{7}{|l|}{ (\% salaire moyen net) } \\
\hline Taux brut de remplacement & 81.2 & 81.2 & 81.2 & 81.2 & 81.2 & 66.7 \\
\hline \multicolumn{7}{|l|}{ (\% salaire individuel brut) } \\
\hline Taux net de remplacement & 84.2 & 82.1 & 84.1 & 84.7 & 85.3 & 72.2 \\
\hline \multicolumn{7}{|l|}{ (\% salaire individuel net) } \\
\hline Patrimoine retraite brut & 12.2 & 12.2 & 12.2 & 12.2 & 12.2 & 10.0 \\
\hline (multiple du salaire individuel brut) & 14.3 & 14.3 & 14.3 & 14.3 & 14.3 & 11.7 \\
\hline Patrimoine retraite net & 10.3 & 10.9 & 10.4 & 10.1 & 9.7 & 7.9 \\
\hline (multiple du salaire individuel brut) & 12.1 & 12.8 & 12.2 & 11.8 & 11.3 & 9.2 \\
\hline
\end{tabular}




\section{États-Unis}

\section{États-Unis : système de retraite en 2006}

Le régime public de retraite, connu sous le nom de "social security", comporte une formule progressive de calcul des prestations. À cela s'ajoute un paiement complémentaire sous conditions de ressources pour les retraités à faible revenu.
Indicateurs essentiels

\begin{tabular}{llrr}
\hline & & États-Unis & OCDE \\
\hline Salaire moyen & USD & 39400 & 35800 \\
& USD & 39400 & 35800 \\
Dépenses publiques & $\%$ du PIB & 6.0 & 7.2 \\
au titre des retraites & & & \\
Espérance de vie & à la naissance & 77.8 & 78.9 \\
Population de plus de 65 ans & à 65 ans & 83.6 & 83.4 \\
& \% de la population & 20.8 & 23.8
\end{tabular}

\section{Conditions d'ouverture des droits}

L'âge normal de la retraite (NRA pour Normal Retirement Age) est de 66 ans en 2006, et sera progressivement porté à 67 ans. L'admissibilité aux prestations de retraite dépend du nombre d'années de cotisation avec un minimum requis de dix ans. Un départ en retraite anticipée avec des prestations réduites est possible à partir de 62 ans.

\section{Calcul des prestations}

\section{Régime lié à la rémunération}

La formule de calcul des prestations est progressive. Pour les premiers 656 USD par mois ouvrant droit à pension, le taux de remplacement est de $90 \%$. Il est de $32 \%$ pour la tranche comprise entre 656 USD et 3955 USD par mois. Ces seuils correspondent respectivement à $20 \%$ et $121 \%$ du salaire moyen. Entre ce dernier seuil et le plafond de rémunération, le taux de remplacement est de $15 \%$. Une majoration de $50 \%$ pour personne à charge s'applique aux couples mariés dans lesquels le deuxième apporteur de revenus a accumulé des droits moindres et où l'enfant à charge remplit les conditions requises.

Les rémunérations des années antérieures sont revalorisées en fonction de la progression du salaire moyen à l'échelle nationale jusqu'aux 60 ans du bénéficiaire. Ensuite, elles ne sont plus revalorisées. La prestation de base est calculée pour une liquidation de la retraite à 62 ans. Par la suite, elle est ajustée en fonction de l'évolution des prix. La prestation est basée sur le salaire moyen des 35 meilleures années (après revalorisation), y compris les années de rémunération nulle si elles sont nécessaires à l'obtention d'un total de 35 ans.

Le plafond de rémunération pour les cotisations comme pour les prestations est de 94200 USD par an, ce qui correspond à $239 \%$ du salaire moyen revalorisé tous les ans sur la base de la progression des salaires à l'échelle nationale.

Les pensions mises en paiement sont ajustées sur la hausse des prix.

\section{Pension minimum}

Il existe une pension minimum dans le cadre du régime de social security. Les personnes dont la rémunération est inférieure à un montant minimum spécifique de 
l'assurance primaire perçoivent une pension minimum qui dépend du nombre total d'années de couverture sur l'ensemble de la carrière et varie entre 33 USD pour 11 ans et 683 USD pour 30 ans de couverture. Le seuil déclenchant le versement de cette pension minimum était en 2006 de 10485 USD, soit $27 \%$ du salaire moyen. (Selon la définition officielle, ce seuil est de $15 \%$ de la base de calcul des cotisations et des prestations dans l'ancien système.) La pension minimum n'a pas d'incidence sur les résultats de la modélisation car la fourchette de rémunération affectée est inférieure à celle présentée.

\section{Régime ciblé}

Les États-Unis accordent aux personnes âgées une prestation sous conditions de ressources appelée revenu complémentaire de sécurité ${ }^{1}$. Les personnes sans conjoint admissible à ces prestations de plus de 65 ans peuvent percevoir jusqu'à 7236 USD par an selon leur patrimoine et autres revenus. Lorsque les deux membres d'un couple ont droit à cette prestation, son montant est de 10848 USD (soit $33 \%$ de plus que pour une personne seule). Ces montants équivalent respectivement à environ $18 \%$ et $28 \%$ du salaire moyen national. La prestation est indexée sur les prix.

Les conditions de patrimoine sont strictes: le plafond d'actifs est de 2000 USD pour les personnes sans conjoint ayant droit à la prestation et de 300 USD pour les couples ayant droit à la prestation, à l'exclusion des effets personnels, de la résidence principale, d'une voiture, d'une assurance pour frais d'obsèques et d'une assurance vie (pour ces deux assurances, la valeur maximum est de 1500 USD). Une franchise minime (20 USD par mois) n'est pas prise en compte dans le calcul des droits à prestations. Au-delà de ce plafond, la prestation est dégressive au taux de $100 \%$ de l'excédent de revenu.

L'analyse est compliquée par le fait que les États peuvent compléter le minimum fixé au niveau fédéral. Si 8 États ne versent que le minimum fédéral, 29 administrent leur propre système, neuf proposent des compléments gérés par la seule administration fédérale de la sécurité sociale (Social Security Administration, SSA) et six proposent des compléments administrés à la fois par l'État et par la SSA. Le paiement complémentaire moyen dans les 15 États où la prestation est administrée par la SSA est de $29 \%$ de la prestation fédérale maximum pour une personne seule et de $50 \%$ pour un couple ${ }^{2}$. On notera que la modélisation ne prend pas en compte ces paiements complémentaires.

\section{Pensions privées facultatives}

Environ $46 \%$ des salariés adhèrent à un régime professionnel de retraite et environ $35 \%$ ont des plans individuels. Du fait que certains ont les deux types de régime, la couverture globale des pensions privées facultatives est de $58 \%$. Les plans à cotisations définies sont devenus beaucoup plus courants. Des indications donnent à penser que les taux de cotisation moyens (salarié plus employeur) sont d'environ $9 \%$ du salaire.

Une minorité de travailleurs continuent d'avoir des plans professionnels à prestations définies. Se fondant sur les résultats d'une étude nationale de ces régimes, la modélisation suppose un taux d'acquisition des droits de $1 \%$ du salaire par année d'activité.

\section{Variantes de carrière}

\section{Retraite anticipée}

Un départ à la retraite anticipée est possible à partir de 62 ans avec une réduction actuarielle. Pour chaque année d'anticipation par rapport à l'âge normal, la prestation est 
réduite de $62 / 3 \%$. Toutefois, au bout de trois ans, la réduction tombe à $5 \%$. Cela s'applique aux retraités pour lesquels l'âge normal de la retraite (NRA) est supérieur à 65 ans. Pour les personnes devenant admissibles à la retraite à 62 ans en 2006, l'âge normal de la retraite est de 66 ans. Il sera progressivement porté à 67 ans pour les personnes devenant admissibles à la retraite en 2022.

\section{Retraite différée}

Le versement initial de la pension peut être reporté après le NRA, et un crédit pour report est accordé jusqu'à 70 ans. La surcote actuarielle pour 2006 est de $7.5 \%$ par année de report. Elle passera à $8 \%$ en 2008.

Le cumul travail-retraite est également possible mais il est soumis à une condition de revenu. Pour les bénéficiaires qui n'ont pas l'âge du NRA, la pension subit une décote de $50 \%$ de l'excédent de salaire au-delà de 12480 USD. Pour les travailleurs ayant atteint leur NRA, il n'y a pas de décote fondée sur la rémunération.

\section{Enfants}

Les périodes d'interruption de la vie professionnelle liées aux enfants ne sont pas validées (excepté pour les travailleurs devenus invalides à un âge précoce, qui peuvent omettre ces années du calcul de leurs prestations).

\section{Chômage}

Les périodes de chômage ne sont pas validées. Mais, dans bien des cas, elles peuvent être omises du calcul de la rémunération pour l'établissement des prestations car seul est pris en considération le salaire des 35 meilleures années. Les périodes d'invalidité ne sont pas prises en compte dans les 35 années considérées.

\section{Notes}

1. Les personnes aveugles ou handicapées admissibles à ces prestations peuvent également recevoir le Supplemental Security Income.

2. À l'exclusion de ceux classés dans les aveugles ou les handicapés. 


\section{Résultats de la modélisation des retraites : Etats-Unis}
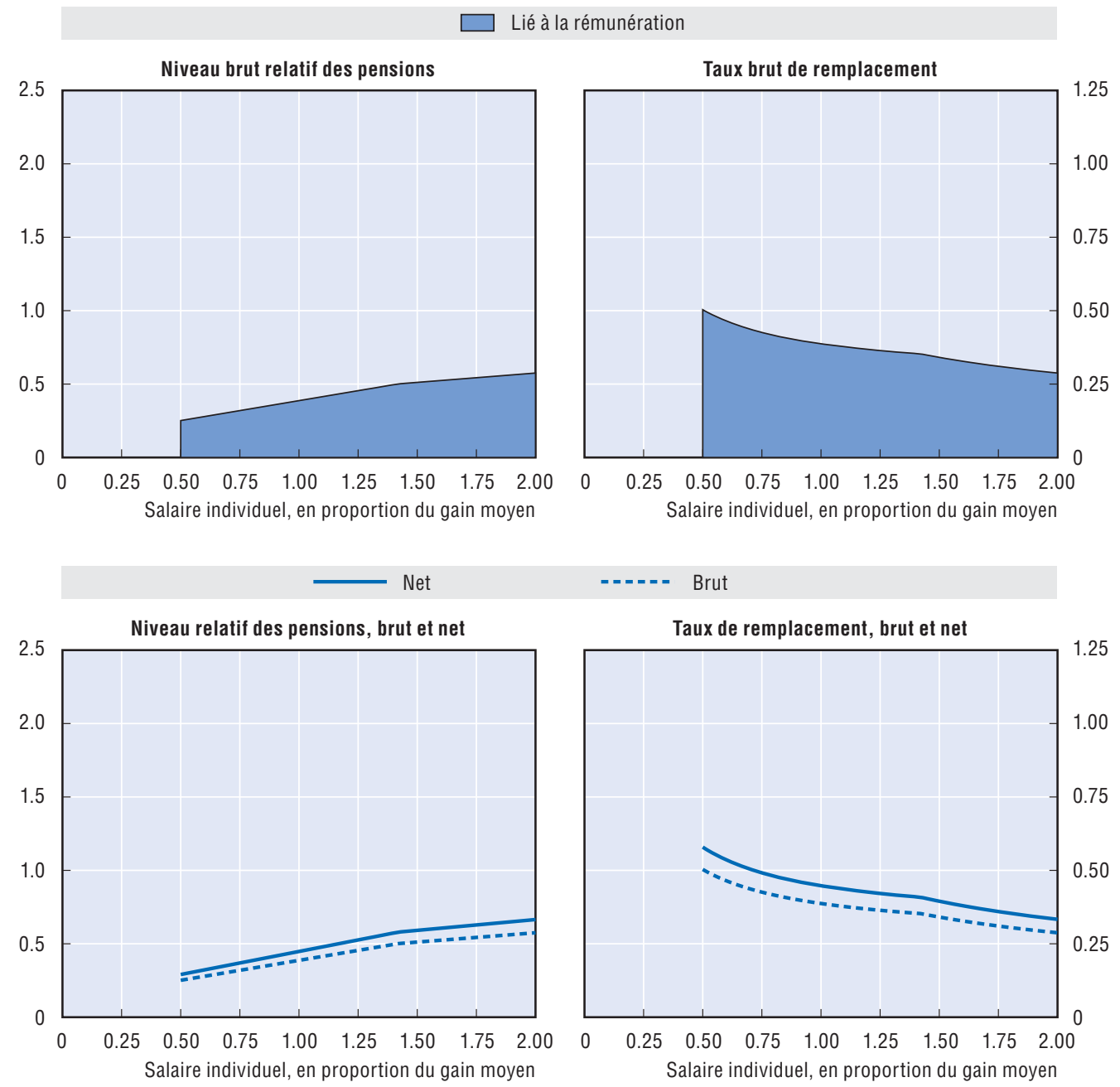

\begin{tabular}{|c|c|c|c|c|c|c|}
\hline \multirow{2}{*}{$\begin{array}{l}\text { Hommes } \\
\text { Femmes (si différent) }\end{array}$} & \multirow{2}{*}{$\begin{array}{l}\text { Salarié à revenu } \\
\text { médian }\end{array}$} & \multicolumn{5}{|c|}{ Salaire individuel, en multiple du salaire moyen } \\
\hline & & 0.5 & 0.75 & 1 & 1.5 & 2 \\
\hline Niveau brut relatif des pensions & 34.7 & 25.2 & 31.9 & 38.7 & 51.2 & 57.5 \\
\hline \multicolumn{7}{|l|}{ (\% salaire moyen brut) } \\
\hline Niveau net relatif des pensions & 40.1 & 29.1 & 36.9 & 44.8 & 59.2 & 66.5 \\
\hline \multicolumn{7}{|l|}{ (\% salaire moyen net) } \\
\hline Taux brut de remplacement & 40.8 & 50.3 & 42.6 & 38.7 & 34.1 & 28.8 \\
\hline \multicolumn{7}{|l|}{ (\% salaire individuel brut) } \\
\hline Taux net de remplacement & 47.1 & 57.9 & 49.2 & 44.8 & 39.5 & 33.3 \\
\hline \multicolumn{7}{|l|}{ (\% salaire individuel net) } \\
\hline Patrimoine retraite brut & 5.8 & 7.2 & 6.1 & 5.5 & 4.9 & 4.1 \\
\hline (multiple du salaire individuel brut) & 6.8 & 8.3 & 7.1 & 6.4 & 5.7 & 4.8 \\
\hline Patrimoine retraite net & 5.8 & 7.2 & 6.1 & 5.5 & 4.9 & 4.1 \\
\hline (multiple du salaire individuel brut) & 6.8 & 8.3 & 7.1 & 6.4 & 5.7 & 4.8 \\
\hline
\end{tabular}




\section{Finlande}

\section{Finlande : système de retraite en 2006}

Le régime finlandais de retraite public est à deux piliers : une pension nationale de base, soumise à une condition de revenu et une série de régimes obligatoires liés à la rémunération, avec des règles très similaires pour les différents groupes. Les régimes des salariés du secteur privé sont pour partie des regimes par capitalisation anticipée tandis que les régimes du secteur public sont des régimes par répartition (avec des fonds de régulation pour lisser les augmentations futures des cotisations de retraite). Une réforme majeure des retraites a été mise en oeuvre en Finlande en 2005 et toutes les règles présentées ici la prennent en compte.

\section{Indicateurs essentiels}

\begin{tabular}{llrr}
\hline & & Finlande & OCDE \\
\hline Salaire & EUR & 33500 & 28600 \\
moyen & USD & 42100 & 35800 \\
Dépenses publiques & $\%$ du PIB & 8.4 & 7.2 \\
au titre des retraites & & & \\
Espérance de vie & à la naissance & 79.5 & 78.9 \\
& à 65 ans & 84.1 & 83.4 \\
Population de plus de 65 ans & \% de la population & 26.9 & 23.8 \\
& d'âge actif & & \\
\hline
\end{tabular}

\section{Conditions d'ouverture des droits}

La pension nationale de base est soumise à une condition de résidence (mais à aucune exigence de cotisation) ; elle est dégressive en fonction du revenu des pensions des régimes liés à la rémunération. La pension nationale de vieillesse est payable à partir de 65 ans. Quarante années de résidence dans le pays (à l'âge adulte) sont nécessaires pour bénéficier de cette pension à taux plein ; des ajustements au pro rata sont opérés pour des durées de résidence plus courtes. Il est possible de partir en retraite anticipée entre 62 et 65 ans (la pension de retraite anticipée peut être perçue à partir du début du mois suivant le $62^{\mathrm{e}}$ anniversaire).

À partir de 2005, l'âge de la retraite est flexible dans le régime lié à la rémunération : entre 63 et 68 ans (autrement dit, il inclut le mois du $68^{\mathrm{e}}$ anniversaire). Il est possible de partir en retraite anticipée entre 62 et 63 ans et la pension de vieillesse peut être différée au-delà de 68 ans. Il n'y a pas de délai d'attente ou de limites (euros) pour acquérir un droit à pension dans le régime lié à la rémunération, même s'il y a un niveau minimum de rémunération pour l'assurance retraite. Les droits à pension ne sont acquis qu'à partir de 18 ans.

\section{Calcul des prestations}

\section{Régime lié à la rémunération}

Parmi les différents régimes liés à la rémunération, le régime des salariés du privé (TEL) est couvert par ces dispositions.

À partir de 2005, le taux d'acquisition des droits est de $1.5 \%$ de la rémunération ouvrant droit à pension entre 18 et 52 ans, de $1.9 \%$ entre 53 et 62 ans et de $4.5 \%$ entre 63 et 
67 ans. Pour un travailleur ayant commencé à travailler à 20 ans et ayant fait une carrière complète jusqu'à son départ à la retraite à 65 ans, le taux d'acquisition des droits sur l'ensemble de sa vie active sera de $77.5 \%$ de la rémunération ouvrant droit à pension (si celle-ci est supposée demeurer constante sur l'ensemble de la carrière).

À partir de 2005, la rémunération ouvrant droit à pension sera calculée sur la base du salaire moyen sur l'ensemble de la carrière. Toutefois, comme l'acquisition des droits à pension est différente selon le groupe d'âge (voir ci-dessus), les gains perçus par les travailleurs seniors pèsent davantage dans la pension totale. Lorsqu'on calcule la rémunération ouvrant droit à pension, le montant des cotisations de retraite du salarié est déduit de ses gains. En 2006, le taux de cotisation était de $4.3 \%$ pour les salariés de moins de 53 ans et de $5.4 \%$ pour les salariés de 53 ans et plus. On notera toutefois que les taux de remplacement indiqués se rapportent à la rémunération brute totale (pour les comparaisons avec les autres pays) plutôt qu'à cette mesure de la rémunération ouvrant droit à pension.

Les gains des années antérieures sont revalorisés selon une formule mixte associant les salaires et les prix à l'échelle nationale. À partir de 2005, le poids de la hausse des salaires est de $80 \%$ et celui de la hausse des prix de $20 \%$. Dans les hypothèses de base pour la hausse des prix et des salaires, cette politique ramène la valeur de la pension à $91.5 \%$ par rapport à une politique qui valoriserait les gains des années antérieures sur la base de la rémunération totale. Après le départ à la retraite, la pension liée à la rémunération est revalorisée à l'aide d'une formule dans laquelle la hausse des salaires intervient pour $20 \%$ et celle des prix pour $80 \%$.

À partir de 2010, les pensions nouvellement liquidées seront réduites en fonction de l'augmentation de l'espérance de vie à partir de 2009. (Les calculs utilisent des chiffres de mortalité décalés : pour 2010, par exemple, les chiffres correspondent à la moyenne de la période 2004-08 tandis que pour l'année de référence ils correspondent à la période 2003-07.) Entre 2002 et 2040, les projections Nations Unies/Banque mondiale de la mortalité impliquent un allongement de l'espérance de vie à 65 ans de 16.8 à 20.4 ans (calculé à partir des taux de mortalité unisexe). L'ajustement prend la forme d'un calcul de rente utilisant un taux d'actualisation de $2 \%$ par an. L'ajustement attendu à l'horizon 2040, sur la base des projections de mortalité, devrait ramener les prestations à $83.1 \%$ de leur montant avant la réforme. Le coefficient d'espérance de vie est calculé pour chaque cohorte à l'âge de 62 ans.

Il n'y a ni plancher de cotisation ni plafonnement des cotisations ou des rémunérations ouvrant droit à pension, ce qui signifie qu'il n'y a pas non plus de plafonnement des pensions. Il existe cependant un seuil de rémunération pour l'assurance retraite.

Le Centre finlandais des retraites coordonne les différents régimes de sorte qu'un seul paiement est effectué même pour les personnes ayant cotisé à différents plans de retraite.

\section{Régime ciblé (pension nationale)}

Les paramètres du système diffèrent d'un groupe de municipalités à l'autre pour refléter les disparités régionales du coût de la vie. En 2006, la prestation mensuelle de base à taux plein était pour une personne seule de 510.80 EUR jusqu'en septembre et ensuite de 515.86 EUR dans le premier groupe de municipalités et de 489.85/494.91 EUR dans le second (soit environ un cinquième du salaire moyen). Pour les couples, les montants correspondants étaient de 450.29/455.34 EUR et de 432.44/437.50 EUR par mois. La pension 
est réduite de $50 \%$ de la différence entre les revenus d'autres pensions et un montant minime de franchise, qui était en 2006 de 567 EUR par an. Elle devient nulle lorsque les revenus d'autres pensions excède un montant compris entre 1 046.08/1 056.17 EUR ou $1004.17 / 1$ 014.25 EUR par mois (selon la municipalité) pour une personne seule. On notera que la modélisation retient la valeur la plus élevée pour la pension nationale.

À partir de 2005, les droits à pension liés à la rémunération (l'emploi) acquis après 63 ans ne seront pas pris en compte dans le calcul des droits à la pension nationale.

La retraite de base, les paramètres de la condition de revenu et les pensions à verser sont revalorisés chaque année en fonction de l'évolution des prix. En pratique, des augmentations supplémentaires ont été opérées sur la base de décisions séparées.

\section{Variantes de carrière}

Pour les carrières interrompues, on utilise un salaire de base pour le calcul de la pension correspondant aux périodes non rémunérées. Si l'acquisition des droits à pension repose sur le salaire sur la base duquel est calculée la prestation, il n'y a pas de déduction de la cotisation de retraite (voir ci-dessus le paragraphe Régime lié à la rémunération). Habituellement, le montant correspondant a déjà été déduit lorsqu'on calcule le salaire pour établir la prestation.

\section{Retraite anticipée}

Une pension nationale de retraite anticipée est disponible à compter du début du mois suivant le $62^{\mathrm{e}}$ anniversaire. Son montant subit une décote permanente (par rapport à la pension de vieillesse ordinaire) de $0.4 \%$ pour chaque mois de pension payé avant l'âge normal de 65 ans. La pension ne passera à son niveau normal que lorsque le bénéficiaire atteindra 65 ans. Ces règles s'appliquent depuis 2005.

Un départ en retraite anticipée est possible à 62 ans dans le régime lié à la rémunération mais avec une réduction de la prestation de $0.6 \%$ par mois d'anticipation jusqu'à 63 ans. Après 63 ans, il n'y a pas de décote mais la constitution des droits à prestations liés à la rémunération est plus rapide (voir ci-dessus).

\section{Retraite différée}

À partir de 2004, la pension nationale peut être reportée après 65 ans ; la pension est alors majorée de $0.6 \%$ par mois de report.

À partir de 2005, dans le régime lié à la rémunération, la surcote pour retraite différée est réduite de $0.4 \%$ par mois ( $4.8 \%$ par an) après 68 ans. Il n'y a pas d'ajustement entre 63 et 68 ans en raison de la constitution accélérée des droits à pension aux âges en quesrtion.

Il est possible de cumuler une retraite et des revenus d'activité. À partir de 2005, après liquidation de la retraite, la rémunération perçue permet d'accumuler des droits à pension supplémentaires au taux de $1.5 \%$ par an jusqu'à 68 ans.

\section{Enfants}

À partir de 2005, pendant les périodes de congé de maternité/paternité et parental, les droits à pension sont acquis sur la base de 1.17 fois le salaire pris comme de base de calcul de l'allocation familiale. La durée maximum du congé parental rémunéré est de 11 mois.

Pour les périodes de non-emploi de l'un ou l'autre des parents liées à l'éducation d'un enfant au cours desquelles une allocation de garde à domicile est demandée, les droits à 
pension s'accumulent comme si la personne percevait un salaire de 556.60 EUR par mois (2006), soit environ un cinquième du salaire moyen. Il en est ainsi jusqu'au troisième anniversaire de l'enfant.

Les personnes en congé parental n'ont pas à cotiser aux régimes de retraite. La pension constituée au titre du congé parental rémunéré est versée par le régime lié à la rémunération. L'État finance la pension au titre des périodes de congé sans solde liées aux enfants.

La partie de la pension qui se fonde sur les périodes de congé sans solde liées aux enfants (et aux études) n'est pas incluse dans la condition de revenu de la pension nationale.

\section{Chômage}

Suite à la réforme de 2005 , les indemnités de chômage liées à la rémunération constituent des droits à pension sur la base de la proportion du salaire $(75 \%)$ sur laquelle est assise la prestation. Seules les indemnités de chômage reçues avant 63 ans génèrent un crédit de retraite.

Les indemnités d'assurance chômage sont versées pendant 500 jours (environ 23 mois, d'une durée moyenne de 21.5 jours). Si un chômeur atteint l'âge de 59 ans avant la fin de cette période, il peut percevoir des indemnités de chômage liées à la rémunération jusqu'à 65 ans. (Du fait des limites d'âge, ces règles ne seront pas appliquées avant 2009.) Les personnes recevant une indemnité au-delà de ce délai de 500 jours sont en droit de choisir de faire valoir leurs droits à une pension de vieillesse à partir de 62 ans. Dans ce cas, il n'y a pas de décote pour retraite anticipée et les indemnités de chômage liées à la rémunération cessent. Après la période d'indemnités de chômage liées à la rémunération, une assistance chômage forfaitaire ou soumise à condition de revenu (sous diverses conditions) peut être demandée mais les périodes pendant lesquelles ces indemnités sont perçues ne sont pas validées pour la retraite. 


\section{Résultats de la modélisation des retraites : Finlande}

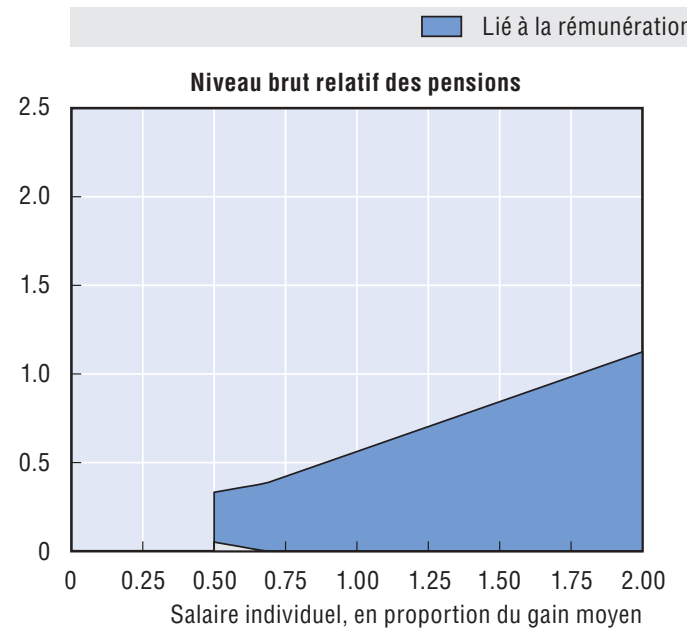

Minimum
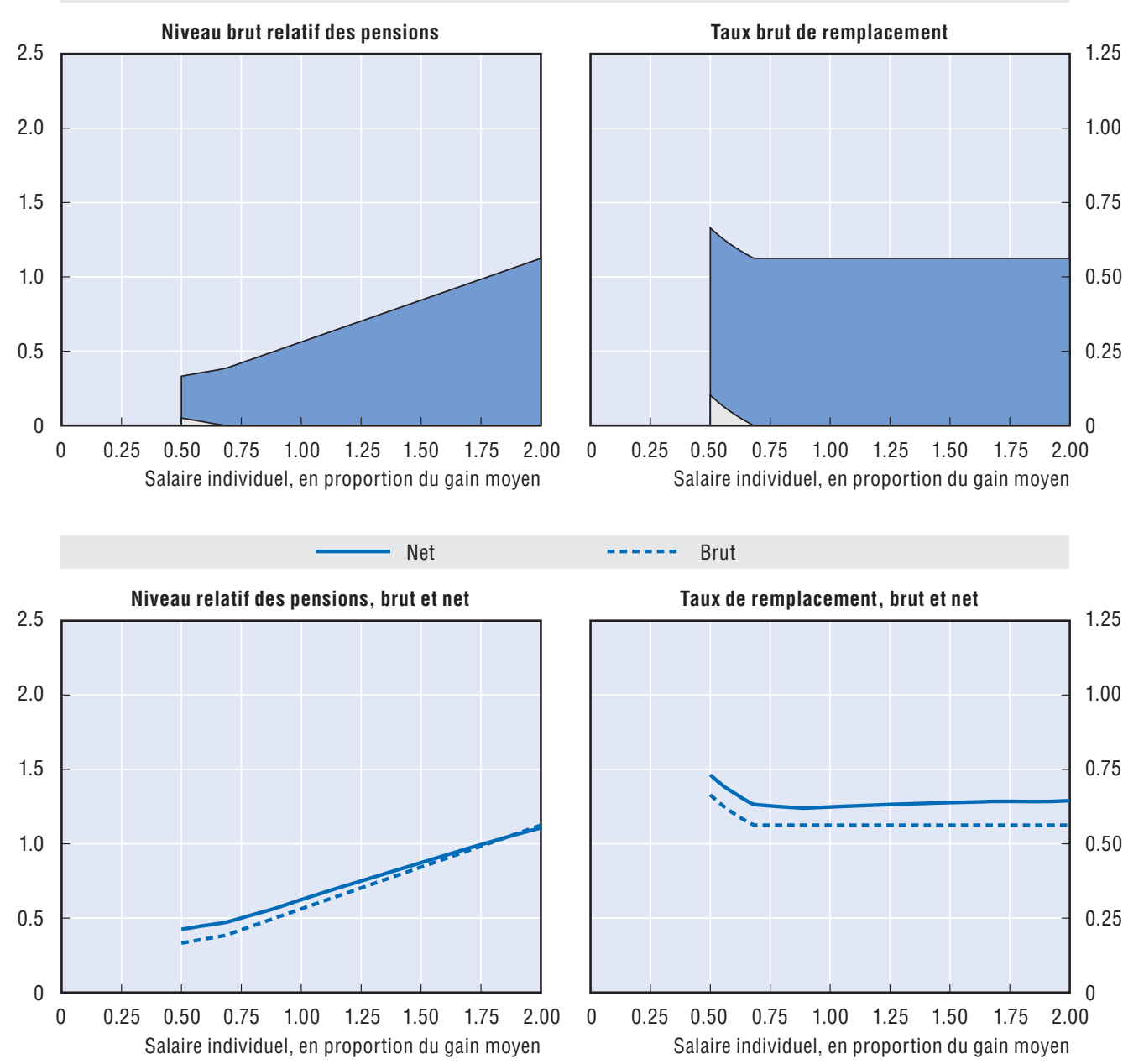

\begin{tabular}{|c|c|c|c|c|c|c|}
\hline \multirow{2}{*}{$\begin{array}{l}\text { Hommes } \\
\text { Femmes (si différent) }\end{array}$} & \multirow{2}{*}{$\begin{array}{l}\text { Salarié à revenu } \\
\text { médian }\end{array}$} & \multicolumn{5}{|c|}{ Salaire individuel, en multiple du salaire moyen } \\
\hline & & 0.5 & 0.75 & 1 & 1.5 & 2 \\
\hline Niveau brut relatif des pensions & 49.5 & 33.2 & 42.2 & 56.2 & 84.3 & 112.5 \\
\hline \multicolumn{7}{|l|}{ (\% salaire moyen brut) } \\
\hline Niveau net relatif des pensions & 56.0 & 42.5 & 50.1 & 62.4 & 87.3 & 110.7 \\
\hline \multicolumn{7}{|l|}{ (\% salaire moyen net) } \\
\hline Taux brut de remplacement & 56.2 & 66.5 & 56.2 & 56.2 & 56.2 & 56.2 \\
\hline \multicolumn{7}{|l|}{ (\% salaire individuel brut) } \\
\hline Taux net de remplacement & 62.0 & 73.2 & 62.7 & 62.4 & 63.8 & 64.5 \\
\hline \multicolumn{7}{|l|}{ (\% salaire individuel net) } \\
\hline Patrimoine retraite brut & 8.8 & 10.4 & 8.8 & 8.8 & 8.8 & 8.8 \\
\hline (multiple du salaire individuel brut) & 10.5 & 12.3 & 10.5 & 10.5 & 10.5 & 10.5 \\
\hline Patrimoine retraite net & 6.8 & 9.0 & 7.1 & 6.6 & 6.2 & 5.9 \\
\hline (multiple du salaire individuel brut) & 8.0 & 10.6 & 8.4 & 7.9 & 7.3 & 7.0 \\
\hline
\end{tabular}




\section{France}

\section{France : système de retraite en 2006}

Le système français de retraite privé repose sur deux piliers : un régime général lié à la rémunération et des plans professionnels obligatoires reposant sur un système de points. Le régime public comporte en outre une pension minimum non soumise à conditions de ressources, le " minimum contributif » et un revenu minimum pour les personnes âgées, le « minimum vieillesse ».

\section{Indicateurs essentiels}

\begin{tabular}{llrr}
\hline & & France & OCDE \\
\hline Salaire moyen & EUR & 31000 & 28600 \\
& USD & 38900 & 35800 \\
Dépenses publiques au titre & \% du PIB & 12.4 & 7.2 \\
des retraites & & 80.9 & 78.9 \\
Espérance de vie & à la naissance & 85.4 & 83.4 \\
& à 65 ans & 28.0 & 23.8 \\
\hline
\end{tabular}

\section{Conditions d'ouverture des droits}

Depuis 2003, 40 années de cotisation contre 37,5 auparavant sont exigées pour percevoir une pension du régime général à taux plein de premier niveau. Entre 2008 et 2012, il est prévu de porter progressivement ce chiffre à 41 ans (révision prévue en 2008). Après 2012, la durée minimum de cotisations pour percevoir une retraite à taux plein devrait être relevée en fonction de l'allongement de l'espérance de vie, de façon que le rapport entre la durée de versement des retraites et la durée du travail demeure constant.

L'âge normal de départ à la retraite est de 60 ans pour la pension du régime général liée à la rémunération. Le minimum contributif est versé lorsque le retraité a cotisé pendant le nombre d'années requis ou lorsqu'il a 65 ans et plus. Dans la modélisation, l'entrée sur le marché du travail intervient à 20 ans et on suppose une durée complète de cotisations (41 ans). Ces hypothèses correspondent à un âge de retraite de 61 ans.

\section{Calcul des prestations}

\section{Régime lié à la rémunération}

L'objectif du régime général est un taux de remplacement de $50 \%$ après une carrière complète ( 40 années de cotisations et plus par la suite, comme indiqué plus haut). Pour chaque trimestre manquant la pension est réduite de deux façons :

- le taux de pension est réduit de $1.25 \%$ (ce qui donne une décote par année manquante de $5 \%$; ces décotes concernent les personnes nées après 1952 ;

- le montant de la pension est, en outre, réduit au prorata $\left(0.625 \%\right.$ soit $1 / 160^{\mathrm{e}}$ par trimestre manquant).

Le salaire de référence est établi sur la base des salaires d'un certain nombre de meilleures années revalorisés en fonction de la hausse des prix. À partir de 2008, la moyenne des salaires considérés sera calculée sur 25 ans, contre 24 en 2007 et 23 en 2006.

Du fait du nombre limité d'années prises en compte dans le salaire de référence pour le calcul des prestations de retraite et de la politique de revalorisation par rapport à l'évolution des prix, le taux de remplacement est très sensible au profil des gains tout au long de la 
carrière. Compte tenu de l'hypothèse retenue ici d'un taux de croissance continu du salaire réel de $2 \%$ sur la carrière d'un travailleur, et du fait que les calculs de l'OCDE prennent comme salaire de référence les gains moyens perçus sur l'ensemble de la carrière et revalorisés, les taux de remplacement obtenus sont inférieurs à ceux que l'on obtiendrait avec la progression des salaires observée en France, pays où les augmentations se concentrent principalement sur la première moitié de la carrière. La réforme de 2003 a introduit pour 2008 et au-delà un objectif de pension équivalant au moins à $85 \%$ du salaire minimum net pour les personnes ayant perçu le salaire minimum durant toute leur carrière.

Le plafond du salaire éligible était en 2006 de 31068 EUR, soit approximativement le salaire moyen tel que le mesure l'OCDE (31 269 EUR). Les pensions mises en paiement sont indexées sur les prix.

\section{Minimum contributif}

Il existe dans le régime général une pension minimum non ciblée qui est versée indépendamment des montants reçus au titre du régime de base ou des régimes complémentaires. Pour l'année civile 2006, son montant était de 6760.82 EUR à partir de 65 ans avec au minimum un trimestre de carrière validé et de 7172.54 EUR si le bénéficiaire justifie d'au minimum 40 années de cotisation effectives, soit $23 \%$ du salaire moyen tel que le mesure l'OCDE. Pour être éligible à une pension à taux plein, il faut être âgé de 65 ans et plus et avoir cotisé pendant 40 années (chiffre qui devrait passer à 41 en 2012) ; pour des durées plus courtes, la pension minimum est calculée au pro rata. La pension minimum est indexée sur les prix.

\section{Régimes professionnels obligatoires}

La majorité des salariés du secteur privé sont couverts par le régime AARCO. Les règles qui s'appliquent aux cadres (c'est-à-dire aux personnes occupant des postes de spécialistes ou d'encadrement) au titre du régime AGIRC sont différentes; les règles décrites ci-dessous s'appliquent aux non-cadres.

Bien que les cotisations effectives soient plus élevées, en dessous du plafond $d u$ régime général, les droits à prestations ne se constituent que sur $6 \%$ du salaire. Entre une et trois fois ce plafond, ils se constituent sur $16 \%$ du salaire. Ainsi le plafond de l'ARRCO est égal à trois fois le plafond du régime général : 93204 EUR. (On notera qu'il n'y a pas de plafond dans le régime AGIRC pour les cadres.)

Le nombre de points acquis chaque année est égal au montant des cotisations divisé par le coût d'un point de retraite. Lors du départ à la retraite, le nombre de points accumulés est multiplié par la valeur du point pour obtenir le montant de la pension. La valeur du point était de 1.1104 EUR entre avril 2005 et avril 2006 et de 1.1287 EUR à partir d'avril 2006, ce qui donne pour l'année civile 2006 le chiffre de 1.1241 EUR. Le coût du point pour cette même année 2006 était de 13.027 EUR.

La revalorisation du coût et de la valeur des points de retraite est négociée entre les partenaires sociaux. Selon l'accord en vigueur, valable jusqu'en 2008, l'augmentation du coût des points de retraite est alignée sur les salaires et l'augmentation de sa valeur est alignée sur les prix. Dans la modélisation, on suppose le maintien de ce différentiel de revalorisation entre le coût et la valeur du point. Là encore, cette politique de revalorisation effective des droits acquis au cours des années antérieures sur les prix aboutit à des prestations inférieures à ce qu'elles seraient avec une revalorisation sur la base des 
salaires. Pour les hypothèses de base, la réduction est ici de $69 \%$ des droits à pension acquis avec une revalorisation sur la base des salaires.

Il est important de noter que la politique d'actualisation de ces deux paramètres affecte à la fois l'évolution des pensions mises en paiement (appelée ici « indexation ") et la variation de la valeur des droits à pension entre le moment où ils ont été acquis et celui où ils sont liquidés (comme pour le processus de « revalorisation » dans les régimes liés à la rémunération).

\section{Régime ciblé : le minimum vieillesse}

Pour les personnes de 65 ans, il existe un revenu minimum soumis à conditions de ressources, qui était en 2006 de 7323.48 EUR par an pour une personne seule et de 13137.69 EUR pour un couple. Cette prestation, qui correspond à $23 \%$ du salaire moyen, tel que le mesure l'OCDE, est ajustée sur l'évolution des prix. Les travailleurs ayant fait une carrière complète sont rarement éligibles au minimum vieillesse dans la mesure où la retraite professionnelle obligatoire complète la prestation du régime général.

\section{Variantes de carrière}

\section{Retraite anticipée}

La préretraite relève d'un régime distinct administré par le Fonds national pour l'emploi (FNE). Un départ en retraite anticipée est possible à partir de 57 ans, et de 56 ans dans certaines circonstances liées aux conditions de travail. Le taux de remplacement est d'environ $80 \%$. À l'âge normal de la retraite (ou à l'âge auquel les travailleurs peuvent prétendre à une pension de vieillesse normale à taux plein jusqu'à 65 ans), les intéressés passent sous le régime général. La période de perception de prestations du FNE est entièrement validée pour le régime général.

Le départ en retraite anticipée (c'est-à-dire avant 60 ans) est autorisé dans le régime général dans les conditions suivantes:

- à 56 ans pour les personnes ayant commencé à travailler avant l'âge de 16 ans et justifiant d'au minimum 42 années validées, dont au moins 42 de cotisation effective ;

- à 58 ans pour les personnes ayant commencé à travailler avant l'âge de 16 ans et justifiant d'au minimum 42 années validées, dont au moins 41 de cotisation effective ;

- à 59 ans pour les personnes ayant commencé à travailler avant l'âge de 17 ans et justifiant d'au minimum 42 années validées, dont au moins 40 de cotisation effective.

Dans le cadre des plans professionnels, un départ en retraite anticipée est également possible, souvent moyennant des réductions en fonction de l'âge de départ à la retraite, du nombre d'années de cotisation ou les deux. Le départ à la retraite est possible à 60 ans sans réduction avec 40 années de cotisation. Avec moins de 40 années, la pension est ajustée comme indiqué dans le tableau, selon la modalité la plus favorable entre l'âge de départ à la retraite et le nombre d'années manquantes. Pour un départ à 61 ans, par exemple, la pension est ramenée à $83 \%$ du taux plein. Toutefois, si la personne part à 61 ans avec 39 années de cotisation, elle n'est ramenée qu'à 96 \% du fait qu'il n'y a qu'une année manquante.

\begin{tabular}{lrrrrrrrrrr}
\hline $\begin{array}{l}\text { Age de départ à la retraite } \\
\text { Années manquantes }\end{array}$ & 55 & 56 & 57 & 58 & 59 & 60 & 61 & 62 & 63 & 64 \\
\begin{tabular}{l} 
Coefficient \\
\hline
\end{tabular} & 0.43 & 0.50 & 0.57 & 0.64 & 0.71 & 0.78 & 0.83 & 0.88 & 0.92 & 0.96 \\
\hline
\end{tabular}




\section{Retraite différée}

Pour les personnes qui travaillent après 60 ans et remplissent les conditions d'ouverture des droits à une pension à taux plein (40 années de cotisation), chaque année supplémentaire augmente la prestation du régime général de $3 \%$. À partir de 2007, cette incitation à différer l'âge de départ à la retraite augmente : elle passe à $4 \%$ par an à partir de la deuxième année de report et à $5 \%$ par an à partir de 65 ans. Pendant la durée du report, les personnes continuent à accumuler des points ARRCO. Le cumul de la retraite et d'un travail peut être autorisé dans certaines limites et à condition que les personnes quittent leur emploi antérieur.

\section{Enfants}

Une mère ayant élevé un enfant pendant au moins neuf ans (avant son $16^{\mathrm{e}}$ anniversaire) est créditée de deux années de cotisation par enfant dans le régime général, qu'elle ait ou non continué à travailler pendant ces années, sous réserve de ne pas avoir une durée de cotisation complète. Dans le régime général, les deux parents peuvent bénéficier d'une majoration de $10 \%$ de leur retraite finale s'ils ont élevé trois enfants et plus pendant au moins neuf ans (avant le $16^{\mathrm{e}}$ anniversaire des enfants).

Les périodes de non emploi ou de travail à temps partiel pour élever un enfant de moins de 3 ans sont également validées dans le régime général et les régimes professionnels. Des crédits sont attribués comme si le parent avait touché le salaire minimum. Le maximum de trois ans s'applique pour les deux premiers enfants : pour les suivants les périodes validées sont plus longues. Pour être éligibles à ces crédits, les parents doivent avoir droit aux prestations familiales et leur rémunération doit être inférieure aux seuils (17 600 EUR pour le premier enfant avec une majoration de $30 \%$ pour les suivants). Ce crédit se cumule avec les deux années par enfant validées dans le régime général.

\section{Chômage}

Les périodes de chômage involontaire sont entièrement validées pour le calcul de la pension du régime général lorsque les personnes sont allocataires du chômage notamment en vertu des programmes suivants : allocation unique dégressive, allocation chômeurs âgés, allocation formation reclassement, allocation de solidarité spécifique, allocation spécifique d'attente et allocation d'insertion. Pour chaque période de 50 jours de chômage sur une année, un quart des cotisations est attribué (avec un maximum de quatre trimestres par an). Ces périodes entrent dans le calcul de la pension en supposant une rémunération de 1438 EUR par trimestre ou de 5752 EUR par an.

La première période de chômage non indemnisé est également validée à concurrence d'une durée maximum d'un an. Les périodes ultérieures de chômage involontaire non indemnisé sont créditées à concurrence d'un maximum d'un an uniquement si elles font suite à une période de chômage indemnisé. En revanche, les périodes pendant lesquelles les personnes reçoivent une aide sociale (revenu minimum d'insertion) ne sont pas validées. 
Résultats de la modélisation des retraites : France

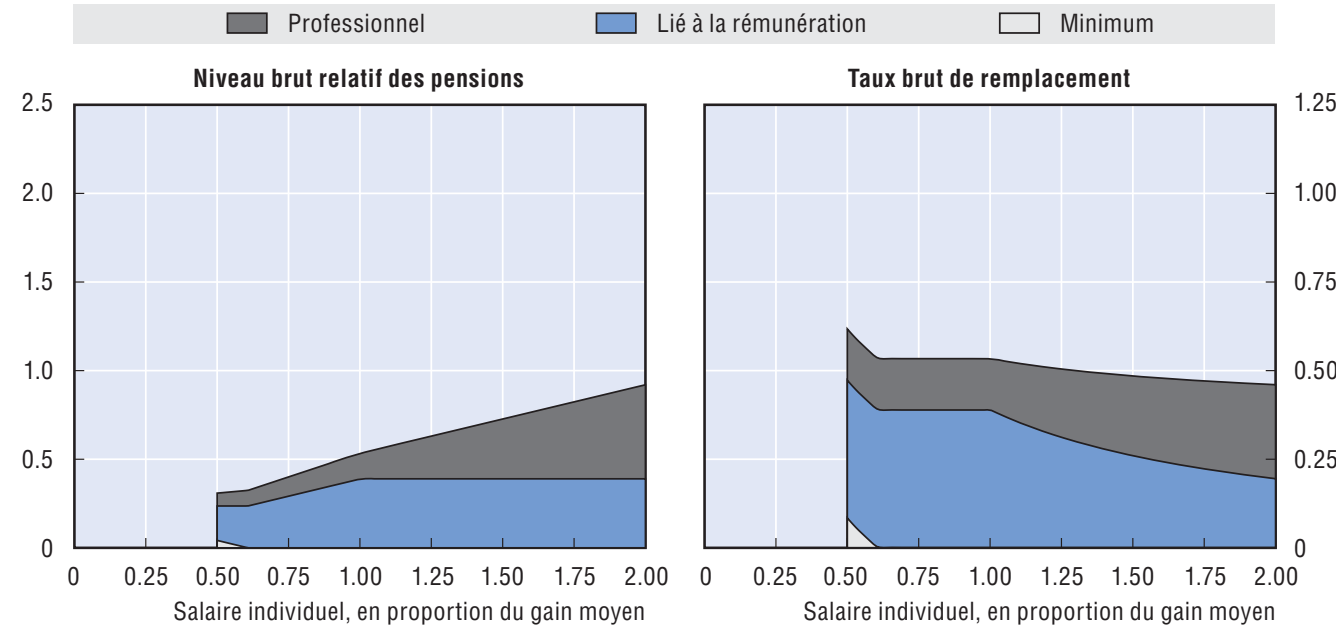

Net

$0-\ldots$ Brut
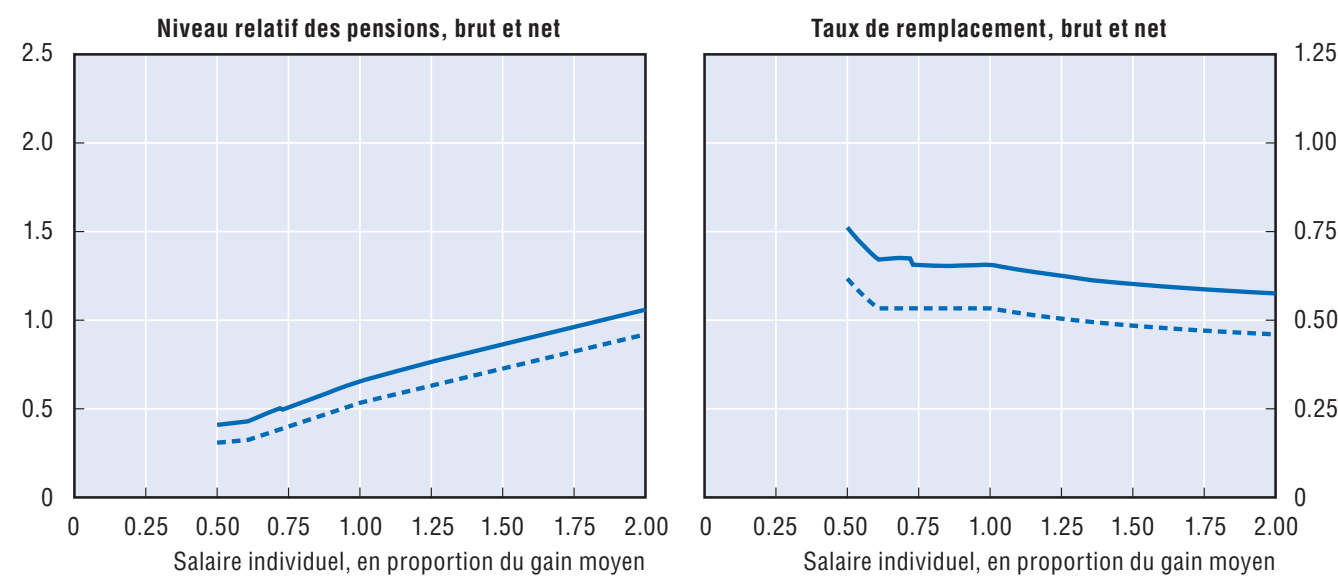

\begin{tabular}{|c|c|c|c|c|c|c|}
\hline \multirow{2}{*}{$\begin{array}{l}\text { Hommes } \\
\text { Femmes (si différent) }\end{array}$} & \multirow{2}{*}{$\begin{array}{l}\text { Salarié à revenu } \\
\text { médian }\end{array}$} & \multicolumn{5}{|c|}{ Salaire individuel, en multiple du salaire moyen } \\
\hline & & 0.5 & 0.75 & 1 & 1.5 & 2 \\
\hline Niveau brut relatif des pensions & 45.3 & 30.9 & 40.0 & 53.3 & 72.7 & 92.0 \\
\hline \multicolumn{7}{|l|}{ (\% salaire moyen brut) } \\
\hline Niveau net relatif des pensions & 56.7 & 40.9 & 50.8 & 65.7 & 86.4 & 106.0 \\
\hline \multicolumn{7}{|l|}{ (\% salaire moyen net) } \\
\hline Taux brut de remplacement & 53.3 & 61.7 & 53.3 & 53.3 & 48.5 & 46.0 \\
\hline \multicolumn{7}{|l|}{ (\% salaire individuel brut) } \\
\hline Taux net de remplacement & 65.3 & 76.2 & 65.6 & 65.7 & 60.2 & 57.5 \\
\hline \multicolumn{7}{|l|}{ (\% salaire individuel net) } \\
\hline Patrimoine retraite brut & 9.3 & 10.8 & 9.3 & 9.3 & 8.5 & 8.0 \\
\hline (multiple du salaire individuel brut) & 10.8 & 12.5 & 10.8 & 10.8 & 9.8 & 9.3 \\
\hline Patrimoine retraite net & 8.3 & 10.2 & 8.4 & 8.2 & 7.1 & 6.6 \\
\hline (multiple du salaire individuel brut) & 9.6 & 11.7 & 9.7 & 9.4 & 8.2 & 7.6 \\
\hline
\end{tabular}




\section{Grèce}

\section{Grèce : système de retraite en 2006}

En Grèce, le système de retraite est un régime public lié à la rémunération à deux composantes auquel s'ajoute une série de pensions minimum/filets de sécurité sociaux.

\section{Indicateurs essentiels}

\begin{tabular}{llrr}
\hline & & Grèce & \multicolumn{1}{c}{ OCDE } \\
\hline Salaire moyens & EUR & 23000 & 28600 \\
& USD & 28900 & 35800 \\
$\begin{array}{l}\text { Dépenses publiques au titre } \\
\text { des retraites }\end{array}$ & 11.5 & 7.2 \\
Espérance de vie & à la naissance & 79.6 & 78.9 \\
& à 65 ans & 83.5 & 83.4 \\
Population de plus de 65 ans & $\%$ de la population d'âge actif & 30.0 & 23.8 \\
\hline
\end{tabular}

\section{Conditions d'ouverture des droits}

L'âge normal de la retraite est de 65 ans pour les hommes comme pour les femmes. Pour percevoir une pension à partir de cet âge, il faut un minimum de 4500 jours de cotisation (soit l'équivalent de 15 ans). Les travailleurs ayant à leur actif 11100 jours ouvrables (37 ans) de cotisation peuvent percevoir une prestation à taux plein quel que soit leur âge de départ à la retraite. Les personnes exerçant des métiers pénibles ou dangereux et les femmes ayant des enfants à charge ou des enfants handicapés bénéficient de dérogations.

La pension sociale minimum requiert 15 années de cotisation.

\section{Calcul des prestations}

\section{Régime lié à la rémunération : composante principale}

Pour les personnes entrées sur le marché du travail à partir de 1993, la pension est de $2 \%$ du salaire pour chaque année de cotisation jusqu'à 35 ans. Le taux de remplacement maximum est donc de 70 \% pour les personnes partant à la retraite à l'âge normal ou plus tôt. Toutefois, pour les personnes qui continuent de travailler après 65 ans et qui ont à leur actif 35 années de cotisation, le taux de constitution de droits est porté à $3.3 \%$ par an pour un maximum de trois ans tandis que pour les personnes travaillant au-delà de ces trois ans il est nul (taux de remplacement maximum de $80 \%$ ).

Le salaire de référence est la moyenne des cinq dernières années précédant le départ à la retraite. La revalorisation de la rémunération des années antérieures suit les augmentations définies dans la politique nationale des revenus.

La pension maximum, qui est égale à quatre fois le PNB par habitant de 1991 (montant fixe de 105.000 drachmes, qui était égal à l'époque au montant du PNB moyen par habitant), est indexée sur les augmentations annuelles des pensions en vertu de la politique des revenus. Pour 2006, ce plafond a été de 2538.28 EUR par mois. Les calculs indiquent que, pour un travailleur ayant une carrière complète, cela équivaut à plafonner les rémunérations ouvrant droit à pension à $325 \%$ du salaire moyen.

L'ajustement des retraites mises en paiement est discrétionnaire. Sur la période 1999-2004, les augmentations des retraites ont été progressives. Depuis 2005, toutes les pensions ont augmenté dans la même proportion (voir ci-dessous). Sur la période 19992001, les augmentations des retraites modestes ont été sensiblement supérieures à 
l'inflation. En 2002, en revanche, elles ont été à la traîne. Etant donné le manque de cohérence des pratiques récentes en matière d'ajustement des prestations, les calculs du patrimoine retraite sont effectués sur la base d'une indexation sur les prix.

\begin{tabular}{|c|c|c|c|c|c|c|c|c|}
\hline Année & 1999 & 2000 & 2001 & 2002 & 2003 & 2004 & 2005 & 2006 \\
\hline Inflation & $2.60 \%$ & $3.20 \%$ & $3.40 \%$ & $3.60 \%$ & $3.00 \%$ & $2.90 \%$ & $3.50 \%$ & $2.90 \%$ \\
\hline Augmentations & $\begin{array}{r}3.9 \% \\
\text { (<733 EUR) }\end{array}$ & $4.00 \%$ & $\begin{array}{r}5.5 \% \\
\text { (<352 EUR) }\end{array}$ & $\begin{array}{r}3.5 \% \\
\text { (< } 400 \text { EUR) }\end{array}$ & $\begin{array}{r}4.0 \% \\
(<500 \text { EUR) }\end{array}$ & $\begin{array}{r}5.0 \% \\
(<500 \text { EUR) }\end{array}$ & $4.0 \%$ & $4.0 \%$ \\
\hline & $\begin{array}{r}3.4 \% \\
\text { (> } 733 \text { EUR) }\end{array}$ & & $\begin{array}{r}2.75 \% \\
\text { (<587 EUR) }\end{array}$ & $\begin{array}{r}1.5 \% \\
(<620 \text { EUR) }\end{array}$ & $\begin{array}{r}2.0 \% \\
(<1000 \text { EUR })\end{array}$ & $\begin{array}{r}3.0 \% \\
(<1000 \text { EUR } \\
\text { ( })\end{array}$ & & \\
\hline & & & $\begin{array}{r}1.4 \%(< \\
880 \text { EUR) }\end{array}$ & $\begin{array}{r}0.75 \% \\
\text { (<910 EUR) }\end{array}$ & $\begin{array}{r}0 \% \\
(>1000 \text { EUR) }\end{array}$ & $\begin{array}{r}0 \% \\
(1000 \text { EUR) }\end{array}$ & & \\
\hline & & & $\begin{array}{r}0 \% \\
(>880 \text { EUR) }\end{array}$ & $\begin{array}{r}0 \% \\
(>910 \text { EUR) }\end{array}$ & & & & \\
\hline
\end{tabular}

\section{Régime lié à la rémunération : composante complémentaire}

La pension complémentaire à taux plein est égale à $20 \%$ de la rémunération prise en compte dans la principale composante du régime lié à la rémunération pour les travailleurs ayant à leur actif 35 années de cotisation. Pour des durées de cotisation plus courtes, la pension est réduite au prorata, ce qui implique un taux linéaire d'acquisition de droits de $0.57 \%$. Sa valeur est augmentée de $1 / 35^{\mathrm{e}}$ par année de cotisation (300 jours) au-delà de 35 .

\section{Pension minimum}

Pour 2006, le montant de la pension minimum a été de 445.37 EUR par mois, soit $27.1 \%$ du salaire moyen. Ce montant est ajusté tous les ans dans le cadre de la politique des revenus. Le montant de la pension minimum complémentaire a été de 111.25 EUR.

\section{Régime soumis à une condition de revenu : prestation de solidarité sociale}

Ce régime, mis en place en 1996, est un régime non contributif soumis à conditions de ressources qui s'applique aux retraités à revenus modestes relevant de la plupart des régimes des retraites (autre que celui des agriculteurs).

Pour être admis à bénéficier de ce régime, désigné sous le nom d'EKAS, il faut que le revenu net total (de toutes provenances) soit inférieur à 7165.7 EUR (2006). Le revenu imposable total ne doit pas excéder 8360.00 EUR et le revenu familial imposable total ne doit pas être supérieur à 13 009.20 EUR.

\begin{tabular}{|c|c|c|c|c|c|}
\hline $\begin{array}{l}\text { Niveau de revenu, limite } \\
\text { inférieure }\end{array}$ & 0 & 6.525.91 EUR & 6781.87 EUR & 6.952.44 EUR & 7165.71 EUR \\
\hline Prestation mensuelle & 160.15 EUR & 120.11 EUR & 80.08 EUR & 40.04 EUR & 0 \\
\hline
\end{tabular}

\section{Variantes de carrière}

\section{Retraite anticipée}

Un départ en retraite anticipée est possible mais avec des réductions des prestations, comme le montre le tableau ci-dessous. L'ajustement est de $1 / 200^{\mathrm{e}}$ par mois d'anticipation, soit $6 \%$ par an. 


\begin{tabular}{ccc}
\hline Nombre d'années & Âge d'ouverture des droits & Conditions \\
\hline 15 & 65 & Sans réduction \\
15 & 60 & Avec réduction $(1 / 200)$ \\
35 & 55 & Avec réduction $(1 / 200)$ \\
37 & Tout âge & Sans réduction \\
\hline
\end{tabular}

\section{Retraite différée}

Il est possible de partir à la retraite après l'âge normal de 65 ans avec une durée de cotisations de 35 années. Un taux de constitution de droits de $3.3 \%$ est appliqué à la principale composante jusqu'à 68 ans et pour un maximum de trois années supplémentaires ; pour les personnes travaillant au-delà de ces trois ans, il est nul (taux de remplacement maximum de $80 \%$ ). La composante complémentaire continue également de constituer des droits

Il est possible de cumuler un travail et une pension de retraite dans la mesure où les personnes n'ont pas moins de 55 ans. Dans ce cas, la partie de leur pension mensuelle excédant 733 EUR est réduite de $70 \%$; il est prévu une surcote pour enfants à charge.

\section{Enfants}

Un système de crédit d'un an pour le premier enfant et de deux ans pour chacun des suivants jusqu'à un maximum de trois (à raison de 300 jours d'assurance par an) a été mis en place en 2003. L'un ou l'autre des parents peut s'en prévaloir et il ne peut être comptabilisé dans la durée minimum d'assurance requise, à savoir pour 37 années d'acquisition, 4500 jours ou 3500 jours.

\section{Chômage}

Un maximum de 200 jours peut être crédité au titre des périodes de chômage sur toute la vie active. Si la période de chômage déborde sur les cinq dernières années prises comme base de calcul de la rémunération ouvrant droit à pension, elle n'est pas prise en compte et la période retenue pour le calcul de la rémunération ouvrant droit à pension est étendue d'autant.

Les chômeurs de longue durée (c'est-à-dire les personnes cumulant un minimum de 12 mois consécutifs de chômage), âgés d'au minimum 60 ans pour les hommes et 55 ans pour les femmes (ou respectivement 55 et 50 ans dans le cas de travaux pénibles et insalubres), qui n'ont pas à leur actif un maximum de 1500 jours ouvrant droit à une pension de vieillesse et/ou une assurance retraite complémentaire servie par l'IKA-ETAM, peuvent choisir de maintenir leur assurance aussi longtemps qu'ils ne remplissent les conditions requises pour la pension minimum. Les cotisations sociales correspondantes sont réglées par un compte spécial du Bureau du travail (LAEK). 


\section{Résultats de la modélisation des retraites : Grèce}
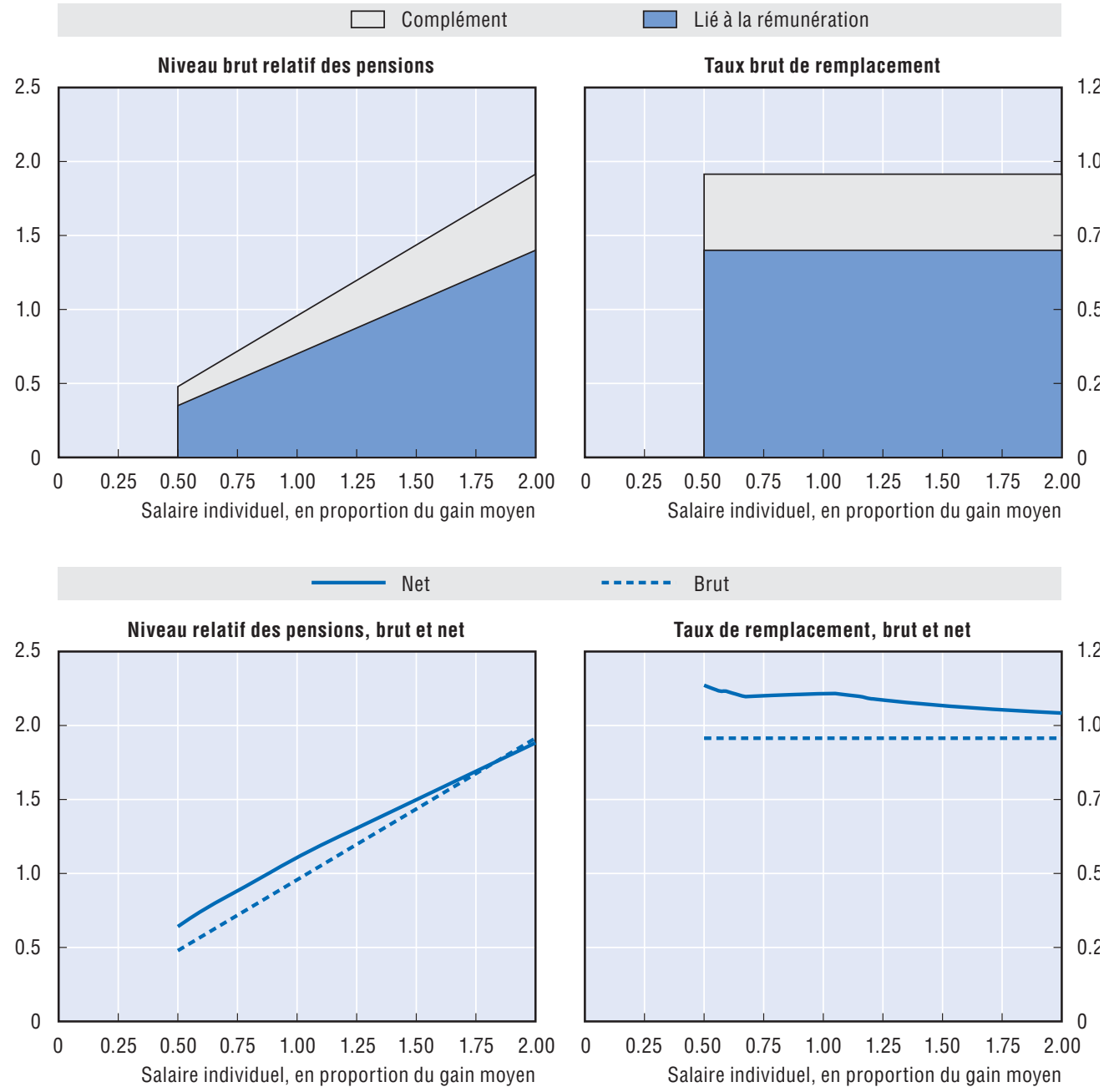

\begin{tabular}{|c|c|c|c|c|c|c|}
\hline \multirow{2}{*}{$\begin{array}{l}\text { Hommes } \\
\text { Femmes (si différent) }\end{array}$} & \multirow{2}{*}{$\begin{array}{l}\text { Salarié à revenu } \\
\text { médian }\end{array}$} & \multicolumn{5}{|c|}{ Salaire individuel, en multiple du salaire moyen } \\
\hline & & 0.5 & 0.75 & 1 & 1.5 & 2 \\
\hline Niveau brut relatif des pensions & 81.4 & 47.9 & 71.8 & 95.7 & 143.6 & 191.4 \\
\hline \multicolumn{7}{|l|}{ (\% salaire moyen brut) } \\
\hline Niveau net relatif des pensions & 97.3 & 64.1 & 88.3 & 110.8 & 149.8 & 188.3 \\
\hline \multicolumn{7}{|l|}{ (\% salaire moyen net) } \\
\hline Taux brut de remplacement & 95.7 & 95.7 & 95.7 & 95.7 & 95.7 & 95.7 \\
\hline \multicolumn{7}{|l|}{ (\% salaire individuel brut) } \\
\hline Taux net de remplacement & 110.4 & 113.6 & 110.1 & 110.8 & 106.7 & 104.2 \\
\hline \multicolumn{7}{|l|}{ (\% salaire individuel net) } \\
\hline Patrimoine retraite brut & 14.3 & 14.3 & 14.3 & 14.3 & 14.3 & 14.3 \\
\hline (multiple du salaire individuel brut) & 16.6 & 16.6 & 16.6 & 16.6 & 16.6 & 16.6 \\
\hline Patrimoine retraite net & 12.8 & 14.3 & 13.1 & 12.3 & 11.1 & 10.5 \\
\hline (multiple du salaire individuel brut) & 14.7 & 16.5 & 15.2 & 14.3 & 12.9 & 12.1 \\
\hline
\end{tabular}




\section{Hongrie}

\section{Hongrie : système de retraite en 2006}

Le nouveau système associe un régime public de retraite lié à la rémunération et des régimes par capitalisation intégrale obligatoires à cotisations définies. Il s'applique aux nouveaux arrivants sur le marché du travail et aux personnes qui étaient âgées d'au maximum 42 ans à l'époque de la réforme. Les travailleurs plus âgés ont eu le choix entre ce régime mixte et un régime public de retraite par répartition pure. La modélisation suppose que les travailleurs sont couverts par le régime mixte.
Indicateurs essentiels

\begin{tabular}{llrr}
\hline & & Hongrie & OCDE \\
\hline Salaire & HUF (millions) & 1.99 & 7.53 \\
moyen & USD & 9500 & 35800 \\
$\begin{array}{l}\text { Dépenses publiques } \\
\text { au titre des retraites }\end{array}$ & 8.5 & 7.2 \\
Espérance de vie & à la naissance & 73.2 & 78.9 \\
& à 65 ans & 80.3 & 83.4 \\
Population de plus de 65 ans & $\%$ de la population d'âge actif & 25.3 & 23.8 \\
\hline
\end{tabular}

\section{Conditions d'ouverture des droits}

L'âge d'ouverture des droits à pension est aligné progressivement sur 62 ans pour les hommes comme pour les femmes (contre 60 et 55 respectivement). Il est passé à 62 ans pour les hommes en 2000 et passera à 62 ans pour les femmes à fin 2009. En outre, 20 années d'activité sont exigées tant pour la pension liée à la rémunération que pour la pension minimum. Pour les personnes partant à la retraite avant le début de l'année 2009, 15 années d'activité sont requises pour avoir droit à une pension partielle.

Le système réformé a été mis en place en janvier 1998. Les personnes qui passaient volontairement au nouveau régime mixte étaient autorisées jusqu'à fin 2002 à revenir au régime par répartition. Par ailleurs, l'obligation pour les nouveaux entrants d'adhérer à un fonds de pension privé a été suspendue en 2002 puis réintroduite en 2003.

\section{Calcul des prestations}

\section{Régime lié à la rémunération}

Pour les personnes couvertes par le régime mixte, le taux d'acquisition des droits est de $1.22 \%$ du salaire par année d'activité (sous réserve du plafond de cotisation indiqué ci-dessous). Pour les personnes relevant uniquement du régime par répartition, ce taux est de $1.65 \%$.

Le salaire de référence, qui est actuellement la rémunération brute-nette (c'est-à-dire la rémunération brute diminuée des cotisations salariales) de toutes les années depuis 1988, se rapproche de la durée de la vie active. À partir de 2008, c'est la rémunération nette qui sera prise comme salaire de référence. Les gains des années antérieures jusqu'à un point situé deux ans avant le départ en retraite, sont revalorisés en fonction du salaire moyen à l'échelle nationale. Les gains des trois dernières années précédant le départ en retraite ne sont pas du tout revalorisés. À partir du $1^{\mathrm{er}}$ janvier 2008, on passera à une 
revalorisation totale (jusqu'à l'année précédant le départ en retraite). L'incidence globale de ces deux changements sera une réduction d'environ $8 \%$.

La rémunération ouvrant droit à pension a été plafonnée en 1992. Depuis 2005, ce plafond est en gros égal à trois fois le salaire brut mais il est fixé d'avance. En 2006, le plafond était de 17330 HUF par jour.

Depuis 2001, les pensions mises en paiement sont indexées pour moitié sur les salaires et pour moitié sur les prix mais des augmentations ponctuelles ultérieures ont été opérées.

Depuis 2006, un mois de pension supplémentaire est versé chaque année.

\section{Pension minimum}

Il existe une pension minimum qui s'établissait en 2006 à 25800 HUF par mois (environ $16 \%$ du salaire moyen brut et environ $30 \%$ du salaire mensuel moyen net). Elle est indexée de la même façon que les prestations du régime lié à la rémunération, c'est-à-dire pour moitié sur les prix et pour moitié sur le salaire moyen.

\section{Régime à cotisations définies}

À partir de 2004, environ $8 \%$ de la rémunération brute ouvrant droit à pension est réorienté vers le régime par capitalisation pour les personnes relevant du régime mixte public-privé (par choix délibéré ou par obligation). Cela représente une augmentation par rapport à la période 1998-2002 de 6 \% et à l'année 2003 de 7 \%. Le capital accumulé doit être converti en rente lors du départ à la retraite. Selon la législation en vigueur, cette rente doit offrir au moins la même indexation mixte de la pension servie que le régime public de retraite. Pour calculer les taux de rente, il faut utiliser des tables de mortalité unisexe. Depuis 2007, le taux de la part patronale des cotisations de retraite a été relevé de 18 à $21 \%$, tandis que celui de la part salariale est demeuré inchangé, à $8.5 \%$.

\section{Variantes de carrière}

\section{Retraite anticipée}

Un départ en retraite anticipée est actuellement possible sans réduction actuarielle à 60 ans pour les hommes et 57 ans pour les femmes. Lorsque l'âge de la retraite sera de 62 ans pour les hommes comme pour les femmes, le départ en retraite anticipée sera possible à partir de 59 ans pour les deux. À partir de 2013, il sera porté à 60 ans sous réserve d'un minimum de 37 années d'activité.

La réduction par mois jusqu'à 62 ans s'établit comme suit :

- $0.3 \%$ par mois manquant entre 61 ans et 62 ans ;

- $3.6 \%+0.4 \%$ par mois manquant jusqu'à 61 ans.

\section{Retraite différée}

Il est possible de différer la liquidation de la retraite liée à la rémunération. La pension est majorée de $0.5 \%$ pour chaque mois de report.

\section{Enfants}

Les régimes de retraite fournissent divers degrés de protection pour les périodes de grossesse et d'éducation des enfants. Les prestations existantes sont l'indemnité de 
grossesse et d'accouchement, l'indemnité et l'allocation de garde d'enfants. Des cotisations de retraite doivent désormais être acquittées par les bénéficiaires de ces prestations et le montant des prestations est inclus dans le calcul de la base de la retraite si cela est avantageux.

L'allocation de grossesse-accouchement (terhességi gyermekágyi segély) est versée pendant 24 semaines (168 jours) durant la grossesse et après la naissance. Elle est égale à $70 \%$ du salaire brut moyen journalier de l'année précédente. L'indemnité de garde d'enfants (gyermekgondozási dij) peut être demandée par l'un des parents à l'expiration de l'allocation de grossesse-accouchement; le droit à prestation prend fin au deuxième anniversaire de l'enfant (maximum 24 mois). Le montant de la prestation est égal à $70 \%$ du salaire brut moyen journalier de l'année précédente jusqu'à concurrence de deux fois le salaire minimum (87 500 HUF par mois en 2006). L'allocation pour garde d'enfants à domicile (gyermekgondozási segély) est versée au parent qui s'ocupe de l'enfant jusqu'à son troisième anniversaire (maximum 36 mois). Le montant mensuel de l'allocation est égal au minimum vieillesse (25 800 HUF en 2006) ; il est indépendant du nombre d'enfants de la famille.

En 2006, le paiement des cotisations de retraite au titre des prestations de garde d'enfants se ventilait comme suit :

\begin{tabular}{lccc}
\hline & Individu & Employeur & Etat \\
\hline Indemnité de garde d'enfants & $\mathrm{X}$ & - & $\mathrm{X}$ \\
Allocation pour garde d'enfants à domicile & $\mathrm{X}$ & - & $\mathrm{X}$ \\
\hline
\end{tabular}

\section{Chômage}

Les chômeurs sont couverts par le régime de retraite lié à la rémunération. Toutes les périodes de chômage sont qualifiées d'activité ouvrant droit à pension. Le salaire de référence pour les périodes de chômage est égal au i) montant des prestations de chômage ou à ii) la moyenne des rémunérations précédentes et ultérieures, si elle est plus favorable.

Durant la phase de transition économique, de nombreux chômeurs de longue durée âgés ont été mis en préretraite (Elõnyugdij) mais aujourd'hui leur nombre n'est plus significatif. Les chômeurs âgés peuvent toutefois bénéficier de prestations particulières de retraite anticipée s'ils remplissent un certain nombre de conditions : avoir été indemnisés oar l'assurance chômage pendant 140 jours, être à cinq ans de l'âge ouvrant droit à pension, avoir épuisé leurs droits à prestations de chômage dans les huit ans les séparant de l'âge ouvrant droit à pension et avoir cotisé aux régimes de retraite pendant un minimum de 20 ans. 


\section{Résultats de la modélisation des retraites : Hongrie}

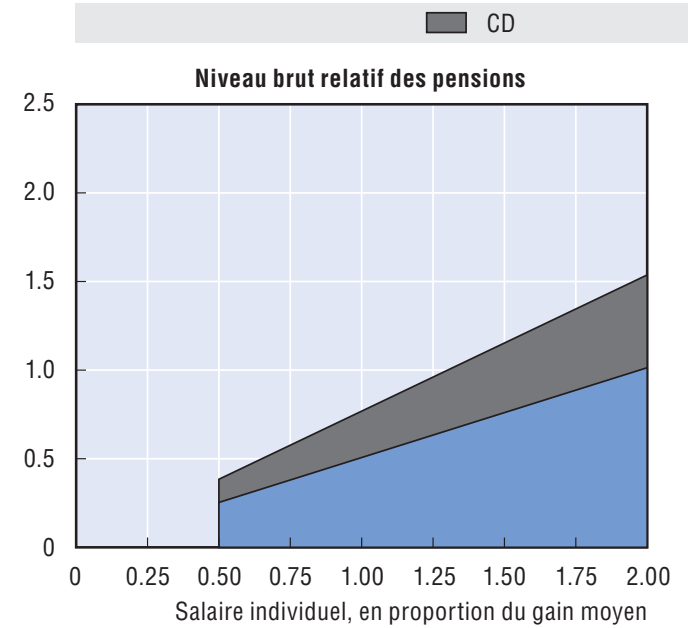

Lié à la rémunération

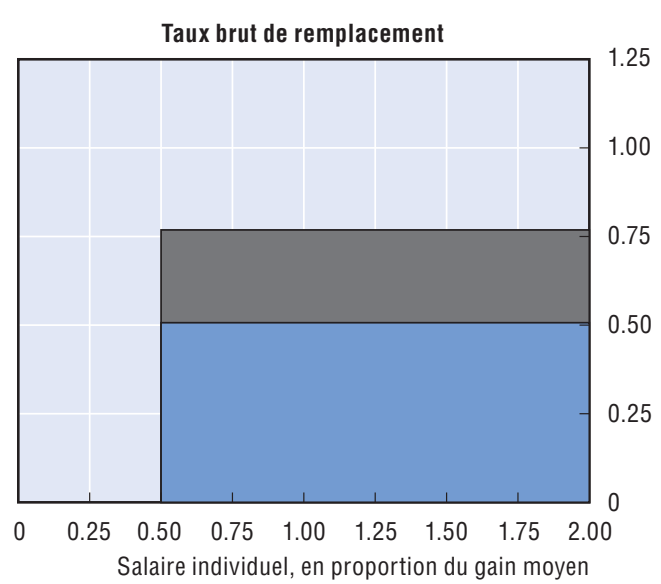

Net

Brut

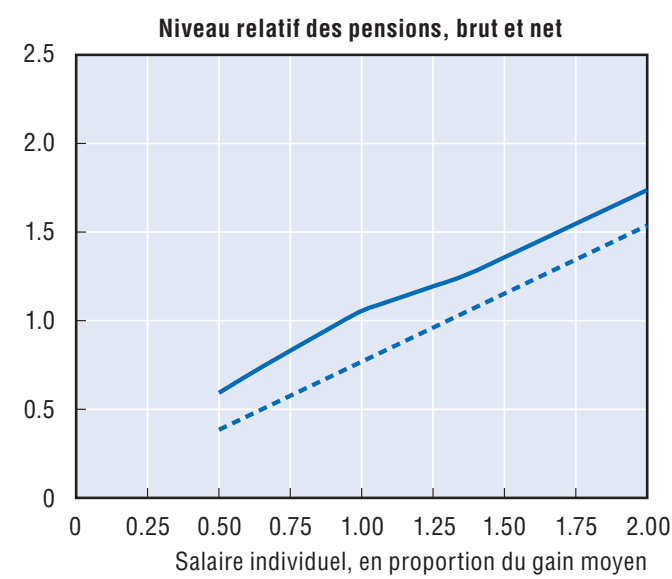

Taux de remplacement, brut et net

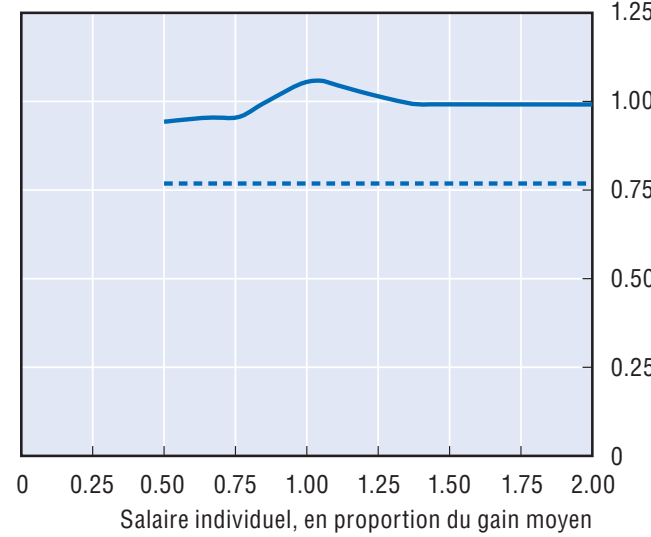

\begin{tabular}{lc|ccccc}
\hline \multirow{2}{*}{$\begin{array}{l}\text { Hommes } \\
\text { Femmes (si différent) }\end{array}$} & $\begin{array}{c}\text { Salarié à revenu } \\
\text { médian }\end{array}$ & 0.5 & 0.75 & 1 & 1.5 & 2 \\
\cline { 3 - 6 } & 56.9 & 38.4 & 57.6 & 76.9 & 115.3 & 153.7 \\
\hline $\begin{array}{l}\text { Niveau brut relatif des pensions } \\
\text { (\% salaire moyen brut) }\end{array}$ & & & & & & 175.7 \\
$\begin{array}{l}\text { Niveau net relatif des pensions } \\
\text { (\% salaire moyen net) }\end{array}$ & 82.1 & 59.3 & 83.1 & 105.5 & 135.7 & \\
$\begin{array}{l}\text { Taux brut de remplacement } \\
\text { (\% salaire individuel brut) }\end{array}$ & 76.9 & 76.9 & 76.9 & 76.9 & 76.9 & 76.9 \\
$\begin{array}{l}\text { Taux net de remplacement } \\
\text { (\% salaire individuel net) }\end{array}$ & 95.4 & 94.3 & 95.4 & 105.5 & 99.2 & 99.2 \\
$\begin{array}{l}\text { Patrimoine retraite brut } \\
\text { (multiple du salaire individuel brut) }\end{array}$ & 12.4 & 12.4 & 12.4 & 12.4 & 12.4 & 12.4 \\
$\begin{array}{l}\text { Patrimoine retraite net } \\
\text { (multiple du salaire individuel brut) }\end{array}$ & 11.6 & 15.4 & 15.4 & 15.4 & 15.4 & 15.4 \\
\hline
\end{tabular}




\section{Irlande}

\section{Irlande : système de retraite en 2006}

Le régime public de retraite est un régime de base qui verse une pension forfaitaire à toutes les personnes remplissant les conditions requises de cotisations. Par ailleurs, une pension soumise à condition de revenu offre un filet de sécurité aux personnes âgées à faibles revenus. Les régimes professionnels facultatifs ont une couverture étendue (environ la moitié des salariés).
Indicateurs essentiels

\begin{tabular}{llrr}
\hline & & Irlande & \multicolumn{1}{c}{ OCDE } \\
\hline Salaire moyen & EUR & 30000 & 28600 \\
& USD & 37600 & 35800 \\
Dépenses publiques au titre & $\%$ du PIB & 3.4 & 7.2 \\
des retraites & & 79.7 & 78.9 \\
Espérance de vie & à la naissance & 83.5 & 83.4 \\
& à 65 ans & 17.7 & 23.8 \\
\hline
\end{tabular}

\section{Conditions d'ouverture des droits}

La pension publique contributive est payable à partir de 66 ans tandis que la pension publique de transition est versée à partir de 65 ans. Pour avoir pleinement droit à ces deux prestations, il faut justifier d'une moyenne de 48 semaines de cotisation ou de crédits par an sur toute la durée de la vie active. En cas de durée de cotisation incomplète, le montant de la pension est réduit au prorata.Toutefois, la pension de vieillesse contributive requiert au minimum une moyenne de 10 semaines de cotisations par an et la pension de retraite de 24 semaines par an. Par ailleurs, la durée totale minimum de cotisation est de 260 semaines, ce qui équivaut à cinq années de couverture complète.

La pension soumise à conditions de ressources est payable à partir de 66 ans.

\section{Calcul des prestations}

\section{Régime de base}

Le montant de la pension de vieillesse contributive, comme celui de la pension de transition est de 193.30 EUR par semaine (sur 53 semaines par an) pour 2006, soit $34.2 \%$ du salaire moyen (tel que le mesure l'OCDE). À cela s'ajoute un montant de 128.8 EUR pour un adulte à charge d'âge actif et de 149.30 EUR pour une personne à charge de 66 ans et plus. Les pensions sont augmentées annuellement sur une base fixée par les pouvoirs publics dans le cadre du budget annuel. Ces dernières années, les augmentations ont été supérieures à la hausse des salaires.

Les retraités ont droit à de nombreuses prestations en nature. Les pouvoirs publics estiment la valeur des produits et services ainsi reçus à 950 EUR par an, hors prestations d'assurance maladie. (On notera que la modélisation prend en compte uniquement les prestations en espèces mais pas les prestations en nature.) 


\section{Régime ciblé}

Pour 2006, le montant maximum de la prestation soumise à conditions de ressources est de 182 EUR par semaine pour une personne seule auxquels s'ajoutent 120.30 EUR pour un adulte à charge. La pension d'une personne seule est égale à $32 \%$ du salaire moyen. Audelà d'une franchise hebdomadaire de faible montant (20 EUR) dans la condition de ressources à laquelle s'ajoute à partir de 2006 une franchise supplémentaire de 100 EUR, la prestation est dégressive à $100 \%$ du revenu. Une condition de patrimoine s'applique également : tout capital supérieur à 35000 EUR est converti en revenu par application d'une formule standard.

Le montant de l'objectif de revenu du filet de sécurité dans le régime soumis à conditions de ressources suit en gros la revalorisation des régimes de base.

\section{Pensions privées facultatives}

Environ $43 \%$ des salariés ont un régime de retraite professionnel. Parmi les adhérents travaillant dans le secteur privé la moitié environ ont un plan à cotisations définies et l'autre moitié un plan à prestations définies. (Ces chiffres se rapportent aux régimes par capitalisation que l'on trouve principalement dans le secteur privé.) Les plans à prestations définies sont supposés verser $1 / 60^{\mathrm{e}}$ du dernier salaire pour chaque année d'activité, avec un taux de cotisation de $1.67 \%$. Les plans à prestations définies sont supposés « intégrés au régime public ", ce qui signifie que le montant de la retraite de base est déduit des droits à prestations. Lorsque les personnes changent d'emploi, la valeur de la retraite professionnelle différée est indexée sur la hausse des prix.

Pour les plans professionnels à cotisations définies, le taux de cotisation moyen est d'environ $10 \%$ de la rémunération. En outre, environ $15 \%$ des personnes ont des plans individuels à cotisations définies.

\section{Variantes de carrière}

\section{Retraite anticipée}

Les pensions ne peuvent être liquidées avant l'âge normal d'ouverture des droits.

\section{Retraite différée}

Le cumul emploi-retraite est possible dans le cadre de la pension de transition, qui est versée pour un an, sous réserve que les gains soient inférieurs à 38 EUR par semaine. En revanche, la pension contributive n'est soumise à aucune condition de gains. Il n'est pas possible de reporter sa liquidation.

\section{Enfants}

Les droits éventuels à une pension publique ne sont pas affectés par les périodes d'interruption de l'activité liées aux enfants.

\section{Chômage}

Les droits éventuels à une pension publique ne sont pas affectés par les périodes de chômage. 
Résultats de la modélisation des retraites : Irlande

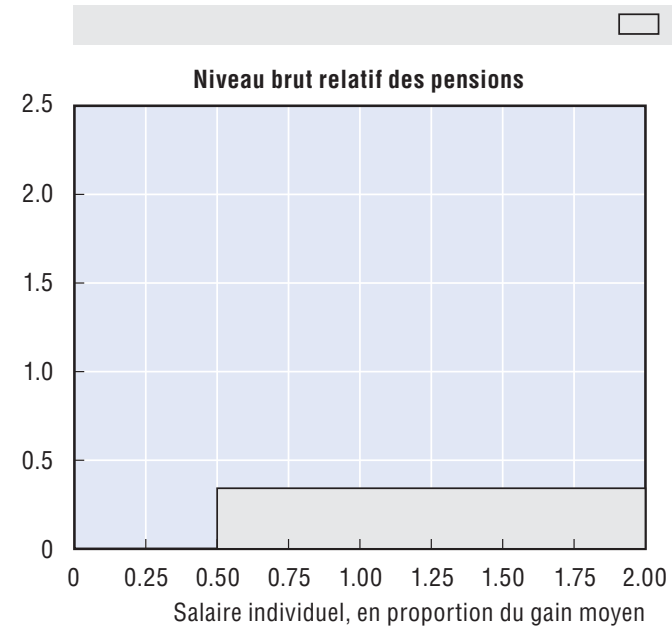

De base
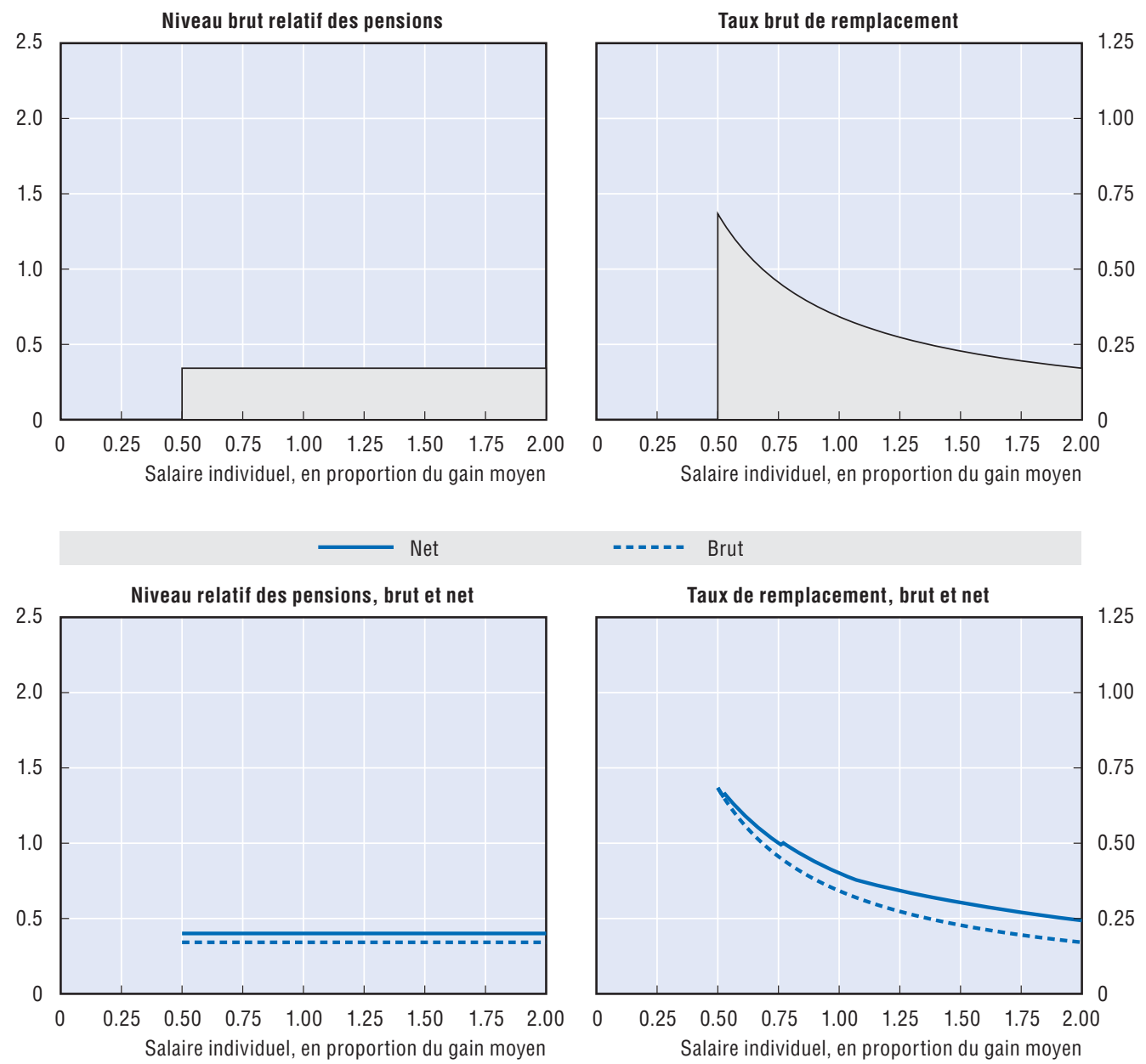

\begin{tabular}{|c|c|c|c|c|c|c|}
\hline \multirow{2}{*}{$\begin{array}{l}\text { Hommes } \\
\text { Femmes (si différent) }\end{array}$} & \multirow{2}{*}{$\begin{array}{l}\text { Salarié à revenu } \\
\text { médian }\end{array}$} & \multicolumn{5}{|c|}{ Salaire individuel, en multiple du salaire moyen } \\
\hline & & 0.5 & 0.75 & 1 & 1.5 & 2 \\
\hline Niveau brut relatif des pensions & 34.2 & 34.2 & 34.2 & 34.2 & 34.2 & 34.2 \\
\hline \multicolumn{7}{|l|}{ (\% salaire moyen brut) } \\
\hline Niveau net relatif des pensions & 40.1 & 40.1 & 40.1 & 40.1 & 40.1 & 40.1 \\
\hline \multicolumn{7}{|l|}{ (\% salaire moyen net) } \\
\hline Taux brut de remplacement & 39.8 & 68.4 & 45.6 & 34.2 & 22.8 & 17.1 \\
\hline \multicolumn{7}{|l|}{ (\% salaire individuel brut) } \\
\hline Taux net de remplacement & 45.6 & 68.4 & 50.1 & 40.1 & 30.3 & 24.4 \\
\hline \multicolumn{7}{|l|}{ (\% salaire individuel net) } \\
\hline Patrimoine retraite brut & 7.1 & 12.1 & 8.1 & 6.1 & 4.0 & 3.0 \\
\hline (multiple du salaire individuel brut) & 8.4 & 14.5 & 9.6 & 7.2 & 4.8 & 3.6 \\
\hline Patrimoine retraite net & 7.1 & 12.1 & 8.1 & 6.1 & 4.0 & 3.0 \\
\hline (multiple du salaire individuel brut) & 8.4 & 14.5 & 9.6 & 7.2 & 4.8 & 3.6 \\
\hline
\end{tabular}




\section{Islande}

\section{Islande : système de retraite en 2006}

Le régime public de retraite a trois composantes: un régime de base et deux régimes soumis à une condition de revenu. À cela s'ajoutent des plans de retraite professionnels obligatoires hybrides (quoique principalement à prestations définies).

\section{Indicateurs essentiels}

\begin{tabular}{llrr}
\hline & & Islande & OCDE \\
\hline Salaire & ISK (millions) & 3.48 & 2.50 \\
moyen & USD & 49800 & 35800 \\
$\begin{array}{l}\text { Dépenses publiques au titre } \\
\text { des retraites }\end{array}$ & 2.0 & 7.2 \\
Espérance de vie & à la naissance & 81.2 & 78.9 \\
& à 65 ans & 84.5 & 83.4 \\
Population de plus de 65 ans & $\%$ de la population d'âge actif & 19.5 & 23.8 \\
\hline
\end{tabular}

\section{Conditions d'ouverture des droits}

L'âge normal de départ à la retraite est de 67 ans. Quarante années de résidence sont nécessaires pour percevoir une retraite de base à taux plein. Pour des durées de résidence plus courte, avec un minimum de trois ans, la pension est réduite au prorata. L'âge de départ à la retraite est également de 67 ans pour les membres de plans professionnels du secteur privé mais il est de 60 ans pour les gens de mer ayant travaillé au minimum 25 ans dans ce secteur.

\section{Calcul des prestations}

\section{Régime de base}

Le montant de la retraite de base à taux plein est de 24131 ISK par mois, soit environ $8 \%$ du salaire moyen. Cette prestation est soumise à une condition de revenu : elle est dégressive à partir du moment où les revenus (autres que ceux provenant de la retraite complémentaire) excèdent 1998438 ISK, soit 57 \% du salaire moyen, et s'annule lorsqu'ils atteignent 2963678 ISK. Cette condition s'applique uniquement aux revenus autres que les pensions, comme les revenus du travail ou de l'aide sociale ainsi qu'à $50 \%$ des revenus du capital.

\section{Régime ciblé}

Un deuxième élément est le complément retraite. Le montant maximum de cette prestation est de 44838 ISK par mois pour une personne seule, soit environ $15 \%$ du salaire moyen. Elle est dégressive à partir du moment où le revenu annuel excède 607850 ISK (environ $17 \%$ du salaire moyen). Toutefois, la retraite de base n'a aucune incidence sur le montant du complément retraite. Le taux de dégressivité appliqué à ce complément en fonction du revenu est de $45 \%$.

Il existe enfin un deuxième complément retraite dont le montant est plafonné à 22109 ISK par mois, soit un peu moins de $8 \%$ du salaire moyen. Ce complément est dégressif au taux de $45 \%$ en fonction de tous les autres revenus et s'annule lorsque ceuxci atteignent 589573 ISK.

Les niveaux des prestations sont ajustés annuellement en fonction du budget de l'État. Les ajustements sont destinés à prendre en compte l'évolution des salaires du secteur 
public (qui est supposée ici identique à celle de l'hypothèse standard de croissance des salaires à l'échelle nationale) et celle des prix par application de l'indice du coût de la vie.

\section{Régimes professionnels obligatoires}

Les régimes d'entreprise sont obligatoires. La loi leur impose un objectif de taux de remplacement de $56 \%$ pour 40 années de cotisation, ce qui donne un taux d'acquisition de $1.4 \%$ par année d'activité. La couverture est obligatoire de 16 à 70 ans. Dans ce calcul, le salaire de référence est le salaire moyen sur la durée de la vie active par année d'adhésion. La rémunération ouvrant droit à pension n'est pas plafonnée. Les salaires antérieurs sont revalorisés en fonction de la hausse des prix plus un taux d'intérêt de $3.5 \%$.

De par la loi, les retraites professionnelles mises en paiement doivent être indexées sur la hausse des prix à la consommation.

Dans la pratique, de nombreux régimes versent davantage que le minimum légal décrit ci-dessus, introduisant habituellement dans le système un élément hybride de cotisations définies/prestations définies. La cotisation minimum aux régimes professionnels est de $12 \%$ du salaire. Le salarié prend à sa charge $4 \%$ et l'employeur $8 \%$. Les cotisations au-delà du niveau nécessaire pour financer les prestations légales décrites ci-dessus peuvent être utilisées pour accroître les droits des régimes à prestations définies ou transférées vers des comptes individuels, assurant ainsi une pension à cotisations définies. Toutefois, la modélisation ne porte que sur la composante obligatoire et pas sur ces prestations extra-légales qui ne sont pas garanties.

\section{Variantes de carrière}

\section{Retraite anticipée}

Dans le régime professionnel obligatoire, les règles régissant le départ à la retraite anticipée varient d'un fonds à l'autre, en fonction de leur composition. Dans le secteur privé, l'âge normal de départ à la retraite est de 67 ans et un départ anticipé est possible à partir de 62 ans. De manière générale, les pensions sont réduites de $7 \%$ par année d'anticipation.

$\mathrm{Ni}$ le régime de base ni les régimes ciblés n'autorisent la liquidation des pensions avant l'âge normal de la retraite.

\section{Retraite différée}

Dans les régimes professionnels obligatoires, les travailleurs peuvent différer leur départ en retraite jusqu'à 70 ans avec une augmentation de leur pension de $9 \%$ par année de report. Les travailleurs qui retardent leur départ à la retraite continuent à cotiser et acquièrent des droits à pension supplémentaires. Dans certains cas, la durée totale de cotisation est limitée à 32 ans.

$\mathrm{Ni}$ le régime de base ni les régimes ciblés n'autorisent le report des pensions au-delà de l'âge normal de la retraite. La retraite de base est soumise à une condition de revenu d'activité (voir ci-dessus) tandis que les régimes ciblés sont soumis à une condition de revenu global incluant les revenus d'activité.

\section{Enfants}

La retraite de base sous condition de résidence et les régimes ciblés protègent automatiquement les femmes qui quittent un emploi rémunéré pour s'occuper de leurs 
enfants. Aucun crédit spécifique n'est accordé au titre des absences liées aux enfants. Les fonds de pension professionnels eux-mêmes ne prévoient rien pour les femmes qui doivent quitter leur emploi pour s'occuper de leurs enfants. Le régime public d'aide sociale comporte des prestations pour les parents (hommes ou femmes) qui doivent s'occuper d'enfants souffrant de longues maladies ou handicapés. De telles prestations sont également prévues pour les personnes devant s'occuper de proches parents : par exemple, cas d'un adulte (homme ou femme) s'occupant d'un parent âgé.

\section{Chômage}

La base de cotisation, sur laquelle est prélévée la cotisation minimum de $10 \%$, inclut les prestations de l'assurance chômage ainsi que les salaires mais exclut toutes les autres prestations. 
Résultats de la modélisation des retraites : Islande
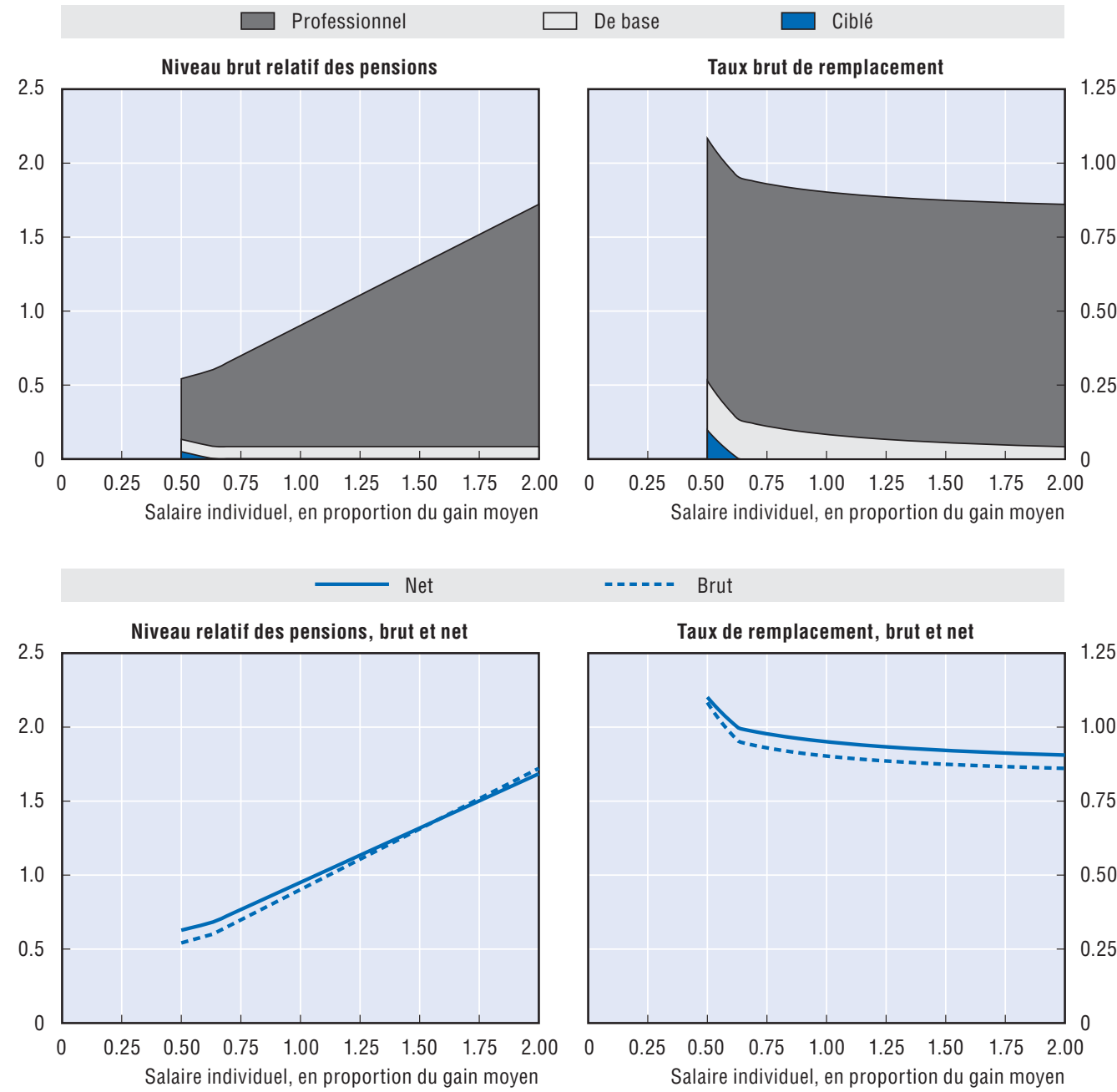

\begin{tabular}{|c|c|c|c|c|c|c|}
\hline \multirow{2}{*}{$\begin{array}{l}\text { Hommes } \\
\text { Femmes (si différent) }\end{array}$} & \multirow{2}{*}{$\begin{array}{c}\text { Salarié à revenu } \\
\text { médian }\end{array}$} & \multicolumn{5}{|c|}{ Salaire individuel, en multiple du salaire moyen } \\
\hline & & 0.5 & 0.75 & 1 & 1.5 & 2 \\
\hline Niveau brut relatif des pensions & 77.9 & 54.2 & 69.8 & 90.2 & 131.2 & 172.1 \\
\hline \multicolumn{7}{|l|}{ (\% salaire moyen brut) } \\
\hline Niveau net relatif des pensions & 84.1 & 62.7 & 76.7 & 95.1 & 131.8 & 168.5 \\
\hline \multicolumn{7}{|l|}{ (\% salaire moyen net) } \\
\hline Taux brut de remplacement & 91.7 & 108.3 & 93.0 & 90.2 & 87.5 & 86.1 \\
\hline \multicolumn{7}{|l|}{ (\% salaire individuel brut) } \\
\hline Taux net de remplacement & 96.5 & 110.1 & 97.7 & 95.1 & 92.1 & 90.6 \\
\hline \multicolumn{7}{|l|}{ (\% salaire individuel net) } \\
\hline Patrimoine retraite brut & 14.0 & 17.0 & 14.2 & 13.7 & 13.2 & 12.9 \\
\hline (multiple du salaire individuel brut) & 15.7 & 19.1 & 15.9 & 15.4 & 14.8 & 14.5 \\
\hline Patrimoine retraite net & 10.6 & 13.9 & 11.0 & 10.2 & 9.3 & 8.9 \\
\hline (multiple du salaire individuel brut) & 11.9 & 15.6 & 12.4 & 11.4 & 10.5 & 10.0 \\
\hline
\end{tabular}




\section{Italie}

\section{Italie : système de retraite en 2006}

Le nouveau régime de retraite italien repose sur un système de comptes notionnels. Le taux de rendement des cotisations est lié à la croissance du PIB. $\mathrm{Au}$ moment du départ en retraite, le capital notionnel accumulé est converti en rente en tenant compte de l'espérance de vie moyenne à cette date. Ce régime s'applique intégralement aux personnes entrées sur le marché du travail à partir de 1996.

\section{Indicateurs essentiels}

\begin{tabular}{llrr}
\hline & & Italie & \multicolumn{1}{c}{ OCDE } \\
\hline Salaire moyen & EUR & 24600 & 28600 \\
& USD & 30900 & 35800 \\
Dépenses publiques au titre & \% du PIB & 14.0 & 7.2 \\
des retraites & & 80.9 & 78.9 \\
Espérance de vie & à la naissance & 84.5 & 83.4 \\
& à 65 ans & 32.5 & 23.8 \\
\hline
\end{tabular}

\section{Conditions d'ouverture des droits}

Dans le nouveau système, l'âge normal de départ à la retraite sera, à partir de 2008, de 65 ans pour les hommes et de 60 ans pour les femmes. Toutefois, un départ anticipé restera possible sous diverses conditions de cotisation (voir ci-dessous). La modélisation de base suppose que les hommes partent à la retraite à 65 ans et les femmes à 60 ans.

\section{Calcul des prestations}

\section{Régime lié à la rémunération}

Dans le régime contributif des salariés du public et du privé, le taux de cotisation est de $33 \%$, dont un tiers environ à la charge du salarié et deux tiers à la charge de l'employeur. Le montant de la pension est obtenu en faisant le produit de deux facteurs : le montant total de cotisation sur l'ensemble de la carrière, capitalisé à l'aide d'une moyenne mobile sur cinq ans du taux de croissance nominal du PIB et le coefficient de conversion, dont le calcul repose principalement sur les probabilités de décès, les probabilités de laisser un veuf/une veuve et le nombre d'années pendant lesquelles une pension de réversion sera perçue. En conséquence, les prestations sont fortement liées à l'âge de départ à la retraite : plus celui-ci sera bas plus la pension sera faible.

Les coefficients de conversion sont révisés tous les trois ans. Ils sont disponibles pour la tranche d'âge 57-65 ans mais les travailleurs ne peuvent pas partir à la retraite avant 65 ans s'ils ne remplissent pas les conditions d'éligibilité établies par la législation en vigueur et si le montant de leur pension n'est pas inférieur à 1.2 fois l'allocation de vieillesse.

\begin{tabular}{lrrrrrrrrr}
\hline Âge de départ à la retraite & 57 & 58 & 59 & 60 & 61 & 62 & 63 & 64 & $65+$ \\
Coefficient de conversion (\%) & 4.014 & 4.113 & 4.217 & 4.328 & 4.446 & 4.572 & 4.705 & 4.847 & 4.999
\end{tabular}

Note : Les coefficients ci-dessus ont été établis à partir des prévisions démographiques ISTAT 2001.

L'hypothèse de base de la modélisation pour tous les pays est un taux de croissance des salaires réels de $2 \%$ par an. Étant donné la baisse prévue de la population active en Italie, une croissance du PIB réel de $1.6 \%$ par an est une hypothèse cohérente. 
Pour les salariés, la rémunération minimum pour le calcul des cotisations est de 171.03 EUR par semaine (36\% du salaire moyen). La rémunération maximum prise en compte pour le calcul des prestations est de 85478 EUR par an dans le nouveau système, soit un peu moins de $347 \%$ du salaire moyen.

L'indexation des pensions mises en paiement est complexe, dans la mesure où les pensions modestes bénéficient d'un traitement plus généreux que celui des pensions élevées. Jusqu'à trois fois la pension minimum, les pensions mises en paiement sont totalement indexées sur les prix. Ce seuil est de 1260 EUR par mois pour 2005 (chiffre utilisé pour l'indexation des pensions en 2006) et de 1283 EUR pour 2006 (pour l'indexation de 2007), soit environ les deux tiers du salaire moyen à l'échelle nationale. Entre trois fois et cinq fois la pension minimum, les pensions mises en paiement sont revalorisées de $90 \%$ de la hausse des prix. Au-delà de ce seuil, l'indexation tombe à $75 \%$ de la hausse des prix. On notera que pour les pensions élevées l'indexation s'applique de façon distincte à chaque tranche.

\section{Aide sociale}

La pension minimum (voir ci-dessous) est supprimée pour les personnes qui relèvent uniquement du nouveau régime, autrement dit qui sont entrées sur le marché du travail après 1996. Cependant, les retraités dont les revenus sont inférieurs au seuil de l'aide sociale peuvent prétendre à 65 ans à une prestation soumise à conditions de ressources. En 2006, la prestation d'aide sociale (assegno sociale), y compris les compléments, s'élevait à 5130 EUR. Pour les personnes de plus de 70 ans, ce montant passe à 7167 EUR. Ces chiffres équivalent respectivement à $21 \%$ et $29 \%$ du salaire moyen.

\section{Pensions privées facultatives}

À cela s'ajoute un régime professionnel complémentaire facultatif composé à la fois de fonds ouverts et de fonds fermés (négociés collectivement). Les fonds fermés peuvent être financés à la fois par les employeurs et par les salariés mais aussi par le TFR. Les fonds ouverts assurent une rente fondée sur des cotisations. Le taux de cotisation au TFR est actuellement de $6.91 \%$. Le nombre de travailleurs inscrits dans un fonds de pension privé reste faible. C'est pourquoi, la Loi de Finances pour 2007 a anticipé (avec quelques changements) la réforme des retraites récemment votée (Loi 243/2004 et Décret Loi 252/2005) qui a mis en place de nouvelles mesures pour accélérer le développement du deuxième pilier : a) renforcement des incitations fiscales et b) acceptation tacite pour le transfert de l'indemnité privée de fin de contrat (TFR). Cette dernière mesure signifie que le pécule accumulé sera transféré à un fonds de pension privé, sauf si le travailleur s'y oppose. L'adhésion aux fonds de pension privés reste toutefois facultative.

En Italie, la couverture des plans professionnels de retraite demeure faible (environ $11 \%$ des salariés). Les pouvoirs publics ont bien encouragé travailleurs et employeurs à passer du régime d'indemnités de fin de contrat (Trattamento di Fine Rapporto, TFR) aux régimes professionnels de retraite mais la faiblesse de ce taux montre qu'ils n'ont guère été suivis.

$\mathrm{Du}$ fait de leur faible couverture, ces plans ne sont pas pris en compte dans la modélisation des pensions privées facultatives. 


\section{Variantes de carrière}

\section{Retraite anticipée}

Dans l'ancien système, les travailleurs pouvaient partir à la retraite à 57 ans s'ils avaient cotisé pendant 35 années. À partir de janvier 2008, l'âge minimum est passé à 58 ans (59 pour les travailleurs indépendants). Une réforme récente, approuvée dans le cadre de la procédure budgétaire 2008, a introduit un système de quotas qui associe des conditions d'âge et d'ancienneté de sorte que l'âge minimum requis pour prétendre à une retraite anticipée (pensions d'ancienneté) sera porté de 57 à 61 ans d'ici à 2013. Il restera toutefois possible de partir à tout âge avec 40 années de cotisation.

\section{Retraite différée}

Les femmes ont le droit de continuer à travailler jusqu'à l'âge normal de la retraite pour les hommes. La retraite à 65 ans n'est pas obligatoire mais les employeurs ont le droit de se séparer des salariés qui atteignent cet âge. Depuis janvier 2009, il est possible de cumuler totalement un salaire et une pension de retraite. S'agissant des pensions qui relèvent d'un régime contributif : a) il est possible de cumuler intégralement des indemnités de chômage et une pension de vieillesse anticipée pour les retraités ayant au minimum 40 ans d'ancienneté ; b) il est possible de cumuler intégralement des indemnités de chômage et une pension de vieillesse pour des retraités de 65 ans et plus, dans le cas d'un homme, de 60 ans et plus dans le cas d'une femme.

Il est possible de reporter la liquidation des droits à pension après 65 ans ; cependant, le coefficient de conversion (voir plus haut) reste le même et les prestations n'augmentent qu'en raison de l'accumulation de nouvelles cotisations et de leur capitalisation (fictive) sur une ou plusieurs années supplémentaires.

\section{Enfants}

La pension est majorée pour les mères auxquelles on attribue un coefficient de conversion plus généreux. Pour les mères d'un ou deux enfants, on prend le coefficient de leur âge réel de départ à la retraite plus un an. Pour les mères de trois enfants et plus on prend leur âge réel de départ à la retraite plus deux ans. En fonction des coefficients de conversion prévus, cela a pour effet de majorer la pension d'environ $3 \%$ pour un ou deux enfants et de $6 \%$ pour trois enfants et plus.

\section{Chômage}

Tous les régimes d'assurance chômage - cassa integrazione guadagni (CIG), indennità di mobilità et indennità di disoccupazione - donnent lieu à des crédits de cotisations pour la période pendant laquelle la prestation est reçue. La pension est calculée sur la base de la rémunération antérieure.

Pour les personnes arrivées sur le marché du travail depuis 1993, la période de crédit maximum est de cinq ans sur l'ensemble de la carrière. Cela affecte uniquement le droit de percevoir une pension d'ancienneté. Par ailleurs, les cotisations créditées au titre de la indennità di disoccupazione, qui est le régime général d'indemnisation du chômage, ne peuvent être prises en compte pour le calcul des 35 années de cotisation exigées même si elles le sont (dans la limite de cinq ans) pour le calcul des 40 années exigées.

Les cotisations sont normalement versées par le gouvernment, à l'exception de l'indennità di mobilità la première année où elle est perçue et de la CIG, qui sont partiellement versées par le salarié à un taux réduit de $5.54 \%$. 
Résultats de la modélisation des retraites : Italie
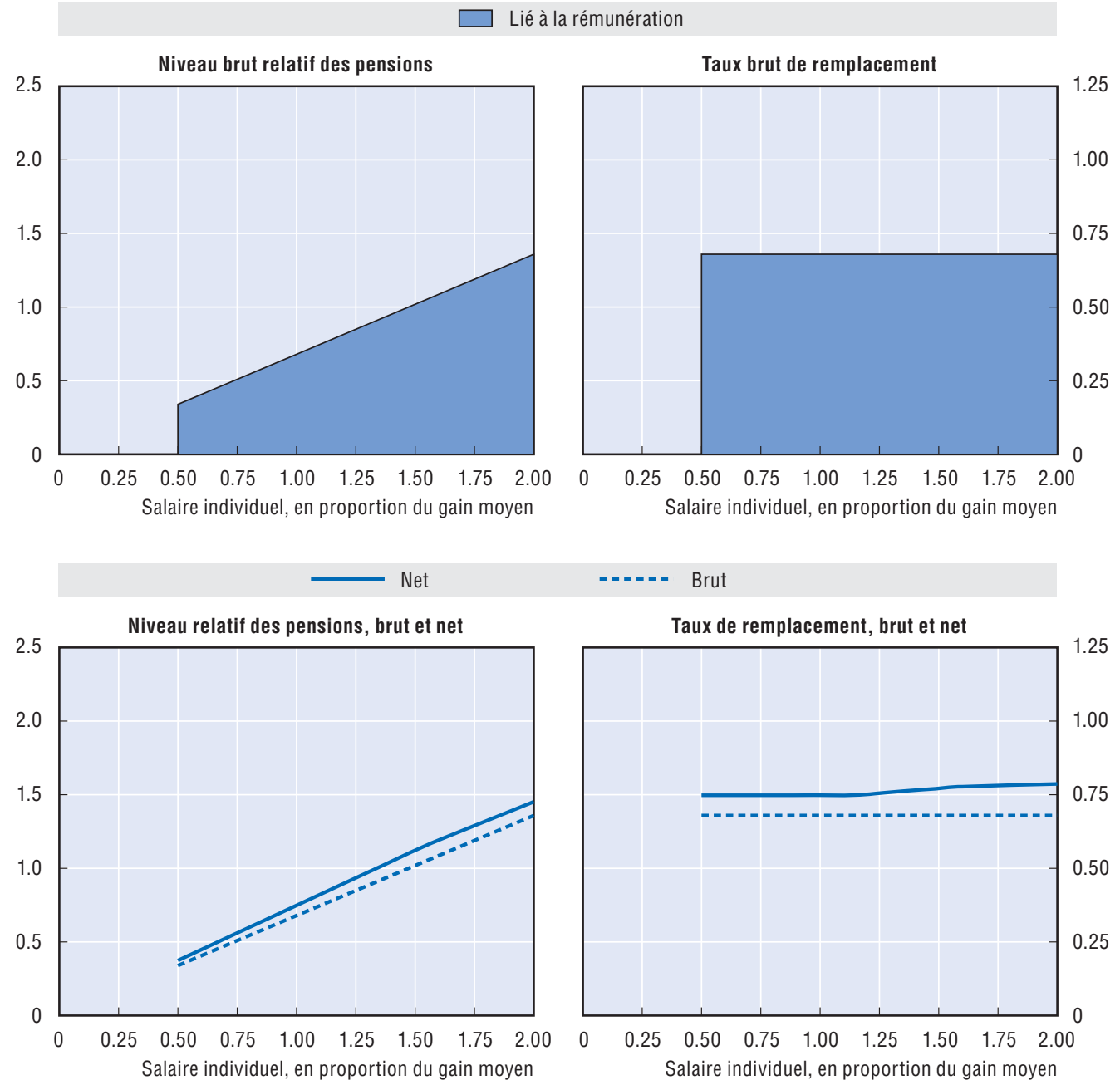

\begin{tabular}{|c|c|c|c|c|c|c|}
\hline \multirow{2}{*}{$\begin{array}{l}\text { Hommes } \\
\text { Femmes (si différent) }\end{array}$} & \multirow{2}{*}{$\begin{array}{l}\text { Salarié à revenu } \\
\text { médian }\end{array}$} & \multicolumn{5}{|c|}{ Salaire individuel, en multiple du salaire moyen } \\
\hline & & 0.5 & 0.75 & 1 & 1.5 & 2 \\
\hline Niveau brut relatif des pensions & 59.8 & 34.0 & 51.0 & 67.9 & 101.9 & 135.9 \\
\hline (\% du salaire moyen brut) & 46.5 & 26.4 & 39.6 & 52.8 & 79.2 & 105.6 \\
\hline Niveau net relatif des pensions & 65.8 & 37.4 & 56.1 & 74.8 & 112.2 & 145.2 \\
\hline (\% du salaire moyen net) & 51.2 & 38.3 & 43.6 & 58.1 & 87.2 & 116.3 \\
\hline Taux brut de remplacement & 67.9 & 67.9 & 67.9 & 67.9 & 67.9 & 67.9 \\
\hline (\% du salaire individuel brut) & 52.8 & 52.8 & 52.8 & 52.8 & 52.8 & 52.8 \\
\hline Taux net de remplacement & 74.8 & 74.8 & 74.8 & 74.8 & 77.1 & 78.7 \\
\hline (\% du salaire individuel net) & 58.1 & 76.6 & 58.1 & 58.1 & 59.9 & 63.0 \\
\hline Patrimoine retraite brut & 10.0 & 10.0 & 10.0 & 10.0 & 9.9 & 9.8 \\
\hline (multiple du salaire individuel brut) & 10.7 & 10.7 & 10.7 & 10.7 & 10.7 & 10.6 \\
\hline Patrimoine retraite net & 7.6 & 7.6 & 7.6 & 7.6 & 7.5 & 7.2 \\
\hline (multiple du salaire individuel brut) & 8.1 & 10.7 & 8.1 & 8.1 & 8.1 & 8.0 \\
\hline
\end{tabular}




\section{Japon}

\section{Japon : système de retraite en 2006}

Le système public de retraite japonais repose sur deux piliers : un régime de base à taux uniforme et un régime lié à la rémunération (retraite des salariés).

\section{Conditions d'ouverture des droits}

\section{Indicateurs essentiels}

\begin{tabular}{llrr}
\hline & & Japon & OCDE \\
\hline Salaire & JPY (millions) & 4.99 & 4.17 \\
moyen & USD & 42900 & 35800 \\
Dépenses publiques & \% du PIB & 8.7 & 7.2 \\
au titre des retraites & & & \\
Espérance de vie & à la naissance & 82.4 & 78.9 \\
& à 65 ans & 86.0 & 83.4 \\
Population de plus de 65 ans & \% de la population & 34.4 & 23.8 \\
& d'âge actif & & \\
\hline
\end{tabular}

La pension de vieillesse de base est versée à partir de 65 ans, avec un minimum de 25 années de cotisation. La pension à taux plein requiert 40 années de cotisation et les prestations sont ajustées au prorata pour des durées de cotisation plus courtes ou plus longues.

La pension liée à la rémunération est versée en sus de la pension de base, avec un minimum d'un mois de cotisation, sous réserve que le bénéficiaire ait droit à la retraite de base. L'âge de la retraite est progressivement relevé de 60 à 65 ans (entre 2001 et 2013 pour les hommes et entre 2006 et 2018 pour les femmes) pour la composante à taux uniforme et de 60 à 65 ans (pour les hommes en 2025 et pour les femmes en 2030) pour la composante liée à la rémunération. La composante liée à la rémunération du régime de retraite des salariés est ajustée pour des durées de cotisation plus courtes ou plus longues.

\section{Calcul des prestations}

\section{Régime de base}

Pour 2006, la pension de base à taux plein était de 792100 JPY par an, soit $15.9 \%$ du salaire moyen. Elle est indexée sur les prix.

\section{Aide sociale}

Les personnes âgées sont couvertes par le régime général d'aide sociale. Le montant de l'aide sociale pour un ménage d'une personne âgée de 60 à 69 ans à Tokyo en 2006 est de 969810 JPY (soit 19 \% du salaire moyen) par an, non compris les prestations logement et autres prestations afférentes.

\section{Régime lié à la rémunération}

Le régime de retraite des salariés comporte une composante à taux uniforme et une composante liée à la rémunération qui est de loin la plus importante. Jusqu'à l'exercice 2002, le taux d'acquisition des droits était de $0.75 \%$ du salaire sans les primes. Depuis l'exercice 2003, la base de calcul de la retraite a été élargie pour inclure les primes. Du fait de cet élargissement, le taux d'acquisition des droits a été ramené à $0.5481 \%$ du salaire (primes incluses). 
Les rémunérations passées sont revalorisées en fonction de l'évolution du salaire net moyen à l'échelle nationale.

La rémunération soumise à cotisations est plafonnée à 620000 JPY par mois, soit $149 \%$ du salaire moyen.

La prestation à taux uniforme dépend de l'année de naissance. En 2006, elle s'échelonnait entre 1676 JPY et 3143 JPY par mois de cotisation. Elle n'est versée qu'aux retraités de 62 à 64 ans et sera progressivement supprimée d'ici à 2013 pour les hommes.

Les retraites mises en paiement sont indexées sur les prix.

\section{Externalisation}

Les employeurs qui emploient au moins 1000 salariés peuvent " externaliser » une partie de la retraite liée à la rémunération (part de substitution) s'ils couvrent eux-mêmes leurs salariés : environ $15 \%$ des salariés participent à ces régimes. L'externalisation impose que les employeurs offrent au moins $150 \%$ (110\% avant 2005) de la prestation qu'aurait assuré le régime public lié à la rémunération. Le calcul de la pension requise pour l'externalisation est basé sur le salaire nominal moyen sur l'ensemble de la carrière. L'indexation des pensions mises en paiement et la revalorisation des rémunérations passées sont financées par l'État.

Le taux de cotisation des régimes externalisés est déterminé par l'État en fonction de la structure des âges des salariés couverts et de l'hypothèse actuarielle. Jusqu'en 1996, toutefois, le taux était uniforme pour tous les régimes. Depuis 2005, il s'échelonne entre $2.4 \%$ et $5 \%$ de la remuneration totale.

Depuis 2001, l'État encourage également les régimes de retraite à cotisations définies et les régimes professionnels à prestations définies, ce qui a conduit à la dissolution de plusieurs fonds de pension de salariés.

\section{Variantes de carrière}

\section{Retraite anticipée}

Jusqu'en 2001, on pouvait bénéficier à 60 ans d'une pension " spéciale " salariés. Celleci est progressivement éliminée et il ne sera plus possible de bénéficier d'une retraite à taux plein avant 65 ans.

Une retraite anticipée à taux réduit est possible aussi bien dans le régime de base que dans les régimes liés à la rémunération. La pension est réduite de $0.5 \%$ par mois d'anticipation soit $6 \%$ par an. Les intéressés peuvent liquider la composante à taux uniforme de la retraite des salariés entre 60 et 65 ans. Les pensions mises en paiement sont indexées sur le salaire moyen net jusqu'à 65 ans et sur les prix après 65 ans.

\section{Retraite différée}

Il est possible de différer la liquidation de la pension de base et de celle liée à la rémunération. La prestation est alors majorée de $0.7 \%$ par mois, soit $8.4 \%$ par an. Les droits à pension continuent à s'accumuler pour chaque année de cotisation au-delà de 65 ans.

À partir de 2006, il est devenu possible de cumuler un emploi et une pension de retraite après 65 ans sous réserve que le revenu total (salaire plus pension) n'excède pas 480000 JPY. Au-delà de cette limite, la pension à taux plein liée à la rémunération sera réduite de la moitié de l'excédent mais la retraite de base sera versée intégralement. 
À partir d'avril 2007, la décote s'est appliquée également aux travailleurs de plus de 70 ans mais ceux-ci n'ont pas de cotisation à payer.

\section{Enfants}

Dans le régime lié à la rémunération, les périodes de non-emploi liées aux enfants sont validées. En 2005, la période maximum a été étendue de un à trois ans. Si d'autres enfants naissent pendant cette période, celle-ci est prolongée jusqu'au troisième anniversaire du dernier enfant. Durant cette période, les cotisations sont considérées reposer totalement sur le salaire perçu juste avant le congé ; pour le calcul du montant de la prestation et l'établissement des conditions d'ouverture des droits l'intégralité de la période d'exemption est validée. Dans le cas de parents travaillant à temps partiel pour pouvoir s'occuper de leurs enfants, la cotisation sera calculée sur la base de la rémunération actuelle mais les prestations de retraite seront calculées sur la base de leur rémunération antérieure à plein-temps.

Si au-delà des trois ans, les personnes restent en dehors du marché du travail et si leur niveau de revenu baisse, les règles d'indemnisation du chômage décrites ci-dessous s'appliquent également.

\section{Chômage}

Les travailleurs qui perdent leur emploi ou dont le revenu est inférieur à un certain niveau n'ont pas à cotiser au régime lié à la rémunération mais ils doivent cotiser au régime de base. Les chômeurs peuvent être exemptés du paiement de l'intégralité, les trois quarts, la moitié ou le quart des cotisations, selon le niveau de revenu du ménage. Une personne seule ayant gagné moins de 570000 JPY l'année précédente est exemptée de toute cotisation. Les personnes dont le revenu est inférieur à 930000 JPY sont autorisées à ne payer qu'un quart de la cotisation, celles dont le revenu est inférieur à 1410000 JPY la moitié et celles dont le revenu est inférieur à 1890000 JPY les trois quarts.

Pour les périodes d'exemption totale, les intéressés sont en droit de percevoir un tiers de la retraite de base. Pour les périodes de paiement d'un quart de la cotisation, ils perçoivent la moitié de la retraite de base. Pour les périodes de paiement de la moitié de la cotisation ils ont droit aux deux tiers de la retraite de base et pour les périodes de paiement des trois quarts de la cotisation, ils sont crédités des cinq sixièmes de la retraite de base. La période d'exemption est comptabilisée comme une période de cotisation intégrale pour l'évaluation des conditions d'ouverture des droits.

Il est possible de payer les cotisations ultérieurement pour percevoir une pension de retraite plus importante. 
Résultats de la modélisation des retraites : Japon
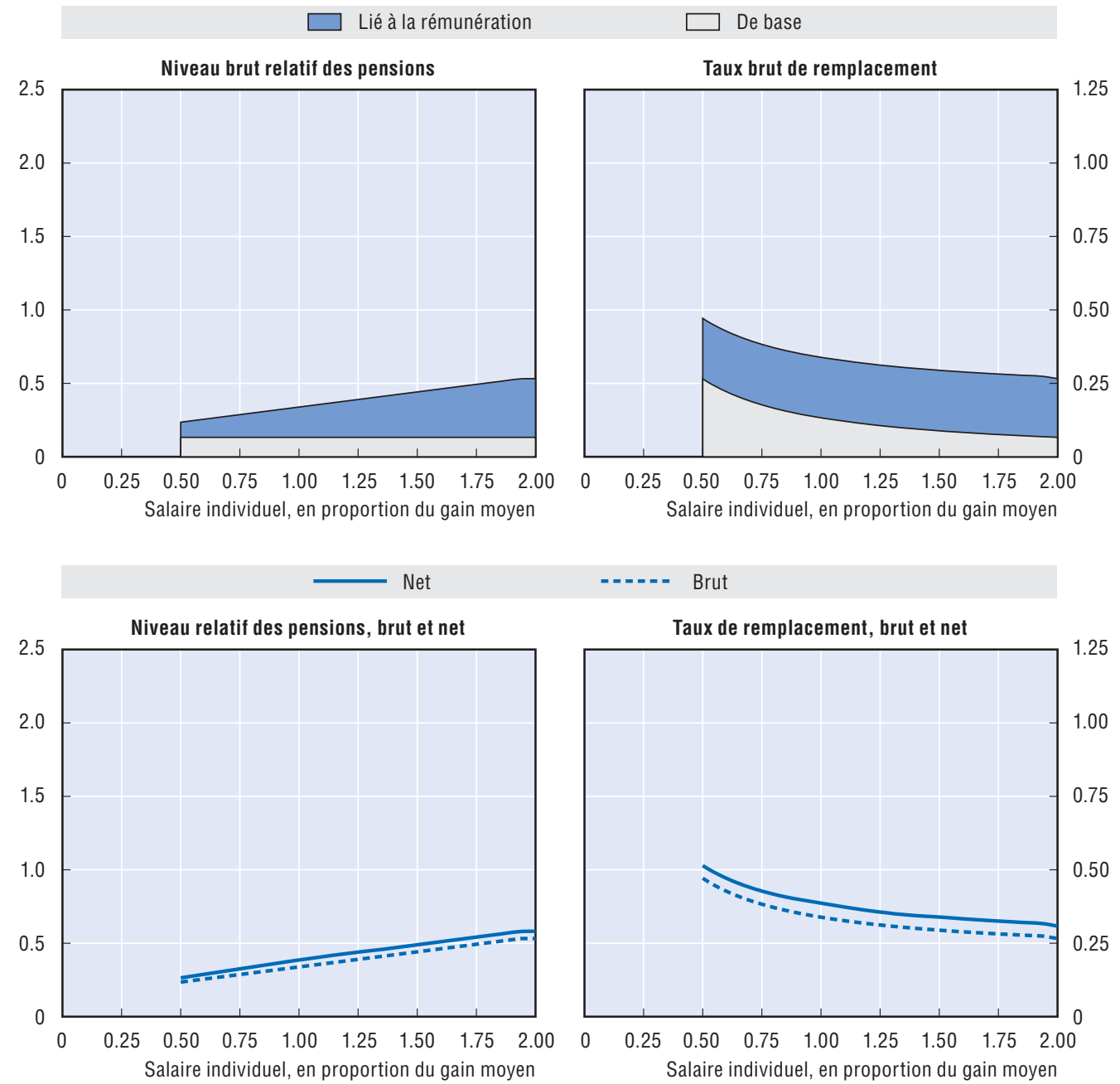

\begin{tabular}{|c|c|c|c|c|c|c|}
\hline \multirow{2}{*}{$\begin{array}{l}\text { Hommes } \\
\text { Femmes (si différent) }\end{array}$} & \multirow{2}{*}{$\begin{array}{l}\text { Salarié à revenu } \\
\text { médian }\end{array}$} & \multicolumn{5}{|c|}{ Salaire individuel, en multiple du salaire moyen } \\
\hline & & 0.5 & 0.75 & 1 & 1.5 & 2 \\
\hline Niveau brut relatif des pensions & 31.4 & 23.6 & 28.7 & 33.9 & 44.2 & 53.2 \\
\hline \multicolumn{7}{|l|}{ ( $\%$ salaire moyen brut) } \\
\hline Niveau net relatif des pensions & 35.8 & 26.4 & 32.6 & 38.7 & 48.9 & 58.1 \\
\hline \multicolumn{7}{|l|}{ (\% salaire moyen net) } \\
\hline Taux brut de remplacement & 35.7 & 47.1 & 38.3 & 33.9 & 29.4 & 26.6 \\
\hline \multicolumn{7}{|l|}{ (\% salaire individuel brut) } \\
\hline Taux net de remplacement & 40.3 & 51.4 & 42.8 & 38.7 & 33.9 & 30.8 \\
\hline \multicolumn{7}{|l|}{ (\% salaire individuel net) } \\
\hline Patrimoine retraite brut & 5.9 & 7.8 & 6.3 & 5.6 & 4.9 & 4.4 \\
\hline (multiple du salaire individuel brut) & 6.7 & 8.8 & 7.1 & 6.3 & 5.5 & 5.0 \\
\hline Patrimoine retraite net & 5.4 & 7.1 & 5.8 & 5.2 & 4.4 & 3.9 \\
\hline (multiple du salaire individuel brut) & 6.1 & 7.9 & 6.5 & 5.8 & 4.9 & 4.4 \\
\hline
\end{tabular}




\section{Luxembourg}

\section{Luxembourg : système de retraite en 2006}

Le régime public de retraite repose sur deux composantes : l'une forfaitaire qui dépend du nombre d'années de couverture et l'autre liée à la rémunération. Il comporte également une pension minimum.

\section{Indicateurs essentiels}

\begin{tabular}{llrr}
\hline & & Luxembourg & OCDE \\
\hline Salaire & EUR & 43600 & 28600 \\
moyen & USD & 54800 & 35800 \\
Dépenses publiques & $\%$ du PIB & 7.2 & 7.2 \\
au titre des retraites & & & \\
Espérance de vie & à la naissance & 79.4 & 78.9 \\
& à 65 ans & 83.7 & 83.4 \\
Population de plus de 65 ans & \% de la population & 22.8 & 23.8 \\
& d'âge actif & & \\
\hline
\end{tabular}

\section{Conditions d'ouverture des droits}

Une pension anticipée peut être versée à partir de 57 ans avec 40 années de cotisation (obligatoire ou facultative). Avec quarante années de cotisation obligatoire ou facultative ou quarante années validées, la pension peut être versée à partir de 60 ans. La modélisation supposant qu'une carrière complète démarre à 20 ans, elle suppose dans l'hypothèse de base que les travailleurs partent à la retraite à 60 ans. Sinon, l'âge normal de la retraite est de 65 ans (sous réserve d'avoir cotisé au minimum dix ans).

\section{Calcul des prestations}

\section{Régime de base}

En 2006, la prestation de base était de 362 EUR par mois, sous réserve de 40 années de couverture, soit l'équivalent d'environ $10 \%$ du salaire moyen. Pour une durée d'assurance incomplète, la prestation est réduite au prorata. (Officiellement, la retraite de base est égale à $23.5 \%$ d'un montant de référence qui était, en 2006, de 1541 EUR.)

À cela s'ajoute une "allocation de fin d'année " qui majore de 49 EUR par mois la pension versée pour 40 années de cotisation. Celle-ci est réduite au prorata pour les périodes d'assurance inférieures à 40 ans, ce qui implique environ 1.23 EUR par mois par année couverte. L'allocation de fin d'année est indexée sur le salaire nominal (voir ci-dessous).

\section{Régime lié à la rémunération}

Pour la pension liée à la rémunération, le taux d'acquisition des droits est de $1.8 \%$. Le salaire de référence utilisé dans la formule de calcul est la rémunération moyenne sur l'ensemble de la carrière revalorisé en fonction de l'évolution du salaire nominal.

Le taux d'acquisition est plus élevé pour les travailleurs âgés et pour ceux qui ont cotisé plus longtemps. Pour chaque année de travail au-delà de 55 ans, il augmente de 0.01 point de pourcentage. En outre, chaque année de cotisation au-delà de 38 entraîne également une augmentation supplémentaire du taux d'acquisition de 0.01 point de pourcentage. Le taux maximum d'acquisition des droits est de $2.05 \%$ par an. Dans l'hypothèse type d'une carrière complète démarrant à 20 ans, il est de $2.01 \%$.

En 2006, la pension maximum était de 6422 EUR par mois (formellement définie comme $25 / 6$ du montant de référence), soit un peu moins de $177 \%$ du salaire moyen. 
Les prestations sont automatiquement indexées sur l'évolution du coût de la vie (si l'inflation cumulée est d'au minimum $2.5 \%$ ). En outre, des ajustements en fonction de la hausse réelle des salaires doivent être envisagés tous les deux ans. La pratique récente a été des hausses proches de celles des salaires et la modélisation suppose la poursuite de cette pratique.

\section{Minimum}

La pension minimum est de 1387 EUR par mois (définis comme $90 \%$ du montant de référence), sous réserve de 40 années de couverture, soit l'équivalent d'environ $38 \%$ du salaire moyen. Pour des périodes de couverture plus courtes, elle est réduite au prorata sous réserve d'un minimum de 20 années d'activité (cotisations obligatoires, facultatives ou périodes validées).

\section{Aide sociale}

Le montant du filet de sécurité que constitue l'aide sociale est de 1098 EUR par mois pour une personne seule.

\section{Variantes de carrière}

\section{Retraite anticipée}

Il est possible de partir à la retraite à 57 ans avec 40 années de cotisations versées et à 60 ans avec 40 années de cotisations versées ou validées. Les personnes parties à la retraite anticipée peuvent travailler de manière occasionnelle sous réserve que leur rémunération n'excède pas un tiers du revenu social minimum. Les prestations de retraite anticipée ne font l'objet d'aucun ajustement actuariel.

Il existe en outre un certain nombre de programmes de préretraite. Nous retiendrons ici les programmes de préretraite-solidarité et de préretraite ajustement. Le premier permet un départ en retraite anticipée à la condition que l'employeur recrute un demandeur d'emploi qui lui est affecté par les services de l'emploi. Le second permet le départ en retraite anticipée des travailleurs âgés perdant leur emploi suite à une restructuration ou à une faillite. Les deux régimes s'appliquent à partir de 57 ans et jusqu'à 60 ans. La prestation de retraite anticipée est de $85 \%$ du salaire antérieur la première année, de $80 \%$ la deuxième et de $75 \%$ la troisième. Le salaire de référence est le salaire des trois derniers mois.

\section{Retraite différée}

La retraite doit être liquidée à l'âge normal de 65 ans. Au-delà, un cumul emploiretraite est possible sans réduction de la prestation de retraite.

\section{Enfants}

Des « années enfants » (deux ans pour un enfant et quatre pour deux) sont validées en années de cotisation. La rémunération ouvrant droit à pension est celle perçue immédiatement avant la demande de validation. La période est prise en compte dans les conditions d'ouverture des droits et elle entre dans la composante forfaitaire de la formule de calcul des pensions. 
Les salariés ne pouvant prétendre à cette validation en raison d'une durée de cotisation insuffisante ont droit à une indemnité mensuelle spéciale de retraite de 89 EUR par enfant.

Les périodes de non-cotisation liées à l'éducation d'enfants de moins de six ans sont prises en compte dans l'établissement des conditions d'ouverture des droits.

\section{Chômage}

Les périodes de chômage indemnisé sont validées : les cotisations de retraite provenant des prestations sont prises en charge par l'État $(2 / 3)$ et par le bénéficiaire $(1 / 3)$. Les périodes de chômage sont prises en compte dans l'établissement des conditions d'ouverture des droits et entrent dans la composante liée à la rémunération de la formule de calcul des pensions. Pour ces périodes, c'est l'allocation de chômage qui sert de base au calcul des pensions. 
Résultats de la modélisation des retraites : Luxembourg

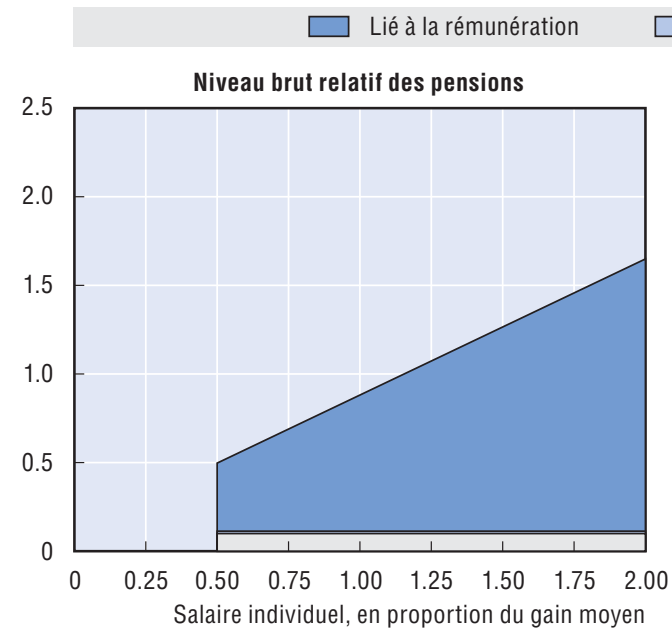

\author{
Fin d'année \\ De base
}
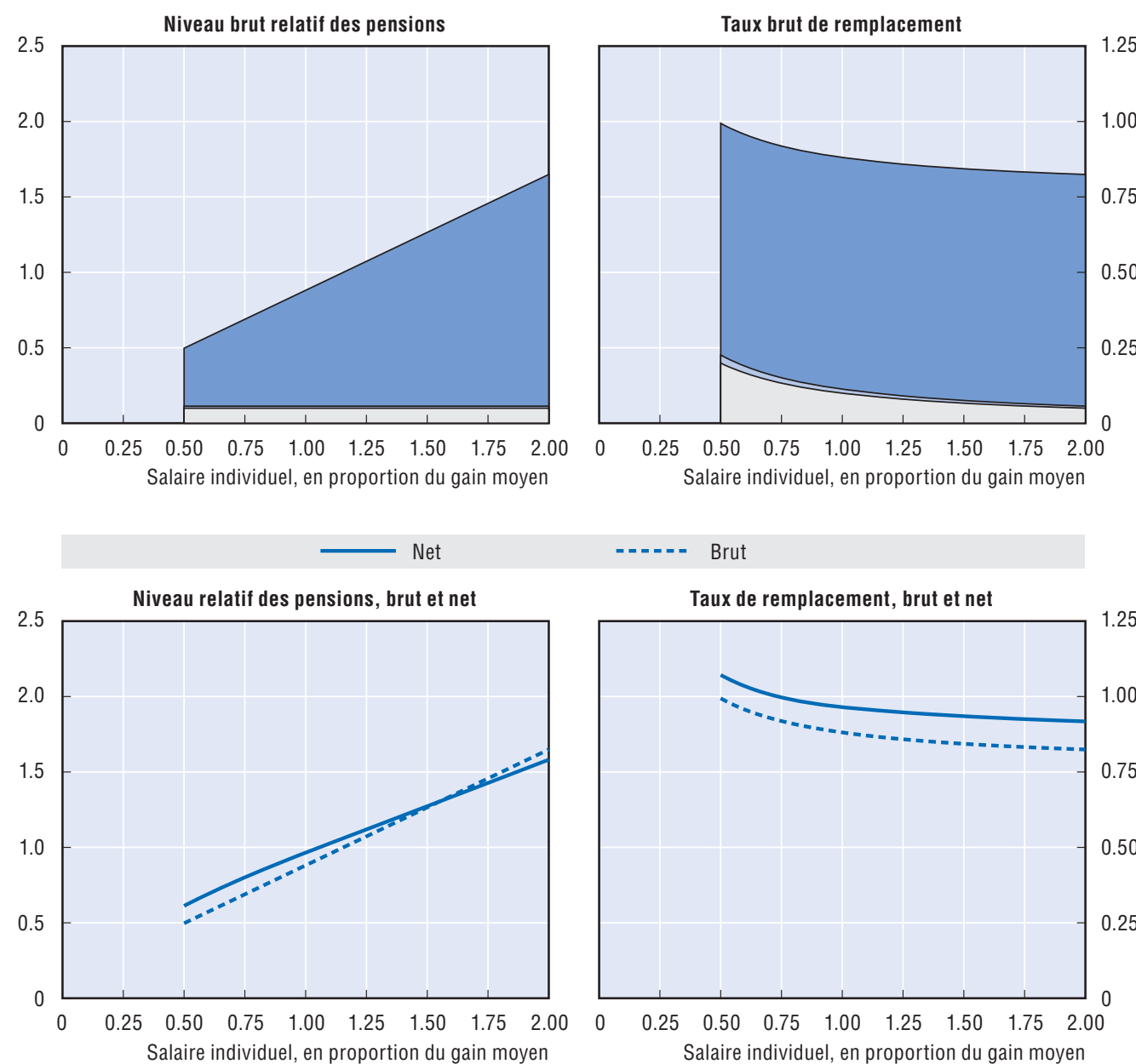

$---=-1$ Brut

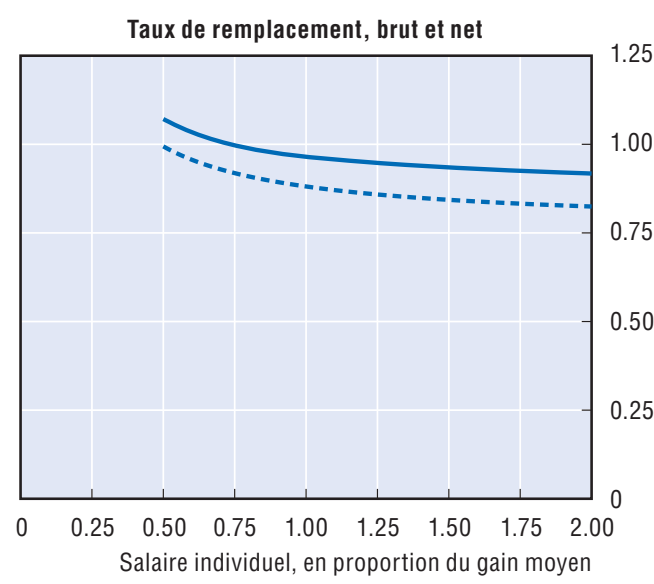

\begin{tabular}{lc|ccccc}
\hline \multirow{2}{*}{$\begin{array}{l}\text { Hommes } \\
\text { Femmes (si différent) }\end{array}$} & $\begin{array}{c}\text { Salarié à revenu } \\
\text { médian }\end{array}$ & \multicolumn{5}{|c}{ Salaire individuel, en multiple du salaire moyen } \\
\cline { 3 - 6 } & & 0.5 & 0.75 & 1 & 1.5 & 2 \\
\hline $\begin{array}{l}\text { Niveau brut relatif des pensions } \\
\text { (\% salaire moyen brut) }\end{array}$ & 76.6 & 49.7 & 68.9 & 88.1 & 126.5 & 164.9 \\
$\begin{array}{l}\text { Niveau net relatif des pensions } \\
\text { (\% salaire moyen net) }\end{array}$ & 87.0 & 61.2 & 80.1 & 96.5 & 127.3 & 158.2 \\
$\begin{array}{l}\text { Taux brut de remplacement } \\
\text { (\% salaire individuel brut) }\end{array}$ & 90.1 & 99.4 & 91.9 & 88.1 & 84.3 & 82.5 \\
$\begin{array}{l}\text { Taux net de remplacement } \\
\text { (\% salaire individuel net) }\end{array}$ & & & & & & \\
Patrimoine retraite brut & 98.1 & 107.1 & 99.7 & 96.5 & 93.5 & 91.8 \\
(multiple du salaire individuel brut) & & & & & & 18.0 \\
Patrimoine retraite net & 19.7 & 21.7 & 20.0 & 19.2 & 18.4 & 22.0 \\
(multiple du salaire individuel brut) & 24.0 & 26.5 & 24.5 & 23.5 & 22.5 & 12.4 \\
\hline
\end{tabular}




\section{Mexique}

\section{Mexique : système de retraite en 2006}

Le système de retraite mexicain est un régime par capitalisation, à gestion privée et cotisations définies, obligatoire pour les travailleurs du secteur privé. Les cotisations sont versées par les salariés, les employeurs et l'État. Il existe également une pension minimum pour les personnes ayant cotisé un minimum de 24 années.
Indicateurs essentiels

\begin{tabular}{llrr}
\hline & & Mexique & OCDE \\
\hline Salaire & MXN & 73200 & 390200 \\
moyen & USD & 6700 & 35800 \\
Dépenses publiques & $\%$ du PIB & 1.3 & 7.2 \\
au titre des retraites & & & \\
Espérance de vie & à la naissance & 75.7 & 78.9 \\
& à 65 ans & 83.0 & 83.4 \\
Population de plus de 65 ans & \% de la population & 9.9 & 23.8 \\
& d'âge actif & &
\end{tabular}

\section{Conditions d'ouverture des droits}

L'âge normal de la retraite est de 65 ans pour les hommes comme pour les femmes sous réserve de pouvoir justifier de 1250 semaines (environ 24 années) de cotisation.

\section{Calcul des prestations}

\section{Régime par capitalisation}

Travailleurs et employeurs versent sur un compte individuel un montant total de cotisations égal à $6.275 \%$ du salaire auquel s'ajoute une contribution de l'État équivalant à $0.225 \%$ du salaire. Une cotisation supplémentaire de $5 \%$ est versée sur un compte logement individuel (régime Infonavit) qui la reverse au compte retraite lorsqu'elle n'est pas utilisée. Enfin, l'État verse sur tous les comptes de retraite individuels $5.5 \%$ du salaire minimum réel de 1997 indexé sur l'inflation ; on considère que c'est l'équivalent d'une pension de base représentant ici $1.4 \%$ du salaire moyen.

Les cotisations sont plafonnées à 24 fois le salaire minimum pour la période allant de juillet 2006 à juin 2007 et à 25 fois le salaire minimum ensuite.

Les calculs supposent qu'à l'âge normal de la retraite l'individu convertit le solde cumulé de son compte (après déduction d'une assurance qui doit être souscrite pour couvrir les prestations de réversion) en rente indexée sur les prix. Les taux de rente varient en fonction du sexe.

\section{Pension minimum}

Le montant de la pension minimum est équivalent au même salaire minimum réel de 1997 indexé sur l'inflation (20 225.40 MXN en 2006). Le fait qu'elle soit liée au salaire minimum réel signifie qu'elle est en fait indexée sur les prix.

\section{Variantes de carrière}

\section{Retraite anticipée}

Un départ en retraite anticipée est possible à partir de 60 ans pour les hommes comme pour les femmes à condition que le travailleur soit sans emploi et qu'il ait cotisé au minimum 1250 semaines. 
Les travailleurs quittant définitivement le marché du travail qui ne remplissent pas les conditions requises pour percevoir la pension minimum peuvent liquider le solde intégral de leur compte à cotisations définies (Afore).

\section{Retraite différée}

Il est possible de différer le départ à la retraite après 65 ans.

\section{Enfants}

Les périodes de non-emploi liées aux enfants ne sont pas validées.

\section{Chômage}

Les périodes de chômage ne sont pas validées.

L'article 191, partie II, de la Loi mexicaine sur la sécurité sociale établit toutefois que lorsqu'un travailleur perd son emploi, il est en droit de tirer de l'argent sur son compte secondaire d'épargne vieillesse/retraite. Le montant qu'il est autorisé à prélever est de 75 jours du salaire de base des 250 dernières semaines ou $10 \%$ du solde de son compte secondaire d'épargne vieillesse/retraite si ce montant est inférieur. Les travailleurs peuvent prétendre à ce montant à compter du quarante-sixième jour de chômage. Ils sont éligibles à cette prestation si leur compte est créditeur et s'ils n'ont effectué aucun retrait durant les cinq dernières années. 
Résultats de la modélisation des retraites : Mexique

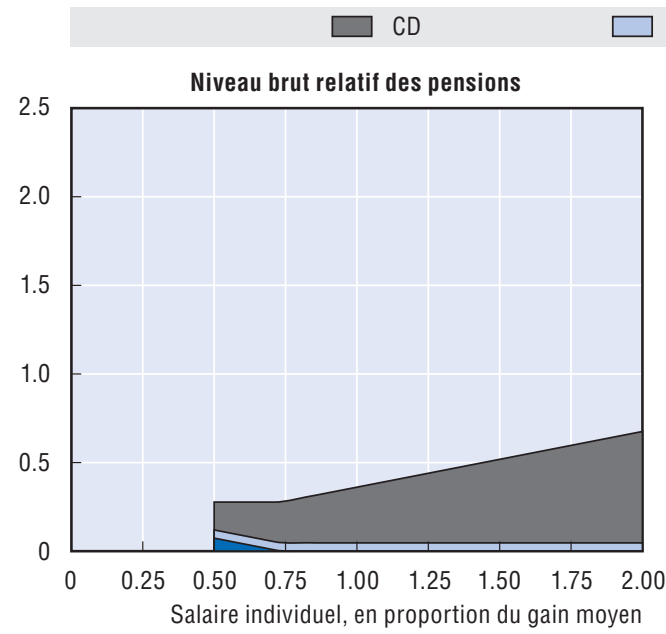

CD fixes

Ciblé
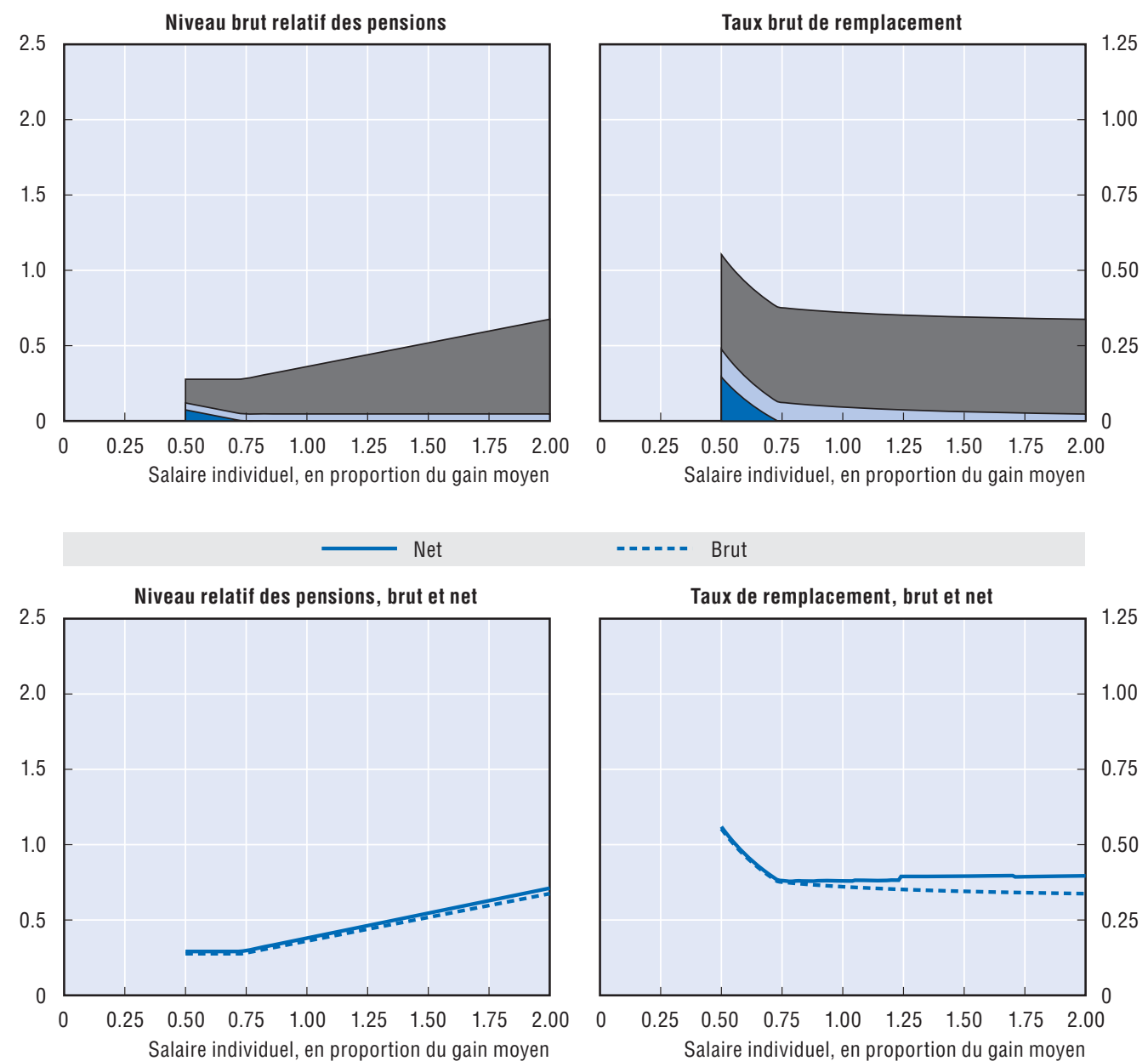

- -n-n'But

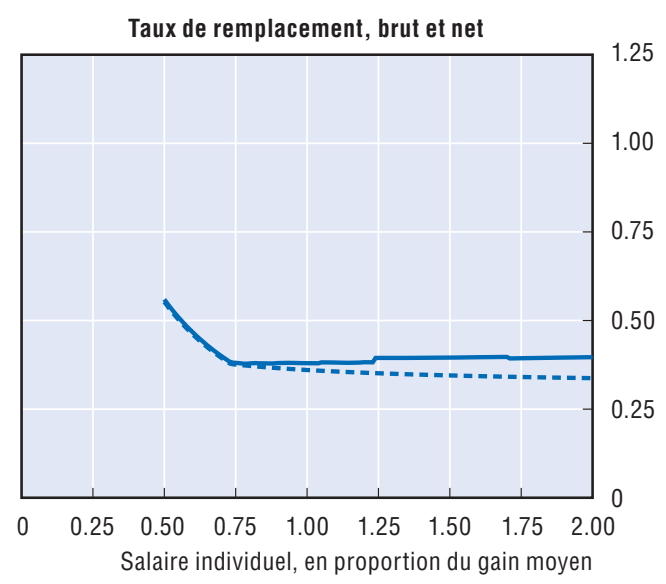

\begin{tabular}{lc|ccccc}
\hline \multirow{2}{*}{$\begin{array}{l}\text { Hommes } \\
\text { Femmes (si différent) }\end{array}$} & $\begin{array}{c}\text { Salarié à revenu } \\
\text { médian }\end{array}$ & \multicolumn{4}{|c}{ Salaire individuel, en multiple du salaire moyen } \\
\cline { 3 - 6 } & & 0.5 & 0.75 & 1 & 1.5 & 2 \\
\hline Niveau brut relatif des pensions & 31.3 & 27.6 & 28.2 & 36.1 & 51.8 & 67.5 \\
(\% salaire moyen brut) & 27.6 & & 27.6 & 29.9 & 42.9 & 56.0 \\
Niveau net relatif des pensions & 33.0 & 29.1 & 29.7 & 38.0 & 54.6 & 71.2 \\
(\% salaire moyen net) & 29.1 & & 29.1 & 31.5 & 45.3 & 59.0 \\
Taux brut de remplacement & 36.9 & 55.3 & 37.6 & 36.1 & 34.5 & 33.7 \\
(\% salaire individuel brut) & 32.5 & & 36.8 & 29.9 & 28.6 & 28.0 \\
Taux net de remplacement & 38.0 & 56.0 & 38.1 & 38.0 & 39.6 & 39.7 \\
(\% salaire individuel net) & 33.5 & & 37.3 & 31.5 & 32.8 & 32.9 \\
Patrimoine retraite brut & 4.9 & 7.3 & 5.0 & 4.8 & 4.6 & 4.5 \\
(multiple du salaire individuel brut) & 5.2 & 8.9 & 5.9 & 4.8 & 4.6 & 4.5 \\
Patrimoine retraite net & 4.9 & 7.3 & 5.0 & 4.8 & 4.6 & 4.5 \\
(multiple du salaire individuel brut) & 5.2 & 8.9 & 5.9 & 4.8 & 4.6 & 4.5 \\
\hline
\end{tabular}




\section{Norvège}

\section{Norvège : système de retraite en 2006}

En Norvège, le système public de retraite se compose d'une pension de base forfaitaire et d'une pension (complémentaire) liée à la rémunération. Les retraités n'ayant pas de retraite complémentaire, ou une retraite complémentaire modeste, ont droit à un complément spécial. Ce complément est soumis à une condition de ressources sur la base de la pension liée à la rémunération. Une retraite professionnelle obligatoire a été récemment mise en place.
Indicateurs essentiels

\begin{tabular}{llrr}
\hline & & Norvège & \multicolumn{1}{c}{ OCDE } \\
\hline Salaire & NOK & 397800 & 229500 \\
moyen & USD & 62000 & 35800 \\
Dépenses publiques & \% du PIB & 4.8 & 7.2 \\
au titre des retraites & & & \\
Espérance de vie & à la naissance & 80.6 & 78.9 \\
& à 65 ans & 84.3 & 83.4 \\
Population de plus de 65 ans & \% de la population & 24.7 & 23.8 \\
& d'âge actif & & \\
\hline
\end{tabular}

\section{Conditions d'ouverture des droits}

Le régime public actuel a été mis en place en 1967. Comme la période d'activité requise pour percevoir une retraite à taux plein est de 40 ans, la première cohorte de travailleurs à remplir cette condition est celle qui est partie à la retraite en 2007.

Lâge normal de la retraite est de 67 ans. Les personnes ayant résidé en Norvège au minimum trois ans entre 17 et 67 ans (inclus) ont droit à la pension minimum qui est composée de la pension de base et du complément spécial. Les prestations à taux plein sont acquises après 40 années de résidence. Pour la pension complémentaire il faut avoir acquis des points de retraite pendant au minimum trois ans et le taux plein est accordé au bout de 40 ans. Ces deux prestations sont réduites au prorata pour des durées d'activité plus courtes.

\section{Calcul des prestations}

\section{Régime de base}

Dans le système national d'assurance norvégien, un grand nombre de prestations sont calculées par rapport à un montant de base, G, qui était en 2006 de 62161 NOK. La pension de base à taux plein pour une personne seule est égale à ce montant de base, qui équivaut à $15.6 \%$ du salaire moyen. Dans le passé, l'indexation du montant de base a généré des augmentations inférieures à la hausse moyenne des salaires. Mais, depuis 2003, le gouvernement lie la valeur de ce montant à l'évolution du salaire moyen. La modélisation suppose la poursuite de cette pratique.

\section{Pension minimum}

La pension minimum se compose de la pension de base décrite ci-dessus et du complément spécial. Elle a été relevée par intermittence lorsque le complément spécial a 
augmenté en valeur réelle. Au fil du temps, elle a eu tendance à augmenter plus vite que les salaires. Depuis 2003, la pension minimum est indexée sur le salaire moyen.

En 2008, toutefois, une nouvelle augmentation du complément spécial a été décidée : de $79.33 \%$ du montant de base en 2007 il a été porté à $94 \%$ en 2008. Il a été convenu de le porter à $100 \%$ du montant de base à l'horizon 2010. Ce complément est soumis à une condition de ressources sur la base de la pension complémentaire liée à la rémunération et la pension minimum correspond à environ $31 \%$ du salaire moyen.

\section{Régime lié à la rémunération}

Dans la mesure où la pension de base remplace la première tranche de salaire, le régime lié à la rémunération ne couvre que les gains au-delà du montant de base. Le complément spécial remplace donc une tranche supplémentaire du salaire, à concurrence de 3.38 fois le montant de base. Le régime lié à la rémunération utilise une formule régressive, autrement dit le taux de remplacement diminue pour les hauts revenus. Pour les rémunérations annuelles comprises entre 3.38 fois et six fois le montant de base, le taux de remplacement est de $42 \%$ (contre $45 \%$ en 1992) ; il correspond pour chaque retraité à la moyenne de ces deux taux pondérée par le nombre d'années d'acquisition de points antérieures à 1992). Entre six et douze fois le montant de base, le taux de remplacement est égal au tiers de ce chiffre. Étant donné que 40 années de cotisation sont nécessaires pour percevoir une retraite à taux plein, cela équivaut à des taux d'acquisition annuels (pour les personnes ayant acquis la totalité de leurs droits après 1992) de $1.05 \%$ et $0.35 \%$ respectivement. Le premier seuil à partir duquel le taux d'acquisition des droits diminue est un peu inférieur au salaire moyen (94\%). Le plafond de rémunération ouvrant droit à prestations est donc un peu inférieur à deux fois le salaire moyen (188\%).

La pension est calculée sur la base des 20 meilleures années en termes de points acquis. La revalorisation des points acquis au cours des années antérieures dépend de la procédure d'ajustement de la valeur du montant de base $(G)$. Comme indiqué précédemment, la modélisation suppose qu'à l'avenir la revalorisation du montant de base sera alignée sur le salaire moyen.

\section{Régime à cotisations définies}

Depuis 2006, les employeurs sont tenus de verser à un plan de retraite à cotisations définies une cotisation minimum égale à $2 \%$ de la rémunération de leurs salariés. (S'ils proposent un régime à prestations définies, le montant des prestations doit être au moins égal au montant attendu de la cotisation obligatoire de $2 \%$. Seule est soumise à cotisations la partie du salaire comprise entre le montant de base $(G)$ et 12 fois ce montant.

Actuellement, les pensions ne peuvent être liquidées qu'à 67 ans et le retrait doit s'étaler sur une période minimum de dix ans. Pour faciliter la comparaison avec les autres pays, on suppose une sortie sous forme de rente indexée sur les prix et calculée à l'aide de tables de mortalité unisexe.

\section{Pensions privées facultatives}

Environ $60 \%$ des salariés sont couverts par des régimes de retraite professionnels facultatifs. Si l'on inclut la cotisation patronale obligatoire de $2 \%$, un régime type aurait un taux de cotisation de $5 \%$ des salaires entre $1 \mathrm{G}$ et $6 \mathrm{G}$ et de $8 \%$ entre $6 \mathrm{G}$ et $12 \mathrm{G}$. 


\section{Variantes de carrière (régime public)}

\section{Retraite anticipée}

Les deux tiers environ des salariés travaillent dans des entreprises qui participent à des programmes de préretraite dans le cadre du régime contractuel de retraite anticipée (AFP). Ce régime, mis en place en 1989, permet un départ à la retraite à partir de 62 ans. Le niveau de la pension servie à ce titre est à peu près identique à celui d'une pension de vieillesse normale, autrement dit à ce que la personne aurait touché si elle avait continué à travailler jusqu'à 67 ans dans l'emploi qu'elle occupait au moment où elle est effectivement partie à la retraite.

Le calcul des pensions AFP diffère selon le secteur. Dans le secteur privé, ces pensions sont calculées de la même façon que la pension d'invalidité permanente: elles donnent droit à des points de retraite pour les années restantes jusqu'à 67 ans. Les retraités perçoivent en outre un " complément AFP » de 11400 NOK par an, qui n'est pas imposable.

L'ouverture des droits est soumise à quelques conditions (la liste n'est pas exhaustive). La première est que le retraité doit avoir été salarié de la même entreprise au cours des trois dernières années (ou couvert par un régime AFP pendant les cinq dernières années). La seconde est que sa rémunération annuelle doit être au moins égale au montant de base (G) au moment où il part à la retraite. Son salaire annuel doit également être supérieur au montant de base $(G)$ durant au moins dix ans à compter de son $50^{\mathrm{e}}$ anniversaire. Et les gains des dix meilleures années de la période allant de 1967 à l'année précédant son départ à la retraite doivent être supérieurs à au moins deux fois le montant de base.

\section{Retraite différée}

Il est possible de partir à la retraite après 67 ans et de continuer à travailler en cumulant un salaire et une pension. Le report de la retraite après 67 ans ne génère pas de surcote supplémentaire.

Initialement, l'âge de la retraite était fixé à 70 ans mais en 1973 il a été ramené à 67 ans. La possibilité d'acquérir des points en fonction d'un revenu du travail jusqu'à 70 ans a été maintenue mais pour la tranche d'âge 69-70 ans, la pension est soumise à une condition de revenu du travail. La pension est réduite de $40 \%$ du revenu excédant deux montants de base $(G)$.

\section{Enfants}

Dans le régime de retraite complémentaire lié à la rémunération, les aidants naturels sont crédités de trois points de retraite par an, ce qui correspond au nombre de points acquis pour un revenu du travail de 248644 NOK. Le terme d'aidants naturels désigne les parents qui élèvent des enfants de moins de sept ans et les individus qui s'occupent de personnes handicapées, malades ou âgées à leur domicile sans recevoir de rémunération en échange.

Les mères qui acquièrent sur l'année moins de trois points de retraite ont droit à un complément. Celles qui en acquièrent plus de trois n'y ont pas droit. La famille peut demander à ce que les points soient attribués au père. Pour l'autre groupe, les points sont attribués sur la base des demandes individuelles.

\section{Chômage}

Les indemnités de chômage, qui sont fixées à $62.4 \%$ de l'ancien salaire jusqu'à six fois le montant de base, valent des points de retraite de la même façon que les revenus salariaux. 
Résultats de la modélisation des retraites : Norvège

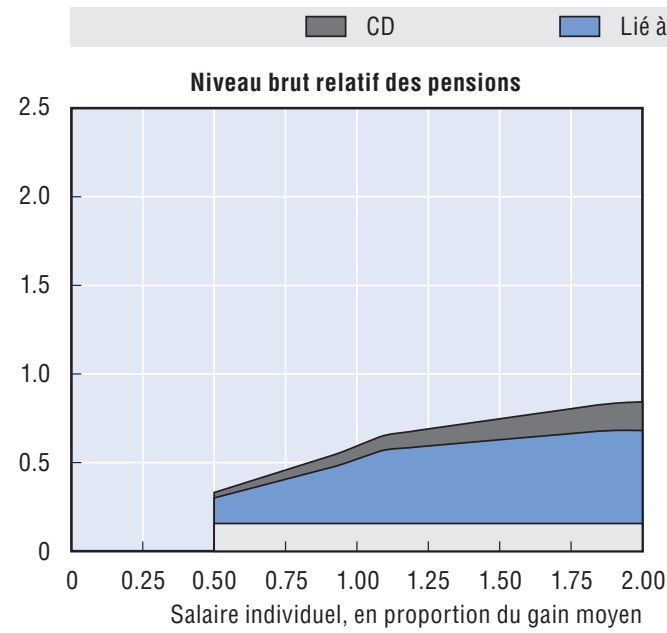

Lié à la rémunération

De base
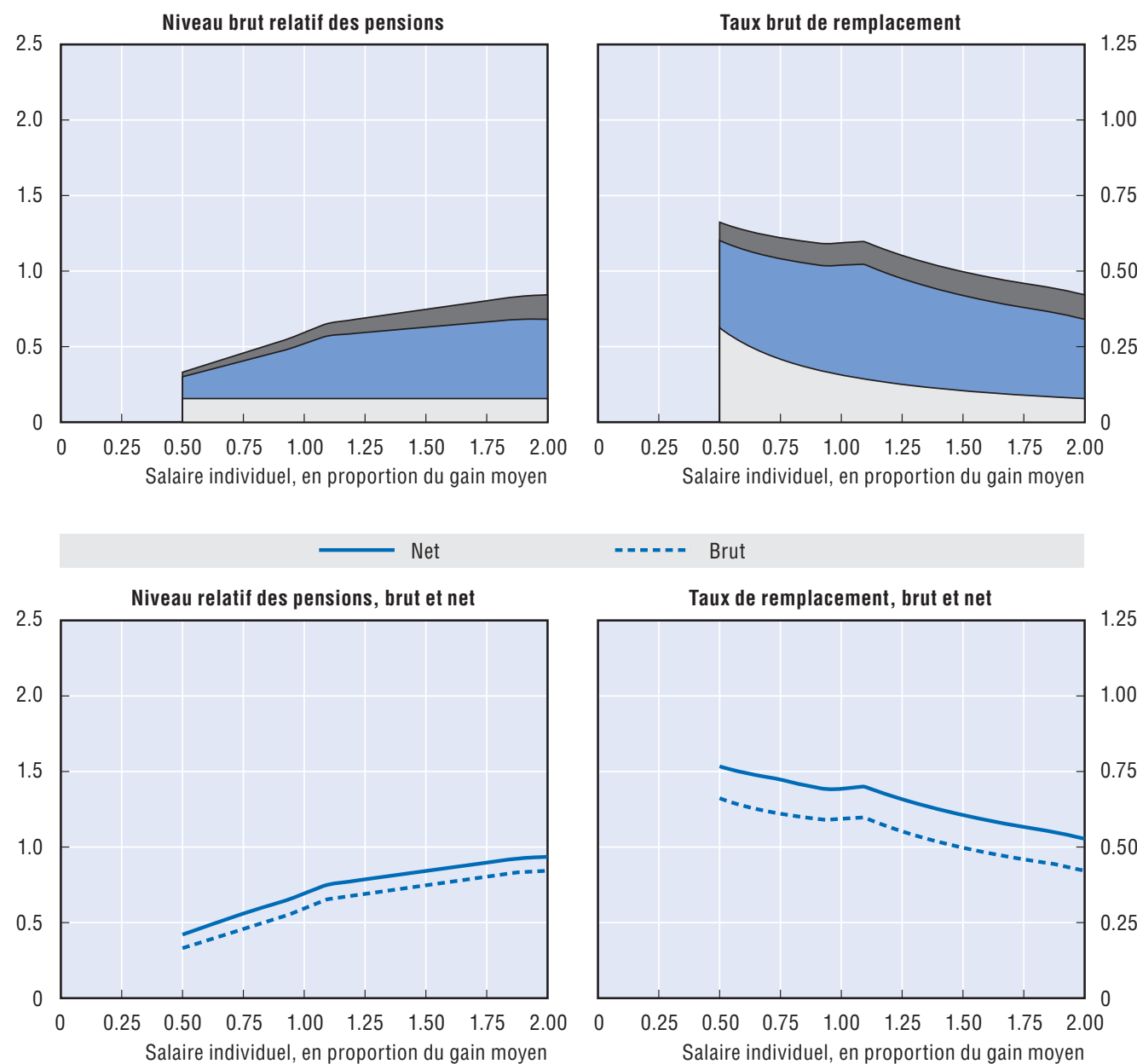

--1---1. Brut

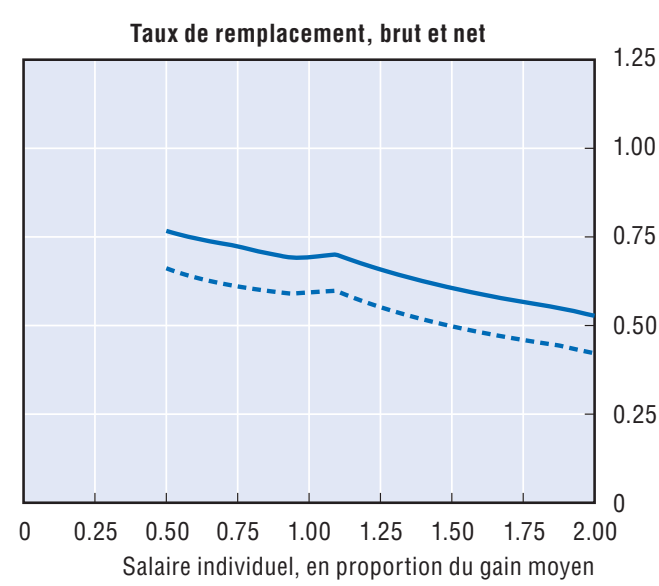

\begin{tabular}{lc|ccccc}
\hline \multirow{2}{*}{$\begin{array}{l}\text { Hommes } \\
\text { Femmes (si différent) }\end{array}$} & $\begin{array}{c}\text { Salarié à revenu } \\
\text { médian }\end{array}$ & \multicolumn{5}{|c}{ Salaire individuel, en multiple du salaire moyen } \\
\cline { 3 - 6 } & & 0.5 & 0.75 & 1 & 1.5 & 2 \\
\hline $\begin{array}{l}\text { Niveau brut relatif des pensions } \\
\text { (\% salaire moyen brut) }\end{array}$ & 51.9 & 33.1 & 45.8 & 59.3 & 74.7 & 84.3 \\
$\begin{array}{l}\text { Niveau net relatif des pensions } \\
\text { (\% salaire moyen net) }\end{array}$ & 62.0 & 42.1 & 56.1 & 69.3 & 84.2 & 93.6 \\
Taux brut de remplacement & & & & & & \\
(\% salaire individuel brut) & 59.6 & 66.2 & 61.0 & 59.3 & 49.8 & 42.2 \\
$\begin{array}{l}\text { Taux net de remplacement } \\
\text { (\% salaire individuel net) }\end{array}$ & 70.2 & 76.7 & 72.3 & 69.3 & 60.6 & 52.8 \\
Patrimoine retraite brut & & & & & & \\
(multiple du salaire individuel brut) & 10.3 & 11.4 & 10.5 & 10.2 & 8.5 & 7.2 \\
Patrimoine retraite net & 12.0 & 13.4 & 12.3 & 11.9 & 9.9 & 8.4 \\
(multiple du salaire individuel brut) & 8.7 & 10.3 & 9.1 & 8.4 & 6.8 & 5.6 \\
\hline
\end{tabular}




\section{Nouvelle-Zélande}

\section{Nouvelle-Zélande : système de retraite en 2006}

Le système repose sur une pension publique forfaitaire soumise à condition de résidence. Il existe certes des régimes professionnels mais leur couverture s'est réduite depuis 1990, passant de $22.6 \%$ de la population active à $14.7 \%$ pour l'exercice clos le 30 juin 2006.
Indicateurs essentiels

\begin{tabular}{llrr}
\hline & & Nouvelle-Zélande & OCDE \\
\hline Salaire moyen & NZD & 43000 & 55100 \\
& USD & 27900 & 35800 \\
Dépenses publiques & $\%$ du PIB & 4.4 & 7.2 \\
au titre des retraites & & & \\
Espérance de vie & à la naissance & 80.2 & 78.9 \\
Population de plus de 65 ans & à 65 ans & 84.4 & 83.4 \\
& d'âge de lactif population & 20.7 & 23.8 \\
& & & \\
\hline
\end{tabular}

\section{Conditions d'ouverture des droits}

Les personnes ayant résidé dix ans dans le pays depuis l'âge de 20 ans (dont cinq après 50 ans) sont en droit de percevoir une retraite publique à 65 ans.

\section{Calcul des prestations}

\section{Régime de base}

Depuis le $1^{\mathrm{er}}$ avril 2007, le montant brut de la pension pour une personne seule est de 336.65 NZD par semaine. Pour l'année 2006/07, il a été de 320.13 NZD. L'augmentation résulte pour partie du processus normal d'ajustement évoqué ci-après et pour partie de l'accord de confiance et de soutien (également évoqué ci-après) passé par le gouvernement avec le parti New Zealand First. Ce qui donne pour l'exercice 2007 une pension totale de 17506 NZD, équivalant à environ 41 \% du salaire moyen.

Les droits à pension publique acquis dans d'autres pays sont pris en compte dans le calcul du montant total à payer.

Le taux de la retraite publique est indexé sur les prix mais avec un plancher et un plafond liés à l'évolution des salaires. Pour un couple, la législation en vigueur impose qu'au $1^{\mathrm{er}}$ avril de chaque année le taux net d'impôt ne soit pas inférieur à $65 \%$ ou supérieur à $72.5 \%$ du salaire de référence hebdomadaire net d'impôt observé lors d'enquêtes. Pour une personne seule, les taux nets d'impôt sont fixés à 65 \% (si elle vit seule) et à 60 \% (si elle est en colocation) du taux net d'impôt pour un couple. Si l'évolution des prix reste constamment inférieure à l'évolution du salaire hebdomadaire net d'impôt observé lors d'enquêtes, c'est ce dernier qui devient l'indice effectif.

Suite à l'accord de confiance et de soutien passé par le gouvernement néozélandais et le parti NZ First après l'élection de 2005, le taux net d'impôt au $1^{\mathrm{er}}$ avril passera de $65 \%$ à 66 \% du salaire de référence net d'impôt pour toute la durée de l'accord.

\section{Pensions privées facultatives}

Depuis quelque temps, la couverture des plans professionnels recule: elle est actuellement de l'ordre de $13 \%$ et environ $5 \%$ de la population d'âge actif cotise à des plans de retraite individuels. Toutefois, le nouveau régime KiwiSaver a atteint un taux de 
couverture de $44 \%$ pour sa première année d'exploitation (depuis juillet 2007). Le taux de cotisation par défaut de ce régime est de $4 \%$ des gains, réparti à parts égales entre salariés et employeurs.

\section{Variantes de carrière}

\section{Retraite anticipée}

Il n'est pas possible de liquider la retraite avant l'âge normal d'ouverture des droits, qui est de 65 ans.

\section{Retraite différée}

La perception de la retraite publique n'est pas subordonnée au départ à la retraite. Il est donc possible de cumuler la retraite et un emploi.

Il n'y a pas d'obligation de liquider la retraite publique à l'âge d'ouverture des droits mais il n'y a aucun avantage à différer cette liquidation.

\section{Enfants}

Les droits éventuels à une retraite publique ne sont pas affectés par les périodes d'interruption de carrière liées aux enfants.

\section{Chômage}

Les droits éventuels à une retraite publique ne sont pas affectés par les périodes de chômage. 


\section{Résultats de la modélisation des retraites : Nouvelle-Zélande}

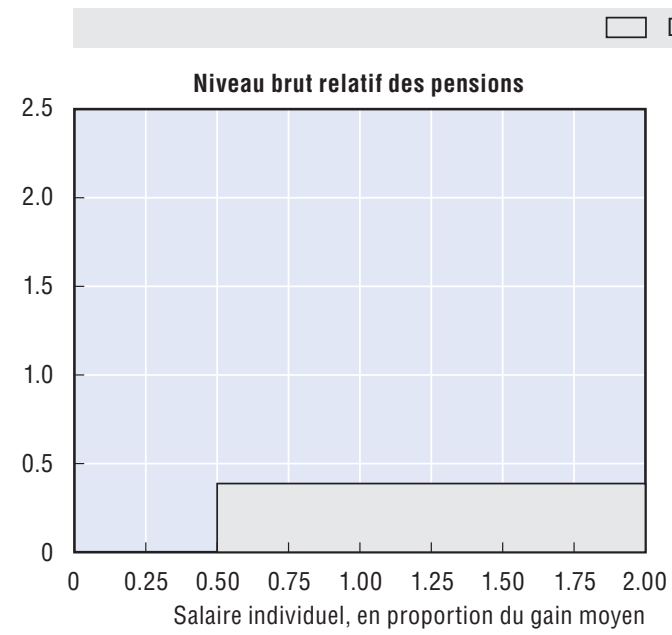

\section{De base}
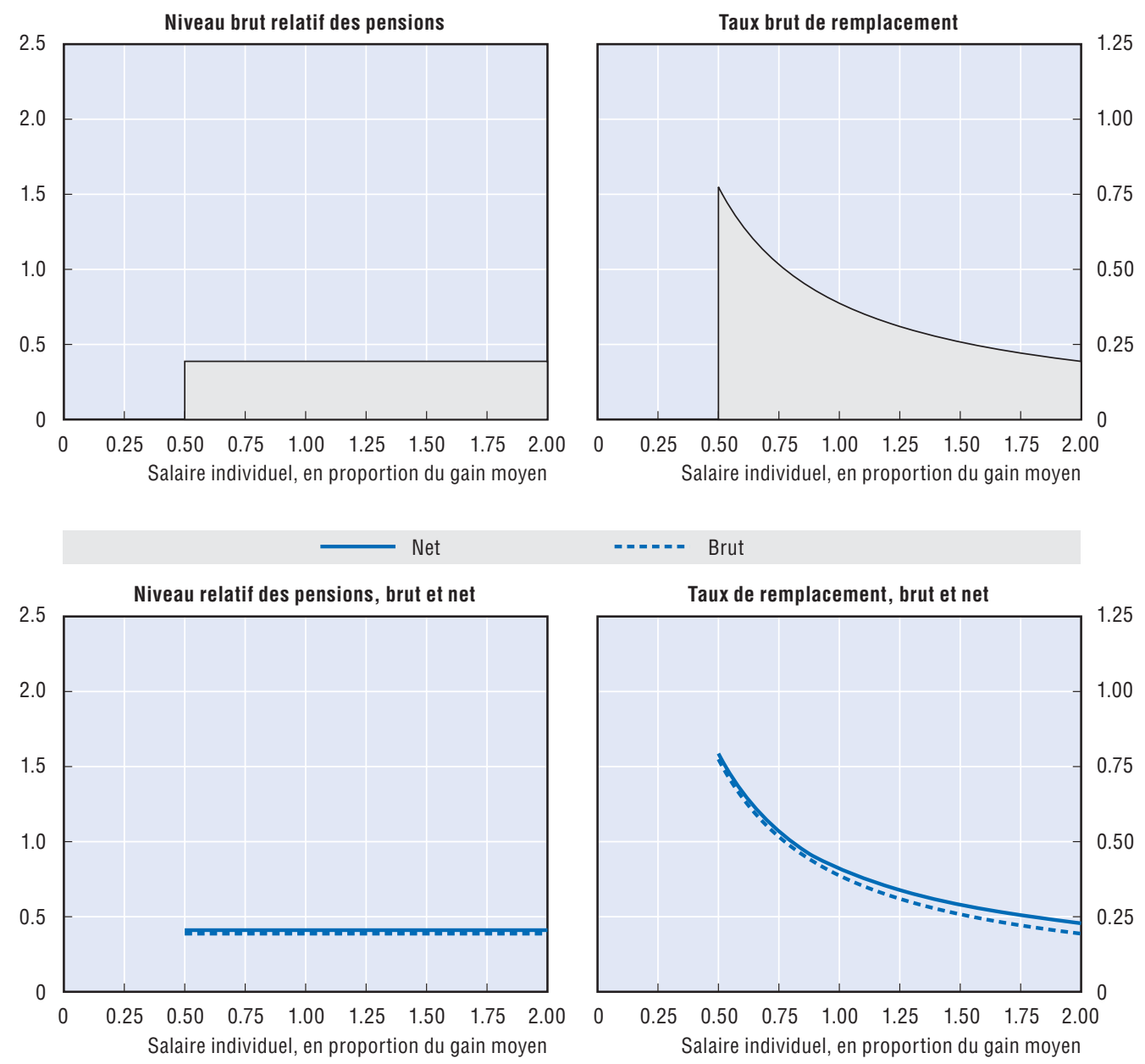

\begin{tabular}{|c|c|c|c|c|c|c|}
\hline \multirow{2}{*}{$\begin{array}{l}\text { Hommes } \\
\text { Femmes (si différent) }\end{array}$} & \multirow{2}{*}{$\begin{array}{l}\text { Salarié à revenu } \\
\text { médian }\end{array}$} & \multicolumn{5}{|c|}{ Salaire individuel, en multiple du salaire moyen } \\
\hline & & 0.5 & 0.75 & 1 & 1.5 & 2 \\
\hline Niveau brut relatif des pensions & 38.7 & 38.7 & 38.7 & 38.7 & 38.7 & 38.7 \\
\hline \multicolumn{7}{|l|}{ (\% salaire moyen brut) } \\
\hline Niveau net relatif des pensions & 41.1 & 41.1 & 41.1 & 41.1 & 41.1 & 41.1 \\
\hline Taux brut de remplacement & 45.6 & 77.5 & 51.6 & 38.7 & 25.8 & 19.4 \\
\hline \multicolumn{7}{|l|}{ (\% salaire individuel brut) } \\
\hline Taux net de remplacement & 47.3 & 79.3 & 53.5 & 41.1 & 29.0 & 22.8 \\
\hline \multicolumn{7}{|l|}{ (\% salaire individuel net) } \\
\hline (multiple du salaire individuel brut) & 8.2 & 13.9 & 9.2 & 6.9 & 4.6 & 3.5 \\
\hline
\end{tabular}




\section{Pays-Bas}

\section{Pays-Bas : système de retraite en 2006}

Le système de retraite néerlandais repose sur deux piliers principaux : un régime public à taux uniforme et des plans professionnels liés à la rémunération. Bien que les employeurs n'aient aucune obligation légale de proposer un régime de retraite à leurs salariés, $91 \%$ des salariés sont couverts dans le cadre d'accords de branche. Il est donc préférable de considérer ces régimes comme quasi obligatoires.

\section{Indicateurs essentiels}

\begin{tabular}{llrr}
\hline & & Pays-Bas & \multicolumn{1}{c}{ OCDE } \\
\hline Salaire & EUR & 39700 & 28600 \\
moyen & USD & 49900 & 35800 \\
Dépenses publiques & \% du PIB & 5.0 & 7.2 \\
au titre des retraites & & 79.8 & 78.9 \\
Espérance de vie & à la naissance & 83.4 & 83.4 \\
& à 65 ans & 23.4 & 23.8 \\
Population de plus de 65 ans & \% de la population d'âge actif & & \\
\hline
\end{tabular}

\section{Conditions d'ouverture des droits}

La pension de vieillesse de base est payable à partir de 65 ans. L'âge normal de la retraite est aussi habituellement de 65 ans dans les plans professionnels. Tous les résidents sont éligibles à cette prestation.

\section{Calcul des prestations}

\section{Régime de base}

Pour une personne seule, le montant brut de la prestation de retraite était en 2006 de 942.33 EUR au premier semestre et de 948.21 EUR au second. À cela s'ajoutait une allocation supplémentaire de congés de 53.22 EUR et 53.15 EUR respectivement. Ce qui nous donne un total annuel de 12017 EUR soit $30 \%$ du salaire moyen. Pour un couple, la prestation annuelle totale s'établirait à 16477 EUR. Le montant de la prestation est lié au salaire minimum net qui est revalorisé deux fois par an.

Le taux d'acquisition des droits à la prestation de base s'établit à $2 \%$ du montant total par année de résidence ou de travail dans le pays. Il existe également un régime d'aide sociale pour les personnes âgées dont le montant est égal au montant net de la pension de base.

\section{Régimes professionnels}

Les Pays-Bas ont également un régime de retraite privé à large couverture composé de 767 fonds de pension (à fin 2006) dont 103 fonds de branche. Sous certaines conditions, les entreprises néerlandaises peuvent s'abstenir d'y adhérer si elles ont leur propre régime offrant des prestations équivalentes. On recense en outre environ 700 plans d'entreprise et 46000 entreprises (en 2005), principalement des petites entreprises, proposent des régimes gérés par des compagnies d'assurances.

Environ $94 \%$ des salariés concernés relèvent d'un régime à prestations définies ; les autres relèvent d'un régime à cotisations définies.

Pour environ $77 \%$ des participants aux régimes à prestations définies, le salaire de référence est la moyenne des gains perçus sur l'ensemble de la carrière et pour $10 \%$ c'est 
le dernier salaire. Pour le restant, c'est soit une formule associant les deux (8\%) soit un montant fixe (1\%).

Il n'y a pas d'obligation légale concernant l'âge d'entrée dans les plans professionnels. En 2006, environ 55 \% des salariés relevaient de régimes de retraite sans âge d'entrée, $7 \%$ de régimes dans lesquels l'âge d'entrée se situe entre 16 et 20 ans et $36 \%$ de régimes dans lesquels il se situe entre 21 et 25 ans.

La plupart des régimes basés sur le dernier salaire donnent $1.75 \%$ de ce salaire par année d'activité, ce qui implique un taux de remplacement de $70 \%$ pour une carrière complète de 40 ans. Dans la plupart des régimes fondés sur le salaire moyen, le taux d'acquisition des droits varie entre $1.75 \%$ et $2 \%$ par année d'activité.

Il n'y a pas d'obligation légale concernant la revalorisation des rémunérations des années antérieures et la pratique varie d'un régime à l'autre selon des règles convenues par les partenaires sociaux. Pour environ $75 \%$ des participants à des régimes fondés sur le salaire moyen, les salaires passés sont revalorisés en fonction de la hausse du salaire moyen tandis que pour $8 \%$ la revalorisation se fait sur la base de l'inflation. La modélisation suppose un régime fondé sur le salaire moyen avec revalorisation sur la base de l'évolution du salaire moyen.

Bien qu'il n'y ait pas d'obligation légale de revalorisation des pensions mises en paiement, la plupart sont également réévaluées chaque année. Près de la moitié des pensions mises en paiement sont indexées sur la hausse des salaires du secteur concerné et $27 \%$ sont indexées sur les prix.

Les droits à pension sont totalement transférables en cas de changement d'employeur. La loi impose d'indexer les droits à pension des personnes qui quittent un régime avant leur départ à la retraite exactement de la même façon que les pensions mises en paiement. Les périodes d'acquisition de droits sont très courtes.

Les rémunérations ouvrant droit à pension ne sont pas plafonnées.

Les retraites professionnelles sont intégrées au régime public de retraite. La réglementation fiscale en vigueur autorise une prestation maximum de $100 \%$ du dernier salaire à 65 ans, pour le régime public comme pour les régimes privés. La plupart des régimes visant un taux de remplacement total de $70 \%$ du dernier salaire, les prestations privées sont réduites d'un montant de franchise qui, en 2006, était en moyenne de 12019 EUR.

\section{Variantes de carrière}

\section{Retraite anticipée}

La pension de base ne peut être liquidée avant 65 ans.

En 2005, le statut fiscal de faveur des programmes de préretraite (VUT) qui conduisait à verser des prestations de retraite anticipée entre 60 et 65 ans a été aboli afin d'encourager les travailleurs âgés à continuer à travailler.

\section{Retraite différée}

Il n'est pas possible de reporter la liquidation de la pension de vieillesse de base audelà de 65 ans mais il est possible de la cumuler avec un emploi.

Les règles de report de la retraite varient d'un plan professionnel à l'autre. Il est possible de cumuler la retraite professionnelle avec un emploi. De fait, certains régimes 
autorisent leurs adhérents à percevoir une retraite et à continuer à travailler pour le même employeur. Il n'y a pas de législation sur ce point.

\section{Enfants}

Dans le régime de base, les périodes d'interruption de carrière sont automatiquement couvertes. Dans les régimes professionnels les périodes d'interruption de carrière liées aux enfants ne sont pas validées mais la constitution de droits à pension continue sur les années d'activité restantes. Toutefois, de nombreux régimes autorisent la cotisation à des dispositifs facultatifs pour couvrir les périodes d'absence mentionnées ci-dessus.

\section{Chômage}

Dans les régimes professionnels les périodes de chômage ne sont pas validées. Là encore, le régime de base couvre automatiquement ces périodes. Par ailleurs, les partenaires sociaux administrent un fonds (FVP) qui permet aux travailleurs âgés d'étendre la constitution de leur retraite sur une certaine période durant leurs épisodes de chômage. Ce fonds n'a aucun lien formel avec l'État. 


\title{
Résultats de la modélisation des retraites : Pays-Bas
}

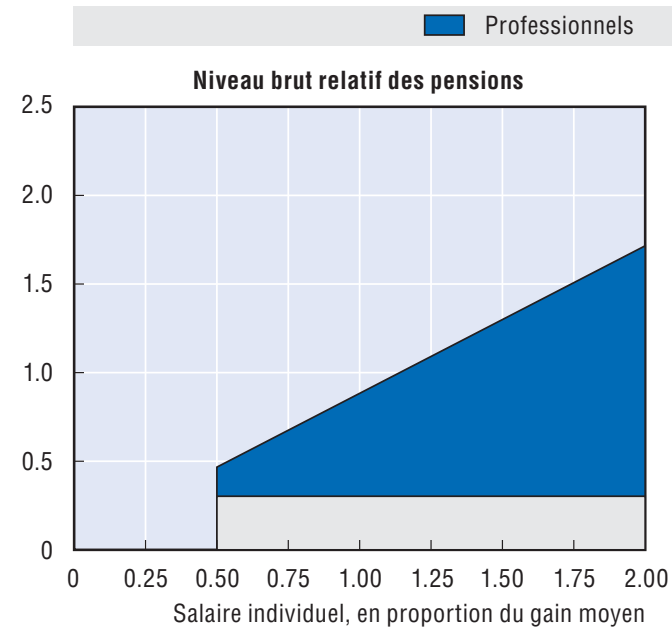

\author{
De base
}

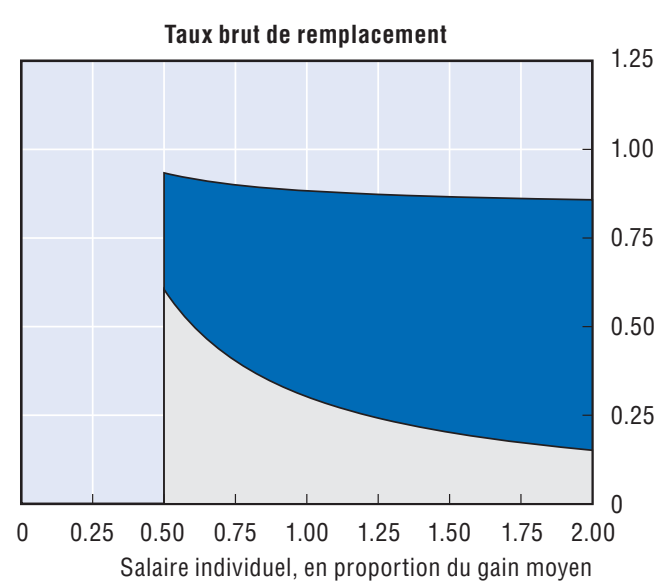

Net

Brut

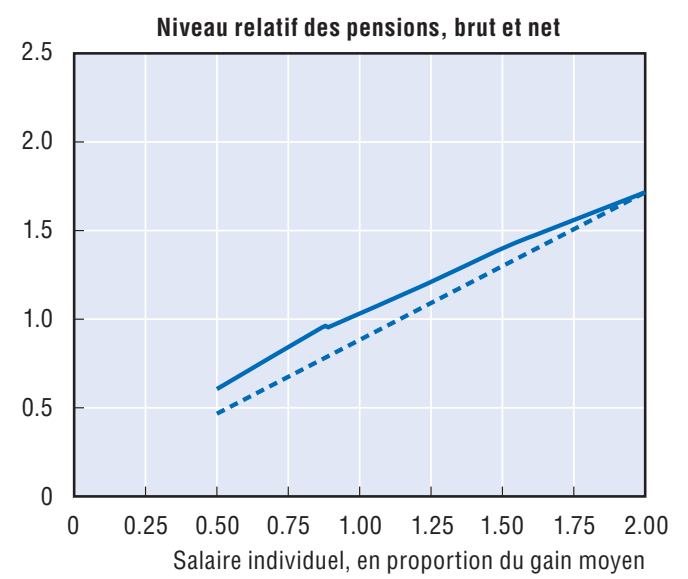

$-2-+-1$ Brut

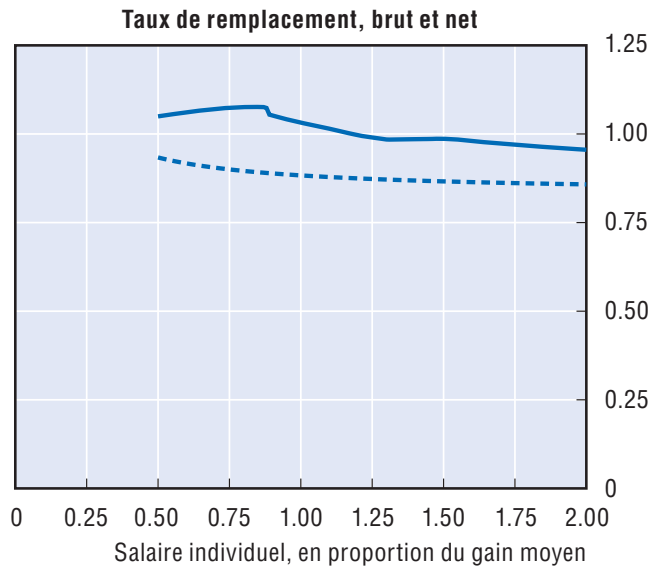

\begin{tabular}{|c|c|c|c|c|c|c|}
\hline \multirow{2}{*}{$\begin{array}{l}\text { Hommes } \\
\text { Femmes (si différent) }\end{array}$} & \multirow{2}{*}{$\begin{array}{l}\text { Salarié à revenu } \\
\text { médian }\end{array}$} & \multicolumn{5}{|c|}{ Salaire individuel, en multiple du salaire moyen } \\
\hline & & 0.5 & 0.75 & 1 & 1.5 & 2 \\
\hline Niveau brut relatif des pensions & 79.2 & 46.7 & 67.5 & 88.3 & 129.9 & 171.6 \\
\hline \multicolumn{7}{|l|}{ (\% salaire moyen brut) } \\
\hline Niveau net relatif des pensions & 95.4 & 60.6 & 84.3 & 103.2 & 140.0 & 171.6 \\
\hline \multicolumn{7}{|l|}{ (\% salaire moyen net) } \\
\hline Taux brut de remplacement & 88.9 & 93.4 & 90.0 & 88.3 & 86.6 & 85.8 \\
\hline \multicolumn{7}{|l|}{ (\% salaire individuel brut) } \\
\hline Taux net de remplacement & 105.5 & 105.0 & 107.4 & 103.2 & 98.6 & 95.5 \\
\hline \multicolumn{7}{|l|}{ (\% salaire individuel net) } \\
\hline Patrimoine retraite brut & 16.4 & 17.2 & 16.6 & 16.3 & 16.0 & 15.8 \\
\hline (multiple du salaire individuel brut) & 19.2 & 20.1 & 19.4 & 19.1 & 18.7 & 18.5 \\
\hline Patrimoine retraite net & 12.6 & 14.2 & 13.2 & 12.1 & 11.0 & 10.1 \\
\hline (multiple du salaire individuel brut) & 14.7 & 16.6 & 15.4 & 14.2 & 12.8 & 11.8 \\
\hline
\end{tabular}




\section{Pologne}

\section{Pologne : système de retraite en 2006}

Le nouveau régime public de retraite mis en place en 1999 s'applique aux personnes nées à partir de 1949. Il repose sur un système de comptes notionnels. Les personnes (nées à partir de1969) qui avaient moins de 30 ans au moment de la réforme doivent également adhérer au régime par capitalisation; celles (nées entre 1949 et 1968) qui avaient entre 30 et 50 ans pouvaient opter pour la capitalisation mais elles devaient le faire en 1999 et leur choix était irrévocable sauf pour les personnes qui ont pu partir en retraite anticipée en 2007 et 2008 du fait de l'extension de la possibilité de départ anticipé accordée par le Parlement face à l'absence de proposition de dispositif de transition. Par ailleurs, en 2005, le système de retraite anticipée des mineurs a été rétabli selon les règles antérieures à 1999.
Indicateurs essentiels

\begin{tabular}{llrr}
\hline & & Pologne & \multicolumn{1}{c}{ OCDE } \\
\hline Salaire & PLN & 29300 & 111000 \\
moyen & USD & 9400 & 35800 \\
Dépenses publiques & \% du PIB & 11.4 & 7.2 \\
au titre des retraites & & 75.3 & 78.9 \\
Espérance de vie & à la naissance & 81.7 & 83.4 \\
& à 65 ans & 21.1 & 23.8 \\
Population de plus de 65 ans & \% de la population d'âge actif & & \\
\hline
\end{tabular}

\section{Conditions d'ouverture des droits}

Dans le nouveau système, l'âge minimum de la retraite est de 65 ans pour les hommes et de 60 ans pour les femmes. Pour la pension minimum, 25 années de cotisation sont requises pour les hommes et 20 pour les femmes.

\section{Calcul des prestations}

\section{Regime lié à la rémunération}

Une cotisation représentant $12.22 \%$ du salaire (ou $19.52 \%$ pour les travailleurs nés entre 1949 et 1968 n'ayant pas opté pour un régime à cotisations définies) sera portée au crédit des comptes notionnels individuels. Initialement, ces cotisations étaient revalorisées entre le moment de leur prélèvement et le moment de la retraite en fonction de la hausse des prix plus $100 \%$ de l'accroissement de la masse salariale réelle couverte. À partir de 2004, le taux d'intérêt théorique a été défini comme égal à $100 \%$ de la progression de la masse salariale réelle couverte sans être inférieur à l'inflation. Ce taux est appliqué rétrospectivement à partir de l'an 2000.

Lors du départ à la retraite, le capital notionnel accumulé est divisé par la "valeur de $g$ " pour obtenir la prestation de retraite. La valeur de g est l'espérance de vie moyenne à l'âge du départ à la retraite : ce processus est équivalent au processus de conversion en rente des régimes par capitalisation. La valeur de g est calculée à l'aide des tables de mortalité publiées par l'Office central de la statistique. Dans la modélisation, les données actuarielles utilisées sont celles de la base de données Nations Unies/Banque mondiale sur la population. 
Les cotisations et les rémunérations ouvrant droit à pension sont plafonnées à 2.5 fois le salaire moyen prévu pour une année donnée de la loi budgétaire. Ce plafond était de 68700 PLN (2.5 fois le salaire moyen) en 2004, 72690 PLN en 2005, 73560 PLN en 2006, 78480 PLN en 2007, 85290 PLN en 2008 et 95790 PLN en 2009.

Entre 1999 et 2004, les pensions mises en paiement ont été revalorisées pour $80 \%$ sur la hausse des prix et pour $20 \%$ sur la hausse du salaire moyen selon les projections pour une année donnée. On notera toutefois qu'à partir de 2005, l'indexation minimum se fait sur la base des prix des années passées, pour les années où l'inflation composée de l'année précédant l'indexation précédente est supérieure à $5 \%$. À partir de 2008 , les pensions mises en paiement sont revalorisées pour $80 \%$ sur la hausse des prix et pour $20 \%$ sur la hausse du salaire moyen de l'année écoulée. L'indexation des pensions au-delà du niveau minimum est négociée avec le Comité tripartite.

\section{Pension minimum}

Dans le régime par répartition, il existe une pension minimum dont le montant est de 597.46 PLN par mois depuis le $1^{\mathrm{er}}$ mars 2006 , soit $24 \%$ du salaire moyen.

\section{Régime à cotisations définies}

Environ 7.3 points de pourcentage de la cotisation totale sont transférés au régime par capitalisation pour les personnes couvertes par ce régime de manière obligatoire ou délibérée. La Loi sur les rentes, votée par le Parlement au début de l'année 2009, suppose la conversion en rente de l'épargne retraite sur la base des tables de mortalité unisexe à l'âge du départ à la retraite mais pas avant 65 ans. Les femmes partant à la retraite avant 65 ans recevront des paiements sur la base de retraits échelonnés jusqu'à 65 ans. Les rentes seront majorées de $90 \%$ du rendement des sociétés de rentes.

On suppose que lors du départ à la retraite le capital accumulé sera converti en rente et qu'au minimum les rentes seront indexées sur les prix (indice utilisé dans la modélisation). Il a été décidé que les taux de rente devront être établis sur la base de tables de mortalité unisexe.

\section{Variantes de carrière}

\section{Retraite anticipée}

Le régime de retraite ne prévoit pas de système de retraite anticipée. L'ancien système (qui s'applique aux personnes nées avant 1949) autorisait diverses formes de retraite anticipée pour des groupes spécifiques tels que les mineurs, les cheminots, les enseignants, les personnes travaillant dans des conditions particulières et les femmes. Les possibilités de départ en retraite anticipée ont été reportées en 2007 et 2008 (anciennement 2006). Cette extension a été la conséquence de l'absence de proposition de dispositif de transition. Par ailleurs, en 2005, le système de retraite anticipée des mineurs a été rétabli dans les règles antérieures à 1999.

Le dispositif de transitions entré en vigueur en 2009 suppose que les personnes ayant des conditions de travail particulières (quelque 270000 travailleurs) recevront une pension de transition pendant un maximum de cinq ans avant l'âge de la retraite. Cette prestation sera financée sur le budget de l'État et calculée suivant la formule de calcul de la pension du régime lié à la rémunération. 


\section{Retraite différée}

Il est possible de différer sans limite d'âge à la fois la composante notionnelle et la composante en capitalisation des régimes à cotisations définies. Les personnes qui reportent la liquidation de leurs droits à pension au-delà de l'âge normal de la retraite cotisent et acquièrent des droits supplémentaires.

Le cumul emploi-retraite est possible. Pour les retraités n'ayant pas atteint l'âge légal de la retraite (dans l'ancien système), il existe des plafonds de revenu. Si le revenu du travail est supérieur à $70 \%$ du salaire moyen, la pension est réduite ; s'il est supérieur à $130 \%$ du salaire moyen, elle est suspendue.

\section{Enfants}

Durant les périodes de congé maternité, les cotisations au régime de retraite sont prises en charge par l'Etat via l'indemnité de maternité, qui est égale au salaire moyen des six derniers mois, net des cotisations de sécurité sociale. À partir de 2004, la période sur laquelle est établi le salaire moyen a été étendue à 12 mois. La durée du congé de maternité est de 16 semaines pour le premier enfant, de 18 semaines pour le deuxième et de 26 semaines en cas de naissances multiples. À partir de décembre 2006, elle est de 18 semaines pour le premier enfant, de 20 semaines pour le deuxième et de 28 semaines pour des naissances multiples. Il a été décidé qu'à partir du $1^{\mathrm{er}}$ janvier 2009 la durée du congé de maternité serait de 20 semaines pour le premier et le deuxième enfants, tandis que pour des naissances multiples elle passerait à 31 semaines, 33 semaines, 35 semaines et 37 semaines selon le nombre d'enfants.

Il est possible de prendre un congé parental d'une durée maximum de 36 mois par enfant. Durant ce congé, des cotisations sont acquittées aux régimes de retraite auxquels adhère la personne et le salaire de référence retenu est le montant de l'allocation sociale, qui correspond à environ $18 \%$ du salaire moyen.

Dans les deux cas, l'État prend en charge les cotisations du parent en congé.

Toutes les périodes pour lesquelles des cotisations sont acquittées ouvrent droit à la retraite minimum garantie.

\section{Chômage}

Il existe un système de prestations de retraite anticipée pour les chômeurs ayant perdu leur emploi suite à une liquidation, une faillite ou une restructuration. Des prestations de retraite anticipée financées sur le budget de l'État sont versées à partir de 55 ans pour les femmes et de 60 ans pour les hommes jusqu'à l'âge de la retraite. Ces règles sont en vigueur depuis mai 2004. Auparavant, ces prestations étaient versées à partir de 50 ans pour les femmes et de 55 ans pour les hommes. Les prestations de retraite anticipée ne sont pas soumises à cotisation au régime de retraite.

Durant les périodes de chômage indemnisé, l'État prend en charge les cotisations au régime de retraite sur la base du montant de l'allocation chômage (12.22 \% de la prestation aux comptes notionnels et $7.3 \%$ au régime à cotisations définies). Toutes les périodes pour lesquelles des cotisations sont versées ouvrent droit à la retraite minimum garantie. 


\section{Résultats de la modélisation des retraites : Pologne}

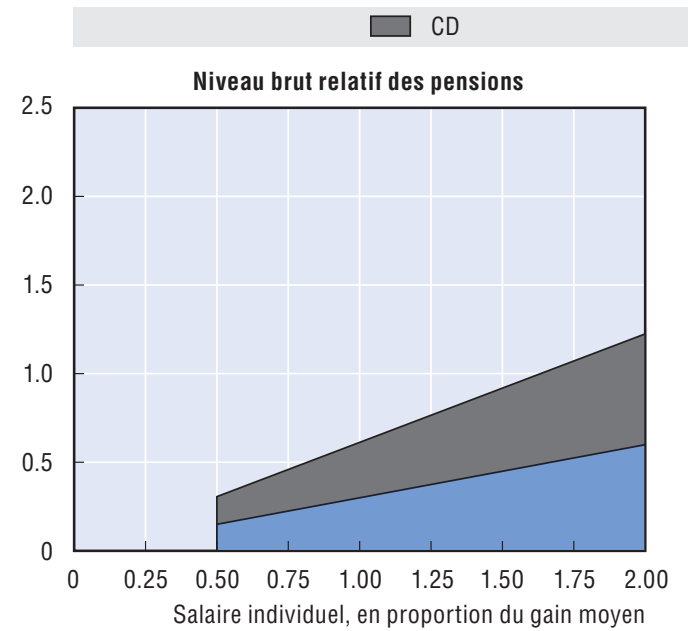

Lié à la rémunération
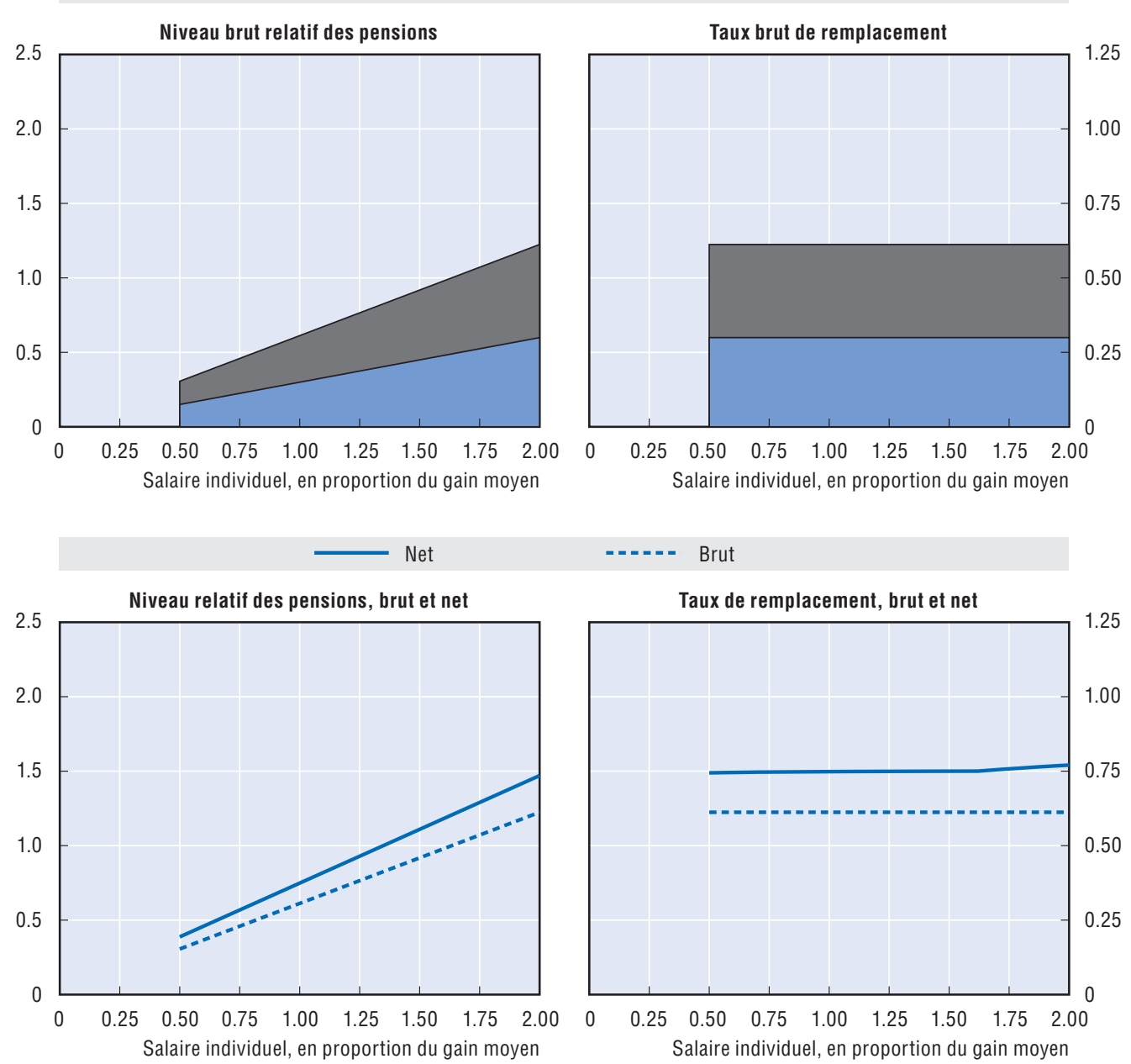

\section{$0-\ldots$ Brut}

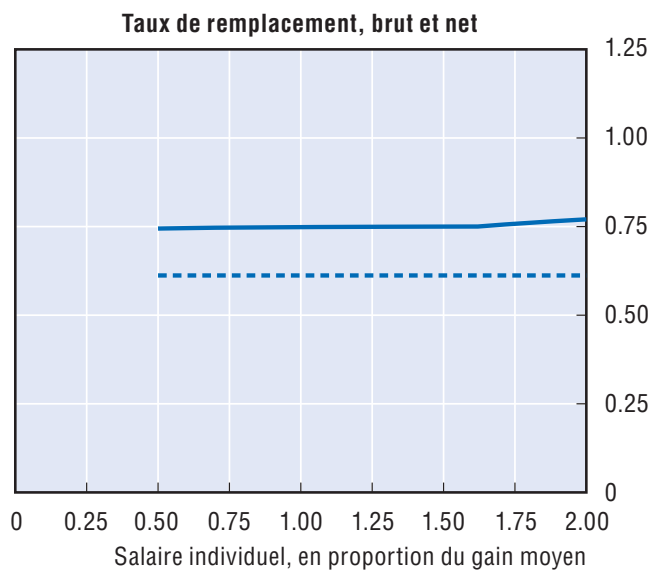

\begin{tabular}{lc|rrrrr}
\hline \multirow{2}{*}{$\begin{array}{l}\text { Hommes } \\
\text { Femmes (si différent) }\end{array}$} & $\begin{array}{c}\text { Salarié à revenu } \\
\text { médian }\end{array}$ & 0.5 & 0.75 & 1 & 1.5 & 2 \\
\cline { 3 - 6 } & & 30.6 & 45.9 & 61.2 & 91.8 & 122.4 \\
\hline Niveau brut relatif des pensions & 50.2 & 24.5 & 33.4 & 44.5 & 66.8 & 89.0 \\
(\% salaire moyen brut) & 36.5 & 38.8 & 56.8 & 74.9 & 110.9 & 147.0 \\
Niveau net relatif des pensions & 61.9 & 31.6 & 42.0 & 55.2 & 81.4 & 107.6 \\
(\% salaire moyen net) & 45.7 & 61.2 & 61.2 & 61.2 & 61.2 & 61.2 \\
Taux brut de remplacement & 61.2 & 49.0 & 44.5 & 44.5 & 44.5 & 44.5 \\
(\% salaire individuel brut) & 44.5 & 74.4 & 74.7 & 74.9 & 75.0 & 77.0 \\
Taux net de remplacement & 74.8 & 60.6 & 55.3 & 55.2 & 55.0 & 56.4 \\
(\% salaire individuel net) & 55.2 & 8.4 & 8.4 & 8.4 & 8.4 & 8.4 \\
Patrimoine retraite brut & 8.4 & 9.5 & 8.6 & 8.6 & 8.6 & 8.6 \\
(multiple du salaire individuel brut) & 8.6 & 7.2 & 7.0 & 7.0 & 6.9 & 6.8 \\
Patrimoine retraite net & 7.0 & 8.3 & 7.3 & 7.2 & 7.1 & 7.1 \\
(multiple du salaire individuel brut) & 7.3 & & & & &
\end{tabular}




\section{Portugal}

\section{Portugal : système de retraite en 2006}

Le Portugal a un régime public de retraite lié à la rémunération avec un filet de sécurité soumis à conditions de ressources.
Indicateurs essentiels

\begin{tabular}{llrr}
\hline & & Portugal & \multicolumn{1}{c}{ OCDE } \\
\hline Salaire moyen & EUR & 15300 & 28600 \\
& USD & 19300 & 35800 \\
Dépenses publiques & \% du PIB & 10.2 & 7.2 \\
au titre des retraites & & 78.9 & 78.9 \\
Espérance de vie & à la naissance & 83.4 & 83.4 \\
& à 65 ans & 27.8 & 23.8 \\
Population de plus de 65 ans & \% de la population d'âge actif & & \\
\hline
\end{tabular}

\section{Conditions d'ouverture des droits}

L'âge normal de la retraite est de 65 ans mais un départ anticipé est possible à partir de 55 ans. Un minimum de 15 ans de cotisations est requis pour un départ à 65 ans. Un départ anticipé est possible avec 30 ans de cotisations.

La retraite sociale est payable à partir de 65 ans.

\section{Calcul des prestations}

\section{Régime lié à la rémunération}

Les droits à pension s'acquièrent au taux de $2 \%$ de la base de rémunération par année de cotisation jusqu'à 20 ans. Pour les bénéficiaires ayant au minimum 21 ans de cotisation, le taux d'acquisition se situe entre $2 \%$ et $2.3 \%$ selon la rémunération. Le barême du taux d'acquisition dépend du salaire individuel par rapport au salaire minimum national (385.90 EUR). Chaque tranche de salaire confère des droits à pension à un taux différent.

\begin{tabular}{lccccc}
\hline Rémunération/salaire minimum & $\leq 1.1$ & $>1.1-2.0$ & $>2.0-4.0$ & $>4.0-8.0$ & $>8.0$ \\
Taux d'acquisition (\%) & 2.3 & 2.25 & 2.2 & 2.1 & 2.0 \\
\hline
\end{tabular}

Les droits à pension s'aquièrent sur un maximum de 40 ans.

Le salaire de référence se calcule actuellement sur les dix meilleures des quinze dernières années. Toutefois, cette base est actuellement élargie et devrait atteindre en 2017 la moyenne des gains perçus sur l'ensemble de la carrière. Pour les personnes qui cotisaient déjà au 31 décembre 2001 et qui remplissaient à cette date les conditions d'ouverture des droits à la pension de vieillesse, cette pension sera calculée selon la plus favorable des trois formules suivantes : 1 ) application des règles antérieures (acquisition au taux de $2 \%$ par année de cotisation sur la base des gains des dix meilleures des quinze dernières années ; 2) application à l'ensemble de la carrière contributive des nouvelles règles décrites ci-dessus; ou 3) application des deux règles au prorata selon la carrière contributive. Pour les personnes qui cotisaient déjà au 31 décembre 2001 mais ne remplissaient pas à cette date les conditions d'ouverture des droits à la pension de vieillesse, cette pension sera calculée selon la plus favorable des trois formules ci-dessus, si elles partent à la retraite entre 2002 et 2016 ; ou selon la plus favorable entre les formules 2) et 3), si elles partent à la retraite après 2016. Les personnes entrées dans le 
régime après 2002 seront entièrement assujetties aux nouvelles règles. Pour les personnes justifiant de plus de 40 années de cotisations, seules les 40 meilleures seront prises en compte dans la formule de calcul.

Depuis le début de l'année 2002, la revalorisation des salaires pour le calcul des pensions se fait selon une formule mixte associant les salaires et les prix, dans laquelle la hausse des prix a un poids de $75 \%$ et celle des salaires de $25 \%$, sous réserve d'une augmentation réelle maximum de $0.5 \%$.

Les pensions mises en paiement sont indexées sur les prix, avec une augmentation plus forte pour les pensions modestes. En décembre 2006, la hausse des pensions déjà mises en paiement était de $3.1 \%$ pour celles n'excédant pas 596.79 EUR, de $2.6 \%$ pour celles comprises entre 596.79 EUR et 2387.16 EUR, de $2.4 \%$ pour celles comprises entre 2387.16 EUR et 4774.32 EUR; et de $0 \%$ pour celles égales ou supérieures à 4774.32 EUR.

\section{Pension minimum}

Pour les travailleurs ayant jusqu'à 15 années de cotisation, le montant de la pension minimum est de 223.24 EUR par mois de janvier à novembre 2006 et de 230.16 EUR à partir de décembre 2006. Pour les travailleurs de la tranche d'âge 15-40 ans, ce montant varie entre une limite inférieure de 249.00 EUR (de janvier à novembre) et 256.72 EUR (à partir de décembre 2006) et une limite supérieure de 343.45 EUR et 354.10 EUR pour les deux mêmes périodes, comme le montre le tableau ci-après.

La pension minimum est versée sur 14 mois.

\begin{tabular}{lcc}
\hline \multirow{2}{*}{ Années de cotisation } & \multicolumn{2}{c}{ Pension minimum (EUR) } \\
\cline { 2 - 3 } & Janvier-novembre & Décembre \\
\hline 15 à 20 & 249 & 256.72 \\
21 à 30 & 274.76 & 283.28 \\
31 et plus & 343.45 & 354.1 \\
\hline
\end{tabular}

\section{Régime ciblé}

Pour les personnes non éligibles au régime lié à la rémunération, le montant de la pension sociale était de 171.73 EUR par mois (janvier à novembre) et de 177.05 EUR (décembre 2006).

Cette pension n'est versée que si le revenu total n'excède pas $30 \%$ du salaire minimum pour une personne seule ou $50 \%$ pour un couple. Là encore, la pension est versée sur 14 mois.

Les bénéficiaires de la pension sociale sont habilités à recevoir le supplément de solidarité en sus de leur pension ; le montant mensuel de cette prestation est de 15.89 EUR (16.38 EUR après le $1^{\mathrm{er}}$ décembre 2006) pour les moins de 70 ans et de 31.77 EUR (32.75 EUR après le $1^{\text {er }}$ décembre 2006) pour les plus de 70 ans).

Début 2006 a été mise en place une nouvelle prestation ciblée, le supplément de solidarité vieillesse (OSS), qui vise à lutter contre la pauvreté des personnes âgées. Pour être éligible à cette prestation, il faut être âgé de 80 ans ou plus en 2006 (extension en 2007 aux personnes de 70 ans et plus et en 2008 aux personnes de 65 ans et plus), percevoir une pension de vieillesse ou de réversion (les ressortissants nationaux n'ayant pas droit à la pension sociale parce qu'ils ne remplissent pas ses conditions de ressources 
peuvent être néanmoins éligibles à cette prestation) et remplir les conditions de ressources de l'OSS.

L'OSS ressemble au revenu social d'insertion en ce sens qu'il s'agit d'un complément égal à la différence entre le revenu du bénéficiaire et un seuil donné, qui est en même temps la condition de ressources. L'OSS est donc égal à la différence entre le revenu du bénéficiaire et les montants de référence (RA) suivants :

- 4200 EUR par an pour une personne seule ;

- 7350 EUR par an pour un couple.

Le revenu du bénéficiaire est constitué par son propre revenu, celui de son conjoint et une partie du revenu des ménages de leurs enfants désignée sous le nom de "solidarité familiale ". Pour établir les droits à prestations et le montant de l'OSS, on ajoute la composante "solidarité familiale " au revenu du bénéficiaire.

Pour calculer la " solidarité familiale ", on prend le revenu annuel total du ménage de chacun des enfants que l'on divise par le nombre d'équivalents adultes présents dans ce ménage (l'échelle d'équivalence est de 1 pour le premier adulte, de 0.7 pour chacun des adultes suivants et de 0.5 pour un mineur) et on détermine ensuite, comme le montre le tableau ci-après, la solidarité familiale en pourcentage du revenu équivalent du ménage. Les personnes dont les enfants ont un revenu de ménage équivalent qui se situe dans le quatrième niveau ne sont pas éligibles à l'OSS.

\begin{tabular}{lcc}
\hline Niveau & Revenu équivalent du ménage & Solidarité familiale (\% du revenu équivalent) \\
\hline $1^{\mathrm{er}}$ & $2.5 \times \mathrm{RA}$ & 0 \\
$2^{\mathrm{e}}$ & $>2.5 \times$ RA et $\leq 3.5 \times$ RA & 5 \\
$3^{\mathrm{e}}$ & $>3.5 \mathrm{RA}$ et $\leq 5 \times$ RA & 10 \\
$4^{\mathrm{e}}$ & $>5 \times$ RA & Exclusion \\
\hline
\end{tabular}

Les montants de la pension minimum et autres prestations sociales seront liés à l'IAS selon le tableau suivant:

\begin{tabular}{lc}
\hline Prestations & Montant (\% de l'IAS) \\
\hline Pension minimum (liée à la rémunération) & \\
15 ans de cotisation & 57.8 \\
15 à 20 ans de cotisation & 64.5 \\
21 à 30 ans de cotisation & 71.2 \\
Plus de 30 ans de cotisation & 89.0 \\
Pension sociale & 44.5 \\
\hline
\end{tabular}

\section{Variantes de carrière}

\section{Retraite anticipée}

À partir d'août 2005, le régime de retraite anticipée a été suspendu. Il n'est donc plus possible d'anticiper le départ à la retraite excepté dans le cas de chômeurs de longue durée (voir ci-après la section « Chômage »). 


\section{Retraite différée}

Il est possible de reporter la liquidation de la retraite jusqu'à 70 ans. La pension est majorée de 12 \% par année de report jusqu'à un maximum de cinq ans.

\section{Enfants}

Les périodes de maternité (congé maternité + travail à temps partiel) sont prises en compte dans le calcul des droits à pension. Ces périodes sont validées pour les conditions d'ouverture des droits. La rémunération ouvrant droit à pension pour ces périodes est établie sur la base de la rémunération des six mois précédant le deuxième mois du congé de maternité.

À partir de 2002, des périodes de travail à temps partiel d'une durée maximum de trois ans liées aux enfants de moins de 12 ans peuvent être comptabilisées comme des périodes de travail à temps plein.

\section{Chômage}

Les périodes de chômage indemnisé sont prises en compte dans le calcul des prestations de retraite. La rémunération ouvrant droit à pension pour ces périodes est établie sur la base de la rémunération des six mois précédant le deuxième mois de chômage. Cela vaut pour le chômage comme pour le traitement social du chômage.

Des règles particulières s'appliquent aux chômeurs de longue durée. Les personnes de 55 ans et plus en chômage de longue durée peuvent partir à la retraite à 60 ans et percevoir une retraite à taux plein sans décote sous réserve d'avoir cotisé le nombre minimum d'années requis et d'être en fin de droits.

Un départ à la retraite anticipée est également possible à partir de 55 ans avec 20 années de cotisation dans le cas de personnes ayant perdu leur emploi à 50 ans et plus. Dans ce cas, la pension est minorée de $4.5 \%$ par an avec une réduction d'au maximum cinq ans.

La subvention d'assistance chômage soumise à conditions de ressources est accordée si la durée de cotisation enregistrée est de plus de 180 jours dans les 12 mois précédant le début du chômage et si la rémunération mensuelle avant l'épisode de chômage est inférieure à $80 \%$ du salaire minimum. Cette allocation peut être maintenue jusqu'à ce que les bénéficiaires remplissent les conditions d'un départ en retraite anticipée sous réserve que ceux-ci n'aient pas moins de 50 ans. 
Résultats de la modélisation des retraites : Portugal
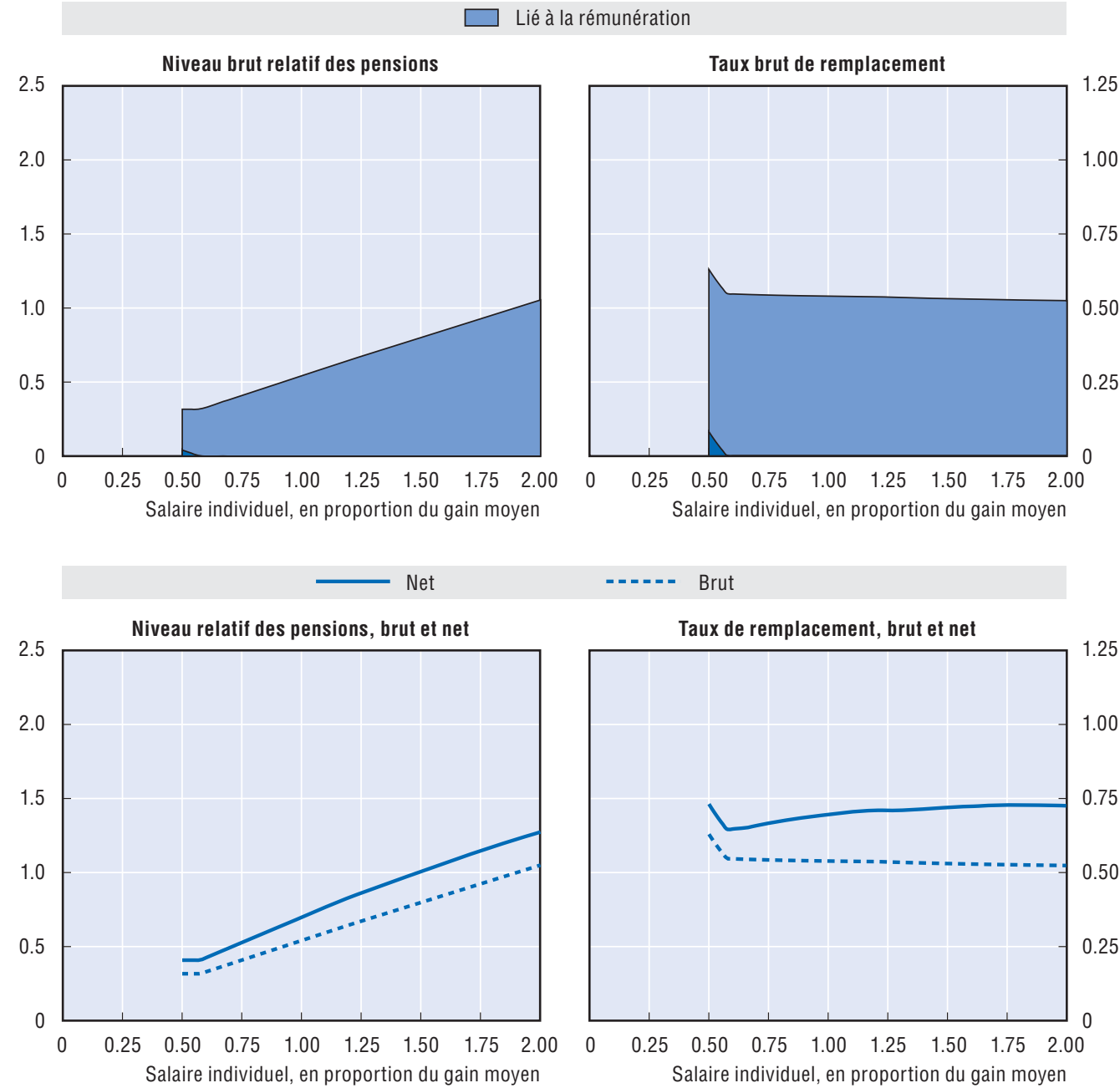

\section{$=-= \pm=1$ Brut}

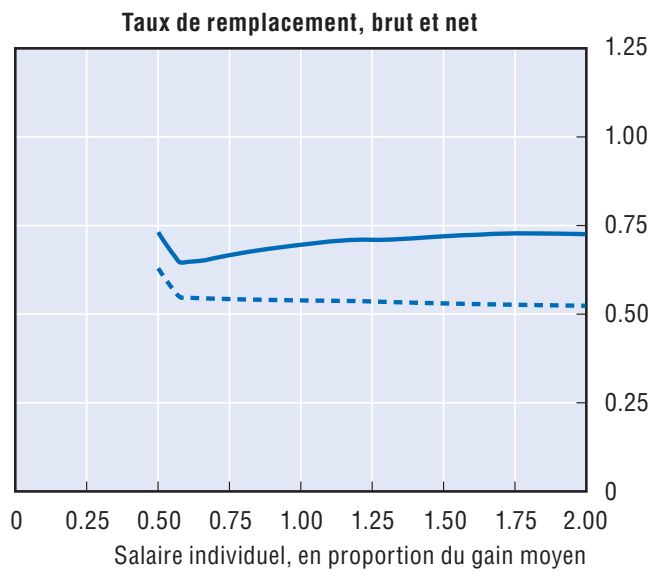

\begin{tabular}{|c|c|c|c|c|c|c|}
\hline $\begin{array}{l}\text { Hommes } \\
\text { Femmes (si différent) }\end{array}$ & $\begin{array}{l}\text { Salarié à revenu } \\
\text { médian }\end{array}$ & \multicolumn{5}{|c|}{ Salaire individuel, en multiple du salaire moyen } \\
\hline \multicolumn{7}{|l|}{ (\% salaire moyen brut) } \\
\hline Niveau net relatif des pensions & 59.4 & 35.4 & 52.6 & 69.6 & 100.5 & 127.2 \\
\hline Taux brut de remplacement & 54.1 & 63.0 & 54.3 & 53.9 & 53.1 & 52.4 \\
\hline \multicolumn{7}{|l|}{ (\% salaire individuel brut) } \\
\hline Taux net de remplacement & 68.0 & 73.2 & 66.7 & 69.6 & 72.0 & 72.6 \\
\hline \multicolumn{7}{|l|}{ (\% salaire individuel net) } \\
\hline Patrimoine retraite brut & 8.1 & 9.2 & 7.9 & 8.1 & 8.0 & 7.9 \\
\hline (multiple du salaire individuel brut) & 9.5 & 10.7 & 9.2 & 9.5 & 9.1 & 8.7 \\
\hline
\end{tabular}




\section{République slovaque}

\section{République slovaque : système de retraite en 2006}

Le régime public de retraite lié à la rémunération est analogue à un système à points, avec des prestations qui dépendent du salaire individuel par rapport au salaire moyen. Il ne prévoit pas de pension minimum mais les travailleurs à faible revenu sont protégés par le fait que la retraite est calculée sur un montant minimum de gains. Tous les retraités sont éligibles aux prestations de l'aide sociale. Des régimes à cotisations définies ont été mis en place au début de l'année 2005. Il est impossible de les évaluer à ce stade.
Indicateurs essentiels

\begin{tabular}{llrr}
\hline & & $\begin{array}{c}\text { République } \\
\text { slovaque }\end{array}$ & \multicolumn{1}{c}{ OCDE } \\
\hline Salaire moyen & SKK & 231200 & 1061500 \\
& USD & 7800 & 35800 \\
Dépenses publiques & $\%$ du PIB & 6.2 & 7.2 \\
au titre des retraites & & 74.3 & 78.9 \\
Espérance de vie & à la naissance & 80.2 & 83.4 \\
Population de plus de 65 ans & \% de la population d'âge actif & 18.4 & 23.8 \\
\hline
\end{tabular}

\section{Conditions d'ouverture des droits}

Dix années de cotisation à une assurance retraite sont nécessaires pour être éligibles à une pension. L'âge de la retraite est progressivement porté à 62 ans, pour les hommes comme pour les femmes. Pour les hommes, il sera de 62 ans en 2006. Pour les femmes, le relèvement s'étalera sur la période 2004-14.

\section{Calcul des prestations}

\section{Régime lié à la rémunération}

Les cotisants acquièrent chaque année des points de retraite que l'on calcule en rapportant les salaires individuels au salaire moyen à l'échelle nationale. Les droits à pension sont obtenus en multipliant la somme des points de retraite sur toute la carrière par la valeur du point.

Celle-ci s'établissait à 214.68 SKK pour 2006. La valeur du point est indexée sur le salaire moyen. En 2006, le salaire moyen national était de 19268 SKK par mois. En divisant la valeur du point par la rémunération, on obtient l'équivalent du taux d'acquisition des droits dans un régime à prestations définies, qui est légèrement inférieur à $1.2 \%$.

Les salaires pris en compte pour le calcul des cotisations et des prestations sont plafonnés à trois fois le salaire moyen. Les données sur les salaires sont décalées de sorte que le plafond pour le premier semestre 2006 a été de trois fois le salaire moyen de 2004 (15 825 SKK par mois). Au second semestre, le plafond a été établi sur la base des données de 2005 pour le salaire moyen (17 274 SKK par mois). (Pour les hypothèses de base relatives à la hausse des salaires et des prix, le décalage signifie que le plafond est légèrement inférieur à trois fois le salaire moyen de la période.)

Les pensions mises en paiement sont indexées sur la moyenne arithmétique de la hausse des salaires et de la hausse des prix. 
Pour les travailleurs qui adhèrent à des régimes à cotisations définies, les prestations du régime public lié à la rémunération sont égales à la moitié de celles des travailleurs restant uniquement dans le régime public. Ces travailleurs sont supposés tirer l'autre moitié de leur retraite du produit d'une assurance vie ou d'une formule mixte associant une assurance vie et une pension de vieillesse.

\section{Pension minimum}

Il n'y a pas de pension minimum. Toutefois, la base d'évaluation des droits à pension est au moins égale au salaire minimum qui, en 2006, à été de 6900 SKK jusqu'au début du mois d'octobre et de 7600 SKK ensuite. Le salaire minimum représente un peu moins de $40 \%$ du salaire moyen.

\section{Régime à cotisations définies}

Le taux de cotisation du régime à cotisations définies est de $9 \%$ du salaire. L'adhésion à ce régime est obligatoire pour les travailleurs entrés sur le marché du travail à partir de janvier 2005 ; tous les autres devaient avoir choisi en juin 2006 de rester exclusivement dans le régime public ou d'adhérer à un régime mixte. La retraite à cotisations définies peut être perçue sous forme de rente ou selon une formule mixte associant des retraits échelonnés et une rente. La modélisation suppose qu'elle est prise sous la forme d'une rente indexée sur les prix à l'aide de taux de rente unisexe.

\section{Variantes de carrière}

\section{Retraite anticipée}

Un départ en retraite anticipée est possible avec des prestations réduites de $0.5 \%$ par mois d'anticipation (ce qui est équivalent à $6 \%$ par an). Un départ anticipé suppose également que la pension correspondante soit au moins égale à 1.2 fois le revenu de subsistance d'un adulte, qui était en 2006 de 4980 SKK par mois. Le minimum de subsistance pour l'année civile 2006 représentait $25.8 \%$ du salaire moyen, ce qui signifie que la pension minimum exigée pour un départ à la retraite anticipée était de 5976 SKK par mois, soit $31 \%$ du salaire moyen. En 2006, la pension moyenne de retraite anticipée était de 8970 SKK par mois, soit $46.7 \%$ du salaire moyen.

Il n'y a actuellement pas de limite d'âge pour partir en retraite anticipée: il est théoriquement possible de le faire à tout âge sous réserve à la fois de justifier de dix années de cotisation et de remplir la condition relative au niveau de la prestation.

\section{Retraite différée}

Il est possible de liquider sa retraite après l'âge normal. La prestation est majorée de $0.5 \%$ pour chaque mois de report (soit $6 \%$ par an). Pour les personnes qui font valoir leurs droits à la retraite et continuent de travailler, la pension sera recalculée au moment du départ définitif et l'on rajoutera la moitié des points acquis durant cette période.

\section{Enfants}

Les interruptions de carrière pour élever des enfants de moins de six ans bénéficient de crédits de retraite, l'État prenant à sa charge les cotisations correspondantes. La base retenue pour le calcul des pensions est de $60 \%$ de la rémunération avant la période d'interruption. Pour le premier semestre de chaque année civile, le calcul est effectué sur 
la base du salaire moyen deux ans avant le début de l'absence. Pour le second semestre, il est effectué sur la base de la rémunération de l'année civile immédiatement antérieure à l'absence. Les personnes s'occupant d'enfants handicapés perçoivent des pensions plus généreuses.

Ces règles s'appliquent également au régime à cotisations définies (pension de vieillesse).

\section{Chômage}

Les chômeurs ne bénéficient pas de crédits de retraite. Ils peuvent toutefois utiliser les dispositions relatives à une assurance retraite facultative. 


\section{Résultats de la modélisation des retraites : République slovaque}

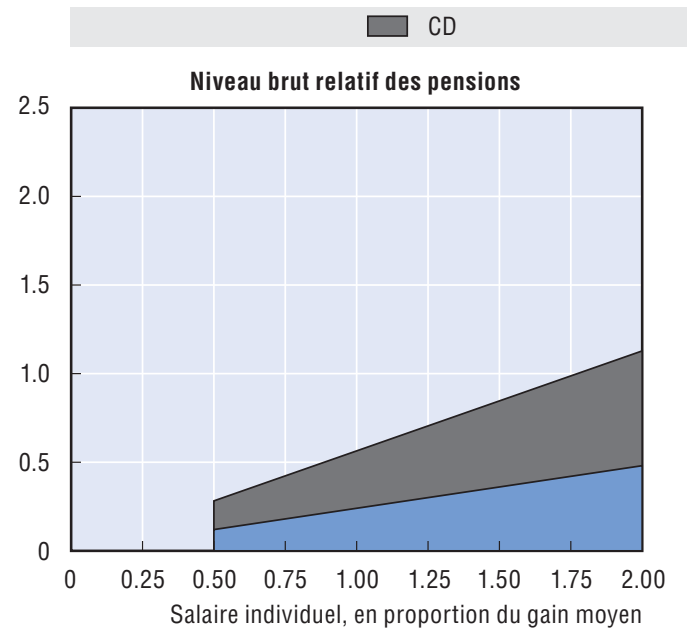

Lié à la rémunération
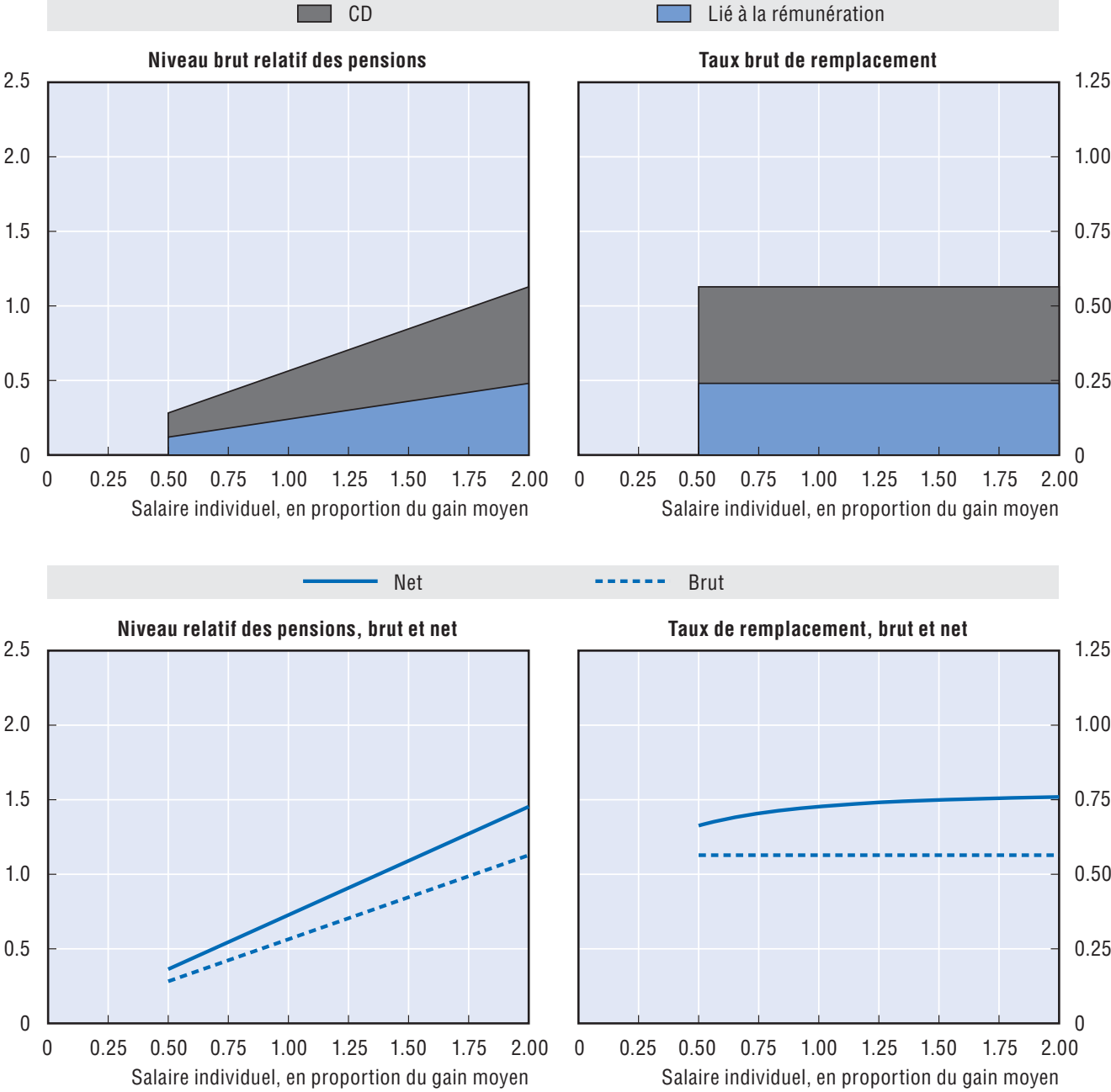

\section{$0 .+-1$ Brut}

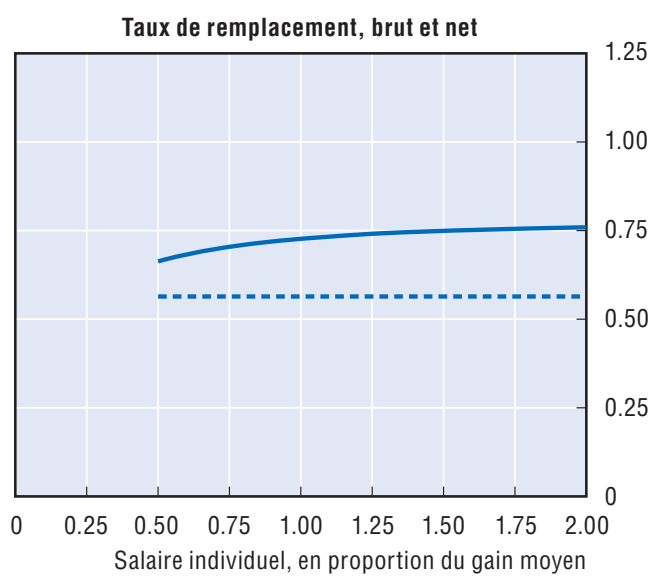

\begin{tabular}{|c|c|c|c|c|c|c|}
\hline \multirow{2}{*}{$\begin{array}{l}\text { Hommes } \\
\text { Femmes (si différent) }\end{array}$} & \multirow{2}{*}{$\begin{array}{l}\text { Salarié à revenu } \\
\text { médian }\end{array}$} & \multicolumn{5}{|c|}{ Salaire individuel, en multiple du salaire moyen } \\
\hline & & 0.5 & 0.75 & 1 & 1.5 & 2 \\
\hline Niveau brut relatif des pensions & 47.9 & 28.2 & 42.3 & 56.4 & 84.6 & 112.8 \\
\hline \multicolumn{7}{|l|}{ (\% salaire moyen brut) } \\
\hline Niveau net relatif des pensions & 61.8 & 36.3 & 54.5 & 72.7 & 109.0 & 145.4 \\
\hline \multicolumn{7}{|l|}{ (\% salaire moyen net) } \\
\hline Taux brut de remplacement & 56.4 & 56.4 & 56.4 & 56.4 & 56.4 & 56.4 \\
\hline \multicolumn{7}{|l|}{ (\% salaire individuel brut) } \\
\hline Taux net de remplacement & 71.5 & 66.3 & 70.4 & 72.7 & 74.9 & 75.9 \\
\hline \multicolumn{7}{|l|}{ (\% salaire individuel net) } \\
\hline Patrimoine retraite brut & 8.8 & 8.8 & 8.8 & 8.8 & 8.8 & 8.8 \\
\hline (multiple du salaire individuel brut) & 10.6 & 10.6 & 10.6 & 10.6 & 10.6 & 10.6 \\
\hline Patrimoine retraite net & 8.8 & 8.8 & 8.8 & 8.8 & 8.8 & 8.8 \\
\hline (multiple du salaire individuel brut) & 10.6 & 10.6 & 10.6 & 10.6 & 10.6 & 10.6 \\
\hline
\end{tabular}




\section{République tchèque}

\section{République tchèque : système de retraite en 2006}

Le régime public de retraite comporte une composante de base et une composante liée à la rémunération calculée selon une formule progressive. Il prévoit également une pension minimum.

\section{Conditions d'ouverture des droits}

L'âge normal de départ à la retraite sera progressivement porté à 65 ans pour les hommes au cours de la phase 1 de la nouvelle réforme des retraites. L'âge d'ouverture des droits à une pension se situera entre 62 et 65 ans pour les femmes avec enfants (en fonction du nombre d'enfants qu'elles auront élevés) ; il sera de 65 ans pour les femmes sans enfant. La couverture minimum requise de 25 ans sera progressivement portée à 35 ans (à raison d'un an de plus tous les ans à partir de 2010) mais les personnes ayant cotisé pendant 15 ans (chiffre qui passera progressivement à 20) peuvent percevoir une pension à partir de 65 ans.

\section{Calcul des prestations}

\section{Régime de base}

Le montant de la pension de base est de $1470 \mathrm{CZK}$ par mois, ce qui équivaut à $7.5 \%$ du salaire moyen. Il n'y a pas d'obligation légale d'indexation du montant de la prestation de base seule mais le montant total des pensions mises en paiement doit être revalorisé au minimum de la hausse des prix plus un tiers de la hausse des salaires réels (voir ci-dessous).

\section{Régime lié à la rémunération}

Le taux de la pension liée à la rémunération est de $1.5 \%$ de la rémunération par année de cotisation. Le salaire de référence est actuellement calculé sur la moyenne de toutes les années depuis 1985 mais la période passera progressivement à 30 ans (en 2015). Les rémunérations des années antérieures sont revalorisées en fonction de la hausse du salaire moyen à l'échelle nationale.

La formule de calcul des prestations est progressive : le taux de remplacement de la première tranche de $9100 \mathrm{CZK}$ par mois est de $100 \%$; pour la tranche comprise entre ce montant et 21800 CZK, il est de 30\% ; au-delà, il tombe à $10 \%$. Le premier seuil, en-dessous duquel le taux de remplacement est de $100 \%$, est équivalent à $46.5 \%$ du salaire moyen,, tandis que le second est de $111.4 \%$ du salaire moyen. Il n'y a pas d'obligation légale d'indexation de ces seuils mais tous deux ont été modifiés chaque année.

Il n'y a pas d'obligation légale spécifique d'indexation de la composante liée à la rémunération des pensions mises en paiement. Toutefois, la prestation globale de retraite 
(composante forfaitaire et composante liée à la rémunération) est ajustée au moins de l'inflation plus un tiers de la hausse des salaires réels.

\section{Pension minimum}

Le montant total de la prestation minimum de retraite est de $2240 \mathrm{CZK}$ par mois, constitué d'une pension minimum liée à la rémunération de $770 \mathrm{CZK}$ à laquelle s'ajoute la composante de base de $1470 \mathrm{CZK}$. Cette retraite minimum est indexée de la manière décrite plus haut. Elle correspond à $11.4 \%$ du salaire moyen.

\section{Aide sociale}

Les personnes âgées sont couvertes par le régime général de l'aide sociale et les prestations en nature correspondantes. Pour un ménage d'une seule personne, l'objectif de revenu du filet de sécurité est de $4420 \mathrm{CZK}$ par mois, soit $22.6 \%$ du salaire moyen. Il se compose d'un montant de $2400 \mathrm{CZK}$ au titre des besoins personnels et d'un montant de 2020 CZK au titre des besoins du ménage.

\section{Pensions privées facultatives}

Environ $45 \%$ des salariés ont une pension professionnelle ou individuelle facultative. Du fait de la limitation des incitations fiscales pour ces régimes, les cotisations tendent à être faibles. Pour la modélisation, on suppose un taux de cotisation de $2.8 \%$ du salaire.

\section{Variantes de carrière}

\section{Retraite anticipée}

Il est possible de partir à la retraite trois ans (chiffre qui passera à cinq sous réserve d'un départ pas avant 60 ans) avant l'âge normal, c'est-à-dire à 60 ans pour les hommes et 59-60 ans pour les femmes à condition d'avoir cotisé 25 ans (durée qui passera à 35 ans comme pour les conditions d'ouverture des droits). Le facteur total d'acquisition de droits (nombre d'années de cotisation multiplié par le taux) est réduit de façon permanente de $0.9 \%$ par trimestre d'anticipation pour les 720 premiers jours de retraite anticipée (3.6\% par an), et de $1.5 \%$ pour les trimestres suivants ( $6 \%$ par an à partir de 2010). Pour un travailleur ayant effectué une carrière complète, cela équivaut à une baisse du niveau de la pension (plutôt que du taux de remplacement) pour un départ anticipé à la retraite de 3.6/64.5 (1.5\% x 43 ans) = 5.6\%.

\section{Retraite différée}

Il est possible de différer la liquidation de ses droits au-delà de l'âge normal de la retraite. Le facteur global d'acquisition des droits (voir plus haut le paragraphe sur la retraite anticipée) est majoré de $1.5 \%$ pour chaque trimestre de report (6\% par an) mais l'intéressé n'acquiert pas de droits à pension supplémentaires pour départ différé. Il est également possible de percevoir une retraite tout en continuant à travailler.

\section{Enfants}

Les femmes sont habilitées à partir plus tôt à la retraite en fonction du nombre d'enfants qu'elles ont eu :

\begin{tabular}{llllc}
\hline Nombre d'enfants & 1 & 2 & 3 & $4+$ \\
Retraite anticipée (ans) & 0 & 1 & 2 & 3 \\
\hline
\end{tabular}


Il existe par ailleurs un système de crédits pour les périodes de sortie du marché de travail pour élever des enfants de moins de quatre ans (ou plus en cas de handicap sévère). Ces années ne sont pas prises en compte dans le calcul de la rémunération aux fins de la retraite de sorte qu'elles ne réduisent pas la base d'évaluation. (Cette approche est utilisée pour toutes les périodes non contributives.)

\section{Chômage}

Les périodes de chômage indemnisé par une assurance chômage liée à la rémunération sont validées. La durée des droits à l'assurance chômage varie avec l'âge : six mois jusqu'à 50 ans, neuf mois entre 50 et 55 ans et 12 mois au-delà de 55 ans. En outre, jusqu'à trois années de chômage sans droits à l'assurance chômage sont également validées. La période de chômage retenue pour le calcul de la pension est ramenée à 80 \%, ce qui signifie que si un individu a eu au cours de sa carrière cinq années de chômage, ce sont quatre années qui seront prises en compte pour l'établissement de sa retraite. Si la période de chômage tombe dans la période de référence (30 années précédant le départ à la retraite à partir de 1986) pour le calcul de la base d'évaluation moyenne, elle n'est pas prise en compte et seul est retenu le revenu sur la base duquel la prime est payée. 


\section{Résultats de la modélisation des retraites : République tchèque}

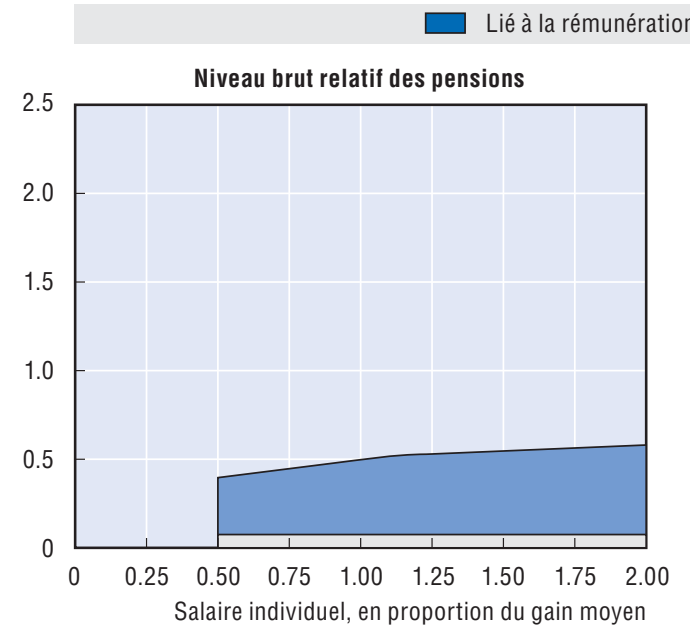

De base

Taux brut de remplacement

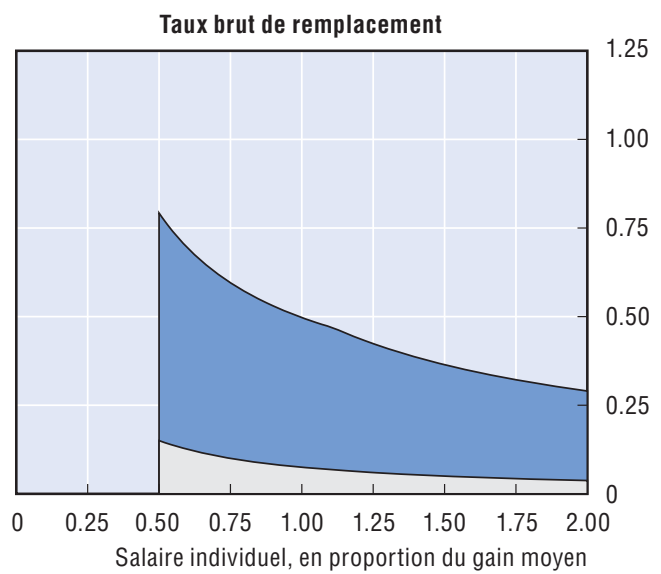

Net

-1-n-1 Brut
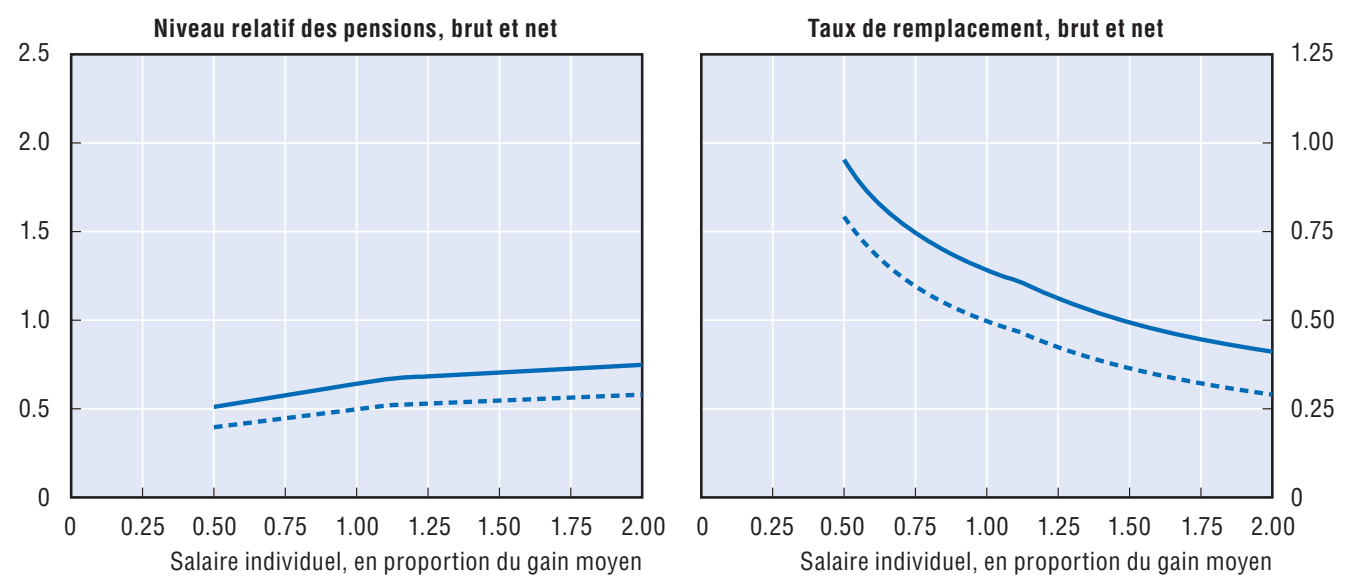

\begin{tabular}{|c|c|c|c|c|c|c|}
\hline \multirow{2}{*}{$\begin{array}{l}\text { Hommes } \\
\text { Femmes (si différent) }\end{array}$} & \multirow{2}{*}{$\begin{array}{c}\text { Salarié à revenu } \\
\text { médian }\end{array}$} & \multicolumn{5}{|c|}{ Salaire individuel, en multiple du salaire moyen } \\
\hline & & 0.5 & 0.75 & 1 & 1.5 & 2 \\
\hline Niveau brut relatif des pensions & 46.7 & 39.6 & 44.7 & 49.7 & 54.7 & 58.0 \\
\hline \multicolumn{7}{|l|}{ (\% salaire moyen brut) } \\
\hline Niveau net relatif des pensions & 60.2 & 51.1 & 57.6 & 64.1 & 70.5 & 74.8 \\
\hline \multicolumn{7}{|l|}{ (\% salaire moyen net) } \\
\hline Taux brut de remplacement & 54.9 & 79.2 & 59.6 & 49.7 & 36.4 & 29.0 \\
\hline \multicolumn{7}{|l|}{ (\% salaire individuel brut) } \\
\hline Taux net de remplacement & 69.8 & 95.3 & 74.7 & 64.1 & 49.4 & 41.1 \\
\hline \multicolumn{7}{|l|}{ (\% salaire individuel net) } \\
\hline Patrimoine retraite brut & 8.4 & 12.1 & 9.1 & 7.6 & 5.6 & 4.4 \\
\hline (multiple du salaire individuel brut) & 9.9 & 14.3 & 10.8 & 9.0 & 6.6 & 5.2 \\
\hline Patrimoine retraite net & 8.4 & 12.1 & 9.1 & 7.6 & 5.6 & 4.4 \\
\hline (multiple du salaire individuel brut) & 9.9 & 14.3 & 10.8 & 9.0 & 6.6 & 5.2 \\
\hline
\end{tabular}




\section{Royaume-Uni}

\section{Royaume-Uni : système de retraite en 2006}

Le Royaume-Uni a un système de retraite complexe qui conjugue une offre publique et une offre privée. Le régime public repose sur deux piliers (une retraite de base forfaitaire et une retraite complémentaire liée à la rémunération) que complète un vaste ensemble de pensions privées facultatives. La plupart des salariés sortent du deuxième pilier $d u$ régime public pour souscrire toutes sortes de retraites privées. Par ailleurs, une prestation liée à la rémunération (Pension Credit) a été mise en place pour les retraités les plus démunis.
Indicateurs essentiels

\begin{tabular}{llrr}
\hline & & Royaume-Uni & \multicolumn{1}{c}{ OCDE } \\
\hline Salaire moyen & GBP & 31500 & 19300 \\
& USD & 58000 & 35800 \\
Dépenses publiques & \% du PIB & 5.7 & 7.2 \\
au titre des retraites & & 79.1 & 78.9 \\
Espérance de vie & à la naissance & 83.3 & 83.4 \\
& à 65 ans & 26.8 & 23.8 \\
Population de plus de 65 ans & \% de la population d'âge actif & & \\
\hline
\end{tabular}

\section{Conditions d'ouverture des droits}

L'âge de la retraite, qui est actuellement de 60 ans pour les femmes nées le 5 avril 1950 ou avant cette date et de 65 ans pour les hommes, sera progressivement aligné sur 65 ans sur la période 2010-2020. Suite à l'adoption du Pensions Act 2007, cet âge sera porté à 66 ans entre 2024 et 2028, à 67 ans entre 2034 et 2036 et à 68 ans entre 2044 et 2046. L'âge d'ouverture des droits à l'élement crédit garanti du Pension credit est de 60 ans; il sera aligné sur l'âge de la retraite des femmes. Le nouvel élément du crédit d'épargne n'est disponible qu'à partir de 65 ans pour les hommes comme pour les femmes

Pour avoir droit à la pension publique de base, il faut cotiser à la sécurité sociale (ou être considéré comme tel) ou avoir acquis des crédits de retraite pour environ les 9/10 de la vie active potentielle ( 39 ans pour les femmes pour lesquelles l'âge de la retraite est de 60 ans ; 44 ans pour les hommes et les femmes pour lesquelles l'âge de la retraite est de 65 ans). Les personnes qui ne remplissent pas la condition d'un taux plein mais uniquement d'un taux minimum de $25 \%$ (dix ans pour les femmes pour lesquelles l'âge de la retraite est de 60 ans ; 11 ans pour les hommes et pour les femmes pour lesquelles cet âge est de 65 ans) perçoivent une pension réduite au prorata. Suite à l'adoption du Pensions Act 2007, le nombre d'années de cotisation ou les crédits requis pour avoir droit à une pension publique de base à taux plein sera abaissé à 30 et les pensions seront réduites au prorata pour un minimum d'une année de cotisation ou de crédits pour les personnes atteignant l'âge de la retraite le 6 avril 2010 ou après cette date.

\section{Calcul des prestations}

\section{Régime de base}

Le montant de la pension publique de base à taux plein pour une personne seule est de 84.25 GBP par semaine en 2006/07, ce qui équivaut à près de $14 \%$ du salaire moyen. 


\section{Régime lié à la rémunération}

Pour les salaires compris entre la limite inférieure (4 368 GBP par an en 2006/07) et le premier seuil de salaire (12 $500 \mathrm{GBP}$ ), le taux de remplacement est de $40 \%$ de la différence. La limite inférieure de rémunération représente près de $14 \%$ du salaire moyen alors que le premier seuil est de $40 \%$. Cela vaut également pour les personnes bénéficiant de crédits de retraite. Cela revient à traiter les personnes dont la rémunération est inférieure à ce premier seuil comme si elles avaient perçu ce niveau de rémunération. Sur la tranche suivante, qui s'arrête à 28800 GBP en 2006/07, le taux de remplacement est de $10 \%$. Entre ce seuil et le plafond, le taux de remplacement est de $20 \%$. Le plafond est de 33540 GBP en 2006/07. Le seuil supérieur représente environ $91 \%$ du salaire moyen et le plafond $106 \%$ du salaire moyen.

La valeur des prestations est calculée sur la base des gains moyens perçus sur l'ensemble de la carrière et les rémunérations antérieures sont revalorisées en fonction du salaire moyen à l'échelle nationale. Après le départ à la retraite, les prestations sont indexées sur les prix.

Suite à l'adoption du Pensions Act 2007, à compter de 2010, les tranches de revenus seront ramenées à deux. Entre la limite inférieure et le premier seuil de salaire, le taux de remplacement sera de $10 \%$. À compter d'une date qui reste à fixer, les revenus de la Tranche 1 donneront droit à une prestation forfaitaire de 1.60 GBP par semaine pour chaque année admissible à la prestation (rémunération 2008/09). De plus, à partir d'avril 2009, le plafond d'acquisition de droits sera gelé via l'introduction d'un point supérieur d'acquisition fixé à 770 GBP par semaine.

\section{Régime ciblé}

Le Pension credit mis en place en 2003 est une prestation hebdomadaire non imposable pour les personnes à faible revenu de 60 ans et plus, qui garantit à tous les retraités un niveau de revenu minimum. Il s'agit d'une prestation liée au revenu, qui ne repose pas sur les cotisations à un régime national d'assurance. Le Pension Credit est composé de deux éléments : le crédit garanti et le crédit d'épargne. Le crédit garanti assure un niveau de revenu minimum en accordant une aide financière aux personnes de 60 ans et plus dont le revenu est inférieur au montant de la garantie minimum type. En 2006/07, ce montant était de 114.05 GBP pour une personne seule et de 174.05 GBP pour un couple (ces montants peuvent être plus importants dans le cas de personnes gravement handicapées, ayant des responsabilités d'aidants ou des coûts de logement particuliers).

Le crédit d'épargne est un montant supplémentaire destiné aux personnes de 65 ans et plus disposant de moyens modestes pour leur retraite. Il vise à ramener le taux effectif de dégressivité des prestations de $100 \%$ dans les régimes précédents à $40 \%$. Les personnes dont le revenu (à l'exclusion du crédit de garantie) est inférieur au revenu minimum du crédit garanti mais supérieur au seuil du crédit d'épargne (respectivement 84.25 GBP pour une personne seule et 134.75 GBP pour un couple en 2006/07), perçoivent $60 \%$ de la différence entre leur revenu et le seuil à concurrence d'un maximum de 17.88 GBP pour une personne seule et de 23.58 GBP pour un couple, respectivement. Pour les personnes dont le revenu est supérieur au revenu minimum (qui donc n'ont pas droit au crédit garanti), le crédit d'épargne maximum est réduit de $40 \%$ de l'excédent de leur revenu sur le niveau garanti.

\section{Pensions privées facultatives}

Environ $47 \%$ des salariés adhèrent à un régime professionnel de retraite et environ $19 \%$ ont des plans individuels. Compte tenu du fait que certaines personnes ont les deux 
types de régime, la couverture globale des pensions privées obligatoires est de $59 \%$. Le plan à prestations définies modélisé verse une pension égale à $1 / 80 \mathrm{du}$ dernier salaire pour chaque année d'activité, ce qui équivaut à un taux d'acquisition de $1.25 \%$. Lorsque les personnes changent d'emploi, la valeur de la pension professionnelle différée est indexée sur les prix.

Toutefois, la plupart des plans professionnels du secteur privé sont désormais à cotisations définies, certains pour les nouveaux adhérents uniquement et d'autres pour tous les adhérents. Le gouvernement va également introduire un nouveau régime national d'épargne retraite. Basé sur les mêmes principes que le KiwiSaver néozélandais, ce régime aura un taux de cotisation par défaut de $8 \%$, soit légèrement inférieur au taux moyen de cotisation de $9 \%$ des plans professionnels à cotisations définies existants. La modélisation suppose un taux de cotisation de $8 \%$ du salaire.

\section{Variantes de carrière}

\section{Retraite anticipée}

Le régime public n'autorise pas le versement d'une pension avant l'âge de la retraite.

\section{Retraite différée}

Jusqu'en avril 2005, il était possible de reporter la liquidation de la retraite publique d'au maximum cinq ans par rapport à l'âge normal avec une majoration d'environ $7.4 \%$ par année de report. Depuis avril 2005, cette limite a été supprimée et la majoration a été portée à environ 10.4 \% par année pleine de report. Il est également possible de percevoir la pension sous la forme d'un montant forfaitaire imposable sous réserve que le report soit d'au minimum 12 mois consécutifs. Ce montant forfaitaire est constitué du montant de la retraite non liquidée pendant la durée du report, majoré d'un taux d'intérêt garanti d'au moins deux points de pourcentage par rapport au taux de base de la Banque d'Angleterre. Le choix doit être fait lors de la liquidation définitive.

\section{Enfants}

Les deux piliers du régime public de retraite (pension publique de base et retraite complémentaire) assurent une protection pour les périodes d'éducation des enfants. Cette protection couvre les personnes en situation de non emploi et celles qui travaillent mais gagnent moins que la limite inférieure et donc ne cotisent pas au régime de retraite. Pour la pension publique de base, ce dispositif appelé Home Responsibilities Protection (HRP) couvre les années consacrées à élever au moins un enfant de moins de seize ans. Ce dispositif réduit le nombre d'années requis pour percevoir une retraite à taux plein de sorte qu'avec un HRP suffisant seules 20 années de travail (incluant les périodes pour lesquelles des cotisations à la sécurité sociale peuvent être validées) sont nécessaires pour percevoir une pension publique de base à taux plein. Pour la retraite complémentaire, les années consacrées à élever un enfant de moins de 6 ans sont validées ; les parents s'occupant de leurs enfants sont réputés avoir une rémunération égale au premier seuil de salaire (12 500 GBP par an en 2006/07).

Suite à l'adoption du Pensions Act 2007, les personnes atteignant l'âge de la retraite publique après 2010 pourront accumuler des droits au titre de la S2P (State Second Pension) si elles élèvent des enfants de moins de 12 ans. 


\section{Chômage}

Les périodes de chômage indemnisé (assurance chômage ou prestations d'aide) sont validées pour la pension publique de base. Pour la State Second Pension, ces périodes de prestations ne sont pas validées.

\section{Résultats de la modélisation des retraites : Royaume-Uni}
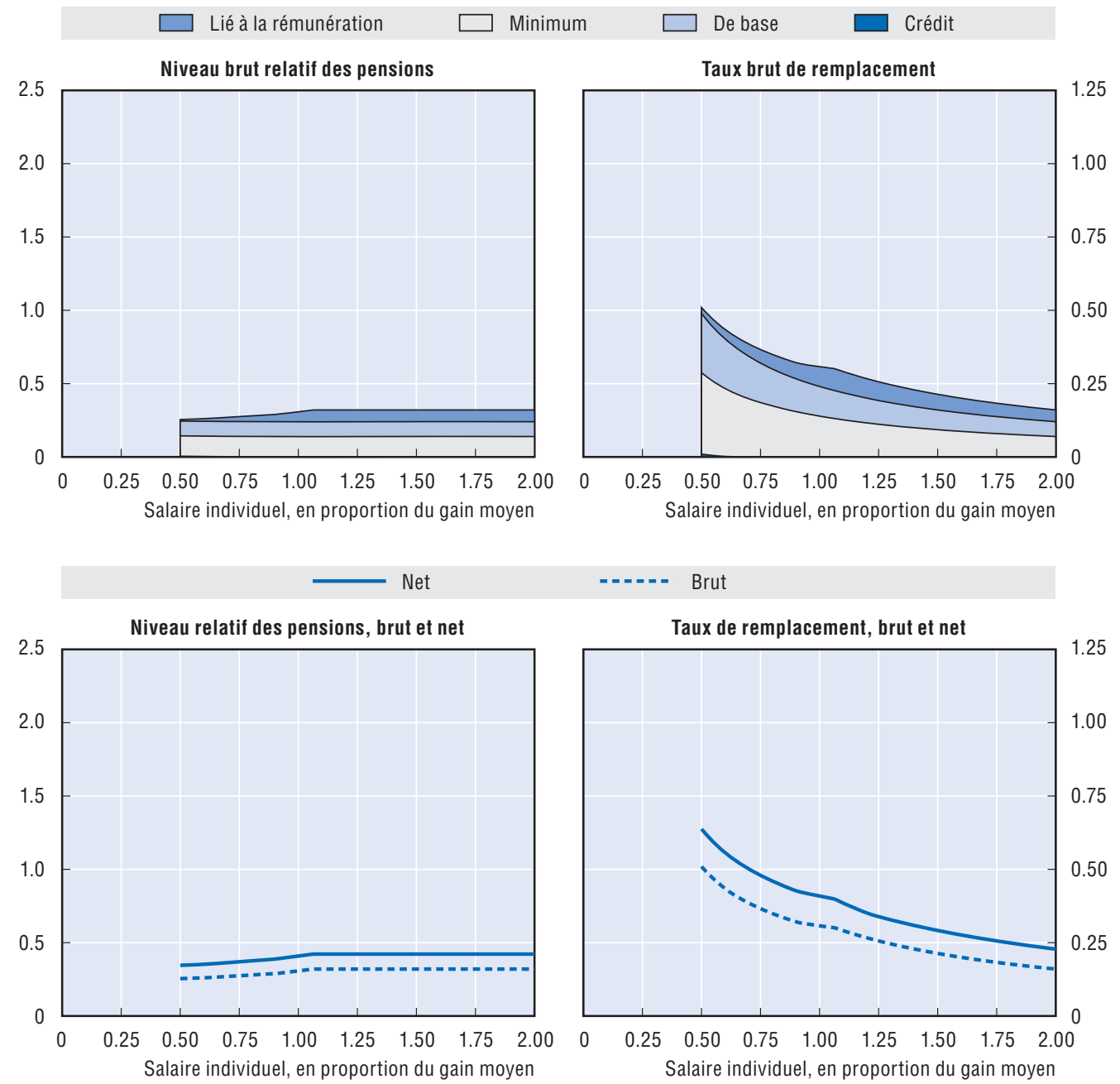

\begin{tabular}{|c|c|c|c|c|c|c|}
\hline \multirow{2}{*}{$\begin{array}{l}\text { Hommes } \\
\text { Femmes (si différent) }\end{array}$} & \multirow{2}{*}{$\begin{array}{c}\text { Salarié à revenu } \\
\text { médian }\end{array}$} & \multicolumn{5}{|c|}{ Salaire individuel, en multiple du salaire moyen } \\
\hline & & 0.5 & 0.75 & 1 & 1.5 & 2 \\
\hline Niveau brut relatif des pensions & 28.4 & 25.5 & 27.5 & 30.8 & 32.0 & 32.0 \\
\hline \multicolumn{7}{|l|}{ (\% salaire moyen brut) } \\
\hline Niveau net relatif des pensions & 38.2 & 34.5 & 37.0 & 40.9 & 42.2 & 42.2 \\
\hline \multicolumn{7}{|l|}{ (\% salaire moyen net) } \\
\hline Taux brut de remplacement & 33.5 & 51.0 & 36.6 & 30.8 & 21.3 & 16.0 \\
\hline \multicolumn{7}{|l|}{ (\% salaire individuel brut) } \\
\hline Taux net de remplacement & 44.3 & 63.8 & 48.0 & 40.9 & 29.2 & 22.8 \\
\hline \multicolumn{7}{|l|}{ (\% salaire individuel net) } \\
\hline Patrimoine retraite brut & 4.5 & 6.8 & 4.9 & 4.1 & 2.9 & 2.1 \\
\hline (multiple du salaire individuel brut) & 5.2 & 7.8 & 5.6 & 4.7 & 3.3 & 2.5 \\
\hline Patrimoine retraite net & 4.4 & 6.8 & 4.8 & 4.0 & 2.8 & 2.1 \\
\hline (multiple du salaire individuel brut) & 5.1 & 7.8 & 5.5 & 4.6 & 3.2 & 2.4 \\
\hline
\end{tabular}




\section{Suède}

\section{Suède : système de retraite en 2006}

Le nouveau régime de retraite, mis en place en 1999, s'applique aux personnes nées à partir de 1954. L'ancien et le nouveau régimes couvrent les travailleurs âgés au prorata : les personnes nées entre 1938 et 1953 relèvent d'un système mixte associant les anciennes et les nouvelles règles. La partie liée à la rémunération repose sur un système de comptes notionnels, auquel s'ajoute une petite cotisation obligatoire à des régimes individuels par capitalisation à cotisations définies. Il existe par ailleurs un complément de retraite soumis à condition de revenu. Les plans professionnels de retraite, qui comportent des éléments à prestations définies et des éléments à cotisations définies, ont une large couverture.

Pour les plans professionnels, le nouveau régime ITP a été adopté en 2006 . Il comporte des dispositions transitoires de longue durée mais s'applique pleinement aux personnes nées à partir de 1979.

\section{Indicateurs essentiels}

\begin{tabular}{llrr}
\hline & & Suède & \multicolumn{1}{c}{ OCDE } \\
\hline Salaire moyen & SEK & 324600 & 263800 \\
& USD & 44000 & 35800 \\
Dépenses publiques & \% du PIB & 7.7 & 7.2 \\
au titre des retraites & & 80.8 & 78.9 \\
Espérance de vie & à la naissance & 84.2 & 83.4 \\
& à 65 ans & 29.4 & 23.8 \\
Population de plus de 65 ans & \% de la population d'âge actif & & \\
\hline
\end{tabular}

\section{Conditions d'ouverture des droits}

La pension liée au revenu et la « premium pension » peuvent être liquidées à partir de 61 ans.

Le droit à une retraite garantie est acquis après trois années de résidence. La liquidation de ce droit est possible à partir de 65 ans.

La retraite garantie maximum est acquise après 40 années de résidence ; pour des durées plus courtes, elle est réduite au prorata. Elle peut être liquidée à partir de 65 ans.

\section{Calcul des prestations}

Des cotisations, égales à $18.5 \%$ de la rémunération ouvrant droit à pension, sont créditées puis revalorisées selon une moyenne mobile sur trois ans du salaire moyen à l'échelle nationale. La rémunération ouvrant droit à pension se définit comme le salaire diminué de la part salariale des cotisations au régime de retraite (c'est-à-dire au système de comptes notionnels comme au Premium Pension System) de $7 \%$ du salaire brut, ce qui donne un taux de cotisation effectif sur le salaire brut de $17.21 \%$, se ventilant en $14.88 \%$ au système de comptes notionnels et $2.33 \%$ aux régimes par capitalisation à cotisations définies. Les cotisations ne sont prélevées que lorsque la rémunération annuelle dépasse un plancher de 16800 SEK en 2006, à peine supérieur à 5.2 \% du salaire moyen, mais elles sont dues sur l'intégralité de la rémunération pour toutes les personnes gagnant plus que 
ce niveau plancher. La rémunération ouvrant droit à pension sur la base de laquelle sont calculées les prestations est plafonnée à 333750 SEK en 2006, ce qui donne un plafond effectif en termes de salaire brut de 359100 SEK en 2006 (environ 111 \% du salaire moyen). Les cotisations patronales sont également soumises à ce plafond mais les rémunérations au-delà de ce plafond sont soumises à une taxe supplémentaire à un taux identique à celui de la cotisation de retraite mais dont le produit va directement sur le budget du gouvernement central et ne donne aucun droit de retraite.

\section{Régime lié à la rémunération}

Le nouveau régime lié à la rémunération utilise un système de comptes notionnels. Les comptes notionnels sont augmentés chaque année du montant des soldes de retraite des personnes décédées qui sont répartis entre les survivants du même âge ("gains hérités »). Les gains hérités des personnes qui décèdent avant l'âge auquel le départ à la retraite devient possible (61 ans) sont importants. Après cet âge, ce facteur est estimé sur la base de la mortalité observée sur une période antérieure (calculée à partir de tables de mortalité unisexe sur cinq ans).

Lors du départ à la retraite, le capital fictif accumulé sera converti en rente. Le calcul utilisera un coefficient qui dépendra de l'âge individuel de départ à la retraite et de l'espérance de vie à cette date (sur la base des tables de mortalité unisexe des cinq années précédentes). Dans ce calcul, on supposera un taux d'actualisation reel de $1.6 \%$ par an. À titre d'exemple, la valeur du coefficient de rente à 65 ans est de 15.4 pour l'année 2000 ; elle passera à 16.8 d'ici à 2020 et à 17.4 d'ici à 2040. Le coefficient de rente est actuellement de 18.0 pour un départ à 61 ans et de 12.8 pour un départ à 70 ans pour les personnes nées en 1940 .

Après le départ à la retraite, les pensions sont revalorisées en fonction de la hausse du salaire moyen nominal, diminuée du taux d'intérêt implicite de $1.6 \%$ pour le nombre d'annuités.

Par ailleurs, un "mécanisme d'équilibrage " a été mis en place : si les actifs (fonds de régulation plus valeur estimée des recettes liées aux cotisations) deviennent inférieurs aux passifs (capital fictif accumulé et valeur en capital des pensions à verser), alors l'indexation des pensions mises en paiement et les rendements portés au crédit des comptes notionnels sont réduits du ratio actifs/passifs. Le ratio d'équilibre a atteint un point bas de 1.0014 en 2004 et s'est maintenu légèrement au-dessus de 1 jusqu'en 2007 (1.0097 en 2003, 1.0014 en 2004, 1.0044 en 2005 et 1.0149 en 2006). En 2008, cependant, il est tombé à 0.9672. On utilise le ratio d'équilibre de l'année t pour calculer le chiffre d'équilibre ou la nécessité d'activer le mécanisme d'équilibrage pour l'année $t+2$. L'activation du mécanisme d'équilibrage génèrerait une baisse des taux de remplacement du régime national mais pourrait également les faire remonter en cas de redressement du régime de retraite et de relèvement du chiffre d'équilibre (l'indice d'équilibre peut être supérieur à l'indice de revenu pendant la période de reprise).

Aux fins de la modélisation, les coefficients de rente sont calculés à l'aide des règles ci-dessus et des données tirées des tables de mortalité de la base de données Nations Unies/Banque mondiale sur la population. Le mécanisme d'équilibrage est supposé ne pas affecter la revalorisation des prestations.

\section{Pension minimum}

La «pension garantie » est un complément soumis à condition de revenu pour les personnes n'ayant qu'un faible montant de prestations au titre des comptes notionnels. 
Pour une personne seule, la prestation totale garantie s'établissait à 84561 SEK en 2006 pour un retraité né après 1938 , soit $26 \%$ du salaire moyen brut.

La pension garantie est dégressive aux taux de $100 \%$ pour les 50022 premiers SEK (2006) de revenu de la pension liée à la rémunération, pour une personne seule, et de $48 \%$ au-delà. Ce seuil est équivalent à $15 \%$ du salaire moyen. Ce n'est que lorsque la pension liée à la rémunération excède 121879 SEK (près de 38 \% du salaire moyen) que le droit à la pension garantie est épuisé.

Dans la législation actuelle, le niveau de la pension garantie est indexé sur les prix. Toutefois, dans la modélisation, l'hypothèse de base pour tous les pays est que la valeur des prestations de retraite au titre du filet de sécurité tendra, avec le temps, à suivre l'évolution du salaire moyen plutôt qu'à diminuer par rapport au niveau de vie général.

À cela s'ajoute une indemnité de logement qui couvre $93 \%$ des frais de logement à concurrence d'un maximum de 5000 SEK par mois pour un retraité. Cette prestation représente une part importante du niveau de vie minimum pour les retraités suédois. Elle est soumise à conditions de ressources et n'est pas prise en compte dans les calculs de la modélisation.

\section{Régime à cotisations définies}

Un pourcentage complémentaire de $2.5 \%$ de la rémunération ouvrant droit à pension (donnant un taux effectif de cotisation de $2.325 \%$ de la rémunération brute) sera versé sur les comptes de retraite individuels : c'est la «premium pension ». Pour l'investissement de ces fonds, les personnes ont le choix entre un large éventail de formules.

Lors de leur départ à la retraite, les intéressés choisissent la manière dont ils percevront les prestations. Ils peuvent tout d'abord convertir la pension en rente afin d'éviter le risque d'investissement. Ils peuvent également opter pour une rente variable, leurs fonds continuant d'être investis par le gestionnaire de fonds qu'ils auront choisi. Ces rentes n'ont pas de valeur garantie. Dans ce cas, le principe de calcul de la pension est le suivant : la valeur du compte est divisée par le nombre d'annuités (sur la base d'une estimation de l'espérance de vie moyenne) et la pension est créditée d'un taux d'intérêt futur estimé de $3 \%$ moins les frais administratifs. Si le rendement est supérieur à $3 \%$, soit un versement complémentaire est effectué soit le solde du compte est plus élevé et par conséquent la base de calcul de la pension annuelle est, elle aussi, plus élevée.

\section{Régimes professionnels quasi obligatoires}

On estime que l'ensemble des régimes professionnels couvre près de $90 \%$ des salariés. On ne recense que quatre grands régimes professionnels. Pour les " cols blancs ", la modélisation a retenu le régime ITP qui associe des éléments à prestations définies et des éléments à cotisations définies. Ce régime a été récemment renégocié. L'ancien système reste en vigueur, avec quelques changements mineurs, pour les personnes nées en 1978 et avant ; le nouveau couvre les personnes nées à partir de 1979.

\section{ITP1}

À partir du $1^{\mathrm{er}}$ janvier 2007, les salariés nés en 1979 et au-delà commencent à acquérir des droits à pension à partir de l'âge de 25 ans dans le nouveau régime ITP1. Il s'agit d'un plan à cotisations définies. La cotisation est égale à $4.5 \%$ du salaire jusqu'à 7.5 fois le revenu de base (333 750 SEK en 2006). Pour les tranches de salaire au-delà de 7.5 fois le 
revenu de base (divisé par 12 pour un mois), la cotisation est de $30 \%$. Le salaire ouvrant droit à pension devient le salaire brut versé en espèces, à l'exclusion des remboursements de frais. Les primes sont payées à partir de la première couronne suédoise (SEK) de salaire.

Le salarié peut choisir la forme d'épargne et le gestionnaire du fonds. Cependant, la moitié au moins de la cotisation est investie dans une assurance retraite traditionnelle. Le salarié peut également choisir une couverture de remboursement et une couverture familiale de un, deux, trois ou quatre fois les montants de base par an sur 5, 10, 15 ou 20 ans. Les cotisations de ceux qui ne spécifient pas leur choix sont investies dans une assurance retraite traditionnelle sans couverture de remboursement ou couverture familiale. C'est ce choix par défaut qui a été retenu dans la modélisation.

Les salariés dont le salaire annuel est supérieur à dix fois le revenu de base (445 000 SEK en 2006) peuvent choisir de relever du nouveau régime avec l'accord de leur employeur. Cette possibilité s'applique que le salarié relève d'un plan ITP2 traditionnel ou qu'il ait opté pour un autre plan ITP.

\section{Variantes de carrière}

\section{Retraite anticipée}

Un départ à la retraite anticipée est possible à partir de 61 ans dans le régime public (pension liée à la rémunération et " premium pension "). Il n'y a pas d'âge fixe de départ en retraite. Les comptes notionnels et les calculs de rentes assurent une réduction actuarielle qui dépend de l'âge de départ à la retraite.

La retraite garantie soumise à condition de revenu ne peut être liquidée avant 65 ans. Dans un système de comptes notionnels, si la retraite est liquidée avant ou après 65 ans, la retraite garantie est toujours calculée comme si elle avait été liquidée à 65 ans.

Dans le nouveau régime ITP1, les pensions sont normalement servies à partir de 65 ans, mais elles peuvent être prises à partir de 55 ans. Les pensions sont à vie mais elles peuvent être servies intégralement ou partiellement pendant une période limitée d'au minimum cinq ans. La modélisation de la rente accorde des paiements à vie. L'importance de la pension est déterminée par le montant de primes versé, le rendement, les droits et taxes, et la durée pendant laquelle la pension doit être versée.

\section{Retraite différée}

La pension basée sur un système de comptes notionnels et la "premium pension" peuvent être différées sans limite d'âge supérieure, là encore avec ajustement actuariel automatique. Il est également possible de cumuler un emploi et une retraite. Enfin, la retraite peut être liquidée partiellement (à 25,50 ou $75 \%$ de la retraite à taux plein). La retraite garantie est ajustée par rapport aux autres pensions du régime vieillesse suédois et aux régimes nationaux étrangers comparables mais elle n'est pas réduite par les revenus salariaux, du capital, des retraites professionnelles ou de l'assurance retraite privée. Il est donc également possible de cumuler un emploi et une retraite garantie.

La retraite professionnelle ITP1 peut être reportée après 65 ans mais aucun droit supplémentaire à pension ne peut être acquis après cet âge.

\section{Enfants}

Dans le régime public de retraite, des années sont créditées au titre des périodes consacrées à l'éducation d'enfants de quatre ans et moins. Dans un ménage biparental, en 
l'absence de choix établi, les crédits vont au parent ayant le revenu le plus bas. Les crédits sont calculés selon la formule la plus intéressante parmi les trois suivantes. 1) Si le revenu est nul ou inférieur aux gains des années précédentes, les crédits sont établis sur la base de la rémunération de l'année précédant la naissance de l'enfant. 2) Pour les travailleurs à faible revenu ou les personnes qui ne travaillaient pas avant d'avoir des responsabilités liées aux enfants, les crédits sont établis sur la base de $75 \%$ du salaire moyen à l'échelle nationale. 3) Si le revenu augmente effectivement ou s'il ne diminue pas de façon sensible après le début des responsabilités liées aux enfants le crédit est fixé à une fois le revenu de base. Dans les trois cas, l'État prend en charge l'intégralité des cotisations au régime de retraite (couvrant à la fois le revenu de retraite et la "premium pension "). Mais il le fait jusqu'au plafond de rémunération dans le système de retraite. Par ailleurs, les allocations versées aux personnes en congé parental de travail sont également considérées comme un revenu ouvrant droit à pension. Le bénéficiaire acquitte la cotisation salariale de retraite de $7 \%$ sur le produit des prestations. L'État prend en charge l'ensemble des « cotisations patronales » de $10.21 \%$ pour les revenus de la sécurité sociale y compris les allocations parentales.

L'allocation parentale est payable comme suit pendant 480 jours :

- 390 jours à $80 \%$ du revenu annuel du parent jusqu'à concurrence d'un plafond égal à dix fois le montant des prix de base (39000 SEK en 2006) ;

- 90 jours à un taux uniforme universellement applicable de 180 SEK/jour.

L'allocation parentale est calculée sur une base journalière. Les parents à revenu modeste ou nul perçoivent une allocation minimum garantie de 180 SEK/jour. Les 480 jours d'allocation parentale sont divisés à parts égales entre les deux parents (240 jours pour chacun). Un parent peut également transférer à l'autre jusqu'à 180 de ses jours.

Dans le régime professionnel ITP, il est recommandé à l'employeur de cotiser pour la retraite d'un salarié pendant des périodes allant jusqu'à 11 mois de congé parental (ce que font la plupart des employeurs).

\section{Chômage}

Les indemnités de chômage et allocations de formation versées aux chômeurs participant à des programmes actifs du marché du travail sont des revenus ouvrant droit à pension et l'État prend à sa charge la part " patronale " de cotisation. Les indemnités de chômage liées à la rémunération sont égales à 80 \% de la rémunération antérieure pendant les 200 premiers jours. Entre le $201^{\mathrm{e}}$ et le $300^{\mathrm{e}}$ jour, l'indemnité est égale à $70 \%$ de la rémunération antérieure. Par la suite, elle est de $65 \%$ de la rémunération antérieure à moins que l'un des deux parents ait un enfant de moins de 18 ans auquel cas l'indemnité correspondante demeure de $70 \%$ de la rémunération antérieure. Les indemnités de chômage sont plafonnées à 680 SEK par jour et assujetties à un paiement minimum de 320 SEK par jour. Elles peuvent, sous certaines conditions, être versées pendant un maximum de 600 jours.

Après avoir perçu des indemnités de chômage pendant un maximum de 600 jours, le bénéficiaire a droit à une garantie d'activité impliquant la perception d'une allocation de formation dans laquelle la rémunération est du niveau de l'indemnité de chômage et permet l'acquisition de droits à pension. La garantie d'activité et l'allocation de formation ultérieure peuvent être obtenues pour une période illimitée jusqu'à 65 ans. Un chômeur choisira rarement de liquider sa pension de vieillesse plutôt que de percevoir une allocation de formation, ce qui se traduirait pour lui par une retraite plus faible. 
Résultats de la modélisation des retraites : Suède

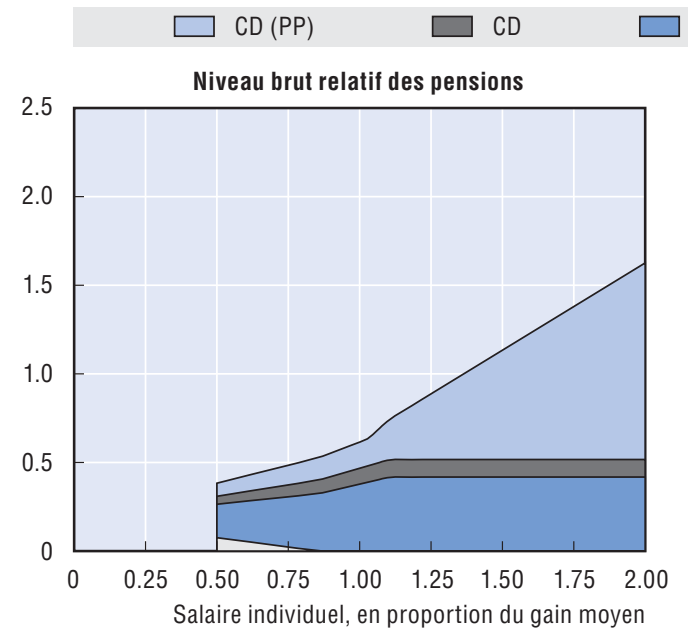

Lié à la rémunération $\square$ Minimum

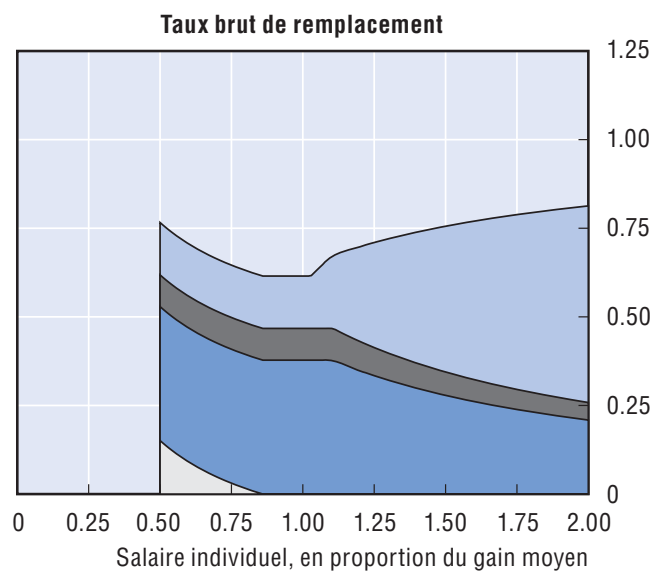

Net

$-----\cdot$ Brut
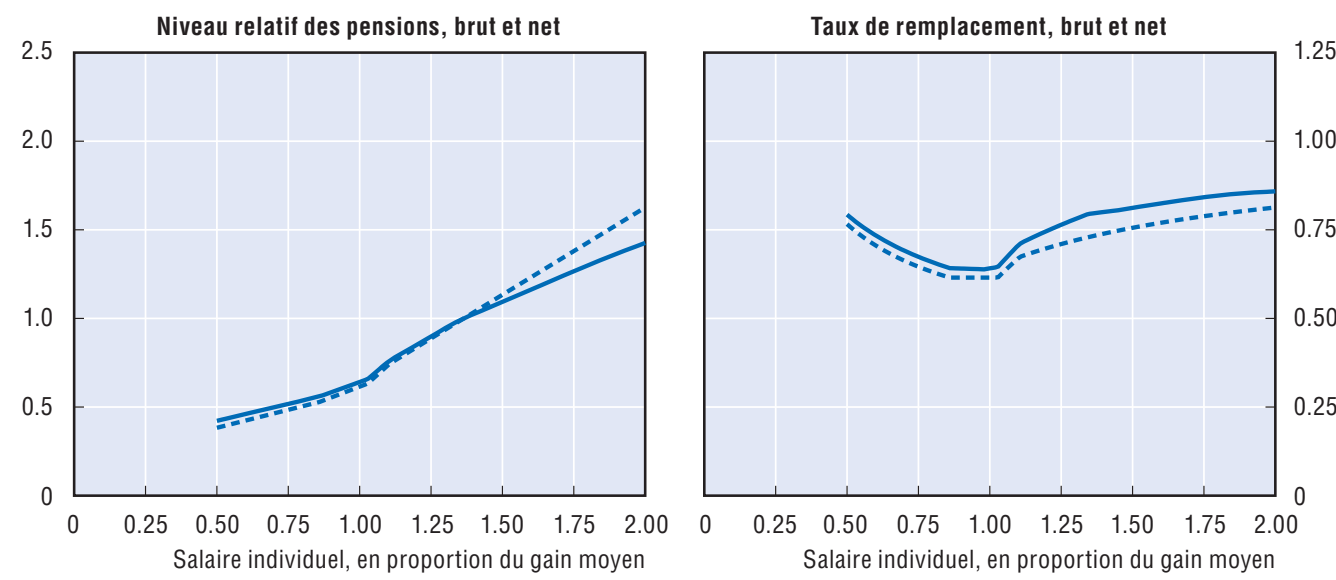

\begin{tabular}{|c|c|c|c|c|c|c|}
\hline \multirow{2}{*}{$\begin{array}{l}\text { Hommes } \\
\text { Femmes (si différent) }\end{array}$} & \multirow{2}{*}{$\begin{array}{l}\text { Salarié à revenu } \\
\text { médian }\end{array}$} & \multicolumn{5}{|c|}{ Salaire individuel, en multiple du salaire moyen } \\
\hline & & 0.5 & 0.75 & 1 & 1.5 & 2 \\
\hline Niveau brut relatif des pensions & 54.1 & 38.3 & 48.4 & 61.5 & 113.3 & 162.6 \\
\hline \multicolumn{7}{|l|}{ (\% salaire moyen brut) } \\
\hline Niveau net relatif des pensions & 57.2 & 42.2 & 51.8 & 64.1 & 109.4 & 142.6 \\
\hline \multicolumn{7}{|l|}{ (\% salaire moyen net) } \\
\hline Taux brut de remplacement & 61.5 & 76.6 & 64.6 & 61.5 & 75.6 & 81.3 \\
\hline \multicolumn{7}{|l|}{ (\% salaire individuel brut) } \\
\hline Taux net de remplacement & 64.1 & 79.3 & 67.4 & 64.1 & 81.2 & 85.9 \\
\hline \multicolumn{7}{|l|}{ (\% salaire individuel net) } \\
\hline Patrimoine retraite brut & 9.9 & 12.2 & 10.3 & 9.9 & 12.0 & 12.9 \\
\hline (multiple du salaire individuel brut) & 11.3 & 14.0 & 11.8 & 11.3 & 13.7 & 14.7 \\
\hline Patrimoine retraite net & 7.2 & 9.3 & 7.6 & 7.1 & 8.0 & 7.8 \\
\hline (multiple du salaire individuel brut) & 8.2 & 10.6 & 8.7 & 8.1 & 9.1 & 8.9 \\
\hline
\end{tabular}




\section{Suisse}

\section{Suisse : système de retraite en 2006}

Le régime de retraite suisse comporte principalement trois composantes : un régime public lié à la rémunération mais selon une formule progressive, des retraites professionnelles obligatoires et une retraite complémentaire soumise à condition de revenu.

\section{Indicateurs essentiels}

\begin{tabular}{lrrr}
\hline & & Suisse & \multicolumn{1}{c}{ OCDE } \\
\hline Salaire & CHF & 72400 & 44800 \\
moyen & USD & 57800 & 35800 \\
$\begin{array}{l}\text { Dépenses publiques } \\
\text { au titre des retraites }\end{array}$ & $\%$ du PIB & 6.8 & 7.2 \\
Espérance de vie & à la naissance & 81.7 & 78.9 \\
& à 65 ans & 85.3 & 83.4 \\
Population de plus de 65 ans & $\%$ de la population d'âge actif & 25.9 & 23.8 \\
\hline
\end{tabular}

\section{Conditions d'ouverture des droits}

Dans le régime public et les retraites professionnelles obligatoires, l'âge ouvrant droit à pension est actuellement de 65 ans pour les hommes et de 64 ans pour les femmes. Pour bénéficier d'une retraite à taux plein, 44 années de cotisation sont nécessaires pour les hommes et 43 pour les femmes.

\section{Calcul des prestations}

\section{Régime lié à la rémunération}

La retraite du régime public est calculée sur la base des gains moyens perçus sur l'ensemble de la carrière. Si ce chiffre est inférieur à 38700 CHF, le droit à pension acquis est de 9546 CHF plus $26 \%$ des gains moyens perçus sur l'ensemble de la carrière. S'il est supérieur à ce seuil; le droit à pension acquis est un montant forfaitaire de 13416 CHF plus $16 \%$ des gains moyens perçus sur l'ensemble de la carrière.

Le montant de la pension minimum est de $12900 \mathrm{CHF}$ et celui de la pension maximum est égal à deux fois ce montant, ce qui équivaut respectivement à $18 \%$ et $36 \%$ du salaire moyen. La prestation maximum est atteinte lorsque les gains moyens perçus sur l'ensemble de la carrière sont de $77400 \mathrm{CHF}$, ce qui équivaut à $107 \%$ du salaire moyen à l'échelle nationale.

Les pensions mises en paiement sont indexées à $50 \%$ sur les prix et à $50 \%$ sur le salaire nominal.

\section{Régimes professionnels obligatoires}

Le système des retraites professionnelles obligatoires a été mis en place en 1985. Il s'articule autour de "crédits définis " à un compte de retraite individuel. Ces crédits varient en fonction du sexe et de l'âge :

\begin{tabular}{lcccc}
\hline Hommes et femmes, âge (à partir de 2005) & $25-34$ & $35-44$ & $45-54$ & $55-64 / 63$ \\
Femmes, âge (1987-2004) & $25-31$ & $32-41$ & $42-51$ & $52-62 / 63$ \\
Crédit (\% du salaire coordonné) & 7 & 10 & 15 & 18 \\
\hline
\end{tabular}

La valeur des crédits accumulés au moment du départ à la retraite dépend naturellement du taux d'intérêt appliqué aux cotisations des années antérieures. Pendant longtemps (jusqu'à fin 2002), ce taux a été de $4 \%$, mais en 2003 il a été ramené à $3.25 \%$ et en 2004 à $2.25 \%$. En 2005, il a été relevé à $2.5 \%$. Si le taux d'intérêt est en gros équivalent 
au taux de progression de la rémunération, un homme ayant fait une carrière complète aura à 65 ans un montant cumulé de crédits égal à $500 \%$ de ses gains. Toutefois, des résultats supérieurs (ou inférieurs) sont possibles si le taux d'intérêt est supérieur (ou inférieur) à la progression de la rémunération. La modélisation suppose que le taux d'intérêt appliqué aux crédits sera équivalent à la rémunération sur le long terme.

Un taux de rente minimum de $7.10 \%$ pour les hommes (65 ans) et de $7.2 \%$ pour les femmes (64 ans) est appliqué à ce capital fictif, ce qui donne pour une carrière complète un taux de remplacement de $500 \times 7.1=35.5 \%$ pour les hommes (sous réserve que le taux d'intérêt soit égal à la progression de la rémunération). À compter de 2005, ce taux est ramené progressivement de $7.10 \%$ à $6.8 \%$ sur dix ans.

Les crédits définis (et donc le taux de remplacement) ne s'appliquent qu'au salaire " coordonné ". Il s'agit de la rémunération comprise entre les trois quarts de la pension maximum du régime public (19350 CHF pour 2006) et trois fois le montant de cette pension (77 $40 \mathrm{CHF}$ pour 2006). Ces seuils sont équivalents à $27 \%$ et $107 \%$ du salaire moyen. La déduction de coordination est de 7/8 de la pension maximum du régime public (22 $575 \mathrm{CHF}$ pour 2006). On notera que le plafond de la rémunération ouvrant droit à pension est le même dans le régime public et dans les régimes professionnels obligatoires. Le minimum de salaire coordonné est de $1 / 8$ de la valeur maximum. Les crédits acquis sont fixés à ce niveau minimum pour les personnes dont le salaire coordonné est inférieur à ce niveau.

\section{Régime ciblé}

Le montant de la prestation annuelle est la part des dépenses constatées excédant les déterminants du revenu. Le montant des dépenses au titre des besoins élémentaires est fixé par la loi ; il est de $17640 \mathrm{CHF}$ pour une personne seule, ce qui équivaut à $24 \%$ du salaire moyen. La prestation complémentaire est indexée de la même façon que les pensions publiques de retraite, à savoir à $50 \%$ sur les prix et à $50 \%$ sur les salaires. Pour les retraités modestes, des compléments d'un montant discrétionnaire peuvent être décidés au niveau des cantons mais la modélisation ne les prend pas en compte.

\section{Variantes de carrière}

\section{Retraite anticipée}

Dans le régime public, le départ en retraite anticipée est possible deux ans avant l'âge normal de la retraite, c'est-à-dire à partir de 63 ans pour les hommes et de 62 ans pour les femmes, en 2005. Pour chaque année d'anticipation, la pension est réduite de $6.8 \%$ de la retraite à taux plein. Ceci équivaut à un ajustement actuariel, tel que l'opèrent d'autres pays, de $4.5 \%$ (puisque $1 / 44=2.3 \%$ de l'ajustement reflète l'année supplémentaire de cotisation).

Pour les femmes nées en 1947 ou avant, la réduction des prestations par rapport à une retraite à taux plein est de $3.4 \%$ par année d'anticipation.

Les plans professionnels autorisent le départ en retraite anticipée. Dans la pratique, ils peuvent autoriser un départ jusqu'à cinq ans avant l'âge normal mais chaque régime peut décider de sa propre politique. De façon générale, le taux de rente obligatoire de $7.1 \%$ à 65 ans ( $7.2 \%$ à 64 ans pour les femmes) est réduit de 0.2 point de pourcentage par année d'anticipation. (On notera que ce taux de conversion sera progressivement ramené à $6.8 \%$ sur la période de dix ans démarrant en 2005.) La réduction de 0.2 point de pourcentage équivaut à un ajustement actuariel, selon la mesure classique, de 2.8 à $3.1 \%$ par année d'anticipation (augmentant avec la durée d'anticipation). Si l'on tient compte également de 
la perte de cotisations et de crédits qu'entraîne le départ anticipé à la retraite, la prestation théorique est inférieure de $8 \%$ à $9.4 \%$ par année d'anticipation. Plus le départ est précoce, plus la perte augmente. (La fourchette indiquée va de 61 à 65 ans.)

\section{Retraite différée}

La retraite du régime public et les retraites professionnelles peuvent être différées audelà de l'âge normal de la retraite. Les pensions sont ajustées de la même façon que pour la retraite anticipée. La pension peut être différée jusqu'à cinq ans après l'âge normal de la retraite. Son montant est majoré selon le barême suivant :

\begin{tabular}{lccccc}
\hline Report & 1 an & 2 ans & 3 ans & 4 ans & 5 ans \\
Ajustement & $5.2 \%$ & $10.8 \%$ & $17.1 \%$ & $24.0 \%$ & $31.5 \%$ \\
\hline
\end{tabular}

Il est également possible de liquider la retraite du régime public à 65 ans et de continuer à travailler.

Pour les personnes travaillant au-delà de 65 ans, il n'est pas prélevé de cotisation si leur rémunération est inférieure à $16800 \mathrm{CHF}$ par an. Pour les rémunérations supérieures à ce niveau, des cotisations sont prélevées si les personnes diffèrent leur retraite ou si elles la liquident tout en continuant à travailler mais aucun droit supplémentaire à pension ne peut être acquis.

Dans les régimes professionnels, le taux de rente est majoré de 0.2 point de pourcentage par année de report conformément à une recommandation de l'Office fédéral des assurances sociales (les fonds de pension fixent librement les points de pourcentage). Dans la pratique, les autorités autorisent un report pouvant aller jusqu'à cinq ans.

En principe, il est possible de cumuler une retraite professionnelle et un emploi. En pratique, il s'agit principalement de personnes n'ayant pas effectué une carrière complète ou de personnes ayant pris une retraite non pas différée mais anticipée. En conséquence, la modélisation suppose que les personnes reportent la liquidation de leur retraite professionnelle si elles continuent à travailler au-delà de l'âge normal de la retraite. Les personnes cessent de cotiser au régime public après 65 ans.

\section{Enfants}

Dans le régime public, les années consacrées à l'éducation d'enfants (de moins de 16 ans) sont créditées comme si la rémunération avait été égale à trois fois la pension minimum de l'année au cours de laquelle le parent part à la retraite. Pour 2006, cette pension était de $38700 \mathrm{CHF}$, soit $53 \%$ du salaire moyen national. Si le parent est marié durant la période considérée, les crédits sont divisés à parts égales entre les conjoints.

L'octroi de crédits liés à l'éducation des enfants n'est pas imposé dans les régimes professionnels.

\section{Chômage}

Les indemnités de chômage sont assujetties au paiement des cotisations de sécurité sociale et comptabilisées comme des salaires pour le calcul de la pension publique. L'assurance chômage verse 80 \% du salaire antérieur. Les personnes sans enfant à charge, qui perçoivent une indemnité journalière totale supérieure à $140 \mathrm{CHF}$ et les personnes ne souffrant pas d'un handicap perçoivent $70 \%$ du salaire assuré. La durée de l'assurance 
chômage varie entre 260 et 520 jours. Un chômeur en fin de droits qui relève de l'aide sociale n'a pas de cotisations à payer. Si son revenu est très bas, ce sont bien souvent les autorités municipales qui payent la cotisation minimum.

Dans les plans professionnels, les périodes de chômage ne sont pas validées.

\section{Résultats de la modélisation des retraites : Suisse}
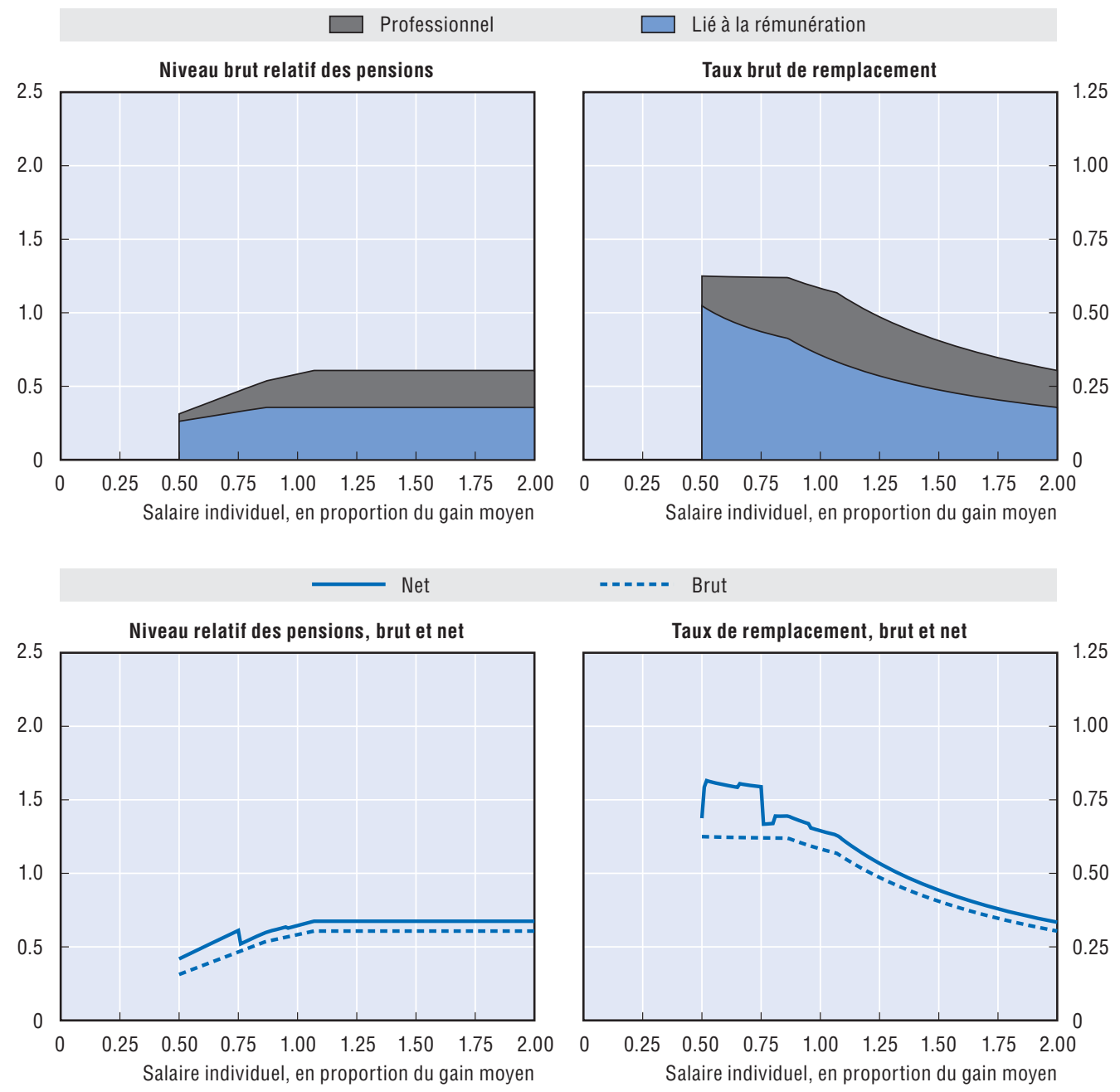

\begin{tabular}{|c|c|c|c|c|c|c|}
\hline \multirow{2}{*}{$\begin{array}{l}\text { Hommes } \\
\text { Femmes (si différent) }\end{array}$} & \multirow{2}{*}{$\begin{array}{c}\text { Salarié à revenu } \\
\text { médian }\end{array}$} & \multicolumn{5}{|c|}{ Salaire individuel, en multiple du salaire moyen } \\
\hline & & 0.5 & 0.75 & 1 & 1.5 & 2 \\
\hline Niveau brut relatif des pensions & 52.7 & 31.2 & 46.6 & 58.3 & 60.8 & 60.8 \\
\hline (\% salaire moyen brut) & 53.2 & 31.4 & 47.0 & 59.0 & 61.5 & 61.5 \\
\hline Niveau net relatif des pensions & 58.8 & 41.8 & 61.1 & 64.5 & 67.5 & 67.5 \\
\hline (\% salaire moyen net) & 59.4 & 42.0 & 51.8 & 65.3 & 68.3 & 68.3 \\
\hline Taux brut de remplacement & 62.0 & 62.5 & 62.1 & 58.3 & 40.5 & 30.4 \\
\hline (\% salaire individuel brut) & 62.6 & 62.8 & 62.6 & 59.0 & 41.0 & 30.7 \\
\hline Taux net de remplacement & 69.5 & 68.8 & 79.4 & 64.5 & 44.3 & 33.4 \\
\hline (\% salaire individuel net) & 70.2 & 69.1 & 67.3 & 65.3 & 44.9 & 33.8 \\
\hline Patrimoine retraite brut & 10.5 & 10.7 & 10.5 & 9.8 & 6.8 & 5.1 \\
\hline (multiple du salaire individuel brut) & 12.8 & 13.1 & 12.9 & 12.0 & 8.3 & 6.2 \\
\hline Patrimoine retraite net & 8.5 & 10.4 & 10.0 & 7.9 & 5.5 & 4.1 \\
\hline (multiple du salaire individuel brut) & 10.4 & 12.7 & 10.3 & 9.6 & 6.7 & 5.0 \\
\hline
\end{tabular}




\section{Turquie}

\section{Turquie : système de retraite en 2006}

Le régime public de retraite turc comporte une partie liée à la rémunération avec un filet de sécurité soumis à condition de revenu et une retraite complémentaire forfaitaire.

\section{Indicateurs essentiels}

\begin{tabular}{lrrr}
\hline & & Turquie & OCDE \\
\hline Salaire moyen & TRY & 15600 & 51200 \\
& USD & 10900 & 35800 \\
Dépenses publiques & $\%$ du PIB & 7.8 & 7.2 \\
au titre des retraites & & 71.6 & 78.9 \\
Espérance de vie & à la naissance & 79.1 & 83.4 \\
& à 65 ans & 10.4 & 23.8 \\
\hline
\end{tabular}

\section{Conditions d'ouverture des droits}

Les personnes entrées dans le système depuis septembre 1999 peuvent faire valoir leurs droits à la retraite à 60 ans pour les hommes et 58 pour les femmes sous réserve d'avoir cotisé 7000 jours, ce qui équivaut à environ 28 années de cotisation pour un emploi continu. Une autre possibilité est d'avoir 25 ans d'assurance avec 4500 jours de cotisation.

La pension soumise à conditions de ressources est payable uniquement aux personnes handicapées ne bénéficiant pas d'autres droits de sécurité sociale ou aux personnes de 65 ans et plus.

\section{Calcul des prestations}

\section{Régime lié à la rémunération}

Dans le nouveau régime, la pension est calculée sur la base des gains moyens perçus sur l'ensemble de la carrière, revalorisés en fonction de la croissance du PIB nominal. La formule de calcul n'est pas linéaire selon les années de couverture. Pour les dix premières années, le taux d'acquisition des droits est de $35 \%$ du salaire ; il augmente ensuite de $2 \%$ par an pendant les 15 années suivantes puis de $1.5 \%$ par année supplémentaire.

Les cotisations sont exigées au-delà d'un plancher qui était, en 2006, de 531 TRY.

Le salaire ouvrant droit à pension est plafonné à 3451.50 TRY.

Conformément à la loi adoptée en 1999, les pensions sont indexées sur l'indice mensuel des prix à la consommation. Mais, depuis 2003, l'indexation des pensions mises en paiement est déterminée une ou deux fois par an par les lois, budgétaires ou autres, ou par le Cabinet ministériel. Pour 2006, les pensions ont été augmentées de $3 \%$ en janvier et de $4.33 \%$ en juillet.

\section{Pension minimum}

Il existe une pension minimum qui a varié entre 463.1 et 483.1 TRY en 2006.

\section{Régime ciblé}

La pension soumise à conditions de ressources est versée sur une base trimestrielle. Pour le premier semestre 2006, elle a été de 68 TRY par mois ; pour le second semestre, elle a été de 71 TRY par mois. 


\section{Variantes de carrière}

\section{Retraite anticipée}

Les salariés de certains secteurs d'activités (secteur minier, par exemple) et les personnes souffrant d'un handicap peuvent partir en retraite anticipée mais les autres ne peuvent liquider leur retraite avant l'âge légal.

\section{Retraite différée}

Il est possible de différer la liquidation de la retraite au-delà de l'âge normal mais jusqu'en 2006 le montant des prestations n'était pas ajusté en conséquence. Depuis 2006, en revanche, l'allongement de la durée de cotisation est pris en compte.

\section{Enfants}

Les périodes d'interruption de carrière liées aux enfants ne sont pas validées.

\section{Chômage}

Les périodes de chômage ne sont pas validées. 


\section{Résultats de la modélisation des retraites : Turquie}

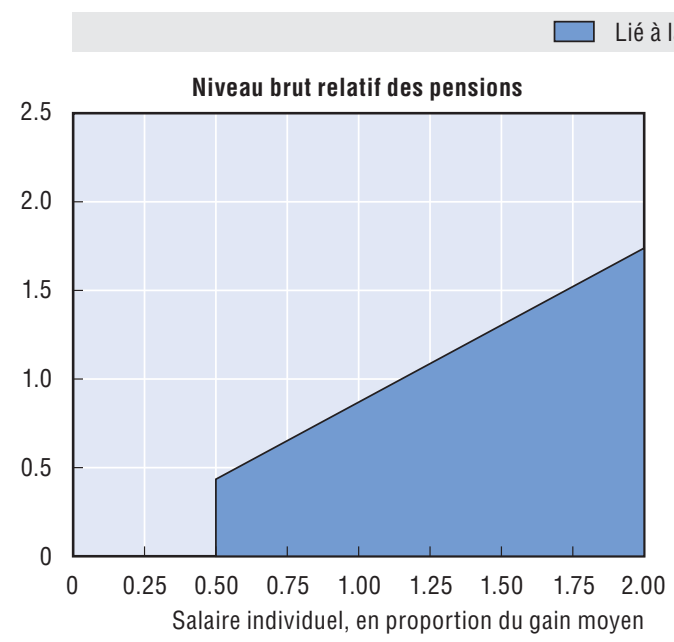

ié à la rémunération
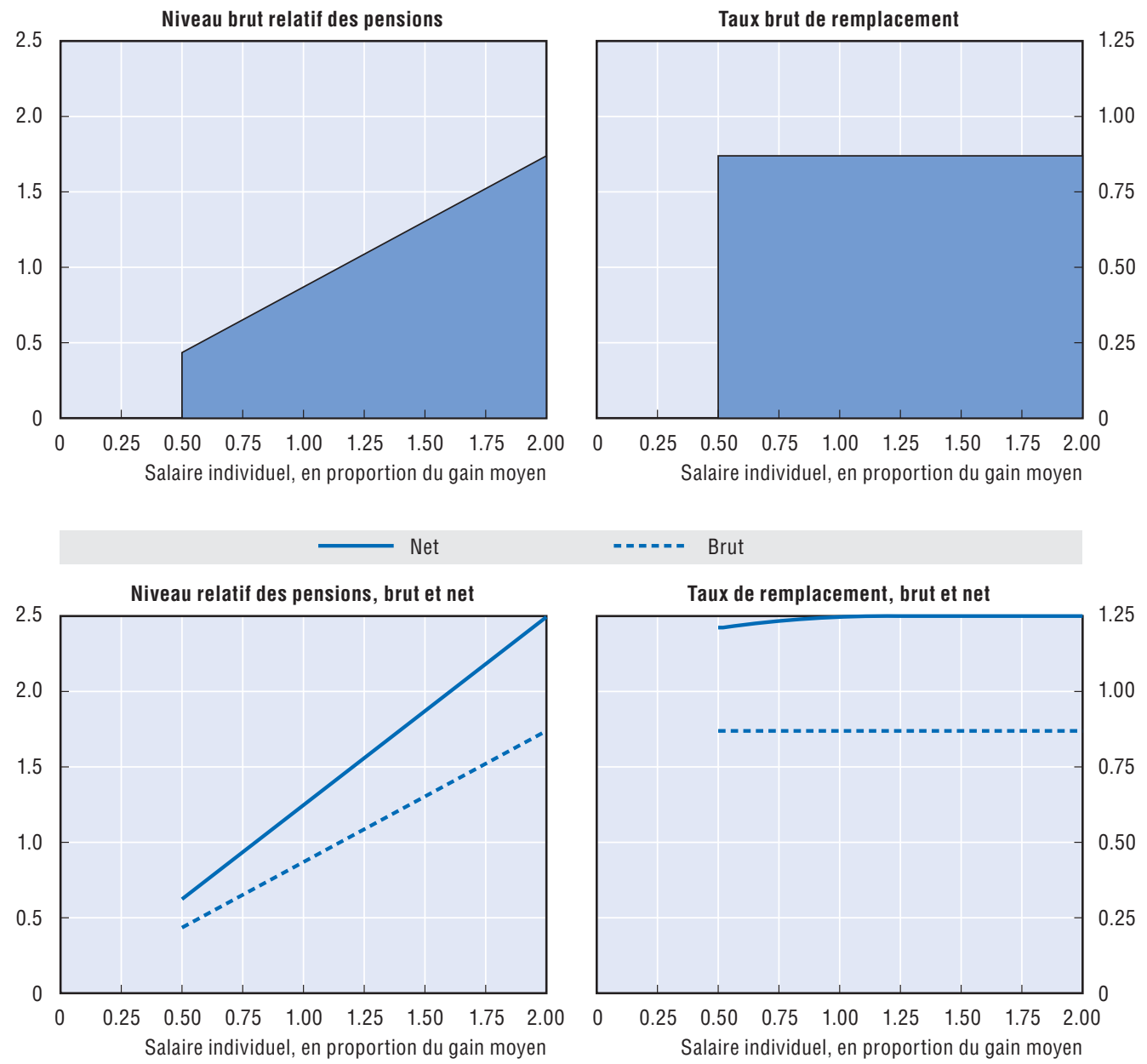

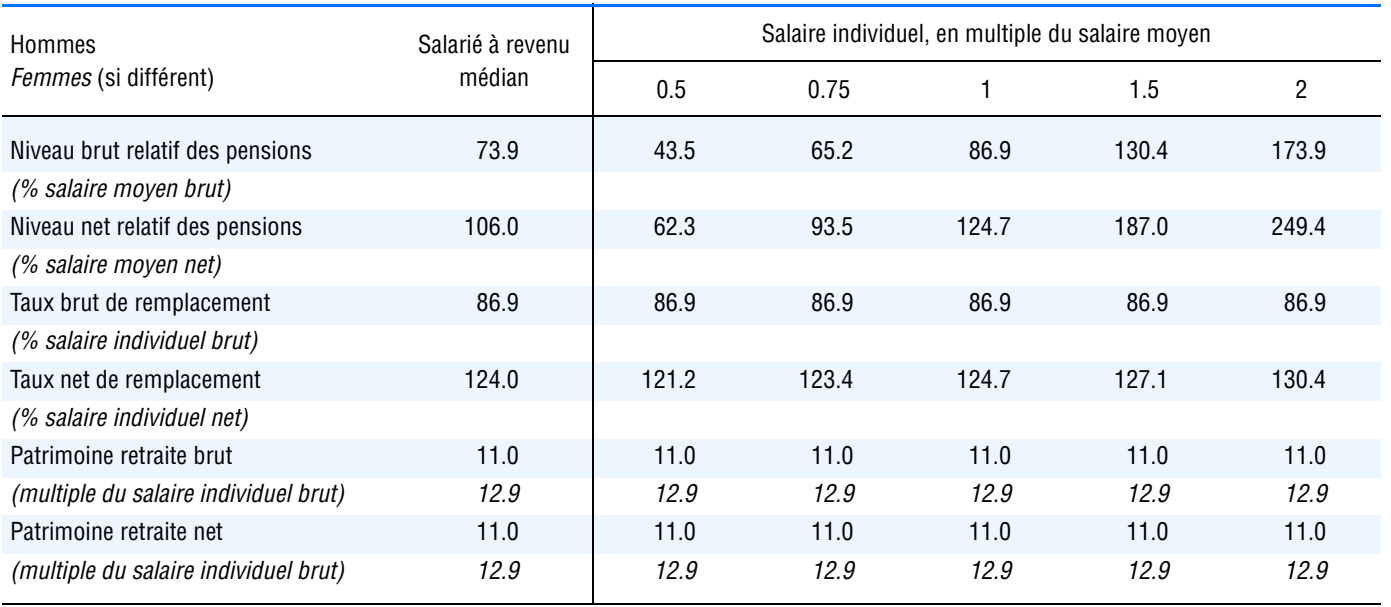


ÉDITIONS OCDE, 2, rue André-Pascal, 75775 PARIS CEDEX 16 IMPRIMÉ EN FRANCE

(81 2009082 P) ISBN 978-92-64-06085-2 - n 567862009 


\section{Les pensions dans les pays de l'OCDE 2009 PANORAMA DES SYSTÈMES DE RETRAITE}

Les politiques menées en matière de pensions et de retraite ont connu des évolutions considérables au cours des dernières années, les pouvoirs publics s'efforçant de concilier deux objectifs : assurer des revenus suffisants aux retraités et garantir la viabilité financière à long terme des systèmes de retraite face au vieillissement de la population. Les pensions dans les pays de l'OCDE 2009 propose un cadre de référence cohérent permettant de comparer les politiques adoptées dans ce domaine par les différents pays de l'OCDE, ainsi qu'un ensemble de données fiables.

Cette troisième édition renferme des informations actualisées sur les principales caractéristiques des pensions versées au sein des pays de l'OCDE et contient des projections relatives aux revenus que percevront demain les salariés d'aujourd'hui. Elle offre également une gamme élargie d'indicateurs, parmi lesquels figurent des données chiffrées concernant les actifs, les performances des investissements, l'ampleur des régimes de retraite privés, les dépenses consacrées au régime public mais aussi le contexte et les perspectives démographiques.

Quatre chapitres thématiques analysent en détail les grandes problématiques liées aux politiques aujourd'hui conduites en matière de retraite. Le premier chapitre étudie les répercussions de la crise économique et financière actuelle sur les systèmes de retraite. Quels sont les pays et les individus les plus touchés ? Quelles mesures les pouvoirs publics peuvent-ils prendre pour les aider et quelles sont les conduites à éviter? Le deuxième chapitre s'intéresse quant à lui aux revenus et à la pauvreté des personnes âgées, en analysant les tendances observées au cours des 20 dernières années. Dans de nombreux pays, la situation des retraités s'est améliorée par rapport à celle de la population prise dans son ensemble, mais il reste néanmoins des poches de pauvreté au sein de la population âgée. Le troisième chapitre contient une étude actualisée des réformes des retraites exposées dans la précédente édition des Pensions dans les pays de l'OCDE. En quoi les systèmes de retraite ont-ils changé au cours de la période 2004-08 ? Enfin, le quatrième et dernier chapitre thématique s'attache à l'ampleur des régimes facultatifs de pensions privées, en élargissant le champ d'analyse aux différences existant en fonction de l'âge et des revenus. II évalue également cinq politiques visant à développer ces systèmes.

" Un recueil extraordinairement utile et rigoureux, rassemblant des informations concernant les retraites dans un large éventail de pays, présentées de manière accessible selon une structure soigneusement étudiée. Félicitons les auteurs qui se sont efforcés de donner une nouvelle dimension aux comparaisons internationales dans ce secteur. Cet ouvrage comparatif est l'un des plus intelligents qui soient, écrit par des spécialistes qui maîtrisent les nuances - et les complexités - inhérentes à ce type de travail dans le domaine des retraites. 》

- Olivia Mitchell, Directrice du Boettner Centre for Pensions and Retirement Research, Wharton School, Université de Pennsylvanie

\section{www.oecd.org/els/social/pensions/PAG-fr}

\footnotetext{
Le texte complet de cet ouvrage est disponible en ligne aux adresses suivantes : www.sourceocde.org/finance/9789264060852 www.sourceocde.org/questionssociales/9789264060852

Les utilisateurs ayant accès à tous les ouvrages en ligne de l'OCDE peuvent également y accéder via : www.sourceocde.org/9789264060852

SourceOCDE est une bibliothèque en ligne qui a reçu plusieurs récompenses. Elle contient les livres, périodiques et bases de données statistiques de l'OCDE. Pour plus d'informations sur ce service ou pour obtenir un accès temporaire gratuit, veuillez contacter votre bibliothécaire ou SourceOECD@oecd.org.
}

éditions OCDE www.oecd.org/editions

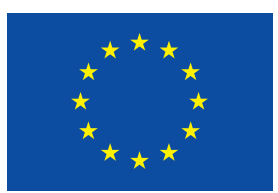

ISBN 978-92-64-06085-2

812009082 P 